MÉMOIRES DE LA SMF 90

\title{
OPTIQUE GÉOMÉTRIQUE POUR DES SYSTĖMES SEMI-LINÉAIRES AVEC INVARIANCE DE JAUGE
}

\author{
Pierre-Yves Jeanne
}

Société Mathématique de France 2002

Publié avec le concours du Centre National de la Recherche Scientifique 
P.- Y. Jeanne

Université Paris-Sud, Bât. 425, Mathématiques, 91405 Orsay cedex, France.

E-mail : pierre-yves.jeanne@math.u-psud.fr

Classification mathématique par sujets (2000). - 78A05, 81T13, 58G17, 58G35, 35L70,35Q75.

Mots clefs. - Optique géométrique, développements WKB, invariance de jauge, invariance relativiste, EDP hyperboliques non linéaires, équation de Yang-Mills, équation de Dirac, équation des ondes.

Travaux supportés par une bourse de thèse DGA/CNRS, allocation de recherches $\mathrm{n}^{\mathrm{O}} 5901798 / \mathrm{C} 16$. 


\title{
OPTIQUE GÉOMÉTRIQUE POUR DES SYSTĖMES SEMI-LINÉAIRES AVEC INVARIANCE DE JAUGE
}

\author{
Pierre-Yves Jeanne
}

Résumé. - L'objet de cet article est de justifier le recours à des méthodes de type « optique géométrique » pour une large classe de systèmes d'équations de champs semi-linéaires, invariants à la fois par transformations de Lorentz et par changements de jauge. Nous construisons explicitement des familles de solutions approchées d'un système modèle, couplant un champ de jauge (équation de Yang-Mills) avec un champ scalaire (équation des ondes) et un champ de spineurs (équation de Dirac), sous forme de développements oscillant à haute fréquence, monophasés, d'amplitude maximale. Nous justifions ensuite notre démarche en prouvant l'existence de solutions exactes, qui prolongent asymptotiquement les développements oscillants obtenus. Les potentiels de Yang-Mills, champ scalaire et champ de spineurs ainsi générés ne restent uniformément bornés que dans - respectivement $-H^{1 / 2}, H^{1 / 2}$ et $L^{2}$. L'obtention de solutions oscillantes de forte amplitude montre que le système étudié peut conserver un comportement non linéaire stable pour toute une classe de champs de très faible régularité.

\footnotetext{
Abstract (Geometric optics for gauge invariant semilinear systems)

In this article, we justify the use of "geometric optics" like methods for a large class of semilinear systems of field equations, that remain invariant under gauge and Lorentz transforms.

In a first time, we consider a model system, coupling a gauge field (Yang-Mills equation) with a scalar field (wave equation) and a spinor field (Dirac equation), and build families of approximate solutions in the shape of single phase expensions, rapidly oscillating at high frequency. Amplitude of such oscillations is maximal with regard to their frequency parameter. Expensions are given to any order.

In a second time, we give exact solutions that remain asymptotic to previous oscillatory expensions. Such solutions may be uniformly bounded only in $H^{1 / 2}, H^{1 / 2}$ and $L^{2}$ - respectively for gauge, scalar and spinor fields.

We give a stability result in the case where oscillatory solutions are obtained as high frequency perturbations of a given smooth solution of the system. It shows that the system can keep a stable nonlinear behaviour, even for very low regularity fields, when nonlinear terms usually lead to destructive interactions.
} 



\section{TABLE DES MATIÈRES}

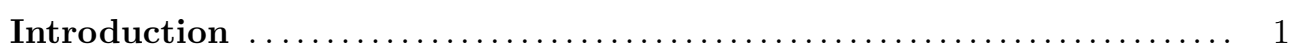

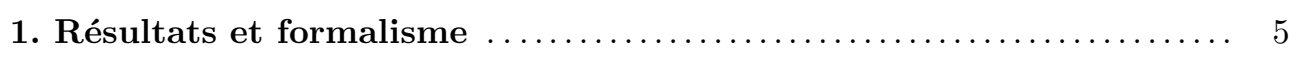

1.1. Formalisme géométrique, système modèle $(Y M) \ldots \ldots \ldots \ldots \ldots \ldots$

1.2. Présentation des résultats, problème de Cauchy oscillant pour le système

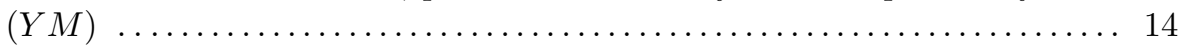

1.3. Quelques remarques préliminaires quant à la structure des équations . . . 15

2. Construction de familles de solutions approchées de $(Y M)$ sous forme de développements oscillant à haute fréquence, monophasés,

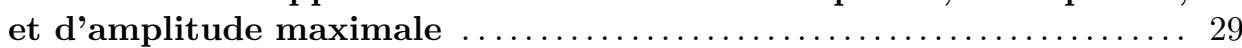

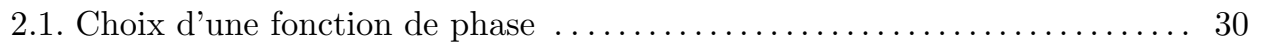

2.2. Recherche d'équations de profils à jauge libre $\ldots \ldots \ldots \ldots \ldots \ldots \ldots \ldots . \ldots 4$

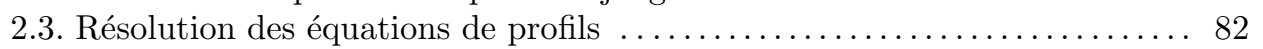

3. Prolongement asymptotique des familles de solutions approchées par

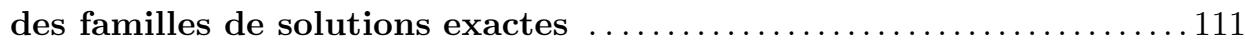

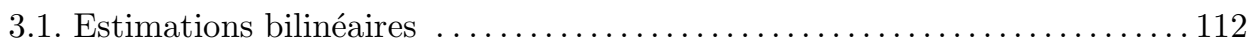

3.2. Résultat d'existence pour un système modèle $\ldots \ldots \ldots \ldots \ldots \ldots \ldots \ldots \ldots$

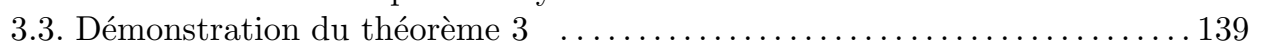

3.4. Conclusion, stabilité des solutions régulières de $(Y M)$ par perturbations

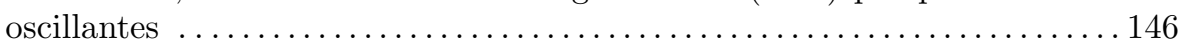

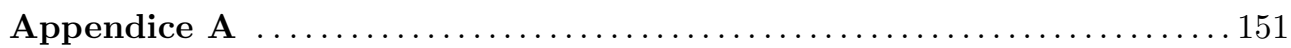

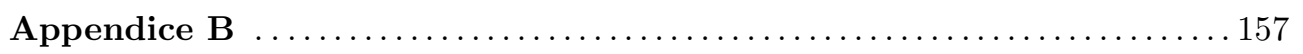

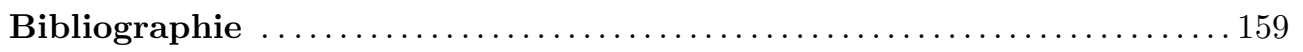





\section{INTRODUCTION}

L'objet de cet article est de justifier le recours à des méthodes de type «optique géométrique» pour une large classe de systèmes d'équations de champs semi-linéaires, invariants à la fois par transformations de Lorentz et par changements de jauge. Nous construisons explicitement des familles de solutions approchées d'un système modèle, couplant un champ de jauge (équation de Yang-Mills) avec un champ scalaire (équation des ondes) et un champ de spineurs (équation de Dirac), sous forme de développements oscillant à haute fréquence, monophasés, d'amplitude maximale. Nous justifions ensuite notre démarche en prouvant l'existence de solutions exactes, qui prolongent asymptotiquement les développements oscillants obtenus. Les potentiels de Yang-Mills, champ scalaire et champ de spineurs ainsi générés ne restent uniformément bornés que dans - respectivement - $H^{1 / 2}, H^{1 / 2}$ et $L^{2}$. L'obtention de solutions oscillantes de forte amplitude montre que le système étudié peut conserver un comportement non linéaire stable pour toute une classe de champs de très faible régularité.

Développement et justification d'une méthode de type "optique géométrique » pour des systèmes semi-linéaires présentant une invariance de jauge. — Les premières tentatives de construire des solutions oscillantes à haute fréquence pour des systèmes hyperboliques d'équations aux dérivées partielles quasi-linéaires remontent à l'article [3] d'Y. Choquet-Bruhat, en 1969. Le but à l'époque était de modifier les développements en ondes planes, utilisés avec succès dans le cas linéaire et célèbres sous le nom de développements « W.K.B. » ou d'optique géométrique, pour donner aux physiciens et aux numériciens des outils plus généraux qui permettent de calculer des développements asymptotiques formels d'équations aux dérivées partielles non linéaires, ou de modéliser les interactions qui se forment entre des «paquets d'ondes » distribués autour de fréquences données. L'article [6] s'inscrit dans ce cadre, proposant des ébauches de développements oscillants pour les équations de champs semi-linéaires avec invariance de jauge qui nous intéressent. La méthode consiste à se ramener, moyennant des 
conditions de polarisation sur certains termes oscillants, au développement d'un système d'équations d'ondes semi-linéaires. En contre partie, le développement s'arrête au premier ordre. Rien ne laisse préjuger que l'on puisse pousser ce développement à tout ordre, et encore moins qu'il existe des solutions exactes qui viendraient prolonger (et du coup justifier) les solutions asymptotiques formelles ainsi obtenues. Enfin, les oscillations envisagées restent de faible amplitude, et ne permettent pas au système de révéler toute la richesse de sa structure algébrique.

Depuis, l'intérêt des méthodes d'optique géométrique pour l'étude de systèmes d'e.d.p. non linéaires issus de lois de conservation a été considérablement renforcé, et des progrès significatifs ont été faits dans la compréhension du comportement asymptotique de perturbations oscillantes de systèmes hyperboliques semi ou quasi-linéaires. Plus qu'un simple moyen de calcul, l'optique géométrique devient un outil efficace pour étudier la propagation ou les interactions d'ondes non linéaires, dans des problèmes où aucun résultat général n'est connu. Il faut cependant attendre 1992 pour voir apparaître les premières justifications rigoureuses de développements W.K.B. monophasés pour des systèmes hyperboliques d'ordre 1 en dimension quelconque dans l'article [13] de J.L. Joly et J. Rauch pour le cas semi-linéaire, ou dans ceux d' O. Guès $[\mathbf{1 1}, \mathbf{1 0}]$ pour le cas quasi-linéaire. L'article [12] de J.L. Joly, G. Métivier et J. Rauch précise et complète ces résultats. Les développements mis aux point par Y. Choquet-Bruhat ont donc été légitimés plus de vingt ans après la parution de [3].

La première raison d'être de cet article est que les systèmes d'équations de champs semi-linéaires avec invariance de jauge envisagés dans [6] (voir aussi [4]) échappent au cadre des études précédentes. Pour cause, ce ne sont pas de vrais systèmes hyperboliques, au sens des articles sus-cités, et ils apparaissent a priori «mal déterminés »... Il est toujours possible de les résoudre sous forme de systèmes d'équations semi-linéaires strictement hyperboliques, mais c'est au prix d'une condition complémentaire sur les inconnues (nous pensons, par exemple, à la jauge de Lorentz). Une façon (optimiste) d'aborder le problème consiste donc à se ramener à un système hyperbolique semilinéaire sur-déterminé, et à le traiter comme si de rien n'était, en espérant que ses degrés de symétrie suffiront pour contourner la difficulté. Cette démarche a déjà fait ses preuve dans le cas de solutions régulières : il existe une relation algébrique sur les équations (héritée des identités de Bianchi sur le champ de jauge) garantissant que certaines «bonnes » contraintes de jauge « hyperbolisantes » se propagent automatiquement, dès lors qu'elles sont satisfaites à $t=0$ ainsi que les contraintes elliptiques de compatibilité structurelle qui pèsent sur les données de Cauchy. Quoiqu'il en soit, il n'est pas acquis que ces principes tiennent encore pour des solutions fortement oscillantes à haute fréquence, et le développement W.K.B. de tels systèmes ne peut se faire directement dans l'esprit de $[\mathbf{1 3}]$ ou $[\mathbf{1 1}, \mathbf{1 0}]$ sans reconsidérer minutieusement la structure algébrique des équations. Enfin, combien même parviendrions nous à résoudre un problème de Cauchy oscillant en s'appuyant sur une contrainte de jauge 
hyperbolisante, il faudrait s'assurer que les oscillations observées ne sont pas artificielles et ne peuvent pas être annulées par un changement de jauge oscillant de même amplitude. Il est nécessaire, à un moment ou un autre, de préciser géométriquement la façon dont se propagent les différentes composantes oscillantes du champ de jauge, en identifiant celles qui sont polarisées (partie sur-déterminée du système liée aux contraintes elliptiques sur les données initiales), libres (partie sous-déterminée du système liée à l'invariance de jauge) ou transportées le long de géodésiques nulles de l'espace-temps (partie dynamique du système se comportant de façon véritablement hyperbolique).

La seconde motivation de l'article provient des résultats d'existence de solutions peu régulières d'équations de champs à non-linéarités dites « compatibles » obtenus il-y-a quelques années par S. Klainerman et M. Machedon dans $[\mathbf{1 4}, \mathbf{1 5}]$, ou par M. Beals et M. Bézard dans [2] en combinant les effets régularisants d'estimations de type Strichartz avec la structure algébrique très particulière des termes non linéaires. Une question assez naturelle est de chercher des développements W.K.B. de solutions aussi peu régulières, et de les justifier convenablement. En fait, nous espérons utiliser l'optique géométrique pour obtenir des informations un peu plus précises sur la façon dont se comportent les solutions lorsque la régularité tombe sous le seuil critique pour lequel les termes non linéaires commencent à jouer un rôle actif. Dans ce but, nous avons cherché à construire les développements oscillants monophasées les plus singuliers possibles : si l'amplitude de ces développements est trop faible, la structure « compatible» des non-linéarités (J.L. Joly, G. Métivier et J. Rauch parlent de conditions de «transparence») fait que les oscillations restent de simples perturbations linéaires d'une solution régulière donnée et ne forment aucune interaction substantielle. Si elle est trop forte, il se forme immédiatement des interactions destructives que nous ne pouvons pas maitriser. Il faut donc trouver l'amplitude d'oscillation critique pour laquelle l'optique géométrique met en évidence de véritables effets non linéaires, tout en restant capables de prouver que les solutions oscillantes que nous construisons restent asymptotiques à des solutions exactes du système. Ce faisant, nous sommes confrontés à des difficultés nouvelles du point de vu de l'analyse et sommes obligés de démontrer des estimations bilinéaires plus fines que celles utilisées dans $[\mathbf{1 1}, \mathbf{1 0}]$.

Plan de l'article. - L'article est divisé en trois sections : la première est consacrée à l'exposition des résultats et à quelques rappels sur la structure des équations, la seconde à la construction de solutions approchées sous forme de développements oscillants monophasés d'amplitude maximale, et la dernière à l'obtention de solutions exactes prolongeant les solutions approchées.

Remerciements. - L'auteur tient particulièrement à remercier Guy Métivier et Patrick Gérard pour les entretiens fructueux qu'il a pu avoir avec eux lors la rédaction de cet article, ainsi que Nicolas Burq pour ses conseils et son dévouement quotidien. 
Il convient également de mentionner l'aide et le soutien de Max Bézard, sans qui ce travail n'aurait pu voir le jour.

La Délégation Générale pour l'Armement a supporté financièrement l'auteur par une bourse de thèse et un accueil chaleureux au sein de l'Unité de Mathématiques Appliquées de l'ENSTA, nous en sommes profondément reconnaissants. 


\section{CHAPITRE 1}

\section{RÉSULTATS ET FORMALISME}

\subsection{Formalisme géométrique, système modèle $(Y M)$}

a) Le cadre géométrique. - Nous nous plaçons dans un cadre géométrique assez favorable pour concentrer notre propos sur des questions d'analyse. Considérons un espace temps $\boldsymbol{E}$ de dimension $n+1$ (le cas qui nous intéresse est $n=3$, mais le propos reste valable pour tout entier $n \geqslant 3$ ) que nous dotons d'une métrique Lorentzienne $\boldsymbol{g}$ de signature $(+,-, \ldots,-)$. Nous supposons au moins que $(\boldsymbol{E}, \boldsymbol{g})$ est régulier $\left(C^{\infty}\right)$ et orientable, et qu'il est alors possible, ne serait-ce que localement, de définir une fonction temps $t, C^{\infty}$, qui génère un feuilletage régulier en hypersurfaces $\Sigma_{t}=\{x \in \boldsymbol{E} \mid t(x)=t\}$ de type espace (le gradient Grad $t$ de la fonction temps et le champ $N_{R}$ des vecteurs normaux unitaires aux $\Sigma_{t}$ orientés vers le futur doivent vérifier $\boldsymbol{g}(\operatorname{Grad} t, \operatorname{Grad} t) \geqslant$ Cte $>0$ et $\alpha=\boldsymbol{g}\left(N_{R}, \operatorname{Grad} t\right) \leqslant$ Cte $)$. Nous pouvons alors identifier, au moins localement, $\boldsymbol{E} \stackrel{\text { diff }}{\longleftrightarrow} \mathbb{R} \times \boldsymbol{\Sigma}$, où $\boldsymbol{\Sigma}$ est une variété de dimension $n$ difféomorphe à chaque $\boldsymbol{\Sigma}_{t}$, et donner un cadre causal à notre étude. Nous avons alors le choix entre deux hypothèses raisonnables :

(1) Nous nous contentons de travailler localement, sur un domaine causal inclus dans un ouvert où la fonction temps est bien définie, sur une tranche de temps finie. Nous évitons délibérément toute discussion sur la structure de $\Sigma$ hors d'un compact

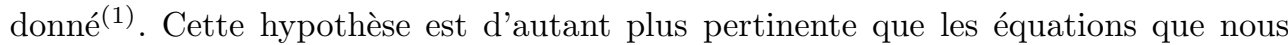
avons à résoudre respectent toutes le principe de «vitesse finie de propagation ». On peut envisager de procéder ultérieurement au recollement de solutions obtenues sur des domaines causaux adjacents. D'autre part, l'optique géométrique est d'ordinaire mise à contribution pour préciser la description de phénomènes propagatoires sur des tranches de temps courtes. Nous assimilons les solutions oscillantes que nous construisons à une perturbation locale de champs réguliers préexistants (background).

(1) En revanche, nous souhaitons conserver un maximum de généralité localement, ayant dans l'idée d'autoriser, dans des travaux ultérieurs, des oscillations de la métrique de base. 
(2) Si l'on désire vraiment travailler globalement en espace, il faut faire en plus des hypothèses d'hyperbolicité globales (La fonction temps définit une coordonnée globale, et les $\Sigma_{t}$ réalisent un feuilletage de type espace sur $\boldsymbol{E}$ tout entier. Nous supposons en particulier que $0<$ cte $\leqslant \alpha^{2}(y, t) \leqslant$ Cte uniformément pour tout $y \in \boldsymbol{\Sigma}$ et localement uniformément pour $t \in \mathbb{R}$ ) et de platitude à l'infini (à l'extérieur d'un compact donné, $\Sigma$ est difféomorphe à $\mathbb{R}^{n}$ privé d'une boule). L'important est de pouvoir disposer de bonnes estimations a priori pour l'équation des ondes et l'équation de Dirac sur toute tranche de temps finie.

Nous supposerons désormais une de ces hypothèses satisfaite. Un point de $\boldsymbol{E}$ sera naturellement désigné par $x=(t, y), t \in \mathbb{R}, y \in \boldsymbol{\Sigma}$. Lorsque nous aurons besoin de nous référer à un choix (local) de coordonnées sur $\mathbb{R} \times \boldsymbol{\Sigma}$, les indices latins $1 \leqslant i \leqslant n$ se rapporterons à $\boldsymbol{\Sigma}$ seule, les indices grecs $0 \leqslant \nu \leqslant n$ à $\mathbb{R} \times \boldsymbol{\Sigma}$ tout entier. Nous utilisons la convention de sommation habituelle sur les indices répétés. La métrique image sur $\mathbb{R} \times \boldsymbol{\Sigma}$ sera elle aussi noté $\boldsymbol{g}$. Pour tout choix de coordonnées locales $y^{i}$ sur $\Sigma$, nous utiliserons systématiquement sur $T^{*}(\mathbb{R} \times \boldsymbol{\Sigma})$ des coordonnées $\xi^{\mu}$ de la forme

$$
\xi^{0}=d t, \text { et } \xi^{i}=d y^{i}+\beta^{i} d t, i=1, \ldots, n
$$

duales aux champs de vecteurs

$$
\partial_{0}=\alpha N_{R}=\frac{\partial}{\partial t}-\beta^{i} \frac{\partial}{\partial y^{i}}, \quad \text { et } \quad \partial_{i}=\frac{\partial}{\partial y^{i}}, i=1, \ldots, n
$$

De telles coordonnées restent partout adaptées au feuilletage que nous avons fait de $\boldsymbol{E}$, et permettent d'écrire la métrique sous sa forme scindée :

$$
g(t, y)=\alpha^{2}(t, y)\left(\xi^{0}\right)^{2}+g_{i j}(t, y) \xi^{i} \xi^{j}
$$

Le scalaire $\alpha(t, y)$ et le vecteur $\beta(t, y)$ sont respectivement nommés « lapse » et «shift » $\mathrm{du}$ feuilletage. Ils correspondent à la projection du vecteur tangent à la ligne de temps respectivement sur la normale de $\Sigma_{t}$ et sur l'espace tangent à $\Sigma_{t}$ (le cas $\beta=0$ correspond à des coordonnées $y(t)$ de $\Sigma_{t}$ transportées par le flot de Grad $t$ ). Nous définissons alors $T^{*} \boldsymbol{\Sigma}=\operatorname{Vect}\left(\xi^{i}, i=1, \ldots, n\right)$, fibré cotangent à $\boldsymbol{\Sigma} \subset \mathbb{R} \times \boldsymbol{\Sigma}$. Nous posons $\boldsymbol{g}_{\Sigma}(t, y)=-g_{i j}(t, y) \xi^{i} \xi^{j}$ la métrique riemannienne induite par $\boldsymbol{g}$ sur $\Sigma$. Nous notons $g^{\nu \mu}$ les éléments de la matrice $\boldsymbol{g}^{-1}$, vérifiant $g^{\nu \mu} g_{\nu \lambda}=\delta_{\lambda}^{\mu}$. Nous utilisons la convention de « relèvement» des indices $A^{\nu}=g^{\nu \mu} A_{\nu}$ (on passe d'une 1-forme $A_{\nu} \xi^{\nu}$ à un vecteur $A^{\nu} \partial_{\nu}$ associé par la métrique). Nous utilisons systématiquement la notation

$$
T=\nabla
$$

pour désigner l'opérateur de dérivation covariante lié à la métrique $\boldsymbol{g}$ sur $\mathbb{R} \times \boldsymbol{\Sigma}$. Sur un champ de vecteur $V=V^{\nu} \partial_{\nu}$, il vient $\nabla_{\mu} V=\left(\nabla_{\mu} V^{\nu}\right) \partial_{\nu}$ avec

$$
\nabla_{\mu} V^{\nu}=\partial_{\mu} V^{\nu}+C_{\lambda \mu}^{\nu} V^{\lambda}
$$

où les coefficients de connexion sont donnés par

$$
C_{\lambda \nu}^{\mu}=\frac{1}{2} g^{\mu \gamma}\left[\partial_{\nu} g_{\lambda \gamma}+\partial_{\lambda} g_{\nu \gamma}-\partial_{\gamma} g_{\lambda \nu}\right]
$$


Pour une 0-forme $\chi$ de $\wedge^{0}(\mathbb{R} \times \boldsymbol{\Sigma}), T \chi=\nabla \chi=d \chi$, où $d$ désigne l'opérateur de dérivation extérieure. En coordonnées locales,

$$
\nabla \chi=\partial_{\nu} \chi \xi^{\nu}
$$

Pour une 1-forme $A=A_{\nu} \xi^{\nu}$, il vient $\nabla_{\mu} A=\left(\nabla_{\mu} A_{\nu}\right) \xi^{\nu}$, avec

$$
\nabla_{\mu} A_{\nu}=\partial_{\mu} A_{\nu}-C_{\mu \nu}^{\lambda} A_{\lambda}
$$

La dérivation covariante ainsi construite est faite pour vérifier $\nabla \boldsymbol{g}=0$, c'est-à-dire pour tous indices, $\nabla_{\nu} g_{\mu \lambda}=0$. Nous notons

$$
R=\nabla^{\star}
$$

l'adjoint de $\nabla$ pour la métrique $\boldsymbol{g}$. Pour une 1-forme $A=A_{\nu} \xi^{\nu}$ de $\wedge^{1}(\mathbb{R} \times \boldsymbol{\Sigma}), R A=$ $\nabla^{\star} A=d^{\star} A$ où $d^{\star}$ désigne l'adjoint de $d$ pour la métrique $\boldsymbol{g}$, soit en coordonnées locales

$$
R A=\nabla^{\nu} A_{\nu}=g^{\nu \mu} \nabla_{\nu} A_{\mu}
$$

Le d'Alembertien associé à $g$ sur $\mathbb{R} \times \boldsymbol{\Sigma}$ est défini par

$$
\square=\nabla^{\star} \nabla=R T
$$

et en coordonnées locales par

$$
\square=\nabla^{\nu} \nabla_{\nu}=\boldsymbol{g}^{\nu \mu} \nabla_{\nu} \nabla_{\mu}=\alpha^{-2}\left(\nabla_{0}\right)^{2}-g_{\Sigma}^{i j} \nabla_{i} \nabla_{j}
$$

Nous renvoyons à [5] pour une présentation générale de l'équation des ondes sur un espace temps courbe (globalement hyperbolique et asymptotiquement plat).

Considérons un groupe de Lie (compact) $\boldsymbol{G}$, que nous nommerons ici «groupe de jauge », et son algèbre de Lie $\mathfrak{g}$. Nous notons $[$,$] le crochet de Lie sur \mathfrak{g}$. Soit $\boldsymbol{P}$ un fibré principal $\left(C^{\infty}\right)$ ayant pour variété de base l'espace temps $\boldsymbol{E}$ et pour structure de groupe $\boldsymbol{G}$.

Nous appelons « connexion de Yang-Mills» une 1-forme $\boldsymbol{A}$ sur $\boldsymbol{P}$. Moyennant la donnée d'une section de référence $s$ sur $\boldsymbol{P}$, que nous désignons ensuite par le terme de «jauge », il est toujours possible d'identifier $\boldsymbol{A}$ à une 1-forme $\operatorname{de} \wedge^{1}(\boldsymbol{E})$ à valeurs dans $\mathfrak{g}$. Réciproquement, nous désignons par «condition de jauge » sur les 1-formes de $\boldsymbol{E}$ à valeurs dans $\mathfrak{g}$ toute condition permettant de se référer de façon univoque aux 1-formes sur $\boldsymbol{P}$. Un « changement de jauge » est caractérisé par la donnée d'une application $u$ de $\boldsymbol{E}$ dans $\boldsymbol{G}$. Le choix d'une jauge sur $\boldsymbol{P}$ et d'un feuilletage sur $\boldsymbol{E}$ nous permet donc de représenter sans ambiguïté une 1-forme $\boldsymbol{A}$ de $\boldsymbol{P}$ par une 1-forme $A$ de $\wedge^{1}(\mathbb{R} \times \boldsymbol{\Sigma})$ (potentiel de Yang-Mills) à valeurs dans $\mathfrak{g}$ :

$$
\boldsymbol{A}(x) \stackrel{s}{\longrightarrow} A(x) d x=A_{0}(x) \xi^{0}+A_{i}(x) \xi^{i}=A_{t}(x) d t+A_{\Sigma}(x) d y
$$

Un calcul immédiat nous donne $A_{i}=\left(A_{\Sigma}\right)_{i}$ et $A_{0}=A_{t}-\beta^{i}\left(A_{\Sigma}\right)_{i}$. Étant donné un couple de 1 -formes $a$ et $A$ à valeurs dans $\mathfrak{g}$, nous posons

$$
d_{a} A=d A+[a, A]
$$


Nous appelons « champ de Yang-Mills» une 2-forme $\Gamma$ de $\wedge^{2}(\boldsymbol{E})$ à valeurs dans $\mathfrak{g}$, correspondant à la courbure d'une connexion de Yang-Mills $\boldsymbol{A}$, et donnée par l'expression

$$
\Gamma(A)=d_{A} A=d A+[A, A]
$$

En représentation $\mathbb{R} \times \boldsymbol{\Sigma}$, la composante de $\Gamma$ conormale à $\boldsymbol{\Sigma}$ est désigné « champ électrique », par analogie avec l'électromagnétisme (cas où $\boldsymbol{G}=U(1)$ ) :

$$
\mathcal{E}_{i}(A)=\Gamma_{0 i}(A)=\nabla_{0} A_{i}-\nabla_{i} A_{0}+\left[A_{0}, A_{i}\right]
$$

Les composantes de $\Gamma$ cotangentes à $\boldsymbol{\Sigma}$ sont données par le « champ magnétique »

$$
\epsilon_{i j k} \mathcal{B}_{k}(A)=\Gamma_{i j}(A)=\nabla_{i} A_{j}-\nabla_{j} A_{i}+\left[A_{i}, A_{j}\right]
$$

La dynamique du champ de Yang-Mills peut donc être décrite, dans le cadre du feuilletage spatio-temporel $\mathbb{R} \times \boldsymbol{\Sigma}$, par la donnée du champ cotangent au feuilletage $A_{\Sigma}$ et par celle du « champ électrique ». Nous définissons l'opérateur de Yang-Mills $\boldsymbol{L}$ par

$$
\boldsymbol{L}(A)=d_{A}{ }^{\star} \Gamma(A)=d_{A}{ }^{\star} d_{A} A
$$

où l'opérateur $d_{A}{ }^{\star}$ désigne l'adjoint de $d_{A}$ pour la métrique $g$. En coordonnées locales, $\boldsymbol{L}_{\mu}(A)=\nabla^{\nu} \nabla_{\nu} A_{\mu}-\nabla^{\nu} \nabla_{\mu} A_{\nu}+2\left[A^{\nu}, \nabla_{\nu} A_{\mu}\right]-\left[A^{\nu}, \nabla_{\mu} A_{\nu}\right]-\left[A_{\mu}, \nabla^{\nu} A_{\nu}\right]+\left[A^{\nu},\left[A_{\nu}, A_{\mu}\right]\right]$ Sous l'action d'un changement de jauge $u: \mathbb{R} \times \boldsymbol{\Sigma} \rightarrow \boldsymbol{G}$,

$$
A \rightsquigarrow A^{\prime}=u^{-1} A u+u^{-1} d u
$$

tandis que $\Gamma \rightsquigarrow \Gamma^{\prime}=u^{-1} \Gamma u$, de même que $\boldsymbol{L}(A) \rightsquigarrow u^{-1} \boldsymbol{L}(A) u$. Il vient naturellement l'identité de Bianchi:

$$
d_{A}{ }^{\star} \boldsymbol{L}(A)=d_{A}{ }^{\star} d_{A}{ }^{\star} d_{A} A \equiv 0
$$

et l'identité duale :

$$
d_{A}{ }^{\star} d_{A} d_{A} \equiv 0
$$

Nous notons de même $L A=d^{\star} d A$, qui correspond à la partie linéaire de $\boldsymbol{L}(A)$. En coordonnées locales

$$
(L A)_{\mu}=\nabla^{\nu} \nabla_{\nu} A_{\mu}-\nabla^{\nu} \nabla_{\mu} A_{\nu}=\square A_{\mu}-\nabla_{\mu} \nabla^{\nu} A_{\nu}-(\mathcal{R} i c .)_{\mu}{ }^{\nu} A_{\nu}
$$

et nous réécrirons

$$
L=R T-T R-(\mathcal{R} i c .)
$$

L'opérateur $(\mathcal{R} i c .)_{\mu}{ }^{\nu}$ désigne l'opérateur de Ricci associé à la métrique $g$. Les identités (2) et (3) donnent

$$
R L A=d^{\star} d^{\star} d A=0
$$

et

$$
L T \chi=d^{\star} d d \chi=0
$$


Nous associons à $\boldsymbol{P}$ un fibré vectoriel complexe (ou réel) par le biais d'une représentation unitaire (respectivement orthogonale) $r$ de $\boldsymbol{G}$. Nous désignons par « champ scalaire » une section $\phi$ de ce fibré. Nous représentons un tel champs $\phi$ par une 0-forme de $\mathbb{R} \times \boldsymbol{\Sigma}$ à valeurs dans $\mathbb{C}^{J}\left(\right.$ ou $\mathbb{R}^{J}$ ). Nous notons $d_{A} \phi=d \phi+r^{\prime}(1) \cdot A \phi$. Remarquons que pour une 0 -forme, $d^{\star} d \phi=\square \phi$. Nous noterons $\bar{\phi}={ }^{t} \phi^{*}$ le conjugué hermitien de $\phi$ (ou le transposé dans le cas réel). Nous posons $|\phi|=(\bar{\phi} \phi)^{1 / 2}$.

Nous supposons qu'il existe sur $\boldsymbol{E}$ une structure spinorielle globale, i.e. un fibré $\boldsymbol{S} \boldsymbol{E}$ ayant pour base $\boldsymbol{E}$ et pour structure de groupe $\operatorname{Spin}(n+1)$ (en dimension $\mathrm{n}=3$, ceci découle directement de l'hypothèse d'hyperbolicité globale, consulter par exemple [9] pour une description précise des espaces temps globalement hyperboliques de dimension $3+1$ et de leur structure spinorielle). Un «champ de spineurs » est une section $\psi$ d'un fibré vectoriel sur $\boldsymbol{E}$ dont les fibres sont de la forme $\mathbb{C}^{K} \times \mathbb{C}^{L}$, où $\mathbb{C}^{K}$ $\left(K=2^{(n+1) / 2}\right.$ si $n$ est impair) correspond à la représentation canonique du groupe $\operatorname{Spin}(n+1)$, tandis que $\mathbb{C}^{L}$ correspond à une représentation unitaire $\rho$ de $\boldsymbol{G}$. Nous représentons un tel champ par une 0 -forme sur $\mathbb{R} \times \boldsymbol{\Sigma}$ à valeurs dans $\mathbb{C}^{K} \times \mathbb{C}^{L}$. Nous considérons des matrices de Dirac $\gamma^{\nu}, \nu=0,1, \ldots, n$, vérifiant dans toutes coordonnées locales :

$$
\gamma^{\nu} \gamma^{\mu}+\gamma^{\mu} \gamma^{\nu}=2 g^{\nu \mu} \operatorname{Id}_{K}
$$

L'opérateur de Dirac se définit alors par

$$
\mathcal{D}=\gamma^{\nu} \nabla_{\nu} \quad \text { et } \quad \mathcal{D}_{A}=\mathcal{D}+\gamma^{\nu} \rho^{\prime}(1) \cdot A_{\nu}
$$

Compte tenu de (7), nous vérifions immédiatement que

$$
\begin{aligned}
\mathcal{D}^{2} & =\gamma^{\mu} \nabla_{\mu} \gamma^{\nu} \nabla_{\nu} \\
& =\square \operatorname{Id}_{K}+\frac{1}{4} \operatorname{tr}(\mathcal{R} i c .)
\end{aligned}
$$

où $\operatorname{tr}(\mathcal{R} i c)=.(\mathcal{R} i c .)^{\nu}{ }_{\nu}$ désigne la courbure scalaire (en d'autres termes, l'opérateur de Dirac est construit, à un terme de courbure près, comme une « racine carrée »du d'Alembertien). L'adjoint d'un champ $\psi$ est donné par $\bar{\psi}={ }^{t} \psi^{*} \kappa$ où $\kappa$ est la matrice $K \times K$ vérifiant $\kappa \gamma^{\mu} \kappa^{-1}={ }^{t}\left(\gamma^{\mu}\right)^{*}$. Nous posons $|\psi|^{2}={ }^{t} \psi^{*} \psi$. Si l'on travaille avec des coordonnées adaptées au feuilletage $\mathbb{R} \times \boldsymbol{\Sigma}$ vérifiant (1), il est intéressant de fixer $\kappa=\alpha \gamma^{0}$. Les conditions (7) deviennent alors

$$
{ }^{t}\left(\gamma^{0}\right)^{*}=\gamma^{0}=\alpha^{-2}\left(\gamma^{0}\right)^{-1} \text { et }\left\{\begin{array}{l}
{ }^{t}\left(\gamma^{i}\right)^{*}=-\gamma^{i} \\
\gamma^{i} \gamma^{j}+\gamma^{j} \gamma^{i}=2 g^{i j} \operatorname{Id}_{K}
\end{array}\right.
$$

Pour un tel choix de matrices de Dirac, adapté au feuilletage $\mathbb{R} \times \boldsymbol{\Sigma}$, il vient

$$
\mathcal{D}=\gamma^{0} \nabla_{0}-\mathcal{D}_{\Sigma}
$$

où $\mathcal{D}_{\Sigma}=-\gamma^{i} \nabla_{i}$ vérifie $\mathcal{D}_{\Sigma}^{2}=g_{\Sigma}^{i j} \nabla_{i} \nabla_{j} \operatorname{Id}_{K}+\frac{1}{4} \operatorname{tr}(\mathcal{R} i c . \mid \Sigma)$, le terme $\operatorname{tr}(\mathcal{R} i c . \mid \Sigma)$ correspondant à la courbure scalaire de $\Sigma$. Nous conservons ce choix de matrices de Dirac 
dans le reste de l'article, et renvoyons à [16] pour une étude plus détaillée de l'opérateur de Dirac sur un espace temps globalement hyperbolique et asymptotiquement plat.

b) Les équations. - Le potentiel de Yang-Mills, le champ scalaire et le champ de spineurs sont couplés entre eux par les équations d'Euler Lagrange associées à un Lagrangien $\Lambda$ invariant par transformations de Lorentz (symétrie externe) et par changements de jauge sur $A$ (symétrie interne) :

$$
\Lambda=\Gamma^{\nu \mu}(A) \Gamma_{\nu \mu}(A)+\left(d_{A} \bar{\phi}\right)^{\nu}\left(d_{A} \phi\right)_{\nu}+i\left(\bar{\psi}\left(\mathcal{D}_{A} \psi\right)-\left(\mathcal{D}_{A} \bar{\psi}\right) \psi\right)+\Lambda_{\text {int. }}(\phi, \psi)
$$

En accord avec [4], nous retiendrons pour le terme de couplage :

$$
\Lambda_{\text {int. }}=\bar{\psi} \mathbb{C} \psi \phi+\overline{\phi \psi}^{t} \mathbb{C}^{*} \psi+\boldsymbol{c} \bar{\phi} \phi
$$

où l'invariance de jauge du couplage est garantie par la relation

$$
{ }^{t} \rho^{\prime}(1)^{*} \mathbb{C}+\mathbb{C} \rho^{\prime}(1)+\mathbb{C} r^{\prime}(1)=0
$$

(ceci autorise un couplage nul). Les équations sont semi-linéaires d'ordre 2 pour le potentiel de Yang-Mills $A$ et le champ scalaire $\phi$, d'ordre 1 pour le champ de spineurs $\psi$, et sont elles aussi invariantes par transformations de Lorentz ou par changements de jauge :

$$
\left\{\begin{aligned}
d_{A}{ }^{\star} d_{A} A & =\mathcal{J} \\
d_{A}{ }^{\star} d_{A} \phi & =\mathcal{C} \\
\mathcal{D}_{A} \psi & =\mathcal{K}
\end{aligned}\right.
$$

Le terme de courant

$$
\mathcal{J}^{\mu}=i \bar{\psi} \gamma^{\mu} \rho^{\prime}(1) \psi+\left(\bar{\phi}^{t} r^{\prime}(1)^{*}\left(d_{A} \phi\right)^{\mu}+\left(d_{A} \bar{\phi}\right)^{\mu} r^{\prime}(1) \phi\right)
$$

doit être compatible avec l'identité de Bianchi : $d_{A}{ }^{\star} \mathcal{J}=0$, et sous l'action d'un changement de jauge $\mathrm{u}: \mathcal{J} \rightsquigarrow \mathcal{J}^{\prime}=u^{-1} \mathcal{J} u$. Les termes

$$
\mathcal{C}=-\bar{\psi}^{t} \mathbb{C}^{*} \psi-\boldsymbol{c}(\bar{\phi} \phi) \phi \quad \text { et } \quad \mathcal{K}=i\left(\mathbb{C} \phi+\bar{\phi}^{t} \mathbb{C}^{*}\right) \psi
$$

correspondent à un couplage éventuel entre $\phi$ et $\psi$. Le lecteur intéressé trouvera le détail de divers couplages physiquement pertinents dans [4], qui donne par ailleurs des résultats d'existence de solutions régulières pour (Y.M.).

En séparant les termes linéaires et non linéaires, nous nous ramenons à étudier un système schématique de la forme :

$$
\left\{\begin{aligned}
L A & =F(A, \partial A, \phi, \partial \phi, \psi) \\
\square \phi & =G(A, \partial A, \phi, \partial \phi, \psi) \\
\mathcal{D} \psi & =h(A, \phi, \psi)
\end{aligned}\right.
$$

Pour récapituler, la partie linéaire est donnée par les opérateurs

$$
(L A)_{\mu}=\nabla^{\nu} \nabla_{\nu} A_{\mu}-\nabla^{\nu} \nabla_{\mu} A_{\nu}, \square \phi=\nabla^{\nu} \nabla_{\nu} \phi \text { et } \mathcal{D} \psi=\gamma^{\nu} \nabla_{\nu} \psi
$$


La partie non linéaire est donnée par des fonctions

et

$$
\begin{aligned}
& F(A, \partial A, \phi, \partial \phi, \psi)=F^{I}(A, \partial A)+F^{I I}(\phi, \partial \phi)+f(A, \phi, \psi) \\
& G(A, \partial A, \phi, \partial \phi, \psi)=G^{I}(A, \partial \phi)+G^{I I}(\partial A, \phi)+g(A, \phi, \psi)
\end{aligned}
$$

où

$$
\begin{aligned}
F^{I}(A, \partial A)_{\mu} & =2\left[\nabla_{\nu} A_{\mu}, A^{\nu}\right]+\left[A^{\nu}, \nabla_{\mu} A_{\nu}\right]+\left[A_{\mu}, \nabla^{\nu} A_{\nu}\right] \\
F^{I I}(\phi, \partial \phi)_{\mu} & =\bar{\phi}^{t} r^{\prime}(1)^{*}\left(\nabla_{\mu} \phi\right)+\left(\nabla_{\mu} \bar{\phi}\right) r^{\prime}(1) \phi \\
f(A, \phi, \psi) & =\left[\left[A_{\nu}, A_{\mu}\right], A^{\nu}\right]+\bar{\phi}^{t} r^{\prime}(1)^{*}\left(r^{\prime}(1) \cdot A_{\mu}\right) \phi+\left(r^{\prime}(1) \cdot A_{\mu}\right) \bar{\phi} r^{\prime}(1) \phi \\
& +i \bar{\psi} \gamma_{\mu} \rho^{\prime}(1) \psi \\
G^{I}(A, \partial \phi) & =-2 r^{\prime}(1) \cdot A_{\nu} \nabla^{\nu} \phi \\
G^{I I}(\partial A, \phi) & =-r^{\prime}(1) \cdot \nabla^{\nu} A_{\nu} \phi \\
g(A, \phi, \psi) & =-\left(r^{\prime}(1) \cdot A^{\nu}\right)\left(r^{\prime}(1) \cdot A_{\nu}\right) \phi-\bar{\psi}^{t} \mathbb{C}^{*} \psi-c(\bar{\phi} \phi) \phi
\end{aligned}
$$

et

$$
h(A, \phi, \psi)=-\rho^{\prime}(1) \cdot A_{\nu} \gamma^{\nu} \psi+i\left(\mathbb{C} \phi+\bar{\phi}^{t} \mathbb{C}^{*}\right) \psi
$$

Les fonctions $F^{I}, F^{I I}, G^{I}$ et $G^{I I}$ sont bilinéaires à coefficients $C^{\infty}$. Les fonctions $f(A, \phi, \psi)$ et $g(A, \phi, \psi)$ sont des polynômes de degré 3 à coefficients $C^{\infty}$, et de degré au plus 2 en $\psi$. Enfin le terme $h(A, \phi, \psi)$ est un polynôme de degré 3 à coefficients $C^{\infty}$, et de degré 1 en $\psi$. Avec les identités (2), (3) et (5), il s'agit des seules hypothèses de structure dont nous avons réellement besoin pour l'analyse des équations.

Ainsi formulé, (Y.M.) est représentatif des équations « à jauge libre » : la donnée d'un potentiel de Yang-Mills $A$ vérifiant le système engendre toute une classe de solutions par changement de jauge. Sous l'action d'un tel changement de jauge caractérisé par une application $u: \mathbb{R} \times \boldsymbol{\Sigma} \rightarrow \boldsymbol{G}$, nous transformons

$$
\begin{aligned}
& A \rightsquigarrow A^{\prime}=u^{-1} A u+u^{-1} d u \\
& \phi \rightsquigarrow \phi^{\prime}=r(u) \phi \\
& \psi \rightsquigarrow \psi^{\prime}=\rho(u) \psi
\end{aligned}
$$

L'invariance de jauge des équations se traduit par :

$$
\left\{\begin{aligned}
L A^{\prime}-F\left(A^{\prime}, \partial A^{\prime}, \phi^{\prime}, \partial \phi^{\prime}, \psi^{\prime}\right) & =u^{-1}(L A-F(A, \partial A, \phi, \partial \phi, \psi)) u \\
\square \phi^{\prime}-G\left(A^{\prime}, \partial A^{\prime}, \phi^{\prime}, \partial \phi^{\prime}, \psi^{\prime}\right) & =r(u)(\square \phi-G(A, \partial A, \phi, \partial \phi, \psi)) \\
\mathcal{D} \psi^{\prime}-h\left(A^{\prime}, \phi^{\prime}, \psi^{\prime}\right) & =\rho(u)(\mathcal{D} \psi-h(A, \phi, \psi))
\end{aligned}\right.
$$

On lève l'indétermination sur le potentiel $A$ en se donnant une condition de jauge, qui apparaît sous la forme d'une équation supplémentaire $J(A)=0$, où $J$ s'identifie d'ordinaire à un opérateur d'ordre 1. Chaque solution du système (YM) à jauge fixée désigne de façon univoque une classe de solutions du système à jauge libre.

Ces propriétés d'invariance par changements de jauge font que le système (YM) semble a priori « mal » déterminé : 
- sous-déterminé à jauge libre,

- sur-déterminé à jauge fixée. Compte tenu de cette «mauvaise » détermination, le système (YM) s'apparente plus à un système quasi-linéaire qu'à un vrai système semi-linéaire. Toute la difficulté consiste à se placer dans des situations où il est techniquement possible de quotienter l'action du groupe de jauge sur les équations, et redonner au système son aspect hyperbolique semi-linéaire « bien déterminé ».

La famille suivante est représentative ${ }^{(2)}$ des conditions de jauge couramment utilisées pour étudier le problème de Cauchy sur le système (YM) :

$$
J_{\lambda}(A)=\lambda \alpha^{-2} \nabla_{0} A_{0}-(1-\lambda) g_{\Sigma}^{i j} \nabla_{i} A_{\Sigma_{j}}=0
$$

où $\lambda$ est un réel pris entre 0 et 1 . On trouve aux extrémités la jauge de Coulomb pour $\lambda=0$ et la jauge temporelle pour $\lambda=1$. Les résultats d'existence de solutions peu régulières d'énergie bornée obtenus dans $[\mathbf{1 4}, \mathbf{1 5}]$ (pour Yang-Mills seul ou pour le système plus simple de Maxwell-Klein-Gordon) utilisent la jauge de Coulomb pour mettre en valeur la structure algébrique des non-linéarités du second membre. L'inconvénient de la jauge de Coulomb est qu'elle scinde le système en une partie elliptique et une partie hyperbolique, et demeure en définitive peu naturelle. En revanche, lorsque $\lambda \in] 0,1\left[\right.$, la condition de jauge $J_{\lambda}(A)=0$ est transverse à $\boldsymbol{T}^{*} \boldsymbol{\Sigma}$, demeure invariante par changement de coordonnées locales sur $\boldsymbol{\Sigma}$, et semble mieux adapté à la résolution du problème de Cauchy avec des données initiales sur $\boldsymbol{\Sigma}$. L'opérateur $L$ se transforme alors en un opérateur hyperbolique symétrisable, auquel on peut espérer appliquer des méthodes d'optique géométrique traditionnelles.

Enfin, on appelle jauge de Lorentz la condition médiane $J_{\frac{1}{2}}(A)=\frac{1}{2} R A=0$. Si $A$ vérifie la jauge de Lorentz, alors $L A=(\square-\mathcal{R} i c$. $) A$, et apparaît sous forme d'un opérateur diagonal strictement hyperbolique. De plus la condition $R A=0$ est invariante par changements de coordonnées sur $\boldsymbol{E}$, et donc indépendante du feuilletage causal choisi pour résoudre le problème de Cauchy (ce n'est en général pas le cas des autres conditions $\left.J_{\lambda}, \lambda \in\left[0, \frac{1}{2}[\cup] \frac{1}{2}, 1\right]\right)$. Le gros inconvénient de cette jauge, du point de vue de l'analyse, est qu'elle semble occulter une partie de la régularité des solutions.

Nous cherchons aussi longtemps que possible à raisonner «à jauge près », afin de souligner le caractère arbitraire et technique du choix de jauge. Le moment venu, c'est avec la jauge de Lorentz que nous prouvons les résultats d'existence qui nécessitent le choix d'une condition de jauge. Deux raisons à cela. D'une part, du point de vue de l'analyse, nous choisissons de nous référer uniquement au sens strict de la notion d'hyperbolicité, pour lesquels les résultats d'optique géométrique sont simples et intuitifs. D'autre part, d'un point de vue géométrique, nous évitons toute ambiguïté liée au choix des coordonnées, ce qui semble important vu le cadre relativiste sous-jacent à

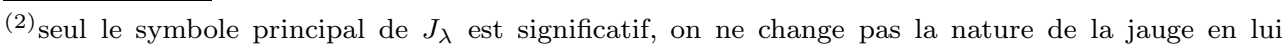
ajoutant un second membre régulier où en modifiant sa partie linéaire d'ordre 0 . 
notre étude ${ }^{(3)}$. En contrepartie, nous montrons comment les résultats obtenus en jauge de Lorentz peuvent être traduits dans n'importe quelle autre jauge $J_{\lambda}$. Nous observons au passage comment l'hyperbolicité du système s'affaiblit pour ne plus concerner que les variables véritablement dynamiques du champ. Nous voyons alors réapparaître la régularité masquée par la jauge de Lorentz. Cette démarche n'est possible qu'en privilégiant par avance une direction isotrope de propagation des singularités (rayons de lumière : c'est le principe même de l'optique géométrique).

c) Les espaces fonctionnels utilisés. - Nous prenons comme Laplacien $\triangle=$ $g_{\Sigma}^{i j} \nabla_{i} \nabla_{j}$. Pour tout réel $s$, nous désignons par $H^{s}(\Sigma)$ l'espace de Sobolev $W^{s, 2}(\Sigma)=$ $(1-\triangle)^{-s / 2} L^{2}(\Sigma)$ (les normes dépendent bien entendu du temps, mais tant que cette dépendance reste $C^{\infty}$, que nous travaillons sur un domaine causal compact ou bien sur un intervalle de temps borné avec bonnes hypothèses à l'infini sur $\boldsymbol{\Sigma}$, elles restent équivalentes). Nous utilisons la notation $\langle D\rangle^{s}=(1-\triangle)^{s / 2}$.

Nous désignerons par $(u)_{\varepsilon}$ toute famille de fonctions $u_{\varepsilon}$ dépendant d'un paramètre réel $\varepsilon, 0<\varepsilon \leqslant \varepsilon_{0}<1$, ayant vocation à devenir arbitrairement petit.

DÉFinition 1.1.1. - Pour tout réels $s$, nous notons $\mathcal{H}_{\varepsilon}^{s}(\Sigma)$ l'ensemble des familles $(u)_{\varepsilon}$ de $H^{s}(\Sigma)$ dépendant du paramètre $\left.\left.\varepsilon \in\right] 0, \varepsilon_{0}\right]$ et dont la norme $H^{s}$ est uniformément bornée en $\varepsilon$. La norme associée est donnée par :

$$
\left|u_{\varepsilon}\right|_{\mathcal{H}_{\varepsilon}^{s}}=\sup _{0<\varepsilon \leqslant \varepsilon_{0}}\left|\langle D\rangle^{s} u_{\varepsilon}\right|_{L^{2}(\Sigma)}
$$

Par la suite, nous nous intéresserons à des familles $(u)_{\varepsilon}$ oscillantes, dont les singularités restent essentiellement conormales à une surface de phase donnée lorsque $\varepsilon$ devient petit. Pour tout entier $m \in \mathbb{N}$ positif, nous dirons qu'une famille $(u)_{\varepsilon} \in \mathcal{H}_{\varepsilon}^{m / 2}(\Sigma)$ admet un développement oscillant de phase $\theta / \varepsilon$ à l'ordre $\varepsilon^{M / 2}$ s'il existe une fonction régulière $u_{0}(y)$ ainsi qu'une suite de fonctions régulières $u_{i}(y, \omega), m \leqslant i<M$, $2 \pi$-périodiques en la variable $\omega$ et un reste $(r)_{\varepsilon} \in \mathcal{H}_{\varepsilon}^{m / 2}(\Sigma)$ tels que :

$$
u_{\varepsilon}(y)=u_{0}(y)+\sum_{i=m}^{M-1} \varepsilon^{i / 2} u_{i}\left(y, \frac{\theta(y)}{\varepsilon}\right)+\varepsilon^{M / 2} r_{\varepsilon}(y)
$$

Pour tout entier $m$ négatif, nous dirons qu'une famille $(u)_{\varepsilon} \in \mathcal{H}_{\varepsilon}^{m / 2}(\Sigma)$ admet un développement oscillant de phase $\theta / \varepsilon$ à l'ordre $\varepsilon^{M / 2}$ s'il existe une suite de fonctions régulières $\stackrel{\star}{u}_{i}(y, \omega), m \leqslant i<0,2 \pi$-périodiques de moyenne nulle en $\omega$ sur toute période de longueur $2 \pi$, ainsi qu'une suite de fonctions régulières $u_{i}(x, \omega), 0 \leqslant i<M$,

(3) il convient de rappeler que les changements de coordonnées sont assimilables à des changements de jauge sur la métrique $\boldsymbol{g}$, qui se superposent aux changements de jauge sur les connexions de Yang-Mills 
$2 \pi$-périodiques en $\omega$ et reste $(r)_{\varepsilon} \in \mathcal{H}_{\varepsilon}^{m / 2}(\Sigma)$ tels que :

$$
u_{\varepsilon}(y)=\sum_{i=m}^{-1} \varepsilon^{i / 2} \stackrel{\star}{u}_{i}\left(y, \frac{\theta(y)}{\varepsilon}\right)+\sum_{i=0}^{M-1} \varepsilon^{i / 2} u_{i}\left(y, \frac{\theta(y)}{\varepsilon}\right)+\varepsilon^{M / 2} r_{\varepsilon}(y)
$$

\subsection{Présentation des résultats, problème de Cauchy oscillant pour le sys- tème $(Y M)$}

L'ensemble de nos résultats se résume dans l'énoncé suivant

THÉORÈme 1. - Le problème de Cauchy oscillant sur $(Y M)$ est bien posé localement en temps dans $\mathcal{H}_{\varepsilon}^{1 / 2} \times \mathcal{H}_{\varepsilon}^{1 / 2} \times \mathcal{H}_{\varepsilon}^{0}(\Sigma)$ :

Pour tout choix de phase $\theta$ non-dégénérée vérifiant les hypothèses 2.1.1 et engendrant un feuilletage régulier d'un domaine $\left[0, t_{0}\right] \times \Sigma$ en hypersurfaces de type nul, pour toutes données initiales

$$
(A, \phi, \psi)_{\varepsilon}(0, y) \in \mathcal{H}_{\varepsilon}^{1 / 2} \times \mathcal{H}_{\varepsilon}^{1 / 2} \times \mathcal{H}_{\varepsilon}^{0}(\Sigma) \text { et }\left(\partial_{t} A, \partial_{t} \phi\right)_{\varepsilon}(0, y) \in \mathcal{H}_{\varepsilon}^{-1 / 2} \times \mathcal{H}_{\varepsilon}^{-1 / 2}(\Sigma)
$$

admettant un développement oscillant de phase $\theta / \varepsilon$ à l'ordre $\varepsilon^{M / 2}, M \geqslant n$, satisfaisant les hypothèses de compatibilité( $e^{(4)} 3.4 .1$ et traduites dans une jauge $J_{\lambda}, \lambda \in[0,1]$ arbitraire, il existe un temps $0<t_{1} \leqslant t_{0}$, et un unique prolongement

$(A, \phi, \psi)_{\varepsilon} \in C^{0}\left(\left[0, t_{1}\right], \mathcal{H}_{\varepsilon}^{1 / 2} \times \mathcal{H}_{\varepsilon}^{1 / 2} \times \mathcal{H}_{\varepsilon}^{0}(\Sigma)\right) \cap C^{1}\left(\left[0, t_{1}\right], \mathcal{H}_{\varepsilon}^{-1 / 2} \times \mathcal{H}_{\varepsilon}^{-1 / 2} \times \mathcal{H}_{\varepsilon}^{-1}(\Sigma)\right)$ solution exacte de $(Y M)$ dans la même jauge $J_{\lambda}$, qui garde la forme d'un développement oscillant de même ordre :

$$
\left\{\begin{array}{l}
A_{\varepsilon}(x)=A_{0}(x)+\sum_{i=1}^{M-1} \varepsilon^{i / 2} A_{i}\left(x, \frac{\theta(x)}{\varepsilon}\right)+\varepsilon^{M / 2} Z_{\varepsilon}(x) \\
\phi_{\varepsilon}(x)=\phi_{0}(x)+\sum_{i=1}^{M-1} \varepsilon^{i / 2} \phi_{i}\left(x, \frac{\theta(x)}{\varepsilon}\right)+\varepsilon^{M / 2} z_{\varepsilon}(x) \\
\psi_{\varepsilon}(x)=\sum_{i=0}^{M-1} \varepsilon^{i / 2} \psi_{i}\left(x, \frac{\theta(x)}{\varepsilon}\right)+\varepsilon^{M / 2} \zeta_{\varepsilon}(x)
\end{array}\right.
$$

où les profils d'oscillation $\left(A_{i}, \phi_{i}, \psi_{i}\right)(x, \omega)$ sont des fonctions $2 \pi$-périodiques en la variable $\omega$, à l'exception de $A_{0}$ et $\phi_{0}$ qui restent indépendants de $\omega$. Le reste est petit devant les autres termes du développement:

$$
(Z, z, \zeta)_{\varepsilon} \in C^{0}\left(\left[0, t_{0}\right], \mathcal{H}_{\varepsilon}^{1 / 2} \times \mathcal{H}_{\varepsilon}^{1 / 2} \times \mathcal{H}_{\varepsilon}^{0}(\Sigma)\right) \cap C^{1}\left(\left[0, t_{0}\right], \mathcal{H}_{\varepsilon}^{-1 / 2} \times \mathcal{H}_{\varepsilon}^{-1 / 2} \times \mathcal{H}_{\varepsilon}^{-1}(\Sigma)\right)
$$

\footnotetext{
${ }^{(4)}$ Ces hypothèses de compatibilité traduisent en termes d'oscillations les contraintes structurelles qui pèsent sur toutes données de Cauchy du système $(Y M)$. Nous rappelons dans la section suivante que toutes les composantes d'un potentiel de Yang-Mills ne sont pas dynamiques, certaines peuvent être fixées librement (invariance de jauge), les autres sont alors propagées de façon hyperbolique ou imposées par des contraintes elliptiques. À $t=0$, ces contraintes elliptiques supposent que des données initiales oscillantes soient correctement polarisées, c'est l'objet des hypothèses 3.4.1.
} 
Pour prouver le théorème 1 , nous employons une méthode de type optique géométrique prenant en compte l'invariance de jauge des équations. Nous construisons explicitement des solutions approchées du système $(Y M)$, sous forme de développements oscillant à haute fréquence, monophasés et d'amplitude maximale. Nous précisons les hypothèses requises sur la phase, détaillons la géométrie des oscillations et précisons le choix des données initiales oscillantes compatibles avec la phase. Le théorème 2, section 2.3.1, rassemble les résultats d'approximation.

Nous montrons ensuite l'existence de familles de solutions exactes, prolongeant asymptotiquement les développements obtenus, et restant uniformément bornées dans $H^{1 / 2} \times H^{1 / 2} \times L^{2}$. Nous justifions ainsi rigoureusement le recours à l'optique géométrique pour le système $(Y M)$. Le théorème 3 , section 3 , rassemble les résultats de prolongement. Mention doit être faite de la proposition 3.1.1, où sont exposées des estimations bilinéaires uniformes en $\varepsilon$ qui jouent un rôle primordial dans la preuve du théorème 3 . Le théorème 1 s'obtient en mettant bout-à-bout les théorèmes 2 et 3 .

Pour conclure, nous vérifions à quelles conditions le terme dominant des solutions oscillantes du théorème 1 peut coïncider avec une solution régulière donnée de $(Y M)$ sur $\left[0, t_{0}\right]$. Nous en déduisons que le système $(Y M)$ reste stable sous l'effet de perturbations oscillantes de forte amplitude, bornées seulement dans $H^{1 / 2} \times H^{1 / 2} \times L^{2}$. Nous vérifions alors que le temps d'existence de la solution oscillante du théorème 1 est bien $t_{1}=t_{0}$, c'est-à-dire que l'optique géométrique garde sa précision jusqu'à l'apparition de caustiques.

Nos résultats restent intimement liés à la nature géométrique (très contraignante) des équations, et aux hypothèses de régularité sur la phase qui excluent l'essentiel des phénomènes néfastes liés à la concentration des singularités. Le système $(Y M)$ conserve ici un comportement linéaire exceptionnel, dans un domaine de régularité où les termes non linéaires jouent d'ordinaire un rôle actif, voire destructeur.

\subsection{Quelques remarques préliminaires quant à la structure des équations}

Nous finissons cette section en rappelant certains résultats élémentaires dont nous faisons grand usage par la suite. En particulier, nous montrons que le comportement du système $(Y M)$ est gouverné par deux types de symétries, qui s'avèrent complémentaires l'une de l'autre. D'une part l'invariance relativiste des équations (le système reste globalement invariant par transformation de Lorentz sur $\boldsymbol{E}$ ), et d'autre part l'invariance de jauge (les solutions du système sont données à un choix de jauge près sur $\boldsymbol{P}$ ). L'exploitation combinée de ces degrés de symétrie, externes et internes, permet de remédier à la mauvaise détermination apparente du système.

1.3.1. Régularité des termes non linéaires, existence de solutions régulières du système. - Avant toutes choses, il est important de préciser un seuil de régularité sur les données $(A, \phi, \psi)$ au dessus duquel nous pouvons estimer efficacement les 
différents termes non linéaires du système sans se soucier de leur structure. Nous dirons qu'une donnée $(A, \phi, \psi)$ est régulière si elle reste bornée dans $H^{s} \times H^{s} \times H^{s-1 / 2}$, avec $s \geqslant \frac{n}{2}+\frac{1}{2}$. Ce seuil est aussi celui pour lequel il est possible de résoudre (cf. [4]) le système $(Y M)$ localement en temps sans avoir à recourir à des effets régularisants de type Stricharz ni à la structure algébrique des seconds membres $(c f .[\mathbf{1 4}, \mathbf{1 5}]$ et [2], qui usent pour leur part de ce genre de méthodes).

Proposition 1.3.1. - Pour tout $s \geqslant \frac{n}{2}+\frac{1}{2}$ :

a) $F^{I}, F^{I I}, G^{I}$ et $G^{I I}$ envoient continûment $H^{s} \times H^{s-1} \hookrightarrow H^{s-1}$ et

$$
\begin{gathered}
\left|F^{I}\left(A, \partial A^{\prime}\right)\right|_{H^{s-1}} \leqslant C|A|_{H^{s}}\left|\partial A^{\prime}\right|_{H^{s-1}} \\
\left|F^{I}\left(\phi, \partial \phi^{\prime}\right)\right|_{H^{s-1}} \leqslant C|\phi|_{H^{s}}\left|\partial \phi^{\prime}\right|_{H^{s-1}} \\
\left|G^{I}(A, \partial \phi)\right|_{H^{s-1}} \leqslant C|A|_{H^{s}}|\partial \phi|_{H^{s-1}} \\
\left|G^{I I}(\partial A, \phi)\right|_{H^{s-1}} \leqslant C|\phi|_{H^{s}}|\partial A|_{H^{s-1}}
\end{gathered}
$$

b) $f$ et $g$ envoient continûment $H^{s} \times H^{s} \times H^{s-1 / 2} \rightarrow H^{s-1}$ et

$$
\begin{aligned}
& |f(A, \phi, \psi)|_{H^{s-1}} \leqslant C_{s}\left(|A|_{H^{s}}^{3}+|\phi|_{H^{s}}^{2}|A|_{H^{s}}+|\psi|_{H^{s-1 / 2}}^{2}\right) \\
& |g(A, \phi, \psi)|_{H^{s-1}} \leqslant C_{s}\left(|\Phi|_{H^{s}}^{3}+|\phi|_{H^{s}}|A|_{H^{s}}^{2}+|\psi|_{H^{s-1 / 2}}^{2}\right)
\end{aligned}
$$

c) $h$ envoie continûment $H^{s} \times H^{s} \times H^{s-1 / 2} \rightarrow H^{s-1 / 2}$ et

$$
|h(A, \phi, \psi)|_{H^{s-1 / 2}} \leqslant C_{s}|\psi|_{H^{s-1 / 2}}\left(|A|_{H^{s}}+|\phi|_{H^{s}}\right)
$$

Démonstration. — Essentiellement, nous remarquons que

$$
H^{s} \times H^{s-1} \hookrightarrow H^{s-1}, \quad H^{s-1 / 2} \times H^{s-1 / 2} \hookrightarrow H^{s-1} \quad \text { et } \quad H^{s} \times H^{s-1 / 2} \hookrightarrow H^{s-1 / 2}
$$

dès que $s \geqslant \frac{n}{2}+\frac{1}{2}$. Nous renvoyons à l'Annexe $\mathrm{B}$, où nous rappelons le lemme de multiplication B.1.

La plus part du temps, ces estimations bilinéaires sont utilisées conjointement avec les propositions suivantes :

Proposition 1.3.2 (résultat d'existence pour l'équation des ondes sur $\mathbb{R} \times \boldsymbol{\Sigma}$ )

Pour tout s réel, le problème de Cauchy

$$
\left\{\begin{array}{l}
\square u=f \in L^{1}\left(\left[0, t_{0}\right], H^{s-1}(\Sigma)\right) \\
u(0, y) \in H^{s}(\Sigma) \text { et } \partial_{t} u(0, y) \in H^{s-1}(\Sigma)
\end{array}\right.
$$

admet une unique solution $u \in C^{0}\left(\left[0, t_{0}\right], H^{s}(\Sigma)\right) \cap C^{1}\left(\left[0, t_{0}\right], H^{s-1}(\Sigma)\right)$. En particulier, il existe une constante finie $C_{g}$ qui ne dépend que de $g$, telle que toute solution de (8) vérifie l'estimation a priori :

$$
\begin{aligned}
& \sup _{t \in\left[0, t_{0}\right]}\left(|u(t, y)|_{H^{s}(\Sigma)}+\left|\partial_{t} u(t, y)\right|_{H^{s-1}(\Sigma)}\right) \\
& \leqslant C_{g}\left(|u(0, y)|_{H^{s}(\Sigma)}+\left|\partial_{t} u(0, y)\right|_{H^{s-1}(\Sigma)}+|f|_{L^{1}\left(\left[0, t_{0}\right], H^{s-1}(\Sigma)\right.}\right)
\end{aligned}
$$

Démonstration. - Se reporter directement à [5], qui s'applique en vertu de nos hypothèses géométriques. 
Proposition 1.3.3 (résultat d'existence pour l'équation de Dirac sur $\mathbb{R} \times \boldsymbol{\Sigma}$ )

Pour tout s réel, le problème de Cauchy

$$
\left\{\begin{array}{l}
\mathcal{D} \psi=h \in L^{1}\left(\left[0, t_{0}\right], H^{s}(\Sigma)\right) \\
\psi(0, y) \in H^{s}(\Sigma)
\end{array}\right.
$$

admet une unique solution $\psi \in C^{0}\left(\left[0, t_{0}\right], H^{s}(\Sigma)\right)$. De plus, il existe une constante finie $C_{g}$ qui ne dépend que de g, telle que toute solution de (10) vérifie l'estimation a priori :

$$
\sup _{t \in\left[0, t_{0}\right]}|\psi(t, y)|_{H^{s}(\Sigma)} \leqslant C_{g}\left(|\psi(0, y)|_{H^{s}(\Sigma)}+|h|_{L^{1}\left(\left[0, t_{0}\right], H^{s}(\Sigma)\right.}\right)
$$

Démonstration. - Se reporter par exemple à [16], qui s'applique en vertu de nos hypothèses géométriques.

1.3.2. Symétrie externe : identité de Bianchi. — L'identité (5) :

$$
R L \equiv 0
$$

suppose implicitement que l'on vérifie

$$
R F(A, \partial A, \phi, \partial \phi, \psi) \equiv 0
$$

pour toute solution $(A, \phi, \psi)$ du système $(Y M)$. Cette dernière identité vient comme sous produit de l'identité de Bianchi (2) et de la nécessaire conservation du terme de courant $\mathcal{J}(A, \phi, \partial \phi, \psi)$. L'identité de Bianchi s'écrit :

$$
d_{A}{ }^{\star} \boldsymbol{L}(A)=R(L A-F+\mathcal{J})+\left[A^{\nu},(L A-F+\mathcal{J})_{\nu}\right]=0
$$

compte tenu de (5), il vient :

$$
R F=R \mathcal{J}+\left[A^{\nu}, \mathcal{J}_{\nu}\right]+\left[A^{\nu},(L A)_{\nu}\right]-\left[A^{\nu}, F_{\nu}\right]
$$

que nous récapitulons par l'identité

$$
R F=d_{A}^{\star} \mathcal{J}+Q_{f}(A, L A-F)
$$

en retenant simplement que $Q_{f}\left(A, A^{\prime}\right)=\left[A^{\nu}, A_{\nu}^{\prime}\right]$ est une forme bilinéaire donnée. La conservation du courant provient des deux équations auxiliaires portant sur le champ scalaire et le champ de spineurs. Il existe aussi des formes bilinéaires $Q_{g}\left(\phi, \phi^{\prime}\right)=$ $\bar{\phi}^{t} r^{\prime}(1)^{*} \phi^{\prime}+\phi r^{\prime}(1) \phi^{\prime}$ et $Q_{h}\left(\psi, \psi^{\prime}\right)=\bar{\psi} \rho^{\prime}(1) \psi^{\prime}$ telles que :

$$
d_{A}{ }^{\star} \mathcal{J}=R \mathcal{J}+\left[A^{\nu}, \mathcal{J}_{\nu}\right]=Q_{g}(\phi, \square \phi-G)+Q_{h}(\psi, \mathcal{D} \psi-h)
$$

En mettant bout à bout ces identités, nous obtenons «l'identité de compatibilité » du système $(Y M)$ avec l'opérateur $L$ :

$$
R F=Q_{f}(A, L A-F)+Q_{g}(\phi, \square \phi-G)+Q_{h}(\psi, \mathcal{D} \psi-h)
$$

Nous aurons plus tard besoin de généraliser cette notion de « compatibilité » à d'autres systèmes extrapolés de $(Y M)$, d'où la définition suivante : 
DÉfinition 1.3.1. - Nous dirons qu'un système de la forme :

(S.Comp.)

$$
\left\{\begin{array}{l}
L A=F(A, \partial A, U) \\
\mathcal{C}(A, \partial A, U)=0
\end{array}\right.
$$

est extérieurement «compatible » avec $L$ si et seulement si il existe des formes bilinéaires $Q$ et $Q^{\prime}$ telles que

$$
R F=Q(A, L A-F)+Q^{\prime}(U, \mathcal{C})
$$

1.3.3. Symétrie interne : invariance de jauge. — L'identité (3) duale de l'identité de Bianchi est liée à l'invariance de jauge du système. Nous utiliserons essentiellement l'identité (6) qui en découle :

$$
L T \equiv 0
$$

Sous l'action d'un «changement de jauge », caractérisé par une application $u: \mathbb{R} \times \boldsymbol{\Sigma} \rightarrow \boldsymbol{G}$, nous transformons :

$$
\begin{aligned}
A & \rightsquigarrow A^{\prime}=u^{-1} A u+u^{-1} d u \\
\phi & \rightsquigarrow \phi^{\prime}=r(u) \phi \\
\psi & \rightsquigarrow \psi^{\prime}=\rho(u) \psi
\end{aligned}
$$

Puisque les équations doivent rester invariantes par ce changement de jauge, il vient nécessairement

$$
\left\{\begin{aligned}
L A^{\prime}-F\left(A^{\prime}, \phi^{\prime}, \psi^{\prime}\right) & =u^{-1}(L A-F(A, \phi, \psi)) u \\
\square \phi^{\prime}-G\left(A^{\prime}, \phi^{\prime}, \psi^{\prime}\right) & =r(u)(\square \phi-G(A, \phi, \psi)) \\
\mathcal{D} \psi^{\prime}-h\left(A^{\prime}, \phi^{\prime}, \psi^{\prime}\right) & =\rho(u)(\mathcal{D} \psi-h(A, \phi, \psi))
\end{aligned}\right.
$$

Par différentiation en $u=1$ (c'est-à-dire en posant $u=1+\epsilon \chi$, où $\chi: \mathbb{R} \times \boldsymbol{\Sigma} \rightarrow \mathfrak{g}$, et en dérivant par rapport à $\epsilon$ en $\epsilon=0$ ) nous déduisons une version infinitésimale de cette relation d'invariance de jauge :

$$
(D . I . J .)\left\{\begin{array}{c}
L([A, \chi]+T \chi)-\frac{\partial}{\partial A} F_{(A, \phi, \psi)} \cdot([A, \chi]+T \chi) \\
-\frac{\partial}{\partial \phi} F_{(A, \phi, \psi)} \cdot r^{\prime}(1) \chi \phi-\frac{\partial}{\partial \psi} F_{(A, \phi, \psi)} \cdot \rho^{\prime}(1) \chi \psi=[L A-F(A, \phi, \psi), \chi] \\
\square\left(r^{\prime}(1) \chi \phi\right)-\frac{\partial}{\partial A} G_{(A, \phi, \psi)} \cdot([A, \chi]+T \chi) \\
-\frac{\partial}{\partial \phi} G_{(A, \phi, \psi)} \cdot r^{\prime}(1) \chi \phi-\frac{\partial}{\partial \psi} G_{(A, \phi, \psi)} \cdot \rho^{\prime}(1) \chi \psi=r^{\prime}(1) \chi(\square \phi-G(A, \phi, \psi)) \\
\left(\mathcal{D} \rho^{\prime}(1) \chi \psi\right)-\frac{\partial}{\partial A} h_{(A, \phi, \psi)} \cdot([A, \chi]+T \chi) \\
-\frac{\partial}{\partial \phi} h_{(A, \phi, \psi)} \cdot r^{\prime}(1) \chi \phi-\frac{\partial}{\partial \psi} h_{(A, \phi, \psi)} \cdot \rho^{\prime}(1) \chi \psi=\rho^{\prime}(1) \chi(\mathcal{D} \psi-h(A, \phi, \psi))
\end{array}\right.
$$


Comme $F^{I}, F^{I I}, G^{I}$ ou $G^{I I}$ sont bilinéaires,

$$
\begin{aligned}
\frac{\partial}{\partial A} F_{(A, \partial A)}^{I} \cdot([A, \chi]+T \chi) & =F^{I}(A, \partial([A, \chi]+T \chi))+F^{I}(([A, \chi]+T \chi), \partial A) \\
\frac{\partial}{\partial \phi} F_{(\phi, \partial \phi)}^{I I} \cdot r^{\prime}(1) \chi \phi & =F^{I I}\left(\phi, \partial\left(r^{\prime}(1) \chi \phi\right)\right)+F^{I I}\left(r^{\prime}(1) \chi \phi, \partial \phi\right) \\
\frac{\partial}{\partial A} G_{(A, \partial \phi)}^{I} \cdot([A, \chi]+T \chi) & =G^{I}(([A, \chi]+T \chi), \partial \phi) \\
\frac{\partial}{\partial \phi} G_{(A, \partial \phi)}^{I} \cdot r^{\prime}(1) \chi \phi & =G^{I}\left(A, \partial\left(r^{\prime}(1) \chi \phi\right)\right) \\
\frac{\partial}{\partial A} G_{(\partial A, \phi)}^{I I} \cdot([A, \chi]+T \chi) & =G^{I I}(\partial([A, \chi]+T \chi), \phi) \\
\frac{\partial}{\partial \phi} G_{(\partial A, \phi)}^{I I} \cdot r^{\prime}(1) \chi \phi & =G^{I I}\left(\partial A, r^{\prime}(1) \chi \phi\right)
\end{aligned}
$$

En tenant compte de l'identité $L T=0$, nous en déduisons des relations traduisant la compatibilité interne des non-linéarités dominantes du système avec l'invariance de jauge :

$$
\begin{aligned}
L[A, \chi]-[L A, \chi]= & F^{I}(A, \partial T \chi)+F^{I}(T \chi, \partial A) \\
& +F^{I}(A, \partial[A, \chi])+F^{I}([A, \chi], \partial A) \\
& +F^{I I}\left(r^{\prime}(1) \chi \phi, \partial \phi\right)+F^{I I}\left(\phi, \partial\left(r^{\prime}(1) \chi \phi\right)\right) \\
& -\left[F^{I}(A, \partial A), \chi\right]-\left[F^{I I}(\phi, \partial \phi), \chi\right] \\
& +\frac{\partial}{\partial A} f_{(A, \phi, \psi)} \cdot(T \chi+[A, \chi])+\frac{\partial}{\partial \phi} f_{(A, \phi, \psi)} \cdot r^{\prime}(1) \chi \phi \\
& +\frac{\partial}{\partial \psi} f_{(A, \phi, \psi)} \cdot \rho^{\prime}(1) \chi \psi-\left[f_{(A, \phi, \psi)}, \chi\right]
\end{aligned}
$$

ainsi que

$$
\begin{aligned}
\square\left(r^{\prime}(1) \chi \phi\right)-r^{\prime}(1) \chi \square \phi= & G^{I}(T \chi, \partial \phi)+G^{I I}(\partial T \chi, \phi) \\
& +G^{I}\left(A, \partial\left(r^{\prime}(1) \chi \phi\right)\right)+G^{I I}(\partial[A, \chi], \phi) \\
& +G^{I}([A, \chi], \partial \phi)+G^{I I}\left(\partial A, r^{\prime}(1) \chi \phi\right) \\
& -r^{\prime}(1) \chi G^{I}(A, \partial \phi)-r^{\prime}(1) \chi G^{I I}(\partial A, \phi) \\
& +\frac{\partial}{\partial A} g_{(A, \phi, \psi)} \cdot(T \chi+[A, \chi])+\frac{\partial}{\partial \phi} g_{(A, \phi, \psi)} \cdot r^{\prime}(1) \chi \phi \\
& +\frac{\partial}{\partial \psi} g_{(A, \phi, \psi)} \cdot \rho^{\prime}(1) \chi \psi-r^{\prime}(1) \chi g_{(A, \phi, \psi)}
\end{aligned}
$$

La dernière identité portant sur $h$ ne nous sera d'aucune utilité par la suite.

1.3.4. Compatibilité des données initiales. - Lorsqu'on s'intéresse au problème de Cauchy pour un système (S.Comp.) compatible avec $L$, il est nécessaire 
d'imposer une contrainte sur les données initiales $\left(A, \partial_{t} A, U\right)_{\left.\right|_{t=0}}(y)$, afin de garder la trace de la condition de compatibilité sur $\boldsymbol{\Sigma}$ à $t=0$ :

$$
\nabla^{i}\left(\nabla_{i} A_{\left.0\right|_{t=0}}-\nabla_{0} A_{\left.i\right|_{t=0}}\right)=F_{0}\left(A_{\left.\right|_{t=0}}, \partial A_{\mid t=0}, U_{\mid t=0}\right)
$$

Pour le système $(Y M)$, cette contrainte s'écrit encore

$$
\nabla^{i} \mathcal{E}_{i}(A)_{\left.\right|_{t=0}}=\left[\mathcal{E}_{i}(A)_{\left.\right|_{t=0}}, A_{\left.\right|_{t=0}}^{i}\right]+\mathcal{J}_{\left.0\right|_{t=0}}
$$

en notant $\mathcal{E}_{i}(A)=\Gamma_{0 i}(A)=\nabla_{0} A_{i}-\nabla_{i} A_{0}+\left[A_{0}, A_{i}\right]$, la composante conormale de la courbure de Yang-Mills $\Gamma$ par rapport à la surface de Cauchy $\Sigma$. En termes physiques, ceci revient à dire qu'à $t=0$, la divergence du vecteur champ «électrique » doit être égale à la «charge» totale induite (somme de la charge extérieure $\mathcal{J}_{0}$ et du terme $\left[\mathcal{E}_{i}(A)_{\mid t=0}, A_{\mid t=0}^{i}\right]$ qui représente une charge auto-induite du champ de jauge sur lui-même). Il est toujours possible de se donner un choix non trivial de données initiales vérifiant la contrainte (15), pour peu que nous les cherchions régulières, i.e. dans $H^{s} \times H^{s} \times H^{s-1 / 2}, s \geqslant \frac{n}{2}+\frac{1}{2}$.

Supposons ensuite que le système est à la fois compatible avec $L$ et invariant par changements de jauge : la contrainte (15) reste alors invariante par changements de jauge, et les données initiales $\left(A, \partial_{t} A, U\right)_{\mid t=0}(y)$ compatibles forment alors des classes d'équivalence, dont les représentants se déduisent par changements de jauge et ont en commun la valeur de $\Gamma_{0 i}(A)=\nabla_{0} A_{i}-\nabla_{i} A_{0}+\left[A_{0}, A_{i}\right]=\mathcal{E}_{i}(A)_{\mid t=0}(y)$ et de $\Gamma_{i j}(A)=\nabla_{i} A_{j}-\nabla_{j} A_{i}+\left[A_{i}, A_{j}\right]=\epsilon_{i j k} \mathcal{B}_{k}(A)_{\mid t=0}(y)$.

Tout choix de données initiales compatibles $\left(A, \partial_{t} A, U\right)_{\left.\right|_{t=0}}(y)$ doit donc impérativement être accompagné d'un choix de jauge.

Une façon naturelle de se donner une jauge à $t=0$ est de fixer une des composantes non dynamiques de $A$ et de la soumettre à une condition différentielle d'ordre 1 , transverse à $T \Sigma$. Par exemple, on peut choisir $A_{\left.0\right|_{t=0}}=0$ et imposer $\nabla_{0} A_{\left.0\right|_{t=0}}=0$ (jauge temps), ou bien $\nabla^{i}\left(A_{\Sigma}\right)_{i_{t=0}}=0$ et $\nabla_{0} \nabla^{i}\left(A_{\Sigma}\right)_{i_{\mid t=0}}=0$ (jauge de Coulomb). Il est encore possible de passer continûment de l'un à l'autre de ces extrêmes en fixant $A_{0}$ (ou de façon équivalente $\left.\nabla_{0} \nabla^{i}\left(A_{\Sigma}\right)_{i}\right|_{t=0}$, puisque les deux composantes son liées par la contrainte 15), et en imposant une condition mixte de type

$$
\lambda \alpha^{-2} \nabla_{0} A_{0}+(1-\lambda) \nabla^{i}\left(A_{\Sigma}\right)_{i}=0,
$$

avec $\lambda \in[0,1]$. Pour être sûr que $J_{1}$ corresponde bien à la jauge temps et $J_{0}$ à la jauge de Coulomb, il faut enfin s'assurer d'une façon ou d'une autre que $A_{0} \rightarrow 0$ lorsque $\lambda \rightarrow 1$ et $\nabla_{0} \nabla^{i}\left(A_{\Sigma}\right)_{i} \rightarrow 0$ lorsque $\lambda \rightarrow 0$. Nous dirons dans ce cas que les données sont traduites en jauge $J_{\lambda}$. Lorsque nous nous intéresserons à la régularité de données initiales traduites en jauge $J_{\lambda}$, nous considérerons la quantité(5)

$$
\left|\left(A, \nabla_{0} A\right)\right|_{s}=|A|_{H^{s}}+\left|\nabla_{0} A\right|_{H^{s-1}}+\sqrt{\frac{\lambda}{1-\lambda}}\left|A_{0}\right|_{H^{s}}+\sqrt{\frac{1-\lambda}{\lambda}}\left|\nabla_{0} \nabla^{i}\left(A_{\Sigma}\right)_{i}\right|_{H^{s-2}}
$$

\footnotetext{
$\overline{(5) \text { il est naturel }}$ d'imposer un certain contrôle sur la régularité initiale des variables de jauge, pour
} ne pas masquer inutilement celle des variables dynamiques. 
1.3.5. Bonnes jauges et hyperbolicité de l'opérateur $L$. - L'hyperbolicité de l'opérateur $L$ est acquise à jauge près, i.e. $L$ ne devient hyperbolique (strictement ou dans un sens plus faible) que si l'on impose sur $A$ certaines «bonnes » conditions de jauge. La définition de ces «bonnes jauges » dépend bien entendu du sens que l'on veut donner au mot hyperbolique. Précisons le cas des conditions de type

$$
J_{\lambda}(A)=\lambda \alpha^{-2} \nabla_{0} A_{0}+(1-\lambda) \nabla^{i} A_{i}=0, \lambda \in[0,1]
$$

Décomposons $A=\left(A_{0}, A_{\Sigma}\right)=\left(A_{0}, A_{(1)}+A_{(2)}\right)$, où $A_{(1)}$ et $A_{(2)}$ sont les projetés respectifs de $A_{\Sigma}$ sur l'ensemble des champs de divergence nulle, vérifiant $\nabla^{i}\left(A_{\Sigma}\right)_{i}=0$, et sur l'ensemble supplémentaire des champs de rotationnel nul, s'écrivant $A_{\Sigma}=\nabla_{\Sigma} \chi$. L'équation $L A=F$ se réécrit alors

$$
\left\{\begin{aligned}
-\triangle A_{0}-\nabla_{0} \nabla^{i}\left(A_{(2)}\right)_{i}-(\mathcal{R} i c . A)_{0} & =F_{0} \\
\square A_{(1)}-(\mathcal{R} i c . A)_{(1)} & =F_{(1)} \\
\alpha^{-2} \nabla_{0}^{2} A_{(2)}-\nabla_{\Sigma} \alpha^{-2} \nabla_{0} A_{0}-(\mathcal{R} i c . A)_{(2)} & =F_{(2)}
\end{aligned}\right.
$$

Lorsque $J_{\lambda} A=0$ avec $\left.\lambda \in\right] 0,1\left[\right.$, il vient $\alpha^{-2} \nabla_{0} A_{0}=\frac{\lambda-1}{\lambda} \nabla^{i}\left(A_{(2)}\right)_{i}$, et alors $A$ satisfait le système

$$
H_{\lambda} A=F
$$

soit avec la décomposition précédente

$$
\left\{\begin{aligned}
\frac{\lambda}{1-\lambda} \alpha^{-2} \nabla_{0}^{2} A_{0}-\triangle A_{0}-(\mathcal{R} i c . A)_{0} & =F_{0} \\
\square A_{(1)}-(\mathcal{R} i c . A)_{(1)} & =F_{(1)} \\
\alpha^{-2} \nabla_{0}^{2} A_{(2)}-\frac{1-\lambda}{\lambda} \triangle A_{(2)}-(\mathcal{R} i c . A)_{(2)} & =F_{(2)}
\end{aligned}\right.
$$

On remarque que pour $\lambda=1 / 2$ (jauge de Lorentz),

$$
H_{\frac{1}{2}} A=(\square-\mathcal{R} i c .) A=F
$$

est diagonal, strictement hyperbolique. Lorsque $A_{0} \equiv 0$, il vient

$$
\left\{\begin{aligned}
-\nabla_{0} \nabla^{i}\left(A_{(2)}\right)_{i}-(\mathcal{R} i c . A)_{0} & =F_{0} \\
\square A_{(1)}-(\mathcal{R} i c . A)_{(1)} & =F_{(1)} \\
\alpha^{-2} \nabla_{0}^{2} A_{(2)}-(\mathcal{R} i c . A)_{(2)} & =F_{(2)}
\end{aligned}\right.
$$

Lorsque $\nabla^{i}\left(A_{(2)}\right)_{i}=0$, soit $A_{(2)} \equiv 0$, il vient

$$
\left\{\begin{aligned}
-\triangle A_{0}-(\mathcal{R} i c . A)_{0} & =F_{0} \\
\square A_{(1)}-(\mathcal{R} i c . A)_{(1)} & =F_{(1)} \\
-\nabla_{\Sigma} \alpha^{-2} \nabla_{0} A_{0}-(\mathcal{R} i c . A)_{(2)} & =F_{(2)}
\end{aligned}\right.
$$

Notons que le système revêt dans ce cas un caractère purement elliptique sur la composante $A_{0}$. Toutes fois, pour tout $\lambda \in[0,1]$, la composante $A_{(1)}$ à divergence nulle est propagée par la même équation d'onde. Le système $H_{\lambda} A=F$ conserve donc toujours une composante strictement hyperbolique. De plus nous bénéficions pour 
l'opérateur $H_{\lambda}$ d'une estimation a priori semblable à celle dont nous disposons pour $\square$, et uniforme en $\lambda$ :

Proposition 1.3.4. - Si $L A=F \in C^{0}\left([0, t], H^{s-1}(\Sigma)\right) \cap C^{1}\left([0, t], H^{s-2}(\Sigma)\right)$ et $J_{\lambda} A=0$ avec $\lambda \in[0,1]$, alors $A$ vérifie l'estimation a priori suivante :

$$
\sup _{[0, t]}\left(|A|_{H^{s}}+\left|\nabla_{0} A\right|_{H^{s-1}}\right) \leqslant C_{g}\left(\left|\left(A, \nabla_{0} A\right)_{\mid t=0}\right|_{s}+C(t)|F|_{C^{0}\left([0, t], H^{s-1}\right) \cap C^{1}\left([0, t], H^{s-2}\right)}\right)
$$

De plus, la constante $C_{g}$ reste indépendante du paramètre de jauge $\lambda$.

Démonstration. - Supposons dans un premier temps que $A$ est supporté sur un domaine causal compact, et pour plus de clarté, que $(\mathcal{R} i c)=$.0 . Remarquons que pour les champs irrotationnels, $\left|\nabla^{i}\left(A_{(2)}\right)_{i}\right|_{H^{s-1}} \sim\left|A_{(2)}\right|_{H^{s}}$.

Si $\lambda \in] 0,1[$ : des trois équations d'ondes (17), nous déduisons :

$$
\sup _{[0, t]}\left(\left|A_{(1)}\right|_{H^{s}}+\left|\nabla_{0} A_{(1)}\right|_{H^{s-1}}\right) \leqslant C_{g}\left(\left.\left|A_{(1)}\right|_{t=0}\right|_{H^{s}}+\left.\left|\nabla_{0} A_{(1)}\right|_{t=0}\right|_{H^{s-1}}+\left|F_{(1)}\right|_{L^{1}\left([0,1], H^{s-1}\right)}\right)
$$

qui correspond à l'estimation (9) pour la composante $A_{(1)}$ (l'équation ne dépend pas de $\lambda$ ), puis

$$
\left[\left|\frac{\lambda}{1-\lambda} \nabla_{0} A_{0}\right|_{L^{2}}^{2}+\frac{\lambda}{1-\lambda}\left|\nabla_{\Sigma} A_{0}\right|_{L^{2}}^{2}\right]_{0}^{t}=\int_{0}^{t}\left\langle\frac{\lambda}{1-\lambda} \nabla_{0} A_{0}, F_{0}\right\rangle
$$

qui conduit à

$$
\begin{aligned}
& \sup _{[0, t]}\left(\left|\frac{\lambda}{1-\lambda} \nabla_{0} A_{0}\right|_{H^{s-1}}+\sqrt{\frac{\lambda}{1-\lambda}}\left|\nabla_{\Sigma} A_{0}\right|_{H^{s-1}}\right) \\
& \left.\quad \leqslant C_{g}\left(\left|\frac{\lambda}{1-\lambda} \nabla_{0} A_{\left.0\right|_{t=0}}\right|_{H^{s-1}}+\sqrt{\frac{\lambda}{1-\lambda}}\left|\nabla_{\Sigma} A_{\left.0\right|_{t=0}}\right|_{H^{s-1}}\right)+\left|F_{0}\right|_{L^{1}\left([0, t], H^{s-1}\right)}\right)
\end{aligned}
$$

et enfin

$$
\left[\left|\nabla_{0} A_{(2)}\right|_{L^{2}}^{2}+\frac{1-\lambda}{\lambda}\left|\nabla_{\Sigma} A_{(2)}\right|_{L^{2}}^{2}\right]_{0}^{t}=\int_{0}^{t}\left\langle\nabla_{0} A_{(2)}, F_{(2)}\right\rangle
$$

qui conduit à

$$
\begin{aligned}
& (22) \sup _{[0, t]}\left(\left|\nabla_{0} A_{(2)}\right|_{H^{s-1}}+\sqrt{\frac{1-\lambda}{\lambda}}\left|\nabla_{\Sigma} A_{(2)}\right|_{H^{s-1}}\right) \\
& \left.\leqslant C_{g}\left(\left.\left|\nabla_{0} A_{(2)}\right|_{t=0}\right|_{H^{s-1}}+\left.\sqrt{\frac{1-\lambda}{\lambda}}\left|\nabla_{\Sigma} A_{(2)}\right|_{t=0}\right|_{H^{s-1}}\right)+\left|F_{(2)}\right|_{L^{1}\left([0, t], H^{s-1}\right)}\right)
\end{aligned}
$$

Lorsque $\lambda \in[1 / 2,1[$, nous utilisons (21) pour majorer

$$
\begin{aligned}
& \sup _{[0, t]}\left(\left|A_{(2)}\right|_{H^{s}}+\left|\nabla_{\Sigma} A_{0}\right|_{H^{s-1}}\right) \leqslant \sup _{[0, t]}\left(\left|\nabla^{i}\left(A_{(2)}\right)_{i}\right|_{H^{s-1}}+\sqrt{\frac{\lambda}{1-\lambda}}\left|\nabla_{\Sigma} A_{0}\right|_{H^{s-1}}\right) \\
& \left.\leqslant C_{g}\left(\left|\nabla^{i}\left(A_{(2)}\right)_{\left.\right|_{\mid t=0}}\right|_{H^{s-1}}+\sqrt{\frac{\lambda}{1-\lambda}}\left|A_{\left.0\right|_{t=0}}\right|_{H^{s}}\right)+\left|F_{0}\right|_{L^{1}\left([0, t], H^{s-1}\right)}\right) \\
& \leqslant C_{g}\left(\left|\left(A, \nabla_{0} A\right)_{\mid t=0}\right|_{s}+\left|F_{0}\right|_{L^{1}\left([0, t], H^{s-1}\right)}\right)
\end{aligned}
$$


du coup

$$
\sup _{[0, t]}\left|\nabla_{0} A_{0}\right|_{H^{s-1}}=\sup _{[0, t]} \frac{1-\lambda}{\lambda}\left|\nabla^{i}\left(A_{(2)}\right)_{i}\right|_{H^{s-1}} \leqslant C_{g}\left(\left|\left(A, \nabla_{0} A\right)_{\left.\right|_{t=0}}\right|_{s}+\left|F_{0}\right|_{L^{1}\left([0, t], H^{s-1}\right)}\right)
$$

nous utilisons enfin (22) pour majorer

$$
\begin{aligned}
\sup _{[0, t]}\left|\nabla_{0} A_{(2)}\right|_{H^{s-1}} & \left.\leqslant C_{g}\left(\left|\nabla_{0} A_{\left.2\right|_{t=0}}\right|_{H^{s-1}}+\left.\sqrt{\frac{1-\lambda}{\lambda}}\left|\nabla_{\Sigma} A_{(2)}\right|_{t=0}\right|_{H^{s-1}}\right)+\left|F_{(2)}\right|_{L^{1}\left([0, t], H^{s-1}\right)}\right) \\
& \leqslant C_{g}\left(\left|\left(A, \nabla_{0} A\right)_{\left.\right|_{t=0}}\right|_{s}+\left|F_{(2)}\right|_{L^{1}\left([0, t], H^{s-1}\right)}\right)
\end{aligned}
$$

Lorsque $\lambda \in] 0,1 / 2[$, nous utilisons (22) pour majorer

$$
\begin{aligned}
& \sup _{[0, t]}\left(\left|A_{(2)}\right|_{H^{s}}+\left|\nabla_{0} A_{(2)}\right|_{H^{s-1}}\right) \\
& \begin{aligned}
\leqslant & C_{g}\left(\left|\nabla_{0} A_{(2)}(t=0)\right|_{H^{s-1}}+\sqrt{\frac{1-\lambda}{\lambda}}\left|\nabla^{i}\left(A_{(2)}\right)\right|_{H^{s-1}}\left|A_{(2)}(t=0)\right|_{H^{s}}^{1 / 2}\right. \\
& \left.+\left|F_{(2)}\right|_{L^{1}\left([0, t], H^{s-1}\right)}\right)
\end{aligned} \\
& \leqslant C_{g}\left(\left|\nabla_{0} A_{(2)}(t=0)\right|_{H^{s-1}}+\left|\nabla_{0} A_{0}\right|_{H^{s-1}}+\left|A_{(2)}(t=0)\right|_{H^{s}}+\left|F_{(2)}\right|_{L^{1}\left([0, t], H^{s-1}\right)}\right) \\
& \leqslant C_{g}\left(\left|\left(A, \nabla_{0} A\right)(t=0)\right|_{s}+\left|F_{(2)}\right|_{L^{1}\left([0, t], H^{s-1}\right)}\right)
\end{aligned}
$$

puis l'équation elliptique

$$
-\triangle A_{0}-\nabla_{0} \nabla^{i}\left(A_{(2)}\right)_{i}=F_{0}
$$

pour majorer

$$
\begin{aligned}
\sup _{[0, t]}\left(\left|A_{0}\right|_{H^{s}}\right) & \leqslant \sup _{[0, t]}\left(\left|\nabla_{0} \nabla^{i}\left(A_{(2)}\right)_{i}\right|_{H^{s-2}}+\left|F_{0}\right|_{H^{s-2}}\right) \\
& \leqslant C_{g}\left(\left|\left(A, \nabla_{0} A\right)(t=0)\right|_{s}+C(t)|F|_{C^{0}\left([0, t], H^{s-1}\right)}\right)
\end{aligned}
$$

en dérivant en temps les équations, nous obtenons de même

$$
\sup _{[0, t]}\left(\left|A_{0}\right|_{H^{s}}\right) \leqslant C_{g}\left(\left|\left(A, \nabla_{0} A\right)(t=0)\right|_{s}+C(t)|F|_{\left.C^{0}\left([0, t], H^{s-1}\right) \cap C^{1}\left([0, t], H^{s-2}\right)\right)}\right.
$$

En rassemblant ces différents termes nous obtenons l'estimation désirée. L'adjonction d'un terme de courbure d'ordre 0 ne change pas notablement les calculs. Les estimations peuvent être étendues globalement en espace avec les réserves géométriques prises dans l'introduction, la constante $C_{g}$ restant un multiple de la constante obtenue pour la proposition 1.3.2. Pour les cas limites, $\lambda=1$ et $\lambda=0$, il n'y a plus que $A_{(1)}$ qui soit encore propagée par une équation d'onde, tandis que les composantes $A_{0}$ et $A_{(2)}$, respectivement, sont nulles. L'estimation a priori s'obtient sans difficulté particulière sur les composantes restantes, en considérant les systèmes (18) et (19).

Une des conséquences de cette proposition est que la régularité est propagée de façon équivalente par l'opérateur $L$ quelle que soit la jauge $J_{\lambda}$ vérifiée. Les résultat d'existence à jauge fixée peuvent donc indifféremment être établis dans une jauge $J_{\lambda}$ donnée, $\lambda \in[0,1]$, et être ensuite généralisés à l'ensemble de la famille des « bonnes » jauges $J_{\lambda}$. Par la suite, nous travaillons avec la jauge de Lorentz $J_{\frac{1}{2}} A=R A=0$ partout où un choix de jauge sera techniquement nécessaire. Cette condition de jauge a le mérite d'être invariante par changements de coordonnées sur l'espace-temps, et nous permet de résoudre directement des systèmes diagonaux d'équations d'ondes. La 
notion d'hyperbolicité à laquelle nous nous rattachons est alors purement géométrique, et provient directement des hypothèse faites sur la métrique $g$. Par la suite, nous nous appuyons à plusieurs reprises sur la proposition suivante :

Proposition 1.3.5 (Hyperbolicité de l'opérateur $L$ ). — Soit

(S.Comp.)

$$
\left\{\begin{array}{l}
L A=F(A, \partial A, U) \\
\mathcal{C}(A, \partial A, U)=0
\end{array}\right.
$$

un système extérieurement compatible avec l'opérateur L. Les propositions suivantes sont équivalentes :

i) $(A, U)$ est solution régulière de (S.Comp.) en jauge de Lorentz.

ii) $(A, U)$ est solution régulière du système hyperbolique :

(S.Hyp.)

$$
\left\{\begin{array}{l}
\square A-(\mathcal{R} i c .) A=F(A, \partial A, U) \\
\mathcal{C}(A, \partial A, U)=0
\end{array}\right.
$$

et satisfait $\grave{a} t=0$ les contraintes

$$
\left\{\begin{array}{l}
\nabla^{i}\left(\nabla_{i} A_{\left.0\right|_{t=0}}-\nabla_{0} A_{\left.i\right|_{t=0}}\right)=F_{0}\left(A_{\left.\right|_{t=0}}, \partial A_{\left.\right|_{t=0}}, U_{\left.\right|_{t=0}}\right) \\
R A_{\mid t=0}=0
\end{array}\right.
$$

Démonstration. - i) implique ii) de façon immédiate. Prouvons la proposition réciproque : à défaut de pouvoir résoudre directement $(\mathcal{S}$.Comp.), supposons que nous disposons sur un intervalle de temps $[0, t]$ d'une solution $(A, U)$ régulière $\left(A \in C^{0}\left([0, t], H^{s}\right) \cap C^{1}\left([0, t], H^{s-1}\right), s \geqslant \frac{n}{2}+\frac{1}{2}\right)$ du système

$$
\text { (S.Hyp.) }
$$

$$
\left\{\begin{array}{l}
\square A-(\mathcal{R} i c .) A=(L+T R) A=F(A, \partial A, U) \\
\mathcal{C}(A, \partial A, U)=0
\end{array}\right.
$$

avec des données de Cauchy compatibles en jauge de Lorentz, qui vérifient

$$
\left\{\begin{array}{l}
\nabla^{i}\left(\nabla_{i} A_{\left.0\right|_{t=0}}-\nabla_{0} A_{\left.i\right|_{t=0}}\right)=F_{0}\left(A_{\left.\right|_{t=0}}, \partial A_{\left.\right|_{t=0}}, U_{\left.\right|_{t=0}}\right) \\
R A_{\mid t=0}=0
\end{array}\right.
$$

Nous calculons alors :

$$
\nabla_{0}\left(R A_{\mid t=0}\right)=\left(\square A_{0 \mid t=0}-(\mathcal{R} i c .)_{0}{ }^{\nu} A_{\nu \mid t=0}\right)-\nabla^{i}\left(\nabla_{i} A_{\left.0\right|_{t=0}}-\nabla_{0} A_{\left.i\right|_{t=0}}\right)=0
$$

Puisque $R L=R(\square-\mathcal{R} i c)-.R T R=0$, il vient

$$
\begin{aligned}
\square(R A) & =R T R A \\
& =R(\square-\mathcal{R} i c .) A \\
& =R F(A, \partial A, U)
\end{aligned}
$$

D'autre part, la compatibilité du système est donnée par une identité de type

$$
R F(A, \partial A, U)=Q(A, L A-F)+Q^{\prime}(U, \mathcal{C})=-Q(A, T(R A))
$$


où $Q$ et $Q^{\prime}$ sont deux formes bilinéaires données. Il en résulte que $(R A)$ est solution du système :

$$
\left\{\begin{array}{l}
\square(R A)=-Q(A, T(R A)) \\
(R A)_{\mid t=0}=0 \\
\partial_{t}(R A)_{\mid t=0}=0
\end{array}\right.
$$

Puisque $Q$ est supposée bilinéaire et que $A \in C^{0}\left([0, t], H^{s}\right) \cap C^{1}\left([0, t], H^{s-1}\right)$ avec $s \geqslant \frac{n}{2}+\frac{1}{2}$ ( $c f$. estimations du lemme B.1 en annexe), nous disposons pour cette équation d'ondes de l'estimation a priori suivante :

$$
|R A|_{L^{\infty}\left([0, t], H^{s}\right)}+\left|\nabla_{0}(R A)\right|_{L^{\infty}\left([0, t], H^{s-1}\right)} \leqslant C\left(\left|R A_{\mid t=0}\right|_{H^{s}}+\left|\nabla_{0}(R A)_{\mid t=0}\right|_{H^{s-1}}\right)
$$

$\mathrm{Vu}$ la nullité des données initiales, il en résulte que l'unique solution est $R A \equiv 0$ sur $[0, t]$. Le système $(\mathcal{S}$.Hyp.) propage donc naturellement la jauge de Lorentz. Mais alors

$$
\square A-(\mathcal{R} \text { ic. }) A=L A+T R A=L A
$$

et il vient naturellement que

$$
(\mathcal{S} . \text { Hyp. })=(\mathcal{S} . \text { Comp. })
$$

Lorsque (S.Comp.) est compatible, invariant par changements de jauge, et que nous disposons de résultats d'existence pour le système hyperbolique $(\mathcal{S}$.Hyp.), la proposition 1.3.5 permet de résoudre le problème de Cauchy pour (S.Comp.) dans n'importe quelle jauge transverse de type $J_{\lambda}$ :

Proposition 1.3.6 (Bonnes conditions de jauge pour l'opérateur $L$ )

Soit $E(\chi)$ une famille de transformations dépendant d'un paramètre $\chi$ à valeurs dans $\mathfrak{g}$, qui transforme

$$
A \stackrel{E(\chi)}{\rightarrow} A^{\prime}=T \chi+A+e(A, \chi)
$$

où e est une fonction analytique vérifiant $e(A, 0)=0$ et linéaire en $A$. Si le système

(S.Comp.)

$$
\left\{\begin{array}{l}
L A=F(A, \partial A, U) \\
\mathcal{C}(A, \partial A, U)=0
\end{array}\right.
$$

est extérieurement compatible avec l'opérateur $L$ et invariant sous l'action de $E(\chi)$, alors la proposition i) implique la proposition ii) :

i) Toutes données initiales $\left(A, \nabla_{0} A, U\right)_{\left.\right|_{t=0}},\left|\left(A, \nabla_{0} A\right)_{\left.\right|_{t=0}}\right|_{s} \leqslant \delta, s \geqslant \frac{n}{2}+\frac{1}{2}$, se prolongent sur un intervalle $\left[0, t_{0}(\delta)\right], 0<t(\delta)$, en une unique solution $(A, U), A \in$ $C^{0}\left(\left[0, t_{0}\right], H^{s}\right) \cap C^{1}\left(\left[0, t_{0}\right], H^{s-1}\right)$ du système hyperbolique

$$
(\text { S.Hyp. }) \quad\left\{\begin{array}{l}
\square A-(\mathcal{R} i c .) A=F(A, \partial A, U) \\
\mathcal{C}(A, \partial A, U)=0
\end{array}\right.
$$

avec $F(A, \partial A, U) \in C^{0}\left(\left[0, t_{0}\right], H^{s-1}\right) \cap C^{1}\left(\left[0, t_{0}\right], H^{s-2}\right)$. 
ii) Il existe un temps $0<t_{1}(\delta) \leqslant t_{0}$ tel que pour tout $\lambda \in[0,1]$, les données initiales

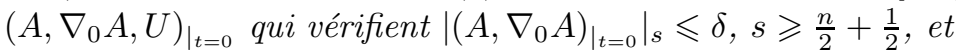

$$
\left\{\begin{array}{l}
\nabla^{i}\left(\nabla_{i} A_{\left.0\right|_{t=0}}-\nabla_{0} A_{\left.i\right|_{t=0}}\right)=F_{0}\left(A_{\left.\right|_{t=0}}, \partial A_{\left.\right|_{t=0}}, U_{\left.\right|_{t=0}}\right) \\
J_{\lambda} A_{\mid t=0}=0,
\end{array}\right.
$$

se prolongent sur $\left[0, t_{1}(\delta)\right]$ en une unique solution $(A, U), A \in C^{0}\left(\left[0, t_{1}\right], H^{s}\right) \cap$ $C^{1}\left(\left[0, t_{1}\right], H^{s-1}\right)$ du système $(\mathcal{S}$.Comp. $)$ vérifiant la condition de jauge $J_{\lambda} A=0$. De plus, si $F(A, \partial A, U)$ est linéaire en $(A, \partial A)$ et si $e(A, \chi)$ est linéaire en $\chi$, alors $t_{1}=t_{0}$.

Démonstration. — En partant de données initiales régulières

$$
\left(A^{\natural}, U^{\natural}\right)_{\left.\right|_{t=0}}, \quad\left|\left(A^{\natural}, \nabla_{0} A^{\natural}\right)_{\left.\right|_{t=0}}\right|_{s} \leqslant \delta, \quad s \geqslant \frac{n}{2}+\frac{1}{2},
$$

vérifiant les contraintes (24), nous souhaitons obtenir une solution $\left(A^{\natural}, U^{\natural}\right)$ de (S.Comp.) en jauge $J_{\lambda} A^{\natural}=0$. Nous allons montrer qu'il est toujours possible de se ramener à des données équivalentes $\left(A^{b}, U^{b}\right)_{\left.\right|_{t=0}}$ en jauge de Lorentz $R A_{\left.\right|_{t=0}}^{b}=0$, de trouver une solution $\left(A^{\mathrm{b}}, U^{\mathrm{b}}\right)$ au problème en jauge de Lorentz $R A^{\mathrm{b}}=0$ grâce à la proposition 1.3.5, et de revenir en jauge $J_{\lambda}$ par une transformation $E(\chi)$ appropriée. Si nous posons à $t=0$ :

$$
\chi_{\mid t=0}=\nabla_{0} \chi_{\mid t=0}=0 \text { et } \alpha^{-2} \nabla_{0}^{2} \chi_{\mid t=0}=R A_{\mid t=0}^{\natural}
$$

nous obtenons des données initiales :

$$
\begin{aligned}
A_{0 \mid t=0}^{b} & =A_{0 \mid t=0}^{\natural} \in H^{s} \\
A_{\Sigma \mid t=0}^{b} & =A_{\Sigma \mid t=0}^{\natural} \in H^{s} \\
\nabla_{0} A_{0 \mid t=0}^{b} & =\nabla_{0} A_{0 \mid t=0}^{\natural}-\nabla_{0}^{2} \chi_{\mid t=0} \in H^{s-1} \\
\nabla_{0} A_{\Sigma \mid t=0}^{b} & =\nabla_{0} A_{\Sigma \mid t=0}^{\natural} \in H^{s-1}
\end{aligned}
$$

qui vérifient la jauge de Lorentz :

$$
R A_{\mid t=0}^{b}=\alpha^{-2} \nabla_{0} A_{0 \mid t=0}^{\natural}+\nabla^{j} A_{\Sigma j \mid t=0}^{\natural}-\alpha^{-2} \nabla_{0}^{2} \chi_{\mid t=0}=0
$$

ainsi que la formule de changement de jauge :

$$
A_{\mid t=0}^{b} \stackrel{E\left(\chi_{\mid t=0}\right)}{\rightsquigarrow} A_{\mid t=0}^{\natural}=T \chi_{\mid t=0}+A^{b}+e\left(A^{b}, \chi\right)_{\mid t=0}
$$

Puisque (S.Comp.) est invariant par $E(\chi)_{\left.\right|_{t=0}}$ à $t=0$, ces données restent compatibles avec $L$ et vérifient en définitive la contrainte (23). La proposition 1.3.5 s'applique, et i) suppose que nous sachions prolonger ces nouvelles données par une unique solution $\left(A^{b}, U^{b}\right)$,

$$
A^{b} \in C^{0}\left(\left[0, t_{0}\right], H^{s}\right) \cap C^{1}\left(\left[0, t_{0}\right], H^{s-1}\right)
$$

vérifiant

$$
R A^{b}=0
$$


soit

$$
J_{\lambda} A^{b}=(2 \lambda-1) \alpha^{-2} \nabla_{0} A_{0}^{b} \in C^{0}\left(\left[0, t_{0}\right], H^{s-1}\right) \cap C^{1}\left(\left[0, t_{0}\right], H^{s-2}\right)
$$

Suivant les valeurs de $\lambda$, l'équation à résoudre sur $\chi$ pour revenir à une solution $\left(A^{\natural}, U^{\natural}\right)$ vérifiant la jauge $J_{\lambda} A^{\natural}=0$ diffère :

$$
J_{\lambda} T \chi=-J_{\lambda} A^{b}-J_{\lambda} e\left(A^{b}, \chi\right)
$$

Lorsque $\lambda \in] 0,1\left[\right.$, il vient $J_{\lambda} T=\alpha^{-2} \lambda \nabla_{0}^{2}-(1-\lambda) \triangle=\square_{\lambda}$ et nous devons résoudre une équation d'onde avec des données initiales nulles :

$$
\left\{\begin{array}{l}
\square_{\lambda} \chi=-J_{\lambda} A_{0}^{b}-J_{\lambda} e\left(A^{b}, \chi\right) \\
\chi_{\mid t=0}=\nabla_{0} \chi_{\mid t=0}=0
\end{array}\right.
$$

Si $e\left(A^{b}, \chi\right)$ est linéaire en $\chi$, cette équation d'onde modifiée admet une unique solution $\chi \in C^{0}\left(\left[0, t_{0}\right], H^{s}\right) \cap C^{1}\left(\left[0, t_{0}\right], H^{s-1}\right)$, le temps d'existence est le temps $t_{0}$ sur lequel $A^{b}$ reste borné dans $H^{s}$. Nous obtenons donc une solution $A^{\natural}$ du système en jauge $J_{\lambda}$. Pour voir que cette solution a bien la régularité espérée, il suffit de reprendre l'inégalité a priori de la proposition 1.3.4. Si $F(A, \partial A, U)$ est linéaire en $(A, \partial A)$, la régularité initiale est automatiquement propagée sur $A^{\natural}$ et on vérifie $A^{\natural} \in C^{0}\left(\left[0, t_{0}\right], H^{s}\right) \cap$ $C^{1}\left(\left[0, t_{0}\right], H^{s-1}\right)$. Du coup, $T \chi+e\left(A^{b}, \chi\right)=A^{\natural}-A^{b} \in C^{0}\left(\left[0, t_{0}\right], H^{s}\right) \cap C^{1}\left(\left[0, t_{0}\right], H^{s-1}\right)$ et on récupère $\chi \in C^{0}\left(\left[0, t_{0}\right], H^{s+1}\right) \cap C^{1}\left(\left[0, t_{0}\right], H^{s}\right)$. Lorsque $e\left(A^{b}, \chi\right)$ n'est pas linéaire en $\chi$, nous passons par une méthode itérative de type point fixe. Nous obtenons $\chi \in C_{l o c .}^{0}\left(\left[0, t_{\lambda, \delta}\left[, H^{s}\right) \cap C_{l o c .}^{1}\left(\left[0, t_{\lambda, \delta}\left[, H^{s-1}\right)\right.\right.\right.\right.$, où $\left[0, t_{\lambda, \delta}[\right.$ est l'intervalle maximum pour lequel $\chi$ reste borné dans $H^{s}$. Nous utilisons ensuite la proposition 1.3.4. Lorsque le second membre $F(A, \partial A, U)$ n'est pas linéaire en $(A, \partial A)$, on peut résoudre par point fixe l'équation $H_{\lambda} A=F(A, \partial A, U)$, et montrer qu'une solution $A^{b}$ de (S.Comp.) à jauge $J_{\lambda}$ fixée reste nécessairement bornée dans $C^{0}\left([0, t], H^{s}\right) \cap C^{1}\left([0, t], H^{s-1}\right)$, pour tout temps $t<t_{1}(\delta)$, avec $t_{1}(\delta)$ ne dépendant que de la taille des données initiale ainsi que de la constante $C_{g}$, mais pas du paramètre de jauge $\lambda$. Si par hasard $t_{\lambda, \delta}<t_{1}(\delta)$, alors $T \chi+e\left(A^{b}, \chi\right)=A^{\natural}-A^{b} \in C^{0}\left(\left[0, t_{\lambda, \delta}\right], H^{s}\right) \cap C^{1}\left(\left[0, t_{\lambda, \delta}\right], H^{s-1}\right)$ et on récupère $\chi \in C^{0}\left(\left[0, t_{\lambda, \delta}\right], H^{s+1}\right) \cap C^{1}\left(\left[0, t_{\lambda, \delta}\right], H^{s}\right)$, donc $t_{\lambda, \delta}$ ne serait pas maximal.

Les cas limites (jauge temps et jauge de Coulomb) s'obtiennent suivant un schéma similaire. Pour $\lambda=1$, nous avons affaire à une équation différentielle ordinaire :

$$
\left\{\begin{array}{l}
\alpha^{-2} \nabla_{0}^{2} \chi=-\alpha^{-2} \nabla_{0} A^{b}-\alpha^{-2} \nabla_{0} e\left(A^{b}, \chi\right)_{0} \\
\chi_{\mid t=0}=\nabla_{0} \chi_{\mid t=0}=0
\end{array}\right.
$$

que l'on résout dans $H^{s-1}$ localement en temps. La régularité $H^{s+1}$ est récupérée grâce au système (18), qui est automatiquement vérifié. L'existence sur l'intervalle de temps maximal $\left[0, t_{1}\right]$ est garantie par un argument similaire à celui développé ci-dessus. Pour $\lambda=0$, il faut résoudre l'équation elliptique :

$$
\triangle \chi=\nabla^{i} A_{i}^{b}+\nabla^{i} e\left(A^{b}, \chi\right)_{i}
$$


cette équation se résout à $t$ fixé dans $H^{s+1}$, la continuité dans le temps étant donnée par celle de $A^{b} \in C^{0}\left(\left[0, t_{1}\right], H^{s}\right) \cap C^{1}\left(\left[0, t_{1}\right], H^{s-1}\right)$ et par le système (19).

Les résultats que nous venons d'énoncer restent vrais tant que nous disposons de bonnes estimations sur les termes non linéaires, ce qui est le cas si nous considérons des données régulières. $[\mathbf{1 5}]$ montre que la situation se détériore brutalement en dessous du seuil que nous nous sommes fixés $\left(s \geqslant \frac{n}{2}+\frac{1}{2}\right)$. Il apparaît, entre autres, que les conditions de jauge ne peuvent pas toujours être imposées globalement en espace. Nous éviterons par la suite ce genre d'inconvénients, en construisant des solutions oscillantes, qui restent très régulières à $\varepsilon$ fixé, et dont les singularités s'accumulent, lorsque $\varepsilon$ devient petit, dans des directions conormales à un feuilletage de $\left[0, t_{0}\right] \times \Sigma$ en hypersurfaces de type nul, fixé une fois pour toute. Notre objectif est de faire un usage systématique de la proposition 1.3.5, en compensant la perte de régularité à $\varepsilon$ petit par un suivi détaillé de la géométrie des oscillations. 


\section{CHAPITRE 2}

\section{CONSTRUCTION DE FAMILLES DE SOLUTIONS APPROCHÉES DE $(Y M)$ SOUS FORME DE DÉVELOPPEMENTS OSCILLANT À HAUTE FRÉQUENCE, MONOPHASÉS, ET D'AMPLITUDE MAXIMALE}

Nous nous proposons de construire des familles de solutions approchées de $(Y M)$ sous forme de développements oscillants monophasés de la forme :

$$
\left\{\begin{array}{l}
A_{\varepsilon}(x)=A_{0}(x)+\sum_{i \geqslant 1} \varepsilon^{i / 2} A_{i}\left(x, \frac{\theta(x)}{\varepsilon}\right) \\
\phi_{\varepsilon}(x)=\phi_{0}(x)+\sum_{i \geqslant 1} \varepsilon^{i / 2} \phi_{i}\left(x, \frac{\theta(x)}{\varepsilon}\right) \\
\psi_{\varepsilon}(x)=\sum_{i \geqslant 0} \varepsilon^{i / 2} \psi_{i}\left(x, \frac{\theta(x)}{\varepsilon}\right)
\end{array}\right.
$$

où $\varepsilon \in] 0,1[$ est un facteur d'échelle destiné à devenir arbitrairement petit et $\theta$ une fonction de phase qui décrit la géométrie des oscillations. Les «profils» $(A, \phi, \psi)_{i}(x, \omega)$ sont des fonctions $2 \pi$-périodiques en la variable $\omega$, à l'exclusion de $\left(A_{0}, \phi_{0}\right)$ que nous choisissons non-oscillantes. Nous recherchons des profils réguliers, au moins bornés dans $C^{s}\left([0,2 \pi], H^{s} \times H^{s} \times H^{s-1 / 2}(\Sigma)\right), s \geqslant \frac{n}{2}+\frac{1}{2}$. La régularité «asymptotique » de la famille est entièrement fixée par le rapport entre l'amplitude et la fréquence des oscillations. Dans le cas que nous envisageons, les oscillations sont d'amplitude maximale et correspondent à des solutions du système $(Y M)$ bornées seulement sur $H^{1 / 2} \times H^{1 / 2} \times L^{2}$ dans les directions conormales aux surfaces de phase. Nous verrons que des développements plus singuliers n'ont aucune chance de donner des solutions oscillantes non triviales du système $(Y M)$.

Les degrés de liberté dont nous disposons pour construire les développements cidessus sont d'une part le choix de la fonction de phase $\theta$, en accord avec la géométrie du problème à un date $t=0$ prise comme origine du temps, et d'autre part une collection de fonctions oscillantes qui complètent la description des données initiales du système. Un certain nombre de contraintes, représentatives des symétries du système, doivent bien entendu s'appliquer à ces données. Nous les détaillerons dans la section 2.2.3. 


\subsection{Choix d'une fonction de phase}

2.1.1. Hypothèses géométriques sur la phase. - Nous commençons par choisir une fonction de phase $\theta(x)$ qui vérifie l'équation eïkonale associée à la métrique hyperbolique $\boldsymbol{g}$ choisie, sur un intervalle de temps $\left[0, t_{0}\right]$ donné :

$$
\boldsymbol{g}(\nabla \theta, \nabla \theta)=g^{\nu \mu} \nabla_{\nu} \theta \nabla_{\mu} \theta=0
$$

Cette équation admet deux racines $\theta^{+}$et $\theta^{-}$, vérifiant respectivement

$$
\nabla_{0} \theta^{ \pm}= \pm \alpha\left|\nabla_{\Sigma} \theta^{ \pm}\right|= \pm \alpha g_{\Sigma}\left(\nabla_{\Sigma} \theta^{ \pm}, \nabla_{\Sigma} \theta^{ \pm}\right)^{1 / 2}
$$

qui peuvent être construites selon la méthode traditionnelle de résolution des équations d'Hamilton-Jacobi à partir de données initiales qui traduisent la géométrie du problème à $t=0$. L'intervalle de temps $\left[0, t_{0}\right]$ est une donnée géométrique du problème, et ne sera essentiellement pas modifié par la suite. Nous cherchons une solution approchée sur un intervalle de temps arbitraire qui ne doit en aucun cas dépendre de $\varepsilon$.

Hypothèse 2.1.1. - Nous supposerons désormais que $\nabla_{0} \theta=+\alpha\left|\nabla_{\Sigma} \theta\right|$ et que les hypothèses de régularité suivantes sont vérifiées :

i) $\theta \in C^{\infty}\left(\left[0, t_{0}\right] \times \Sigma\right)$

ii) $\left|\nabla_{0} \theta\right| \in\left[c_{0}^{-1}, c_{0}\right], c_{0}>0$ sur tout $\left[0, t_{0}\right] \times \Sigma$,

iii) $\nabla^{2} \theta \in C^{\infty}\left(\left[0, t_{0}\right], H^{\infty}(\Sigma)\right)$

En faisant ces hypothèses, nous choisissons de travailler avant l'apparition de caustiques. En particulier, il est important que le champ de vecteurs

$$
l=g^{\mu \nu} \nabla_{\nu} \theta \partial_{\mu}
$$

reste régulier sur $\left[0, t_{0}\right] \times \boldsymbol{\Sigma}$. Au vu de l'équation eïkonale, il est clair que

$$
g(l, l)=g_{\mu \nu} l^{\mu} l^{\nu}=g^{\mu \nu} \nabla_{\mu} \theta \nabla_{\nu} \theta=0
$$

c'est-à-dire que $l$ est un champ de vecteurs nuls, et ses courbes intégrales des géodésiques nulles de $\left[0, t_{0}\right] \times \boldsymbol{\Sigma}$ (en termes physiques, il s'agit des « rayons de lumière »). Posons $\frac{d}{d s}=\nabla_{(l)}=g^{\mu \nu} \nabla_{\mu} \theta \nabla_{\nu}$. Dire que le flot de $l$ est géodésique revient à écrire

$$
\frac{d l}{d s}=0
$$

Nous notons

$$
\mathbb{C}_{\vartheta}=\left\{(t, y) \in\left[0, t_{0}\right] \times \boldsymbol{\Sigma}, \theta(t, y)=\vartheta\right\}
$$

les hypersurfaces de phase dans $\left[0, t_{0}\right] \times \boldsymbol{\Sigma}$, et

$$
\mathbb{C}_{\vartheta, t}=\{y \in \boldsymbol{\Sigma}, \theta(t, y)=\vartheta\}
$$

les surfaces de phase à temps fixé dans $\{t\} \times \boldsymbol{\Sigma}$. Les surfaces $\mathbb{C}_{\vartheta, t}$ caractérisent donc l'intersection entre deux feuilletages complémentaires de $\boldsymbol{E}$, l'un (de type spatial) par la fonction temps $t$, l'autre (de type nul) par la fonction de phase $\theta$. Naturellement, le champ de vecteurs nuls $l$ est tangent aux $\mathbb{C}_{\vartheta}$, et le flot de $l$ engendre les $\mathbb{C}_{\vartheta}$ à partir des surfaces de phase initiales $\mathbb{C}_{\vartheta, 0}$. Remarquons que $\theta, l$ et $\mathbb{C}_{\vartheta}$ sont des objets 
indépendants du choix des coordonnées, et on peut s'y référer indifféremment sur $\boldsymbol{E}$ ou sur sa représentation $\mathbb{R} \times \boldsymbol{\Sigma}$. Pour finir de décrire le feuilletage de $\boldsymbol{E}$ par les surfaces de phase, définissons

$$
N_{\Sigma}=\frac{\alpha}{\nabla_{0} \theta} g^{i j} \nabla_{j} \theta \partial_{i}
$$

le champ des normales unitaires sortantes à $\mathbb{C}_{\vartheta, t}$ dans $T \Sigma$, de sorte que

$$
g\left(N_{R}, N_{R}\right)=1, g\left(N_{\Sigma}, N_{\Sigma}\right)=-1 \text { et } g\left(N_{R}, N_{\Sigma}\right)=0
$$

Posons

$$
e_{1}=\frac{1}{2}\left(N_{R}+N_{\Sigma}\right)=\frac{\alpha}{\nabla_{0} \theta} l \quad \text { et } \quad e_{2}=\frac{1}{2}\left(N_{R}-N_{\Sigma}\right)=\frac{\nabla_{0} \theta}{\alpha} l^{\prime}
$$

qui vérifient

$$
g\left(e_{1}, e_{1}\right)=0, g\left(e_{2}, e_{2}\right)=0 \text { et } g\left(e_{1}, e_{2}\right)=2
$$

ou de façon équivalente

$$
g(l, l)=0, g\left(l^{\prime}, l^{\prime}\right)=0 \text { et } g\left(l, l^{\prime}\right)=2
$$

Les champs $l$ et $l^{\prime}$ sont définis globalement sur $\left[0, t_{0}\right] \times \Sigma$. Par ailleurs, tout choix local d'un repère orthonormé $\left(e_{I}\right), I=3, \ldots, n+1$, sur $\mathbb{C}_{\vartheta, 0}$ vérifie

$$
g\left(N_{R}, e_{I}\right)=g\left(N_{\Sigma}, e_{I}\right)=g\left(e_{1}, e_{I}\right)=g\left(e_{2}, e_{I}\right)=0
$$

et peut être prolongé le long des rayons de lumière sur un voisinage cylindrique de $\mathbb{C}_{\vartheta}$ en résolvant l'équation différentielle

$$
\frac{d e_{I}}{d s}=C_{I} e_{1}
$$

avec

$$
C_{I}=-g\left(e_{I}, \frac{d N_{R}}{d s}\right)
$$

Puisque $g\left(N_{R}, e_{1}\right)=1$ et que $g\left(N_{\Sigma}, e_{1}\right)=-1$, il vient alors

$$
\begin{aligned}
\frac{d}{d s} g\left(N_{R}, e_{I}\right) & =g\left(N_{R}, \frac{d e_{I}}{d s}\right)+g\left(\frac{d N_{R}}{d s}, e_{I}\right) \\
& =C_{I} g\left(N_{R}, e_{1}\right)+g\left(e_{I}, \frac{d N_{R}}{d s}\right) \\
& =0
\end{aligned}
$$

de même que

$$
\begin{aligned}
\frac{d}{d s} g\left(N_{\Sigma}, e_{I}\right) & =g\left(N_{\Sigma}, \frac{d e_{I}}{d s}\right)+g\left(\frac{d N_{\Sigma}}{d s}, e_{I}\right) \\
& =C_{I} g\left(N_{\Sigma}, e_{1}\right)+g\left(e_{I}, \frac{d N_{\Sigma}}{d s}\right) \\
& =-C_{I}-g\left(e_{I}, \frac{d N_{R}}{d s}\right) \\
& =0
\end{aligned}
$$


Le champ $e_{I}$ reste donc orthogonal à $N_{R}, N_{\Sigma}, e_{1}$ et $e_{2}$ tout le long des rayons de lumière. Du coup,

$$
\begin{aligned}
\frac{d}{d s} g\left(e_{I}, e_{J}\right) & =g\left(e_{I}, \frac{d e_{J}}{d s}\right)+g\left(\frac{d e_{I}}{d s}, e_{J}\right) \\
& =C_{J} g\left(e_{I}, e_{1}\right)+C_{I} g\left(e_{1}, e_{J}\right) \\
& =0
\end{aligned}
$$

c'est-à-dire que

$$
g\left(e_{I}, e_{J}\right)=\delta_{J}^{I}
$$

Il en résulte que $\left(e_{I}\right)_{\vartheta, t}, I=3, \ldots, n+1$, est bien un repère orthonormé sur $\mathbb{C}_{\vartheta, t}$, $t \in\left[0, t_{0}\right]$. Calculons

$$
\frac{d e_{1}}{d s}=\frac{d}{d s}\left(\frac{\alpha}{\nabla_{0} \theta} l\right)=\left(\frac{d}{d s} \frac{\alpha}{\nabla_{0} \theta}\right) l+\frac{\alpha}{\nabla_{0} \theta} \frac{d l}{d s}=\left(\frac{d}{d s} \frac{\alpha}{\nabla_{0} \theta}\right) \frac{\nabla_{0} \theta}{\alpha} e_{1}
$$

soit

$$
\frac{d e_{1}}{d s}=C_{1} e_{1}
$$

Enfin, puisque

$$
g\left(e_{2}, e_{2}\right)=0, g\left(e_{1}, e_{2}\right)=2, \text { et } g\left(e_{2}, e_{I}\right)=0
$$

il vient successivement

$$
\begin{gathered}
g\left(\frac{d e_{2}}{d s}, e_{2}\right)=0 \\
g\left(\frac{d e_{2}}{d s}, e_{1}\right)=-g\left(\frac{d e_{1}}{d s}, e_{2}\right)=-g\left(\left(\frac{d}{d s} \frac{\alpha}{\nabla_{0} \theta}\right) l, e_{2}\right)=0
\end{gathered}
$$

et

$$
g\left(\frac{d e_{2}}{d s}, e_{I}\right)=-g\left(e_{2}, \frac{d e_{I}}{d s}\right)=-2 C_{I}
$$

ce qui nous permet de conclure que

$$
\frac{d e_{2}}{d s}=-2 \sum_{I=3}^{n+1} C_{I} e_{I}
$$

Nommons $\left(e^{\mu}\right)=\left(e_{\mu}\right)^{*}, \mu=1, \ldots, n+1$, les coordonnées de $T^{*} \boldsymbol{E}$ associées au repère que nous venons de définir et vérifiant $e^{\nu} e_{\mu}=\delta_{\mu}^{\nu}$. Nous en déduisons la décomposition :

$$
\boldsymbol{T}^{*} \boldsymbol{E}=\mathcal{E}^{\vee} \oplus \mathcal{E}^{0} \oplus \mathcal{E}^{\wedge}
$$

où

$$
\begin{aligned}
\mathcal{E}^{\vee} & =\left\{A \in \boldsymbol{T}^{*} \boldsymbol{E} \mid A=A_{1} e^{1}\right\} \\
\mathcal{E}^{\wedge} & =\left\{A \in \boldsymbol{T}^{*} \boldsymbol{E} \mid A=A_{2} e^{2}\right\} \\
\mathcal{E}^{0} & =\left\{A \in \boldsymbol{T}^{*} \boldsymbol{E} \mid A=\sum_{I=3}^{n+1} A_{I} e^{I}\right\}
\end{aligned}
$$


soit, de façon équivalente sur $\boldsymbol{T}^{*}(\mathbb{R} \times \boldsymbol{\Sigma})$ :

$$
\begin{aligned}
& \mathcal{E}^{\vee}=\left\{A \in \boldsymbol{T}^{*}(\mathbb{R} \times \boldsymbol{\Sigma}) \mid A_{\Sigma}=-\frac{A_{0}}{\nabla_{0} \theta} \nabla_{\Sigma} \theta\right\} \\
& \mathcal{E}^{\wedge}=\left\{A \in \boldsymbol{T}^{*}(\mathbb{R} \times \boldsymbol{\Sigma}) \mid A_{\Sigma}=\frac{A_{0}}{\nabla_{0} \theta} \nabla_{\Sigma} \theta\right\} \\
& \mathcal{E}^{0}=\left\{A \in \boldsymbol{T}^{*}(\mathbb{R} \times \boldsymbol{\Sigma}) \mid A_{0}=0 \text { et } g_{\Sigma}\left(A_{\Sigma}, \nabla_{\Sigma} \theta\right)=0\right\}
\end{aligned}
$$

Nous définissons enfin

$$
\begin{aligned}
& \mathcal{E}^{\#}=\mathcal{E}^{\wedge} \oplus \mathcal{E}^{0} \\
& \mathcal{E}^{=}=\mathcal{E}^{\vee} \oplus \mathcal{E}^{0}
\end{aligned}
$$

soit, de façon équivalente sur $\boldsymbol{T}^{*}(\mathbb{R} \times \boldsymbol{\Sigma})$ :

$$
\begin{aligned}
& \mathcal{E}^{\#}=\left\{A \in \boldsymbol{T}^{*}(\mathbb{R} \times \boldsymbol{\Sigma}) \mid A_{0}=\frac{\alpha^{2}}{\nabla_{0} \theta} g_{\Sigma}\left(\nabla_{\Sigma} \theta, A_{\Sigma}\right)\right\} \\
& \mathcal{E}^{=}=\left\{A \in \boldsymbol{T}^{*}(\mathbb{R} \times \boldsymbol{\Sigma}) \mid A_{0}=-\frac{\alpha^{2}}{\nabla_{0} \theta} g_{\Sigma}\left(\nabla_{\Sigma} \theta, A_{\Sigma}\right)\right\}
\end{aligned}
$$

En dérivant le long des rayons de lumière chacune des relations $e^{\mu} e_{\nu}=\delta_{\nu}^{\mu}$, il vient

$$
\begin{aligned}
& \frac{d e^{1}}{d s} e_{1}=-e^{1} \frac{d e_{1}}{d s}=-C_{1} e^{1} e_{1}=-C_{1} \\
& \frac{d e^{1}}{d s} e_{2}=-e^{1} \frac{d e_{2}}{d s}=2 e^{1} \sum_{I=3}^{n+1} C_{I} e_{I}=0 \\
& \frac{d e^{1}}{d s} e_{I}=-e^{1} \frac{d e_{I}}{d s}=-C_{I} e^{1} e_{1}=-C_{I}
\end{aligned}
$$

donc

$$
\frac{d e^{1}}{d s}=-C_{1} e^{1}-\sum_{I=3}^{n+1} C_{I} e^{I}
$$

puis

donc

$$
\begin{gathered}
\frac{d e^{2}}{d s} e_{1}=-e^{2} \frac{d e_{1}}{d s}=-C_{1} e^{2} e_{1}=0 \\
\frac{d e^{2}}{d s} e_{2}=-e^{2} \frac{d e_{2}}{d s}=2 e^{2} \sum_{I=3}^{n+1} C_{I} e_{I}=0 \\
\frac{d e^{2}}{d s} e_{I}=-e^{2} \frac{d e_{I}}{d s}=-C_{I} e^{2} e_{1}=0
\end{gathered}
$$

$$
\frac{d e^{2}}{d s}=0
$$

et enfin

$$
\frac{d e^{I}}{d s} e_{1}=-e^{I} \frac{d e_{1}}{d s}=-C_{1} e^{I} e_{1}=0
$$


donc

$$
\begin{gathered}
\frac{d e^{I}}{d s} e_{2}=-e^{I} \frac{d e_{2}}{d s}=2 e^{I} \sum_{J=3}^{n+1} C_{J} e_{J}=2 C_{I} \\
\frac{d e^{I}}{d s} e_{J}=-e^{I} \frac{d e_{J}}{d s}=-C_{J} e^{I} e_{1}=0
\end{gathered}
$$

$$
\frac{d e^{I}}{d s}=2 C_{I} e^{2}
$$

2.1.2. Identités fondamentales. - Une fois spécifiée la fonction de phase $\theta$, la géométrie du problème est définitivement figée sur $\left[0, t_{0}\right] \times \Sigma$. Il est désormais utile de caractériser les opérateurs linéaires du système par leur action sur des fonctions oscillantes de phase $\theta$. Nous nommons respectivement $\Pi^{y . m .}, \Pi^{c . j}, \Pi^{s c .}$ et $\Pi^{s p .}$ l'ensemble des potentiels de Yang-Mills, des changements de jauge infinitésimaux (0-forme de $\boldsymbol{E}$ à valeurs dans $\mathfrak{g})$, des champs scalaires et des champs de spineurs dépendant de façon $2 \pi$-périodique d'un paramètre réel $\omega$, dont la moyenne en $\omega$ est nulle sur toute période de longueur $2 \pi$. Nous définissons les fibrés

$$
\begin{aligned}
\Pi^{y . m .}{ }_{(\theta / \varepsilon)} & =\left\{\boldsymbol{A}_{\varepsilon}(x)=A\left(x, \frac{\theta(x)}{\varepsilon}\right) \text { où } A(x, \omega) \in \Pi^{y . m .}\right\} \\
\Pi^{c . j .}{ }_{(\theta / \varepsilon)} & =\left\{\boldsymbol{\chi}_{\varepsilon}(x)=\chi\left(x, \frac{\theta(x)}{\varepsilon}\right) \text { où } \chi(x, \omega) \in \Pi^{c . j .}\right\} \\
\Pi^{s c .}{ }_{(\theta / \varepsilon)} & =\left\{\boldsymbol{\Phi}_{\varepsilon}(x)=\phi\left(x, \frac{\theta(x)}{\varepsilon}\right) \text { où } \phi(x, \omega) \in \Pi^{s c .}\right\} \\
\Pi^{s p .}{ }_{(\theta / \varepsilon)} & =\left\{\boldsymbol{\Psi}_{\varepsilon}(x)=\psi\left(x, \frac{\theta(x)}{\varepsilon}\right) \text { où } \psi(x, \omega) \in \Pi^{s p .}\right\}
\end{aligned}
$$

Nous définissons les opérateurs $M$ (d'ordre 1) et $L_{\theta}$ (ordre 0 ), agissant sur les potentiels réguliers de $\Pi^{y \cdot m \cdot}{ }_{(\theta / \varepsilon)}$, par l'identité :

$$
L \boldsymbol{A}_{\varepsilon}(x)=L A\left(x, \frac{\theta(x)}{\varepsilon}\right)+\varepsilon^{-1} M \partial_{\omega} A\left(x, \frac{\theta(x)}{\varepsilon}\right)+\varepsilon^{-2} L_{\theta} \partial_{\omega}^{2} A\left(x, \frac{\theta(x)}{\varepsilon}\right)
$$

Nous définissons l'opérateur $R_{\theta}$ (d'ordre 0) agissant sur les potentiels réguliers de $\Pi^{y \cdot m .}(\theta / \varepsilon)$, par l'identité :

$$
R \boldsymbol{A}_{\varepsilon}(x)=R A\left(x, \frac{\theta(x)}{\varepsilon}\right)+\varepsilon^{-1} R_{\theta} \partial_{\omega} A\left(x, \frac{\theta(x)}{\varepsilon}\right)
$$

Nous définissons l'opérateur $T_{\theta}$ (d'ordre 0) agissant sur les potentiels réguliers de $\Pi^{c . j .}{ }_{(\theta / \varepsilon)}$, par l'identité :

$$
T \chi_{\varepsilon}(x)=T \chi\left(x, \frac{\theta(x)}{\varepsilon}\right)+\varepsilon^{-1} T_{\theta} \partial_{\omega} \chi\left(x, \frac{\theta(x)}{\varepsilon}\right)
$$

Nous définissons l'opérateur $X$ (d'ordre 1) sur les champs réguliers de $\Pi^{s c .}{ }_{\theta / \varepsilon}$ (ou de $\left.\Pi^{c . j .}{ }_{(\theta / \varepsilon)}\right)$ par l'identité :

$$
\square \Phi_{\varepsilon}(x)=\square \phi\left(x, \frac{\theta(x)}{\varepsilon}\right)+\varepsilon^{-1} X \partial_{\omega} \phi\left(x, \frac{\theta(x)}{\varepsilon}\right)
$$


(le coefficient de $\varepsilon^{-2}$ est identiquement nul, puisque $\theta$ vérifie l'équation eïkonale) Nous définissons enfin l'opérateur $\mathcal{D}_{\theta}$ (d'ordre 0) agissant sur les potentiels réguliers de $\Pi^{s p .}{ }_{(\theta / \varepsilon)}$, par l'identité :

$$
\mathcal{D} \Psi_{\varepsilon}(x)=\mathcal{D} \psi\left(x, \frac{\theta(x)}{\varepsilon}\right)+\varepsilon^{-1} \mathcal{D}_{\theta} \partial_{\omega} \psi\left(x, \frac{\theta(x)}{\varepsilon}\right)
$$

nous en déduisons les formules explicites en coordonnées locales :

$$
\begin{aligned}
& X \phi=2 \nabla^{\nu} \theta \nabla_{\nu} \phi+(\square \theta) \phi=2 \frac{d}{d s} \phi+(\square \theta) \phi \\
& \mathcal{D}_{\theta} \psi=\gamma^{\nu} \nabla_{\nu} \theta \psi \\
& R_{\theta} A=\nabla^{\nu} \theta A_{\nu} \\
& T_{\theta} \chi_{\mu}=\nabla_{\mu} \theta \chi \\
& L_{\theta} A_{\mu}=\nabla_{\mu} \theta \nabla^{\nu} \theta A_{\nu} \text { soit } L_{\theta}=T_{\theta} R_{\theta} \\
& M A_{\mu}=2 \nabla^{\nu} \theta \nabla_{\nu} A_{\mu}+\square \theta A_{\mu}-\nabla_{\mu}\left(\nabla^{\nu} \theta A_{\nu}\right)-\nabla_{\mu} \theta \nabla^{\nu} A_{\nu} \\
& \text { soit } \\
& M A=X A-T\left(R_{\theta} A\right)-T_{\theta} R A
\end{aligned}
$$

Si $\boldsymbol{A} \in \Pi^{y \cdot m .}{ }_{(\theta / \varepsilon)}$ et $\Phi \in \Pi^{s c .}{ }_{(\theta / \varepsilon)}$, nous posons :

$$
\begin{gathered}
F^{I}\left(\boldsymbol{A}_{\varepsilon}, \partial \boldsymbol{A}_{\varepsilon}\right)(x)=F^{I}(A, \partial A)\left(x, \frac{\theta(x)}{\varepsilon}\right)+\varepsilon^{-1} F^{I}{ }_{\theta}\left(A, \partial_{\omega} A\right)\left(x, \frac{\theta(x)}{\varepsilon}\right) \\
F^{I I}\left(\Phi_{\varepsilon}, \partial \Phi_{\varepsilon}\right)(x)=F^{I I}(\phi, \partial \phi)\left(x, \frac{\theta(x)}{\varepsilon}\right)+\varepsilon^{-1} F^{I I}{ }_{\theta}\left(\phi, \partial_{\omega} \phi\right)\left(x, \frac{\theta(x)}{\varepsilon}\right) \\
G^{I}\left(\boldsymbol{A}_{\varepsilon}, \partial \Phi_{\varepsilon}\right)(x)=G^{I}(A, \partial \phi)\left(x, \frac{\theta(x)}{\varepsilon}\right)+\varepsilon^{-1} G^{I}{ }_{\theta}\left(A, \partial_{\omega} \phi\right)\left(x, \frac{\theta(x)}{\varepsilon}\right) \\
G^{I I}\left(\partial \boldsymbol{A}_{\varepsilon}, \Phi_{\varepsilon}\right)(x)=G^{I I}(\partial A, \phi)\left(x, \frac{\theta(x)}{\varepsilon}\right)+\varepsilon^{-1} G^{I I}{ }_{\theta}\left(\partial_{\omega} A, \phi\right)\left(x, \frac{\theta(x)}{\varepsilon}\right)
\end{gathered}
$$

Ainsi définis, $F_{\theta}^{I}, F^{I I}{ }_{\theta}, G_{\theta}^{I}$ et $G_{\theta}^{I I}$ sont des formes bilinéaires à coefficients $C^{\infty}$. En coordonnées locales,

$$
\begin{aligned}
F_{\theta}^{I}\left(A, \partial_{\omega} a\right)_{\mu} & =2\left[\nabla_{\nu} \theta \partial_{\omega} a_{\mu}, A^{\nu}\right]+\left[A^{\nu}, \nabla_{\mu} \theta \partial_{\omega} a_{\nu}\right]+\left[A_{\mu}, \nabla^{\nu} \theta \partial_{\omega} a_{\nu}\right] \\
F_{\theta}^{I I}\left(\phi, \partial_{\omega} \varphi\right)_{\mu} & =\bar{\phi}^{t} r^{\prime}(1)^{*}\left(\nabla_{\mu} \theta \partial_{\omega} \varphi\right)+\left(\nabla_{\mu} \theta \partial_{\omega} \bar{\varphi}\right) r^{\prime}(1) \phi \\
G^{I}\left(A, \partial_{\omega} \varphi\right) & =-2 r^{\prime}(1) \cdot A_{\nu} \nabla^{\nu} \theta \partial_{\omega} \varphi \\
G^{I I}\left(\partial_{\omega} a, \phi\right) & =-r^{\prime}(1) \cdot \nabla^{\nu} \theta \partial_{\omega} a_{\nu} \phi
\end{aligned}
$$

Proposition 2.1.1. - Pour tout $s \geqslant \frac{n}{2}+\frac{1}{2}, F_{\theta}^{I}, F^{I I}{ }_{\theta}, G^{I}{ }_{\theta}$ et $G^{I I} \theta$ envoient continûment $H^{s} \times H^{s} \rightarrow H^{s}$ et

$$
\begin{gathered}
\left|F_{\theta}^{I}\left(A, A^{\prime}\right)\right|_{H^{s}} \leqslant C|A|_{H^{s}}\left|A^{\prime}\right|_{H^{s}},\left|F^{I I}{ }_{\theta}\left(\phi, \phi^{\prime}\right)\right|_{H^{s}} \leqslant C|\phi|_{H^{s}}\left|\phi^{\prime}\right|_{H^{s}} \\
\left|G_{\theta}^{I}(A, \phi)\right|_{H^{s}} \leqslant C|A|_{H^{s}}|\phi|_{H^{s}},\left|G^{I I}{ }_{\theta}(A, \phi)\right|_{H^{s}} \leqslant C|\phi|_{H^{s}}|A|_{H^{s}}
\end{gathered}
$$


Démonstration. - Compte tenu des hypothèses 2.1.1, le résultat vient immédiatement du lemme de multiplication B.1.

Reprenons maintenant les identités fondamentales du système en tenant compte des oscillations. L'équation eïkonale (26)

$$
\nabla^{\nu} \theta \nabla_{\nu} \theta=0
$$

donne :

$$
R_{\theta} T_{\theta} \equiv 0
$$

L'identité de Bianchi $R L=\nabla^{\mu}\left(\nabla^{\nu} \nabla_{\nu} \cdot{ }_{\mu}-\nabla_{\mu}\left(\nabla^{\nu}{ }_{\nu}\right)\right) \equiv 0$ se développe, par action sur un potentiel $\boldsymbol{A}_{\varepsilon} \in \Pi^{y \cdot m \cdot}{ }_{(\theta / \varepsilon)}$, de la façon suivante :

$$
\begin{aligned}
R L \boldsymbol{A}_{\varepsilon}(x)= & \varepsilon^{-3} R_{\theta} L_{\theta} \partial_{\omega}^{3} A\left(x, \frac{\theta(x)}{\varepsilon}\right) \\
& +\varepsilon^{-2}\left(R L_{\theta}+R_{\theta} M\right) \partial_{\omega}^{2} A\left(x, \frac{\theta(x)}{\varepsilon}\right) \\
& +\varepsilon^{-1}\left(R M+R_{\theta} L\right) \partial_{\omega} A\left(x, \frac{\theta(x)}{\varepsilon}\right) \\
& +R L A\left(x, \frac{\theta(x)}{\varepsilon}\right) \\
\equiv & 0
\end{aligned}
$$

En exigeant que cette relation reste vraie pour tout $\varepsilon \in] 0,1]$, nous obtenons les identités fondamentales suivantes :

$$
\left\{\begin{array}{l}
R M+R_{\theta} L=0 \\
R L_{\theta}+R_{\theta} M=0 \\
R_{\theta} L_{\theta}=0
\end{array}\right.
$$

L'identité duale $L T=0$ se développe de la même façon et donne lieu à des résultats symétriques

$$
\left\{\begin{array}{l}
M T_{\theta}+L_{\theta} T=0 \\
L T_{\theta}+M T=0 \\
L_{\theta} T_{\theta}=0
\end{array}\right.
$$

L'identité $\mathcal{D}^{2}=\square \operatorname{Id}_{K}+\frac{1}{4} \operatorname{tr}(\mathcal{R} i c$. ) fournit quant à elle

$$
\left\{\begin{array}{l}
\mathcal{D}_{\theta}^{2}=0(\text { eïkonale }) \\
\mathcal{D D}_{\theta}+\mathcal{D}_{\theta} \mathcal{D}=X
\end{array}\right.
$$


2.1.3. Géométrie des oscillations. - Les hypothèses 2.1.1 sur la fonction de phase $\theta$ nous garantissent que les opérateurs $L_{\theta}, R_{\theta}$ et $T_{\theta}$ sont de rang constant sur tout $\left[0, t_{0}\right]$.

Nous aurons besoin par la suite de décomposer le noyau et l'image de l'opérateur $L_{\theta}$ dans $\Pi^{y \cdot m \cdot}{ }_{(\theta / \varepsilon)}$. Puisque $L_{\theta}=T_{\theta} R_{\theta}$,

$$
\operatorname{Ker} L_{\theta}=\operatorname{Ker} R_{\theta} \quad \text { et } \quad \operatorname{Im} L_{\theta}=\operatorname{Im} T_{\theta}
$$

Par ailleurs, sur $\boldsymbol{T}(\mathbb{R} \times \boldsymbol{\Sigma})$, nous identifions

$$
\operatorname{Ker} R_{\theta}=\mathcal{E}^{\#} \quad \text { et } \quad \operatorname{Im} T_{\theta}=\mathcal{E}^{\wedge}
$$

Nous en déduisons les fibrés :

$$
\begin{aligned}
\Pi_{\#} & =\operatorname{Ker} R_{\theta} \cap \Pi^{y \cdot m \cdot}(\theta / \varepsilon) \\
& =\left\{\boldsymbol{A} \in \Pi^{y \cdot m \cdot}{ }_{(\theta / \varepsilon)} \mid A_{0}=\frac{\alpha^{2}}{\nabla_{0} \theta} g_{\Sigma}\left(\nabla_{\Sigma} \theta, A_{\Sigma}\right)\right\} \\
& =\mathcal{E}^{\#} \cap \Pi^{y \cdot m \cdot}{ }_{(\theta / \varepsilon)}
\end{aligned}
$$

et

$$
\begin{aligned}
\Pi_{\wedge} & =\operatorname{Im} T_{\theta} \cap \Pi^{y \cdot m \cdot}{ }_{(\theta / \varepsilon)} \\
& =\left\{\boldsymbol{A} \in \Pi^{y \cdot m \cdot}{ }_{(\theta / \varepsilon)} \mid A_{\Sigma}=\frac{A_{0}}{\nabla_{0} \theta} \nabla_{\Sigma} \theta\right\} \\
& =\mathcal{E}^{\wedge} \cap \Pi^{y \cdot m \cdot}{ }_{(\theta / \varepsilon)}
\end{aligned}
$$

Compte tenu de l'équation eïkonale $R_{\theta} T_{\theta} \equiv 0$, nous vérifions bien entendu l'inclusion

$$
\Pi_{\wedge} \subset \Pi_{\#}
$$

Nous définissons de même les fibrés :

$$
\begin{aligned}
\Pi_{0} & =\mathcal{E}^{0} \cap \Pi^{y \cdot m \cdot}(\theta / \varepsilon) \\
& =\left\{\boldsymbol{A} \in \Pi^{y \cdot m \cdot}(\theta / \varepsilon) \mid A_{0}=0 \text { et } g_{\Sigma}\left(A_{\Sigma}, \nabla_{\Sigma} \theta\right)=0\right\}
\end{aligned}
$$

et

$$
\begin{aligned}
\Pi_{\vee} & =\mathcal{E}^{\vee} \cap \Pi^{y \cdot m \cdot}{ }_{(\theta / \varepsilon)} \\
& =\left\{\boldsymbol{A} \in \Pi^{y \cdot m .}{ }_{(\theta / \varepsilon)} \mid A_{\Sigma}=-\frac{A_{0}}{\nabla_{0} \theta} \nabla_{\Sigma} \theta\right\}
\end{aligned}
$$

d'où les décompositions :

$$
\Pi_{(\theta / \varepsilon)}^{y \cdot m \cdot}=\Pi_{\#} \oplus \Pi_{\vee} \quad \text { et } \quad \Pi_{\#}=\Pi_{\wedge} \oplus \Pi_{0}
$$

soit en définitive :

$$
\Pi^{y \cdot m \cdot}{ }_{(\theta / \varepsilon)}=\Pi_{\wedge} \oplus \Pi_{0} \oplus \Pi_{\vee}
$$

Remarquons enfin que

$$
\frac{\alpha^{2}}{2 \nabla_{0} \theta} R_{\theta} A=\frac{\alpha^{2}}{2 \nabla_{0} \theta}\left(\alpha^{-2} \nabla_{0} \theta A_{0}+\frac{A_{0}}{\nabla_{0} \theta} g_{\Sigma}\left(\nabla_{\Sigma} \theta, \nabla_{\Sigma} \theta\right)\right)=A_{0}
$$


donc

on peut encore écrire

$$
\pi^{\vee}=\frac{\alpha^{2}}{2\left(\nabla_{0} \theta\right)^{2}}\left(\begin{array}{c}
\nabla_{0} \theta \\
-\nabla_{\Sigma} \theta
\end{array}\right) R_{\theta}
$$

$$
\pi^{\vee}=L_{\theta}^{-1} L_{\theta}=\left(L_{\theta}^{-1} T_{\theta}\right) R_{\theta}=0
$$

La proposition suivante résume l'essentiel des résultats géométriques dont nous aurons besoin par la suite

Proposition 2.1.2. - a) $\pi^{\vee} X \pi^{\vee}-X_{\left.\right|_{\Pi_{\vee}}}$ et $\pi^{0} X \pi^{\vee}$ sont des opérateurs linéaires d'ordre 0 tandis que $\pi^{\wedge} X \pi^{\vee}=0$.

b) $\pi^{\vee} X \pi^{0}=0$ tandis que $\pi^{0} X \pi^{0}=X_{\left.\right|_{\Pi_{0}}}$ et que $\pi^{\wedge} X \pi^{0}$ est un opérateur linéaire d'ordre 0 .

c) $\pi^{\vee} X \pi^{\wedge}=0$ et $\pi^{0} X \pi^{\wedge}=0$ tandis que $\pi^{\wedge} X \pi^{\wedge}=X_{\left.\right|_{\Pi_{\wedge}}}$.

d) il en résulte entre autres que

$$
\pi^{0} M \pi^{0}=X_{\left.\right|_{\Pi_{0}}}
$$

tandis que

$$
\pi^{\vee} M \pi^{\#}=0 \quad \text { et } \quad \pi^{0} M \pi^{\wedge}=0
$$

Démonstration. - Nous nous référons localement aux coordonnées radiatives $\left(e^{1}, e^{2}\right)$ et $\left(e^{I}\right), I=3, \ldots, n+1$ telles que nous les avons construites précédemment.

a) Pour tout $A \in \Pi^{y \cdot m .}{ }_{(\theta / \varepsilon)}$,

$$
\pi^{\vee} A=A_{1} e^{1}
$$

et compte tenu de (32), il en résulte que

$$
\begin{aligned}
X\left(\pi^{\vee} A\right) & =\left(X A_{1}\right) e^{1}+2 A_{1} \frac{d}{d s} e^{1} \\
& =\left(X A_{1}-2 C_{1} A_{1}\right) e^{1}-2 A_{1} \sum_{I=3}^{n+1} C_{I} e^{I}
\end{aligned}
$$

Il vient immédiatement que $\pi^{\wedge} X \pi^{\vee}=0$. Puisque $X_{\Pi_{\vee}} A=\left(X A_{1}\right) e^{1}$ il apparaît clairement que $\pi^{\vee} X \pi^{\vee} A-X_{\left.\right|_{\vee}} A=-2 C_{1} A_{1} e^{1}$ est d'ordre 0 en $\mathrm{A}$, de même que $\pi^{0} X \pi^{\vee}=-2 A_{1} \sum C_{I} e^{I}$.

b) Pour tout $A \in \Pi^{y \cdot m .}(\theta / \varepsilon)$ :

$$
\pi^{0} A=\sum_{I=3}^{n+1} A_{I} e^{I}
$$

compte tenu de (34), il en résulte que

$$
\begin{aligned}
X\left(\pi^{0} A\right) & =\sum_{I=3}^{n+1}\left(X A_{I}\right) e^{I}+\sum_{I=3}^{n+1} A_{I}\left(2 \frac{d}{d s} e^{I}\right) \\
& =\sum_{I=3}^{n+1}\left(X A_{I}\right) e^{I}+\left(\sum_{I=3}^{n+1} 4 C_{I} A_{I}\right) e^{2}
\end{aligned}
$$


donc $\pi^{0} X \pi^{0} A=\sum_{I=3}^{n+1}\left(X A_{I}\right) e^{I}$ et en définitive $\pi^{0} X \pi^{0}=X_{\left.\right|_{\Pi_{0}}}$. On montre de la même façon que $\pi^{\vee} X \pi^{0}=0$ tandis que $\pi^{\wedge} X \pi^{0}=\left(\sum 4 C_{I} A_{I}\right) e^{2}$ est d'ordre 0 en A.

c) Pour tout $A \in \Pi^{y \cdot m \cdot}(\theta / \varepsilon)$,

$$
\pi^{\wedge} A=A_{2} e^{2}
$$

et compte tenu de (32), il en résulte que

$$
\begin{aligned}
X\left(\pi^{\wedge} A\right) & =\left(X A_{2}\right) e^{2}+2 A_{2} \frac{d}{d s} e^{2} \\
& =\left(X A_{2}\right) e^{2}
\end{aligned}
$$

Il vient immédiatement que $\pi^{\vee} X \pi^{\wedge}=0$ et $\pi^{0} X \pi^{\wedge}=0$, puis que $\pi^{\wedge} X \pi^{\wedge}=$ $\left(X A_{2}\right) e^{2}=X_{\left.\right|_{\Pi \wedge}} A$.

d) $\quad M=X-T R_{\theta}-R T_{\theta} \quad$ et $\quad \pi^{\vee} T_{\theta}=\pi^{0} T_{\theta}=R_{\theta} \pi^{0}=R_{\theta} \pi^{\wedge}=0$

donc

$$
\begin{aligned}
\pi^{0} M \pi^{0} & =\pi^{0} X \pi^{0}=X_{\left.\right|_{\Pi \vee}} \\
\pi^{0} M \pi^{\wedge} & =\pi^{0} X \pi^{\wedge}=0 \\
\pi^{\vee} M \pi^{\wedge} & =\pi^{\vee} X \pi^{\wedge}=0 \\
\pi^{\vee} M \pi^{0} & =\pi^{\vee} X \pi^{0}=0
\end{aligned}
$$

ce qui achève la preuve.

Il est également utile de décrire $\operatorname{Ker} \mathcal{D}_{\theta}$ et $\operatorname{Im} \mathcal{D}_{\theta}$ dans $\Pi^{s p .}{ }_{(\theta / \varepsilon)}$.

$$
\mathcal{D}_{\theta}=\gamma^{0}\left(\nabla_{0} \theta-\alpha^{2} \gamma^{0}\left(\mathcal{D}_{\Sigma}\right)_{\theta}\right)
$$

où $\left(\mathcal{D}_{\Sigma}\right)_{\theta}=-\gamma^{i} \nabla_{i} \theta$ vérifie $\left(\mathcal{D}_{\Sigma}\right)_{\theta}{ }^{2}=\left|\nabla_{0} \theta\right|^{2} \operatorname{Id}_{K}$. Compte tenu de $(7)$, il vient

$$
\left(\alpha^{2} \gamma^{0}\left(\mathcal{D}_{\Sigma}\right)_{\theta}\right)^{2}=\alpha^{2}\left|\nabla_{0} \theta\right| \operatorname{Id}_{K}
$$

et les valeurs propres de $\alpha^{2} \gamma^{0}\left(\mathcal{D}_{\Sigma}\right)_{\theta}$ sont donc $\pm \alpha\left|\nabla_{0} \theta\right|$. Comme ${ }^{t}\left(\gamma^{i}\right)^{*}=-\gamma^{i}$, il vient par ailleurs ${ }^{t}\left(\mathcal{D}_{\Sigma}\right)_{\theta}^{*}=-\left(\mathcal{D}_{\Sigma}\right)_{\theta}$, et puisque ${ }^{t}\left(\gamma^{0}\right)^{*}=\gamma^{0}$, nous obtenons

$$
{ }^{t}\left(\alpha^{2} \gamma^{0}\left(\mathcal{D}_{\Sigma}\right)_{\theta}\right)^{*}=-\left(\alpha^{2} \gamma^{0}\left(\mathcal{D}_{\Sigma}\right)_{\theta}\right)
$$

Les deux sous-espaces propres $S^{ \pm}$associés respectivement aux valeurs propres $\pm \alpha\left|\nabla_{\Sigma} \theta\right|$ sont orthogonaux et de même dimension $K / 2$. Puisque $\theta$ vérifie $\nabla_{0} \theta=$ $\alpha\left|\nabla_{\Sigma} \theta\right|$, on en déduit que $\operatorname{Ker} \mathcal{D}_{\theta}=S^{+}$et $\operatorname{Im} \mathcal{D}_{\theta}=\gamma^{0} S^{-}$. L'identité $\mathcal{D}_{\theta}^{2}=0$ issue de l'eïkonale impose $\operatorname{Im} \mathcal{D}_{\theta} \subset \operatorname{Ker} \mathcal{D}_{\theta}$. Il en résulte que

$$
\operatorname{Im} \mathcal{D}_{\theta}=\operatorname{Ker} \mathcal{D}_{\theta}=S^{+}=\gamma^{0} S^{-}
$$

Nous posons

$$
\Pi_{+}=\Pi^{s p .}{ }_{(\theta / \varepsilon)} \cap S^{+} \text {et } \Pi_{-}=\Pi^{s p .}{ }_{(\theta / \varepsilon)} \cap S^{-}
$$

de sorte que

$$
\Pi_{(\theta / \varepsilon)}^{s p \cdot}=\Pi_{+} \oplus \Pi_{-}
$$

Nous nommons

$$
\pi^{+}=\frac{\alpha^{2}}{2 \nabla_{0} \theta} \mathcal{D}_{\theta} \gamma^{0} \quad \text { et } \quad \pi^{-}=\frac{\alpha^{2}}{2 \nabla_{0} \theta} \gamma^{0} \mathcal{D}_{\theta}
$$


Il est clair que $\operatorname{Ker} \pi^{-}=\operatorname{Ker} \mathcal{D}_{\theta}=S^{+}$et que $\operatorname{Im} \pi^{+}=\operatorname{Im} \mathcal{D}_{\theta}=S^{+}$. En utilisant (7), il est facile de vérifier que

$$
\begin{aligned}
\pi^{+}+\pi^{-} & =\frac{\alpha^{2}}{2 \nabla_{0} \theta}\left(2 \gamma^{0} \gamma^{0} \nabla_{0} \theta+\left(\gamma^{0} \gamma^{i}+\gamma^{i} \gamma^{0}\right) \nabla_{i} \theta\right) \\
& =\frac{\alpha^{2}}{2 \nabla_{0} \theta}\left(2 \alpha^{-2} \nabla_{0} \theta+0\right) \\
& =\operatorname{Id}_{K}
\end{aligned}
$$

On voit également que

$$
\pi^{+} \pi^{-}=\left(\frac{\alpha^{2}}{2 \nabla_{0} \theta}\right)^{2} \gamma^{0} \mathcal{D}_{\theta}^{2} \gamma^{0}=0 \quad \text { et } \quad \pi^{-} \pi^{+}=\left(\frac{\alpha^{2}}{2 \nabla_{0} \theta}\right)^{2} \mathcal{D}_{\theta} \gamma^{0} \gamma^{0} \mathcal{D}_{\theta}=\left(\frac{1}{2 \nabla_{0} \theta}\right)^{2} \mathcal{D}_{\theta}^{2}=0
$$

Nous en déduisons

$$
\left(\pi^{ \pm}\right)^{2}=\pi^{ \pm}\left(\operatorname{Id}_{K}-\pi^{\mp}\right)=\pi^{ \pm}
$$

Enfin

$$
\begin{aligned}
{ }^{t}\left(\pi^{+}\right)^{*} & =\frac{\alpha^{2}}{2 \nabla_{0} \theta} \alpha \gamma^{0} \gamma^{0}\left(\alpha \gamma^{0}\right)^{-1} \alpha \gamma^{0} \gamma^{\mu} \nabla_{\mu} \theta\left(\alpha \gamma^{0}\right)^{-1} \\
& =\frac{\alpha^{2}}{2 \nabla_{0} \theta} \alpha^{-1} \gamma^{\mu} \nabla_{\mu} \theta \alpha \gamma^{0} \\
& =\pi^{+}
\end{aligned}
$$

un calcul analogue donne

$$
{ }^{t}\left(\pi^{-}\right)^{*}=\pi^{-}
$$

Il en résulte que $\pi^{ \pm}$forment une paire de projecteurs orthogonaux, respectivement, $\operatorname{sur} \Pi_{ \pm}$

Proposition 2.1.3. - $\quad \pi^{-} \mathcal{D} \pi^{+}=\frac{\alpha^{2}}{2 \nabla_{0} \theta} \gamma^{0} X \pi^{+}$ ou, d'une façon équivalente

$$
X \pi^{+}=\mathcal{D}_{\theta} \mathcal{D} \pi^{+}
$$

Démonstration. - Comme $\mathcal{D}_{\theta} \pi^{+}=0$ et que $\frac{\alpha^{2}}{2 \nabla_{0} \theta} \gamma^{0} \mathcal{D}_{\theta}=\pi^{-}$, il suffit alors de composer l'identité (38) à droite par $\pi^{+}$et gauche par $\frac{\alpha^{2}}{2 \nabla_{0} \theta} \gamma^{0}$ pour obtenir

$$
\frac{\alpha^{2}}{2 \nabla_{0} \theta} \gamma^{0} X \pi^{+}=\frac{\alpha^{2}}{2 \nabla_{0} \theta} \gamma^{0} \mathcal{D}_{\theta} \mathcal{D} \pi^{+}+\frac{\alpha^{2}}{2 \nabla_{0} \theta} \gamma^{0} \mathcal{D} \mathcal{D}_{\theta} \pi^{+}=\pi^{-} \mathcal{D} \pi^{+}
$$

Comme $\pi^{-} \mathcal{D}_{\theta}=0$, l'identité (38) donne également

$$
\pi^{-} X \pi^{+}=\pi^{-} \mathcal{D}_{\theta} \mathcal{D} \pi^{+}+\pi^{-} \mathcal{D} \mathcal{D}_{\theta} \pi^{+}=0
$$

il en résulte que $\Pi_{+}$est stable sous l'action de $X$

$$
\pi^{+} X \pi^{+}=X \pi^{+}=X_{\left.\right|_{\Pi^{+}}}+\left(X \frac{\alpha^{2}}{2 \nabla_{0} \theta} \mathcal{D}_{\theta} \gamma^{0}\right)
$$

Il vient alors

$$
\frac{\alpha^{2}}{2 \nabla_{0} \theta} \gamma^{0} X \pi^{+}=\pi^{-} \mathcal{D} \pi^{+}
$$


Examinons maintenant la façon dont l'invariance de jauge se manifeste sur la structure des fonctions $F^{I}{ }_{\theta}, G^{I}{ }_{\theta}$ et $G^{I I}{ }_{\theta}$.

Proposition 2.1.4. - a) Pour tout couple $\left(A_{0}, \phi_{0}\right)(x)$ de fonctions régulières nonoscillantes, pour toute fonction $a^{\wedge} \in \Pi_{\wedge}$ :

$$
F_{\theta}^{I}\left(A_{0}, a^{\wedge}\right) \in \Pi_{\wedge} \quad \text { et } \quad G^{I I}{ }_{\theta}\left(a^{\wedge}, \phi_{0}\right)=0
$$

b) Pour tout couple $\left(A_{1}^{\#}, \phi_{1}\right)(x, \omega)$ de fonctions régulières $2 \pi$-périodiques en $\omega$ avec $A_{1}^{\#} \in \Pi_{\#}$, pour toute fonction $a^{\wedge} \in \Pi_{\wedge}$ :

$$
F^{I}{ }_{\theta}\left(A_{1}^{\#}, \partial_{\omega} a^{\wedge}\right)+F^{I}{ }_{\theta}\left(a^{\wedge}, \partial_{\omega} A_{1}^{\#}\right)=0 \quad \text { et } \quad G^{I}{ }_{\theta}\left(a^{\wedge}, \partial_{\omega} \phi_{1}\right)+G^{I I}{ }_{\theta}\left(\partial_{\omega} a^{\wedge}, \phi_{1}\right)=0
$$

Ces identités traduisent en termes d'oscillations la compatibilité interne des nonlinéarités dominantes du système avec l'invariance de jauge.

Démonstration. - Considérons un changement de jauge oscillant $\chi_{\varepsilon} \in \Pi^{c . j .}{ }_{(\theta / \varepsilon)}$, ainsi que des fonctions $(A, \phi, \psi)_{\varepsilon}=(A, \phi, \psi)(x, \theta(x) / \varepsilon)$, où $(A, \phi, \psi)(x, \omega)$ sont régulières et $2 \pi$-périodique en $\omega$, éventuellement constantes en $\omega$. Pour ces fonctions, nous développons l'identité (13) en puissances de $\varepsilon$, et ne gardons que les termes dominants d'ordre $\varepsilon^{-2}$. Nous obtenons l'identité suivante :

$$
F^{I}{ }_{\theta}\left(A, T_{\theta} \partial_{\omega}^{2} \chi\right)+F_{\theta}^{I}\left(T_{\theta} \partial_{\omega} \chi, \partial_{\omega} A\right)=L_{\theta}\left(\partial_{\omega}^{2}[A, \chi]-\left[\partial_{\omega}^{2} A, \chi\right]\right)
$$

En développant de la même façon l'identité (14) et en gardant uniquement les termes d'ordre $\varepsilon^{-2}$, il vient, puisque $\theta$ vérifie l'équation eïkonale (26) associée à la métrique $g$ :

$$
G_{\theta}^{I}\left(T_{\theta} \partial_{\omega} \chi, \partial_{\omega} \phi\right)+G_{\theta}^{I I}\left(T_{\theta} \partial_{\omega}^{2} \chi, \phi\right)=0
$$

Il suffit alors de remarquer que tout élément de $\Pi_{\wedge}=\operatorname{Im} T_{\theta} \cap \Pi^{y \cdot m .}{ }_{(\theta / \varepsilon)}$ peut s'écrire $a^{\wedge}=T_{\theta} \partial_{\omega} \chi$. Le a) vient en posant $A=A_{0}, \phi=\phi_{0}$, vérifiant $\partial_{\omega} A_{0}=0$ et $\partial_{\omega} \phi_{0}=0$, tandis que le b) s'obtient en prenant $A=A_{1}^{\#} \in \operatorname{Ker} L_{\theta}$ et $\phi=\phi_{1}$. Nous aurions pu donner ces formules par calcul explicite en coordonnées locales, mais il nous a paru intéressant de montrer qu'il s'agit d'une conséquence directe des règles de changement

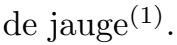

\subsection{Recherche d'équations de profils à jauge libre}

La phase des oscillations étant fixée, nous cherchons maintenant à préciser les équations que doivent vérifier les profils $\left(A_{i}, \phi_{i}, \psi_{i}\right)$ pour qu'une solution oscillante $(A, \phi, \psi)_{\varepsilon}$ de la forme $(25)$ vérifie le système (Y.M.) à un ordre $\varepsilon^{M / 2}$ quelconque, indépendamment des conditions de jauge retenues.

\footnotetext{
(1) Nous souhaitons mener à bien l'analyse du système en s'appuyant uniquement sur l'invariance des équations. Dans un premier temps, nous détaillons le moins possible l'écriture des termes non linéaires, notre objectif étant d'abord d'arriver à justifier nos développements. Nous revenons sur l'expression exacte des premiers termes dans notre conclusion, une fois assurés qu'ils correspondent bien à la partie dominante d'une vraie solution du système
} 
- Pour ce faire, nous commençons par développer en puissances de $\varepsilon$ le système $(Y . M$.$) appliqué à (A, \phi, \psi)_{\varepsilon}$. Ce développement primaire à pour but de révéler une relation de dépendance causale entre les profils d'ordre croissant, et nous conduit à une succession d'équations de profils à jauge libre.

- Nous entreprenons alors de développer les identités de compatibilité de (Y.M.), et de réduire les équations de profils en les quotientant par les composantes oscillantes du champ $A_{\varepsilon}$ qui correspondent à des oscillations de la jauge. Nous séparons ainsi les composantes véritablement dynamiques (transportées le long des rayons de lumière) des composantes libres (partie sous déterminée du système) ou polarisées (partie surdéterminée du système) des oscillations.

- Nous finissons la section en donnant un analogue de la proposition (1.3.5) pour chaque système d'équations de profils, qui deviennent du coup exploitables.

Les résultats de cette section sont donnés pour des profils que nous supposons réguliers (au sens de la section 1.3.1). Nous vérifierons dans la section suivante l'existence de tels profils.

2.2.1. Développement primaire. — Le développement primaire du système nécessite trois étapes :

a) Développement de Taylor du système. - La première étape consiste à faire un développement de Taylor du système en puissances de $\varepsilon$. Nous dirons que $(A, \phi, \psi)_{\varepsilon}$ est une famille de solutions approchées de (Y.M.) à l'ordre $O\left(\varepsilon^{M / 2}\right)$ si et seulement si

$$
\left\{\begin{array}{l}
\mathcal{O}_{\varepsilon}=L A_{\varepsilon}-F\left(A_{\varepsilon}, \phi_{\varepsilon}, \psi_{\varepsilon}\right)=\varepsilon^{M / 2} \sum_{k \geqslant 0} \varepsilon^{i / 2} \mathcal{O}_{k}\left(x, \frac{\theta(x)}{\varepsilon}\right) \\
\mathcal{P}_{\varepsilon}=\square \phi_{\varepsilon}-G\left(A_{\varepsilon}, \phi_{\varepsilon}, \psi_{\varepsilon}\right)=\varepsilon^{M / 2} \sum_{k \geqslant 0} \varepsilon^{i / 2} \mathcal{P}_{k}\left(x, \frac{\theta(x)}{\varepsilon}\right) \\
\mathcal{Q}_{\varepsilon}=\mathcal{D} \psi_{\varepsilon}-h\left(A_{\varepsilon}, \phi_{\varepsilon}, \psi_{\varepsilon}\right)=\varepsilon^{M / 2} \sum_{k \geqslant 0} \varepsilon^{i / 2} \mathcal{Q}_{k}\left(x, \frac{\theta(x)}{\varepsilon}\right)
\end{array}\right.
$$

ou, de façon équivalente, si chaque coefficient de $\varepsilon^{i / 2},-3 \leqslant i<M$ est nul dans le développement de Taylor du système. Un calcul explicite nous fournit les expressions suivantes :

Terme d'ordre $\varepsilon^{-3 / 2}$ :

$$
L_{\theta} \partial_{\omega}^{2} A_{1}=0
$$

Terme d'ordre $\varepsilon^{-1}$ :

$$
\left\{\begin{array}{l}
L_{\theta} \partial_{\omega}^{2} A_{2}=0 \\
\mathcal{D}_{\theta} \partial_{\omega} \psi_{0}=0
\end{array}\right.
$$

Terme d'ordre $\varepsilon^{-1 / 2}$ :

$$
\left\{\begin{aligned}
L_{\theta} \partial_{\omega}^{2} A_{3}+M \partial_{\omega} A_{1} & =F^{I}{ }_{\theta}\left(A_{0}, \partial_{\omega} A_{1}\right)+F^{I I}{ }_{\theta}\left(\phi_{0}, \partial_{\omega} \phi_{1}\right) \\
X \partial_{\omega} A_{1} & =G^{I}{ }_{\theta}\left(\phi_{0}, \partial_{\omega} A_{1}\right)+G^{I I}{ }_{\theta}\left(A_{0}, \partial_{\omega} \phi_{1}\right) \\
\mathcal{D}_{\theta} \partial_{\omega} \psi_{1} & =0
\end{aligned}\right.
$$


Terme d'ordre $\varepsilon^{0}$ :

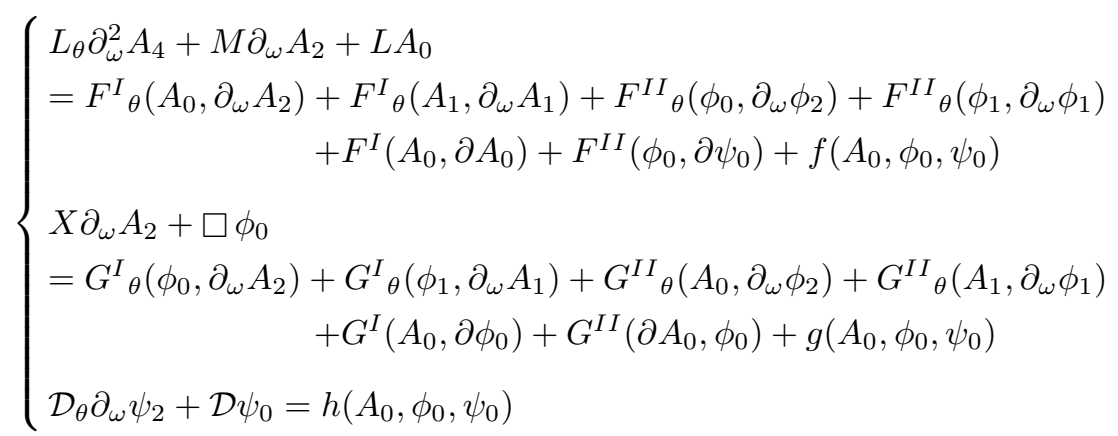

puis, pour les indices $1 \leqslant i<M$, les terme d'ordre $\varepsilon^{i / 2}$ sont de la forme :

$$
\left\{\begin{array}{l}
L_{\theta} \partial_{\omega}^{2} A_{i+4}+M \partial_{\omega} A_{i+2}+L A_{i} \\
=F^{I}{ }_{\theta}\left(A_{0}, \partial_{\omega} A_{i+2}\right)+F^{I}{ }_{\theta}\left(A_{i+1}, \partial_{\omega} A_{1}\right)+F_{\theta}^{I}\left(A_{1}, \partial_{\omega} A_{i+1}\right) \\
+F^{I I}{ }_{\theta}\left(\phi_{0}, \partial_{\omega} \phi_{i+2}\right)+F^{I I}{ }_{\theta}\left(\phi_{i+1}, \partial_{\omega} \phi_{1}\right)+F^{I I}{ }_{\theta}\left(\phi_{1}, \partial_{\omega} \phi_{i+1}\right) \\
+F^{I}\left(A_{i}, \partial A_{0}\right)+F^{I I}\left(\phi_{i}, \partial \psi_{0}\right)+F^{I}\left(A_{0}, \partial A_{i}\right)+F^{I I}\left(\phi_{0}, \partial \psi_{i}\right) \\
+f_{0}^{\prime} \cdot\left(A_{i}, \phi_{i}, \psi_{i}\right)+\boldsymbol{F}_{i}\left(A_{k}, \partial A_{k}, \partial_{\omega} A_{k+1}, \phi_{k}, \partial \phi_{k}, \partial_{\omega} \phi_{k+1}, \psi_{k}, k \leqslant i-1\right) \\
X \partial_{\omega} \phi_{i+2}+\square \phi_{i} \\
=G^{I}{ }_{\theta}\left(\phi_{0}, \partial_{\omega} A_{i+2}\right)+G_{\theta}^{I}\left(\phi_{i+1}, \partial_{\omega} A_{1}\right)+G^{I}{ }_{\theta}\left(\phi_{1}, \partial_{\omega} A_{i+1}\right) \\
+G^{I I}{ }_{\theta}\left(A_{0}, \partial_{\omega} \phi_{i+2}\right)+G^{I I}{ }_{\theta}\left(A_{i+1}, \partial_{\omega} \phi_{1}\right)+G^{I I}{ }_{\theta}\left(A_{1}, \partial_{\omega} \phi_{i+1}\right) \\
+G^{I}\left(A_{0}, \partial \phi_{i}\right)+G^{I I}\left(\partial A_{0}, \phi_{i}\right)+G^{I}\left(A_{i}, \partial \phi_{0}\right)+G^{I I}\left(\partial A_{i}, \phi_{0}\right) \\
+g_{0}^{\prime} \cdot\left(A_{i}, \phi_{i}, \psi_{i}\right)+\boldsymbol{G}_{i}\left(A_{k}, \partial A_{k}, \partial_{\omega} A_{k+1}, \phi_{k}, \partial \phi_{k}, \partial_{\omega} \phi_{k+1}, \psi_{k}, k \leqslant i-1\right) \\
\mathcal{D}_{\theta} \partial_{\omega} \psi_{i+2}+\mathcal{D} \psi_{i}=h_{0}^{\prime} \cdot\left(A_{i}, \phi_{i}, \psi_{i}\right)+\boldsymbol{H}_{i}\left(A_{k}, \phi_{k}, \psi_{k}, k \leqslant i-1\right)
\end{array}\right.
$$

$\boldsymbol{F}_{i}, \boldsymbol{G}_{i}$ et $\boldsymbol{H}_{i}$ désignent des fonctions polynomiales à coefficients $C^{\infty}$, qui regroupent les termes de plus bas indices. Nous avons noté

$$
f_{0}^{\prime} \cdot\left(A_{i}, \phi_{i}, \psi_{i}\right)=\frac{\partial f}{\partial A}\left(A_{0}, \phi_{0}, \psi_{0}\right) A_{i}+\frac{\partial f}{\partial \phi}\left(A_{0}, \phi_{0}, \psi_{0}\right) \phi_{i}+\frac{\partial f}{\partial \psi}\left(A_{0}, \phi_{0}, \psi_{0}\right) \psi_{i}
$$

qui est donc un terme linéaire en $\left(A_{i}, \phi_{i}, \psi_{i}\right)$. Définitions similaires pour $g_{0}^{\prime}$ et $h_{0}^{\prime}$.

b) Séparation des parties moyennes et oscillantes du développement. — La deuxième étape consiste consiste à décomposer chacun des termes précédents en sa partie moyenne et sa partie oscillante. Notons $\sim$ ou Moy $(\cdot)$ la partie moyenne d'un terme donné, $2 \pi$-périodique en la variable $\omega: \widetilde{u}(x)=\int_{0}^{2 \pi} u(x, \omega) d \omega$. La partie purement oscillante (de moyenne nulle) sera désignée par $\star$ ou $O s c(\cdot)$ et définie par $: \stackrel{\star}{u}(x, \omega)=$ $u(x, \omega)-\widetilde{u}(x)$, la décomposition obtenue est univoque. Rappelons que les fonctions $A_{0}$ et $\phi_{0}$ sont des fonctions non-oscillantes, i.e. $\stackrel{\star}{A}_{0}=0$ et $\stackrel{\star}{\phi_{0}}=0$, tandis que $\psi_{0}$ a 
une partie moyenne et une partie oscillante. Rappelons enfin que $\partial_{\omega}$ est inversible sur l'ensemble des fonctions $2 \pi$-périodiques de moyenne nulle. Le noyau de $\partial_{\omega}$ est bien sûr l'ensemble des fonctions moyennes.

Nous dirons que $(A, \phi, \psi)_{\varepsilon}$ est une famille de solutions approchées de (Y.M.) à l'ordre $O\left(\varepsilon^{M / 2}\right)$ en partie moyenne, à l'ordre $O\left(\varepsilon^{(M-1) / 2}\right)$ en partie oscillante sur les $\left(A_{i}, \phi_{i}\right)$, à l'ordre $O\left(\varepsilon^{M / 2}\right)$ en partie oscillante sur les $\left(\psi_{i}\right)$, si et seulement si

$$
\left\{\begin{array}{l}
\mathcal{O}_{\varepsilon}=L A_{\varepsilon}-F\left(A_{\varepsilon}, \phi_{\varepsilon}, \psi_{\varepsilon}\right)=\varepsilon^{M / 2} \sum_{i \geqslant 0} \varepsilon^{i / 2}\left(\varepsilon^{-1 / 2} \stackrel{\star}{\mathcal{O}}_{i-1}\left(x, \frac{\theta(x)}{\varepsilon}\right)+\widetilde{\mathcal{O}}_{i}(x)\right) \\
\mathcal{P}_{\varepsilon}=\square \phi_{\varepsilon}-G\left(A_{\varepsilon}, \phi_{\varepsilon}, \psi_{\varepsilon}\right)=\varepsilon^{M / 2} \sum_{0 \leqslant i \leqslant M^{\prime}} \varepsilon^{i / 2}\left(\varepsilon^{-1 / 2} \stackrel{\star}{\mathcal{P}}_{i-1}\left(x, \frac{\theta(x)}{\varepsilon}\right)+\widetilde{\mathcal{P}}_{i}(x)\right) \\
\mathcal{Q}_{\varepsilon}=\mathcal{D} \psi_{\varepsilon}-h\left(A_{\varepsilon}, \phi_{\varepsilon}, \psi_{\varepsilon}\right)=\varepsilon^{M / 2} \sum_{0 \leqslant i \leqslant M^{\prime}} \varepsilon^{i / 2}\left(\widetilde{\mathcal{Q}}_{i}(x)+\stackrel{\star}{\mathcal{Q}}_{i}\left(x, \frac{\theta(x)}{\varepsilon}\right)\right)
\end{array}\right.
$$

ou, de façon équivalente, si les systèmes suivants sont satisfaits :

$$
\left\{\begin{array}{c}
L_{\theta} \partial_{\omega}^{2} \stackrel{\star}{A_{1}}=0 \\
\mathcal{D}_{\theta} \partial_{\omega}{\stackrel{\star}{\psi_{0}}}^{2}=0
\end{array}\right.
$$

$$
\left\{\begin{array}{c}
L_{\theta} \partial_{\omega}^{2} \stackrel{\star}{A_{2}}=0 \\
\mathcal{D}_{\theta} \partial_{\omega} \stackrel{\star}{\psi_{1}}=0
\end{array}\right.
$$

(que nous interpréterons comme des conditions de polarisation des premiers termes oscillants), puis

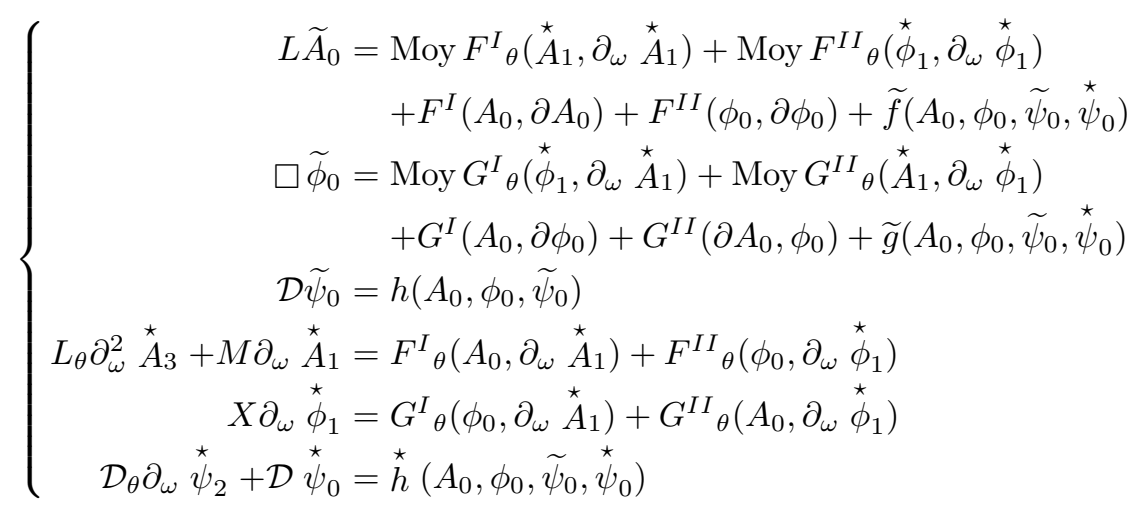


puis pour les indices $1 \leqslant i<M$ :

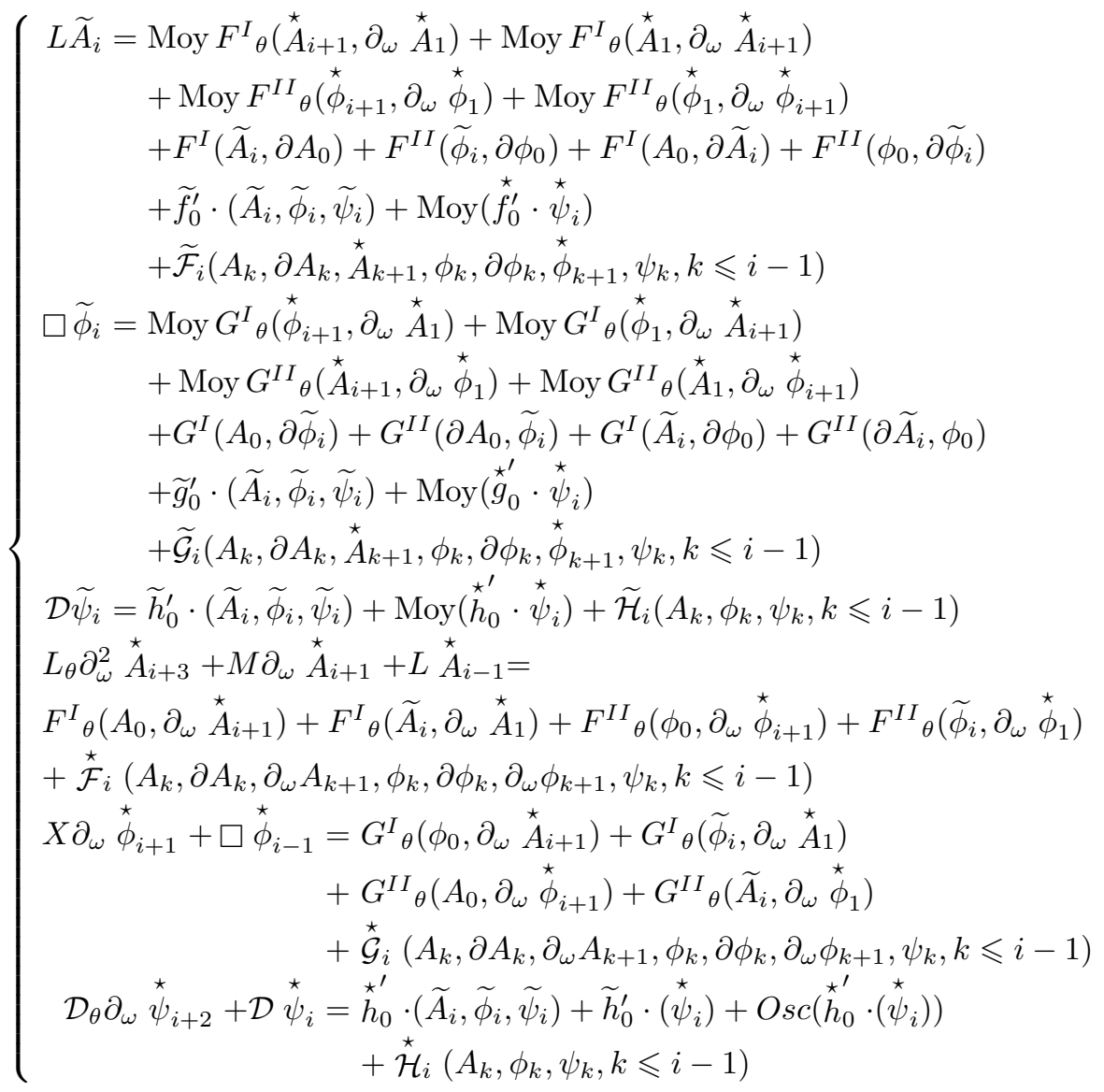

Les nouvelles fonctions $\stackrel{\star}{\mathcal{F}}_{i}, \widetilde{\mathcal{F}}_{i}, \stackrel{\star \mathcal{G}}{i}_{i}, \widetilde{\mathcal{G}}_{i}, \stackrel{\mathcal{H}}{i}_{i}$, et $\widetilde{\mathcal{H}}_{i}$ sont des polynômes à coefficients $C^{\infty}$, qui regroupent les profils $\left(A_{k}, \partial A_{k}, \partial_{\omega} A_{k+1}, \phi_{k}, \partial \phi_{k}, \partial_{\omega} \phi_{k+1}, \psi_{k}\right)$ d'ordre $k \leqslant i-1$, et que nous ne souhaitons pas détailler d'avantage ici. Réécrivons les systèmes $(S, i)$ précédents sous une forme plus synthétique :

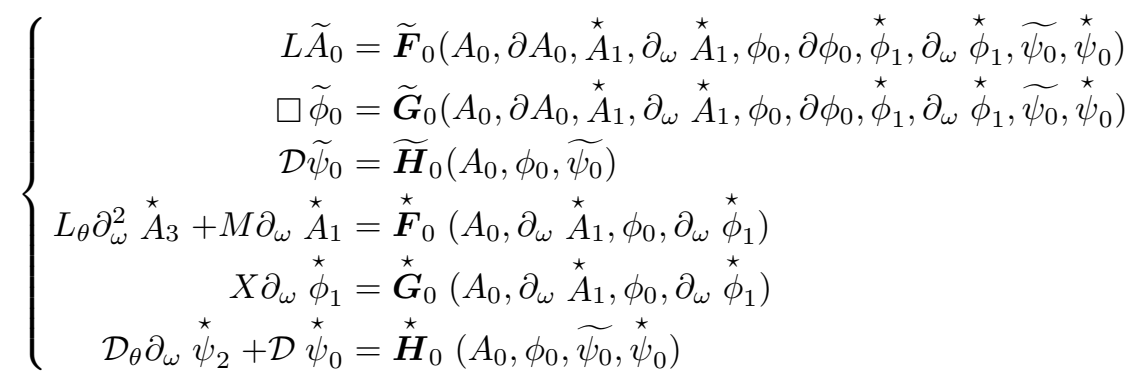


Puis pour les indices $1 \leqslant i \leqslant 2 M$ :

$$
\begin{aligned}
& \int L \widetilde{A}_{i}=\widetilde{\boldsymbol{F}}_{0}^{\prime} \cdot\left(\widetilde{A}_{i}, \partial \widetilde{A}_{i}, \partial_{\omega} \stackrel{\star}{A}_{i+1}, \widetilde{\phi}_{i}, \partial \widetilde{\phi}_{i}, \partial_{\omega} \stackrel{\star}{\phi}_{i+1}, \widetilde{\psi}_{i}, \stackrel{\star}{\psi}_{i}\right) \\
& +\widetilde{\mathcal{F}}_{i}\left(A_{k}, \partial A_{k}, \stackrel{\star}{A}_{k+1}, \phi_{k}, \partial \phi_{k}, \stackrel{\star}{\phi}_{k+1}, \psi_{k}, k \leqslant i-1\right) \\
& \square \widetilde{\phi}_{i}=\widetilde{\boldsymbol{G}}_{0}^{\prime} \cdot\left(\widetilde{A}_{i}, \partial \widetilde{A}_{i}, \partial_{\omega} \stackrel{\star}{A} i+1, \widetilde{\phi}_{i}, \partial \widetilde{\phi}_{i}, \partial_{\omega} \stackrel{\star}{\phi}_{i+1}, \widetilde{\psi}_{i}, \stackrel{\star}{\psi}_{i}\right) \\
& +\widetilde{\mathcal{G}}_{i}\left(A_{k}, \partial A_{k}, \stackrel{\star}{A}_{k+1}, \phi_{k}, \partial \phi_{k}, \stackrel{\star}{\phi}_{k+1}, \psi_{k}, k \leqslant i-1\right) \\
& \mathcal{D} \widetilde{\psi}_{i}=\widetilde{\boldsymbol{H}}_{0}^{\prime} \cdot\left(\widetilde{A}_{i}, \widetilde{\phi}_{i}, \widetilde{\psi}_{i}, \stackrel{\psi}{i}_{i}\right)+\widetilde{\mathcal{H}}_{i}\left(A_{k}, \phi_{k}, \psi_{k}, k \leqslant i-1\right) \\
& L_{\theta} \partial_{\omega}^{2} \stackrel{\star}{A}_{i+3}+M \partial_{\omega} \stackrel{\star}{A}_{i+1}+L \stackrel{\star}{A}_{i-1} \\
& ={\stackrel{\star \star *}{\boldsymbol{F}^{\prime}}}_{0} \cdot\left(\widetilde{A}_{i}, \partial \widetilde{A}_{i}, \partial_{\omega} \stackrel{\star}{A}_{i+1}, \widetilde{\phi}_{i}, \partial \widetilde{\phi}_{i}, \partial_{\omega} \stackrel{\star}{\phi}_{i+1}, \widetilde{\psi}_{i}, \stackrel{\star}{\psi}_{i}\right) \\
& +\stackrel{\star}{\mathcal{F}}_{i}\left(A_{k}, \partial A_{k}, \stackrel{\star}{A}_{k+1}, \phi_{k}, \partial \phi_{k}, \stackrel{\star}{\phi}_{k+1}, \psi_{k}, k \leqslant i-1\right) \\
& X \partial_{\omega} \stackrel{\star}{\phi}_{i+1}+\square \stackrel{\star}{\phi}_{i-1}=\stackrel{\star \star}{\boldsymbol{G}}_{0}^{\prime} \cdot\left(\widetilde{A}_{i}, \partial \widetilde{A}_{i}, \partial_{\omega} \stackrel{\star}{A}_{i+1}, \widetilde{\phi}_{i}, \partial \widetilde{\phi}_{i}, \partial_{\omega} \stackrel{\star}{\phi}_{i+1}, \widetilde{\psi}_{i}, \stackrel{\star}{\psi}_{i}\right) \\
& +\stackrel{\star}{\mathcal{G}}_{i}\left(A_{k}, \partial A_{k}, \stackrel{\star}{A}_{k+1}, \phi_{k}, \partial \phi_{k}, \stackrel{\star}{\phi}_{k+1}, \psi_{k}, k \leqslant i-1\right) \\
& \mathcal{D}_{\theta} \partial_{\omega} \stackrel{\star}{\psi}_{i+2}+\mathcal{D} \stackrel{\star}{\psi}_{i}=\stackrel{\star \star}{\boldsymbol{H}}_{0}^{\prime} \cdot\left(\widetilde{A}_{i}, \widetilde{\phi}_{i}, \widetilde{\psi}_{i}, \stackrel{\star}{\psi}_{i}\right) \\
& +\stackrel{\star}{\mathcal{H}}_{i}\left(A_{k}, \phi_{k}, \psi_{k}, k \leqslant i-1\right)
\end{aligned}
$$

Nous avons posé

$$
\begin{aligned}
& \widetilde{\boldsymbol{F}}_{0}\left(A_{0}, \partial A_{0}, \stackrel{\star}{A}, \partial_{\omega} \stackrel{\star}{A_{1}}, \phi_{0}, \partial \phi_{0}, \stackrel{\star}{\phi_{1}}, \partial_{\omega} \stackrel{\star}{\phi_{1}}, \widetilde{\psi_{0}}, \stackrel{\star}{\psi_{0}}\right) \\
& =\operatorname{Moy} F_{\theta}^{I}\left(\stackrel{\star}{A}_{1}, \partial_{\omega} \stackrel{\star}{A}_{1}\right)+\operatorname{Moy} F^{I I}{ }_{\theta}\left(\stackrel{\star}{\phi}_{1}, \partial_{\omega} \stackrel{\star}{\phi}_{1}\right)+F^{I}\left(A_{0}, \partial A_{0}\right)+F^{I I}\left(\phi_{0}, \partial \phi_{0}\right) \\
& +\widetilde{f}\left(A_{0}, \phi_{0}, \widetilde{\psi}_{0}, \stackrel{\star}{\psi}_{0}\right) \\
& \widetilde{\boldsymbol{G}}_{0}\left(A_{0}, \partial A_{0}, \stackrel{\star}{A} 1, \partial_{\omega} \stackrel{\star}{A} 1, \phi_{0}, \partial \phi_{0}, \stackrel{\star}{\phi_{1}}, \partial_{\omega} \stackrel{\star}{\phi}_{1}, \widetilde{\psi_{0}}, \stackrel{\star}{\psi_{0}}\right) \\
& =\operatorname{Moy} G_{\theta}^{I}\left(\stackrel{\star}{\phi}_{1}, \partial_{\omega} \stackrel{\star}{A}_{1}\right)+\operatorname{Moy} G_{\theta}^{I I}\left(\stackrel{\star}{A}_{1}, \partial_{\omega} \stackrel{\star}{\phi}_{1}\right)+G^{I}\left(A_{0}, \partial \phi_{0}\right)+G^{I I}\left(\partial A_{0}, \phi_{0}\right) \\
& +\widetilde{g}\left(A_{0}, \phi_{0}, \widetilde{\psi}_{0}, \stackrel{\star}{\psi}_{0}\right) \\
& \widetilde{\boldsymbol{H}}_{0}\left(A_{0}, \phi_{0}, \widetilde{\psi_{0}}\right)=h\left(A_{0}, \phi_{0}, \widetilde{\psi}_{0}\right) \\
& {\stackrel{\star}{\boldsymbol{F}_{0}}}\left(A_{0}, \partial_{\omega} \stackrel{\star}{A}_{1}, \phi_{0}, \partial_{\omega} \stackrel{\star}{\phi}_{1}\right)=F^{I}{ }_{\theta}\left(A_{0}, \partial_{\omega} \stackrel{\star}{A}_{1}\right)+F^{I I}{ }_{\theta}\left(\phi_{0}, \partial_{\omega} \stackrel{\star}{\phi}_{1}\right) \\
& \stackrel{\star}{\boldsymbol{G}}_{0}\left(A_{0}, \partial_{\omega} \stackrel{\star}{A}_{1}, \phi_{0}, \partial_{\omega} \stackrel{\star}{\phi}_{1}\right)=G^{I}{ }_{\theta}\left(\phi_{0}, \partial_{\omega} \stackrel{\star}{A}_{1}\right)+G^{I I}{ }_{\theta}\left(A_{0}, \partial_{\omega} \stackrel{\star}{\phi}_{1}\right) \\
& \stackrel{\star}{\boldsymbol{H}}_{0}\left(A_{0}, \phi_{0}, \widetilde{\psi}_{0}, \stackrel{\star}{\psi}{ }_{0}\right)=\stackrel{\star}{h}\left(A_{0}, \phi_{0}, \widetilde{\psi}_{0}, \stackrel{\star}{\psi}_{0}\right)
\end{aligned}
$$

qui sont des termes non linéaires, polynomiaux en

$$
\left(A_{0}, \partial A_{0}, \stackrel{\star}{A_{1}}, \partial_{\omega} \stackrel{\star}{A_{1}}, \phi_{0}, \partial \phi_{0}, \stackrel{\star}{\phi_{1}}, \partial_{\omega} \stackrel{\star}{\phi_{1}}, \widetilde{\psi_{0}}, \stackrel{\star}{\psi_{0}}\right)
$$


à coefficients $C^{\infty}$, puis

$$
\begin{aligned}
& \widetilde{\boldsymbol{F}}_{0}^{\prime} \cdot\left(\widetilde{A}_{i}, \partial \widetilde{A}_{i}, \partial_{\omega} \stackrel{\star}{A}_{i+1}, \widetilde{\phi}_{i}, \partial \widetilde{\phi}_{i}, \partial_{\omega} \stackrel{\star}{\phi}_{i+1}, \widetilde{\psi}_{i}, \stackrel{\star}{\psi}_{i}\right) \\
& =-\operatorname{Moy} F^{I}{ }_{\theta}\left(\partial_{\omega} \stackrel{\star}{A}_{i+1}, \stackrel{\star}{A}_{1}\right)+\operatorname{Moy} F^{I}{ }_{\theta}\left(\stackrel{\star}{A}_{1}, \partial_{\omega} \stackrel{\star}{A}_{i+1}\right) \\
& -\operatorname{Moy} F_{\theta}^{I I}\left(\partial_{\omega} \stackrel{\star}{\phi}_{i+1}, \stackrel{\star}{\phi}_{1}\right)+\operatorname{Moy} F^{I I}{ }_{\theta}\left(\stackrel{\star}{\phi}_{1}, \partial_{\omega} \stackrel{\star}{\phi}_{i+1}\right) \\
& +F^{I}\left(\widetilde{A}_{i}, \partial A_{0}\right)+F^{I I}\left(\widetilde{\phi}_{i}, \partial \phi_{0}\right)+F^{I}\left(A_{0}, \partial \widetilde{A}_{i}\right)+F^{I I}\left(\phi_{0}, \partial \widetilde{\phi}_{i}\right) \\
& +\widetilde{f}_{0}^{\prime} \cdot\left(\widetilde{A}_{i}, \widetilde{\phi}_{i}, \widetilde{\psi}_{i}\right)+\operatorname{Moy}\left(\stackrel{\star}{f_{0}^{\prime}} \cdot\left(\stackrel{\star}{\psi}_{i}\right)\right) \\
& \widetilde{\boldsymbol{G}}_{0}^{\prime} \cdot\left(\widetilde{A}_{i}, \partial \widetilde{A}_{i}, \partial_{\omega} \stackrel{\star}{A}_{i+1}, \widetilde{\phi}_{i}, \partial \widetilde{\phi}_{i}, \partial_{\omega} \stackrel{\star}{\phi}_{i+1}, \widetilde{\psi}_{i}, \stackrel{\star}{\psi}_{i}\right) \\
& =-\operatorname{Moy} G_{\theta}^{I}\left(\partial_{\omega} \stackrel{\star}{\phi}_{i+1}, \stackrel{\star}{A}_{1}\right)+\operatorname{Moy} G_{\theta}^{I}\left(\stackrel{\star}{\phi}_{1}, \partial_{\omega} \stackrel{\star}{A}_{i+1}\right) \\
& -\operatorname{Moy} G_{\theta}^{I I}\left(\partial_{\omega} \stackrel{\star}{A}_{i+1}, \stackrel{\star}{\phi}_{1}\right)+\operatorname{Moy} G_{\theta}^{I I}\left(\stackrel{\star}{A}_{1}, \partial_{\omega} \stackrel{\star}{\phi}_{i+1}\right) \\
& +G^{I}\left(A_{0}, \partial \widetilde{\phi}_{i}\right)+G^{I I}\left(\partial A_{0}, \widetilde{\phi}_{i}\right)+G^{I}\left(\widetilde{A}_{i}, \partial \phi_{0}\right)+G^{I I}\left(\partial \widetilde{A}_{i}, \phi_{0}\right) \\
& +\widetilde{g}_{0}^{\prime} \cdot\left(\widetilde{A}_{i}, \widetilde{\phi}_{i}, \widetilde{\psi}_{i}\right)+\operatorname{Moy}\left(\stackrel{\star}{g}_{0}^{\prime} \cdot\left(\stackrel{\star}{\psi}_{i}\right)\right. \\
& \widetilde{\boldsymbol{H}}_{0}^{\prime} \cdot\left(\widetilde{A}_{i}, \widetilde{\phi}_{i}, \widetilde{\psi}_{i}, \stackrel{\star}{\psi}_{i}\right)=\widetilde{h}_{0}^{\prime} \cdot\left(\widetilde{A}_{i}, \widetilde{\phi}_{i}, \widetilde{\psi}_{i}\right)+\operatorname{Moy}\left(\stackrel{\star}{h}_{0}^{\prime} \cdot\left(\stackrel{\star}{\psi}_{i}\right)\right) \\
& {\stackrel{\star}{\boldsymbol{F}^{\prime}}}_{0} \cdot\left(\widetilde{A}_{i}, \partial \widetilde{A}_{i}, \partial_{\omega} \stackrel{\star}{A_{i+1}}, \widetilde{\phi}_{i}, \partial \widetilde{\phi}_{i}, \partial_{\omega} \stackrel{\star}{\phi}_{i+1}, \widetilde{\psi}_{i}, \stackrel{\star}{\psi}_{i}\right) \\
& =F_{\theta}^{I}\left(A_{0}, \partial_{\omega} \stackrel{\star}{A}_{i+1}\right)+F_{\theta}^{I}\left(\widetilde{A}_{i}, \partial_{\omega} \stackrel{\star}{A}_{1}\right)+F_{\theta}^{I I}\left(\phi_{0}, \partial_{\omega} \stackrel{\star}{\phi}_{i+1}\right)+F_{\theta}^{I I}\left(\widetilde{\phi}_{i}, \partial_{\omega} \stackrel{\star}{\phi}_{1}\right) \\
& \stackrel{\star}{\boldsymbol{G}}_{0}^{\prime} \cdot\left(\widetilde{A}_{i}, \partial \widetilde{A}_{i}, \partial_{\omega} \stackrel{\star}{A}_{i+1}, \widetilde{\phi}_{i}, \partial \widetilde{\phi}_{i}, \partial_{\omega} \stackrel{\star}{\phi}_{i+1}, \widetilde{\psi}_{i}, \stackrel{\star}{\psi}_{i}\right) \\
& =G_{\theta}^{I}\left(\phi_{0}, \partial_{\omega} \stackrel{\star}{A}_{i+1}\right)+G_{\theta}^{I}\left(\widetilde{\phi}_{i}, \partial_{\omega} \stackrel{\star}{A}_{1}\right)+G_{\theta}^{I I}\left(A_{0}, \partial_{\omega} \stackrel{\star}{\phi}_{i+1}\right)+G^{I I}{ }_{\theta}\left(\widetilde{A}_{i}, \partial_{\omega} \stackrel{\star}{\phi}_{1}\right) \\
& {\stackrel{\star}{\boldsymbol{H}_{0}^{\prime}}}_{0} \cdot\left(\widetilde{A}_{i}, \widetilde{\phi}_{i}, \widetilde{\psi}_{i}, \stackrel{\star}{\psi}_{i}\right)=\stackrel{\star \star}{h}_{0}^{\prime} \cdot\left(\widetilde{A}_{i}, \widetilde{\phi}_{i}, \widetilde{\psi}_{i}\right)+\widetilde{h}_{0}^{\prime} \cdot\left(\stackrel{\star}{\psi}_{i}\right)+\operatorname{Osc}\left(\stackrel{\star \star}{h}_{0}^{\prime} \cdot\left(\stackrel{\star}{\psi}_{i}\right)\right)
\end{aligned}
$$

qui sont linéaires en $\left(\widetilde{A}_{i}, \partial \widetilde{A}_{i}, \partial_{\omega} \stackrel{\star}{A}_{i+1}, \widetilde{\phi}_{i}, \partial \widetilde{\phi}_{i}, \partial_{\omega} \stackrel{\star}{\phi}_{i+1}, \widetilde{\psi}_{i}, \stackrel{\star}{\psi}_{i}\right)$, et qui dépendent de façon $C^{\infty}$ des profils dominants $\left(A_{0}, \stackrel{\star}{A}_{1}, \phi_{0}, \stackrel{\star}{\phi}_{1}, \psi_{0}\right)$. Nous userons parfois d'une notation simplifiée de type $\widetilde{\boldsymbol{F}_{0}^{\prime}}(i)$, par mesure d'économie, pour désigner ces termes dans les calculs où nulle ambiguïté n'est possible. Si $U$ et $V$ sont deux fonctions oscillantes $2 \pi$-périodiques en $\omega$, alors $\operatorname{Moy}\left(U \partial_{\omega} V\right)=-\operatorname{Moy}\left(V \partial_{\omega} U\right)$. Nous retrouvons bien

$$
\begin{aligned}
\operatorname{Moy} F^{I}{ }_{\theta}\left(\stackrel{\star}{A}_{i+1}, \partial_{\omega} \stackrel{\star}{A}_{1}\right) & =-\operatorname{Moy} F_{\theta}^{I}\left(\partial_{\omega} \stackrel{\star}{A}_{i+1}, \stackrel{\star}{A}_{1}\right) \\
\operatorname{Moy} F^{I I}{ }_{\theta}\left(\stackrel{\star}{\phi}_{i+1}, \partial_{\omega} \stackrel{\star}{\phi}_{1}\right) & =-\operatorname{Moy} F^{I I}{ }_{\theta}\left(\partial_{\omega} \stackrel{\star}{\phi}_{i+1}, \stackrel{\star}{\phi}_{1}\right) \\
\operatorname{Moy} G^{I}{ }_{\theta}\left(\stackrel{\star}{\phi}_{i+1}, \partial_{\omega} \stackrel{\star}{A}_{1}\right) & =-\operatorname{Moy} G^{I}{ }_{\theta}\left(\partial_{\omega} \stackrel{\star}{\phi}_{i+1}, \stackrel{\star}{A}_{1}\right) \\
\operatorname{Moy} G^{I I}{ }_{\theta}\left(\stackrel{\star}{A}_{i+1}, \partial_{\omega} \stackrel{\star}{\phi}_{1}\right) & =-\operatorname{Moy} G^{I I}{ }_{\theta}\left(\partial_{\omega} \stackrel{\star}{A}_{i+1}, \stackrel{\star}{\phi}_{1}\right)
\end{aligned}
$$


Enfin (ceci nous servira lors de la résolution des équations de profils),

Remarque 2.2.1. - Nous retiendrons que

$$
\partial_{\omega}^{-1} \stackrel{\star}{\boldsymbol{F}_{0}}\left(A_{0}, \partial_{\omega} \stackrel{\star}{A}, \phi_{0}, \partial_{\omega} \stackrel{\star}{\phi_{1}}\right)=\stackrel{\star}{\boldsymbol{F}_{0}}\left(A_{0}, \stackrel{\star}{A_{1}}, \phi_{0}, \stackrel{\star}{\phi_{1}}\right)
$$

de même que

$$
\partial_{\omega}^{-1} \stackrel{\star}{\boldsymbol{G}_{0}}\left(A_{0}, \partial_{\omega} \stackrel{\star}{A_{1}}, \phi_{0}, \partial_{\omega} \stackrel{\star}{\phi_{1}}\right)=\stackrel{\star}{\boldsymbol{G}_{0}}\left(A_{0}, \stackrel{\star}{A_{1}}, \phi_{0}, \stackrel{\star}{\phi_{1}}\right)
$$

c) Polarisation des oscillations. - La dernière étape consiste à reformuler chaque $(S, i)$ sous forme d'un système $(C, i)$ portant exclusivement sur les inconnues $\left(\widetilde{A}_{i}, \widetilde{\phi}_{i}, \widetilde{\psi}_{i}, \pi^{\#} \stackrel{\star}{A}_{i+1}, \stackrel{\star}{\phi}_{i+1}, \pi^{+} \stackrel{\star}{\psi}_{i}\right)$, accompagné d'une condition de polarisation $(P, i)$ portant sur les composantes oscillantes $\left(\pi^{\vee} \stackrel{\star}{A} i+3, \pi^{-} \stackrel{\star}{\psi}_{i}\right)$. Pour tout indice $1 \leqslant i$, décomposons la partie oscillante de chaque $A_{i}$ en :

$$
\stackrel{\star}{A_{i}}=a_{i}^{\#}+a_{i}^{\vee}=a_{i}^{\wedge}+a_{i}^{0}+a_{i}^{\vee}
$$

en adoptant la notation $a_{i}^{\#}=\pi^{\#} \stackrel{\star}{A_{i}} \in \Pi_{\#}, a_{i}^{\wedge}=\pi^{\wedge} \stackrel{\star}{A_{i}} \in \Pi_{\wedge}, a_{i}^{0}=\pi^{0} \stackrel{\star}{A_{i}} \in \Pi_{0}$ et $a_{i}^{\vee}=\pi^{\vee} \stackrel{\star}{A}_{i} \in \Pi_{\vee}$, conformément aux définitions de la section 2.1. Les deux premières équations

$$
L_{\theta} \partial_{\omega}^{2} \stackrel{\star}{A} 1=L_{\theta} \partial_{\omega}^{2} \stackrel{\star}{A}{ }_{2}=0
$$

sont équivalentes à

$$
\stackrel{\star}{A} \in \Pi_{\#} \text { soit } a_{1}^{\vee}=0 \quad \text { et } \quad \stackrel{\star}{A}{ }_{2} \in \Pi_{\#} \text { soit } a_{2}^{\vee}=0
$$

De même, pour tout indice $0 \leqslant i \leqslant N$, nous décomposons la partie oscillante de chaque spineur $\psi_{i}$ en

$$
\stackrel{\star}{\psi_{i}}=\stackrel{\star}{\psi_{i}^{+}}+\stackrel{\star}{\psi_{i}^{-}}
$$

en notant $\stackrel{\star}{\psi_{i}^{+}}=\pi^{+} \stackrel{\star}{\psi}_{i} \in \Pi_{+}$et $\stackrel{\star}{\psi_{i}^{-}}=\pi^{-} \stackrel{\star}{\psi}_{i} \in \Pi_{-}$. Conformément aux remarques de la section 2.1, les équations

$$
\mathcal{D}_{\theta} \partial_{\omega} \stackrel{\star}{\psi}_{0}=\mathcal{D}_{\theta} \partial_{\omega} \stackrel{\star}{\psi}_{1}=0
$$

sont équivalentes à

$$
\stackrel{\star}{\psi_{0}} \in \Pi_{+}, \text {c'est-à-dire } \stackrel{\star}{\psi_{0}^{-}}=0 \text {, et } \quad \stackrel{\star}{\psi}_{1} \in \Pi_{+} \text {, c'est-à-dire } \stackrel{\star}{\psi_{1}^{-}}=0
$$

Pour les profils d'indice $i \geqslant 3$, la condition de polarisation n'est que partielle, car il faut absorber une composante résiduelle issue de l'interaction non linéaire des profils d'indice inférieur. Puisque

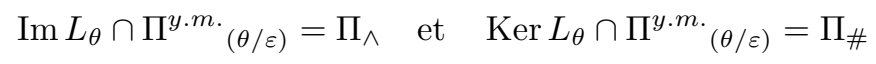

toute équation du type

$$
L_{\theta} U+V=0
$$


où $U$ et $V \in \Pi^{y \cdot m .}{ }_{(\theta / \varepsilon)}$ sont des fonctions oscillantes de phase $\theta / \varepsilon$, est équivalente aux conditions :

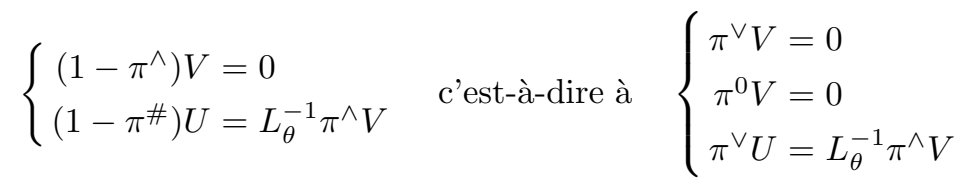

D'une façon similaire, les équations de type

$$
\mathcal{D}_{\theta} \mathcal{U}+\mathcal{V}=0
$$

où $U$ et $V \in \Pi^{s p .}{ }_{(\theta / \varepsilon)}$, sont équivalentes à

Il en résulte que l'équation :

$$
\left\{\begin{array}{l}
\pi^{-} \mathcal{V}=0 \\
\pi^{-} \mathcal{U}=\mathcal{D}_{\theta}^{-1} \pi^{+} \mathcal{V}
\end{array}\right.
$$

$$
L_{\theta} \partial_{\omega}^{2} \stackrel{\star}{A}{ }_{3}+M \partial_{\omega} \stackrel{\star}{A_{1}}=\stackrel{\star}{\boldsymbol{F}_{0}}\left(A_{0}, \partial_{\omega} \stackrel{\star}{A_{1}}, \phi_{0}, \partial_{\omega} \stackrel{\star}{\phi_{1}}\right)
$$

est équivalente à :

$$
\left\{\begin{array}{l}
\pi^{0}\left(M \partial_{\omega} \stackrel{\star}{A_{1}}\right)=\pi^{0}\left(\stackrel{\star}{\boldsymbol{F}_{0}}\left(A_{0}, \partial_{\omega} \stackrel{\star}{A_{1}}, \phi_{0}, \partial_{\omega} \stackrel{\star}{\phi_{1}}\right)\right) \\
\pi^{\vee}\left(M \partial_{\omega} \stackrel{\star}{A_{1}}\right)=\pi^{\vee}\left(\stackrel{\star}{\boldsymbol{F}}_{0}\left(A_{0}, \partial_{\omega} \stackrel{\star}{A}_{1}, \phi_{0}, \partial_{\omega} \stackrel{\star}{\phi}_{1}\right)\right)
\end{array}\right.
$$

et

$$
\begin{aligned}
a_{3}^{\vee} & =L_{\theta}^{-1} \partial_{\omega}^{-2} \pi^{\wedge}\left(-M \partial_{\omega} \stackrel{\star}{A_{1}}+\stackrel{\star \star \star}{F}_{0}\left(A_{0}, \partial_{\omega} \stackrel{\star}{A_{1}}, \phi_{0}, \partial_{\omega} \stackrel{\star}{\phi_{1}}\right)\right) \\
& =a_{3}^{\vee}\left(A_{0}, \partial A_{0}, \stackrel{\star}{A_{1}}, \phi_{0}, \partial \phi_{0}, \stackrel{\phi}{\phi}_{1}, \psi_{0}\right)
\end{aligned}
$$

Puis, pour $2 \leqslant i \leqslant 2 M$, l'équation

$$
\begin{aligned}
L_{\theta} \partial_{\omega}^{2} \stackrel{\star}{A}_{i+3}+M \partial_{\omega} \stackrel{\star}{A}_{i+1}+L \stackrel{\star}{A}{ }_{i-1} & =\stackrel{\star \star \star}{\mathbf{F}}_{\mathbf{0}} \cdot\left(\widetilde{A}_{i}, \partial \widetilde{A}_{i}, \partial_{\omega} \stackrel{\star}{A}_{i+1}, \widetilde{\phi}_{i}, \partial \widetilde{\phi}_{i}, \partial_{\omega} \stackrel{\star}{\phi}_{i+1}, \widetilde{\psi}_{i}, \stackrel{\star}{\psi_{i}}\right) \\
& +\stackrel{\star}{\mathcal{F}}_{i}\left(A_{k}, \partial A_{k}, \stackrel{\star}{A}_{k+1}, \phi_{k}, \partial \phi_{k}, \stackrel{\star}{\phi}_{k+1}, \psi_{k}, k \leqslant i-1\right)
\end{aligned}
$$

équivaut à

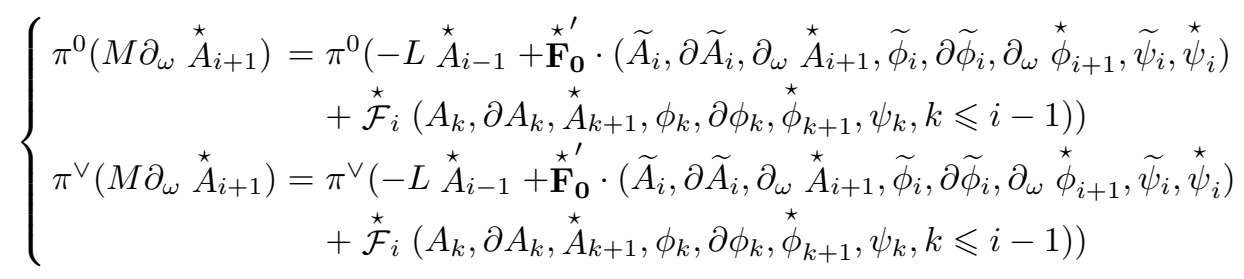

et

$$
\begin{aligned}
& a_{i+3}^{\vee}=L_{\theta}^{-1} \partial_{\omega}^{-2} \pi^{\wedge}\left(-M \partial_{\omega} \stackrel{\star}{A_{i+1}}-L \stackrel{\star}{A}_{i-1}\right. \\
& +\stackrel{\star \star}{\mathbf{F}} \mathbf{0}_{\mathbf{0}} \cdot\left(\widetilde{A}_{i}, \partial \widetilde{A}_{i}, \partial_{\omega} \stackrel{\star}{A_{i+1}}, \widetilde{\phi}_{i}, \partial \widetilde{\phi}_{i}, \partial_{\omega} \stackrel{\star}{\phi}_{i+1}, \widetilde{\psi}_{i}, \stackrel{\star}{\psi}_{i}\right) \\
& \left.+\stackrel{\star}{\mathcal{F}}_{i}\left(A_{k}, \partial A_{k}, \stackrel{\star}{A}_{k+1}, \phi_{k}, \partial \phi_{k}, \stackrel{\star}{\phi}_{k+1}, \psi_{k}, k \leqslant i-1\right)\right) \\
& =a_{i+3}^{\vee}\left(A_{k}, \partial A_{k}, \stackrel{\star}{A}_{k+1}, \phi_{k}, \partial \phi_{k}, \stackrel{\star}{\phi}_{k+1}, \psi_{k}, k \leqslant i\right)
\end{aligned}
$$


L'équation

$$
\mathcal{D}_{\theta} \partial_{\omega} \stackrel{\star}{\psi}_{2}+\mathcal{D} \stackrel{\star}{\psi}_{0}=\stackrel{\star}{\boldsymbol{H}}_{0}\left(A_{0}, \phi_{0}, \widetilde{\psi_{0}}, \stackrel{\star}{\psi_{0}}\right)
$$

est quant à elle équivalente à :

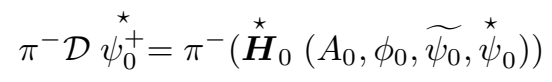

et

$$
\begin{aligned}
{\stackrel{\star}{\psi_{2}^{-}}}^{-} & =\mathcal{D}_{\theta}^{-1} \partial_{\omega}^{-1} \pi^{+}\left(\mathcal{D} \stackrel{\star}{\psi_{0}}-\stackrel{\star}{\boldsymbol{H}}_{0}\left(A_{0}, \phi_{0}, \widetilde{\psi_{0}}, \stackrel{\star}{\psi_{0}}\right)\right) \\
& =\psi_{2}^{-}\left(A_{0}, \phi_{0}, \psi_{0}\right)
\end{aligned}
$$

En appliquant la proposition 2.1.3, il vient que $\pi^{-} \mathcal{D} \pi^{+} \stackrel{\star}{\psi_{0}}=\frac{\alpha^{2}}{2 \nabla_{0} \theta} \gamma^{0} X \pi^{+} \stackrel{\star}{\psi_{0}^{+}}$. La dernière condition se réécrit alors

$$
X \stackrel{\star}{\psi_{0}^{+}}=\mathcal{D}_{\theta} \stackrel{\star}{\boldsymbol{H}_{0}}\left(A_{0}, \phi_{0}, \widetilde{\psi_{0}}, \stackrel{\star}{\psi_{0}}\right)
$$

Pour les indices $2 \leqslant i \leqslant 2 M$, l'équation

$$
\mathcal{D}_{\theta} \partial_{\omega} \stackrel{\star}{\psi}_{i+2}+\mathcal{D} \stackrel{\star}{\psi}_{i}={\stackrel{\star}{\mathbf{H}^{\prime}}}_{\mathbf{0}} \cdot\left(\widetilde{A}_{i}, \widetilde{\phi}_{i}, \widetilde{\psi}_{i}, \stackrel{\star}{\psi}_{i}\right)+\stackrel{\star}{\mathcal{H}}_{i}\left(A_{k}, \phi_{k}, \psi_{k}, k \leqslant i-1\right)
$$

est équivalente à

$$
\begin{aligned}
X \stackrel{\star}{\psi_{i}^{+}=} & \mathcal{D}_{\theta}\left(\stackrel{\star}{\mathbf{H}^{\prime}}{ }_{\mathbf{0}} \cdot\left(\widetilde{A}_{i}, \widetilde{\phi}_{i}, \widetilde{\psi}_{i}, \stackrel{\star}{\psi}_{i}\right)\right. \\
& \left.+\stackrel{\star}{\mathcal{H}}_{i}\left(A_{k}, \phi_{k}, \psi_{k}, k \leqslant i-1\right)-\mathcal{D} \psi_{i}^{-}\left(A_{k}, \phi_{k}, \psi_{k}, k \leqslant i-1\right)\right)
\end{aligned}
$$

et

$$
\begin{aligned}
\psi_{i+2}^{\stackrel{\star}{-}} & =\mathcal{D}_{\theta}^{-1} \partial_{\omega}^{-1} \pi^{+}\left(\mathcal{D} \stackrel{\star}{\psi}_{i}-{\stackrel{\star}{\mathbf{H}^{\prime}}}_{\mathbf{0}} \cdot\left(\widetilde{A}_{i}, \widetilde{\phi}_{i}, \widetilde{\psi}_{i}, \stackrel{\star}{\psi}_{i}\right)-\stackrel{\star}{\mathcal{H}}_{i}\left(A_{k}, \phi_{k}, \psi_{k}, k \leqslant i-1\right)\right) \\
& =\psi_{i+2}^{-}\left(A_{k}, \phi_{k}, \psi_{k}, k \leqslant i\right)
\end{aligned}
$$

Il en résulte que le système $(S, 0)$ est équivalent au système

$$
\begin{aligned}
& (C, 0) \quad\left\{\pi^{0}\left(M \partial_{\omega} \stackrel{\star}{A_{1}}\right)=\pi^{0}\left(\stackrel{\star}{F}_{0}\left(A_{0}, \partial_{\omega} \stackrel{\star}{A_{1}}, \phi_{0}, \partial_{\omega} \stackrel{\star}{\phi_{1}}\right)\right)\right. \\
& \pi^{\vee}\left(M \partial_{\omega} \stackrel{\star}{A_{1}}\right)=\pi^{\vee}\left({\stackrel{\star}{\boldsymbol{F}_{0}}}\left(A_{0}, \partial_{\omega} \stackrel{\star}{A_{1}}, \phi_{0}, \partial_{\omega} \stackrel{\star}{\phi}_{1}\right)\right) \\
& X \partial_{\omega} \stackrel{\star}{\phi}_{1}=\stackrel{\star}{\boldsymbol{G}_{0}}\left(A_{0}, \partial_{\omega} \stackrel{\star}{A_{1}}, \phi_{0}, \partial_{\omega} \stackrel{\star \star}{\phi_{1}}\right) \\
& \stackrel{\star}{\psi}_{0}=\stackrel{\star}{\psi}_{0}^{+} \\
& X \stackrel{\star}{\psi_{0}^{+}}=\mathcal{D}_{\theta} \stackrel{\star}{\boldsymbol{H}_{0}}\left(A_{0}, \phi_{0}, \widetilde{\psi_{0}}, \stackrel{\star}{\psi_{0}}\right)
\end{aligned}
$$


accompagné des polarisations

$$
\left\{\begin{array}{c}
a_{3}^{\vee}=L_{\theta}^{-1} \partial_{\omega}^{-2} \pi^{\wedge}\left(-M \partial_{\omega} \stackrel{\star}{A}_{1}+\stackrel{\star}{\boldsymbol{F}}_{0}\left(A_{0}, \partial_{\omega} \stackrel{\star}{A_{1}}, \phi_{0}, \partial_{\omega} \stackrel{\star}{\phi}_{1}\right)\right) \\
\psi_{2}^{-}=\mathcal{D}_{\theta}^{-1} \partial_{\omega}^{-1} \pi^{+}\left(\stackrel{\star}{\boldsymbol{H}}_{0}\left(A_{0}, \bar{\phi}_{0}, \widetilde{\psi_{0}}, \stackrel{\star}{\psi}_{0}\right)-\mathcal{D}{\stackrel{\star}{\psi_{0}}}^{-}\right.
\end{array}\right.
$$

puis pour tout indice $1 \leqslant i<M$, le système $(S, i)$ est équivalent au système

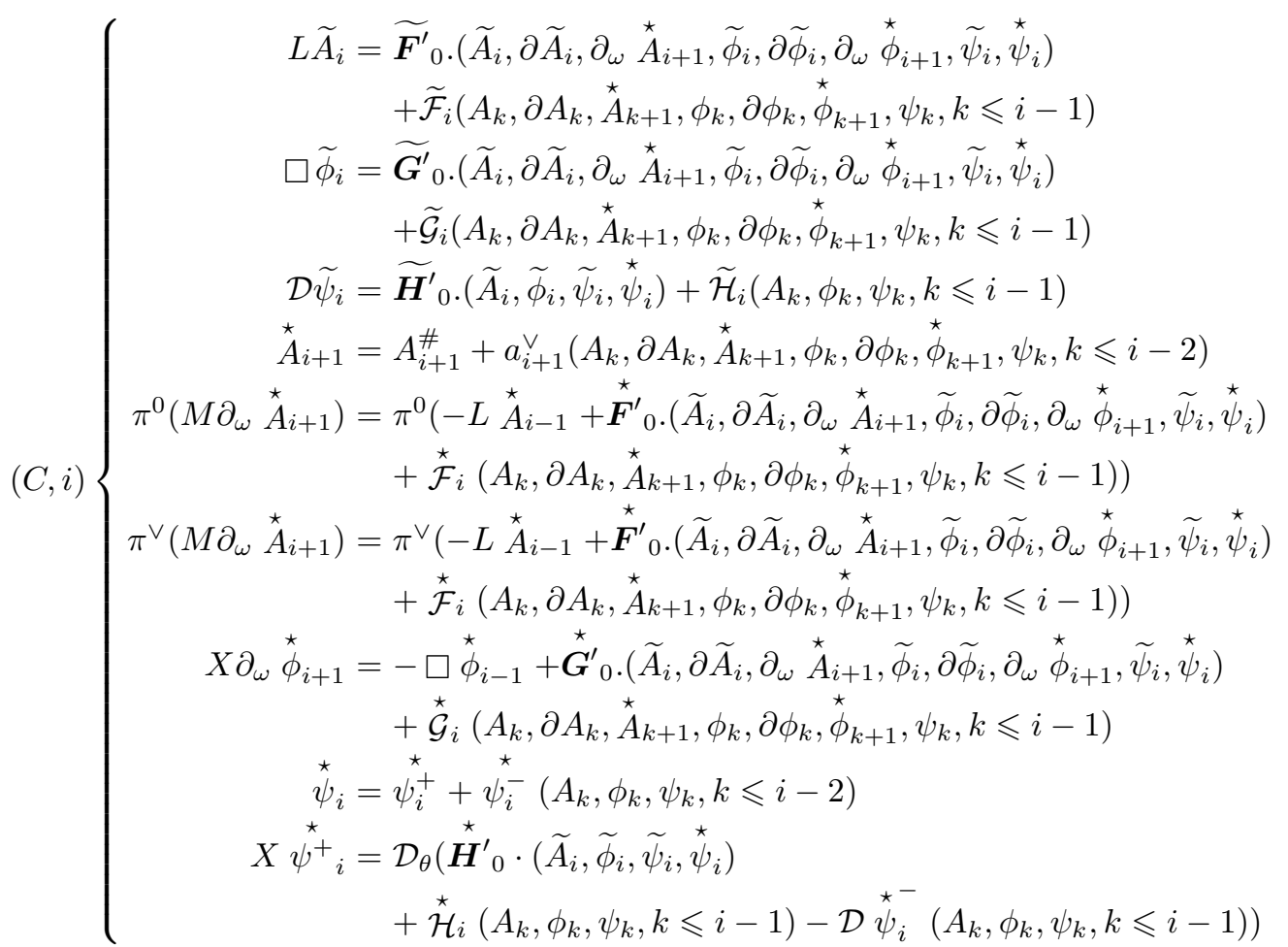

accompagné des polarisations

$$
(P, i)\left\{\begin{aligned}
a_{i+3}^{\vee}= & L_{\theta}^{-1} \partial_{\omega}^{-2} \pi^{\wedge}\left(-M \partial_{\omega} \stackrel{\star}{A}_{i+1}-L \stackrel{\star}{A}_{i-1}\right. \\
& +\stackrel{\text { F }}{\prime}_{0} \cdot\left(\widetilde{A}_{i}, \partial \widetilde{A}_{i}, \partial_{\omega} \stackrel{\star}{A}_{i+1}, \widetilde{\phi}_{i}, \partial \widetilde{\phi}_{i}, \partial_{\omega} \stackrel{\star}{\phi}_{i+1}, \widetilde{\psi}_{i}, \stackrel{\star}{\psi}_{i}\right) \\
& \left.+\stackrel{\star}{\mathcal{F}}_{i}\left(A_{k}, \partial A_{k}, \stackrel{\star}{A}_{k+1}, \phi_{k}, \partial \phi_{k}, \stackrel{\star}{\phi}_{k+1}, \psi_{k}, k \leqslant i-1\right)\right) \\
{ }_{i+2}^{\star}= & \mathcal{D}_{\theta}^{-1} \partial_{\omega}^{-1} \pi^{+}\left(\stackrel{\star}{H}_{0}^{\prime} \cdot\left(\widetilde{A}_{i}, \widetilde{\phi}_{i}, \widetilde{\psi}_{i}, \stackrel{\star}{\psi}_{i}\right)+\stackrel{\star}{\mathcal{H}}_{i}\left(A_{k}, \phi_{k}, \psi_{k}, k \leqslant i-1\right)-\mathcal{D} \stackrel{\star}{\psi}_{i}\right)
\end{aligned}\right.
$$

(On convient que les profils d'indice négatif qui apparaissent dans les expressions sont nuls). Les systèmes $(C, i)$ tels qu'ils apparaissent ci-dessus semblent nettement plus satisfaisant que les $(S, i)$, en ce sens qu'ils ne portent plus que sur les inconnues $\left(\widetilde{A}_{i}, \stackrel{\star}{A}_{i+1}^{\#}, \widetilde{\phi}_{i}, \stackrel{\star}{\phi}_{i+1}, \widetilde{\psi}_{i}, \stackrel{\star}{\psi}_{i}^{+}\right)$, et apparaissent clairement découplés les uns des autres. Il nous reste cependant à prouver la compatibilité de chacun de ces systèmes avec 
l'opérateur $L$, et à vérifier que les polarisations successives des profils n'entravent pas d'éventuelles oscillations de la jauge.

\subsubsection{Développement des conditions de compatibilité du système et ré- duction des équations de profils}

Développement de l'identité de Bianchi. - La relation de compatibilité (2) donne, pour les solutions approchées que nous envisageons,

$$
\begin{aligned}
& R\left(F^{I}\left(A_{\varepsilon}, \partial A_{\varepsilon}\right)+\right.\left.F^{I I}\left(\phi_{\varepsilon}, \partial \phi_{\varepsilon}\right)+f\left(A_{\varepsilon}, \phi_{\varepsilon}, \psi_{\varepsilon}\right)\right) \\
&=Q_{f}\left(A_{\varepsilon}, L A_{\varepsilon}-F^{I}\left(A_{\varepsilon}, \partial A_{\varepsilon}\right)-F^{I I}\left(\phi_{\varepsilon}, \partial \phi_{\varepsilon}\right)-f\left(A_{\varepsilon}, \phi_{\varepsilon}, \psi_{\varepsilon}\right)\right) \\
&+Q_{g}\left(\phi_{\varepsilon}, \square \phi_{\varepsilon}-G^{I}\left(A_{\varepsilon}, \partial \phi_{\varepsilon}\right)-G^{I I}\left(\partial A_{\varepsilon}, \phi_{\varepsilon}\right)-g\left(A_{\varepsilon}, \phi_{\varepsilon}, \psi_{\varepsilon}\right)\right) \\
&+Q_{h}\left(\psi_{\varepsilon}, \mathcal{D} \psi_{\varepsilon}-h\left(A_{\varepsilon}, \phi_{\varepsilon}, \psi_{\varepsilon}\right)\right)
\end{aligned}
$$

que nous développons en puissances de $\varepsilon$, en rappelant que $Q_{f}, Q_{g}$ et $Q_{h}$ sont bilinéaires. Pour les parties oscillantes du développement, il vient

- à l'ordre $\varepsilon^{-3 / 2}$ :

$$
R_{\theta} \partial_{\omega} \stackrel{\star}{\boldsymbol{F}}_{0}=Q_{f}\left(A_{0}, L_{\theta} \partial_{\omega}^{2} \stackrel{\star}{A}{ }_{1}\right)
$$

- à l'ordre $\varepsilon^{-1}$ :

$$
\left.R_{\theta} \partial_{\omega}\left({\stackrel{\star}{\mathbf{F}_{0}^{\prime}}}_{\mathbf{0}}(1)+\stackrel{\star}{\mathcal{F}}\right)_{1}\right)=Q_{f}\left(A_{0}, L_{\theta} \partial_{\omega}^{2} \stackrel{\star}{A}_{2}\right)+O s c\left(Q_{f}\left(A_{1}, L_{\theta} \partial_{\omega}^{2} \stackrel{\star}{A}_{1}\right)+Q_{h}\left(\psi_{0}, \mathcal{D}_{\theta} \partial_{\omega} \stackrel{\star}{\psi}_{0}\right)\right)
$$

- à l'ordre $\varepsilon^{-1 / 2}$ :

$$
\begin{aligned}
& R_{\theta} \partial_{\omega}\left(\stackrel{\star}{\mathbf{F}}_{\mathbf{0}}^{\prime}(2)+\stackrel{\star}{\mathcal{F}}_{2}\right)+R \stackrel{\star}{\boldsymbol{F}}_{0} \\
& =Q_{f}\left(A_{0}, L_{\theta} \partial_{\omega}^{2} \stackrel{\star}{A}_{3}+M \partial_{\omega} \stackrel{\star}{A}_{1}-\stackrel{\star}{\boldsymbol{F}}_{0}\right)+Q_{g}\left(\phi_{0}, X \partial_{\omega} \stackrel{\star}{\phi}_{1}-\stackrel{\star}{\boldsymbol{G}}_{0}\right) \\
+ & O s c\left(Q_{f}\left(A_{1}, L_{\theta} \partial_{\omega}^{2} \stackrel{\star}{A}_{2}\right)+Q_{f}\left(A_{2}, L_{\theta} \partial_{\omega}^{2} \stackrel{\star}{A}_{1}\right)+Q_{h}\left(\psi_{0}, \mathcal{D}_{\theta} \partial_{\omega} \stackrel{\star}{\psi}_{1}\right)+Q_{h}\left(\psi_{1}, \mathcal{D}_{\theta} \partial_{\omega} \stackrel{\star}{\psi}_{0}\right)\right)
\end{aligned}
$$

- à l'ordre $\varepsilon^{0}$ :

$$
\begin{aligned}
& R_{\theta} \partial_{\omega}\left(\stackrel{\star}{\mathbf{F}}_{\mathbf{0}}^{\prime}(3)+\stackrel{\star}{\mathcal{F}}\right)+R\left({\stackrel{\star}{\mathbf{F}_{0}^{\prime}}}_{3}(1)+\stackrel{\star}{\mathcal{F}}_{1}\right) \\
& =Q_{f}\left(A_{0}, L_{\theta} \partial_{\omega}^{2} \stackrel{\star}{A}_{4}+M \partial_{\omega} \stackrel{\star}{A}_{2}-\stackrel{\star}{\mathbf{F}}_{\mathbf{0}}^{\prime}(1)-\stackrel{\star}{\mathcal{F}}_{1}\right)+Q_{g}\left(\phi_{0}, X \partial_{\omega} \stackrel{\star}{\phi}_{2}-\stackrel{\star}{\mathbf{G}}_{\mathbf{0}}^{\prime}(1)-\stackrel{\star}{\mathcal{G}}_{1}\right) \\
& +\operatorname{Osc}\left(Q_{f}\left(A_{1}, L_{\theta} \partial_{\omega}^{2} \stackrel{\star}{A}_{3}+M \partial_{\omega} \stackrel{\star}{A}_{1}-\stackrel{\star}{\boldsymbol{F}}_{0}\right)\right)+O s c\left(Q_{g}\left(\stackrel{\star}{\phi}_{1}, X \partial_{\omega} \stackrel{\star}{\phi}_{1}-\stackrel{\star}{\boldsymbol{G}}_{0}\right)\right) \\
& +\operatorname{Osc}\left(Q_{h}\left(\psi_{0}, \mathcal{D}_{\theta} \partial_{\omega} \stackrel{\star}{\psi}_{2}+\mathcal{D} \psi_{0}-\boldsymbol{H}_{0}\right)\right)+\operatorname{Osc}\left(Q_{f}\left(A_{2}, L_{\theta} \partial_{\omega}^{2} \stackrel{\star}{A}_{2}\right)+Q_{f}\left(A_{3}, L_{\theta} \partial_{\omega}^{2} \stackrel{\star}{A}_{1}\right)\right) \\
& +\operatorname{Osc}\left(Q_{h}\left(\psi_{1}, \mathcal{D}_{\theta} \partial_{\omega} \stackrel{\star}{\psi}_{1}\right)+Q_{h}\left(\psi_{2}, \mathcal{D}_{\theta} \partial_{\omega} \stackrel{\star}{\psi}_{0}\right)\right)
\end{aligned}
$$


- à l'ordre $\varepsilon^{1 / 2}$ :

$$
\begin{aligned}
& R_{\theta} \partial_{\omega}\left({\stackrel{\star}{\mathbf{F}_{\mathbf{0}}^{\prime}}}^{\prime}(4)+\stackrel{\star}{\mathcal{F}_{4}}\right)+R\left({\stackrel{\star}{\mathbf{F}_{\mathbf{0}}^{\prime}}}(2)+\stackrel{\star}{\mathcal{F}}_{2}\right)= \\
& Q_{f}\left(A_{0}, L_{\theta} \partial_{\omega}^{2} \stackrel{\star}{A}_{5}+M \partial_{\omega} \stackrel{\star}{A}_{3}-\stackrel{\star}{\mathbf{F}}_{\mathbf{0}}^{\prime}(2)-\stackrel{\star}{\mathcal{F}}_{2}\right)+Q_{g}\left(\phi_{0}, X \partial_{\omega} \stackrel{\star}{\phi}_{3}+\square \stackrel{\star}{\phi}_{1}-\stackrel{\star}{\mathbf{G}}_{\mathbf{0}}^{\prime}(2)-\stackrel{\star}{\mathcal{G}}_{2}\right) \\
& +\operatorname{Osc}\left(Q_{h}\left(\psi_{0}, \mathcal{D}_{\theta} \partial_{\omega} \stackrel{\star}{*}_{3}+\mathcal{D} \psi_{1}-\boldsymbol{H}_{0}^{\prime}(1)-\mathcal{H}_{1}\right)\right) \\
& \left.+Q_{f}\left(\stackrel{\star}{A}_{1}, L \widetilde{A}_{0}-\widetilde{\boldsymbol{F}}_{0}\right)\right)+\operatorname{Osc}\left(Q_{f}\left(A_{1}, L_{\theta} \partial_{\omega}^{2} \stackrel{\star}{A}_{4}+M \partial_{\omega} \stackrel{\star}{A}_{2}-\stackrel{\star}{*}_{\mathbf{0}}^{\prime}(1)-\stackrel{\star}{\mathcal{F}}_{1}\right)\right) \\
& +Q_{g}\left(\stackrel{\star}{\phi}_{1}, \square \widetilde{\phi}_{0}-\widetilde{\boldsymbol{G}}_{0}\right)+\operatorname{Osc}\left(Q_{g}\left(\stackrel{\star}{\phi}_{1}, X \partial_{\omega} \stackrel{\star}{\phi}_{2}-{\stackrel{\star}{\mathbf{G}_{0}^{\prime}}}_{\mathbf{0}}(1)-\stackrel{\star}{\mathcal{G}}_{1}\right)\right) \\
& +\operatorname{Osc}\left(Q_{h}\left(\psi_{1}, \mathcal{D}_{\theta} \partial_{\omega} \stackrel{\star}{\psi}_{2}+\mathcal{D} \psi_{0}-\boldsymbol{H}_{0}\right)\right) \\
& +\operatorname{Osc}\left(Q_{f}\left(A_{2}, L_{\theta} \partial_{\omega}^{2} \stackrel{\star}{A}_{3}+M \partial_{\omega} \stackrel{\star}{A}{ }_{1}-\stackrel{\star}{\boldsymbol{F}}_{0}\right)\right)+\operatorname{Osc}\left(Q_{g}\left(\stackrel{\star}{\phi}_{2}, X \partial_{\omega} \stackrel{\star}{\phi}_{1}-\stackrel{\star}{\boldsymbol{G}}_{0}\right)\right) \\
& +\operatorname{Osc}\left(Q_{f}\left(A_{3}, L_{\theta} \partial_{\omega}^{2} \stackrel{\star}{A} 2\right)+Q_{f}\left(A_{4}, L_{\theta} \partial_{\omega}^{2} \stackrel{\star}{A}_{1}\right)\right) \\
& +\operatorname{Osc}\left(Q_{h}\left(\psi_{2}, \mathcal{D}_{\theta} \partial_{\omega} \stackrel{\star}{\psi}_{1}\right)+Q_{h}\left(\psi_{3}, \mathcal{D}_{\theta} \partial_{\omega} \stackrel{\star}{\psi}_{0}\right)\right) \\
& \text { - à l'ordre } \varepsilon^{i / 2}, i>1 \text { : }
\end{aligned}
$$

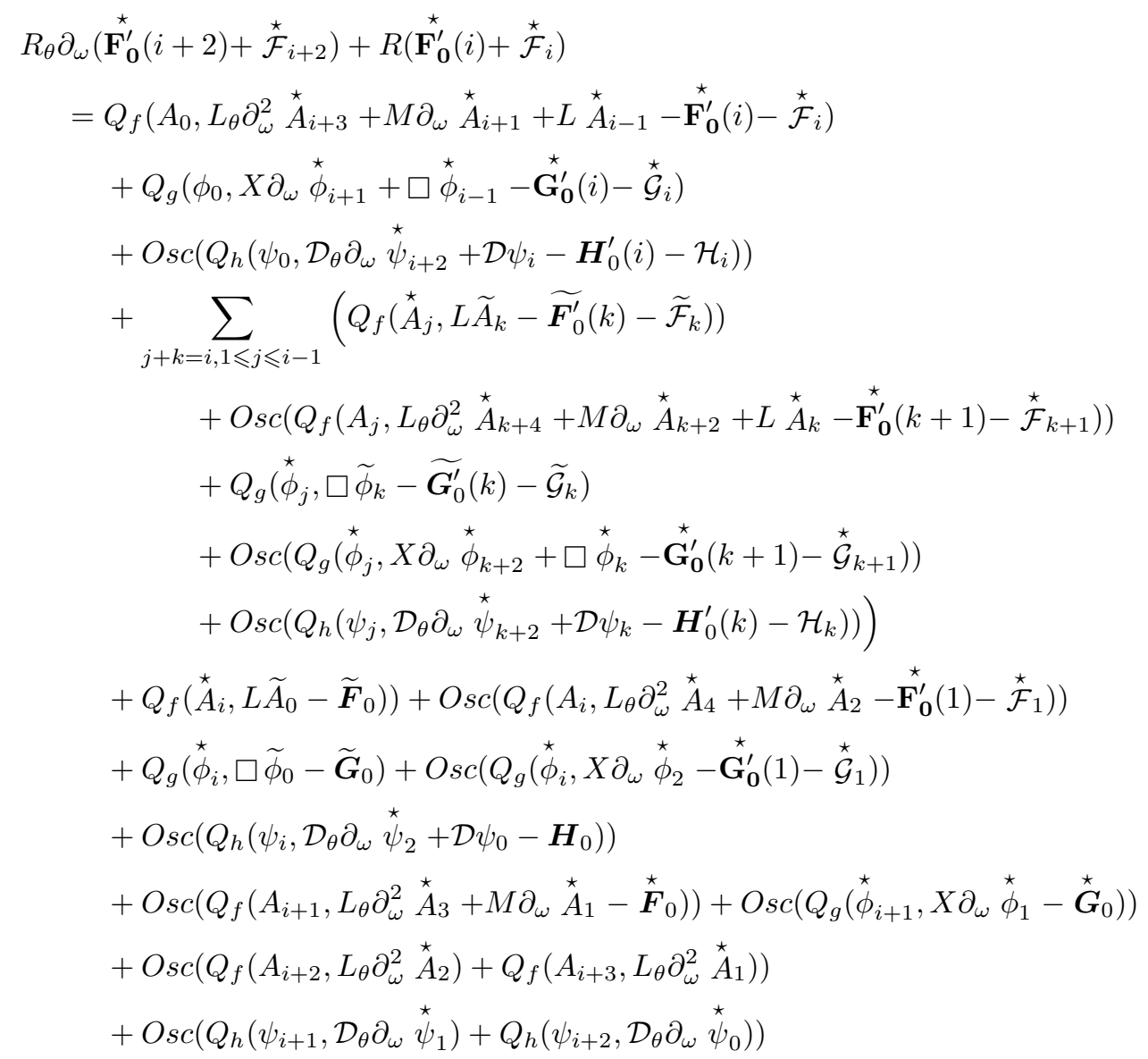


Nous en déduisons :

Proposition 2.2.1. - a) Si $a_{1}^{\vee}=0$, alors

$$
R_{\theta} \stackrel{\star}{\boldsymbol{F}}_{0}=0
$$

Si $a_{1}^{\vee}=a_{2}^{\vee}=0$ et $\psi_{0}^{\star}=0$, alors

$$
R_{\theta}\left({\stackrel{\star}{\mathbf{F}_{0}^{\prime}}}_{\mathbf{0}}(1)+\stackrel{\star}{\mathcal{F}}_{1}\right)=0
$$

b) $S i a_{1}^{\vee}=a_{2}^{\vee}=0$ et $\psi_{0}^{\star}=\psi_{1}^{-}=0$, si $\left(A_{0}, a_{1}^{\#}, \phi_{0}, \stackrel{\star}{\phi}_{1}, \widetilde{\psi}_{0},{\stackrel{\star}{\psi_{0}^{+}}}^{+}\right.$est solution de $(C, 0)$ et si $\left(a_{3}^{\vee}, \psi_{2}^{-}\right)$satisfont à la polarisation $(P, 0)$, alors

$$
R_{\theta}\left({\stackrel{\star}{\mathbf{F}_{0}^{\prime}}}^{(2)}+\stackrel{\star}{\mathcal{F}}_{2}\right)+R \partial_{\omega}^{-1} \stackrel{\star}{\boldsymbol{F}}_{0}=0
$$

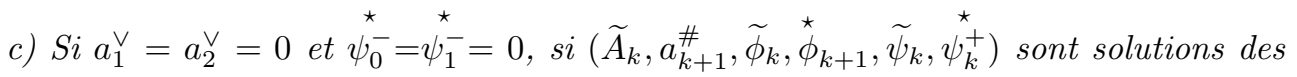
systèmes $(C, k)$ successifs et si $\left(a_{k+3}^{\vee}, \psi_{k+2}^{\star}\right)$ satisfont aux polarisations $(P, k)$, pour tout $0 \leqslant k \leqslant i-2$, alors

$$
R_{\theta}\left({\stackrel{\star}{\mathbf{F}_{\mathbf{0}}^{\prime}}}^{(i+2)}+\stackrel{\star}{\mathcal{F}}_{i+2}\right)+R \partial_{\omega}^{-1}\left({\stackrel{\star}{\mathbf{F}_{\mathbf{0}}^{\prime}}}^{(i)}+\stackrel{\star}{\mathcal{F}}_{i}\right)=0
$$

Nous utiliserons ce résultat pour montrer que les systèmes $(C, i)$ successifs nous laissent libre choix de la composante $a_{i+1}^{\wedge}$ de chaque $\stackrel{\star}{A}_{i+1}$. Pour les parties moyennes du développement, nous obtenons les expressions suivantes :

- à l'ordre $\varepsilon^{-1}$ :

$$
\operatorname{Moy}\left(Q_{f}\left(A_{1}, L_{\theta} \partial_{\omega}^{2} \stackrel{\star}{A}\right)\right)+\operatorname{Moy}\left(Q_{h}\left(\psi_{0}, \mathcal{D}_{\theta} \partial_{\omega} \stackrel{\star}{\psi_{0}}\right)\right)=0
$$

- à l'ordre $\varepsilon^{-1 / 2}$ :

$$
\begin{aligned}
\operatorname{Moy}\left(Q_{f}\left(A_{1}, L_{\theta} \partial_{\omega}^{2} \stackrel{\star}{A}_{2}\right)+Q_{f}(\right. & \left.\left.A_{2}, L_{\theta} \partial_{\omega}^{2} \stackrel{\star}{A}_{1}\right)\right) \\
& +\operatorname{Moy}\left(Q_{h}\left(\stackrel{\star}{\psi}_{0}, \mathcal{D}_{\theta} \partial_{\omega} \stackrel{\star}{\psi_{1}}\right)+Q_{h}\left(\stackrel{\star}{\psi}_{1}, \mathcal{D}_{\theta} \partial_{\omega} \stackrel{\star}{\psi_{0}}\right)\right)=0
\end{aligned}
$$

(Ces deux premières identités ne nous apportent aucun renseignement utile, dès lors qu'elles recoupent les conditions de polarisation des premiers profils oscillants).

- à l'ordre $\varepsilon^{0}$ :

$$
\begin{aligned}
R \widetilde{\boldsymbol{F}}_{0}= & Q_{f}\left(A_{0}, L A_{0}-\widetilde{\boldsymbol{F}}_{0}\right)+\operatorname{Moy}\left(Q_{f}\left(\stackrel{\star}{A}_{1}, L_{\theta} \partial_{\omega}^{2} \stackrel{\star}{A}_{3}+M \partial_{\omega} \stackrel{\star}{A} 1-\stackrel{\star}{\boldsymbol{F}}_{0}\right)\right) \\
& +Q_{g}\left(\phi_{0}, \square \phi_{0}-\widetilde{\boldsymbol{G}}_{0}\right)+\operatorname{Moy}\left(Q_{g}\left(\stackrel{\star}{\phi}_{1}, X \partial_{\omega} \stackrel{\star}{\phi}_{1}-\stackrel{\star}{\boldsymbol{G}}_{0}\right)\right) \\
& +\operatorname{Moy}\left(\left(Q_{h}\left(\psi_{0}, \mathcal{D}_{\theta} \partial_{\omega} \stackrel{\star}{\psi}_{2}+\mathcal{D} \psi_{0}-\boldsymbol{H}_{0}\right)\right)\right. \\
& +\operatorname{Moy}\left(Q_{f}\left(A_{2}, L_{\theta} \partial_{\omega}^{2} \stackrel{\star}{A}_{2}\right)+Q_{f}\left(A_{3}, L_{\theta} \partial_{\omega}^{2} \stackrel{\star}{A}_{1}\right)\right) \\
& +\operatorname{Moy}\left(Q_{h}\left(\stackrel{\star}{\psi}_{1}, \mathcal{D}_{\theta} \partial_{\omega} \stackrel{\star}{\psi}_{1}\right)+Q_{h}\left(\stackrel{\star}{\psi}_{2}, \mathcal{D}_{\theta} \partial_{\omega} \stackrel{\star}{\psi}_{0}\right)\right)
\end{aligned}
$$


- à l'ordre $\varepsilon^{1 / 2}$;

$$
\begin{aligned}
& R\left(\widetilde{\boldsymbol{F}_{0}^{\prime}}(1)+\widetilde{\mathcal{F}}_{1}\right)=Q_{f}\left(A_{0}, L \widetilde{A}_{1}-\widetilde{\boldsymbol{F}_{0}^{\prime}}(1)-\widetilde{\mathcal{F}}_{1}\right) \\
& +\operatorname{Moy}\left(Q_{f}\left(\stackrel{\star}{A}_{1}, L_{\theta} \partial_{\omega}^{2} \stackrel{\star}{A}_{4}+M \partial_{\omega} \stackrel{\star}{A_{2}}-\stackrel{\star}{\mathbf{F}}_{\mathbf{0}}^{\prime}(1)-\stackrel{\star}{\mathcal{F}}_{1}\right)\right) \\
& +Q_{g}\left(\phi_{0}, \square \widetilde{\phi}_{1}-\widetilde{\boldsymbol{G}_{0}^{\prime}}(1)-\widetilde{\mathcal{G}}_{1}\right)+\operatorname{Moy}\left(Q_{g}\left(\stackrel{\star}{\phi}_{1}, X \partial_{\omega} \stackrel{\star}{\phi}_{2}-\stackrel{\mathbf{G}}{\mathbf{0}}_{\mathbf{0}}(1)-\stackrel{\star}{\mathcal{G}}_{1}\right)\right) \\
& +\operatorname{Moy}\left(Q_{h}\left(\psi_{0}, \mathcal{D}_{\theta} \partial_{\omega} \stackrel{\star}{\psi}_{3}+\mathcal{D} \psi_{1}-\boldsymbol{H}_{0}^{\prime}(1)-\mathcal{H}_{1}\right)\right) \\
& +Q_{f}\left(\widetilde{A}_{1}, L \widetilde{A}_{0}-\widetilde{\boldsymbol{F}}_{0}\right)+\operatorname{Moy}\left(Q_{f}\left(\stackrel{\star}{A_{2}}, L_{\theta} \partial_{\omega}^{2} \stackrel{\star}{A_{3}}+M \partial_{\omega} \stackrel{\star}{A_{1}}-\stackrel{\star}{\boldsymbol{F}}_{0}\right)\right) \\
& +Q_{g}\left(\widetilde{\phi}_{1}, \square \widetilde{\phi}_{0}-\widetilde{\boldsymbol{G}}_{0}\right)+\operatorname{Moy}\left(Q_{g}\left(\stackrel{\star}{\phi}_{2}, X \partial_{\omega} \stackrel{\star}{\phi}_{1}-\stackrel{\star}{\boldsymbol{G}}_{0}\right)\right) \\
& +\operatorname{Moy}\left(Q_{h}\left(\psi_{1}, \mathcal{D}_{\theta} \partial_{\omega} \stackrel{\star}{\psi}_{2}+\mathcal{D} \psi_{0}-\boldsymbol{H}_{0}\right)\right) \\
& +\operatorname{Moy}\left(Q_{f}\left(A_{3}, L_{\theta} \partial_{\omega}^{2} \stackrel{\star}{A}{ }_{2}\right)+Q_{f}\left(A_{4}, L_{\theta} \partial_{\omega}^{2} \stackrel{\star}{A_{1}}\right)\right) \\
& +\operatorname{Moy}\left(Q_{h}\left(\stackrel{\star}{\psi}_{2}, \mathcal{D}_{\theta} \partial_{\omega} \stackrel{\star}{\psi}_{1}\right)+Q_{h}\left(\stackrel{\star}{\psi}_{3}, \mathcal{D}_{\theta} \partial_{\omega} \stackrel{\star}{\psi}_{0}\right)\right)
\end{aligned}
$$

- enfin, à l'ordre $\varepsilon^{i / 2}, i>1$ :

$$
\begin{aligned}
& R\left(\widetilde{\boldsymbol{F}_{0}^{\prime}}(i)+\widetilde{\mathcal{F}}_{i}\right)=Q_{f}\left(A_{0}, L \widetilde{A}_{i}-\widetilde{\boldsymbol{F}_{0}^{\prime}}(i)-\widetilde{\mathcal{F}}_{i}\right) \\
& +\operatorname{Moy}\left(Q_{f}\left(\stackrel{\star}{A}_{1}, L_{\theta} \partial_{\omega}^{2} \stackrel{\star}{A} i+3+M \partial_{\omega} \stackrel{\star}{A} i_{i+1}+L \stackrel{\star}{A_{i-1}}-\stackrel{\star}{\mathbf{F}}_{\mathbf{0}}(i)-\stackrel{\star}{\mathcal{F}}_{i}\right)\right) \\
& +Q_{g}\left(\phi_{0}, \square \widetilde{\phi_{i}}-\widetilde{\boldsymbol{G}_{0}^{\prime}}(i)-\widetilde{\mathcal{G}}_{i}\right)+\operatorname{Moy}\left(Q_{g}\left(\stackrel{\star}{\phi}_{1}, X \partial_{\omega}{\stackrel{\star}{\phi_{i+1}}}+\square \stackrel{\star}{\phi}_{i-1}-{\stackrel{\star}{\mathbf{G}_{0}^{\prime}}}^{\prime}(i)-\stackrel{\star}{\mathcal{G}}_{i}\right)\right) \\
& +\operatorname{Moy}\left(Q_{h}\left(\psi_{0}, \mathcal{D}_{\theta} \partial_{\omega} \stackrel{\star}{\psi}_{i+2}+\mathcal{D} \psi_{i}-\boldsymbol{H}_{0}^{\prime}(i)-\mathcal{H}_{i}\right)\right) \\
& +\sum_{j+k=i, 1 \leqslant j \leqslant i-1}\left(Q_{f}\left(\widetilde{A}_{j}, L \widetilde{A}_{k}-\widetilde{\boldsymbol{F}}_{0}^{\prime}(k)-\widetilde{\mathcal{F}}_{k}\right)\right. \\
& +\operatorname{Moy}\left(Q_{f}\left(\stackrel{\star}{A}_{j+1}, L_{\theta} \partial_{\omega}^{2} \stackrel{\star}{A}_{k+3}+M \partial_{\omega} \stackrel{\star}{A}_{k+1}+L \stackrel{\star}{A}_{k-1}-\stackrel{\star}{\mathbf{F}}_{\mathbf{0}}^{\prime}(k)-\stackrel{\star}{\mathcal{F}}_{k}\right)\right) \\
& +Q_{g}\left(\widetilde{\phi}_{j}, \square \widetilde{\phi}_{k}-{\widetilde{G_{0}^{\prime}}}_{0}(k)-\widetilde{\mathcal{G}}_{k}\right) \\
& +\operatorname{Moy}\left(Q_{g}\left(\stackrel{\star}{\phi}_{j+1}, X \partial_{\omega} \stackrel{\star}{\phi}_{k+1}+\square \stackrel{\star}{\phi}_{k-1}-{\stackrel{\star}{\mathbf{G}_{0}^{\prime}}}^{\prime}(k)-\stackrel{\star}{\mathcal{G}}_{k}\right)\right) \\
& \left.+\operatorname{Moy}\left(Q_{h}\left(\psi_{j}, \mathcal{D}_{\theta} \partial_{\omega} \stackrel{\star}{\psi}_{k+2}+\mathcal{D} \psi_{k}-\boldsymbol{H}_{0}^{\prime}(k)-\mathcal{H}_{k}\right)\right)\right) \\
& +Q_{f}\left(\widetilde{A}_{i}, L \widetilde{A}_{0}-\widetilde{\boldsymbol{F}}_{0}\right)+\operatorname{Moy}\left(Q_{f}\left(\stackrel{\star}{A}_{i+1}, L_{\theta} \partial_{\omega}^{2} \stackrel{\star}{A}{ }_{3}+M \partial_{\omega} \stackrel{\star}{A_{1}}-\stackrel{\star}{\boldsymbol{F}}_{0}\right)\right) \\
& +Q_{g}\left(\widetilde{\phi}_{i}, \square \widetilde{\phi}_{0}-\widetilde{\boldsymbol{G}}_{0}\right)+\operatorname{Moy}\left(Q_{g}\left(\stackrel{\star}{\phi}_{i+1}, X \partial_{\omega} \stackrel{\star}{\phi}_{1}-\stackrel{\star}{\boldsymbol{G}}_{0}\right)\right) \\
& +\operatorname{Moy}\left(Q_{h}\left(\psi_{i}, \mathcal{D}_{\theta} \partial_{\omega} \stackrel{\star}{\psi}_{2}+\mathcal{D} \psi_{0}-\boldsymbol{H}_{0}\right)\right) \\
& +\operatorname{Moy}\left(Q_{f}\left(A_{i+2}, L_{\theta} \partial_{\omega}^{2} \stackrel{\star}{A} 2\right)+Q_{f}\left(A_{i+3}, L_{\theta} \partial_{\omega}^{2} \stackrel{\star}{A} 1\right)\right) \\
& +\operatorname{Moy}\left(Q_{h}\left(\stackrel{\star}{\psi}_{i+1}, \mathcal{D}_{\theta} \partial_{\omega} \stackrel{\star}{\psi}_{1}\right)+Q_{h}\left(\stackrel{\star}{\psi}_{i+2}, \mathcal{D}_{\theta} \partial_{\omega} \stackrel{\star}{\psi}_{0}\right)\right)
\end{aligned}
$$


Nous en déduisons la proposition :

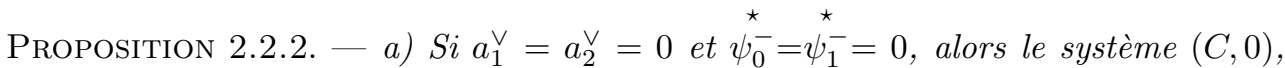
pris conjointement avec les polarisations $(P, 0)$ qui l'accompagnent, est compatible extérieurement avec l'opérateur $L$ (au sens de la définition 1.3.1). La relation de compatibilité s'écrit :

$$
\begin{aligned}
R \widetilde{\boldsymbol{F}}_{0}= & Q_{f}\left(A_{0}, L A_{0}-\widetilde{\boldsymbol{F}}_{0}\right)+\operatorname{Moy}\left(Q_{f}\left(\stackrel{\star}{A}_{1}, L_{\theta} \partial_{\omega}^{2} \stackrel{\star}{A_{3}}+M \partial_{\omega} \stackrel{\star}{A_{1}}-\stackrel{\star}{\boldsymbol{F}}_{0}\right)\right) \\
& +Q_{g}\left(\phi_{0}, \square \phi_{0}-\widetilde{\boldsymbol{G}}_{0}\right)+\operatorname{Moy}\left(Q_{g}\left(\stackrel{\star}{\phi}_{1}, X \partial_{\omega} \stackrel{\star}{\phi}_{1}-\stackrel{\star}{\boldsymbol{G}}_{0}\right)\right) \\
& +\operatorname{Moy}\left(\left(Q_{h}\left(\psi_{0}, \mathcal{D}_{\theta} \partial_{\omega} \stackrel{\star}{\psi_{2}}+\mathcal{D} \psi_{0}-\boldsymbol{H}_{0}\right)\right)\right.
\end{aligned}
$$

b) Si $a_{1}^{\vee}=a_{2}^{\vee}=0$ et $\psi_{0}^{-}=\psi_{1}^{-}=0$, si $\left(\widetilde{A}_{k}, a_{k+1}^{\#}, \widetilde{\phi}_{k}, \stackrel{\star}{\phi}_{k+1}, \widetilde{\psi}_{k}, \stackrel{\star}{*}+^{+}\right)$sont solutions des systèmes $(C, k)$ successifs et si $\left(a_{k+3}^{\vee}, \psi_{k+2}^{-}\right)$satisfont aux polarisations $(P, k)$, pour tout $0 \leqslant k<i$, alors le système $(C, i)$ accompagné des polarisations $(P, i)$ est compatible extérieurement avec l'opérateur $L$, et sa relation de compatibilité s'écrit :

$$
\begin{aligned}
R\left(\widetilde{\boldsymbol{F}}_{0}^{\prime}(i)+\widetilde{\mathcal{F}}_{i}\right)= & Q_{f}\left(A_{0}, L \widetilde{A}_{i}-\widetilde{\boldsymbol{F}}_{0}^{\prime}(i)-\widetilde{\mathcal{F}}_{i}\right) \\
& +\operatorname{Moy}\left(Q_{f}\left(\stackrel{\star}{A}_{1}, L_{\theta} \partial_{\omega}^{2} \stackrel{\star}{A}_{i+3}+M \partial_{\omega} \stackrel{\star}{A_{i+1}}+L \stackrel{\star}{A_{i-1}}-\stackrel{\star}{\mathbf{F}}_{\mathbf{0}}(i)-\stackrel{\star}{\mathcal{F}}_{i}\right)\right) \\
& +Q_{g}\left(\phi_{0}, \square \widetilde{\phi}_{i}-\widetilde{\boldsymbol{G}}_{0}^{\prime}(i)-\widetilde{\mathcal{G}}_{i}\right) \\
& +\operatorname{Moy}\left(Q_{g}\left(\stackrel{\star}{\phi}_{1}, X \partial_{\omega} \stackrel{\star}{\phi_{i+1}}+\square \stackrel{\star}{\phi_{i-1}}-\stackrel{\star}{\mathbf{G}_{\mathbf{0}}^{\prime}}(i)-\stackrel{\star}{\mathcal{G}}\right)\right) \\
& +\operatorname{Moy}\left(Q_{h}\left(\psi_{0}, \mathcal{D}_{\theta} \partial_{\omega} \stackrel{\star}{\psi}_{i+2}+\mathcal{D} \psi_{i}-\boldsymbol{H}_{0}^{\prime}(i)-\mathcal{H}_{i}\right)\right)
\end{aligned}
$$

Remarque 2.2.2. - Les résultats des propositions 2.2.1 et 2.2.2 relèvent du bon sens, puisqu'ils nous disent simplement que le développement de l'identité de compatibilité d'un système donne bien les identités de compatibilité du développement de ce même système (en d'autres termes, le développement de Taylor d'une loi de composition donne bien, au moins globalement, la composée des développements de chacun de ses membres) ... Encore faut-il vérifier que ce résultat reste vrai terme à terme lorsqu'on tronque les développements à un ordre arbitraire. Il est indispensable que la condition de compatibilité de chaque $(C, i)$ n'implique que des profils donnés par par les $(C, k)$ d'indice $k \leqslant i$. Or nous sommes précisément dans le cas limite où chaque $(C, i)$ porte sa propre condition de compatibilité, ce qui rend son intérêt au calcul explicite qui précède. Des développements oscillants plus singuliers que ceux que nous envisageons conduiraient fatalement à rompre la chaîne de dépendance causale qui gouverne les $(C, i)$.

Comme corollaire immédiat de la proposition 2.2.2 et de la proposition 1.3.5, nous concluons que les systèmes $(C, i)$ successifs propagent n'importe quelle bonne jauge sur la partie moyenne des profils $\widetilde{A_{i}}, i \geqslant 0$, ce qui deviendra, le temps venu, une condition importante pour résoudre les $(C, i)$. 
Réduction du système. - Nous allons maintenant exploiter la proposition 2.2.1 pour achever la description géométrique des oscillations du champ de jauge.

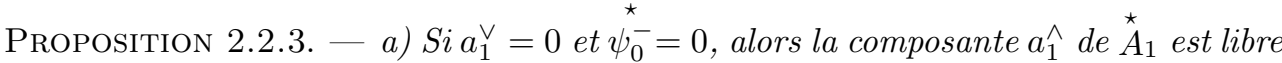
et n'intervient pas dans les équations du système $(C, 0)$. La composante dynamique $a_{1}^{0}$ de $\stackrel{\star}{A_{1}}$ est entièrement déterminée par une équation de transport sur le fibré $\Pi_{0}$ :

$$
X_{\left.\right|_{\Pi_{0}}} \partial_{\omega} a_{1}^{0}=\pi^{0}{\stackrel{\star}{\boldsymbol{F}_{0}}}
$$

Si $a_{1}^{\vee}=a_{2}^{\vee}=0$ et $\psi_{0}^{-}=\psi_{1}^{-}=0$, alors la composante $a_{2}^{\wedge}$ de $\stackrel{\star}{A}$ est libre et n'intervient pas dans les équations du système $(C, 1)$. La composante dynamique $a_{2}^{0}$ de $\stackrel{\star}{A}_{2}$ est entièrement déterminée par une équation de transport sur le fibré $\Pi_{0}$ :

$$
X_{\left.\right|_{\Pi_{0}}} \partial_{\omega} a_{2}^{0}=\pi^{0}\left(\stackrel{\star}{\mathbf{F}_{\mathbf{0}}^{\prime}}(1)+\stackrel{\star}{\mathcal{F}}{ }_{1}\right)
$$

b) si $a_{1}^{\vee}=a_{2}^{\vee}=0$ et $\stackrel{\star}{*}_{0}^{-}=\psi_{1}^{-}=0$, si $\left(\widetilde{A}_{k}, a_{k+1}^{0}, \widetilde{\phi}_{k}, \stackrel{\star}{\phi}_{k+1}, \widetilde{\psi}_{k}, \stackrel{\star}{*}+_{k}^{+}\right)$sont solutions des systèmes $(C, k)$ successifs et si $\left(a_{k+3}^{\vee}, \psi_{k+2}^{-}\right)$satisfont aux polarisations $(P, k)$, pour tout $0 \leqslant k \leqslant i-2$, alors :

- la composante $a_{i+1}^{\vee}$ de $\stackrel{\star}{A}_{i+1}$, fixée d'avance par la contrainte $(P, i-2)$, est une fonction polynomiale des $\left(\widetilde{A}_{k}, \stackrel{\star}{A}_{k+1}, \widetilde{\phi}_{k}, \stackrel{\star}{\phi}_{k+1}, \widetilde{\psi}_{k}, \stackrel{\star}{\psi}_{k}\right), k \leqslant i-2$,

- le choix de la composante $a_{i+1}^{\wedge}$ de $\stackrel{\star}{A}_{i+1}$ est libre et n'intervient pas dans les équations du système $(C, i)$.

- La composante dynamique $a_{i+1}^{0}$ de $\stackrel{\star}{A}_{i+1}$ est entièrement déterminée par une équation de transport sur le fibré $\Pi_{0}$ :

$$
X_{\left.\right|_{\Pi_{0}}} \partial_{\omega} a_{i+1}^{0}=\pi^{0}\left({\stackrel{\star}{\mathbf{F}_{\mathbf{0}}^{\prime}}}^{\prime}(i)+\stackrel{\star}{\mathcal{F}}{ }_{i}\right)
$$

Cette proposition montre qu'il est possible de réduire l'étude des oscillations de chaque profil $A_{i+1}, i \geqslant 1$, au fibré $\Pi_{0}$ en quotientant au sein de chaque $(C, i)$ l'action des sections de $\Pi_{\wedge}$. Les composantes dynamiques du système sont portées par $\Pi_{0}$. En identifiant les composantes libres de $\Pi_{\wedge}$ avec des oscillations de la jauge, il sera possible de construire des solutions approchées du système $(Y M)$ qui préservent à tout ordre les conditions de jauge de type $J_{\lambda}$. Les composantes résiduelles de $\Pi_{\vee}$ sont figées, et représentent la partie surdéterminée du système. Nous commençons par prouver la proposition intermédiaire suivante :

Proposition 2.2.4. - a) Si $a_{1}^{\vee}=0$, alors

$$
\pi^{\vee} L_{\theta} \partial_{\omega}^{2} \stackrel{\star}{A}_{3}=\pi^{\vee}\left(M \partial_{\omega} \stackrel{\star}{A}{ }_{1}\right)=\pi^{\vee}\left(\stackrel{\star}{F}_{0}\left(A_{0}, \partial_{\omega} \stackrel{\star}{A_{1}}, \phi_{0}, \partial_{\omega} \stackrel{\star}{\phi_{1}}\right)\right)=0
$$

indépendamment des valeurs prises par les variables $\left(A_{0}, a_{1}^{\#}, \phi_{0}, \stackrel{\star}{\phi_{1}}, \widetilde{\psi}_{0},{\stackrel{\star}{\psi_{0}^{+}}}^{+}\right.$. 
Si $a_{1}^{\vee}=a_{2}^{\vee}=0$ et $\psi_{0}^{\star}=0$, alors

$$
\pi^{\vee}\left(L_{\theta} \partial_{\omega}^{2} \stackrel{\star}{A}_{4}+M \partial_{\omega} \stackrel{\star}{A}_{2}\right)=\pi^{\vee}\left({\stackrel{\star}{\mathbf{F}_{0}^{\prime}}}_{\mathbf{0}}(1)+\stackrel{\star}{\mathcal{F}}_{1}\right)=0
$$

indépendamment des valeurs prises par les variables $\left(\widetilde{A}_{1}, a^{\star}{ }_{2}, \widetilde{\phi}_{1}, \stackrel{\star}{\phi}_{2}, \widetilde{\psi}_{1},{\stackrel{\star}{\psi_{1}^{+}}}^{+}\right)$.

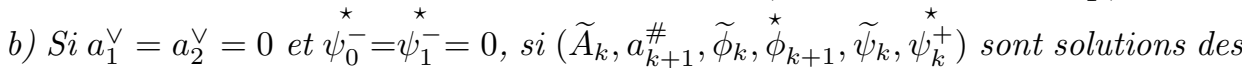
systèmes $(C, k)$ successifs et si $\left(a_{k+3}^{\vee}, \psi_{k+2}^{-}\right)$satisfont aux polarisations $(P, k)$, pour tout $0 \leqslant k \leqslant i-2$, alors l'équation

$$
\begin{aligned}
& \pi^{\vee}\left(L_{\theta} \partial_{\omega}^{2} \stackrel{\star}{A}_{i+3}+M \partial_{\omega} \stackrel{\star}{A}_{i+1}+L \stackrel{\star}{A}_{i-1}\right) \\
& =\pi^{\vee}\left({\stackrel{\star}{\mathbf{F}_{\mathbf{0}}^{\prime}}}^{\prime} \cdot\left(\widetilde{A}_{i}, \partial \widetilde{A}_{i}, \partial_{\omega} \stackrel{\star}{A_{i+1}}, \widetilde{\phi}_{i}, \partial \widetilde{\phi}_{i}, \partial_{\omega} \stackrel{\star}{\phi}_{i+1}, \widetilde{\psi}_{i}, \stackrel{\star}{\psi}_{i}\right)\right. \\
& \left.+\stackrel{\star}{\mathcal{F}}_{i}\left(A_{k}, \partial A_{k}, \stackrel{\star}{A}_{k+1}, \phi_{k}, \partial \phi_{k}, \stackrel{\star}{\phi}_{k+1}, \psi_{k}, k \leqslant i-1\right)\right)
\end{aligned}
$$

est automatiquement satisfaite, indépendamment des valeurs prises par les variables $\left(\widetilde{A}_{i}, a_{i+1}^{\#}, \widetilde{\phi}_{i}, \stackrel{\phi}{\phi}_{i+1}, \widetilde{\psi}_{i}, \psi_{i}^{\star}\right)$.

Démonstration. - $\pi^{\vee} L_{\theta}=0$ compte tenu de la décomposition (39), section 2.1. D'après la proposition 2.1.2, $\pi^{\vee} M \pi^{\#}=0$, d'où, pour tout indice $i \geqslant 0$ :

$$
\pi^{\vee} M \partial_{\omega} \stackrel{\star}{A}_{i+1}=\pi^{\vee} M \pi^{\vee} \partial_{\omega} \stackrel{\star}{A}_{i+1}
$$

Rappelons également que

$$
\pi^{\vee}=L_{\theta}^{-1} L_{\theta}=\left(L_{\theta}^{-1} T_{\theta}\right) R_{\theta}=\frac{\alpha^{2}}{2\left(\nabla_{0} \theta\right)^{2}}\left(\begin{array}{c}
\nabla_{0} \theta \\
-\nabla_{\Sigma} \theta
\end{array}\right) R_{\theta}
$$

a) pour $i=0$, si $a_{1}^{\vee}=0$, alors

$$
\pi^{\vee} M \partial_{\omega} \stackrel{\star}{A}{ }_{1}=\pi^{\vee} M \partial_{\omega} a_{1}^{\vee}=0
$$

tandis que

$$
\pi^{\vee} \stackrel{\star}{\boldsymbol{F}}_{0}=\left(L_{\theta}^{-1} T_{\theta}\right) R_{\theta} \stackrel{\star}{\boldsymbol{F}}_{0}\left(A_{0}, \partial_{\omega} \stackrel{\star}{A_{1}}, \phi_{0}, \partial_{\omega} \stackrel{\star}{\phi}_{1}\right)=0
$$

en en vertu de la proposition 2.2.1, a). De même, pour $i=1$, si les conditions de polarisation initiales $a_{1}^{\vee}=a_{2}^{\vee}=0$ et $\psi_{0}^{\star}=0$ sont respectée, il vient

$$
\pi^{\vee} M \partial_{\omega} \stackrel{\star}{A}{ }_{2}=\pi^{\vee} M \partial_{\omega} a_{2}^{\vee}=0
$$

et toujours par la proposition 2.2.1, a)

$$
\pi^{\vee}\left({\stackrel{\star}{\mathbf{F}_{0}^{\prime}}}_{\mathbf{0}}(1)+\stackrel{\star}{\mathcal{F}}_{1}\right)=\left(L_{\theta}^{-1} T_{\theta}\right) R_{\theta}\left(\stackrel{\star}{\mathbf{F}}_{\mathbf{0}}^{\prime}(1)+\stackrel{\star}{\mathcal{F}}_{1}\right)=0
$$

b) En faisant systématiquement appel aux identités (36) et (37), section 2.1, nous effectuons les calculs suivants : 


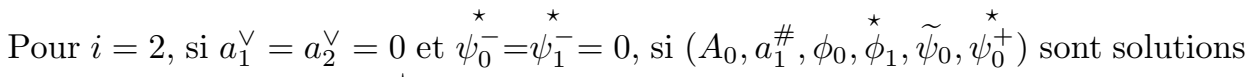
du système $(C, 0)$ et si $\left(a_{3}^{\vee}, \psi_{2}^{\star}\right)$ satisfont aux conditions de polarisation $(P, 0)$, alors :

$$
\begin{aligned}
& \pi^{\vee}\left(L_{\theta} \partial_{\omega}^{2} \stackrel{\star}{A}_{5}+M \partial_{\omega} \stackrel{\star}{A}_{3}+L \stackrel{\star}{A}_{1}-\stackrel{\star}{*}_{\mathbf{0}}^{\prime}(2)-\stackrel{\star}{\mathcal{F}}_{2}\right) \\
& =\pi^{\vee}\left(M \partial_{\omega} a_{3}^{\vee}+L \stackrel{\star}{A_{1}}-\stackrel{\star}{*}_{\mathbf{0}}^{\prime}(2)-\stackrel{\star}{\mathcal{F}}_{2}\right) \\
& =\left(L_{\theta}^{-1} T_{\theta}\right)\left(R_{\theta} M L_{\theta}^{-1} \pi^{\wedge} \partial_{\omega}^{-1}\left(-M \partial_{\omega} \stackrel{\star}{A} 1+\stackrel{\star}{\boldsymbol{F}}_{0}\right)+R_{\theta} L \stackrel{\star}{A_{1}}-R_{\theta} \stackrel{\star}{\mathbf{F}}_{\mathbf{0}}(2)-R_{\theta} \stackrel{\star}{\mathcal{F}} 2\right) \\
& =\left(L_{\theta}^{-1} T_{\theta}\right)\left(R L_{\theta} L_{\theta}^{-1} \pi^{\wedge} \partial_{\omega}^{-1}\left(M \partial_{\omega} \stackrel{\star}{A}_{1}-\stackrel{\star}{\boldsymbol{F}}_{0}\right)+R_{\theta} L \stackrel{\star}{A}_{1}-R_{\theta} \stackrel{\star}{\mathbf{F}}_{\mathbf{0}}^{\prime}(2)-R_{\theta} \stackrel{\star}{\mathcal{F}}_{2}\right) \\
& =\left(L_{\theta}^{-1} T_{\theta}\right)\left(R M \stackrel{\star}{A}{ }_{1}-R \partial_{\omega}^{-1} \stackrel{\star}{\boldsymbol{F}}_{0}-R M \stackrel{\star}{A} 1-R_{\theta} \stackrel{\star}{*}_{\mathbf{0}}^{\prime}(2)-R_{\theta} \stackrel{\star}{\mathcal{F}}_{2}\right) \\
& =-\left(L_{\theta}^{-1} T_{\theta}\right)\left(R \stackrel{\star}{\boldsymbol{F}_{0}}+R_{\theta} \partial_{\omega} \stackrel{\star}{\mathbf{F}}_{\mathbf{0}}^{\prime}(2)+R_{\theta} \partial_{\omega} \stackrel{\stackrel{\star}{\mathcal{F}}_{2}}{)}\right.
\end{aligned}
$$

Cette dernière expression est nulle en vertu de la proposition 2.2.1, b). Pour les indices

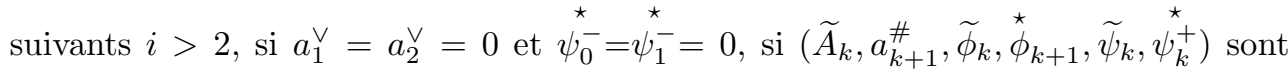
solutions des systèmes $(C, k)$ successifs et si $\left(a_{k+3}^{\vee}, \psi_{k+2}^{-}\right)$satisfont aux polarisations $(P, k)$, pour tout $0 \leqslant k \leqslant i-2$, alors

$$
\begin{aligned}
& \pi^{\vee}\left(L_{\theta} \partial_{\omega}^{2} \stackrel{\star}{A}_{i+3}+M \partial_{\omega} \stackrel{\star}{A}_{i+1}+L \stackrel{\star}{A}_{i-1}-{\stackrel{\star}{\mathbf{F}_{0}^{\prime}}}_{\mathbf{0}}(i)-\stackrel{\star}{\mathcal{F}}_{i}\right) \\
& =\pi^{\vee}\left(M \partial_{\omega} a_{i+1}^{\vee}+L \stackrel{\star}{A} i-1-\stackrel{\star}{\mathbf{F}}_{\mathbf{0}}^{\prime}(i)-\stackrel{\star}{\mathcal{F}}_{i}\right) \\
& =\left(L_{\theta}^{-1} T_{\theta}\right)\left(R_{\theta} M L_{\theta}^{-1} \pi^{\wedge} \partial_{\omega}^{-1}\left(-M \partial_{\omega} \stackrel{\star}{A}_{i-1}-L \stackrel{\star}{A}_{i-3}+\stackrel{\star}{\mathbf{F}}_{\mathbf{0}}(i-2)+\stackrel{\star}{\mathcal{F}}_{i-2}\right)\right. \\
& \left.+R_{\theta} L \stackrel{\star}{A}_{i-1}-R_{\theta} \stackrel{\star}{\prime}_{\mathbf{0}}^{\prime}(i)-R_{\theta} \stackrel{\star}{\mathcal{F}}_{i}\right) \\
& =\left(L_{\theta}^{-1} T_{\theta}\right)\left(R L_{\theta} L_{\theta}^{-1} \pi^{\wedge} \partial_{\omega}^{-1}\left(M \partial_{\omega} \stackrel{\star}{A}_{i-1}+L \stackrel{\star}{A}_{i-3}-\stackrel{\star}{\mathbf{F}}_{\mathbf{0}}^{\prime}(i-2)-\stackrel{\star}{\mathcal{F}}_{i-2}\right)\right. \\
& \left.+R_{\theta} L \stackrel{\star}{A}_{i-1}-R_{\theta} \stackrel{\star}{\mathbf{F}}_{\mathbf{0}}^{(i)}-R_{\theta} \stackrel{\star}{\mathcal{F}}_{i}\right) \\
& =\left(L_{\theta}^{-1} T_{\theta}\right)\left(R M \stackrel{\star}{A}_{i-1}+R L \stackrel{\star}{A}_{i-3}-R \partial_{\omega}^{-1}{\stackrel{\star}{\mathbf{F}_{\mathbf{0}}^{\prime}}}(i-2)-R \partial_{\omega}^{-1} \stackrel{\star}{\mathcal{F}}_{i-2}\right. \\
& \left.-R M \stackrel{\star}{A}_{i-1}-R_{\theta} \stackrel{\star}{\mathbf{F}}_{\mathbf{0}}(i)-R_{\theta} \stackrel{\star}{\mathcal{F}}_{i}\right) \\
& =-\left(L_{\theta}^{-1} T_{\theta}\right)\left(R \stackrel{\star}{\prime}_{\mathbf{0}}^{\prime}(i-2)+R \stackrel{\star}{\mathcal{F}}{ }_{i-2}+R_{\theta} \partial_{\omega}{\stackrel{\star}{\mathbf{F}_{\mathbf{0}}^{\prime}}}^{(i)}+R_{\theta} \partial_{\omega} \stackrel{\star}{\mathcal{F}}_{i}\right)
\end{aligned}
$$

La proposition $2.2 .1 \mathrm{c}$ ) s'applique, et ce terme s'annule également.

Démonstration de la proposition 2.2.3. - Nous prenons immédiatement en compte la proposition 2.2.4 précédente. Pour finir de quotienter l'action de $a_{i+1}^{\wedge}$ sur le reste du système, il ne reste plus qu'à vérifier :

- que Moy $F_{\theta}^{I}\left(a_{i+1}^{\wedge}, \partial_{\omega} \stackrel{\star}{A}_{1}\right)+\operatorname{Moy} F_{\theta}^{I}\left(\stackrel{\star}{A}_{1}, \partial_{\omega} a_{i+1}^{\wedge}\right)=0$. C'est une conséquence directe de la proposition 2.1 .4 , b) 
- que Moy $G_{\theta}^{I}\left(\stackrel{\phi}{\phi}_{1}, \partial_{\omega} a_{i+1}^{\wedge}\right)+\operatorname{Moy} G^{I I}{ }_{\theta}\left(a_{i+1}^{\wedge}, \partial_{\omega} \stackrel{\star}{\phi}_{1}\right)=0$. Ceci tient directement de la proposition 2.1.4, b)

- que $\pi^{0} M a_{i+1}^{\wedge}=0$. Ceci résulte de la proposition 2.1.2, qui nous donne l'identité $\pi^{0} M \pi^{\wedge}=0$.

- que $\pi^{0} F_{\theta}^{I}\left(A_{0}, \partial_{\omega} a_{i+1}^{\wedge}\right)=0$. Ceci découle une fois de plus de la proposition 2.1.4, a)

$$
F^{I}{ }_{\theta}\left(A_{0}, \partial_{\omega} a_{i+1}^{\wedge}\right) \in \Pi^{\wedge}
$$

- que $G^{I}{ }_{\theta}\left(\phi_{0}, \partial_{\omega} a_{i+1}^{\wedge}\right)=0$. Ceci vient immédiatement en appliquant une dernière fois la proposition 2.1.4, a).

Enfin, la proposition 2.1.2 nous dit que $\pi^{0} M \pi^{0}=X_{\left.\right|_{\Pi_{0}}}$. La preuve de la proposition 2.2.3 est complète.

2.2.3. Équations de profils à jauge libre. - Les résultats de la section 2.2.1, complétés par la proposition 2.2.3, nous permettent d'énoncer :

Proposition 2.2.5. - Soit $\theta$ une fonction de phase vérifiant les hypothèses 2.1.1 et $M \geqslant 1$ un entier arbitraire. Le développement oscillant de phase $\theta$

$$
\left\{\begin{array}{l}
A_{\varepsilon}(x)=\sum_{i \geqslant 0} \varepsilon^{i / 2}\left(\widetilde{A}_{i}(x)+\varepsilon^{1 / 2} \stackrel{\star}{A}_{i+1}\left(x, \frac{\theta(x)}{\varepsilon}\right)\right) \\
\phi_{\varepsilon}(x)=\sum_{i \geqslant 0} \varepsilon^{i / 2}\left(\widetilde{\phi}_{i}(x)+\varepsilon^{1 / 2} \stackrel{\star}{\phi}_{i+1}\left(x, \frac{\theta(x)}{\varepsilon}\right)\right) \\
\psi_{\varepsilon}(x)=\sum_{i \geqslant 0} \varepsilon^{i / 2}\left(\widetilde{\psi}_{i}(x)+\stackrel{\star}{\psi}_{i}\left(x, \frac{\theta(x)}{\varepsilon}\right)\right)
\end{array}\right.
$$

vérifie le système (Y.M.) à l'ordre $O\left(\varepsilon^{M / 2}\right)$ en partie moyenne, à l'ordre $O\left(\varepsilon^{(M-1) / 2}\right)$ en partie oscillante sur les $\left(A_{i}, \phi_{i}\right)$, à l'ordre $O\left(\varepsilon^{M / 2}\right)$ en partie oscillante sur les $\left(\psi_{i}\right)$ :

$$
\left\{\begin{array}{l}
\mathcal{O}_{\varepsilon}=L A_{\varepsilon}-F\left(A_{\varepsilon}, \phi_{\varepsilon}, \psi_{\varepsilon}\right)=\varepsilon^{M / 2} \sum_{i \geqslant 0} \varepsilon^{i / 2}\left(\varepsilon^{-1 / 2} \stackrel{\star}{\mathcal{O}}_{i-1}\left(x, \frac{\theta(x)}{\varepsilon}\right)+\widetilde{\mathcal{O}}_{i}(x)\right) \\
\mathcal{P}_{\varepsilon}=\square \phi_{\varepsilon}-G\left(A_{\varepsilon}, \phi_{\varepsilon}, \psi_{\varepsilon}\right)=\varepsilon^{M / 2} \sum_{0 \leqslant i \leqslant M^{\prime}} \varepsilon^{i / 2}\left(\varepsilon^{-1 / 2} \stackrel{\star}{\mathcal{P}}_{i-1}\left(x, \frac{\theta(x)}{\varepsilon}\right)+\widetilde{\mathcal{P}}_{i}(x)\right) \\
\mathcal{Q}_{\varepsilon}=\mathcal{D} \psi_{\varepsilon}-h\left(A_{\varepsilon}, \phi_{\varepsilon}, \psi_{\varepsilon}\right)=\varepsilon^{M / 2} \sum_{0 \leqslant i \leqslant M^{\prime}} \varepsilon^{i / 2}\left(\widetilde{\mathcal{Q}}_{i}(x)+\stackrel{\star}{\mathcal{Q}}_{i}\left(x, \frac{\theta(x)}{\varepsilon}\right)\right)
\end{array}\right.
$$

si et seulement si

- les composantes $\left(a_{1}^{\vee}, \psi_{0}^{\star}\right)$ et $\left(a_{2}^{\vee}, \psi_{1}^{\star}\right)$ satisfont aux polarisations initiales $(P,-2)$ et $(P,-1)$ 
- les profils $\left(A_{0}, a_{1}^{0}, \phi_{0}, \stackrel{\star}{\phi_{1}}, \widetilde{\psi}_{0}, \stackrel{\star}{\psi_{0}^{+}}\right)$vérifient

$(C, 0)$

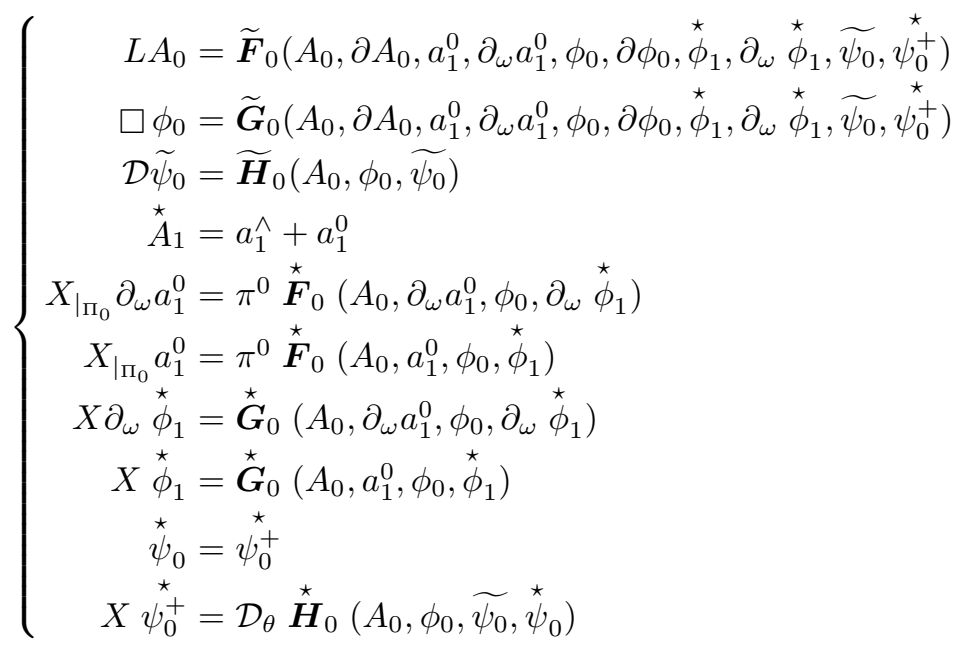

et les composantes $\left(a_{3}^{\vee}, \psi_{2}^{-}\right)$satisfont aux conditions de polarisations

$$
(P, 0)\left\{\begin{array}{c}
a_{3}^{\vee}=L_{\theta}^{-1} \partial_{\omega}^{-2} \pi^{\wedge}\left(-M \partial_{\omega} \stackrel{\star}{A}_{1}+\stackrel{\star}{\boldsymbol{F}_{0}}\left(A_{0}, \partial_{\omega} a_{1}^{0}, \phi_{0}, \partial_{\omega} \stackrel{\star}{\phi}_{1}\right)\right) \\
\psi_{2}^{-}=\mathcal{D}_{\theta}^{-1} \partial_{\omega}^{-1} \pi^{+}\left(-\mathcal{D} \stackrel{\star}{\psi_{0}}+\stackrel{\star}{\boldsymbol{H}}_{0}\left(A_{0}, \phi_{0}, \widetilde{\psi_{0}}, \stackrel{\star}{\psi}_{0}\right)\right)
\end{array}\right.
$$

- puis, pour les indices $1 \leqslant i<M$, les profils $\left(\widetilde{A}_{i}, a_{i+1}^{0}, \widetilde{\phi}_{i}, \stackrel{\star}{\phi}_{i+1}, \widetilde{\psi}_{i},,_{i}^{\star}\right)$ vérifient

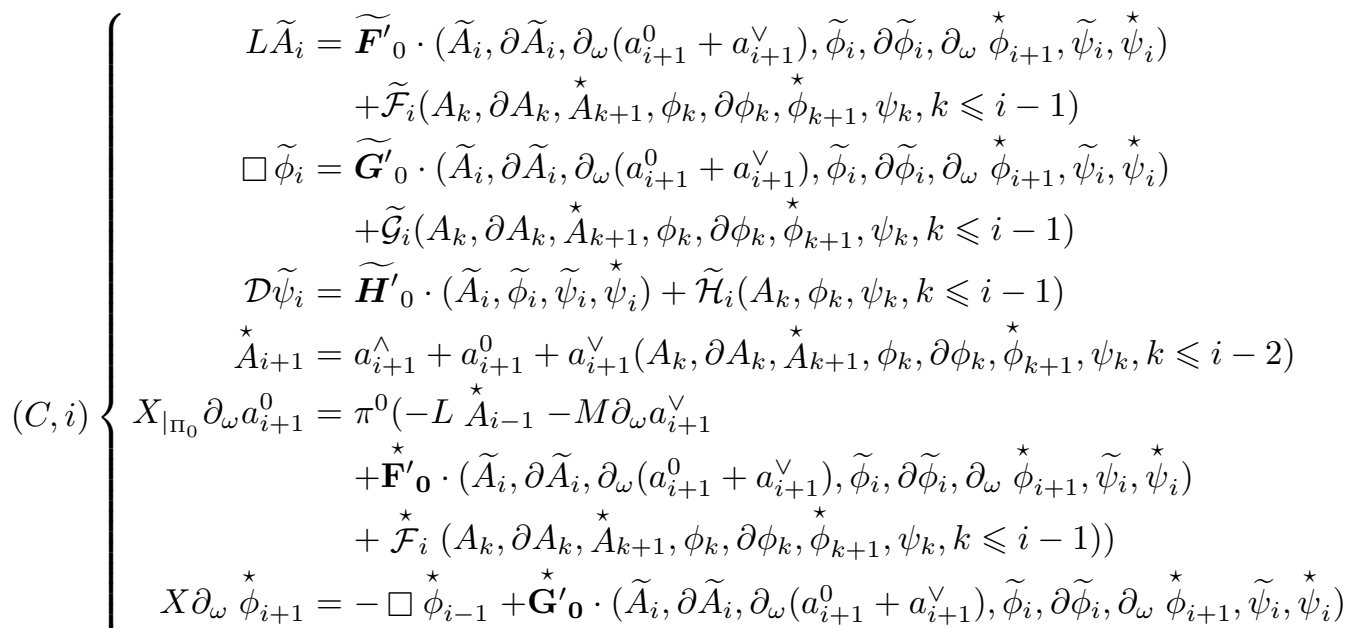

$$
\begin{aligned}
& +\stackrel{\star}{\mathcal{G}}_{i}\left(A_{k}, \partial A_{k}, \stackrel{\star}{A}_{k+1}, \phi_{k}, \partial \phi_{k}, \stackrel{\star}{\phi}_{k+1}, \psi_{k}, k \leqslant i-1\right) \\
& \stackrel{\star}{\psi}_{i}=\stackrel{\star}{\psi_{i}^{+}}+\stackrel{\star}{\psi_{i}^{-}}\left(A_{k}, \phi_{k}, \psi_{k}, k \leqslant i-2\right) \\
& \left.X \stackrel{\star}{\psi_{i}^{+}}=\mathcal{D}_{\theta}\left({\stackrel{\star}{\mathbf{H}^{\prime}}}_{\mathbf{0}} \cdot\left(\widetilde{A}_{i}, \widetilde{\phi}_{i}, \widetilde{\psi}_{i}, \stackrel{\star}{\psi}_{i}\right)\right)+\stackrel{\star}{\mathcal{H}}_{i}\left(A_{k}, \phi_{k}, \psi_{k}, k \leqslant i-1\right)-\mathcal{D} \stackrel{\star}{*}_{i}^{-}\right)
\end{aligned}
$$


et les composantes $\left(a_{i+3}^{\vee}, \psi_{i+2}^{\star}\right)$ satisfont aux conditions de polarisations

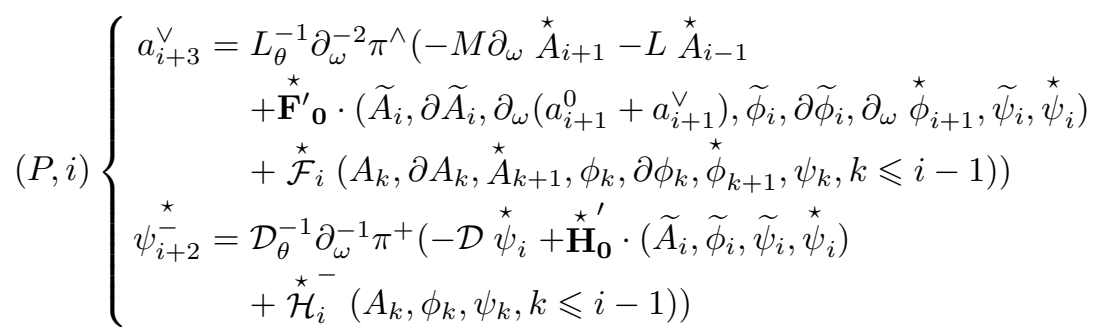

Invariance de jauge des $(C, i)$. — L'invariance de jauge des équations de profils est à prendre au sens suivant

Proposition 2.2.6. - a) Le système $(C, 0)$ et la condition de polarisation $(P, 0)$ qui l'accompagne restent invariants lorsque $(A, \phi, \psi)_{\varepsilon}$ sont soumis à un changement de jauge oscillant de la forme

$$
u_{\varepsilon}(x)=u_{0}(x)+\varepsilon^{3 / 2} \stackrel{\star}{\chi}_{3}\left(x, \frac{\theta(x)}{\varepsilon}\right)
$$

où $u_{0}(x)$ est un changement de jauge non-oscillant tandis que $\stackrel{\star}{\chi}_{3}(x, \omega)$ est une fonction oscillante à valeurs dans $\mathfrak{g}, 2 \pi$-périodiques de moyenne nulle en $\omega$.

b) les systèmes $(C, k)$ et les conditions de polarisation $(P, k)$ qui les accompagnent, d'indices $0 \leqslant k \leqslant i$, restent invariants lorsque $(A, \phi, \psi)_{\varepsilon}$ sont soumis à un changement de jauge oscillant de la forme

$$
u_{\varepsilon}(x)=1_{G}+\varepsilon^{i / 2} \widetilde{\chi}_{i}(x)+\varepsilon^{(i+3) / 2} \stackrel{\star}{\chi}_{i+3}\left(x, \frac{\theta(x)}{\varepsilon}\right)
$$

où les $\widetilde{\chi}_{i}(x)$ est une fonction non-oscillante à valeurs dans $\mathfrak{g}$, et $\stackrel{\star}{\chi}_{i+3}(x, \omega)$ est une fonction oscillante à valeurs dans $\mathfrak{g}, 2 \pi$-périodique de moyenne nulle en $\omega$.

Nous dirons que de tels changements de jauge sont «admissibles » pour les $(C, k)$, $k \leqslant i$.

Démonstration. - a) Sous l'action de $u_{\varepsilon}$,

$$
\left\{\begin{aligned}
A_{\varepsilon} \rightsquigarrow A_{\varepsilon}^{\prime} & =u_{\varepsilon}^{-1} A_{\varepsilon} u_{\varepsilon}+u_{\varepsilon}^{-1} d u_{\varepsilon} \\
\phi_{\varepsilon} \rightsquigarrow \phi_{\varepsilon}^{\prime} & =r\left(u_{\varepsilon}\right) \phi_{\varepsilon} \\
\psi_{\varepsilon} \rightsquigarrow \psi_{\varepsilon}^{\prime} & =\rho\left(u_{\varepsilon}\right) \psi_{\varepsilon}
\end{aligned}\right.
$$

avec

et

$$
\begin{aligned}
u_{\varepsilon}^{-1} & =u_{0}^{-1}-\varepsilon^{3 / 2} u_{0}^{-1} \stackrel{\star}{\chi}_{3} u_{0}^{-1}+o\left(\varepsilon^{3 / 2}\right) \\
d u_{\varepsilon} & =d u_{0}+\varepsilon^{1 / 2} T_{\theta} \partial_{\omega} \stackrel{\star}{\chi}_{3}+\varepsilon^{3 / 2} T \stackrel{\star}{\chi}_{3}+o\left(\varepsilon^{3 / 2}\right) \\
r\left(u_{\varepsilon}\right) & =r\left(u_{0}\right)+\varepsilon^{3 / 2} r\left(u_{0}\right) r^{\prime}(1) u_{0}^{-1} \stackrel{\star}{\chi}_{3}+o\left(\varepsilon^{3 / 2}\right) \\
\rho\left(u_{\varepsilon}\right) & =\rho\left(u_{0}\right)+\varepsilon^{3 / 2} \rho\left(u_{0}\right) \rho^{\prime}(1) u_{0}^{-1} \stackrel{\star}{\chi}_{3}+o\left(\varepsilon^{3 / 2}\right)
\end{aligned}
$$

MÉMOIRES DE LA SMF 90 
Nous identifions alors :

$$
\begin{aligned}
A_{0}^{\prime} & =u_{0}^{-1} A_{0} u_{0}+u_{0}^{-1} d u_{0} \\
A_{1}^{\prime} & =u_{0}^{-1} \stackrel{\star}{A} u_{0}+u_{0}^{-1} T_{\theta} \partial_{\omega} \stackrel{\star}{\chi}_{3} \\
\phi_{0}^{\prime} & =r\left(u_{0}\right) \phi_{0} \\
\stackrel{\star}{*} & =r\left(u_{0}\right) \stackrel{\star}{\phi_{1}} \\
\phi_{1}^{\prime} & =\rho\left(u_{0}\right) \psi_{0} \\
\psi_{0}^{\prime} & \stackrel{\star}{A_{2}^{\prime}}=u_{0}^{-1} \stackrel{\star}{A_{2}} u_{0} \\
\stackrel{\star}{A_{3}^{\prime}} & =u_{0}^{-1} \stackrel{\star}{A} u_{0}+u_{0}^{-1} T \stackrel{\star}{\varkappa_{3}}-u_{0}^{-1} \stackrel{\star}{\chi}_{3} u_{0}^{-1} d u_{0}+\left[u_{0}^{-1} A_{0} u_{0}, u_{0}^{-1} \stackrel{\star}{\chi}_{3}\right] \\
\psi_{1}^{\prime} & =\rho\left(u_{0}\right) \psi_{1} \\
\psi_{2}^{\prime} & =\rho\left(u_{0}\right) \psi_{2}
\end{aligned}
$$

Nous vérifions avant toute chose que les polarisations initiales ne sont pas affectées par le changement de jauge. Si $a_{1}^{\vee}=a_{2}^{\vee}=0$, alors

$$
\begin{aligned}
& R_{\theta} \stackrel{\star}{A_{1}^{\prime}}=u_{0}^{-1} R_{\theta} \stackrel{\star}{A} u_{0}+u_{0}^{-1} R_{\theta} T_{\theta} \partial_{\omega} \stackrel{\star}{\chi_{3}}=0 \\
& \text { et } \quad R_{\theta} \stackrel{\star}{A_{2}^{\prime}}=u_{0}^{-1} R_{\theta} \stackrel{\star}{A_{2}} u_{0}=0
\end{aligned}
$$

De même, si $\psi_{0}^{-}=\psi_{1}^{-}=0$, alors ${\psi_{0}^{\star}}^{{ }^{\prime}}=\rho\left(u_{0}\right){ }^{\star}{ }_{0}^{-}=0$ et ${\psi_{1}^{-}}^{\prime}=\rho\left(u_{0}\right){ }_{1}^{\star}=0$. Nous notons $(C, 0)^{\prime}$ le système obtenu en remplaçant $\left(A_{0}, \stackrel{\star}{A_{1}}, \phi_{0}, \stackrel{\star}{\phi}_{1}, \psi_{0}\right) \operatorname{par}\left(A_{0}^{\prime},{\stackrel{\star}{A^{\prime}}}_{1}, \phi_{0}^{\prime},{\stackrel{\star}{\phi^{\prime}}}_{1}, \psi_{0}^{\prime}\right)$ dans $(C, 0)$. La composante $T_{\theta} u_{0}^{-1} \partial_{\omega} \stackrel{\star}{\chi}_{3} \in \Pi_{\wedge}$ de ${\stackrel{\star}{A_{1}^{\prime}}}^{\prime}$ 'intervient pas dans les équations de $(C, 0)^{\prime}$, en vertu de la proposition 2.2.3. En développant les identités $(I . J$.$) pour$ $(A, \phi, \psi)=\left(A_{0}+\varepsilon^{1 / 2} \stackrel{\star}{A} 1, \phi_{0}+\varepsilon^{1 / 2} \stackrel{\star}{\phi}_{1}, \psi_{0}\right)$ et $u=u_{0}$, il vient - à l'ordre $\varepsilon^{-1 / 2}$ :

$$
\left\{\begin{aligned}
M \partial_{\omega}\left(u_{0}^{-1} \stackrel{\star}{A}_{1} u_{0}\right) & -F^{I}{ }_{\theta}\left(u_{0}^{-1} A_{0} u_{0}+u_{0}^{-1} d u_{0}\right) \partial_{\omega} u_{0}^{-1} \stackrel{\star}{A}_{1} u_{0} \\
& -F^{I I}{ }_{\theta}\left(r\left(u_{0}\right) \phi_{0}\right) \partial_{\omega} r\left(u_{0}\right) \stackrel{\star}{\phi}_{1} \\
& =u_{0}^{-1}\left(M \partial_{\omega} \stackrel{\star}{A}_{1}-F^{I}{ }_{\theta}\left(A_{0}\right) \partial_{\omega} \stackrel{\star}{A}_{1}-F^{I I}{ }_{\theta}\left(\phi_{0}\right) \partial_{\omega} \stackrel{\star}{\phi}_{1}\right) u_{0} \\
X \partial_{\omega}\left(r\left(u_{0}\right) \stackrel{\star}{\phi}_{1}\right) & -G^{I}{ }_{\theta}\left(r\left(u_{0}\right) \phi_{0}\right) \partial_{\omega} u_{0}^{-1} \stackrel{\star}{A}_{1} u_{0} \\
& -G^{I I}{ }_{\theta}\left(u_{0}^{-1} A_{0} u_{0}+u_{0}^{-1} d u_{0}\right) \partial_{\omega} r\left(u_{0}\right) \stackrel{\star}{\phi}_{1} \\
& =r\left(u_{0}\right)\left(X \partial_{\omega} \stackrel{\star}{\phi}_{1}-G^{I}{ }_{\theta}\left(\phi_{0}\right) \cdot \partial_{\omega} \stackrel{\star}{A}_{1}+G^{I I}{ }_{\theta}\left(A_{0}\right) \cdot \partial_{\omega} \stackrel{\star}{\phi}_{1}\right)
\end{aligned}\right.
$$


- à l'ordre $\varepsilon^{0}$ :

(42)

$$
\begin{aligned}
& \left(\begin{array}{l}
L\left(u_{0}^{-1} A_{0} u_{0}+u_{0}^{-1} d u_{0}\right) \\
-\operatorname{Moy}\left(F^{I}{ }_{\theta}\left(u_{0}^{-1} \stackrel{\star}{A} u_{1}\right) \partial_{\omega} u_{0}^{-1} \stackrel{\star}{A} u_{1} u_{0}\right)-\operatorname{Moy}\left(F^{I I}{ }_{\theta}\left(r\left(u_{0}\right) \stackrel{\star}{\phi}\right) \partial_{\omega} r\left(u_{0}\right) \stackrel{\star}{\phi_{1}}\right) \\
-F^{I}\left(u_{0}^{-1} A_{0} u_{0}+u_{0}^{-1} d u_{0}, d\left(u_{0}^{-1} A_{0} u_{0}+u_{0}^{-1} d u_{0}\right)\right)-F^{I I}\left(r\left(u_{0}\right) \phi_{0}, d\left(r\left(u_{0}\right) \phi_{0}\right)\right) \\
-\widetilde{f}\left(u_{0}^{-1} A_{0} u_{0}+u_{0}^{-1} d u_{0} r\left(u_{0}\right) \phi_{0} \rho\left(u_{0}\right) \phi_{0}\right)
\end{array}\right. \\
& -\widetilde{f}\left(u_{0}^{-1} A_{0} u_{0}+u_{0}^{-1} d u_{0}, r\left(u_{0}\right) \phi_{0}, \rho\left(u_{0}\right) \psi_{0}\right) \\
& =u_{0}^{-1}\left(L A_{0}-\operatorname{Moy}\left(F^{I} \theta\left(\stackrel{\star}{A_{1}}\right) \partial_{\omega} \stackrel{\star}{A_{1}}\right)-\operatorname{Moy}\left(F^{I I}{ }_{\theta}\left(\star_{1}\right) \partial_{\omega} \stackrel{\star}{\phi_{1}}\right)\right. \\
& \left.-F^{I}\left(A_{0}, d A_{0}\right)-F^{I I}\left(\phi_{0}, d \phi_{0}\right)-\widetilde{f}\left(A_{0}, \phi_{0}, \psi_{0}\right)\right) u_{0} \\
& \square\left(r\left(u_{0}\right) \phi_{0}\right)-\operatorname{Moy}\left(G^{I} \theta_{\theta}\left(r\left(u_{0}\right) \stackrel{\star}{\phi_{1}}\right) \partial_{\omega} r\left(u_{0}\right) \stackrel{\star}{A} 1\right) \\
& -\operatorname{Moy}\left(G^{I I}{ }_{\theta}\left(u_{0}^{-1} \stackrel{\star}{A} u_{0}\right) \partial_{\omega} r\left(u_{0}\right) \stackrel{\star}{\phi_{1}}\right) \\
& -G^{I}\left(u_{0}^{-1} A_{0} u_{0}+u_{0}^{-1} d u_{0}, d\left(r\left(u_{0}\right) \phi_{0}\right)\right)-G^{I I}\left(d\left(u_{0}^{-1} A_{0} u_{0}+u_{0}^{-1} d u_{0}\right), r\left(u_{0}\right) \phi_{0}\right) \\
& -\widetilde{g}\left(u_{0}^{-1} A_{0} u_{0}+u_{0}^{-1} d u_{0}, r\left(u_{0}\right) \phi_{0}, \rho\left(u_{0}\right) \psi_{0}\right) \\
& =r\left(u_{0}\right)\left(\square \phi_{0}-\operatorname{Moy}\left(G^{I}{ }_{\theta}\left(\stackrel{\star}{\phi_{1}}\right) \partial_{\omega} \stackrel{\star}{A_{1}}\right)-\operatorname{Moy}\left(G^{I I}{ }_{\theta}\left(\stackrel{\star}{A_{1}}\right) \partial_{\omega} \stackrel{\star}{\phi_{1}}\right)\right. \\
& \left.-G^{I}\left(A_{0}, d \phi_{0}\right)-G^{I I}\left(d A_{0}, \phi_{0}\right)-\widetilde{g}\left(A_{0}, \phi_{0}, \psi_{0}\right)\right) \\
& \mathcal{D}\left(\rho\left(u_{0}\right) \psi_{0}\right)-\widetilde{h}\left(u_{0}^{-1} A_{0} u_{0}+u_{0}^{-1} d u_{0}, r\left(u_{0}\right) \phi_{0}, \rho\left(u_{0}\right) \psi_{0}\right) \\
& =\rho\left(u_{0}\right)\left(\mathcal{D} \psi_{0}-\widetilde{h}\left(A_{0}, \phi_{0}, \psi_{0}\right)\right)
\end{aligned}
$$

Du coup, nous vérifions que

$$
(C, 0)^{\prime} \sim(C, 0)
$$

La condition de polarisation qui $(P, 0)$ qui accompagne $(C, 0)$ n'est pas non plus affectée par le changement de jauge. Détaillons :

$$
\begin{aligned}
-L_{\theta}^{-1} \pi^{\wedge} M T_{\theta} u_{0}^{-1} \stackrel{\star}{\chi}_{3} & =L_{\theta}^{-1} L_{\theta} T u_{0}^{-1} \stackrel{\star}{\chi}_{3} \\
& =-\pi^{\vee}\left(u_{0}^{-1}\left(d u_{0}\right) u_{0}^{-1} \stackrel{\star}{\chi}_{3}\right)+\pi^{\vee}\left(u_{0}^{-1} T \stackrel{\star}{\chi_{3}}\right)
\end{aligned}
$$

puis, par l'égalité (40) section 2.1,

$$
\begin{aligned}
L_{\theta}^{-1} \pi^{\wedge} F^{I}{ }_{\theta}\left(u_{0}^{-1}\right. & \left.A_{0} u_{0}+u_{0}^{-1} d u_{0}, T_{\theta} u_{0}^{-1} \stackrel{\star}{\chi}_{3}\right) \\
& =L_{\theta}^{-1} L_{\theta}\left[u_{0}^{-1} A_{0} u_{0}+u_{0}^{-1} d u_{0}, u_{0}^{-1} \stackrel{\star}{\chi}_{3}\right] \\
& =\pi^{\vee}\left[u_{0}^{-1} A_{0} u_{0}, u_{0}^{-1} \stackrel{\star}{\chi}_{3}\right]+\pi^{\vee}\left(u_{0}^{-1}\left(d u_{0}\right) u_{0}^{-1} \stackrel{\star}{\chi}_{3}\right)-\pi^{\vee}\left(u_{0}^{-1} \stackrel{\star}{\chi}_{3} u_{0}^{-1} d u_{0}\right)
\end{aligned}
$$

de sorte que

$$
\begin{aligned}
L_{\theta}^{-1} \pi^{\wedge}\left(F^{I}{ }_{\theta}\left(u_{0}^{-1} A_{0} u_{0}+u_{0}^{-1} d u_{0}, T_{\theta} u_{0}^{-1} \stackrel{\star}{\chi}_{3}\right)-M T_{\theta} u_{0}^{-1} \stackrel{\star}{\chi}_{3}\right) \\
=\pi^{\vee}\left(u_{0}^{-1} T \stackrel{\star}{\chi}_{3}-u_{0}^{-1} \stackrel{\star}{\chi}_{3} u_{0}^{-1} d u_{0}+\left[u_{0}^{-1} A_{0} u_{0}, u_{0}^{-1} \stackrel{\star}{\chi}_{3}\right]\right)
\end{aligned}
$$


du coup, en tenant compte du développement précédent (41) des identités (I.J.), il vient

$$
\begin{gathered}
\pi^{\vee}{\stackrel{\star}{A^{\prime}}}_{3}-L_{\theta}^{-1} \pi^{\wedge}\left(F^{I}{ }_{\theta}\left(A_{0}^{\prime}, \partial_{\omega}^{-1}{\stackrel{\star}{A^{\prime}}}_{1}\right)+F^{I I}{ }_{\theta}\left(\phi_{0}^{\prime}, \partial_{\omega}^{-1}{\stackrel{\star}{\phi^{\prime}}}_{1}\right)-M \partial_{\omega}^{-1} \stackrel{\star}{A}^{\prime}{ }_{1}\right) \\
=u_{0}^{-1} \pi^{\vee} \stackrel{\star}{A}_{3} u_{0}-L_{\theta}^{-1} \pi^{\wedge} F^{I}{ }_{\theta}\left(u_{0}^{-1} A_{0} u_{0}+u_{0}^{-1} d u_{0}, \partial_{\omega}^{-1} u_{0}^{-1} \stackrel{\star}{A}_{1} u_{0}\right) \\
\quad-L_{\theta}^{-1} \pi^{\wedge} F^{I I}{ }_{\theta}\left(r\left(u_{0}\right) \phi_{0}, \partial_{\omega}^{-1} r\left(u_{0}\right) \stackrel{\star}{\phi_{1}}\right)+L_{\theta}^{-1} \pi^{\wedge} M \partial_{\omega}^{-1} u_{0}^{-1} \stackrel{\star}{A}_{1} u_{0} \\
\quad+\pi^{\vee}\left(u_{0}^{-1} T \stackrel{\star}{\chi}_{3}-u_{0}^{-1} \stackrel{\star}{\chi}_{3} u_{0}^{-1} d u_{0}+\left[u_{0}^{-1} A_{0} u_{0}, u_{0}^{-1} \stackrel{\star}{\chi}_{3}\right]\right) \\
\quad-L_{\theta}^{-1} \pi^{\wedge} F^{I}{ }_{\theta}\left(u_{0}^{-1} A_{0} u_{0}+u_{0}^{-1} d u_{0}, T_{\theta} u_{0}^{-1} \stackrel{\star}{\chi}_{3}\right)+L_{\theta}^{-1} \pi^{\wedge} M T_{\theta} u_{0}^{-1} \stackrel{\star}{\chi_{3}} \\
=u_{0}^{-1}\left(\pi^{\vee} \stackrel{\star}{A}{ }_{3}-L_{\theta}^{-1} \pi^{\wedge}\left(F^{I}{ }_{\theta}\left(A_{0}, \partial_{\omega}^{-1} \stackrel{\star}{A}_{1}\right)+F^{I I}{ }_{\theta}\left(\phi_{0}, \partial_{\omega}^{-1} \stackrel{\star}{\phi}_{1}\right)-M \partial_{\omega}^{-1} \stackrel{\star}{A}\right) u_{0}\right)
\end{gathered}
$$

Enfin, nous vérifions directement sur le développement (41) des identités (I.J.) que la condition de polarisation sur $\psi_{2}^{-}$reste elle aussi invariante. Dès lors,

$$
(P, 0)^{\prime} \sim(P, 0)
$$

b) Sous l'action de $u_{\varepsilon}, A_{\varepsilon} \rightsquigarrow A_{\varepsilon}^{\prime}, \phi_{\varepsilon} \rightsquigarrow \phi_{\varepsilon}^{\prime}$, et $\psi_{\varepsilon} \rightsquigarrow \psi_{\varepsilon}^{\prime}$ avec, pour $0 \leqslant k<i$,

$$
\widetilde{A}_{k}^{\prime}=\widetilde{A}_{k},{\stackrel{\star}{A^{\prime}}}_{k+1}^{\prime}=\stackrel{\star}{A}_{k+1}, \widetilde{\phi}_{k}^{\prime}=\widetilde{\phi}_{k}, \stackrel{\star}{\phi}_{k+1}^{\prime}=\stackrel{\star}{\phi}_{k+1}, \text { et } \psi_{k}^{\prime}=\psi_{k}
$$

tandis que

$$
\begin{aligned}
&{\widetilde{A^{\prime}}}_{i}=\widetilde{A}_{i}+\left[A_{0}, \widetilde{\chi}_{i}\right]+T \widetilde{\chi}_{i} \\
& \widetilde{A}^{\prime}{ }_{i+1}\left.=\stackrel{\star}{A}^{*}+\stackrel{\star}{A}_{1}, \widetilde{\chi}_{i}\right]+T_{\theta} \partial_{\omega} \stackrel{\star}{\chi}_{i+3} \\
& \widetilde{\phi}^{\prime}=\widetilde{\phi}_{i}+r^{\prime}(1) \widetilde{\chi}_{i} \phi_{0} \\
& \phi_{i+1}^{\star}={ }^{\star} \\
& \psi_{i+1}+r^{\prime}(1) \widetilde{\chi}_{i}{ }^{\star} \\
& \psi_{i}^{\prime} \\
&=\psi_{i}+\rho^{\prime}(1) \widetilde{\chi}_{i} \psi_{0}
\end{aligned}
$$

puis

$$
\begin{aligned}
{\stackrel{\star}{A^{\prime}}}_{i+2} & =\stackrel{\star}{A}_{i+2}+\left[\stackrel{\star}{A}, \widetilde{\chi}_{i}\right] \\
{\stackrel{\star}{A^{\prime}}}_{i+3} & =\stackrel{\star}{A}_{i+3}+\left[A_{0}, \stackrel{\star}{\chi}_{i+3}\right]+T \stackrel{\star}{\chi}_{i+3}+\left[\stackrel{\star}{A}_{3}, \widetilde{\chi}_{i}\right] \\
\psi_{i+1}^{\prime} & =\psi_{i+i}+\rho^{\prime}(1) \widetilde{\chi}_{i} \psi_{1} \\
\psi_{i+2}^{\prime} & =\psi_{i+2}+\rho^{\prime}(1) \widetilde{\chi}_{i} \psi_{2}
\end{aligned}
$$

Il est clair que les $(C, k)$ et les $(P, k-2)$ d'indices $0 \leqslant k<i$ ne sont pas affectés par le changement de jauge, tandis que

$$
\begin{aligned}
& a_{i+1}^{\vee}{ }^{\prime}=a_{i+1}^{\vee}+\left[a_{1}^{\vee}, \tilde{\chi}_{i}\right]+\pi^{\vee} T_{\theta} \partial_{\omega} \stackrel{\star}{\chi}_{i+3}=a_{i+1}^{\vee} \\
& a_{i+2}^{\vee}{ }^{\prime}=a_{i+2}^{\vee}+\left[a_{2}^{\vee}, \tilde{\chi}_{i}\right]=a_{i+2}^{\vee} \\
& \psi_{i}^{{ }^{\prime}}{ }^{\prime}=\psi_{i}^{-}+\rho^{\prime}(1) \tilde{\chi}_{i} \psi_{0}^{\star}=\psi_{i}^{-} \\
& \psi_{i+1}^{-}{ }^{\prime}=\psi_{i+1}^{-}+\rho^{\prime}(1) \widetilde{\chi}_{i} \psi_{1}^{\star}=\psi_{i+1}^{-}
\end{aligned}
$$


Du coup, $(P, i-2)$ et $(P, i-1)$ restent également inchangées. Nous notons $(C, i)^{\prime}$ le système obtenu en remplaçant $\left(\widetilde{A}_{i}, \stackrel{\star}{A}_{i+1}, \widetilde{\phi}_{i}, \stackrel{\star}{\phi}_{i+1}, \psi_{i}\right) \operatorname{par}\left(\widetilde{A^{\prime}} i,{\stackrel{\star}{A^{\prime}}}_{i+1}, \widetilde{\phi}_{i}^{\prime},{\stackrel{\star}{\phi^{\prime}}}_{i+1}, \psi_{i}^{\prime}\right)$ dans $(C, i)$. La composante $T_{\theta} \partial_{\omega} \stackrel{\star}{\chi}_{i+3} \in \Pi_{\wedge}$ de ${\stackrel{\star}{A^{\prime}}}_{i+1}$ n'intervient pas dans $(C, i)^{\prime}$ en vertu de la proposition 2.2.3. En développant les identités (D.I.J.) pour $(A, \phi, \psi)=$ $\left(A_{0}+\varepsilon^{1 / 2} \stackrel{\star}{A}, \phi_{0}+\varepsilon^{1 / 2} \stackrel{\star}{\phi_{1}}, \psi_{0}\right)$ et $\chi=\widetilde{\chi}_{i}$ solution de $(C, 0)$ et en supposant la polarisation $(P, 0)$ satisfaite, il vient

- à l'ordre $\varepsilon^{-1 / 2}$ :

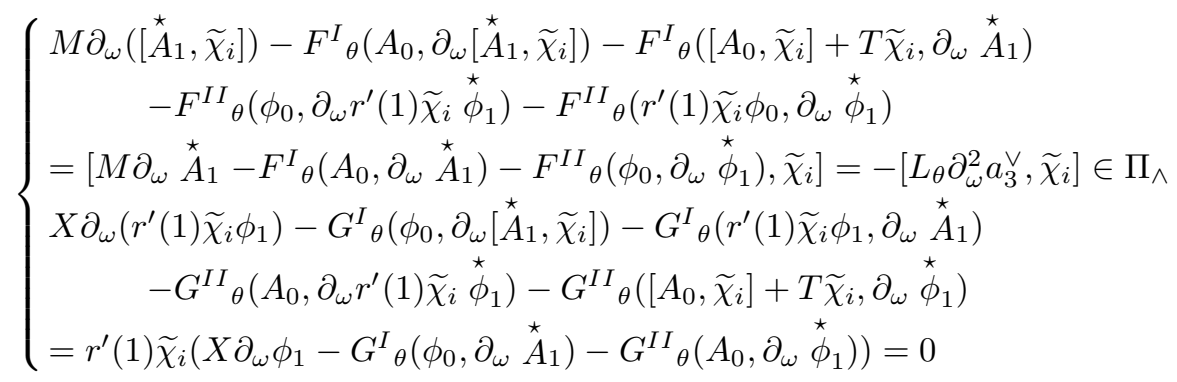

- à l'ordre $\varepsilon^{0}$ :

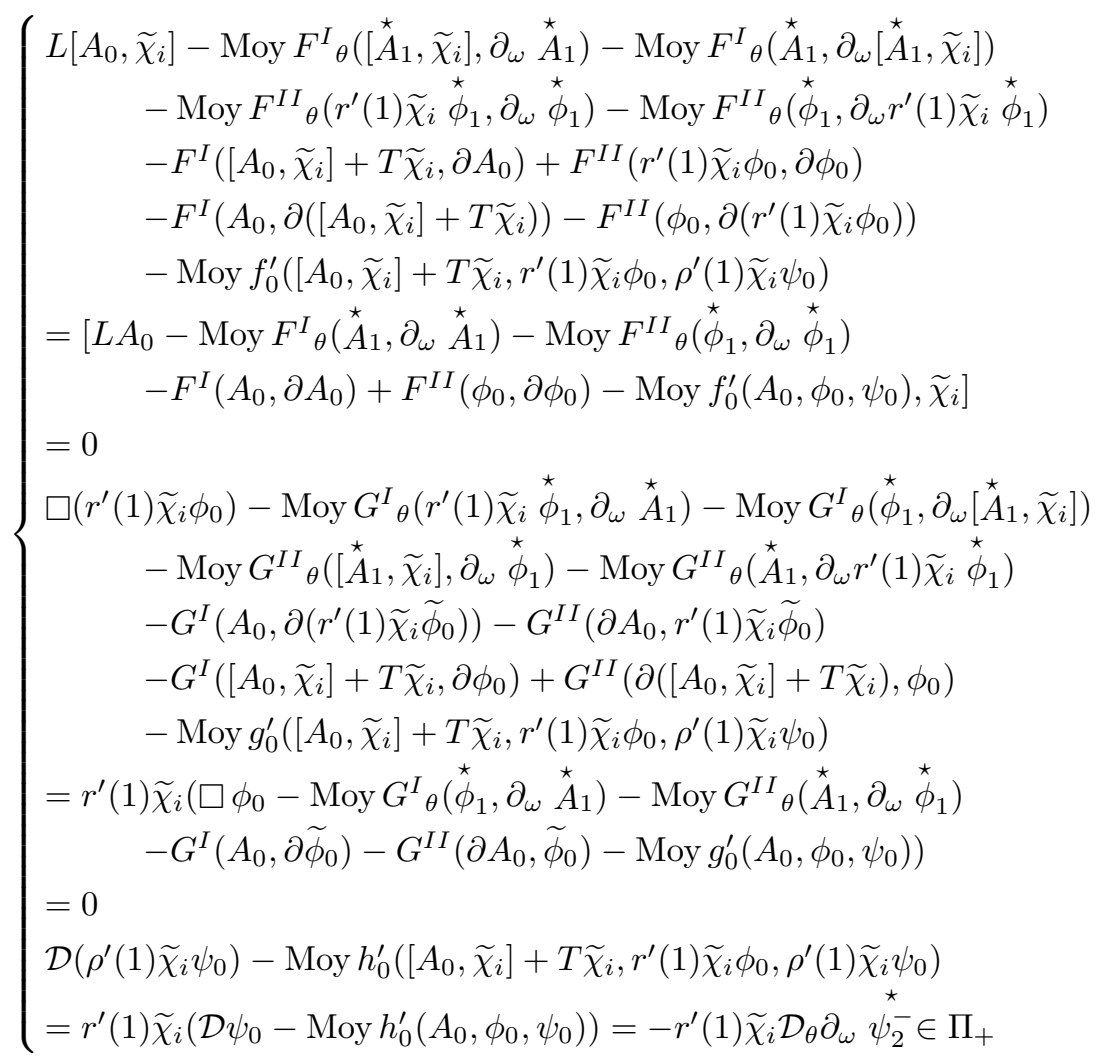


Il en résulte que

$$
(C, i)^{\prime}=(C, i)
$$

La condition de polarisation qui $(P, i)$ qui accompagne $(C, i)$ n'est pas non plus affectée par le changement de jauge. Détaillons :

$$
-L_{\theta}^{-1} \pi^{\wedge} M T_{\theta} \stackrel{\star}{\chi}_{i+3}=L_{\theta}^{-1} L_{\theta} T \stackrel{\star}{\chi}_{i+3}=\pi^{\vee}\left(T \stackrel{\star}{\chi}_{i+3}\right)
$$

puis, par l'égalité (40) section 2.1,

$$
L_{\theta}^{-1} \pi^{\wedge} F_{\theta}^{I}\left(A_{0}, T_{\theta} \stackrel{\star}{\chi}_{i+3}\right)=L_{\theta}^{-1} L_{\theta}\left[A_{0}, \stackrel{\star}{\chi}_{i+3}\right]=\pi^{\vee}\left[A_{0}, \stackrel{\star}{\chi}_{i+3}\right]
$$

du coup, en tenant compte du développement précédent (43) des identités (D.I.J.), il vient

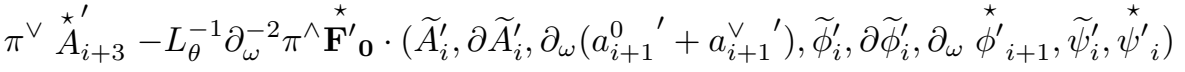

$$
\begin{aligned}
& -L_{\theta}^{-1} \partial_{\omega}^{-2} \pi^{\wedge} \stackrel{\star}{\mathcal{F}}_{i}\left(A_{k}^{\prime}, \partial A_{k}^{\prime},{\stackrel{\star}{A^{\prime}}}_{k+1}, \phi_{k}^{\prime}, \partial \phi_{k}^{\prime},{\stackrel{\star}{\phi^{\prime}}}_{k+1}, \psi_{k}^{\prime}, k \leqslant i-1\right) \\
& +L_{\theta}^{-1} \partial_{\omega}^{-1} \pi^{\wedge} M{\stackrel{\star}{A^{\prime}}}_{i+1}+L_{\theta}^{-1} \partial_{\omega}^{-2} L A_{i-1}^{\star} \\
& =\pi^{\vee} \stackrel{\star}{A}_{i+3}-L_{\theta}^{-1} \partial_{\omega}^{-2} \pi^{\wedge} \stackrel{\mathbf{F}}{\prime}_{\mathbf{0}}^{\prime} \cdot\left(\widetilde{A}_{i}, \partial \widetilde{A}_{i}, \partial_{\omega}\left(a_{i+1}^{0}+a_{i+1}^{\vee}\right), \widetilde{\phi}_{i}, \partial \widetilde{\phi}_{i}, \partial_{\omega} \stackrel{\star}{\prime}_{i+1}^{\prime}, \widetilde{\psi}_{i}^{\prime},{ }_{\psi^{\prime}}{ }_{i}\right) \\
& -L_{\theta}^{-1} \partial_{\omega}^{-2} \pi^{\wedge} \stackrel{\star}{\mathcal{F}}_{i}\left(A_{k}, \partial A_{k}, \stackrel{\star}{A}_{k+1}, \phi_{k}, \partial \phi_{k}, \stackrel{\star}{\phi}_{k+1}, \psi_{k}, k \leqslant i-1\right) \\
& +L_{\theta}^{-1} \partial_{\omega}^{-1} \pi^{\wedge} M \partial_{\omega} \stackrel{\star}{A}_{i+1}+L_{\theta}^{-1} \partial_{\omega}^{-2} L \stackrel{\star}{A}_{i-1} \\
& +\pi^{\vee}\left(u_{0}^{-1} T \stackrel{\star}{\chi}_{i+3}\right)+\pi^{\vee}\left[A_{0}, \stackrel{\star}{\chi}_{i+3}\right]-L_{\theta}^{-1} \pi^{\wedge} F^{I}{ }_{\theta}\left(A_{0}, T_{\theta} \stackrel{\star}{\chi}_{i+3}\right)+L_{\theta}^{-1} \pi^{\wedge} M T_{\theta} \stackrel{\star}{\chi}_{i+3} \\
& +\left[\pi^{\vee} \stackrel{\star}{A}_{3}, \widetilde{\chi}_{i}\right]-L_{\theta}^{-1} \partial_{\omega}^{-1} \pi^{\wedge}\left(F_{\theta}^{I}{ }_{\theta}\left(A_{0},\left[\stackrel{\star}{A}_{1}, \widetilde{\chi}_{i}\right]\right)-F_{\theta}^{I}\left(\left[A_{0}, \widetilde{\chi}_{i}\right]+T \widetilde{\chi}_{i}, \stackrel{\star}{A}_{1}\right)\right. \\
& \left.-F^{I I}{ }_{\theta}\left(\phi_{0}, r^{\prime}(1) \widetilde{\chi}_{i} \stackrel{\star}{\phi}_{1}\right)-F^{I I}{ }_{\theta}\left(r^{\prime}(1) \widetilde{\chi}_{i} \phi_{0}, \stackrel{\star}{\phi_{1}}\right)+M \partial_{\omega}\left[\stackrel{\star}{A}{ }_{1}, \widetilde{\chi}_{i}\right]\right) \\
& =\pi^{\vee} \stackrel{\star}{A}_{i+3}-L_{\theta}^{-1} \partial_{\omega}^{-2} \pi^{\wedge} \stackrel{\star}{*}^{\prime}{ }_{0} \cdot\left(\widetilde{A}_{i}, \partial \widetilde{A}_{i}, \partial_{\omega}\left(a_{i+1}^{0}+a_{i+1}^{\vee}\right), \widetilde{\phi}_{i}, \partial \widetilde{\phi}_{i}, \partial_{\omega} \stackrel{\star}{\prime}_{i+1}^{\prime}, \widetilde{\psi}_{i}^{\prime}, \stackrel{\star}{*}_{i}^{\prime}\right) \\
& -L_{\theta}^{-1} \partial_{\omega}^{-2} \pi^{\wedge} \stackrel{\star}{\mathcal{F}}_{i}\left(A_{k}, \partial A_{k}, \stackrel{\star}{A}_{k+1}, \phi_{k}, \partial \phi_{k}, \stackrel{\star}{\phi}_{k+1}, \psi_{k}, k \leqslant i-1\right) \\
& +L_{\theta}^{-1} \partial_{\omega}^{-1} \pi^{\wedge} M \partial_{\omega} \stackrel{\star}{A}_{i+1}+L_{\theta}^{-1} \partial_{\omega}^{-2} \pi^{\wedge} L \stackrel{\star}{A}_{i-1}
\end{aligned}
$$

La condition de polarisation sur $\psi_{2}^{-}$reste invariante, toujours en vertu du développement (43) des identités (D.I.J.). Dès lors,

$$
(P, i)^{\prime}=(P, i)
$$


Données de Cauchy oscillantes compatibles pour les équations de profils à jauge libre

Le choix des données initiales à $t=0$ pour $(C, 0)$ se résume à la donnée de

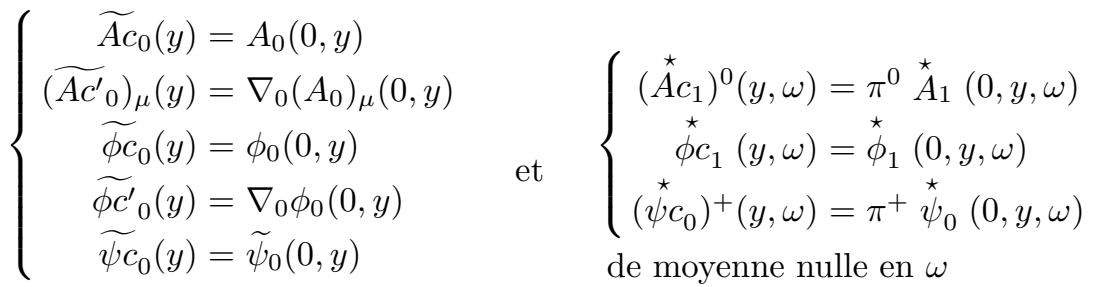

constantes en $\omega$,

vérifiant la contrainte de compatibilité( ${ }^{(2)}$

$$
\nabla^{j}\left(\nabla_{j}\left(\widetilde{A c_{0}}\right)_{0}-\left(\widetilde{A c^{\prime}}\right)_{j}\right)=\left(\widetilde{\boldsymbol{F}_{0}}\right)_{0}\left(\widetilde{A c_{0}}, \widetilde{A c^{\prime}}{ }_{0}, \widetilde{\phi c_{0}}, \widetilde{\phi c^{\prime}}{ }_{0}, \widetilde{\psi} c_{0}, \stackrel{\star A}{A c_{1}}, \stackrel{\star}{\phi} c_{1}, \stackrel{\star}{\psi} c_{0}^{+}\right)
$$

Il existe toujours un choix non trivial de données initiales compatibles pour le système $(C, 0)$, pour peu qu'on les prenne suffisamment régulières pour résoudre la contrainte (45).

Les composantes $a_{1}^{\vee}$ et $\psi_{0}^{\star}$ sont sujettes à polarisation et doivent nécessairement rester nulles. $a_{1}^{\wedge}$ est une variable de jauge libre a priori.

Si $\left(\widetilde{A}_{k}, a_{k+1}^{\#}, \widetilde{\phi}_{k}, \stackrel{\star}{\phi}_{k+1}, \widetilde{\psi}_{k}, \stackrel{\star}{*}+_{k}^{+}\right)$sont prises parmi les données initiales compatibles des systèmes $(C, k)$ successifs, $0 \leqslant k<i$, et si $\left(a_{k+3}^{\vee}, \psi_{k+2}^{\stackrel{\star}{-}}\right)$ satisfont aux polarisations $(P, k)$ à $t=0$, pour tout $0 \leqslant k<i$, alors le choix de données initiales compatibles pour le système $(C, i)$ se résume à la donnée de

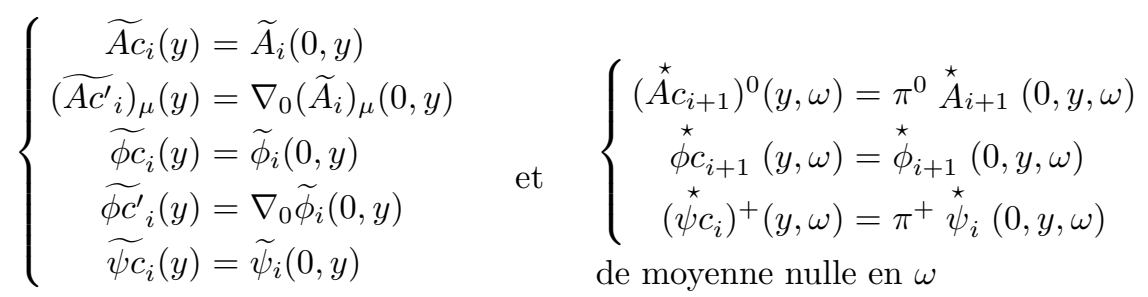

constantes en $\omega$,

vérifiant la contrainte de compatibilité

$$
\begin{array}{r}
\left.\nabla^{j}\left(\nabla_{j}\left(\widetilde{A c_{i}}\right)_{0}-\widetilde{\left(A c^{\prime}\right.}{ }_{i}\right)_{j}\right) \\
=\left(\widetilde{\boldsymbol{F}^{\prime}}\left(\widetilde{A c_{i}}, \widetilde{A c^{\prime}}{ }_{i}, \widetilde{\phi c_{i}}, \widetilde{\phi c^{\prime}}{ }_{i}, \widetilde{\psi c_{0}},\left(\stackrel{\star}{A c_{i+1}}\right)^{0}+a_{i+1}^{\vee}(t=0), \stackrel{\star}{\phi} c_{i+1},{ }^{\star}+c_{i}\right)\right. \\
\left.\quad+\widetilde{\mathcal{F}}_{i}\left(A_{k}, \partial A_{k}, \stackrel{\star}{A}_{k+1}, \phi_{k}, \partial \phi_{k}, \stackrel{\star}{\phi}_{k+1}, \psi_{k}, k \leqslant i-1\right)\right)_{0}
\end{array}
$$

\footnotetext{
(2) Notons que la contrainte (45) diffère en général de la contrainte usuelle (15) pour une solution $\left(A_{0}, \phi_{0}, \psi_{0}\right)$ non-oscillante de $(Y . M$.$) , cf. section 3.4, où nous développons précisément le cas où ces$ deux contraintes coïncident
} 
Le dernier terme étant entièrement déterminé en reportant les données initiales d'ordre inférieur dans les équations. Pour peu que nous disposions données suffisamment régulières pour les $(C, k), 0 \leqslant k<i$, il est possible de résoudre la contrainte (46), et il existe un choix non trivial de données initiales régulières compatibles pour chaque $(C, i)$.

Les composantes $a_{i+1}^{\vee}$ et $\psi_{i}^{-}$sont nécessairement fixées par polarisation, et nous sont données par $(P, i-2)$ à $t=0$. La composante $a_{i+1}^{\wedge}$ est une variable de jauge libre a priori.

Les contraintes de compatibilité ci-dessus sont invariantes par changements de jauge « admissibles », au sens de la proposition 2.2.6. Il est donc possible

- d'imposer une contrainte de jauge complémentaire sur $\widetilde{A c_{i}}$ et $\widetilde{A c^{\prime}}{ }_{i}, i \geqslant 0$, en plus des contraintes (45) ou (46), par exemple la jauge $J_{\lambda}, \lambda \in[0,1]$, en partie moyenne :

$$
\alpha^{-2} \lambda\left({\widetilde{A c^{\prime}}}_{i}\right)_{0}+(1-\lambda) \nabla^{j}\left(A c_{i}\right)_{j}=0
$$

- d'imposer arbitrairement le choix de $a_{i+1}^{\wedge}, i \geqslant 0$, à $t=0$. Ce degré de liberté est illusoire et disparaît dès que l'on veut vérifier une jauge $J_{\lambda}$ donnée $\lambda \in\left[0, \frac{1}{2}[\cup] \frac{1}{2}, 1\right]$, à l'ordre $O\left(\varepsilon^{i / 2}\right)$ en partie oscillante. Il est alors nécessaire de poser

$$
a_{1}^{\wedge}=a_{2}^{\wedge}=0
$$

puis pour $2 \leqslant i \leqslant M$

$$
\left.J_{\lambda \theta} \partial_{\omega} \stackrel{\star}{A}_{i+1}\right|_{t=0}+\left.J_{\lambda} \stackrel{\star}{A}_{i-1}\right|_{t=0}=0
$$

soit

$$
a_{i+\left.1\right|_{t=0}}^{\wedge}=\frac{\alpha^{2} T_{\theta}}{(1-2 \lambda)\left(\nabla_{0} \theta\right)^{2}}\left(\frac{1}{2} R_{\theta} a_{i+\left.1\right|_{t=0}}^{\vee}+J_{\lambda} \partial_{\omega}^{-1} \stackrel{\star}{A}_{i-\left.1\right|_{t=0}}\right)
$$

Le cas de la jauge de Lorentz est exceptionnel, et nous laisse totalement libre du choix initial des $a_{i+1}^{\wedge}$, cf. propositions suivantes.

Nous dirons que les données des $(C, i)$ sont traduites en jauge $J_{\lambda}$ si les contraintes (47) et (48) sont vérifiées.

Hyperbolicité des équations de profils. — L'hyperbolicité des équations de profils est donnée à un changement de jauge « admissible » près. Nous énonçons dans un premier temps deux propositions importantes, prouvant que les systèmes $(C, i)$ résolus l'un après l'autre propagent correctement la jauge de Lorentz, en partie moyenne comme en partie oscillante. Nous concluons en énonçant l'analogue de la proposition 1.3.5 pour les solutions approchées obtenues en résolvant les $(C, i)$.

Proposition 2.2.7. - La jauge de Lorentz est une bonne jauge pour $(C, 0)$ :

a) Partie moyenne - si $a_{1}^{\vee}=a_{2}^{\vee}=0$ et $\psi_{0}^{-}=\psi_{1}^{-}=0$, alors les propositions suivantes sont équivalentes : 


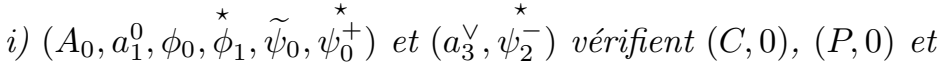

$$
R \widetilde{A}_{0}=0
$$

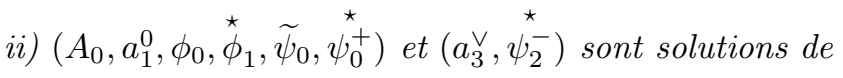

$$
\left\{\begin{aligned}
\square A_{0}-(\mathcal{R} i c .) A_{0} & =\widetilde{\boldsymbol{F}}_{0} \\
\square \phi_{0} & =\widetilde{\boldsymbol{G}}_{0} \\
\mathcal{D} \widetilde{\psi}_{0} & =\widetilde{\boldsymbol{H}}_{0} \\
X_{\left.\right|_{\Pi_{0}}} \partial_{\omega} a_{1}^{0} & =\pi^{0} \stackrel{\star}{\boldsymbol{F}}_{0} \\
X \partial_{\omega}{\stackrel{\star}{\phi_{1}}}^{\star} & =\stackrel{\boldsymbol{G}}{*}_{0} \\
X \stackrel{\star}{\psi_{0}^{+}} & =\mathcal{D}_{\theta} \stackrel{\star}{\boldsymbol{H}}_{0}
\end{aligned}\right.
$$

et de $(P, 0)$, et vérifient à $t=0$ les contraintes

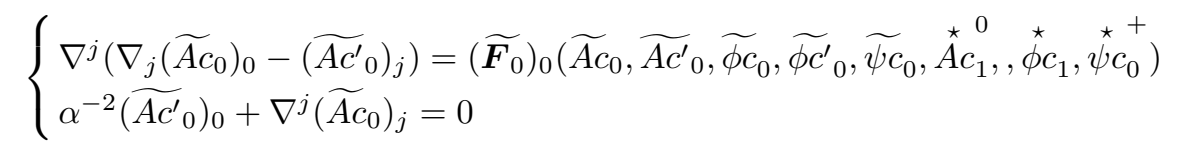

b) Partie oscillante - si $a_{1}^{\vee}=a_{2}^{\vee}=0$ et $\stackrel{\star}{-}_{0}^{-}=\psi_{1}^{-}=0$, alors les propositions suivantes sont équivalentes :

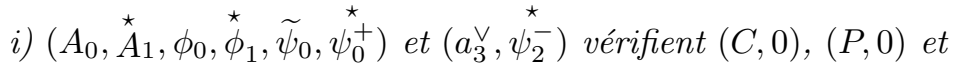

$$
R_{\theta} \partial_{\omega} a_{3}^{\vee}+R \stackrel{\star}{A}{ }_{1}=0
$$

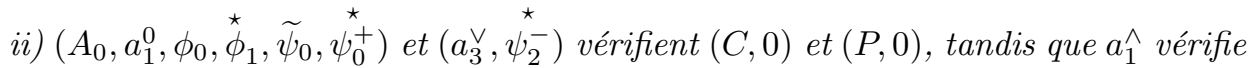
l'équation de transport

$$
X_{\mid \Pi_{\wedge}} \partial_{\omega} a_{1}^{\wedge}=\pi^{\wedge}{\stackrel{\star}{\boldsymbol{F}_{0}}}_{0}-\pi^{\wedge} X \partial_{\omega} a_{1}^{0}
$$

Démonstration. - La compatibilité de $(C, 0)$ est donnée par la proposition 2.2.2, et le a) est donc un corollaire de la proposition 1.3.5. La jauge de Lorentz est propagée sur $A_{0}$ pour peu que que la contrainte de jauge $\alpha^{-2}\left(\widetilde{A c^{\prime}}{ }_{0}\right)_{0}-\nabla^{j}\left(\widetilde{A c_{0}}\right)_{j}=0$ soit vérifiée à $t=0$.

b) Les conditions de polarisation initiales $a_{1}^{\vee}=a_{2}^{\vee}=0$ se confondent avec la jauge de Lorentz jusqu'à l'ordre $O\left(\varepsilon^{1 / 2}\right)$ en partie oscillante : $R_{\theta} \stackrel{\star}{A_{1}}=R_{\theta} \stackrel{\star}{A_{2}}=0$. Si l'on cherche à propager la jauge de Lorentz à l'ordre $O(\varepsilon)$ près en partie oscillante, la seule ressource dont nous disposons est de fixer convenablement la composante $a_{1}^{\wedge}$, qui est libre en vertu de la proposition 2.2.3.

Si $a_{1}^{\vee}=0$, et si $a_{3}^{\vee}=-\partial_{\omega}^{-2} L_{\theta}^{-1} \pi^{\wedge}\left(M \partial_{\omega} \stackrel{\star}{A_{1}}-\stackrel{\star}{\boldsymbol{F}}_{0}\right)$, l'équation

$$
R_{\theta} \partial_{\omega} \stackrel{\star}{A_{3}}+R \stackrel{\star}{A_{1}}=0
$$


équivaut à

$$
-R_{\theta} \partial_{\omega}^{-1} L_{\theta}^{-1} \pi^{\wedge}\left(M \partial_{\omega}\left(a_{1}^{0}+a_{1}^{\wedge}\right)-\stackrel{\boldsymbol{F}}{\star}_{0}\right)+R\left(a_{1}^{0}+a_{1}^{\wedge}\right)=0
$$

soit, en multipliant par $T_{\theta} \partial_{\omega}$, et en détaillant $M=X-T_{\theta} R-T R_{\theta}$, à :

$$
L_{\theta} L_{\theta}^{-1} \pi^{\wedge}\left(\left(X-T_{\theta} R-T R_{\theta}\right) \partial_{\omega}\left(a_{1}^{\wedge}+a_{1}^{0}\right)-\stackrel{\star}{\boldsymbol{F}}_{0}\right)+\pi^{\wedge} T_{\theta} R \partial_{\omega}\left(a_{1}^{0}+a_{1}^{\wedge}\right)=0
$$

Or $a_{1}^{\wedge}+a_{1}^{0} \in \operatorname{Ker} R_{\theta}$, donc cette dernière équation équivaut à

$$
\pi^{\wedge} X \partial_{\omega}\left(a_{1}^{0}+a_{1}^{\wedge}\right)=\pi^{\wedge} \stackrel{\star}{\boldsymbol{F}_{0}}
$$

La proposition 2.1.2 précise que $\pi^{\wedge} X \pi^{\wedge}=X_{\mid \Pi_{\wedge}}$ tandis que $\pi^{\wedge} X \pi^{0}$ est linéaire d'ordre 0. L'équation (50) est alors équivalente à

$$
X_{\mid \Pi_{\wedge}} \partial_{\omega} a_{1}^{\wedge}=\pi^{\wedge}{\stackrel{\star}{\boldsymbol{F}_{0}}}-\pi^{\wedge} X \partial_{\omega} a_{1}^{0}
$$

Nous remarquons que le choix de la valeur initiale de $a_{1}^{\wedge}$ est parfaitement libre en jauge de Lorentz, contrairement à n'importe quelle autre jauge $J_{\lambda}, \lambda \in\left[0, \frac{1}{2}[\cup] \frac{1}{2}, 1\right]$, pour laquelle il vient $a_{1}^{\wedge}=0$ (la jauge de Lorentz dissimule de la régularité dans la direction $\left.\operatorname{Im} T_{\theta}\right)$.

Proposition 2.2.8. - La jauge de Lorentz est une bonne jauge pour les $(C, i)$, $i \geqslant 1$ :

a) Partie moyenne - si $a_{1}^{\vee}=a_{2}^{\vee}=0$ et $\psi_{0}^{\star}=\psi_{1}^{-}=0$, si $\left(\widetilde{A}_{k}, a_{k+1}^{0}, \widetilde{\phi}_{k}, \stackrel{\phi}{\phi}_{k+1}, \widetilde{\psi}_{k},{ }_{\psi_{k}^{+}}^{\star \star}\right)$ sont solutions des $(C, k)$ successifs, et si $\left(a_{k+3}^{\vee}, \psi_{k+2}^{-}\right)$satisfont aux polarisations $(P, k)$ associées, pour tout $0 \leqslant k<i$, alors les propositions suivantes sont équivalentes :

i) $\left(\widetilde{A}_{i}, a_{i+1}^{0}, \widetilde{\phi}_{i}, \stackrel{\phi}{i+1}_{i}, \widetilde{\psi}_{i}, \psi_{i}^{+}\right)$et $\left(a_{i+3}^{\vee}, \psi_{i+2}^{\star}\right)$ vérifient $(C, i),(P, i)$ et

$$
R \widetilde{A}_{i}=0
$$

ii) $\left(\widetilde{A}_{i}, a_{i+1}^{0}, \widetilde{\phi}_{i}, \stackrel{\star}{\phi}_{i+1}, \widetilde{\psi}_{i}, \stackrel{\star}{*}+^{+}\right)$et $\left(a_{i+3}^{\vee}, \psi_{i+2}^{\stackrel{\star}{-}}\right)$ sont solutions de

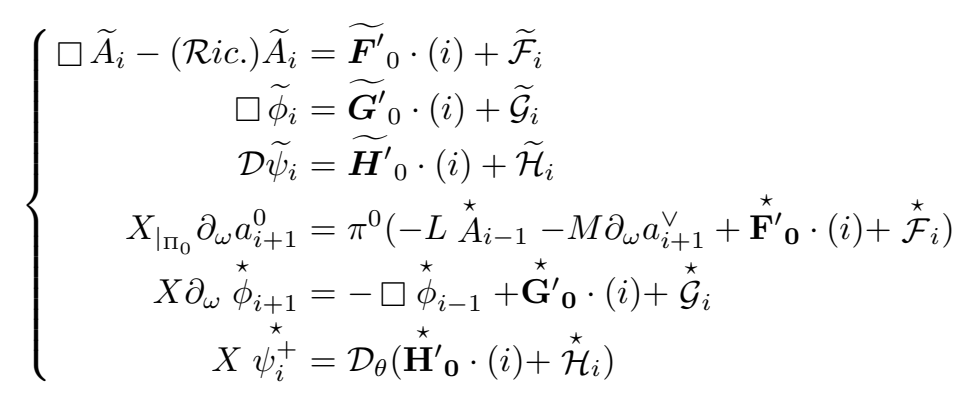


et de $(P, i)$, et vérifient à $t=0$ les contraintes

(51)

$$
\left\{\begin{aligned}
\nabla^{j}\left(\nabla_{j}\left(\widetilde{A c_{i}}\right)_{0}-\left(\widetilde{A c^{\prime}}{ }_{i}\right)_{j}\right)= & \operatorname{Moy}\left(\boldsymbol { F } _ { 0 } ^ { \prime } \left(\widetilde{A c_{i}}, \widetilde{A c^{\prime}}{ }_{i}, \widetilde{\phi c_{i}}, \widetilde{\phi c^{\prime}}{ }_{i}, \widetilde{\psi} c_{i},{\left.\left.\stackrel{\star A}{A} c_{i+1}, \stackrel{\star}{\phi} c_{i+1},{\stackrel{\star}{\psi} c_{i}}^{+}\right)\right)_{0}}+\left(\widetilde{\mathcal{F}}_{i}\right)_{0}\left(A_{k}, \partial A_{k}, \stackrel{\star}{A}_{k+1}, \phi_{k}, \partial \phi_{k},{ }_{k+1}, \psi_{k}, k \leqslant i-1\right)\right.\right. \\
\alpha^{-2}\left(\widetilde{A c^{\prime}}{ }_{i}\right)_{0}+\nabla^{j}\left(\widetilde{A c_{i}}\right)_{j}= & 0
\end{aligned}\right.
$$

b) Partie oscillante - si $a_{1}^{\vee}=a_{2}^{\vee}=0$ et $\psi_{0}^{\star}=\psi_{1}^{-}=0$, si $\left(\widetilde{A}_{k}, a_{k+1}^{0}, \widetilde{\phi}_{k}, \stackrel{\star}{\phi}_{k+1}, \widetilde{\psi}_{k},{ }_{\psi_{k}^{+}}^{\star}\right)$ sont solutions des $(C, k)$ successifs, si $\left(a_{k+3}^{\vee}, \psi_{k+2}^{-}\right)$satisfont aux polarisations $(P, k)$ associées, pour tout $0 \leqslant k<i$, et si

$$
R_{\theta} \partial_{\omega} a_{i+1}^{\vee}+R \stackrel{\star}{A}_{i-1}=0
$$

alors les propositions suivantes sont équivalentes :

i) $\left(\widetilde{A}_{i}, \stackrel{\star}{A}_{i+1}, \widetilde{\phi}_{i}, \stackrel{\star}{\phi}_{i+1}, \widetilde{\psi}_{i}, \stackrel{\star}{*}_{i}^{+}\right)$et $\left(a_{i+3}^{\vee}, \psi_{i+2}^{\stackrel{\star}{-}}\right)$ vérifient $(C, i),(P, i)$ et

$$
R_{\theta} \partial_{\omega} a_{i+3}^{\vee}+R \stackrel{\star}{A}_{i+1}=0
$$

ii) $\left(\widetilde{A}_{i}, a_{i+1}^{0}, \widetilde{\phi}_{i}, \stackrel{\phi}{\phi}_{i+1}, \widetilde{\psi}_{i},{ }_{\psi_{i}^{+}}^{\star}\right)$ et $\left(a_{i+3}^{\vee}, \psi_{i+2}^{\star}\right)$ vérifient $(C, i),(P, i)$ et $a_{i+1}^{\wedge}$ vérifie l'équation de transport

$$
(E, i): X_{\left.\right|_{\Pi_{\curlywedge}}} \partial_{\omega} a_{i+1}^{\wedge}=\pi^{\wedge}\left(-\square \stackrel{\star}{A_{i-1}}+(\mathcal{R} i c .) \stackrel{\star}{A}_{i-1}-X \partial_{\omega} a_{i+1}^{0}+{\stackrel{\star}{\boldsymbol{F}^{\prime}}}_{0}(i)+\stackrel{\star}{\mathcal{F}}_{i}\right)
$$

Démonstration. - La compatibilité de $(C, i)$ est donnée par la proposition 2.2.2, et le a) est un corollaire de la proposition 1.3.5. La jauge de Lorentz est propagée sur $\widetilde{A}_{i}$ pour peu que que la contrainte de jauge $\alpha^{-2}\left(\widetilde{A c^{\prime}}{ }_{i}\right)_{0}-\nabla^{j}\left(\widetilde{A c_{i}}\right)_{j}=0$ soit vérifiée à $t=0$.

b) Si l'on cherche à propager la jauge à l'ordre $O\left(\varepsilon^{(i+2) / 2}\right)$ en partie oscillante, il faut faire usage de la composante $a_{i+1}^{\wedge}$, qui est libre en vertu de la proposition 2.2.3. $\mathrm{Si}$

$$
a_{i+3}^{\vee}=-\partial_{\omega}^{-2} L_{\theta}^{-1} \pi^{\wedge}\left(M \partial_{\omega} A_{i+1}^{\star}+L \stackrel{\star}{A_{i-1}}-\stackrel{\star}{\boldsymbol{F}_{0}^{\prime}}(i)-\stackrel{\star}{\mathcal{F}}_{i}\right)
$$

l'équation

$$
R_{\theta} \partial_{\omega} a_{i+3}^{\vee}+R A_{i+1}^{\star}=0
$$

est équivalente à

$$
\begin{aligned}
-R_{\theta} \partial_{\omega}^{-1} L_{\theta}^{-1} \pi^{\wedge}\left(M \partial_{\omega}\left(a_{i+1}^{0}+a_{i+1}^{\wedge}+a_{i+1}^{\vee}\right)+L \stackrel{\star}{A_{i-1}}-{\left.\stackrel{\star}{\boldsymbol{F}_{0}^{\prime}}(i)-\stackrel{\star}{\mathcal{F}}_{i}\right)}+R\left(a_{i+1}^{0}+a_{i+1}^{\wedge}+a_{i+1}^{\vee}\right)=0\right.
\end{aligned}
$$


soit, en multipliant par $T_{\theta} \partial_{\omega}$, et en détaillant $M=X-T_{\theta} R-T R_{\theta}$, à

$$
\begin{aligned}
L_{\theta} L_{\theta}^{-1} \pi^{\wedge}\left(( X - T _ { \theta } R - T R _ { \theta } ) \partial _ { \omega } \left(a_{i+1}^{0}+a_{i+1}^{\wedge}+\right.\right. & \left.\left.a_{i+1}^{\vee}\right)-\stackrel{\star}{\boldsymbol{F}_{0}^{\prime}}(i)-\stackrel{\star}{\mathcal{F}}\right) \\
& +T_{\theta} R \partial_{\omega}\left(a_{i+1}^{0}+a_{i+1}^{\wedge}+a_{i+1}^{\vee}\right)=0
\end{aligned}
$$

Puisque $a_{i+1}^{\wedge}+a_{i+1}^{0} \in \operatorname{Ker} R_{\theta}$, que $\pi^{\wedge} X a_{i+1}^{\vee}=0$ et $\pi^{\wedge} X \pi^{\wedge}=X_{\left.\right|_{\wedge} \wedge}$ (proposition 2.1.2), cette dernière équation équivaut à

$$
X_{\Pi_{\wedge}} \partial_{\omega} a_{i+1}^{\wedge}=\pi^{\wedge}\left(-T R_{\theta} \partial_{\omega} a_{i+1}^{\vee}-L \stackrel{\star}{A}{ }_{i-1}-X \partial_{\omega} a_{i+1}^{0}+\stackrel{\star}{\boldsymbol{F}_{0}^{\prime}}(i)+\stackrel{\star}{\mathcal{F}}\right)
$$

Ayant supposé la jauge vérifiée à l'ordre $O\left(\varepsilon^{i / 2}\right)$ en partie oscillante $: R_{\theta} \partial_{\omega} a_{i+1}^{\vee}+$ $R A_{i-1}^{\star}=0$, l'équation (52) équivaut à

$$
X_{\left.\right|_{\Pi_{\curlywedge}}} \partial_{\omega} a_{i+1}^{\wedge}=\pi^{\wedge}\left(-\square \stackrel{\star}{A} i-1+(\mathcal{R} i c .) \stackrel{\star}{A}{ }_{i-1}-X \partial_{\omega} a_{i+1}^{0}+{\stackrel{\star}{F_{0}^{\prime}}}_{0}(i)+\stackrel{\star}{\mathcal{F}}_{i}\right)
$$

Nous remarquons de nouveau que le choix de la valeur initiale de $a_{i+1}^{\wedge}$ est parfaitement libre en jauge de Lorentz, contrairement à n'importe quelle autre jauge $J_{\lambda}, \lambda \in\left[0, \frac{1}{2}[\cup\right.$ ]$\left.\frac{1}{2}, 1\right]$.

Proposition 2.2.9. - Pour tout indice entier $i \geqslant 0$, les propositions suivantes sont équivalentes :

i) $a_{1}^{\vee}=a_{2}^{\vee}=0, \psi_{0}^{-}=\psi_{1}^{-}=0$, puis pour tout indice $0 \leqslant k \leqslant i$,

- les profils $\left(\widetilde{A}_{k}, a_{k+1}^{0}, \widetilde{\phi}_{k}, \stackrel{\star}{\phi}_{k+1}, \widetilde{\psi}_{k}, \stackrel{\star}{k}+^{+}\right)$sont solutions (régulières) des systèmes $(C, k)$ successifs, et vérifient les conditions de jauge $R \widetilde{A}_{k}=0$.

- les profils $\left(a_{k+3}^{\vee}, \psi_{k+2}^{\star}\right)$ vérifient les polarisations $(P, k)$,

- les composantes $a_{k+1}^{\wedge}$ vérifient les équations de transport $(E, k)$.

ii) $\hat{\psi}_{0}^{-}=\psi_{1}^{-}=0$, les profils $\left(A_{0}, \stackrel{\star}{A_{1}}, \phi_{0}, \stackrel{\star}{\phi}_{1}, \widetilde{\psi}_{0}, \stackrel{\star}{*}_{0}^{+},,_{2}^{\star}\right)$ sont solutions (régulières) $d u$ système

$$
(H, 0 \text { - Lorentz Fixé - }) \quad\left\{\begin{aligned}
\square A_{0}-(\mathcal{R} i c .) A_{0} & =\widetilde{\boldsymbol{F}_{0}} \\
\square \phi_{0} & =\widetilde{\boldsymbol{G}_{0}} \\
\mathcal{D} \widetilde{\psi_{0}} & =\widetilde{\mathbf{h}_{\mathbf{0}}} \\
X \partial_{\omega} \stackrel{\star}{A_{1}} & =\stackrel{\star}{*}_{0} \\
X \partial_{\omega}^{\star} \stackrel{\star}{\phi}_{1} & =\stackrel{\star}{\boldsymbol{G}_{0}} \\
X \stackrel{\star}{\psi_{0}^{+}} & =\mathcal{D}_{\theta} \mathbf{h}_{\mathbf{0}}^{\star} \\
\star & \stackrel{\star}{\psi_{2}^{-}}=\mathcal{D}_{\theta}^{-1} \partial_{\omega}^{-1} \pi^{+}\left(-\mathcal{D} \psi_{0}^{+}+\stackrel{\star}{\mathbf{h}_{\mathbf{0}}}\right)
\end{aligned}\right.
$$

et vérifient les contraintes initiales $\grave{a} t=0$ :

$$
\left\{\begin{array}{l}
(49) \\
R_{\theta} \stackrel{\star}{A} c_{1}=0
\end{array}\right.
$$


puis pour tout $1 \leqslant k \leqslant i,\left(\widetilde{A}_{k}, \stackrel{\star}{A}_{k+1}, \widetilde{\phi}_{k}, \stackrel{\star}{\phi}_{k+1}, \widetilde{\psi}_{k}, \stackrel{\star}{\psi}_{k}^{+}, \psi_{k+2}^{-}\right)$sont solutions des systèmes

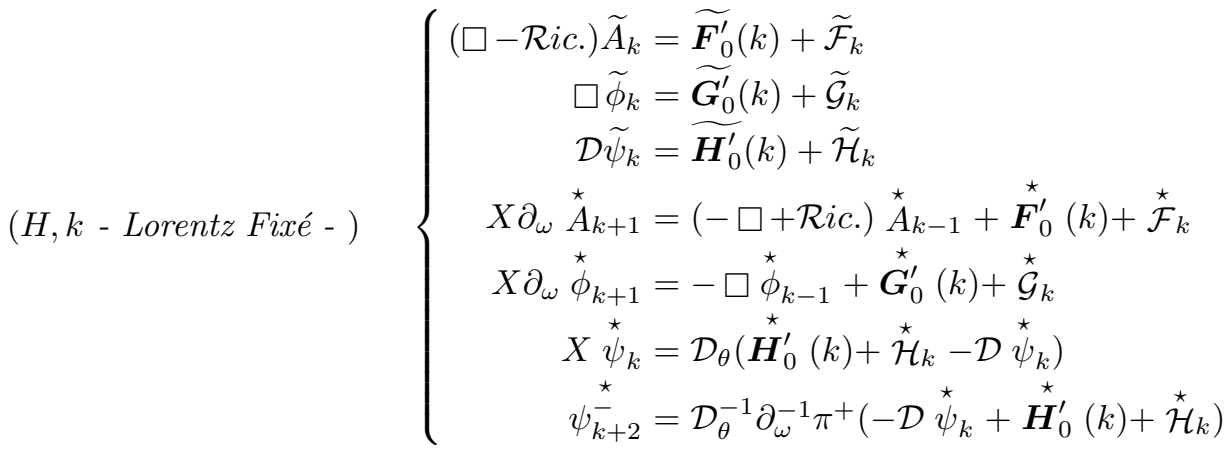

et satisfait $\grave{a} t=0$ les contraintes initiales

$$
\left\{\begin{array}{l}
(51) \\
R_{\theta} \partial_{\omega} \pi^{\vee} \stackrel{\star}{A} c_{k+1}+R \stackrel{\star}{A} c_{k-1}=0
\end{array}\right.
$$

et enfin $a_{i+2}^{\vee}$ et $a_{i+3}^{\vee}$ satisfont les contraintes de jauge

$$
\begin{aligned}
R_{\theta} \partial_{\omega} a_{i+2}^{\vee}+R \stackrel{\star}{A} & =0 \\
R_{\theta} \partial_{\omega} a_{i+3}^{\vee}+R \stackrel{\star}{A} & =0
\end{aligned}
$$

Les systèmes $(H, k$ - Lorentz Fixé - ) sont les équations de profils que l'on obtient en développant (Y.M.) sous sa forme strictement hyperbolique, à jauge de Lorentz fixée. La proposition 2.2.9 nous permet de vérifier que le résultat de la proposition 1.3.5 reste valable, à un ordre arbitraire, pour les solutions oscillantes (d'amplitude critique) que nous envisageons. Nous obtenons la même solution approchée

i) en développant le système sous sa forme mal déterminée, à jauge libre, tout en fixant judicieusement les composantes non dynamiques du champ de jauge pour vérifier in fine la jauge de Lorentz.

ii) en développant directement le système sous sa forme hyperbolique, à jauge de Lorentz fixée.

Encore une fois, le résultat semble de bon sens, mais vu l'amplitude critique des oscillations, sa démonstration requiert quelque attention. Nous avons privilégié jusqu'ici le développement à jauge libre pour étendre nos résultats à la famille de jauges transverses $J_{\lambda}$, et ne pas dépendre exclusivement de la jauge de Lorentz, qui demeure singulière et masque certaines informations ${ }^{(3)}$.

\footnotetext{
${ }^{(3)}$ Notons qu'en dépit des apparences, il n'est pas plus simple de commencer par développer le système sous sa forme hyperbolique, à jauge de Lorentz fixée : il est nécessaire, à un moment ou un autre, de décrire le comportement des différentes composantes oscillatoires du champ de jauge.
} 
Démonstration de la proposition 2.2.9. - Nous procédons par récurrence. Montrons i) $\Rightarrow$ ii)

- Pour $i=0$, si l'hypothèse i) est satisfaite, alors $R_{\theta} \partial_{\omega} a_{2}^{\vee}=0$ et la proposition 2.2 .7 b) garantit que $a_{3}^{\vee}$ vérifie $R_{\theta} \partial_{\omega} a_{3}^{\vee}+R \stackrel{\star}{A_{1}}=0$. Reste à montrer que ( H, 0 - Lorentz Fixé - ) est satisfait ainsi que les contraintes initiales (53). La propo-

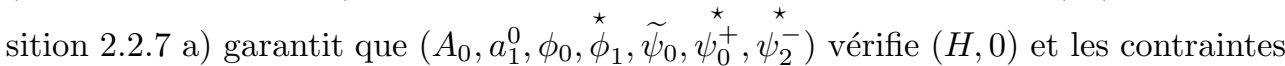
initiales (49). Puisque $a_{1}^{\vee}=0$, la proposition 2.2.1 donne $\pi^{\vee} \stackrel{\star}{\boldsymbol{F}_{0}}=0$, et nous vérifions

$$
\left\{\begin{array}{l}
\pi^{\vee} X \partial_{\omega} a_{1}^{\vee}=\pi^{\vee} \stackrel{\boldsymbol{F}}{\boldsymbol{F}}_{0} \\
a_{\left.1\right|_{t=0} ^{\vee}}=0
\end{array}\right.
$$

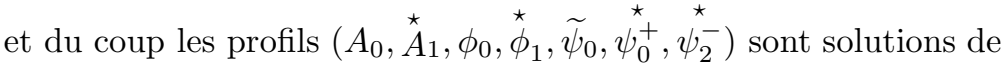

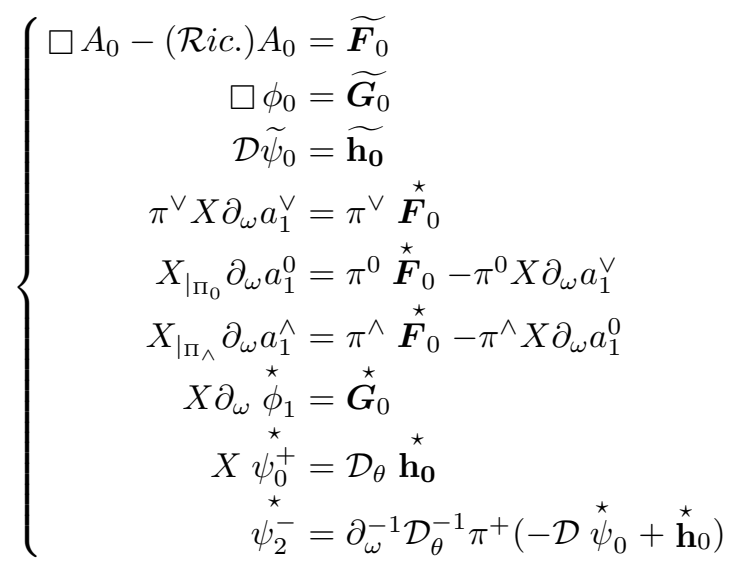

et satisfont à $t=0$ les contraintes (53). Enfin, toujours par la proposition (2.1.2), nous savons que $\pi^{\wedge} X \pi^{\wedge}=X_{\left.\right|_{\Pi_{\wedge}}}$ et $\pi^{0} X \pi^{0}=X_{\mid \Pi_{0}}$ tandis que $\pi^{\vee} X \pi^{0}=0, \pi^{\vee} X \pi^{\wedge}=0$, $\pi^{0} X \pi^{\wedge}=0$ et $\pi^{\wedge} X \pi^{\vee}=0$. Il en résulte que l'hypothèse i) implique ii) pour l'indice $i=0$.

- Supposons maintenant que i) implique ii) pour un indice $i-1 \geqslant 0$ donné. Nous sommes assurés que

$$
\begin{gathered}
R_{\theta} \partial_{\omega} a_{i+1}^{\vee}+R \stackrel{\star}{A}_{i-1}=0 \\
R_{\theta} \partial_{\omega} a_{i+2}^{\vee}+R \stackrel{\star}{A}{ }_{i}=0
\end{gathered}
$$

et que les profils

$$
\left(\widetilde{A}_{k}, \stackrel{\star}{A}_{k+1}, \widetilde{\phi}_{k}, \stackrel{\star}{\phi}_{k+1}, \widetilde{\psi}_{k}, \stackrel{\star}{*}+_{k}^{+}, \stackrel{\star}{*}_{k+2}^{\star}\right)
$$


vérifient les systèmes $(H, k$ - Lorentz Fixé - ) successifs pour $k \leqslant i-1$ et les contraintes initiales (54) associées. Si l'hypothèse i) est satisfaite pour l'indice $i$, alors la proposition 2.2 .7 b) garantit que $a_{i+3}^{\vee}$ vérifie

$$
R_{\theta} \partial_{\omega} a_{i+3}^{\vee}+R \stackrel{\star}{A}{ }_{i+1}=0
$$

Reste à montrer que $(H, i$ - Lorentz Fixé - ) est satisfait ainsi que les contraintes initiales (54) associées. La proposition 2.2 .7 a) garantit que $\left(\widetilde{A}_{i}, a_{i+1}^{0}, \widetilde{\phi}_{i}, \stackrel{\star}{\phi}_{i+1}, \widetilde{\psi}_{i}, \hat{\psi}_{i}^{+}\right.$ ,$\left.\psi_{i+2}^{\star}\right)$ vérifie $(H, i)$ et les contraintes initiales (51) associées. Lorsque i) est vérifiée pour l'indice $i$, la proposition 2.2.4 nous garantit que

$$
\pi^{\vee} M \partial_{\omega} \stackrel{\star}{A_{i+1}}=\pi^{\vee}\left(-L \stackrel{\star}{A} i-1+\stackrel{\star}{\mathbf{F}}_{\mathbf{0}}^{\prime}(i)+\stackrel{\star}{\mathcal{F}}_{i}\right)
$$

est toujours vérifiée. La proposition 2.1.2 donne par ailleurs $\pi^{\vee} X \pi^{0}=\pi^{\vee} X \pi^{\wedge}=0$, et la jauge de Lorentz est vérifiée à l'ordre $\varepsilon^{i-1 / 2}$ en partie oscillante (56), donc

$$
\pi^{\vee} M \partial_{\omega} \stackrel{\star}{A}_{i+1}=\pi^{\vee}\left(X \partial_{\omega} a_{i+1}^{\vee}-T R_{\theta} \partial_{\omega} a_{i+1}^{\vee}\right)=\pi^{\vee} X \partial_{\omega} a_{i+1}^{\vee}+\pi^{\vee} T R \stackrel{\star}{A}
$$

nous en déduisons immédiatement que

$$
\begin{aligned}
& \pi^{\vee} X \partial_{\omega} a_{i+1}^{\vee}=\pi^{\vee}\left((-L-T R) \stackrel{\star}{A_{i-1}}+\stackrel{\star}{\mathbf{F}}_{\mathbf{0}}(i)+\stackrel{\star}{\mathcal{F}}_{i}\right) \\
& =\pi^{\vee}\left(-\square \stackrel{\star}{A}_{i-1}+(\mathcal{R} i c .) \stackrel{\star}{A} i-1+\stackrel{\star}{*}_{0}^{\prime}(i)+\stackrel{\star}{\mathcal{F}}_{i}\right)
\end{aligned}
$$

De même, (56) nous permet de simplifier

$$
\pi^{0} M \partial_{\omega} a_{i+1}^{\vee}=-\pi^{0}\left(X-T R_{\theta}\right) \partial_{\omega} a_{i+1}^{\vee}=\pi^{0}\left(X \partial_{\omega} a_{i+1}^{\vee}+T R \stackrel{\star}{A_{i-1}}\right)
$$

donc l'équation de $(H, i)$

$$
X_{\left.\right|_{\Pi_{0}}} \partial_{\omega} a_{i+1}^{0}=\pi^{0}\left(-L \stackrel{\star}{A_{i-1}}-M \partial_{\omega} a_{i+1}^{\vee}+\stackrel{\star}{F}_{0}^{\prime}(i)+\stackrel{\star}{\mathcal{F}}_{i}\right)
$$

devient

$$
X_{\left.\right|_{\Pi_{0}}} \partial_{\omega} a_{i+1}^{0}=\pi^{0}\left((-\square+\mathcal{R} i c .) \stackrel{\star}{A} i-1-X \partial_{\omega} a_{i+1}^{\vee}+\stackrel{\star}{F_{0}^{\prime}}(i)+\stackrel{\star}{\mathcal{F}} i\right)
$$


Il en résulte que les profils $\left(\widetilde{A}_{i}, \stackrel{\star}{A} i+1, \widetilde{\phi}_{i}, \stackrel{\star}{\phi}_{i+1}, \widetilde{\psi}_{i}, \psi_{i}^{+}, \psi_{i+2}^{-}\right)$sont solutions de

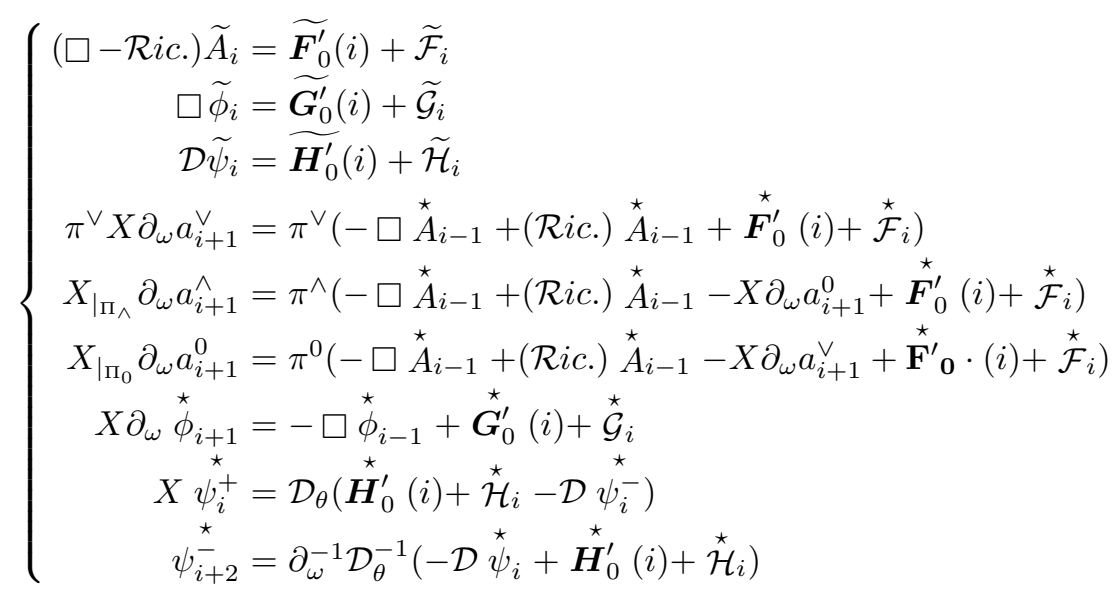

et satisfont à $t=0$ les contraintes (54) pour l'indice $i$. En s'appuyant de nouveau sur la proposition (2.1.2) (qui nous donne $\pi^{\wedge} X \pi^{\wedge}=X_{\left.\right|_{\Lambda}}$ et $\pi^{0} X \pi^{0}=X_{\mid \Pi_{0}}$ tandis que $\pi^{\vee} X \pi^{0}=0, \pi^{\vee} X \pi^{\wedge}=0, \pi^{0} X \pi^{\wedge}=0$ et $\left.\pi^{\wedge} X \pi^{\vee}=0\right)$ nous concluons immédiatement que $(H, i$ - Lorentz Fixé - ) est vérifié et que l'hypothèse i) implique ii) pour l'indice $i$. Montrons maintenant ii) $\Rightarrow$ i)

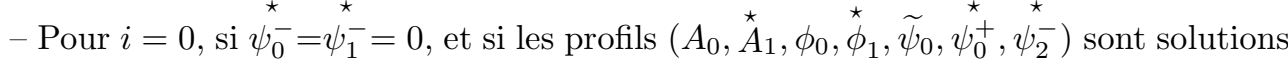
(régulières) du système ( $H, 0$ - Lorentz Fixé- ) et vérifient les contraintes initiales (53), alors

$$
a_{1}^{\vee}=0
$$

En effet, l'équation

$$
X \partial_{\omega} \stackrel{\star}{A}{ }_{1}=\stackrel{\star}{F}_{0}
$$

est satisfaite. Par projection sur $\Pi_{\vee}$, en remarquant que $\pi^{\vee} X \pi^{0}=\pi^{\vee} X \pi^{\wedge}=0$, et compte tenu des contraintes (53), il vient alors

$$
\left\{\begin{array}{l}
\pi^{\vee} X \partial_{\omega} a_{1}^{\vee}=\pi^{\vee} \stackrel{\star}{\boldsymbol{F}_{0}} \\
\left.a_{1}^{\vee}\right|_{t=0}=0
\end{array}\right.
$$

Le développement de l'identité de Bianchi, section 2.2.2, nous donne

$$
\begin{aligned}
\pi^{\vee}{\stackrel{\star}{\boldsymbol{F}_{0}}}_{0} & =L_{\theta}^{-1} T_{\theta} R_{\theta} \stackrel{\star}{F_{0}} \\
& =L_{\theta}^{-1} T_{\theta} Q_{f}\left(A_{0}, L_{\theta} \partial_{\omega} a_{1}^{\vee}\right)
\end{aligned}
$$

qui est donc un terme linéaire en $a_{1}^{\vee}$. Donc pour tout $s \geqslant \frac{n}{2}+\frac{1}{2}$, il vient

$$
\left|\pi^{\vee} \stackrel{\star}{\boldsymbol{F}_{0}}\right|_{H^{s}} \leqslant C\left|A_{0}\right|_{H^{s}}\left|a_{1}^{\vee}\right|_{H^{s}}
$$


La proposition (2.1.2) nous montre que $\pi^{\vee} X a_{1}^{\vee}-X_{\left.\right|_{\Upsilon \vee}} a_{1}^{\vee}$ est également linéaire d'ordre 0 en $a_{1}^{0}$. Sur tout $H^{s}, s \geqslant \frac{n}{2}+\frac{1}{2}$, et quel que soit $A_{0} \in H^{s}$, l'unique solution de l'équation de transport linéaire

$$
\left\{\begin{array}{l}
X_{\left.\right|_{\Pi \vee}} \partial_{\omega} a_{1}^{\vee}=L_{\theta}^{-1} T_{\theta} Q_{f}\left(A_{0}, L_{\theta} \partial_{\omega} a_{1}^{\vee}\right)-\left(\pi^{\vee} X a_{1}^{\vee}-X_{\left.\right|_{\Pi \vee}} a_{1}^{\vee}\right) \\
a_{\left.\right|_{t=0}}^{\vee}=0
\end{array}\right.
$$

est bien entendu la solution identiquement nulle

$$
a_{1}^{\vee}=0
$$

Si l'hypothèse ii) est satisfaite pour l'indice $i=0$, nous vérifions en plus

$$
a_{2}^{\vee}=0 \quad \text { et } \quad R_{\theta} \partial_{\omega} a_{3}^{\vee}+R \stackrel{\star}{A_{1}}=0
$$

Il en résulte que

$$
\begin{aligned}
L_{\theta} \partial_{\omega}^{2} \stackrel{\star}{A}+M \partial_{\omega} \stackrel{\star \star}{A}- & \stackrel{\star}{\boldsymbol{F}_{0}}=-T_{\theta} R_{\theta} \partial_{\omega}^{2} a_{3}^{\vee}+\left(X-T R_{\theta}-T_{\theta} R\right) \partial_{\omega} \stackrel{\star}{A_{1}}-\stackrel{\star}{\boldsymbol{F}_{0}} \\
& =-T_{\theta} \partial_{\omega}\left(R_{\theta} \partial_{\omega} a_{3}^{\vee}+R \stackrel{\star}{A_{1}}\right)-T\left(R_{\theta} \partial_{\omega} a_{1}^{\vee}\right)+\left(X \partial_{\omega} \stackrel{\star}{A_{1}}-{\stackrel{\star}{\boldsymbol{F}_{0}}}\right) \\
& =0
\end{aligned}
$$

En projetant cette équation sur $\Pi_{\wedge}, \Pi_{0}$ et $\Pi_{0}$, nous en déduisons que la polarisation $(P, 0)$, l'équation $(E, 0)$ et le système $(H, 0)$ sont vérifiés. La proposition 2.2 .7 a) garantit que $(C, 0)$ l'est également. Ainsi, ii) $\Rightarrow$ i) pour l'indice $i=0$.

- Pour l'indice $i=1$, nous montrons de façon analogue que si $\psi_{0}^{-}=\psi_{1}^{-}=0$, et si les profils $\left(\widetilde{A}_{k}, \stackrel{\star}{A}_{k+1}, \widetilde{\phi}_{k}, \stackrel{\star}{\phi}_{k+1}, \widetilde{\psi}_{k}, \stackrel{\star}{k}_{k}^{+}, \psi_{k+2}^{-}\right)$sont solutions (régulières) des systèmes $(H, k$ - Lorentz Fixé- ), $0 \leqslant k \leqslant 1$, et vérifient les contraintes initiales (53) et (54) associées, alors

$$
a_{1}^{\vee}=a_{2}^{\vee}=0
$$

Si l'hypothèse ii) est satisfaite pour l'indice $i=0$, nous vérifions en plus

$$
R_{\theta} \partial_{\omega} a_{3}^{\vee}+R \stackrel{\star}{A_{1}}=0 \quad \text { et } \quad R_{\theta} \partial_{\omega} a_{4}^{\vee}+R \stackrel{\star}{A_{2}}=0
$$

donc, exactement comme pour $i=0$, nous en tirons

$$
\begin{aligned}
L_{\theta} \partial_{\omega}^{2} \stackrel{\star}{A}+M \partial_{\omega} \stackrel{\star \star}{A} 1-\stackrel{\star}{*}_{0} \\
\quad=-T_{\theta} \partial_{\omega}\left(R_{\theta} \partial_{\omega} a_{3}^{\vee}+R \stackrel{\star}{A} A_{1}\right)-T\left(R_{\theta} \partial_{\omega} a_{1}^{\vee}\right)+\left(X \partial_{\omega} \stackrel{\star}{A}{ }_{1}-\stackrel{\star}{\boldsymbol{F}_{0}}\right)=0
\end{aligned}
$$

et

$$
\begin{aligned}
L_{\theta} \partial_{\omega}^{2} \stackrel{\star}{A}_{4} & +M \partial_{\omega} \stackrel{\star \star}{A}{ }_{2}-\mathbf{F}_{\mathbf{0}}^{\prime}(1)-\stackrel{\star}{\mathcal{F}}_{1} \\
& =-T_{\theta} \partial_{\omega}\left(R_{\theta} \partial_{\omega} a_{4}^{\vee}+R \stackrel{\star}{A_{2}}\right)-T\left(R_{\theta} \partial_{\omega} a_{2}^{\vee}\right)+\left(X \partial_{\omega} \stackrel{\star}{A_{2}}-{\stackrel{\star}{\mathbf{F}_{\mathbf{0}}^{\prime}}}^{(1)}-\stackrel{\star}{\mathcal{F}} 1\right)=0
\end{aligned}
$$

En projetant ces équations sur $\Pi_{\wedge}, \Pi_{0}$ et $\Pi_{0}$, nous en déduisons que les polarisations $(P, 0),(P, 1)$, les équations $(E, 0),(E, 1)$ et les systèmes $(H, 0),(H, 1)$ sont vérifiés. La 
proposition 2.2 .7 a) garantit que $(C, 0)$, puis $(C, 1)$, le sont également. Ainsi, ii) $\Rightarrow$ i) pour l'indice $i=1$.

- Pour l'indice suivant $i=2$, si $\psi_{0}^{-}=\psi_{1}^{-}=0$, et si les profils $\left(\widetilde{A}_{k}, \stackrel{\star}{A}_{k+1}, \widetilde{\phi}_{k}, \stackrel{\star}{\phi}_{k+1}\right.$ $\left., \widetilde{\psi}_{k}, \psi_{k}^{+}, \psi_{k+2}^{\star}\right)$ sont solutions (régulières) des systèmes $(H, k$ - Lorentz Fixé- $), 0 \leqslant$ $k \leqslant 2$, et vérifient les contraintes initiales (53) et (54) associées, alors

$$
a_{1}^{\vee}=a_{2}^{\vee}=0, \quad R_{\theta} \partial_{\omega} a_{3}^{\vee}+R \stackrel{\star}{A} 1=0 \quad \text { et } \quad R A_{0}=0
$$

Du coup, l'hypothèse i) est vérifiée pour l'indice $i=0$.

En effet : $R_{\theta} M+R L_{\theta}=0$ et $\pi^{\vee} M=\pi^{\vee}\left(X-T R_{\theta}\right)$. Si $(H, 2$ - Lorentz Fixé - ) est vérifié, il vient

$$
\begin{aligned}
0 & =R_{\theta} M \partial_{\omega} \stackrel{\star}{A}_{3}+R L_{\theta} \partial_{\omega} \stackrel{\star}{A}_{3} \\
& =R_{\theta}\left(X \partial_{\omega} \stackrel{\star}{A}{ }_{3}-T R_{\theta} \partial_{\omega} a_{3}^{\vee}\right)+R L_{\theta} \partial_{\omega} a_{3}^{\vee} \\
& =R_{\theta}\left(\stackrel{\star}{\mathbf{F}}_{\mathbf{0}}(2)+\stackrel{\star}{\mathcal{F}}_{2}-(\square-\mathcal{R} i c .) \stackrel{\star}{A}{ }_{1}\right)-\left(R_{\theta} T+R T_{\theta}\right) R_{\theta} \partial_{\omega} a_{3}^{\vee} \\
& =R_{\theta}\left(\stackrel{\star}{\mathbf{F}}_{\mathbf{0}}^{\prime}(2)+\stackrel{\star}{\mathcal{F}}_{2}-(\square-\mathcal{R} i c .) \stackrel{{ }^{*}}{A_{i}}\right)-X R_{\theta} \partial_{\omega} a_{3}^{\vee}
\end{aligned}
$$

De même, $R M+R_{\theta} L=0$. Si (H,0 - Lorentz Fixé - ) est vérifié, nous en déduisons

$$
\begin{aligned}
& 0=R M \stackrel{\star}{A} 1+R_{\theta} L \stackrel{\star}{A_{1}} \\
& =R\left(X-T_{\theta} R-T R_{\theta}\right) \stackrel{\star}{A} 1+R_{\theta}(\square-(\mathcal{R} i c .)-T R) \stackrel{\star}{A} 1 \\
& =R \partial_{\omega}^{-1} \stackrel{\star}{\boldsymbol{F}}_{0}-\left(R T R_{\theta} \stackrel{\star}{A_{1}}\right)-\left(R T_{\theta}+R_{\theta} T\right) R \stackrel{\star}{A}{ }_{1}+R_{\theta}(\square-\mathcal{R} i c .) \stackrel{\star}{A} 1 \\
& =R \partial_{\omega}^{-1} \stackrel{\star}{\boldsymbol{F}}_{0}+R T R_{\theta} \stackrel{\star}{A}{ }_{1}-X R \stackrel{\star}{A_{1}}+R_{\theta}(\square-\mathcal{R} i c .) \stackrel{\star}{A_{1}} \\
& =R \partial_{\omega}^{-1} \stackrel{\star}{*}_{0}-X R \stackrel{\star}{A} 1+R_{\theta}(\square-\mathcal{R} i c .) \stackrel{\star}{A} 1
\end{aligned}
$$

Il en résulte que :

$$
X\left(R_{\theta} \partial_{\omega} a_{3}^{\vee}+R \stackrel{\star}{A} 1\right)=R_{\theta}\left(\stackrel{\star}{*}_{\mathbf{0}}^{\prime}(2)+\stackrel{\star}{\mathcal{F}}_{2}\right)+R \partial_{\omega}^{-1}{\stackrel{\star}{\boldsymbol{F}_{0}}}
$$

Nous déduisons également de (H,0 - Lorentz Fixé- ) :

$$
R A_{0}=R \widetilde{\boldsymbol{F}_{0}}
$$

En s'appuyant sur le développement de l'identité de Bianchi, section 2.2.2, et compte tenu des contraintes initiales $(53,54)$, nous en déduisons le système linéaire

$$
\left\{\begin{aligned}
X\left(R_{\theta} \partial_{\omega} a_{3}^{\vee}+R \stackrel{\star}{A}_{1}\right) & =Q_{f}\left(A_{0}, T_{\theta}\left(R_{\theta} \partial_{\omega} a_{3}^{\vee}+R \stackrel{\star}{A}_{1}\right)\right) \\
\square R A_{0} & =Q_{f}\left(A_{0}, T\left(R A_{0}\right)\right)+\operatorname{Moy} Q_{f}\left(\stackrel{\star}{A}_{1}, T_{\theta}\left(R_{\theta} \partial_{\omega} a_{3}^{\vee}+R \stackrel{\star}{A}_{1}\right)\right) \\
\left(R_{\theta} \partial_{\omega} a_{3}^{\vee}+R \stackrel{\star}{A_{1}}\right)_{\left.\right|_{t=0}} & =0 \\
R A_{\left.0\right|_{t=0}} & =0
\end{aligned}\right.
$$

dont l'unique solution est $R_{\theta} \partial_{\omega} a_{3}^{\vee}+R \stackrel{\star}{A}{ }_{1}=0$ et $R A_{0}=0$ dès lors que $\left(A_{0}, \stackrel{\star}{A}{ }_{1}\right)$ sont des données régulières. 
Si nous supposons que l'hypothèse ii) est vérifiée pour l'indice $i=2$, nous vérifions en plus

$$
R_{\theta} \partial_{\omega} a_{4}^{\vee}+R \stackrel{\star}{A}{ }_{2}=0 \quad \text { et } \quad R_{\theta} \partial_{\omega} a_{5}^{\vee}+R \stackrel{\star}{A}{ }_{3}=0
$$

nous en tirons

$$
\begin{aligned}
L_{\theta} \partial_{\omega}^{2} \stackrel{\star}{A}_{4} & +M \partial_{\omega} \stackrel{\star}{A}_{2}-{\stackrel{\star}{\mathbf{F}_{\mathbf{0}}^{\prime}}}^{(1)}-\stackrel{\star}{\mathcal{F}}_{1} \\
& =-T_{\theta} \partial_{\omega}\left(R_{\theta} \partial_{\omega} a_{4}^{\vee}+R \stackrel{\star}{A}{ }_{2}\right)-T\left(R_{\theta} \partial_{\omega} a_{2}^{\vee}\right)+\left(X \partial_{\omega} \stackrel{\star}{A}_{2}-\stackrel{\star}{*}_{\mathbf{0}}^{\prime}(1)-\stackrel{\star}{\mathcal{F}}_{1}\right)=0
\end{aligned}
$$

et

$$
\begin{aligned}
& L_{\theta} \partial_{\omega}^{2} \stackrel{\star}{A}_{5}+M \partial_{\omega} \stackrel{\star}{A}_{3}-{\stackrel{\star}{\mathbf{F}_{0}^{\prime}}}_{\mathbf{0}}(2)-\stackrel{\star}{\mathcal{F}}_{2} \\
& =-T_{\theta} \partial_{\omega}\left(R_{\theta} \partial_{\omega} a_{5}^{\vee}+R \stackrel{\star}{A}_{3}\right)-T\left(R_{\theta} \partial_{\omega} a_{3}^{\vee}+R \stackrel{\star}{A}_{1}\right)+\left(X \partial_{\omega} \stackrel{\star}{A}_{3}-\stackrel{\star}{\mathbf{F}}_{\mathbf{0}}^{\prime}(2)-\stackrel{\star}{\mathcal{F}}_{2}\right)=0
\end{aligned}
$$

En projetant ces équations sur $\Pi_{\wedge}, \Pi_{0}$ et $\Pi_{0}$, nous en déduisons que les polarisations $(P, 1),(P, 2)$, les équations $(E, 1),(E, 2)$ et les systèmes $(H, 1),(H, 2)$ sont vérifiés. La proposition 2.2 .8 a) garantit que $(C, 1)$, puis $(C, 2)$, le sont également. Ainsi, ii) $\Rightarrow$ i) pour l'indice $i=2$.

- Supposons maintenant que si $\psi_{0}^{-}=\psi_{1}^{-}=0$, et si les profils

$$
\left(\widetilde{A}_{k}, \stackrel{\star}{A}_{k+1}, \widetilde{\phi}_{k}, \stackrel{\star}{\phi}_{k+1}, \widetilde{\psi}_{k}, \psi_{k}^{+}, \psi_{k+2}^{-}\right)
$$

sont solutions (régulières) des systèmes $(H, k$ - Lorentz Fixé- ), $0 \leqslant k \leqslant i-1$, et vérifient les contraintes initiales (53) et (54) associées, alors

$$
a_{1}^{\vee}=a_{2}^{\vee}=0, \quad \text { puis } \quad R_{\theta} \partial_{\omega} a_{k+1}^{\vee}+R \stackrel{\star}{A}_{k-1}=0 \quad \text { et } \quad R \widetilde{A}_{k}=0
$$

pour tout $0 \leqslant k \leqslant i-3$, et du coup, que l'hypothèse i) est vérifiée pour l'indice $i-3$.

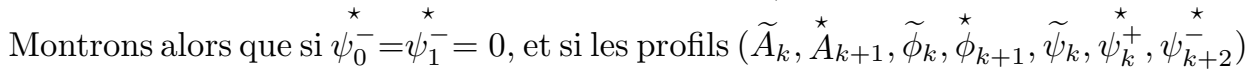
sont solutions (régulières) des systèmes $(H, k$ - Lorentz Fixé- ), $0 \leqslant k \leqslant i$, et vérifient les contraintes initiales (53) et (54) associées, alors

$$
a_{1}^{\vee}=a_{2}^{\vee}=0, \quad \text { puis } \quad R_{\theta} \partial_{\omega} a_{k+1}^{\vee}+R \stackrel{\star}{A}_{k-1}=0 \quad \text { et } \quad R \widetilde{A}_{k}=0
$$

pour tout $0 \leqslant k \leqslant i-2$, donc finalement, que l'hypothèse i) est vérifiée pour l'indice $i-2$.

Comme $R_{\theta} M+R L_{\theta}=0, \pi^{\vee} M=\pi^{\vee}\left(X-T R_{\theta}\right)$ et que $(H, i$ - Lorentz Fixé - ) est supposé vérifié, il vient

$$
\begin{aligned}
0 & =R_{\theta} M \partial_{\omega} \stackrel{\star}{A}_{i+1}+R L_{\theta} \partial_{\omega} \stackrel{\star}{A}_{i+1} \\
& =R_{\theta}\left(\pi^{\vee} X \partial_{\omega} a_{i+1}^{\vee}-\pi^{\vee} T R_{\theta} \partial_{\omega} a_{i+1}^{\vee}\right)+R L_{\theta} \partial_{\omega} a_{i+1}^{\vee} \\
& =R_{\theta}\left(\stackrel{\star}{\mathbf{F}}_{\mathbf{0}}^{\prime}(i)+\stackrel{\star}{\mathcal{F}}_{i}-(\square-\mathcal{R} i c .) \stackrel{\star}{A}_{i-1}\right)-\left(R_{\theta} T+R T_{\theta}\right) R_{\theta} \partial_{\omega} a_{i+1}^{\vee} \\
& =R_{\theta}\left(\stackrel{\star}{\mathbf{F}}_{\mathbf{0}}^{\prime}(i)+\stackrel{\star}{\mathcal{F}}_{i}-(\square-\mathcal{R} i c .) \stackrel{\star}{A}_{i-1}\right)-X R_{\theta} \partial_{\omega} a_{i+1}^{\vee}
\end{aligned}
$$


De même, $R M+R_{\theta} L=0$, et $(H, i-2$ - Lorentz - ) est vérifié. Nous en déduisons

$$
\begin{aligned}
& 0=R M \stackrel{\star}{A} i-1+R_{\theta} L \stackrel{\star}{A} i-1 \\
& =R\left(X-T_{\theta} R-T R_{\theta}\right) \stackrel{\star}{A}_{i-1}+R_{\theta}(\square-(\mathcal{R} i c .)-T R) \stackrel{\star}{A}_{i-1} \\
& =R \partial_{\omega}^{-1}\left({\stackrel{\star}{\mathbf{F}_{0}^{\prime}}}_{\mathbf{0}}(i-2)+\stackrel{\star}{\mathcal{F}}_{i-2}\right)-\left(R \square \partial_{\omega}^{-1} \stackrel{\star}{A}_{i-3}+R T R_{\theta} \stackrel{\star}{A}_{i-1}\right)-\left(R T_{\theta}+R_{\theta} T\right) R \stackrel{\star}{A} \stackrel{\star}{i-1}_{1} \\
& +R_{\theta}(\square-\mathcal{R} \text { ic. }) \stackrel{\star}{A}{ }_{i-1} \\
& =R \partial_{\omega}^{-1}\left(\stackrel{\star}{\mathbf{F}}_{\mathbf{0}}^{\prime}(i-2)+\stackrel{\star}{\mathcal{F}}_{i-2}\right)+R T\left(R \partial_{\omega}^{-1} \stackrel{\star}{A}_{i-3}+R_{\theta} \stackrel{\star}{A}_{i-1}\right)-X R \stackrel{\star}{A}_{i-1} \\
& +R_{\theta}(\square-\mathcal{R} i c .) \stackrel{\star}{A}{ }_{i-1} \\
& =R \partial_{\omega}^{-1}\left({\stackrel{\star}{\mathbf{F}_{0}}}_{\mathbf{0}}(i-2)+\stackrel{\star}{\mathcal{F}}_{i-2}\right)-X R \stackrel{\star}{A}_{i-1}+R_{\theta}(\square-\mathcal{R} i c .) \stackrel{\star}{A}_{i-1}
\end{aligned}
$$

Il en résulte que :

$$
X\left(R_{\theta} \partial_{\omega} a_{i+1}^{\vee}+R \stackrel{\star}{A}_{i-1}\right)=R_{\theta}\left(\stackrel{\star}{\mathbf{F}}_{\mathbf{0}}^{\prime}(i)+\stackrel{\star}{\mathcal{F}}_{i}\right)+R \partial_{\omega}^{-1}\left({\stackrel{\star}{\mathbf{F}_{\mathbf{0}}^{\prime}}}(i-2)+\stackrel{\star}{\mathcal{F}}_{i-2}\right)
$$

Par ailleurs, $(H, i-2$ - Lorentz Fixé- ) nous donne

$$
\square\left(R \widetilde{A}_{i-2}\right)=R \widetilde{F}_{0}^{\prime}(i-2)+R \widetilde{\mathcal{F}}_{i-2}
$$

En s'appuyant sur le développement de l'identité de Bianchi, section 2.2.2, et compte tenu des contraintes initiales (54), nous en déduisons que le système linéaire suivant est vérifié :

$$
\left\{\begin{aligned}
X\left(R_{\theta} \partial_{\omega} a_{i+1}^{\vee}+R \stackrel{\star}{A}_{i-1}\right)= & Q_{f}\left(A_{0}, T_{\theta}\left(R_{\theta} \partial_{\omega} a_{i+1}^{\vee}+R \stackrel{\star}{A}_{i-1}\right)\right) \\
\square\left(R \widetilde{A}_{i-2}\right)= & Q_{f}\left(A_{0}, T\left(R \widetilde{A}_{i-2}\right)\right) \\
& +\operatorname{Moy} Q_{f}\left(\stackrel{\star}{A}_{1}, T_{\theta}\left(R_{\theta} \partial_{\omega} a_{i+1}^{\vee}+R \stackrel{\star}{A}_{i-1}\right)\right) \\
\left(R \partial_{\omega} a_{i+1}^{\vee}+R \stackrel{\star}{A}_{i-1}\right)_{\left.\right|_{t=0}}= & 0 \\
\left.R \widetilde{A}_{i-2}\right|_{t=0}= & 0
\end{aligned}\right.
$$

dont la seule solution est

$$
R_{\theta} \partial_{\omega} a_{i+1}^{\vee}+R \stackrel{\star}{A}_{i-1}=0 \quad \text { et } \quad R \widetilde{A}_{i-2}=0
$$

dès lors que $\left(A_{0}, \stackrel{\star}{A_{1}}\right)$ sont des données régulières.

Si l'hypothèse ii) est vérifiée pour l'indice $i=2$, nous supposons en plus que

$$
R_{\theta} \partial_{\omega} a_{i+2}^{\vee}+R \stackrel{\star}{A}_{i}=0 \quad \text { et } \quad R_{\theta} \partial_{\omega} a_{i+3}^{\vee}+R \stackrel{\star}{A}_{i+1}=0
$$

Nous en tirons

$$
\begin{aligned}
& L_{\theta} \partial_{\omega}^{2} \stackrel{\star}{A}_{i+2}+M \partial_{\omega} \stackrel{\star}{A}_{i}-\stackrel{\star}{\mathbf{F}}_{\mathbf{0}}(i-1)-\stackrel{\star}{\mathcal{F}}_{i-1} \\
& =-T_{\theta} \partial_{\omega}\left(R_{\theta} \partial_{\omega} a_{i+2}^{\vee}+R \stackrel{\star}{A} i\right)-T\left(R_{\theta} \partial_{\omega} a_{i}^{\vee}+R \stackrel{\star}{A}{ }_{i-2}\right)+\left(X \partial_{\omega} \stackrel{\star}{A_{i}}-{\stackrel{\star}{\mathbf{F}_{0}^{\prime}}}_{\mathbf{0}}(i-1)-\stackrel{\star}{\mathcal{F}}_{i-1}\right) \\
& =0
\end{aligned}
$$


et

$$
\begin{aligned}
& L_{\theta} \partial_{\omega}^{2} \stackrel{\star}{A}_{i+3}+M \partial_{\omega} \stackrel{\star}{A}{ }_{i+1}-\stackrel{\star}{\mathbf{F}}_{\mathbf{0}}(i)-\stackrel{\star}{\mathcal{F}}_{i} \\
& =-T_{\theta} \partial_{\omega}\left(R_{\theta} \partial_{\omega} a_{i+3}^{\vee}+R \stackrel{\star}{A_{i+1}}\right)-T\left(R_{\theta} \partial_{\omega} a_{i+1}^{\vee}+R \stackrel{\star}{A}_{i-1}\right)+\left(X \partial_{\omega} \stackrel{\star}{A}_{i+1}-\stackrel{\star}{\mathbf{F}}_{\mathbf{0}}(i)-\stackrel{\star}{\mathcal{F}}_{i}\right) \\
& =0
\end{aligned}
$$

En projetant ces équations sur $\Pi_{\wedge}, \Pi_{0}$ et $\Pi_{0}$, nous en déduisons que les polarisations $(P, i-1),(P, i)$, les équations $(E, i-1),(E, i)$ et les systèmes $(H, i-1),(H, i)$ sont vérifiés. La proposition 2.2 .8 a) garantit que $(C, i-1)$, puis $(C, i)$, le sont également. Ainsi, ii) $\Rightarrow$ i) pour l'indice $i$.

\subsection{Résolution des équations de profils}

2.3.1. Solutions approchées pour une jauge $J_{\lambda}$ arbitraire, $\lambda \in[0,1]$

Hypothèse 2.3.1. - Nous nommons «données de Cauchy oscillantes » compatibles à l'ordre $M$, de phase $\theta / \varepsilon$, tout choix de fonctions $\left(A_{\varepsilon}, \phi_{\varepsilon}, \psi_{\varepsilon}\right)$ et $\left(\partial_{t} A_{\varepsilon}, \partial_{t} \phi_{\varepsilon}\right)$ de la forme

$$
\left\{\begin{array}{l}
A_{\varepsilon}(0, y)=\sum_{i \geqslant 0} \varepsilon^{i / 2}\left(\widetilde{A} c_{i}(y)+\varepsilon^{1 / 2} \stackrel{\star}{A} c_{i+1}\left(y, \frac{\theta(0, y)}{\varepsilon}\right)\right) \\
\phi_{\varepsilon}(0, y)=\sum_{i \geqslant 0} \varepsilon^{i / 2}\left(\widetilde{\phi} c_{i}(y)+\varepsilon^{1 / 2} \stackrel{\star}{\phi} c_{i+1}\left(y, \frac{\theta(0, y)}{\varepsilon}\right)\right) \\
\psi_{\varepsilon}(0, y)=\sum_{i \geqslant 0} \varepsilon^{i / 2}\left(\widetilde{\psi} c_{i}(y)+{\stackrel{\psi}{\psi} c_{i}}^{\star}\left(y, \frac{\theta(0, y)}{\varepsilon}\right)\right)
\end{array}\right.
$$

et

$$
\begin{aligned}
& \nabla_{0}\left(A_{\varepsilon}\right)_{\mu}(0, y)=\sum_{i \geqslant 0}\left(\varepsilon^{-1 / 2} \nabla_{0} \theta \partial_{\omega}\left(\stackrel{\star}{A} c_{i+1}\right)_{\mu}\left(y, \frac{\theta(0, y)}{\varepsilon}\right)+\left(\widetilde{A} c_{i}^{\prime}\right)_{\mu}(y)\right. \\
& \begin{aligned}
& \left.+\varepsilon^{1 / 2} \nabla_{0}\left(\stackrel{\star}{A} c_{i+1}\right)_{\mu}\left(y, \frac{\theta(0, y)}{\varepsilon}\right)\right) \\
\nabla_{0} \phi_{\varepsilon}(0, y)=\sum_{i \geqslant 0}\left(\varepsilon^{-1 / 2} \nabla_{0} \theta \partial_{\omega} \stackrel{\star}{\phi} c_{i+1}\left(y, \frac{\theta(0, y)}{\varepsilon}\right)+\right. & \widetilde{\phi} c_{i}^{\prime}(y) \\
& \left.+\varepsilon^{1 / 2} \nabla_{0} \stackrel{\star}{\phi} c_{i+1}\left(y, \frac{\theta(0, y)}{\varepsilon}\right)\right)
\end{aligned}
\end{aligned}
$$

où les profils $\left(\widetilde{A} c_{i}, \widetilde{A} c_{i}^{\prime}, \widetilde{\phi} c_{i}, \widetilde{\phi} c_{i}^{\prime}, \widetilde{\psi} c_{i}\right)(y)$ et $\left(A \stackrel{\star}{A c_{i+1}^{0}}, \stackrel{\star}{\phi} c_{i+1}, \stackrel{\star}{*} c_{i}^{+}\right)(y, \omega)$ sont pris parmi les données de Cauchy compatibles des $(C, i)$ successifs, $0 \leqslant i<M$, telles que décrites section 2.2.3, $A^{\star} c_{1}^{\vee}=A^{\star} c_{2}^{\vee}=0, \psi c_{0}^{-}=\psi c_{1}^{-}=0$ puis les composantes $\left(A c_{i+3}^{\star}, \psi c_{i+2}^{-}\right)(y, \omega)$ satisfont les polarisations $(P, i)$ à $t=0$, pour tout $0 \leqslant i<M$, tandis que les valeurs $\nabla_{0} \stackrel{\star}{A} c_{i+1}$ et $\nabla_{0} \stackrel{\star}{\phi} c_{i+1}$ se déduisent des équations de chaque $(C, i), 0 \leqslant i<M$.

Il est maintenant possible d'énoncer le principal résultat de cette section : 
THÉORÈme 2 (Existence de solutions approchées). - Soit $\theta$ une fonction de phase satisfaisant les hypothèses 2.1 .1 sur un intervalle $\left[0, t_{0}\right]$, un réel $s \geqslant \frac{n}{2}+\frac{1}{2}$, et un entier $M \geqslant 1$ arbitraire. Toutes données de Cauchy oscillantes, compatibles à l'ordre $M$, de phase $\theta / \varepsilon$, traduites dans une jauge $J_{\lambda}, \lambda \in[0,1]$, et vérifiant

(59) $\left|\left(\widetilde{A} c_{i}, \widetilde{A} c_{i}^{\prime}\right)\right|_{s(i)}+\left|\widetilde{\phi} c_{i}\right|_{H^{s(i)}}+\left|\widetilde{\phi} c_{i}^{\prime}\right|_{H^{s(i)-1}}+\left|\widetilde{\psi} c_{i}\right|_{H^{s(i)-\frac{1}{2}}}$

$+\left|A c_{i+1}^{\star}\right|_{C^{s}\left([0,2 \pi],\left(H^{s(i)}\right)\right)}+\left|\phi c_{i+1}^{\star}\right|_{C^{s}\left([0,2 \pi],\left(H^{s(i)}\right)\right)}+\left|\psi^{\star} c_{i}\right|_{C^{s}\left([0,2 \pi],\left(H^{s(i)-\frac{1}{2}}\right)\right)} \leqslant \delta_{i} / 2$, avec $s(i)=s+M-1-i$ pour $0 \leqslant i<M(s(i)=s$ pour tout autre indice) se prolongent sur un intervalle non-nul $\left[0, t_{1}\right], 0<t_{1} \leqslant t_{0}$, en une solution approchée $e^{(4)}$

$$
\left\{\begin{array}{l}
A_{\varepsilon}(x)=\sum_{i \geqslant 0} \varepsilon^{i / 2}\left(\widetilde{A}_{i}(x)+\varepsilon^{1 / 2} \stackrel{\star}{A}_{i+1}\left(x, \frac{\theta(x)}{\varepsilon}\right)\right) \\
\phi_{\varepsilon}(x)=\sum_{i \geqslant 0} \varepsilon^{i / 2}\left(\widetilde{\phi}_{i}(x)+\varepsilon^{1 / 2} \stackrel{\star}{\phi}_{i+1}\left(x, \frac{\theta(x)}{\varepsilon}\right)\right) \\
\psi_{\varepsilon}(x)=\sum_{i \geqslant 0} \varepsilon^{i / 2}\left(\widetilde{\psi}_{i}(x)+\stackrel{\star}{\psi}_{i}\left(x, \frac{\theta(x)}{\varepsilon}\right)\right)
\end{array}\right.
$$

vérifiant le système $(Y M)$ à l'ordre $O\left(\varepsilon^{M / 2}\right)$ en partie moyenne, à l'ordre $O\left(\varepsilon^{(M-1) / 2}\right)$ en partie oscillante sur les $\left(A_{i}, \phi_{i}\right)$, à l'ordre $O\left(\varepsilon^{M / 2}\right)$ en partie oscillante sur les $\left(\psi_{i}\right)$, et vérifiant la jauge $J_{\lambda}$ à l'ordre $O\left(\varepsilon^{M / 2}\right)$ en partie moyenne et à l'ordre $O\left(\varepsilon^{(M+1) / 2}\right)$ en partie oscillante :

$$
\left\{\begin{array}{l}
\mathcal{O}_{\varepsilon}=L A_{\varepsilon}-F\left(A_{\varepsilon}, \phi_{\varepsilon}, \psi_{\varepsilon}\right)=\varepsilon^{M / 2} \sum_{i \geqslant 0} \varepsilon^{i / 2}\left(\varepsilon^{-1 / 2} \stackrel{\star}{\mathcal{O}}_{i-1}\left(x, \frac{\theta(x)}{\varepsilon}\right)+\widetilde{\mathcal{O}}_{i}(x)\right) \\
\mathcal{P}_{\varepsilon}=\square \phi_{\varepsilon}-G\left(A_{\varepsilon}, \phi_{\varepsilon}, \psi_{\varepsilon}\right)=\varepsilon^{M / 2} \sum_{0 \leqslant i \leqslant M^{\prime}} \varepsilon^{i / 2}\left(\varepsilon^{-1 / 2} \stackrel{\star}{\mathcal{P}}_{i-1}\left(x, \frac{\theta(x)}{\varepsilon}\right)+\widetilde{\mathcal{P}}_{i}(x)\right) \\
\mathcal{Q}_{\varepsilon}=\mathcal{D} \psi_{\varepsilon}-h\left(A_{\varepsilon}, \phi_{\varepsilon}, \psi_{\varepsilon}\right)=\varepsilon^{M / 2} \sum_{0 \leqslant i \leqslant M^{\prime}} \varepsilon^{i / 2}\left(\widetilde{\mathcal{Q}}_{i}(x)+\stackrel{\mathcal{Q}}{*}_{i}\left(x, \frac{\theta(x)}{\varepsilon}\right)\right)
\end{array}\right.
$$

et

$$
\mathcal{J}_{\varepsilon}=J_{\lambda} A_{\varepsilon}=J_{\lambda} \sum_{M \leqslant i} \varepsilon^{i / 2}\left(\widetilde{A}_{i}(x)+\varepsilon^{3 / 2} \stackrel{\star}{A}_{i+3}\left(x, \frac{\theta(x)}{\varepsilon}\right)\right)
$$

De plus, si $\delta_{0}$ est suffisamment petit, alors $t_{1}=t_{0}$. De plus, si $\delta_{i} \leqslant \delta_{M}, \forall i<M$, et si $\delta_{M}$ est assez petit, alors la solution approchée construite reste petite :

$$
\begin{aligned}
& \left|\widetilde{A}_{i}\right|_{L^{\infty}\left(\left[0, t_{1}\right], H^{s(i)}\right)}+\left|\nabla_{0} \widetilde{A}_{i}\right|_{L^{\infty}\left(\left[0, t_{1}\right], H^{s(i)-1}\right)} \\
& +\left|\widetilde{\phi}_{i}\right|_{L^{\infty}\left(\left[0, t_{1}\right], H^{s(i)}\right)}+\left|\nabla_{0} \widetilde{\phi}_{i}\right|_{L^{\infty}\left(\left[0, t_{1}\right], H^{s(i)-1}\right)}^{\star} \\
& +\left|\stackrel{\star}{A}_{i+1}\right|_{L^{\infty}\left(\left[0, t_{1}\right], C^{s}\left([0,2 \pi],\left(H^{s(i)}\right)\right)\right)}+\left|\stackrel{\star}{\phi}_{i+1}\right|_{L^{\infty}\left(\left[0, t_{1}\right], C^{s}\left([0,2 \pi],\left(H^{s(i)}\right)\right)\right)} \\
& +\left|\widetilde{\psi}_{i}\right|_{L^{\infty}\left(\left[0, t_{1}\right], H^{\left.s(i)-\frac{1}{2}\right)}\right.}+\mid{\stackrel{\phi}{\phi_{i+1}}}_{L^{\infty}\left(\left[0, t_{1}\right], C^{s}\left([0,2 \pi],\left(H^{s(i)-\frac{1}{2}}\right)\right)\right)} \leqslant \delta_{M}
\end{aligned}
$$

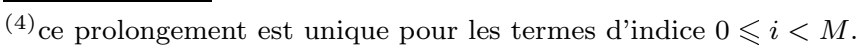


avec $s(i)=s+M-1-i$ pour tout $0 \leqslant i<M(s(i)=s$ pour tout autre indice), et

$$
\left|(\mathcal{O}, P, Q)_{i}\right|_{L^{\infty}\left(\left[0, t_{1}\right], C^{s-1}\left([0,2 \pi],\left(H^{s-1}\right)\right)\right)} \leqslant C \delta_{M}, \forall i, i \geqslant-1
$$

Avant de prouver le théorème 2, nous énonçons deux propositions qui contiennent les résultats d'existence dont nous avons besoin pour résoudre les équations de profils successives.

Proposition 2.3.1 (Résolution du système hyperbolique semi-linéaire)

$(H, 0)$. Si $\theta$ vérifie les hypothèses 2.1 .1 sur l'intervalle $\left[0, t_{0}\right]$ et $s \geqslant \frac{n}{2}+\frac{1}{2}$, alors il existe un temps $0<t_{1} \leqslant t_{0}$ et une unique solution

$$
\begin{aligned}
& A_{0} \in C^{0}\left(\left[0, t_{1}\right], H^{s}(\Sigma)\right) \cap C^{1}\left(\left[0, t_{1}\right], H^{s-1}(\Sigma)\right) \\
& \phi_{0} \in C^{0}\left(\left[0, t_{1}\right], H^{s}(\Sigma)\right) \cap C^{1}\left(\left[0, t_{1}\right], H^{s-1}(\Sigma)\right) \\
& \widetilde{\psi}_{0} \in C^{0}\left(\left[0, t_{1}\right], H^{s-1 / 2}(\Sigma)\right) \\
& a_{1}^{0} \in C^{1}\left(\left[0, t_{1}\right], C^{s}\left([0,2 \pi], H^{s-1}(\Sigma)\right)\right) \\
& \stackrel{\star}{\phi}_{1} \in C^{0}\left(\left[0, t_{1}\right], C^{s}\left([0,2 \pi], H^{s-1}(\Sigma)\right)\right)
\end{aligned}
$$

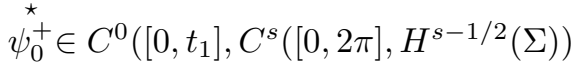

du système $(H, 0)$, coïncidant à $t=0$ avec les données de Cauchy

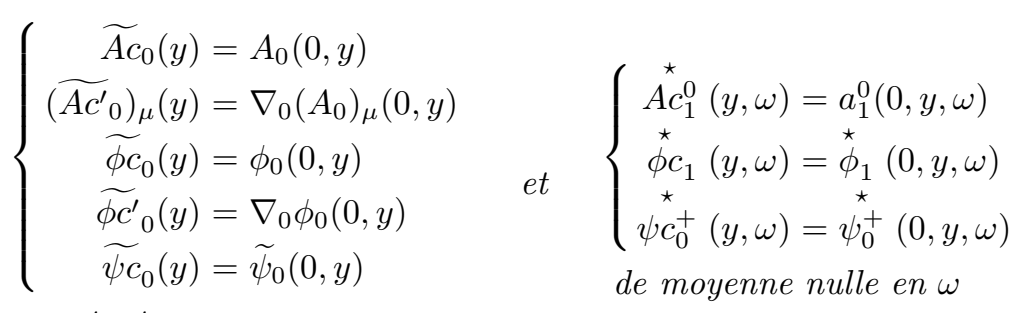

constantes en $\omega$,

vérifiant

$$
\begin{aligned}
& \left|A_{c 0}\right|_{H^{s}(\Sigma)}+\left|A_{c 0}^{\prime}\right|_{H^{s-1}(\Sigma)}+\left|\phi_{c_{0}}\right|_{H^{s}(\Sigma)}+\left|\phi_{c 0}^{\prime}\right|_{H^{s-1}(\Sigma)}+\left|\widetilde{\psi} c_{0}\right|_{\left.H^{s-1 / 2}(\Sigma)\right)} \\
& +\left|\stackrel{\star}{\star} A_{1}^{0}\right|_{C^{s}\left([0,2 \pi], H^{s}(\Sigma)\right)}+\left|\stackrel{\star}{\phi_{1}}\right|_{C^{s}\left([0,2 \pi], H^{s}(\Sigma)\right)}+\left|\psi_{0}^{+}\right|_{C^{s}\left([0,2 \pi], H^{s-1 / 2}(\Sigma)\right)} \leqslant \delta_{0} / 2
\end{aligned}
$$

De plus, si $\delta_{0}$ est assez petit, alors $t_{1}=t_{0}$ et

$$
\begin{aligned}
& \left|A_{0}\right|_{L^{\infty}\left(\left[0, t_{0}\right], H^{s}\right)}+\left|\nabla_{0} A_{0}\right|_{L^{\infty}\left(\left[0, t_{0}\right], H^{s-1}\right)}+\left|\phi_{0}\right|_{L^{\infty}\left(\left[0, t_{0}\right], H^{s}\right)}+\left|a_{1}^{0}\right|_{L^{\infty}\left(\left[0, t_{0}\right], C^{s}\left([0,2 \pi], H^{s}\right)\right)} \\
& +\left|\nabla_{0} \phi_{0}\right|_{L^{\infty}\left(\left[0, t_{0}\right], H^{s-1}\right)}+\mid \stackrel{\phi_{1}}{\phi_{L^{\infty}\left(\left[0, t_{0}\right], C^{s}\left([0,2 \pi], H^{s}\right)\right)}} \\
& +\left|\psi_{0}\right|_{L^{\infty}\left(\left[0, t_{0}\right], H^{s-1 / 2}\right)}+\left|\psi_{0}^{+}\right|_{L^{\infty}\left(\left[0, t_{0}\right], C^{s}\left([0,2 \pi], H^{s-1 / 2}\right)\right)} \leqslant \delta_{0}
\end{aligned}
$$


Démonstration de la proposition 2.3.1. - Nous devons résoudre le problème de Cauchy semi-linéaire suivant,

$$
\left\{\begin{aligned}
(\square-\mathcal{R} i c .) A & =\widetilde{\boldsymbol{F}_{0}}\left(A, \partial A, a, \alpha, \Phi, \partial \Phi, \phi, \varphi, \Psi, \mathcal{D}_{\theta} \psi\right) \\
\square \Phi & =\widetilde{\boldsymbol{G}_{0}}\left(A, \partial A, a, \alpha, \Phi, \partial \Phi, \phi, \varphi, \Psi, \mathcal{D}_{\theta} \psi\right) \\
\mathcal{D} \Psi & =\widetilde{\mathbf{h}_{0}}\left(A, \Phi, \Psi, \mathcal{D}_{\theta} \psi\right) \\
X_{\left.\right|_{\Pi_{0}}} a & =\pi^{0} \stackrel{\star}{\boldsymbol{F}_{0}}(A, a, \Phi, \phi) \\
X_{\left.\right|_{\Pi_{0}}} \alpha & =\pi^{0} \stackrel{\star}{*}_{0}(A, \alpha, \Phi, \varphi) \\
X \phi & =\stackrel{\boldsymbol{G}}{0}_{0}(A, a, \Phi, \phi) \\
X \varphi & =\stackrel{\star}{\boldsymbol{G}_{0}}(A, \alpha, \Phi, \varphi) \\
X \psi & =\pi^{-} \stackrel{\mathbf{h}}{\mathbf{0}}^{\star}\left(A, \Phi, \Psi, \mathcal{D}_{\theta} \psi\right)
\end{aligned}\right.
$$

avec pour données à $t=0$ :

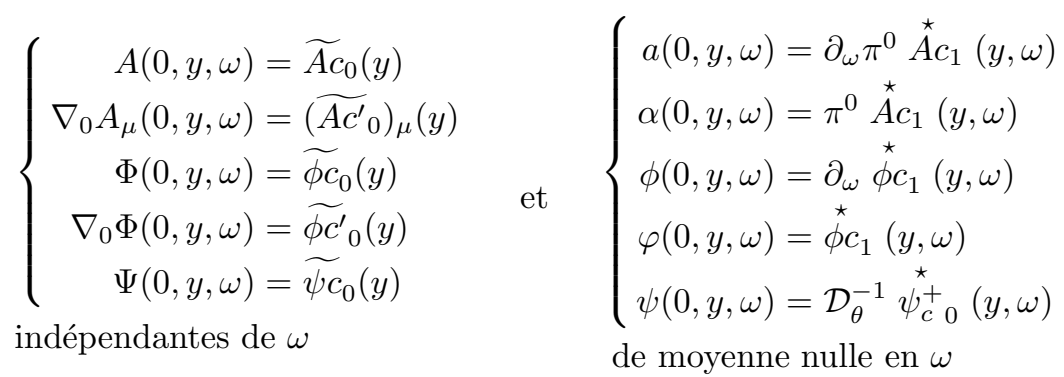

Pour tout intervalle $[0, t] \subset\left[0, t_{0}\right]$ sur lequel les hypothèses 1 sur la phase $\theta$ restent valides, nous disposons de l'estimation a priori suivante :

$$
\begin{aligned}
& \sup _{\varsigma \in[0, t]} e^{-\lambda \varsigma}\left(|A|_{H^{s}}+\left|\nabla_{0} A\right|_{H^{s-1}}+|a|_{C^{s-1}\left(H^{s}\right)}+|\alpha|_{C^{s}\left([0,2 \pi], H^{s}\right)}\right. \\
& +|\Phi|_{H^{s}}+\left|\nabla_{0} \Phi\right|_{H^{s-1}}+|\phi|_{C^{s-1}\left([0,2 \pi], H^{s}\right)}+|\varphi|_{C^{s}\left([0,2 \pi], H^{s}\right)} \\
& \left.+|\Psi|_{H^{s-1 / 2}}+|\psi|_{C^{s}\left([0,2 \pi], H^{s-1 / 2}\right)}\right) \\
& \leqslant\left|\widetilde{A_{c 0}}\right|_{H^{s}}+\left|\widetilde{A_{c 0}^{\prime}}\right|_{H^{s-1}}+\left|\widetilde{\phi_{c 0}}\right|_{H^{s}}+\left|\widetilde{\phi_{c 0}^{\prime}}\right|_{H^{s-1}}+\left|\widetilde{\psi_{c 0}}\right|_{H^{s-1 / 2}} \\
& +\left|A_{c 1}^{\star}\right|_{C^{s}\left([0,2 \pi], H^{s}\right)}+\left|{\stackrel{\star}{\phi_{c 1}}}^{\star}\right|_{C^{s}\left([0,2 \pi], H^{s}\right)}+\left|\psi_{c_{0}}^{\star}\right|_{C^{s}\left([0,2 \pi], H^{s-1 / 2}\right)} \\
& +\int_{0}^{t} e^{-\lambda \varsigma}\left(\left|\widetilde{\boldsymbol{F}}_{0}(A, \partial A, a, \alpha, \Phi, \partial \Phi, \phi, \varphi, \Psi, \psi)\right|_{H^{s-1}}\right. \\
& +\left|\widetilde{\boldsymbol{G}}_{0}(A, \partial A, a, \alpha, \Phi, \partial \Phi, \phi, \varphi, \Psi, \psi)\right|_{H^{s-1}}+\left|\widetilde{\boldsymbol{H}}_{0}(A, \Phi, \Psi)\right|_{H^{s-1 / 2}} \\
& +\left|\pi^{0} \stackrel{\star}{\boldsymbol{F}}_{0}(A, a, \Phi, \phi)\right|_{C^{s-1}\left([0,2 \pi], H^{s}\right)}+\left|\pi^{0} \stackrel{\star}{\boldsymbol{F}}_{0}(A, \alpha, \Phi, \varphi)\right|_{C^{s}\left([0,2 \pi], H^{s}\right)} \\
& +\left|\stackrel{\star}{\boldsymbol{G}}_{0}(A, a, \Phi, \phi)\right|_{C^{s-1}\left([0,2 \pi], H^{s}\right)}+\left|\stackrel{\star}{\boldsymbol{G}}_{0}(A, \alpha, \Phi, \varphi)\right|_{C^{s}\left([0,2 \pi], H^{s}\right)} \\
& \left.+\left|\pi^{-} \stackrel{\star}{\boldsymbol{H}}_{0}(A, \Phi, \Psi, \psi)\right|_{C^{s}\left([0,2 \pi], H^{s-1 / 2}\right)}\right) d \varsigma
\end{aligned}
$$


Les termes non linéaires sont polynomiaux et s'annulent au moins à l'ordre 2 en 0 . Il peuvent être correctement estimés dès que $s \geqslant \frac{n}{2}+\frac{1}{2}$ conformément aux propositions 1.3.1 et 2.1.1 :

$$
\begin{aligned}
\mid \widetilde{\boldsymbol{F}}_{0}(A, \partial A, a, \alpha, \Phi, & \partial \Phi, \phi, \varphi, \Psi, \psi)\left.\right|_{H^{s-1}} \\
\leqslant C_{s}\left(|A|_{H^{s}}\left|\partial_{t} A\right|_{H^{s-1}}+|\alpha|_{C^{s}\left([0,2 \pi], H^{s}\right)}|a|_{C^{s-1}\left([0,2 \pi], H^{s}\right)}\right. & \\
& +|\Phi|_{H^{s}}\left|\partial_{t} \Phi\right|_{H^{s-1}}+|\varphi|_{C^{s}\left([0,2 \pi], H^{s}\right)}|\phi|_{C^{s-1}\left([0,2 \pi], H^{s}\right)} \\
& \left.+|A|_{H^{s}}^{3}+|\Phi|_{H^{s}}^{2}|A|_{H^{s}}+|\Psi|_{H^{s-1 / 2}}^{2}+|\psi|_{C^{s}\left([0,2 \pi], H^{s-1 / 2}\right)}^{2}\right) \\
\mid \widetilde{\boldsymbol{G}}_{0}(A, \partial A, a, \alpha, \Phi, & \partial \Phi, \phi, \varphi, \Psi, \psi)\left.\right|_{H^{s-1}} \leqslant C_{s}\left(|A|_{H^{s}}\left|\partial_{t} \Phi\right|_{H^{s-1}}+|\Phi|_{H^{s}}\left|\partial_{t} A\right|_{H^{s-1}}\right. \\
& +|\alpha|_{C^{s}\left([0,2 \pi], H^{s}\right)}|\phi|_{C^{s-1}\left([0,2 \pi], H^{s}\right)}+|\varphi|_{C^{s}\left([0,2 \pi], H^{s}\right)}|a|_{C^{s-1}\left([0,2 \pi], H^{s}\right)} \\
& \left.+|\Phi|_{H^{s}}^{3}+|\Phi|_{H^{s}}|A|_{H^{s}}^{2}+|\Psi|_{H^{s-1 / 2}}^{2}+|\psi|_{C^{s}\left([0,2 \pi], H^{s-1 / 2}\right)}^{2}\right)
\end{aligned}
$$

et

$$
\begin{aligned}
& \left|\pi^{0} \stackrel{\star}{\boldsymbol{F}}_{0}(A, a, \Phi, \phi)\right|_{C^{s-1}\left([0,2 \pi], H^{s}\right)} \leqslant C_{s}\left(|A|_{H^{s}}|a|_{C^{s-1}\left([0,2 \pi], H^{s}\right)}+|\Phi|_{H^{s}}|\phi|_{C^{s-1}\left([0,2 \pi], H^{s}\right)}\right) \\
& \left|\pi^{0} \stackrel{\star}{\boldsymbol{F}}_{0}(A, \alpha, \Phi, \varphi)\right|_{C^{s}\left([0,2 \pi], H^{s}\right)} \leqslant C_{s}\left(|A|_{H^{s}}|\alpha|_{C^{s}\left([0,2 \pi], H^{s}\right)}+|\Phi|_{H^{s}}|\varphi|_{C^{s}\left([0,2 \pi], H^{s}\right)}\right) \\
& \left|\stackrel{\star}{\boldsymbol{G}}_{0}(A, a, \Phi, \phi)\right|_{C^{s-1}\left([0,2 \pi], H^{s}\right)} \leqslant C_{s}\left(|\Phi|_{H^{s}}|a|_{C^{s-1}\left([0,2 \pi], H^{s}\right)}+|A|_{H^{s}}|\phi|_{C^{s-1}\left([0,2 \pi], H^{s}\right)}\right) \\
& \left|\stackrel{\star}{G}_{0}(A, \alpha, \Phi, \varphi)\right|_{C^{s}\left([0,2 \pi], H^{s}\right)} \leqslant C_{s}\left(|\Phi|_{H^{s}}|\alpha|_{C^{s}\left([0,2 \pi], H^{s}\right)}+|A|_{H^{s}}|\varphi|_{C^{s}\left([0,2 \pi], H^{s}\right)}\right) \\
& \left|\pi^{-} \stackrel{\star}{\boldsymbol{H}}_{0}(A, \Phi, \Psi, \psi)\right|_{C^{s}\left([0,2 \pi], H^{s-1 / 2}\right)} \leqslant C_{s}|\psi|_{C^{s}\left([0,2 \pi], H^{s-1 / 2}\right)}\left(|A|_{H^{s}}+|\Phi|_{H^{s}}\right)
\end{aligned}
$$

La résolution du problème semi-linéaire se fait donc par une méthode de point fixe traditionnelle, conduisant à l'existence d'une unique solution

$$
\begin{aligned}
(A, \Phi) & \in C^{0}\left(\left[0, t_{1}\right], H^{s}(\Sigma)\right) \cap C^{1}\left(\left[0, t_{1}\right], H^{s-1}(\Sigma)\right) \\
\Psi & \in C^{0}\left(\left[0, t_{1}\right], H^{s-1 / 2}(\Sigma)\right) \\
(\alpha, \varphi) & \in C^{0}\left(\left[0, t_{1}\right], C^{s}\left([0,2 \pi], H^{s}(\Sigma)\right)\right) \\
(a, \phi) & \in C^{0}\left(\left[0, t_{1}\right], C^{s-1}\left([0,2 \pi], H^{s-1}(\Sigma)\right)\right) \\
\text { et } \quad \psi & \in C^{0}\left(\left[0, t_{1}\right], C^{s}\left([0,2 \pi], H^{s-1 / 2}(\Sigma)\right)\right)
\end{aligned}
$$

sur un intervalle de temps $\left[0, t_{1}\right]$ qui ne dépend que de la taille des données initiales dans $H^{s}$ et $H^{s-1}$ respectivement. En particulier, on vérifie dans le point fixe que si

$$
\begin{aligned}
& \left|A_{c_{0}}\right|_{H^{s}(\Sigma)}+\left|A_{c 0}^{\prime}\right|_{H^{s-1}(\Sigma)}+\left|\phi_{c_{0}}\right|_{H^{s}(\Sigma)}+\left|\phi_{c 0}^{\prime}\right|_{H^{s-1}(\Sigma)}+\left|\widetilde{\psi} c_{0}\right|_{\left.H^{s-1 / 2}(\Sigma)\right)} \\
& \quad+\left|\stackrel{\star}{A_{1}^{0}}\right|_{C^{s}\left([0,2 \pi], H^{s}(\Sigma)\right)}+\left|\stackrel{\star}{\phi}_{1}\right|_{C^{s}\left([0,2 \pi], H^{s}(\Sigma)\right)}+\left|\stackrel{\psi_{0}^{+}}{{ }^{+}}\right|_{C^{s}\left([0,2 \pi], H^{s-1 / 2}(\Sigma)\right)} \leqslant \delta_{0} / 2
\end{aligned}
$$


pour un certain $\delta_{0}$ assez petit, alors $t_{1}=t_{0}$ et

$$
\begin{aligned}
& \sup _{\varsigma \in[0, t]} e^{-\lambda \varsigma}\left(|A|_{H^{s}}+\left|\nabla_{0} A\right|_{H^{s-1}}+|a|_{C^{s-1}\left(H^{s}\right)}+|\alpha|_{C^{s}\left([0,2 \pi], H^{s}\right)}\right. \\
&+|\Phi|_{H^{s}}+\left|\nabla_{0} \Phi\right|_{H^{s-1}}+|\phi|_{C^{s-1}\left([0,2 \pi], H^{s}\right)}+|\varphi|_{C^{s}\left([0,2 \pi], H^{s}\right)} \\
&\left.+|\Psi|_{H^{s-1 / 2}}+|\psi|_{C^{s}\left([0,2 \pi], H^{s-1 / 2}\right)}\right) \leqslant \delta_{0}
\end{aligned}
$$

Posons enfin $a^{\prime \prime}=a-\partial_{\omega} \alpha$ et $\phi^{\prime \prime}=\phi-\partial_{\omega} \varphi$, qui vérifient les équations

$$
\left\{\begin{aligned}
X_{\left.\right|_{0}} a^{\prime \prime} & =\pi^{0}\left(\stackrel{\star}{\boldsymbol{F}}_{0}(A, a, \Phi, \phi)-\partial_{\omega}{\stackrel{\star}{\boldsymbol{F}_{0}}}_{0}(A, \alpha, \Phi, \phi)\right)=0 \\
X \phi^{\prime \prime} & =\stackrel{\star}{\boldsymbol{G}_{0}}(A, a, \Phi, \phi)-\partial_{\omega} \stackrel{\star}{\boldsymbol{G}}_{0}(A, \alpha, \Phi, \phi)=0
\end{aligned}\right.
$$

avec des données initiales nulles. L'unique solution de ce problème est bien sûr $a^{\prime \prime}=0$ et $\phi^{\prime \prime}=0$ sur tout $\left[0, t_{1}\right]$. De même, il est facile de vérifier que $\psi_{0}^{+}=\mathcal{D}_{\theta} \psi \in \Pi^{+}$est l'unique solution de l'équation

$$
\mathcal{D} \stackrel{\star}{*}_{0}^{+}=\mathcal{D}_{\theta}{\stackrel{\mathbf{h}_{0}}{\star}}^{*}
$$

avec $\psi_{0}^{\star}(0, y, \omega)={\stackrel{\psi_{c}^{+}}{+}}_{0}(y, \omega)$, en remarquant simplement que $\mathcal{D}_{\theta} \pi^{-}=\mathcal{D}_{\theta}$ et que

$$
X \mathcal{D}_{\theta}=\mathcal{D}_{\theta} X=\mathcal{D}_{\theta} \mathcal{D} \mathcal{D}_{\theta}
$$

Nous pouvons légitimement poser

$$
\begin{array}{rlrlrl}
A_{0}(x) & =A(x) & & a_{1}^{0}(x, \omega) & =\alpha(x, \omega) \\
\phi_{0}(x) & =\Phi(x) & \text { et } & \star^{\star}(x, \omega) & =\varphi(x, \omega) \\
\widetilde{\phi}_{0} & =\Psi(x) & & { }^{\star} & \psi_{0}^{+}(x, \omega) & =\mathcal{D}_{\theta} \psi(x, \omega)
\end{array}
$$

ce qui achève la preuve de la proposition 2.3.1.

Proposition 2.3.2 (Résolution des systèmes hyperboliques linéaires $(H, i)$, pour tout indice $i \geqslant 1$ )

Si $\theta$ vérifie les hypothèses 2.1 .1 sur l'intervalle $0 \leqslant t \leqslant t_{1} \leqslant t_{0}$, si $s \geqslant \frac{n}{2}+\frac{1}{2}$, et si l'on dispose de données

$$
\begin{aligned}
\widetilde{A}_{k} & \in C^{0}\left(\left[0, t_{1}\right], H^{s+i-k}(\Sigma)\right) \cap C^{1}\left(\left[0, t_{1}\right], H^{s-1+i-k}(\Sigma)\right) \\
\widetilde{\phi}_{k} & \in C^{0}\left(\left[0, t_{1}\right], H^{s+i-k}(\Sigma)\right) \cap C^{1}\left(\left[0, t_{1}\right], H^{s-1+i-k}(\Sigma)\right) \\
\widetilde{\psi}_{k} & \in C^{0}\left(\left[0, t_{1}\right], H^{s-\frac{1}{2}+i-k}(\Sigma)\right) \\
\stackrel{\star}{A}_{k+1} & \in C^{0}\left(\left[0, t_{1}\right], C^{s}\left([0,2 \pi], H^{s+i-k}(\Sigma)\right)\right) \\
\stackrel{\star}{\phi}_{k+1} & \in C^{0}\left(\left[0, t_{1}\right], C^{s}\left([0,2 \pi], H^{s+i-k}(\Sigma)\right)\right) \\
\star_{k} & \in C^{0}\left(\left[0, t_{1}\right], C^{s}\left([0,2 \pi], H^{s-\frac{1}{2}+i-k}(\Sigma)\right)\right)
\end{aligned}
$$


vérifiant

$$
\begin{aligned}
& \left|\widetilde{A}_{k}\right|_{L^{\infty}\left(\left[0, t_{1}\right], H^{s+i-k}\right)}+\left|\nabla_{0} \widetilde{A}_{k}\right|_{L^{\infty}\left(\left[0, t_{1}\right], H^{s-1+i-k}\right)} \\
& +\left|\widetilde{\phi}_{k}\right|_{L^{\infty}\left(\left[0, t_{1}\right], H^{s+i-k}\right)}+\left|\nabla_{0} \widetilde{\phi}_{k}\right|_{L^{\infty}\left(\left[0, t_{1}\right], H^{s-1+i-k}\right)} \\
& +\left|\stackrel{\star}{A}_{k+1}\right|_{L^{\infty}\left(\left[0, t_{1}\right], C^{s}\left([0,2 \pi],\left(H^{s+i-k}\right)\right)\right)}+\left|\stackrel{\star}{\phi}_{k+1}\right|_{L^{\infty}\left(\left[0, t_{1}\right], C^{s}\left([0,2 \pi],\left(H^{s+i-k}\right)\right)\right)} \\
& +\left|\widetilde{\psi}_{k}\right|_{L^{\infty}\left(\left[0, t_{1}\right], H^{s-\frac{1}{2}+i-k}\right)}+\left|\star_{k}\right|_{L^{\infty}\left(\left[0, t_{1}\right], C^{s}\left([0,2 \pi],\left(H^{s-\frac{1}{2}+i-k}\right)\right)\right)} \leqslant \delta_{k}
\end{aligned}
$$

pour tout indice $0 \leqslant k<i$, ainsi que

$$
\begin{aligned}
a_{i+1}^{\vee} & \in C^{0}\left(\left[0, t_{1}\right], C^{s+1}\left([0,2 \pi], H^{s}(\Sigma)\right)\right) \\
\star_{\star}^{-} & \in C^{0}\left(\left[0, t_{1}\right], C^{s+1}\left([0,2 \pi], H^{s+\frac{1}{2}}(\Sigma)\right)\right)
\end{aligned}
$$

vérifiant

$$
\left|a_{i+1}^{\vee}\right|_{L^{\infty}\left(\left[0, t_{1}\right], C^{s+1}\left([0,2 \pi],\left(H^{s}\right)\right)\right)}+\left|\psi_{i}^{\star}\right|_{L^{\infty}\left(\left[0, t_{1}\right], C^{s+1}\left([0,2 \pi],\left(H^{s-\frac{1}{2}+i-k}\right)\right)\right)} \leqslant \delta_{i}
$$

alors il existe une unique solution

$$
\begin{aligned}
\widetilde{A}_{i} & \in C^{0}\left(\left[0, t_{1}\right], H^{s}(\Sigma)\right) \cap C^{1}\left(\left[0, t_{1}\right], H^{s-1}(\Sigma)\right) \\
\widetilde{\phi}_{i} & \in C^{0}\left(\left[0, t_{1}\right], H^{s}(\Sigma)\right) \cap C^{1}\left(\left[0, t_{1}\right], H^{s-1}(\Sigma)\right) \\
\widetilde{\psi}_{i} & \in C^{0}\left(\left[0, t_{1}\right], H^{s-\frac{1}{2}}(\Sigma)\right) \\
a_{i+1}^{0} & \in C^{0}\left(\left[0, t_{1}\right], C^{s}\left([0,2 \pi], H^{s}(\Sigma)\right)\right) \\
{\stackrel{\star}{\phi_{i+1}}}_{\star}^{\star} & \in C^{0}\left(\left[0, t_{1}\right], C^{s}\left([0,2 \pi], H^{s}(\Sigma)\right)\right) \\
\psi_{i}^{+} & \in C^{0}\left(\left[0, t_{1}\right], C^{s}\left([0,2 \pi], H^{s-\frac{1}{2}}(\Sigma)\right)\right)
\end{aligned}
$$

du système $(H, i)$ coïncidant à $t=0$ avec les données de Cauchy

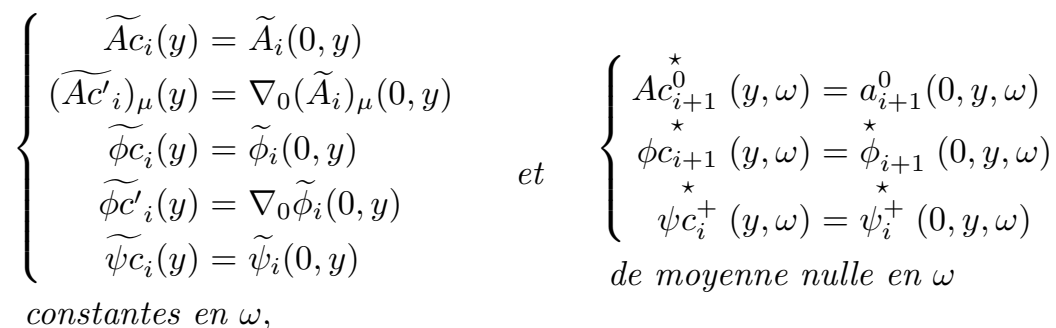

vérifiant

(61) $\left|\widetilde{A_{c i}}\right|_{H^{s}}+\left|\widetilde{A_{c i}^{\prime}}\right|_{H^{s-1}}+\left|\widetilde{\phi_{c i}}\right|_{H^{s}}+\left|\widetilde{\phi_{c i}}\right|_{H^{s-1}}+\left|\widetilde{\psi_{c i}}\right|_{H^{s-1 / 2}}$

$$
+\left|A c_{i+1}^{\star}\right|_{C^{s}\left([0,2 \pi],\left(H^{s}\right)\right)}+\left|\phi c_{i+1}^{\star}\right|_{C^{s}\left([0,2 \pi],\left(H^{s}\right)\right)}+\left|\psi{ }^{\star} c_{i}^{+}\right|_{C^{s}\left([0,2 \pi],\left(H^{s-1 / 2}\right)\right)} \leqslant \delta_{i}
$$


De plus, si $\delta_{k} \leqslant \delta / 2$ pour tout $0 \leqslant k \leqslant i$, avec $\delta$ assez petit, alors

(62) $\left|\widetilde{A}_{i}\right|_{L^{\infty}\left(\left[0, t_{1}\right], H^{s}\right)}+\left|\nabla_{0} \widetilde{A}_{i}\right|_{L^{\infty}\left(\left[0, t_{1}\right], H^{s-1}\right)}+\left|\widetilde{\phi}_{i}\right|_{L^{\infty}\left(\left[0, t_{1}\right], H^{s}\right)}$

$$
\begin{aligned}
+\left|\nabla_{0} \widetilde{\phi}_{i}\right|_{L^{\infty}\left(\left[0, t_{1}\right], H^{s-1}\right)} & +\left|a_{i+1}^{0}\right|_{L^{\infty}\left(\left[0, t_{1}\right], C^{s}\left([0,2 \pi],\left(H^{s}\right)\right)\right)}+\left|{\stackrel{\star}{\phi_{i+1}}}\right|_{L^{\infty}\left(\left[0, t_{1}\right], C^{s}\left([0,2 \pi],\left(H^{s}\right)\right)\right)} \\
& +\left|\widetilde{\psi}_{i}\right|_{L^{\infty}\left(\left[0, t_{1}\right], H^{s-1 / 2}\right)}+\left|\hat{\psi}_{i}^{0}\right|_{L^{\infty}\left(\left[0, t_{1}\right], C^{s}\left([0,2 \pi],\left(H^{s-1 / 2}\right)\right)\right)} \leqslant \delta
\end{aligned}
$$

Démonstration de la proposition 2.3.2. - Nous devons résoudre le problème de Cauchy linéaire suivant :

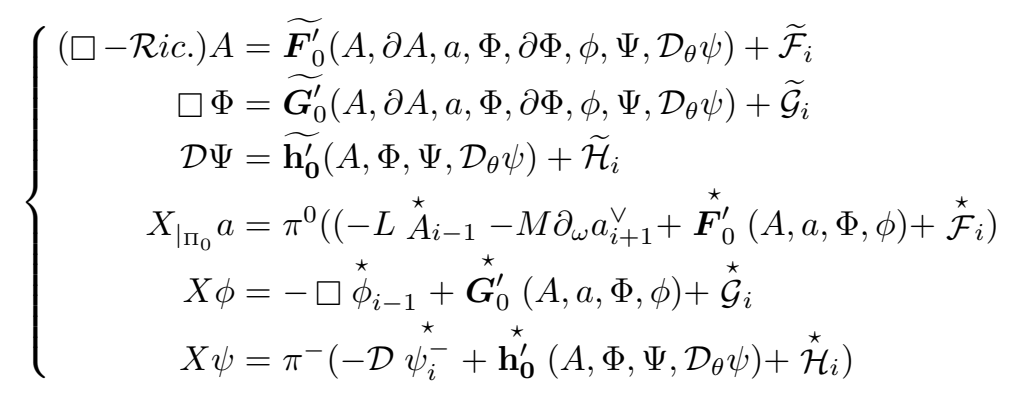

avec pour données à $t=0$ :

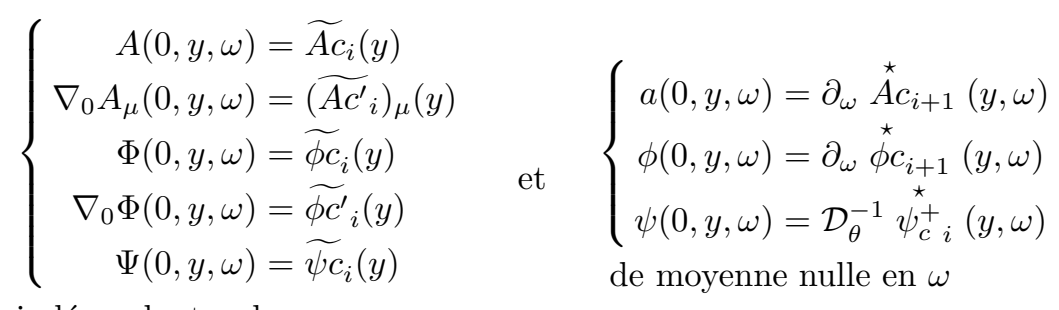

indépendantes de $\omega$

En vertu des propositions 1.3.1 et 2.1.1, les termes linéaires peuvent-être convenablement estimés si les profils dominants d'indice $k=0$ restent eux-mêmes réguliers :

$$
\begin{aligned}
& \left(\left|{\widetilde{\boldsymbol{F}^{\prime}}}_{0}\left(A, \partial A, a, \Phi, \partial \Phi, \phi, \Psi, \mathcal{D}_{\theta} \psi\right)\right|_{H^{s-1}}+\left|{\widetilde{\boldsymbol{G}^{\prime}}}_{0}\left(A, \partial A, a, \Phi, \partial \Phi, \phi, \Psi, \mathcal{D}_{\theta} \psi\right)\right|_{H^{s-1}}\right. \\
& +\left|\widetilde{\boldsymbol{H}}_{0}^{\prime}\left(A, \Phi, \Psi, \mathcal{D}_{\theta} \psi\right)\right|_{H^{s-1 / 2}}+\left|\pi^{0}{\stackrel{\star}{\boldsymbol{F}^{\prime}}}_{0}(A, a, \Phi, \phi)\right|_{C^{s-1}\left(H^{s}\right)} \\
& \left.+\left|{\stackrel{\star}{\boldsymbol{G}^{\prime}}}_{0}(A, a, \Phi, \phi)\right|_{C^{s-1}\left(H^{s}\right)}+\left|\pi^{-}{\stackrel{\star}{\boldsymbol{H}^{\prime}}}_{0}\left(A, \Phi, \Psi, \mathcal{D}_{\theta} \psi\right)\right|_{C^{s}\left(H^{s-1 / 2}\right)}\right) \\
& \leqslant C_{s} \delta_{0}\left(1+\delta_{0}\right)\left(|A|_{H^{s}}+\left|\partial_{t} A\right|_{H^{s-1}}+|a|_{C^{s-1}\left(H^{s}\right)}+|\Phi|_{H^{s}}+\left|\partial_{t} \Phi\right|_{H^{s-1}}\right. \\
& \left.+|\phi|_{C^{s-1}\left(H^{s}\right)}+|\Psi|_{H^{s-1 / 2}}+|\psi|_{C^{s}\left(H^{s-1 / 2}\right)}\right)
\end{aligned}
$$


Pour tout intervalle $[0, t] \subset\left[0, t_{1}\right]$ sur lequel les hypothèses 2.1 .1 sur la phase $\theta$ restent valides, nous disposons de l'estimation a priori suivante :

$$
\begin{aligned}
& \sup _{\varsigma \in[0, t]} e^{-\lambda\left(\delta_{0}\right) \varsigma}\left(|A|_{H^{s}}+\left|\nabla_{0} A\right|_{H^{s-1}}+|a|_{C^{s-1}\left(H^{s}\right)}+|\Phi|_{H^{s}}\right. \\
& \left.+\left|\nabla_{0} \Phi\right|_{H^{s-1}}+|\phi|_{C^{s-1}\left(H^{s}\right)}+|\Psi|_{H^{s-1 / 2}}+|\psi|_{C^{s}\left(H^{s-1 / 2}\right)}\right) \\
& \leqslant\left|\widetilde{A_{c i}}\right|_{H^{s}}+\left|\widetilde{A_{c i}^{\prime}}\right|_{H^{s-1}}+\left.\left|\pi^{0} \stackrel{A}{c i+1}_{C^{s}\left(H^{s}\right)}+\right| \widetilde{\phi_{c i}}\right|_{H^{s}}+\left|\widetilde{\phi_{c i}^{\prime}}\right|_{H^{s-1}} \\
& +\left|\hat{\phi}_{c_{i+1}}\right|_{C^{s}\left(H^{s}\right)}+\left|\psi_{c_{i}}\right|_{C^{s}\left(H^{s-1 / 2}\right)} \\
& +\int_{0}^{t} e^{-\lambda\left(\delta_{0}\right) \varsigma}\left(\left|\widetilde{\mathcal{F}}_{i}\right|_{H^{s-1}}+\left|\widetilde{\mathcal{G}}_{i}\right|_{H^{s-1}}+\left|\widetilde{\mathcal{H}}_{i}\right|_{H^{s-1 / 2}}+\left|\pi^{0}\left(-L \stackrel{\star}{A} i-1-M \partial_{\omega} a_{i+1}^{\vee}+\stackrel{\star}{\mathcal{F}} i\right)\right|_{C^{s-1}\left(H^{s}\right)}\right.
\end{aligned}
$$

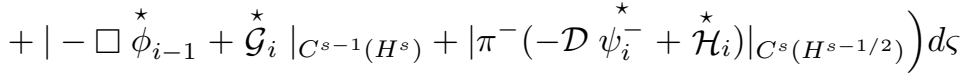

Les termes source qui dépendent uniquement des profils d'ordre inférieur à $i$ peuventêtre estimés directement par les propositions 1.3 .1 et 2.1.1 :

$$
\begin{aligned}
\left|\widetilde{\mathcal{F}}_{i}\right|_{H^{s-1}} & +\left|\widetilde{\mathcal{G}}_{i}\right|_{H^{s-1}}+\left|\widetilde{\mathcal{H}}_{i}\right|_{H^{s-1 / 2}}+\left|\pi^{0}\left(-L \stackrel{\star}{A_{i-1}}-M \partial_{\omega} a_{i+1}^{\vee}+\stackrel{\star}{\mathcal{F}}_{i}\right)\right|_{C^{s-1}\left(H^{s}\right)} \\
+ & +\square \stackrel{\star}{\phi_{i-1}}+\left.\stackrel{\star}{\mathcal{G}}_{i}\right|_{C^{s-1}\left(H^{s}\right)}+\left|\pi^{-}\left(-\mathcal{D} \psi_{i}^{-}+\stackrel{\star}{\mathcal{H}}_{i}\right)\right|_{C^{s}\left(H^{s-1 / 2}\right)} \leqslant C_{s} \delta^{2}(1+\delta)
\end{aligned}
$$

Nous observons une déperdition inévitable de régularité entre les profils oscillants d'ordre $i-1$ et les profils d'ordre $i+1$ : la présence de termes source $L \stackrel{\star}{A}{ }_{i-1}$ et $\square \stackrel{\star}{\phi_{i-1}}$ nous coûte systématique deux dérivées sur $\stackrel{\star}{A}_{i-1}$ et $\stackrel{\star}{\phi}_{i-1}$. Le problème linéaire admet finalement une unique solution

$$
\begin{aligned}
(A, \Phi) & \in C^{0}\left(\left[0, t_{1}\right], H^{s}\left(\Sigma \times S^{1}\right)\right) \cap C^{1}\left(\left[0, t_{1}\right], H^{s-1}\left(\Sigma \times S^{1}\right)\right) \\
\Psi & \in C^{0}\left(\left[0, t_{1}\right], H^{s-1 / 2}(\Sigma)\right) \\
\psi & \in C^{0}\left(\left[0, t_{1}\right], C^{s}\left([0,2 \pi], H^{s-1 / 2}(\Sigma)\right)\right) \\
\text { et } \quad(a, \phi) & \in \cap_{r=0 \text { ou }{ }_{1} C^{0}\left(\left[0, t_{1}\right], C^{s-1}\left([0,2 \pi], H^{s}(\Sigma)\right)\right)}
\end{aligned}
$$

sur l'intervalle de temps $\left[0, t_{1}\right]$. Nous posons finalement

$$
\begin{aligned}
& \widetilde{A}_{i}(x)=A(x) \\
& \pi^{0} \stackrel{\star}{A}_{i+1}(x, \omega)=\partial_{\omega}^{-1} a(x, \omega) \\
& \widetilde{\phi}_{i}(x)=\Phi(x) \quad \text { et } \quad \stackrel{\star}{\phi}_{i+1}(x, \omega)=\partial_{\omega}^{-1} \phi(x, \omega) \\
& \widetilde{\phi}_{i}=\Psi(x) \quad{\stackrel{\star}{*}{ }_{i}(x, \omega)}^{*} \mathcal{D}_{\theta} \psi(x, \omega)
\end{aligned}
$$

En reprenant l'inégalité a priori, on vérifie aisément le résultat additionnel de petitesse formulé dans la proposition.

Démonstration du théorème 2. - Par récurrence sur l'entier $M$.

Lorsque $M=1$, quelque soit la jauge $J_{\lambda}$ choisie, nous commençons par fixer $a_{1}^{\vee}=A c_{1}^{\vee}=0, a_{2}^{\vee}=A c_{2}^{\vee}=0, \psi_{0}^{-}=\psi{ }^{\star} c_{0}^{-}=0$ et $\psi_{1}^{-}=\psi{ }^{\star} c_{1}^{-}=0$ sur l'intervalle $\left[0, t_{0}\right]$. 
- Lorsque la jauge choisie est la jauge de Lorentz $(\lambda=1 / 2)$, nous pouvons utiliser directement les résultats de la proposition 2.3.1. Les données de Cauchy oscillantes sont compatibles à l'ordre $M=1$ et traduites en jauge de Lorentz, donc nous vérifions les contraintes 49 :

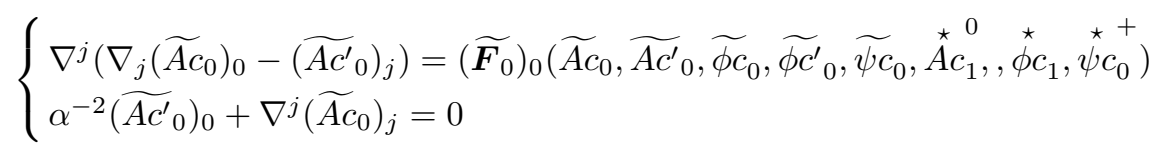

Du coup, la proposition 2.2 .7 a) s'applique et il suffit de résoudre le système $(H, 0)$ par la proposition 2.3.1. Les données $\left(A_{0}, \phi_{0}, \widetilde{\psi}_{0}, a_{1}^{0}, \hat{\phi}_{1},{ }_{\psi_{0}^{*}}\right)$ que nous obtenons sont nécessairement solutions régulières de $(\mathrm{C}, 0)$ et satisfont la condition de jauge $R A_{0}=0$. Nous appliquons ensuite la proposition $2.2 .7 \mathrm{~b}$ ) qui nous dit que $R_{\theta} \partial_{\omega} a_{3}^{\vee}+R \stackrel{\star}{A_{1}}=0$ si et seulement si $a_{1}^{\wedge}$ vérifie l'équation de transport $(E, 0)$ :

$$
X_{\mid \Pi_{\wedge}} \partial_{\omega} a_{1}^{\wedge}=\pi^{\wedge} \stackrel{\star}{\boldsymbol{F}}_{0}-\pi^{\wedge} X \partial_{\omega} a_{1}^{0}
$$

Cette équation de transport est linéaire en $a_{1}^{\wedge}$, et le second membre s'estime à l'aide de la proposition 1.3 .1 en fonction des profils $\left(A_{0}, a_{1}^{0}, \phi_{0}, \stackrel{\star}{\phi}_{1}\right)$ que nous venons d'obtenir :

$$
\begin{aligned}
& \left|\pi^{\wedge} \stackrel{\star}{\boldsymbol{F}}_{0}\right|_{C^{s-1}\left([0,2 \pi], H^{s}\right)} \\
& \quad \leqslant C_{s}\left(\left|A_{0}\right|_{H^{s}}\left(\left|a_{1}^{0}\right|_{C^{s-1}\left([0,2 \pi], H^{s}\right)}+\left|a_{1}^{\wedge}\right|_{C^{s}\left([0,2 \pi], H^{s}\right)}\right)+\left|\Phi_{0}\right|_{H^{s}}\left|\stackrel{\star}{\phi}_{1}\right|_{C^{s-1}\left([0,2 \pi], H^{s}\right)}\right)
\end{aligned}
$$

Compte tenu des hypothèses 2.1.1, $(E, 0)$ admet une unique solution

$$
a_{1}^{\wedge} \in C^{0}\left(\left[0, t_{1}\right], C^{s}\left([0,2 \pi], H^{s}(\Sigma)\right)\right)
$$

pour tout choix initial de $a_{1}^{\wedge}(0, y, \omega)=\pi^{\wedge} \stackrel{\star}{A}_{c 1}(y, \omega) \in C^{s}\left([0,2 \pi], H^{s}(\Sigma)\right)$.

- Lorsque la jauge choisie n'est pas la jauge de Lorentz $\left(\lambda \in\left[0, \frac{1}{2}[\cup] \frac{1}{2}, 1\right]\right)$, il est toujours possible d'avoir recours à un changement de jauge admissible

$$
u_{\varepsilon}=\widetilde{u}_{0}+\varepsilon^{3 / 2} \stackrel{\star}{\chi}_{3}
$$

au sens de la proposition 2.2.6. Nous fixons la partie moyenne de ce changement de jauge exactement comme nous l'avons fait section 1.3.5: en posant $u_{0}=e^{\chi}$, il vient $A_{0} \stackrel{u_{0}}{\rightsquigarrow} A_{0}^{\prime}=T \chi+e^{-\chi} A_{0} e^{\chi}$ et nous appliquons directement la proposition 1.3.6 conjointement avec le résultat d'existence de la proposition 2.3.1. Nous obtenons une

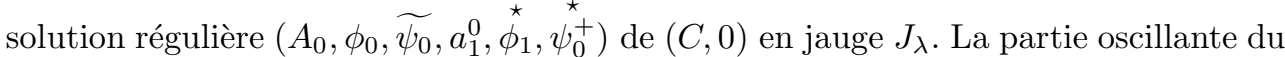
changement de jauge admissible permet quant à elle de modifier la composante libre $a_{1}^{\wedge}$, qui n'intervient pas dans les équations de $(C, 0)$. Nous fixons alors :

$$
a_{1}^{\wedge}=0
$$

(le degré de liberté dont nous semblions disposer sur le choix initial de $a_{1}^{\wedge}$ en jauge de Lorentz est illusoire et disparaît dans toute autre jauge de la famille $J_{\lambda}$ ). Nous 
choisissons ensuite

$$
a_{2}^{\wedge}=0 \quad \text { et } \quad a_{3}^{\wedge}=\frac{\alpha^{2} T_{\theta}}{(1-2 \lambda)\left(\nabla_{0} \theta\right)^{2}}\left(\frac{1}{2} R_{\theta} a_{3}^{\vee}+J_{\lambda} \partial_{\omega}^{-1} \stackrel{\star}{A_{1}}\right)
$$

de sorte que la jauge $J_{\lambda}$ est vérifiée à l'ordre $O(\varepsilon)$ en partie oscillante :

et

$$
\begin{gathered}
\lambda \alpha^{-2} \nabla_{0} \theta \partial_{\omega}\left(\stackrel{\star}{A}_{1}\right)_{0}+(1-\lambda) \nabla^{j} \theta \partial_{\omega}\left(\stackrel{\star}{A}_{1}\right)_{j}=(\lambda-1) g_{\Sigma}\left(\nabla_{\Sigma} \theta, \partial_{\omega} a_{1}^{0}\right)=0 \\
\lambda \alpha^{-2} \nabla_{0} \theta \partial_{\omega}(\stackrel{\star}{A})_{0}+(1-\lambda) \nabla^{j} \theta \partial_{\omega}(\stackrel{\star}{A})_{j}=0 \\
\lambda \alpha^{-2} \nabla_{0} \theta \partial_{\omega}\left(\stackrel{\star}{A}_{3}\right)_{0}+(1-\lambda) \nabla^{j} \theta \partial_{\omega}(\stackrel{\star}{A})_{j}+J_{\lambda} \stackrel{\star}{A_{1}}=0
\end{gathered}
$$

- Enfin, quelque soit la jauge, il ne reste plus qu'à fixer les composantes $a_{3}^{\vee}$ et $\psi_{2}^{\star}$ pour satisfaire la polarisation $(P, 0)$, et nous sommes assurés que le système $(Y M)$ est vérifié à l'ordre $\varepsilon^{1 / 2}$ en partie moyenne, à l'ordre $O\left(\varepsilon^{0}\right)$ en partie oscillante pour les champs de jauge et scalaire, à l'ordre $O\left(\varepsilon^{1 / 2}\right)$ en partie oscillante pour le champ spinoriel, et la jauge à l'ordre $O\left(\varepsilon^{1 / 2}\right)$ en partie moyenne, à l'ordre $O(\varepsilon)$ en partie oscillante. Les clauses de petitesse du théorème 2 découlent directement de celle de la proposition 2.3.1. Le théorème est prouvé à l'ordre $M=1$.

Considérons ensuite des données de Cauchy oscillantes, compatibles à un ordre $M+$ $1>1$, de phase $\theta / \varepsilon$, traduites dans une jauge $J_{\lambda}, \lambda \in[0,1]$, et vérifiant, pour un réel $s \geqslant \frac{n}{2}+\frac{1}{2}$ donné :

$$
\begin{aligned}
& \left|\left(\widetilde{A}_{i}, \partial_{t} \widetilde{A}_{i}\right)\right|_{s+M-i}+\left|\widetilde{\phi}_{i}\right|_{H^{s+M-i}}+\left|\partial_{t} \widetilde{\phi}_{i}\right|_{H^{s-1+M-i}}+\left|\widetilde{\psi}_{i}\right|_{H^{s-\frac{1}{2}+M-i}} \\
& +\left|\stackrel{\star}{A}_{i+1}\right|_{C^{s}\left([0,2 \pi],\left(H^{s+M-i}\right)\right)}+\left|\stackrel{\star}{\phi}_{i+1}\right|_{C^{s}\left([0,2 \pi],\left(H^{s+M-i}\right)\right)}+\left|\stackrel{\hat{\psi}_{i}}{ }\right|_{C^{s}\left([0,2 \pi],\left(H^{s-\frac{1}{2}+M-i}\right)\right)} \\
& \leqslant \delta_{i} / 2
\end{aligned}
$$

pour chaque $0 \leqslant i<M$. Supposons le théorème 2 prouvé à l'ordre $M$. Il existe alors une suite ${ }^{(5)}$

$$
\begin{aligned}
\widetilde{A}_{i} & \in C^{0}\left(\left[0, t_{1}\right], H^{s+M-i}(\Sigma)\right) \cap C^{1}\left(\left[0, t_{1}\right], H^{s-1}(\Sigma)\right) \\
\widetilde{\phi}_{i} & \in C^{0}\left(\left[0, t_{1}\right], H^{s+M-i}(\Sigma)\right) \cap C^{1}\left(\left[0, t_{1}\right], H^{s-1}(\Sigma)\right) \\
\widetilde{\psi}_{i} & \in C^{0}\left(\left[0, t_{1}\right], H^{s-\frac{1}{2}+M-i}(\Sigma)\right) \\
a_{i+1}^{0} & \in C^{0}\left(\left[0, t_{1}\right], C^{s}\left([0,2 \pi], H^{s+M-i}(\Sigma)\right)\right) \\
{\stackrel{\phi}{\phi_{i+1}}}_{\star} & \in C^{0}\left(\left[0, t_{1}\right], C^{s}\left([0,2 \pi], H^{s+M-i}(\Sigma)\right)\right) \\
\psi_{i}^{+} & \in C^{0}\left(\left[0, t_{1}\right], C^{s}\left([0,2 \pi], H^{s-\frac{1}{2}+M-i}(\Sigma)\right)\right)
\end{aligned}
$$

$0 \leqslant i \leqslant M+1$, telle que les $(C, k)$ et $(P, k)$ successifs soient vérifiés sur un intervalle fixe $\left[0, t_{1}\right], 0<t_{1} \leqslant t_{0}$, pour tout $0 \leqslant k<M$, ainsi que les conditions de jauge

$$
\lambda \alpha^{-2} \nabla_{0} \theta \partial_{\omega}(\stackrel{\star}{A})_{0}+(1-\lambda) \nabla^{j} \theta \partial_{\omega}\left(\stackrel{\star}{A}_{1}\right)_{j}=0
$$

${ }^{(5)}$ unique pour les termes d'indice $i<M$ 


$$
\lambda \alpha^{-2} \nabla_{0} \theta \partial_{\omega}(\stackrel{\star}{A})_{0}+(1-\lambda) \nabla^{j} \theta \partial_{\omega}\left(\stackrel{\star}{A}_{2}\right)_{j}=0
$$

puis

$$
J_{\lambda} \widetilde{A}_{i}=0 \quad \text { et } \quad \lambda \alpha^{-2} \nabla_{0} \theta \partial_{\omega}\left(\stackrel{\star}{A}_{k+3}\right)_{0}+(1-\lambda) \nabla^{j} \theta \partial_{\omega}\left(\stackrel{\star}{A}_{k+3}\right)_{j}+J_{\lambda} \stackrel{\star}{A}_{k+1}=0
$$

pour tout $0 \leqslant k<M$. De plus, si $\delta_{0}$ est suffisamment petit, alors $t_{1}=t_{0}$. De plus, si $\delta_{i} \leqslant \delta_{M-1}, \forall i<M$ avec $\delta_{M}$ assez petit, alors

$$
\begin{aligned}
\left|\widetilde{A}_{i}\right|_{L^{\infty}\left(\left[0, t_{1}\right], H^{s}\right)} & +\left|\partial_{t} \widetilde{A}_{i}\right|_{L^{\infty}\left(\left[0, t_{1}\right], H^{s-1}\right)}+\left|\widetilde{\phi}_{i}\right|_{L^{\infty}\left(\left[0, t_{1}\right], H^{s}\right)}+\left|\partial_{t} \widetilde{\phi}_{i}\right|_{L^{\infty}\left(\left[0, t_{1}\right], H^{s-1}\right)} \\
& +\left|\stackrel{\star}{A}_{i+1}\right|_{L^{\infty}\left(\left[0, t_{1}\right], C^{s}\left(H^{s}\right)\right)}+\left|\stackrel{\star}{\phi}_{i+1}\right|_{L^{\infty}\left(\left[0, t_{1}\right], C^{s}\left(H^{s}\right)\right)} \\
+ & \left|\widetilde{\psi}_{i}\right|_{L^{\infty}\left(\left[0, t_{1}\right], H^{s-1 / 2}\right)}+\left|\stackrel{\phi}{\phi}_{i+1}\right|_{L^{\infty}\left(\left[0, t_{1}\right], C^{s}\left(H^{s-1 / 2}\right)\right)} \leqslant \delta_{M-1}, \forall i<M
\end{aligned}
$$

- Lorsque la jauge choisie est la jauge de Lorentz $(\lambda=1 / 2)$, nous pouvons utiliser directement les résultats de la proposition 2.3.2. Les données de Cauchy oscillantes sont compatibles à l'ordre $M+1$ et traduites en jauge de Lorentz, donc nous vérifions les contraintes (51):

$$
\begin{aligned}
& \nabla^{j}\left(\nabla_{j}\left(\widetilde{A c}_{M}\right)_{0}-\left({\widetilde{A c^{\prime}}}_{M}\right)_{j}\right) \\
& =\operatorname{Moy}\left(\boldsymbol{F}_{0}^{\prime}\left(\widetilde{A c_{M}}, \widetilde{A c^{\prime}} M, \widetilde{\phi c_{M}},{\widetilde{\phi c^{\prime}}}_{M}, \widetilde{\psi c_{M}}, \stackrel{\stackrel{A}{A}^{0}}{M+1}, \stackrel{\star}{\phi} c_{M+1}, \stackrel{\star}{\psi} c_{M}^{+}\right)\right)_{0} \\
& +\left(\widetilde{\mathcal{F}}_{M}\right)_{0}\left(A_{k}, \partial A_{k}, \stackrel{\star}{A}_{k+1}, \phi_{k}, \partial \phi_{k}, \stackrel{\star}{\phi}_{k+1}, \psi_{k}, k \leqslant M-1\right) \\
& \alpha^{-2}\left(\widetilde{A c^{\prime}} M\right)_{0}+\nabla^{j}\left(\widetilde{A c_{M}}\right)_{j}=0
\end{aligned}
$$

Du coup, la proposition 2.2 .8 a) s'applique et il suffit de résoudre le système $(H, M)$ par la proposition 2.3.2. Les données $\left(\widetilde{A}_{M}, \widetilde{\phi}_{M}, \widetilde{\psi}_{M}, a_{M+1}^{0}, \stackrel{\star}{\phi}_{M+1},{ }_{\psi_{M}^{+}}^{+}\right)$que nous obtenons sont nécessairement solutions régulières de $(\mathrm{C}, \mathrm{M})$ et satisfont la condition de jauge $R \widetilde{A}_{M}=0$. Nous appliquons ensuite la proposition $2.2 .8 \mathrm{~b}$ ) qui nous dit que $R_{\theta} \partial_{\omega} a_{M+3}^{\vee}+R \stackrel{\star}{A}_{M+1}=0$ si et seulement si $a_{M+1}^{\wedge}$ vérifie l'équation de transport $(E, M)$ :

$$
X_{\left.\right|_{\Pi \wedge}} \partial_{\omega} a_{M+1}^{\wedge}=\pi^{\wedge}\left(-\square \stackrel{\star}{A}{ }_{M-1}+(\mathcal{R} i c .) \stackrel{\star}{A}_{M-1}-X \partial_{\omega} a_{M+1}^{0}+{\stackrel{\star}{\boldsymbol{F}_{0}^{\prime}}}_{0}(M)+\stackrel{\star}{\mathcal{F}}_{M}\right)
$$

Cette équation de transport est linéaire en $a_{M+1}^{\wedge}$, et le second membre s'estime à l'aide de la proposition 1.3.1 et 2.1.1 en fonction des profils déjà connus :

$$
\begin{aligned}
& \left|\pi^{\wedge}\left(-\square \stackrel{\star}{A}_{M-1}+(\mathcal{R} i c .) \stackrel{\star}{A}_{M-1}-X \partial_{\omega} a_{M+1}^{0}+\stackrel{\star}{\prime}_{0}^{\prime}(M)+\stackrel{\star}{\mathcal{F}}_{M}\right)\right|_{C^{s-1}\left([0,2 \pi], H^{s}\right)} \\
& \leqslant C_{s} \delta_{M}\left(1+\left|a_{M+1}^{0}\right|_{C^{s-1}\left([0,2 \pi], H^{s}\right)}+\delta_{M}+\delta_{M}^{2}\right)
\end{aligned}
$$

Compte tenu des hypothèses 2.1.1, $(E, M)$ admet une unique solution

$$
a_{M+1}^{\wedge} \in C^{0}\left(\left[0, t_{1}\right], C^{s}\left([0,2 \pi], H^{s}(\Sigma)\right)\right)
$$

pour tout choix initial de $a_{M+1}^{\wedge}(0, y, \omega)=\pi^{\wedge} \stackrel{\star}{A}_{c M+1}(y, \omega) \in C^{s}\left([0,2 \pi], H^{s}(\Sigma)\right)$. 
- Lorsque la jauge choisie n'est pas la jauge de Lorentz $\left(\lambda \in\left[0, \frac{1}{2}[\cup] \frac{1}{2}, 1\right]\right)$, il est toujours possible d'avoir recours à un changement de jauge admissible

$$
u_{\varepsilon}=1+\varepsilon^{M / 2} \widetilde{\chi}_{M}+\varepsilon^{(M+3) / 2} \stackrel{\star}{\chi}_{M+3}
$$

au sens de la proposition 2.2.6. La partie moyenne de ce changement de jauge transforme $A_{M} \stackrel{u_{\xi}}{\rightsquigarrow} A_{M}^{\prime}=T \widetilde{\chi}_{M}+A_{M}+\left[A_{0}, \widetilde{\chi}_{M}\right]$ et nous pouvons appliquer directement la proposition 1.3.6 conjointement avec le résultat d'existence de la proposition 2.3.2. Nous obtenons une solution régulière $\left(\widetilde{A}_{M}, \widetilde{\phi}_{M}, \widetilde{\psi_{M}}, a_{M+1}^{0}, \phi_{M+1}^{\star}, \psi_{M}^{+}\right)$de $(C, M)$ en jauge $J_{\lambda}$. La partie oscillante du changement de jauge admissible permet quant à elle de modifier la composante libre $a_{M+1}^{\wedge}$, qui n'intervient pas dans les équations de $(C, M)$. Nous fixons alors

$$
a_{M+1}^{\wedge}=\frac{\alpha^{2} T_{\theta}}{(1-2 \lambda)\left(\nabla_{0} \theta\right)^{2}}\left(\frac{1}{2} R_{\theta} a_{M+1}^{\vee}+J_{\lambda} \partial_{\omega}^{-1} \stackrel{\star}{A}_{M-1}\right)
$$

(le degré de liberté dont nous semblions disposer sur le choix initial de $a_{M+1}^{\wedge}$ en jauge de Lorentz est illusoire et disparaît dans toute autre jauge de la famille $J_{\lambda}$ ). Nous choisissons ensuite

$$
a_{M+2}^{\wedge}=\frac{\alpha^{2} T_{\theta}}{(1-2 \lambda)\left(\nabla_{0} \theta\right)^{2}}\left(\frac{1}{2} R_{\theta} a_{M+2}^{\vee}+J_{\lambda} \partial_{\omega}^{-1} \stackrel{\star}{A}{ }_{M}\right)
$$

et

$$
a_{M+3}^{\wedge}=\frac{\alpha^{2} T_{\theta}}{(1-2 \lambda)\left(\nabla_{0} \theta\right)^{2}}\left(\frac{1}{2} R_{\theta} a_{M+3}^{\vee}+J_{\lambda} \partial_{\omega}^{-1} \stackrel{\star}{A}_{M+1}\right)
$$

de sorte que la jauge $J_{\lambda}$ est vérifiée à l'ordre $O\left(\varepsilon^{\frac{M+2}{2}}\right)$ en partie oscillante :

$$
\begin{aligned}
& \lambda \alpha^{-2} \nabla_{0} \theta \partial_{\omega}\left(\stackrel{\star}{A}_{1}\right)_{0}+(1-\lambda) \nabla^{j} \theta \partial_{\omega}(\stackrel{\star}{A})_{j}=0 \\
& \lambda \alpha^{-2} \nabla_{0} \theta \partial_{\omega}\left(\stackrel{\star}{A}_{2}\right)_{0}+(1-\lambda) \nabla^{j} \theta \partial_{\omega}\left(\stackrel{\star}{A}_{2}\right)_{j}=0
\end{aligned}
$$

puis

$$
\lambda \alpha^{-2} \nabla_{0} \theta \partial_{\omega}\left(\stackrel{\star}{A}_{i+3}\right)_{0}+(1-\lambda) \nabla^{j} \theta \partial_{\omega}\left(\stackrel{\star}{A}_{i+3}\right)_{j}+J_{\lambda} \stackrel{\star}{A}_{i+1}=0
$$

pour tout $0 \leqslant i<M+1$.

- Enfin, quelque soit la jauge, il ne reste plus qu'à fixer les composantes $a_{M+3}^{\vee}$ et $\psi_{M+2}^{-}$pour satisfaire la polarisation $(P, M)$, et nous sommes assurés que le système $(Y M)$ est vérifié à l'ordre $O\left(\varepsilon^{(M+1) / 2}\right)$ en partie moyenne, à l'ordre $O\left(\varepsilon^{\frac{M}{2}}\right)$ en partie oscillante pour les champs de jauge et scalaire, à l'ordre $O\left(\varepsilon^{(M+1) / 2}\right)$ en partie oscillante pour le champ spinoriel, et la jauge à l'ordre $O\left(\varepsilon^{(M+1) / 2}\right)$ en partie moyenne, à l'ordre $O\left(\varepsilon^{\frac{M+2}{2}}\right)$ en partie oscillante. Les clauses de petitesse du théorème 2 découlent directement de celle de la proposition 2.3.2. Le théorème est prouvé à l'ordre $M+1$. 
2.3.2. Régularité des familles de solutions approchées obtenues, estimations uniformes en $\varepsilon$. - Nous finissons cette section par quelques considérations sur la régularité des solutions approchées que nous venons de construire, lorsque $\varepsilon$ devient arbitrairement petit. Il est important de remarquer que les familles de fonctions $(A, \phi)_{\varepsilon}$ du théorème 2 ne sont pas uniformément lipschitziennes lorsque $\varepsilon \rightarrow 0$. Ceci sera source de difficultés pour l'obtention des solutions exactes asymptotiques du théorème 3. Ces fonctions ont en fait une « demi-dérivée » bornée uniformément en $\varepsilon:$ c'est ce que nous souhaitons caractériser ici pour en faire usage dans la proposition 3.1.1.

Nous posons $\langle\varepsilon D\rangle^{s}=\left(1-\varepsilon^{2} \triangle\right)^{s / 2}$. L'espace BMO apparaît de façon naturelle dans les estimations bilinéaires de la partie suivante. Rappelons qu'une fonction $f$ est dans l'espace BMO si et seulement si

$$
|f|_{\mathrm{BMO}}=\sup _{B} \frac{1}{\operatorname{Vol}(B)} \int_{B}\left|f(y)-f_{B}\right| d y<C
$$

où $f_{B}$ désigne la moyenne de $f$ sur la boule $B$. En particulier,

$$
|f|_{\mathrm{BMO}} \leqslant 2|f|_{L^{\infty}}
$$

DÉfinition 2.3.1. - Pour tous réels $s$ et $m$, pour toute famille de fonctions $(u)_{\varepsilon}$ de $H^{s+m}(\Sigma)$ dépendant d'un paramètre $\varepsilon, 0<\varepsilon \leqslant \varepsilon_{0}<1$, nous définissons la norme suivante :

$$
\left\|u_{\varepsilon}\right\|_{s, m, 2}=\sup _{0<\varepsilon \leqslant \varepsilon_{0}}\left|\langle D\rangle^{s}\langle\varepsilon D\rangle^{m} u_{\varepsilon}\right|_{\mathbf{L}^{2}}
$$

Nous désignons par $\mathcal{W}_{\varepsilon}^{s, m, 2}(\Sigma)$ l'ensemble des familles $(u)_{\varepsilon}$ de $H^{s+m}(\Sigma)$ dépendant d'un paramètre $\varepsilon$ dont la norme $\left\|u_{\varepsilon}\right\|_{s, m, 2}$ est bornée. Pour tout $M \in \mathbb{R}$, nous faisons usage de la notation $\varepsilon^{M} \mathcal{W}_{\varepsilon}^{s, m, 2}(\Sigma)$ pour désigner l'ensemble des familles $(u)_{\varepsilon}$ de $H^{s+m}(\Sigma)$ telles que $\left\|\varepsilon^{-M} u_{\varepsilon}\right\|_{s, m, 2}$ est bornée.

Pour tous $s$ et $m$, ces espaces sont complets pour la norme $\|\cdot\|_{s, m, 2}$ (et conviennent donc à la mise en œuvre d'un point fixe). Ils contiennent des familles uniformément bornées dans $H^{s}$ et nous serviront dans la partie suivante à estimer les termes de reste prolongeant la solution approchée du théorème 2 en une solution exacte. Nous définissons d'une façon similaire des espaces $\mathcal{W}_{\varepsilon}^{s, m, \infty}$, construits sur l'espace BMO, et non plus sur $L^{2}$.

DÉfinition 2.3.2. - Pour tout réels $s$ et $m$, pour toute famille de fonctions $(u)_{\varepsilon}$, $0<\varepsilon \leqslant \varepsilon_{0}<1$, nous définissons la norme :

$$
\left\|u_{\varepsilon}\right\|_{s, m, \infty}=\sup _{0<\varepsilon \leqslant \varepsilon_{0}}\left|\langle D\rangle^{s}\langle\varepsilon D\rangle^{m} u_{\varepsilon}\right|_{\mathrm{BMO}}
$$

Nous nommons $\mathcal{W}_{\varepsilon}^{s, m, \infty}(\Sigma)$ l'ensemble des familles $(u)_{\varepsilon}$ dont la norme $\left\|u_{\varepsilon}\right\|_{s, m, \infty}$ est bornée. 
Enfin, nous définissons les espaces qui nous permettent d'estimer uniformément la régularité de développements oscillants dépendant du paramètre $\varepsilon$.

DÉFInItion 2.3.3. - Nous nommons $\mathcal{Z}_{\varepsilon}^{(s, m)}$ l'ensemble des familles $(u)_{\varepsilon}$ de $\mathcal{W}_{\varepsilon}^{s, m, 2}$ qui peuvent se décomposer en

$$
(u)_{\varepsilon}=\left(u^{\prime}\right)_{\varepsilon}+\left(u^{\prime \prime}\right)_{\varepsilon} \operatorname{avec}\left(u^{\prime}\right)_{\varepsilon} \in \mathcal{W}_{\varepsilon}^{s+m, 0,2} \text { et }\left(u^{\prime \prime}\right)_{\varepsilon} \in \mathcal{W}_{\varepsilon}^{s, m, \infty}
$$

Nous prenons comme norme sur $\mathcal{Z}_{\varepsilon}^{(s, m)}$ la quantité

$$
\left\|u_{\varepsilon}\right\|_{(s, m)}=\left\|u_{\varepsilon}\right\|_{s, m, 2}+\inf _{\left(u^{\prime}+u^{\prime \prime}=u\right)}\left(\left\|u_{\varepsilon}^{\prime}\right\|_{s+m, 0,2}+\left\|u_{\varepsilon}^{\prime \prime}\right\|_{s, m, \infty}\right)
$$

où $\inf _{\left(u^{\prime}+u^{\prime \prime}=u\right)}$ désigne l' inf pour toutes les décompositions possibles.

En vue des estimations bilinéaires de la section suivante, nous précisons alors le théorème 2 :

Proposition 2.3.3 (Estimations uniformes sur les solutions approchées et les termes de reste)

La famille de solutions approchées $(A, \phi, \psi)_{\varepsilon}$ du théorème 2 vérifie

$$
\left\{\begin{array}{l}
(A)_{\varepsilon} \in C^{0}\left([0, t], \mathcal{Z}_{\varepsilon}^{\frac{1}{2}, \frac{n}{2}}(\Sigma)\right) \cap C^{1}\left([0, t], \mathcal{Z}_{\varepsilon}^{-\frac{1}{2}, \frac{n}{2}}(\Sigma)\right) \\
(\phi)_{\varepsilon} \in C^{0}\left([0, t], \mathcal{Z}_{\varepsilon}^{\frac{1}{2}, \frac{n}{2}}(\Sigma)\right) \cap C^{1}\left([0, t], \mathcal{Z}_{\varepsilon}^{-\frac{1}{2}, \frac{n}{2}}(\Sigma)\right) \\
(\psi)_{\varepsilon} \in C^{0}\left([0, t], \mathcal{Z}_{\varepsilon}^{0, \frac{n}{2}}(\Sigma)\right)
\end{array}\right.
$$

Le reste $(\mathcal{O}, \mathcal{P}, \mathcal{Q})_{\varepsilon}$ vérifie

$$
\left\{\begin{array}{l}
(\mathcal{O})_{\varepsilon} \in C^{0}\left([0, t], \varepsilon^{M / 2} \mathcal{W}_{\varepsilon}^{-\frac{1}{2}, \frac{n}{2}, 2}(\Sigma)\right) \\
(\mathcal{P})_{\varepsilon} \in C^{0}\left([0, t], \varepsilon^{M / 2} \mathcal{W}_{\varepsilon}^{-\frac{1}{2}, \frac{n}{2}, 2}(\Sigma)\right) \\
(\mathcal{Q})_{\varepsilon} \in C^{0}\left([0, t], \varepsilon^{M / 2} \mathcal{W}_{\varepsilon}^{0, \frac{n}{2}, 2}(\Sigma)\right)
\end{array}\right.
$$

Enfin

$$
(\mathcal{J})_{\varepsilon}=\lambda \alpha^{-2} \nabla_{0} \mathcal{J}_{0}+(1-\lambda) \nabla^{i}\left(\mathcal{J}_{\Sigma}\right)_{i}
$$

avec

$$
\left(\mathcal{J}_{0}, \mathcal{J}_{\Sigma}\right) \in C^{0}\left([0, t], \varepsilon^{M / 2} \mathcal{W}_{\varepsilon}^{\frac{3}{2}, \frac{n}{2}, 2}(\Sigma)\right) \cap C^{1}\left([0, t], \varepsilon^{M / 2} \mathcal{W}_{\varepsilon}^{\frac{1}{2}, \frac{n}{2}, 2}(\Sigma)\right)
$$

De plus, si la clause de petitesse est respectée dans le théorème 2, alors

$$
\begin{array}{r}
\left.\left\|(A, \phi)_{\varepsilon}\right\|\right|_{\left(\frac{1}{2}, \frac{n}{2}\right)}+\left.\left\|\left(\partial_{t} A, \partial_{t} \phi\right)_{\varepsilon}\right\|\right|_{\left(-\frac{1}{2}, \frac{n}{2}\right)}+\left\|\psi_{\varepsilon}\right\| \|_{\left(0, \frac{n}{2}\right)} \leqslant C \delta_{M} \\
\left\|\varepsilon^{-M / 2}(\mathcal{O}, P)_{\varepsilon}\right\|_{-\frac{1}{2}, \frac{n}{2}, 2}+\left\|\varepsilon^{-M / 2} \mathcal{Q}_{\varepsilon}\right\|_{0, \frac{n}{2}, 2} \leqslant C \delta_{M} \\
\left\|\varepsilon^{-M / 2}\left(\mathcal{J}_{0}, \mathcal{J}_{\Sigma}\right)\right\|_{\frac{3}{2}, \frac{n}{2}, 2}+\left\|\varepsilon^{-M / 2}\left(\partial_{t} \mathcal{J}_{0}, \partial_{t} \mathcal{J}_{\Sigma}\right)\right\|_{\frac{1}{2}, \frac{n}{2}, 2} \leqslant C \delta_{M}
\end{array}
$$


Démonstration de la proposition 2.3.3. - En décomposant chaque fonction du théorème 2 en sa partie moyenne et oscillante, puis en comptant les puissances de $\varepsilon$, la proposition 2.3.3 s'obtient comme corollaire de la proposition 2.3.4 suivante :

Proposition 2.3.4. - Soit un réel $0 \leqslant s \leqslant \frac{n}{2}$ et $\theta$ une fonction de phase $C^{\infty}$ vérifiant $c_{0}^{-1} \leqslant|\operatorname{grad} \theta| \leqslant c_{0}$, il existe des constantes $C_{\theta}$ ne dépendant que d'un nombre fini de semi-normes de $\theta$ dans $C^{\infty}$, telles que :

a) pour toute fonction $u(x, \omega) \in C^{\frac{n}{2} \pm s}\left([0,2 \pi], H^{\frac{n}{2} \pm s}(\Sigma)\right) 2 \pi$-périodique de moyenne nulle en $\omega$ :

$$
\left\|\varepsilon^{ \pm s} u\left(x, \frac{\theta(x)}{\varepsilon}\right)\right\|_{ \pm s, \frac{n}{2}, 2} \leqslant C_{\theta}|u(x, \omega)|_{C^{\frac{n}{2} \pm s}\left(H^{\frac{n}{2} \pm s}\right)}
$$

b) pour toute fonction $u(x, \omega) \in C^{\frac{n}{2}+s}\left([0,2 \pi], H^{\frac{n}{2}+s}(\Sigma)\right) 2 \pi$-périodique de moyenne nulle en $\omega$ :

$$
\|\left|\varepsilon^{s} u\left(x, \frac{\theta(x)}{\varepsilon}\right)\right|||_{\left(s, \frac{n}{2}\right)} \leqslant C_{\theta}|u(x, \omega)|_{C^{\frac{n}{2}+s}\left(H^{\frac{n}{2}+s}\right)}
$$

c) pour toute fonction $u(x, \omega) \in C^{\frac{n}{2}+s}\left([0,2 \pi], H^{\frac{n}{2}-s}(\Sigma)\right) 2 \pi$-périodique de moyenne nulle en $\omega$ :

$$
\left\|\left|\varepsilon^{s} u\left(x, \frac{\theta(x)}{\varepsilon}\right)\right|\right\|_{\left(-s, \frac{n}{2}\right)} \leqslant C_{\theta}|u(x, \omega)|_{C^{\frac{n}{2}+s}\left(H^{\frac{n}{2}-s}\right)}
$$

d) pour toute fonction $u(x, \omega) \in C^{\frac{n}{2}-s}\left([0,2 \pi], H^{\frac{n}{2}+s}(\Sigma)\right) 2 \pi$-périodique de moyenne nulle en $\omega$ :

$$
\left\|\left.|| \varepsilon^{-s} u\left(x, \frac{\theta(x)}{\varepsilon}\right)\left|\|_{\left(-s, \frac{n}{2}\right)} \leqslant C_{\theta}\right| u(x, \omega)\right|_{C^{\frac{n}{2}-s}\left(H^{\frac{n}{2}+s}\right)}\right.
$$

La preuve de cette proposition nécessite quelques étapes intermédiaires. Nous utiliserons dorénavant des décompositions diadiques de Littlewood-Paley, dont nous rappelons ici le cadre standard. Soit $\psi \in C_{0}^{\infty}\left(\mathbb{R}^{n}\right)$, vérifiant $\psi(\xi)=1$ pour $|\xi| \leqslant 1 / 2$ et $\psi(\xi)=0$ pour $|\xi| \geqslant 1$. Posons $\varphi(\xi)=\psi(\xi / 2)-\psi(\xi) . \varphi$ est supportée dans la couronne $1 / 2 \leqslant|\xi| \leqslant 2$, et pour tout $\xi \in \mathbb{R}^{n}, 1=\psi(\xi)+\sum_{k \geqslant 0} \varphi\left(2^{-k} \xi\right)$. Pour tout $u \in \mathcal{S}^{\prime}\left(\mathbb{R}^{n}\right)$, posons alors $\Delta_{k} u=\varphi\left(2^{-k} D\right) u$ pour $k \geqslant 0$ et $\Delta_{-1} u=\psi(D) u$. Nous noterons $S_{k} u=\sum_{j=-1}^{k-1} \Delta_{j} u$. Nous rappelons ci-dessous quelques propriétés classiques de ce type de décompositions diadiques:

Lemme 2.3.5. - a) Pour toute fonction $u \in L^{2}$,

$$
\sum_{k \geqslant-1}\left|\Delta_{k} u\right|_{L^{2}}^{2} \leqslant|u|_{L^{2}}^{2} \leqslant 2 \sum_{k \geqslant-1}\left|\Delta_{k} u\right|_{L^{2}}^{2}
$$

En particulier, il existe une suite $\left(c_{k}\right)(u)$ vérifiant $\sum c_{k}^{2} \leqslant 1$ et telle que, pour tout $k \geqslant-1,\left|\Delta_{k} u\right|_{L^{2}} \leqslant c_{k}|u|_{L^{2}}$. Réciproquement, si, pour $k \geqslant-1,\left|\Delta_{k} u\right|_{L^{2}} \leqslant c_{k}$ avec $\sum c_{k}^{2} \leqslant C / 2$, alors $|u|_{L^{2}}^{2} \leqslant C$.

b) Il existe une constante $C$ telle que, pour tout $s \in \mathbb{R}, k \geqslant 0$ et $u \in H^{s}$,

$$
\frac{1}{C} 2^{s k}\left|\Delta_{k} u\right|_{L^{2}} \leqslant\left|\Delta_{k} u\right|_{H^{s}} \leqslant C 2^{s k}\left|\Delta_{k} u\right|_{L^{2}}
$$


soit, en tenant compte du a)

$$
\frac{1}{C} \sum_{k \geqslant-1}\left|2^{s k} \Delta_{k} u\right|_{L^{2}}^{2} \leqslant|u|_{H^{s}}^{2} \leqslant 2 C \sum_{k \geqslant-1}\left|2^{s k} \Delta_{k} u\right|_{L^{2}}^{2}
$$

b') Pour tout $m \in \mathbb{R}$, il existe une constante $C$ telle que pour tout $k \geqslant 0, \varepsilon \in] 0,1]$ et pour tout $u \in H^{m}$,

$$
\frac{1}{C}\left|\left\langle\varepsilon 2^{k}\right\rangle^{m} \Delta_{k} u\right|_{L^{2}}^{2} \leqslant\left|\langle\varepsilon D\rangle^{m} \Delta_{k} u\right|_{L^{2}}^{2} \leqslant C\left|\left\langle\varepsilon 2^{k}\right\rangle^{m} \Delta_{k} u\right|_{L^{2}}^{2}
$$

c) Il existe une constante $C$ telle que, pour tout $s \geqslant 0, k \geqslant-1$ et $u \in H^{s}$,

$$
\left|S_{k} u\right|_{H^{s}} \leqslant C 2^{k s}|u|_{L^{2}}
$$

$\left.c^{\prime}\right)$. Pour tout $m \geqslant 0$, il existe une constante $C$ telle que pour tout $\left.\left.k \geqslant 0, \varepsilon \in\right] 0,1\right]$ et $u \in H^{m}$,

$$
\left|\langle\varepsilon D\rangle^{m} S_{k} u\right|_{L^{2}}^{2} \leqslant C\left|\left\langle\varepsilon 2^{k}\right\rangle^{m} u\right|_{L^{2}}^{2}
$$

d) Il existe une constante $C$ telle que, pour tout $k \geqslant-1$ et $u \in L^{\infty}$,

$$
\left|S_{k} u\right|_{L^{\infty}} \leqslant C|u|_{L^{\infty}}
$$

Démonstration. - consulter par exemple [1, p.92-94]. Le a) exprime la quasiorthogonalité des termes de la décomposition diadique. L'essentiel est de bien vérifier $C^{-1} \leqslant \sum\left|\psi_{k}\right|^{2} \leqslant C$. Le b) et le c) traduisent la sensibilité des termes de la décomposition diadique aux dérivations. On les obtient immédiatement en passant en transformée de Fourier par le théorème de Plancherel.

L'espace BMO se laisse bien appréhender par décomposition diadique :

LEMme 2.3.6 (caractérisation diadique de BMO). - Il existe des constantes positives $c$ et $C$ telles que

$$
c|u|_{\mathrm{BMO}}^{2} \leqslant \sup _{y} \sup _{k} 2^{k n} \int_{B\left(y, 2^{-k}\right)} \sum_{j \geqslant k}\left|\Delta_{j} u\left(y^{\prime}\right)\right|^{2} d y^{\prime} \leqslant C|u|_{\mathrm{BMO}}^{2}
$$

Démonstration. - Consulter [17]. Nous reprenons le membre de droite de l'inégalité dans l'annexe A en détaillant le cas de décompositions diadiques légèrement plus générales.

Proposition 2.3.7 (Injection de Sobolev limite). - L'espace $\dot{H}^{n / 2}=(-\triangle)^{n / 4} L^{2}$ s'injecte de façon continue dans BMO. Il existe une constante positive $C$ telle que pour tout $U \in \dot{H}^{n / 2}$,

$$
|U|_{\mathrm{BMO}} \leqslant C|U|_{\dot{H}^{n / 2}}
$$


Démonstration. - Pour toute boule $B\left(y_{0}, 2^{-k}\right)$ de centre $y_{0}$ et de rayon $2^{k}$ arbitraires,

$$
\begin{aligned}
2^{k n} \int_{B\left(y_{0}, 2^{-k}\right)} \sum_{j \geqslant k}\left|\Delta_{j} U(y)\right|^{2} d y & \leqslant \int_{B\left(y_{0}, 2^{-k}\right)} \sum_{j \geqslant k} 2^{j n}\left|\Delta_{j} U(y)\right|^{2} d y \\
& \leqslant \sum_{j}\left|2^{j \frac{n}{2}} \Delta_{j} U(y)\right|_{L^{2}}^{2} d y \\
& \leqslant|U|_{\dot{H}^{n / 2}}^{2}
\end{aligned}
$$

La proposition découle alors directement de la caractérisation diadique de l'espace BMO, lemme 2.3.6. Remarquons par la même occasion :

LEMme 2.3.8. - L'espace $\mathcal{Z}_{\varepsilon}^{\left(s, \frac{n}{2}\right)}$ s'injecte de façon continue dans $\mathcal{W}_{\varepsilon}^{s, 0, \infty}$.

Démonstration. - nous décomposons $u_{\varepsilon} \in \mathcal{Z}_{\varepsilon}^{\left(s, \frac{n}{2}\right)}$ en

$$
u_{\varepsilon}=u_{\varepsilon}^{\prime}+u_{\varepsilon}^{\prime \prime}
$$

de sorte que

$$
\left\|u_{\varepsilon}^{\prime}\right\|_{s+\frac{n}{2}, 0,2} \leqslant 2 \mid\left\|u_{\varepsilon}\right\|_{\left(s, \frac{n}{2}\right)} \quad \text { et } \quad\left\|u_{\varepsilon}^{\prime \prime}\right\|_{s, \frac{n}{2}, \infty} \leqslant 2\left|\left\|u_{\varepsilon}\right\|\right|_{\left(s, \frac{n}{2}\right)}
$$

Il est évident que

$$
\left\|u_{\varepsilon}^{\prime \prime}\right\|_{s, 0, \infty} \leqslant\left\|u_{\varepsilon}^{\prime \prime}\right\|_{s, \frac{n}{2}, \infty}
$$

tandis que l'injection de Sobolev $H^{n / 2} \hookrightarrow$ BMO, proposition 2.3.7, nous donne pour tout $\varepsilon$ :

$$
\left|\langle D\rangle^{s} u_{\varepsilon}^{\prime}\right|_{\mathrm{BMO}} \leqslant C\left|\langle D\rangle^{s+\frac{n}{2}} u_{\varepsilon}^{\prime}\right|_{L^{2}}
$$

soit, en prenant le $\sup \operatorname{sur} \varepsilon$,

$$
\left\|u_{\varepsilon}^{\prime}\right\|_{s, 0, \infty} \leqslant C\left\|u_{\varepsilon}^{\prime}\right\|_{s+\frac{n}{2}, 0,2}
$$

ce qui achève la preuve du lemme.

Les fonctions oscillantes de la forme $e^{i \lambda \theta}$, où $\theta$ est une fonction de phase régulière de gradient non nul, sont essentiellement supportées en fréquence dans une couronne de taille $\lambda|\operatorname{grad} \theta|$ :

Lemme 2.3.9. - Soit $\theta$ une fonction de phase $C^{\infty}$ vérifiant $c_{0}^{-1} \leqslant|\operatorname{grad} \theta| \leqslant c_{0}$. Quelque soit $N>0$, il existe $C_{N, \theta}>0$ ne (ne dépendant que des semi-normes de $\theta$ dans $C^{N}$ ) tel que, pour tout réel $\lambda>0$ et tout entier $j$ vérifiant

a) $2^{j} \notin\left[\frac{\lambda}{2 c_{0}}, 2 c_{0} \lambda\right]$, on $a$

$$
\left|\Delta_{j} e^{i \lambda \theta}\right|_{L^{\infty}} \leqslant C_{N, \theta}\left(\sup \left\{2^{j}, \lambda\right\}\right)^{-N}
$$

b) $2^{j}<\frac{\lambda}{2 c_{0}}$, on a alors

$$
\left|S_{j} e^{i \lambda \theta}\right|_{L^{\infty}} \leqslant C_{N, \theta} \lambda^{-N}
$$


Démonstration. - a) Le résultat s'obtient sans difficulté par le lemme de la phase non stationnaire. Prenons $\chi \in C_{0}^{\infty}$ valant 1 dans sur $B\left(y_{0}, 1\right)$.

$$
\begin{aligned}
\Delta_{j} e^{i \lambda \theta\left(y_{0}\right)}= & \int e^{i\left(y_{0}-y\right) \xi+i \lambda \theta(y)} \psi\left(\frac{\xi}{2^{j}}\right) \chi(y) d \xi d y \\
& +\int e^{i\left(y_{0}-y\right) \xi+i \lambda \theta(y)} \psi\left(\frac{\xi}{2^{j}}\right)(1-\chi(y)) d \xi d y \\
= & I_{1}+I_{2}
\end{aligned}
$$

$I_{1}$ est à support compact en $y$, et sa phase ne stationne pas en $y$ par hypothèses, car nous avons choisi $2^{j} \notin\left[\frac{\lambda}{2 c_{0}}, 2 c_{0} \lambda\right]$ avec $c_{0}^{-1} \leqslant|\operatorname{grad} \theta| \leqslant c_{0}$, donc $|\xi-\lambda \operatorname{grad} \theta|>0$. Pour $I_{2}$, la phase ne stationne ni en $y$, ni en $\xi$, car cette fois $\left|y-y_{0}\right| \geqslant 1$. Nous ne sommes plus à support compact en $\mathrm{y}$, mais un nombre suffisant d'intégrations par parties en $\xi$ nous donnent la décroissance voulue en $y$ pour faire converger l'intégrale. Pour achever la preuve, il suffit de constater que a) $\Rightarrow$ b) de façon immédiate.

Choisissons une fonction $\sigma \in C^{\infty}$ vérifiant $\sigma(\xi)=1$ pour $\xi \notin\left[\frac{1}{2^{4} c_{0}}, 2^{4} c_{0}\right]$ et $\sigma(\xi)=0$ pour $\xi \in\left[\frac{1}{2^{3} c_{0}}, 2^{3} c_{0}\right]$. Pour tout réel $\lambda \geqslant 1$, nous posons $\sigma_{\lambda}(\xi)=\sigma\left(\frac{\xi}{\lambda}\right)$. nous définissons

$$
\sigma_{\lambda} u=\sigma_{\lambda}(D) u
$$

de sorte que $\sigma_{\lambda}$ agit comme un filtre « coupe-bande» en fréquence, dans la zone $|\xi| \approx \lambda$.

Proposition 2.3.10. - Soit une fonction de phase $\theta \in C^{\infty}$ vérifiant $c_{0}^{-1} \leqslant$ $|\operatorname{grad} \theta| \leqslant c_{0}$. Il existe des constantes $C_{\theta}$ (ne dépendant que d'un nombre fini de semi-normes de $\theta$ dans $\left.C^{\infty}\right)$ telles que :

a) pour toute fonction $u \in H^{s+r}$, $s$ et $r$ réels, $r \geqslant 0$ :

$$
\left|\langle D\rangle^{s} \sigma_{\lambda}\left(e^{i \lambda \theta} u\right)\right|_{L^{2}} \leqslant \lambda^{-r} C_{\theta}|u|_{H^{s+r}}
$$

b) pour toute fonction $u \in L^{2}$ et pour tout $s$ réel :

$$
\left|\langle D\rangle^{s}\left(1-\sigma_{\lambda}\right)\left(e^{i \lambda \theta} u\right)\right|_{L^{2}} \leqslant \lambda^{s} C_{\theta}|u|_{L^{2}}
$$

Démonstration. - Pour le a), avec $r \geqslant 0$. Posons $v_{\lambda}=e^{i \lambda \theta}$. Le produit de deux fonctions $u v_{\lambda}$ se décompose en

$$
u v_{\lambda}=T_{(u)} v_{\lambda}+T_{\left(v_{\lambda}\right)} u+R\left(u, v_{\lambda}\right)
$$

où

$$
T_{(u)} v_{\lambda}=\sum_{j \geqslant 2} S_{j-2} u \Delta_{j} v_{\lambda}, T_{\left(v_{\lambda}\right)} u=\sum_{j \geqslant 2} S_{j-2} v_{\lambda} \Delta_{j} u \text { et } R\left(u, v_{\lambda}\right)=\sum_{\left|j-j^{\prime}\right| \leqslant 2} \Delta_{j^{\prime}} u \Delta_{j} v_{\lambda}
$$

Commençons par examiner le terme $T_{\left(v_{\lambda}\right)} u$. Le spectre de chaque terme $S_{j-2} v_{\lambda} \Delta_{j} u$ est supporté dans une couronne $2^{j-2} \leqslant|\xi| \leqslant 2^{j+2}$. Par conséquent, si $2^{j} \in\left[\frac{\lambda}{2 c_{0}}, 2 c_{0} \lambda\right]$, le spectre de chaque terme $S_{j-2} v_{\lambda} \Delta_{j} u$ est supporté dans la couronne $c_{0}^{-1} 2^{-3} \lambda \leqslant|\xi| \leqslant$ $c_{0} \lambda 2^{3}$, et nous sommes assurés que

$$
\sigma_{\lambda}\left(S_{j-2} v_{\lambda} \Delta_{j} u\right)=0
$$


lorsque

$$
2^{j} \in\left[\frac{\lambda}{2 c_{0}}, 2 c_{0} \lambda\right]
$$

En considérant une fonction test $h \in \mathcal{S},|h|_{H^{-s}} \leqslant 1$, en utilisant le b) et le d) du lemme 2.3.5 puis en appliquant le lemme $2.3 .9 \mathrm{~b}$ ), il vient

$$
\begin{aligned}
&\left|\left\langle\sigma_{\lambda}\left(T_{\left(v_{\lambda}\right)} u\right), h\right\rangle\right| \leqslant \sum_{2^{j} \notin\left[\frac{\lambda}{2 c_{0}}, 2 c_{0} \lambda\right]} \sum_{j-3 \leqslant j^{\prime} \leqslant j+3}\left\langle\left|S_{j-2} v_{\lambda} \Delta_{j} u\right|,\left|\Delta_{j^{\prime}} h\right|\right\rangle \\
& \leqslant C\left(\sum_{2^{j}<\frac{\lambda}{2 c_{0}}}\left|S_{j-2} v_{\lambda}\right|_{L^{\infty}}^{2} 2^{2 j s}\left|\Delta_{j} u\right|_{L^{2}}^{2}\right)^{1 / 2}\left(\sum_{j} 2^{-2 j s}\left|\Delta_{j} h\right|_{L^{2}}^{2}\right)^{1 / 2} \\
&+C\left(\sum_{2^{j}>2 c_{0} \lambda} \lambda^{-2 r} 2^{2 j(s+r)}\left|S_{j-2} v_{\lambda}\right|_{L^{\infty}}^{2}\left|\Delta_{j} u\right|_{L^{2}}^{2}\right)^{1 / 2}\left(\sum_{j} 2^{-2 j s}\left|\Delta_{j} h\right|_{L^{2}}^{2}\right)^{1 / 2} \\
& \leqslant C_{\theta, N} \lambda^{-N}|h|_{H^{-s}}\left(\sum_{2^{j}<\frac{\lambda}{2 c_{0}}} 2^{2 j s}\left|\Delta_{j} u\right|_{L^{2}}^{2}\right)^{1 / 2} \\
&+C \lambda^{-r}|h|_{H^{-s}}\left(\sum_{2^{j}>2 c_{0} \lambda} 2^{2 j(s+r)}\left|\Delta_{j} u\right|_{L^{2}}^{2}\right)^{1 / 2}
\end{aligned}
$$

Comme $N$ est arbitraire, il suffit de fixer $N \geqslant r$ pour récupérer :

$$
\left|\sigma_{\lambda}\left(T_{(u)} v_{\lambda}\right)\right|_{H^{s}} \leqslant \lambda^{-r} C_{\theta}|u|_{H^{s+r}}
$$

Pour $T_{(u)} v_{\lambda}$, nous remarquons de nouveau que le spectre de chaque terme $S_{j-2} u \Delta_{j} v_{\lambda}$ est supporté dans une couronne $2^{j-2} \leqslant|\xi| \leqslant 2^{j+2}$. Prenons dans un premier temps $r \geqslant 0$ et $s \geqslant 0$. En considérant une fonction test $h \in \mathcal{S},|h|_{H^{-s}} \leqslant 1$, en utilisant le b) et le d) du lemme 2.3.5 puis en appliquant le lemme 2.3 .9 a), il vient :

$$
\begin{aligned}
& \left|\left\langle\sigma_{\lambda}\left(T_{(u)} v_{\lambda}\right), h\right\rangle\right| \leqslant \sum_{2^{j} \notin\left[\frac{\lambda}{2 c_{0}}, 2 c_{0} \lambda\right]} \sum_{j-3 \leqslant j^{\prime} \leqslant j+3}\left\langle\left|S_{j-2} u \Delta_{j} v_{\lambda}\right|,\left|\Delta_{j^{\prime}} h\right|\right\rangle \\
& \leqslant C\left(\sum_{2^{j}<\frac{\lambda}{2 c_{0}}} \lambda^{2 s}\left|\Delta_{j} v_{\lambda}\right|_{L^{\infty}}^{2}\left|S_{j-2} u\right|_{L^{2}}^{2}\right)^{1 / 2}\left(\sum_{j} 2^{-2 j s}\left|\Delta_{j} h\right|_{L^{2}}^{2}\right)^{1 / 2} \\
& \quad+C\left(\sum_{2^{j}>2 c_{0} \lambda} \lambda^{-2 r} 2^{2 j(s+r)}\left|\Delta_{j} v_{\lambda}\right|_{L^{\infty}}^{2}\left|S_{j-2} u\right|_{L^{2}}^{2}\right)^{1 / 2}\left(\sum_{j} 2^{-2 j^{\prime} s}\left|\Delta_{j^{\prime}} h\right|_{L^{2}}^{2}\right)^{1 / 2} \\
& \leqslant C_{\theta, N}|h|_{H^{-s}}|u|_{L^{2}}\left(\left(\sum_{2^{j}<\frac{\lambda}{2 c_{0}}} \lambda^{2(s-N)}\right)^{1 / 2}+\left(\sum_{2^{j}>2 c_{0} \lambda} \lambda^{-2 r} 2^{2 j(s+r-N)}\right)^{1 / 2}\right)
\end{aligned}
$$

Comme $N$ est arbitraire, il suffit de fixer $N>s+r+1$ pour obtenir :

$$
\left|\sigma_{\lambda}\left(T_{(u)} v_{\lambda}\right)\right|_{H^{s}} \leqslant C_{\theta} \lambda^{-r}|u|_{L^{2}}
$$

Prenons ensuite $r \geqslant 0$ et $s \leqslant 0$. Dans ce cas, le lemme 2.3.5, c) nous donne

$$
\sup _{j}\left|2^{j s} S_{j} u\right|_{L^{2}} \leqslant C|u|_{H^{s}}
$$


En considérant une fonction test $h \in \mathcal{S},|h|_{H^{-s}} \leqslant 1$, en utilisant le b) et le d) du lemme 2.3.5 puis en appliquant le lemme 2.3.9 a), il vient alors :

$$
\begin{aligned}
& \left|\left\langle\sigma_{\lambda}\left(T_{(u)} v_{\lambda}\right), h\right\rangle\right| \leqslant \sum_{2^{j} \notin\left[\frac{\lambda}{2 c_{0}}, 2 c_{0} \lambda\right]} \sum_{j-3 \leqslant j^{\prime} \leqslant j+3}\left\langle\left|S_{j-2} u \Delta_{j} v_{\lambda}\right|,\left|\Delta_{j^{\prime}} h\right|\right\rangle \\
& \leqslant C\left(\sum_{2^{j}<\frac{\lambda}{2 c_{0}}}\left|\Delta_{j} v_{\lambda}\right|_{L^{\infty}}^{2} 2^{2 j s}\left|S_{j-2} u\right|_{L^{2}}^{2}\right)^{1 / 2}\left(\sum_{j} 2^{-2 j s}\left|\Delta_{j} h\right|_{L^{2}}^{2}\right)^{1 / 2} \\
& \quad+C\left(\sum_{2^{j}>2 c_{0} \lambda} \lambda^{-2 r} 2^{2 j r}\left|\Delta_{j} v_{\lambda}\right|_{L^{\infty}}^{2} 2^{2 j s}\left|S_{j-2} u\right|_{L^{2}}^{2}\right)^{1 / 2}\left(\sum_{j} 2^{-2 j s}\left|\Delta_{j} h\right|_{L^{2}}^{2}\right)^{1 / 2} \\
& \quad \leqslant C_{\theta, N}|h|_{H^{-s}}|u|_{H^{s}}\left(\left(\sum_{2^{j}<\frac{\lambda}{2 c_{0}}} \lambda^{-2 N}\right)^{1 / 2}+\left(\sum_{2^{j}>2 c_{0} \lambda} \lambda^{-2 r} 2^{2 j(r-N)}\right)^{1 / 2}\right)
\end{aligned}
$$

Comme $N$ est arbitraire, il suffit de fixer $N>r+1$ pour obtenir :

$$
\left|\sigma_{\lambda}\left(T_{(u)} v_{\lambda}\right)\right|_{H^{s}} \leqslant C_{\theta} \lambda^{-r}|u|_{H^{s}}
$$

Pour le terme $R\left(u, v_{\lambda}\right)$, nous remarquons que chaque $\Delta_{j^{\prime}} u \Delta_{j} v_{\lambda},\left|j-j^{\prime}\right| \leqslant 2$ est supporté en fréquence dans une boule $|\xi| \leqslant 2^{j+4}$. Prenons $s \geqslant 0$ et $r \geqslant 0$. Considérons une fonction test $h \in \mathcal{S},|h|_{H^{-s}} \leqslant 1$ :

$$
\begin{aligned}
&\left|\left\langle R\left(u, v_{\lambda}\right), h\right\rangle\right| \leqslant \sum_{\left|j-j^{\prime}\right| \leqslant 2}\left\langle\left|\Delta_{j^{\prime}} u \Delta_{j} v_{\lambda}\right|,\left|S_{j+5} h\right|\right\rangle \\
& \leqslant C\left(\sum_{j} 2^{2 j s}\left|\Delta_{j} u\right|_{L^{2}}^{2}\right)^{1 / 2}\left(\sum_{2^{j}<\frac{\lambda}{2 c_{0}}}\left|\Delta_{j} v_{\lambda}\right|_{L^{\infty}}^{2} 2^{-2(j+5) s}\left|S_{j+5} h\right|_{L^{2}}^{2}\right)^{1 / 2} \\
&+C\left(\sum_{j} 2^{2 j(s+r)}\left|\Delta_{j} u\right|_{L^{2}}^{2}\right)^{1 / 2}\left(\sum_{2^{j} \geqslant \frac{\lambda}{2 c_{0}}} \lambda^{-2 r}\left|\Delta_{j} v_{\lambda}\right|_{L^{\infty}}^{2} 2^{-2(j+5) s}\left|S_{j+5} h\right|_{L^{2}}^{2}\right)^{1 / 2}
\end{aligned}
$$

Le lemme 2.3.5, c) nous donne, lorsque $s \geqslant 0$ :

$$
\sup _{j}\left|2^{-j s} S_{j} h\right|_{L^{2}} \leqslant C|h|_{H^{-s}}
$$

de sorte que :

$$
\begin{aligned}
& \left|\left\langle R\left(u, v_{\lambda}\right), h\right\rangle\right| \\
& \quad \leqslant C|u|_{H^{s+r}}|h|_{H^{-s}}\left(\left(\sum_{2^{j}<\frac{\lambda}{2 c_{0}}}\left|\Delta_{j} v_{\lambda}\right|_{L^{\infty}}^{2}\right)^{1 / 2}+\left(\sum_{2^{j} \geqslant \frac{\lambda}{2 c_{0}}} \lambda^{-2 r}\left|\Delta_{j} v_{\lambda}\right|_{L^{\infty}}^{2}\right)^{1 / 2}\right)
\end{aligned}
$$

et nous finissons en appliquant le lemme 2.3.9 a) :

$$
\sum_{2^{j}<\frac{\lambda}{2 c_{0}}}\left|\Delta_{j} v_{\lambda}\right|_{L^{\infty}}^{2} \leqslant C_{\theta, N} \sum_{2^{j}<\frac{\lambda}{2 c_{0}}} \lambda^{-2 N} \leqslant C_{\theta} \lambda^{-2 r}
$$


dès que $N>r+1$, puis

$$
\sum_{2^{j} \geqslant \frac{\lambda}{2 c_{0}}} \lambda^{-2 r}\left|\Delta_{j} v_{\lambda}\right|_{L^{\infty}}^{2} \leqslant C \sum_{2^{j} \in\left[\frac{\lambda}{2 c_{0}}, 2 c_{0} \lambda\right]} \lambda^{-2 r}+C_{\theta, N} \sum_{2^{j}>2 c_{0} \lambda} \lambda^{-2 r} 2^{-2 j N} \leqslant C_{\theta} \lambda^{-2 r}
$$

Prenons maintenant $s \leqslant 0$ et $r \geqslant 0$. Considérons une fonction test $h \in \mathcal{S},|h|_{H^{-s}} \leqslant 1$ :

$$
\begin{aligned}
& \left|\left\langle R\left(u, v_{\lambda}\right), h\right\rangle\right| \leqslant \sum_{\left|j-j^{\prime}\right| \leqslant 2}\left\langle\left|\Delta_{j^{\prime}} u \Delta_{j} v_{\lambda}\right|,\left|S_{j+5} h\right|\right\rangle \\
& \leqslant C\left(\sum_{j} 2^{2 j s}\left|\Delta_{j} u\right|_{L^{2}}^{2}\right)^{1 / 2}\left(\sum_{2^{j}<\frac{\lambda}{2 c_{0}}}\left|\Delta_{j} v_{\lambda}\right|_{L^{\infty}}^{2} \lambda^{-2 s}\left|S_{j+5} h\right|_{L^{2}}^{2}\right)^{1 / 2} \\
& \quad+C\left(\sum_{j} 2^{2 j(s+r)}\left|\Delta_{j} u\right|_{L^{2}}^{2}\right)^{1 / 2}\left(\sum_{2^{j} \geqslant \frac{\lambda}{2 c_{0}}} \lambda^{-2 r} 2^{-2 j s}\left|\Delta_{j} v_{\lambda}\right|_{L^{\infty}}^{2}\left|S_{j+5} h\right|_{L^{2}}^{2}\right)^{1 / 2} \\
& \quad \leqslant C|u|_{H^{s+r}}|h|_{L^{2}}\left(\left(\sum_{2^{j}<\frac{\lambda}{2 c_{0}}} \lambda^{-2 s}\left|\Delta_{j} v_{\lambda}\right|_{L^{\infty}}^{2}\right)^{1 / 2}+\left(\sum_{2^{j} \geqslant \frac{\lambda}{2 c_{0}}} \lambda^{-2 r} 2^{-2 j s}\left|\Delta_{j} v_{\lambda}\right|_{L^{\infty}}^{2}\right)^{1 / 2}\right)
\end{aligned}
$$

nous finissons en appliquant le lemme 2.3.9 a) :

$$
\sum_{2^{j}<\frac{\lambda}{2 c_{0}}} \lambda^{-2 s}\left|\Delta_{j} v_{\lambda}\right|_{L^{\infty}}^{2} \leqslant C_{\theta, N} \sum_{2^{j}<\frac{\lambda}{2 c_{0}}} \lambda^{-2(N+s)} \leqslant C_{\theta} \lambda^{-2 r}
$$

dès que $N+s>r+1$. Enfin

$$
\begin{aligned}
\sum_{2^{j} \geqslant \frac{\lambda}{2 c_{0}}} \lambda^{-2 r} 2^{-2 j s}\left|\Delta_{j} v_{\lambda}\right|_{L^{\infty}}^{2} & \leqslant C \sum_{2^{j} \in\left[\frac{\lambda}{2 c_{0}}, 2 c_{0} \lambda\right]} \lambda^{-2 r} 2^{-2 j s}+C_{\theta, N} \sum_{2^{j}>2 c_{0} \lambda} \lambda^{-2 r} 2^{-2 j(N+s)} \\
& \leqslant C_{\theta} \lambda^{-2 r}
\end{aligned}
$$

pour $N$ assez grand. Nous achevons ainsi la preuve du a).

Pour prouver le b) de la proposition, prenons $s$ réel quelconque, et une fonction test $h \in \mathcal{S},|h|_{H^{-s}} \leqslant 1$ :

$$
\begin{aligned}
\left|\left\langle\left(1-\sigma_{\lambda}\right)\left(u v_{\lambda}\right), h\right\rangle\right| & \leqslant\left\langle\left|\left(1-\sigma_{\lambda}\right)\left(u v_{\lambda}\right)\right|, \sum_{2^{j} \in\left[\frac{\lambda}{2^{5} c_{0}}, 2^{5} c_{0} \lambda\right]}\left|\Delta_{j} h\right|\right\rangle \\
& \leqslant C|u|_{L^{2}}\left|v_{\lambda}\right|_{L^{\infty}} \sum_{2^{j} \in\left[\frac{\lambda}{2^{5} c_{0}}, 2^{5} c_{0} \lambda\right]} \lambda^{s}\left|2^{-j s} \Delta_{j} h\right|_{L^{2}} \\
& \leqslant C \lambda^{s}|u|_{L^{2}}|h|_{H^{-s}}
\end{aligned}
$$

d'où l'inégalité attendue.

Un fonction supportée en fréquence dans une couronne fixe de taille $2^{j}$ ne peut pas « concentrer » une partie importante de sa norme $L^{2}$ sur une boule de rayon $2^{-k}$ inférieur à la longueur d'onde critique $2^{-j}$. Nous utiliserons l'estimation suivante qui traduit cette remarque : 
LEMME 2.3.11. - Il existe $C>0$, tel que pour tout $u \in L^{2}$, pour tous entiers $j<k$ et toute boule $B\left(2^{-k}\right)$ de rayon $2^{-k}<2^{-j}$ :

$$
2^{k n} \int_{B\left(2^{-k}\right)}\left|\Delta_{j} u(y)\right|^{2} d y \leqslant C 2^{j n}\left|\Delta_{j} u\right|_{L^{2}}^{2}
$$

Démonstration. - Rappelons que $\Delta_{j} u$ est une fonction $C^{\infty}$ et que

$$
\left|\Delta_{j} u\right|_{C^{1}} \leqslant C_{1} 2^{j\left(\frac{n}{2}+1\right)}\left|\Delta_{j} u\right|_{L^{2}}
$$

par injection de Sobolev.

Nous considérons une boule $B\left(y_{0}, 2^{-k}\right)$ de centre $y$ et de rayon $2^{-k}$. Fixons un entier $\mathrm{N}$ et supposons que

$$
\int_{B\left(y_{0}, 2^{-k}\right)}\left|\Delta_{j} u(y)\right|^{2} d y>2^{N} 2^{(j-k) n}\left|\Delta_{j} u\right|_{L^{2}}^{2}
$$

Nous remarquons que la boule $B\left(y_{0}, 2^{-j}\right)$ de rayon $2^{-j}$ peut toujours contenir au moins $c_{n} 2^{(k-j) n}$ boules de rayon $2^{-k}$ disjointes (ou au pire tangentes), avec une constante $c_{n} \geqslant 2^{-n}$ (on peut toujours placer $2^{(k-j-1) n}$ boules de rayon $2^{-k}$ dans un n-cube de demi-arrête $2^{-j-1}$, lequel est toujours compris dans une boule de rayon $\left.2^{-j} \ldots\right)$. On considère alors une collection $\left(B\left(y_{\alpha}, 2^{-k}\right)\right)_{1 \leqslant \alpha \leqslant c_{n} 2^{(k-j) n}}$ de telles boules. Il existe nécessairement une boule $B\left(y_{\alpha_{1}}, 2^{-k}\right)$ de centre $y_{\alpha_{1}}$ tel que $\left|y_{\alpha_{1}}-y_{0}\right| \leqslant 2^{-j}$ et vérifiant

$$
\int_{B\left(y_{\left.\alpha_{1}, 2^{-k}\right)}\right.}\left|\Delta_{j} u(y)\right|^{2} d y<2\left(c_{n}\right)^{-1} 2^{(j-k) n}\left|\Delta_{j} u\right|_{L^{2}}^{2}
$$

(sinon $\sum_{\alpha} \int_{B\left(y_{1}, 2^{-k}\right)}\left|\Delta_{j} u(y)\right|^{2} d y>2\left|\Delta_{j} u\right|_{L^{2}}^{2}$, ce qui est absurde vu que $\left.\sum_{\alpha} \int_{B\left(y_{1}, 2^{-k}\right)}\left|\Delta_{j} u(y)\right|^{2} d y \leqslant\left|\Delta_{j} u\right|_{L^{2}}^{2}\right)$. Prenons $y_{0}^{\prime} \in B\left(y_{0}, 2^{-j}\right)$ et $y_{\alpha_{1}}^{\prime} \in B\left(y_{\alpha_{1}}, 2^{-j}\right)$ tels que

$$
\left|\Delta_{j} u\left(y_{0}^{\prime}\right)\right|>2^{N / 2} 2^{j \frac{n}{2}}\left|\Delta_{j} u\right|_{L^{2}} \quad \text { et } \quad\left|\Delta_{j} u\left(y_{\alpha_{1}}^{\prime}\right)\right|<2\left(c_{n}\right)^{-1 / 2} 2^{j \frac{n}{2}}\left|\Delta_{j} u\right|_{L^{2}}
$$

Il existe alors un $t \in[0,1]$ tel que

$$
\operatorname{grad} \Delta_{j} u\left(t y_{0}^{\prime}-(1-t) y_{\alpha_{1}}^{\prime}\right) \cdot\left(y_{0}^{\prime}-y_{\alpha_{1}}^{\prime}\right)=\Delta_{j} u\left(y_{0}^{\prime}\right)-\Delta_{j} u\left(y_{\alpha_{1}}^{\prime}\right)
$$

comme $\left|y_{0}^{\prime}-y_{\alpha_{1}}^{\prime}\right| \leqslant 2^{-j}$, il vient finalement

$$
\left|\operatorname{grad} \Delta_{j} u\left(t y_{0}^{\prime}-(1-t) y_{\alpha_{1}}^{\prime}\right)\right| \geqslant\left|2^{N / 2}-(C)^{-1 / 2}\right| 2^{j\left(\frac{n}{2}+1\right)}\left|\Delta_{j} u\right|_{L^{2}}
$$

L'inégalité (65) contredit alors (64) dès que nous fixons $N$ assez grand pour que $\left|2^{N / 2}-(C)^{-1 / 2}\right|>C_{1}$. D'où le lemme.

Proposition 2.3.12. - Soit $\theta$ une fonction de phase $C^{\infty}$ vérifiant $c_{0}^{-1} \leqslant|\operatorname{grad} \theta| \leqslant$ $c_{0}$. Il existe des constantes $C_{\theta}$ ne dépendant que des semi-normes de $\theta$ dans $C^{\infty}$, telles que :

a) pour tous réel $s$ et $r, r \geqslant 0$, pour toute fonction $u \in H^{\frac{n}{2}+s+r}$ :

$$
\left|\langle D\rangle^{s} \sigma_{\lambda}\left(e^{i \lambda \theta} u\right)\right|_{\mathrm{BMO}} \leqslant C_{\theta} \lambda^{-r}|u|_{H^{\frac{n}{2}+s+r}}
$$


b) pour tous réels $s$ et $r, r \geqslant 0$, pour toute fonction $u \in H^{\frac{n}{2}-r}$ :

$$
\left|\langle D\rangle^{s}\left(1-\sigma_{\lambda}\right)\left(e^{i \lambda \theta} u\right)\right|_{\mathrm{BMO}} \leqslant C_{\theta} \lambda^{s+r}|u|_{H^{\frac{n}{2}-r}}
$$

Démonstration. - a) La proposition 2.3.10 a) nous donne

$$
\left|\langle D\rangle^{s} \sigma_{\lambda}\left(e^{i \lambda \theta} u\right)\right|_{H^{n / 2}} \leqslant C_{\theta} \lambda^{-r}|u|_{H^{\frac{n}{2}+s+r}}
$$

la constante $C_{\theta}$ ne dépendant que d'un nombre fini de semi-normes de $\theta$ dans $C^{\infty}$. Nous utilisons directement l'injection de Sobolev limite $H^{n / 2} \hookrightarrow$ BMO, proposition 2.3.7 :

$$
\forall \lambda, \quad\left|\langle D\rangle^{s} \sigma_{\lambda}\left(e^{i \lambda \theta} u\right)\right|_{\mathrm{BMO}} \leqslant C\left|\langle D\rangle^{s} \sigma_{\lambda}\left(e^{i \lambda \theta} u\right)\right|_{H^{n / 2}} \leqslant C_{\theta} \lambda^{-r}|u|_{H^{\frac{n}{2}+s+r}}
$$

b) Nous gardons la notation $v_{\lambda}=e^{i \lambda \theta}$ et rappelons que $\Delta_{j^{\prime}}\left(1-\sigma_{\lambda}\right)=0$ dès que $2^{j^{\prime}} \notin\left[c_{0}{ }^{-1} 2^{j-5} \lambda, c_{0} 2^{j+5} \lambda\right]$. Considérons une boule $B\left(y_{0}, 2^{-k}\right)$ arbitraire et majorons :

$$
\begin{aligned}
& 2^{k n} \int_{B\left(y_{0}, 2^{-k}\right)} \sum_{j^{\prime} \geqslant k}\left|\Delta_{j^{\prime}}\langle D\rangle^{s}\left(1-\sigma_{\lambda}\right)\left(T_{(u)} v_{\lambda}\right)(y)\right|^{2} d y \\
& \leqslant 2^{k n} \int_{B\left(y_{0}, 2^{-k}\right)} \sum_{2^{j^{\prime}} \in\left[\frac{\lambda}{2^{5} c_{0}}, 2^{5} c_{0} \lambda\right], j^{\prime} \geqslant k} 2^{2 j^{\prime}(s+r)}\left|\Delta_{j^{\prime}}\left(\sum_{j^{\prime}-3 \leqslant j \leqslant j^{\prime}+3} 2^{-j r} S_{j-2} u \Delta_{j} v_{\lambda}\right)(y)\right|^{2} d y \\
& \leqslant C \lambda^{2(s+r)} 2^{k n} \int_{B\left(y_{0}, 2^{-k}\right)} \sum_{2^{j} \in\left[\frac{\lambda}{2^{8} c_{0}}, 2^{8} c_{0} \lambda\right], k \leqslant j}\left|2^{-(j) r} S_{j-2} u(y)\right|^{2} d y \\
& \leqslant C \lambda^{2(s+r)}\left(2^{k n} \int_{B\left(y_{0}, 2^{-k}\right)}\left|2^{-k r} S_{k-1} u(y)\right|^{2} d y+\sum_{j \geqslant k}\left|2^{j\left(\frac{n}{2}-r\right)} \Delta_{i} u\right|_{L^{2}}^{2}\right)
\end{aligned}
$$

Le dernier terme du membre de droite s'estime aisément

$$
\sum_{j \geqslant k}\left|2^{j\left(\frac{n}{2}-r\right)} \Delta_{i} u(y)\right|_{L^{2}}^{2} d y \leqslant C|u|_{H^{\frac{n}{2}-r}}^{2}
$$

tandis que pour les basses fréquences, nous bénéficions du lemme 2.3.11 qui nous donne

$$
\begin{aligned}
2^{k n} \int_{B\left(y_{0}, 2^{-k}\right)} 2^{-2 k r}\left|S_{k-1} u(y)\right|^{2} d y & \leqslant C \sum_{j<k} 2^{k n} \int_{B\left(y_{0}, 2^{-k}\right)} 2^{-2 j r}\left|\Delta_{j} u(y)\right|^{2} d y \\
& \leqslant C \sum_{j<k} 2^{j(n-2 r)}\left|\Delta_{j} u(y)\right|_{L^{2}}^{2} d y \\
& \leqslant C|u|_{H^{\frac{n}{2}-r}}^{2}
\end{aligned}
$$


Pour les termes restants, il suffit d'estimer

$$
\begin{aligned}
& 2^{k n} \int_{B\left(y_{0}, 2^{-k}\right)} \sum_{j^{\prime} \geqslant k}\left|\Delta_{j^{\prime}}\langle D\rangle^{s}\left(1-\sigma_{\lambda}\right)\left(T_{\left(v_{\lambda}\right)} u\right)(y)\right|^{2} d y \\
& \leqslant C 2^{k n} \int_{B\left(y_{0}, 2^{-k}\right)} \sum_{2^{j^{\prime}} \in\left[\frac{\lambda}{2^{5} c_{0}}, 2^{5} c_{0} \lambda\right], j^{\prime} \geqslant k} 2^{2 j^{\prime}(s+r)}\left|\Delta_{j^{\prime}}\left(\sum_{j^{\prime}-3 \leqslant j \leqslant j^{\prime}+3} 2^{-j r} S_{j-2} v_{\lambda} \Delta_{j} u\right)(y)\right|^{2} d y \\
& \leqslant C \lambda^{2(s+r)} \sum_{2^{j} \in\left[\frac{\lambda}{2^{8} c_{0}}, 2^{8} c_{0} \lambda\right], k \leqslant j} 2^{j(n-2 r)}\left|\Delta_{j} u(y)\right|_{L^{2}}^{2} d y \\
& \leqslant C \lambda^{2(s+r)}|u|_{H^{\frac{n}{2}-r}}^{2}
\end{aligned}
$$

puis, en appliquant le lemme 2.3.9 a),

$$
\begin{aligned}
& 2^{k n} \int_{B\left(y_{0}, 2^{-k}\right)} \sum_{j^{\prime} \geqslant k}\left|\Delta_{j^{\prime}}\langle D\rangle^{s}\left(1-\sigma_{\lambda}\right) R\left(v_{\lambda}, u\right)(y)\right|^{2} d y \\
& \leqslant C 2^{k n} \int_{B\left(y_{0}, 2^{-k}\right)} \sum_{2^{j^{\prime}} \in\left[\frac{\lambda}{2^{5} c_{0}}, 2^{5} c_{0} \lambda\right], j^{\prime} \geqslant k} 2^{2 j^{\prime}(s)}\left|\Delta_{j^{\prime}}\left(\sum_{j^{\prime}-3 \leqslant j} \Delta_{j} v_{\lambda} \Delta_{j} u\right)(y)\right|^{2} d y \\
& \leqslant C \lambda^{2(s+r)} \sum_{2^{j} \in\left[\frac{\lambda}{2^{8} c_{0}}, 2 c_{0} \lambda\right]} 2^{j(n-2 r)}\left|\Delta_{j} u\right|_{L^{2}}^{2}+C_{\theta, N} \sum_{2^{j}>2 c_{0} \lambda} 2^{j(n-2 r-2 N)}\left|\Delta_{j} u\right|_{L^{2}}^{2} \\
& \leqslant C_{\theta} \lambda^{2(s+r)}|u|_{H^{\frac{n}{2}-r}}^{2}
\end{aligned}
$$

La preuve de b) est achevée.

Démonstration de la proposition 2.3.4. - a) Identifions la fonction $2 \pi$-périodique $u(x, \omega) \in C^{\frac{n}{2}+\varsigma}\left([0,2 \pi], H^{\frac{n}{2}+\varsigma}(\Sigma)\right)$ avec sa série de Fourrier. Comme $u(x, \omega)$ est de moyenne nulle, le premier coefficient de cette série est nul :

$$
u(x, \omega)=\sum_{|k| \geqslant 1} e^{i k \omega} u_{k}(x)
$$

soit

$$
u\left(x, \frac{\theta(x)}{\varepsilon}\right)=\sum_{|k| \geqslant 1} e^{i k \frac{\theta(x)}{\varepsilon}} u_{k}(x)
$$

Les fonctions $u_{k}$ sont uniformément bornées dans $H^{\frac{n}{2}+\varsigma}$ et satisfont à la condition de sommation (égalité de Parseval)

$$
\sum_{|k| \geqslant 1} k^{n+2 \varsigma} c_{k}^{2}\left|u_{k}\right|_{H^{\frac{n}{2}+\varsigma}}^{2}=|u|_{\left.C \frac{n}{2}+\varsigma^{(} H^{\frac{n}{2}+\varsigma}\right)}^{2}
$$

Nous appliquons directement la proposition 2.3.10 a) et b) :

$$
\begin{gathered}
\varepsilon^{2 \sigma}\left|\langle D\rangle^{\varsigma}\langle\varepsilon D\rangle^{n / 2} u\left(x, \frac{\theta(x)}{\varepsilon}\right)\right|_{L^{2}}^{2} \leqslant \sum_{|k| \geqslant 1}\left(\varepsilon^{2 \varsigma}\left|\langle D\rangle^{\varsigma} e^{i k \frac{\theta}{\varepsilon}} u_{k}\right|_{L^{2}}^{2}+\varepsilon^{2 \varsigma+n}\left|\langle D\rangle^{\frac{n}{2}+\varsigma} e^{i k \frac{\theta}{\varepsilon}} u_{k}\right|_{L^{2}}^{2}\right) \\
\leqslant C_{\theta} \sum_{|k| \geqslant 1}\left(\varepsilon^{2(\varsigma-r)}\left|u_{k}\right|_{H^{\varsigma+r}}^{2}+k^{2 \varsigma}\left|u_{k}\right|_{L^{2}}^{2}+\varepsilon^{2 \varsigma+n}\left|u_{k}\right|_{H^{\frac{n}{2}+\varsigma}}^{2}+k^{n+2 \varsigma}\left|u_{k}\right|_{L^{2}}^{2}\right)
\end{gathered}
$$


Si $\varsigma=+s \geqslant 0$, nous prenons $r=0$. Si $\varsigma=-s \leqslant 0$ avec $0 \leqslant s \leqslant \frac{n}{2}$, nous prenons $r=+s$. Il vient dans les deux cas

$$
\varepsilon^{ \pm 2 s}\left|\langle D\rangle^{ \pm s}\langle\varepsilon D\rangle^{n / 2} u\left(x, \frac{\theta(x)}{\varepsilon}\right)\right|_{L^{2}}^{2} \leqslant C_{\theta}|u|_{C^{\frac{n}{2} \pm s}\left(H^{\frac{n}{2} \pm s}\right)}^{2}
$$

En prenant le $\sup$ en $\varepsilon$, nous obtenons

$$
\left\|\varepsilon^{ \pm s} u\left(x, \frac{\theta(x)}{\varepsilon}\right)\right\|_{ \pm s, \frac{n}{2}, 2} \leqslant C_{\theta}|u(x, \omega)|_{C^{\frac{n}{2} \pm s}\left(H^{\frac{n}{2} \pm s}\right)}
$$

b) Identifions la fonction $2 \pi$-périodique $u(x, \omega) \in C^{\frac{n}{2}+s}\left([0,2 \pi], H^{\frac{n}{2}+s}(\Sigma)\right)$ avec sa série de Fourrier.

$$
u\left(x, \frac{\theta(x)}{\varepsilon}\right)=\sum_{|k| \geqslant 1} e^{i k \frac{\theta(x)}{\varepsilon}} u_{k}(x)
$$

Nous séparons

avec

$$
u\left(x, \frac{\theta(x)}{\varepsilon}\right)=U_{\varepsilon}^{\prime}(x)+U_{\varepsilon}^{\prime \prime}(x)
$$

$$
U_{\varepsilon}^{\prime}(x)=\sum_{|k| \geqslant 1} \sigma_{\frac{k}{\varepsilon}}\left(e^{i k \frac{\theta(x)}{\varepsilon}} u_{k}\right)(x) \quad \text { et } \quad U_{\varepsilon}^{\prime \prime}(x)=\sum_{|k| \geqslant 1}\left(1-\sigma_{\frac{k}{\varepsilon}}\right)\left(e^{i k \frac{\theta(x)}{\varepsilon}} u_{k}\right)(x)
$$

Nous utilisons successivement la propositions 2.3.10 a) :

$$
\begin{aligned}
\left|\langle D\rangle^{s+\frac{n}{2}} U_{\varepsilon}^{\prime}\right|_{L^{2}}^{2} & \leqslant \sum_{|k| \geqslant 1}\left|\langle D\rangle^{s+\frac{n}{2}} \sigma_{\frac{k}{\varepsilon}}\left(e^{i k \frac{\theta}{\varepsilon}} u_{k}\right)\right|_{L^{2}}^{2} \\
& \leqslant C_{\theta} \sum_{|k| \geqslant 1}\left|u_{k}\right|_{H^{\frac{n}{2}+s}}^{2} \\
& \leqslant C_{\theta}|u|_{C^{0}\left(H^{\frac{n}{2}+s}\right)}^{2}
\end{aligned}
$$

puis la proposition 2.3.12 b) :

$$
\begin{aligned}
& \varepsilon^{2 s}\left|\langle D\rangle^{s}\langle\varepsilon D\rangle^{\frac{n}{2}} U_{\varepsilon}^{\prime \prime}\right|_{\mathrm{BMO}}^{2} \leqslant \sum_{|k| \geqslant 1} \varepsilon^{2 s}\left|\langle D\rangle^{s}\langle\varepsilon D\rangle^{\frac{n}{2}}\left(1-\sigma_{\frac{k}{\varepsilon}}\right)\left(e^{i k \frac{\theta}{\varepsilon}} u_{k}\right)\right|_{\mathrm{BMO}}^{2} \\
& \quad \leqslant \sum_{|k| \geqslant 1} \varepsilon^{2 s}\left|\langle D\rangle^{s}\left(1-\sigma_{\frac{k}{\varepsilon}}\right)\left(e^{i k \frac{\theta}{\varepsilon}} u_{k}\right)\right|_{\mathrm{BMO}}^{2}+\sum_{|k| \geqslant 1} \varepsilon^{2 s+n}\left|\langle D\rangle^{s+\frac{n}{2}}\left(1-\sigma_{\frac{k}{\varepsilon}}\right)\left(e^{i k \frac{\theta}{\varepsilon}} u_{k}\right)\right|_{\mathrm{BMO}}^{2} \\
& \quad \leqslant C_{\theta} \sum_{|k| \geqslant 1} k^{2 s+n}\left|u_{k}\right|_{H^{\frac{n}{2}+s}}^{2} \\
& \leqslant C_{\theta}|u|_{C^{\frac{n}{2}+s}\left(H^{\frac{n}{2}+s}\right)}^{2}
\end{aligned}
$$

En prenant le sup en $\varepsilon$, nous obtenons

$$
\left\|U_{\varepsilon}^{\prime}\right\|_{s+\frac{n}{2}, 0,2} \leqslant C_{\theta}|u(x, \omega)|_{C^{0}\left(H^{\frac{n}{2}+s}\right)} \quad \text { et } \quad\left\|\varepsilon^{s} U_{\varepsilon}^{\prime \prime}\right\|_{s, \frac{n}{2}, \infty} \leqslant C_{\theta}|u(x, \omega)|_{C^{\frac{n}{2}+s}\left(H^{\frac{n}{2}+s}\right)}
$$

Le a) ci-dessus nous donne directement

$$
\left\|\varepsilon^{s} u\left(x, \frac{\theta(x)}{\varepsilon}\right)\right\|_{s, \frac{n}{2}, 2} \leqslant C_{\theta}|u(x, \omega)|_{C^{\frac{n}{2}+s}\left(H^{\frac{n}{2}+s}\right)}
$$


Il vient alors

$$
\|\left|\varepsilon^{s} u\left(x, \frac{\theta(x)}{\varepsilon}\right)\right|||_{\left(s, \frac{n}{2}\right)} \leqslant C_{\theta}|u(x, \omega)|_{C^{\frac{n}{2}+s}\left(H^{\frac{n}{2}+s}\right)}
$$

c) Nous utilisons la même décomposition pour $u(x, \omega) \in C^{\frac{n}{2}+s}\left([0,2 \pi], H^{\frac{n}{2}-s}(\Sigma)\right)$, puis majorons avec la propositions 2.3 .10 a) :

$$
\begin{aligned}
\left|\varepsilon^{s}\langle D\rangle^{-s+\frac{n}{2}} U_{\varepsilon}^{\prime}\right|_{L^{2}}^{2} & \leqslant \sum_{|k| \geqslant 1}\left|\varepsilon^{s}\langle D\rangle^{-s+\frac{n}{2}} \sigma_{\frac{k}{\varepsilon}}\left(e^{i k \frac{\theta}{\varepsilon}} u_{k}\right)\right|_{L^{2}}^{2} \\
& \leqslant C_{\theta} \sum_{|k| \geqslant 1}\left|u_{k}\right|_{H^{\frac{n}{2}-s}}^{2} \\
& \leqslant C_{\theta}|u|_{C^{0}\left(H^{\frac{n}{2}-s}\right)}^{2}
\end{aligned}
$$

La proposition 2.3.12 b) donne ensuite :

$$
\begin{aligned}
\left|\varepsilon^{s}\langle D\rangle^{-s}\langle\varepsilon D\rangle^{\frac{n}{2}} U_{\varepsilon}^{\prime \prime}\right|_{\mathrm{BMO}}^{2} \leqslant & \sum_{|k| \geqslant 1} \varepsilon^{2 s}\left|\langle D\rangle^{-s}\langle\varepsilon D\rangle^{\frac{n}{2}}\left(1-\sigma_{\frac{k}{\varepsilon}}\right)\left(e^{i k \frac{\theta}{\varepsilon}} u_{k}\right)\right|_{\mathrm{BMO}}^{2} \\
\leqslant & \sum_{|k| \geqslant 1} \varepsilon^{2 s}\left|\langle D\rangle^{-s}\left(1-\sigma_{\frac{k}{\varepsilon}}\right)\left(e^{i k \frac{\theta}{\varepsilon}} u_{k}\right)\right|_{\mathrm{BMO}}^{2} \\
& \quad+\sum_{|k| \geqslant 1} \varepsilon^{2 s+n} \mid\langle D\rangle^{-s+\frac{n}{2}}\left\langle\left.\left(1-\sigma_{\frac{k}{\varepsilon}}\right)\left(e^{i k \frac{\theta}{\varepsilon}} u_{k}\right)\right|_{\mathrm{BMO}} ^{2}\right. \\
& \leqslant C_{\theta} \sum_{|k| \geqslant 1} k^{2 s+n}\left|u_{k}\right|_{H^{\frac{n}{2}-s}}^{2} \\
& \leqslant C_{\theta}|u|_{C^{\frac{n}{2}+s}\left(H^{\frac{n}{2}-s}\right)}^{2}
\end{aligned}
$$

En prenant le sup en $\varepsilon$, nous obtenons

$$
\left\|\varepsilon^{s} U_{\varepsilon}^{\prime}\right\|_{-s+\frac{n}{2}, 0,2} \leqslant C_{\theta}|u(x, \omega)|_{C^{0}\left(H^{\frac{n}{2}-s}\right)}
$$

puis

et enfin, par le a) ci-dessus,

$$
\left\|\varepsilon^{s} U_{\varepsilon}^{\prime \prime}\right\|_{-s, \frac{n}{2}, \infty} \leqslant C_{\theta}|u(x, \omega)|_{C^{\frac{n}{2}+s}\left(H^{\frac{n}{2}-s}\right)}
$$

$$
\left\|\varepsilon^{-s} u\left(x, \frac{\theta(x)}{\varepsilon}\right)\right\|_{-s, \frac{n}{2}, 2} \leqslant C_{\theta}|u(x, \omega)|_{C^{\frac{n}{2}-s}\left(H^{\frac{n}{2}-s}\right)}
$$

Il en résulte que

$$
\|\left|\varepsilon^{s} u\left(x, \frac{\theta(x)}{\varepsilon}\right)\right|||_{\left(-s, \frac{n}{2}\right)} \leqslant C_{\theta}|u(x, \omega)|_{C^{\frac{n}{2}+s}\left(H^{\frac{n}{2}-s}\right)}
$$

d) Nous utilisons la même décomposition pour $u(x, \omega) \in C^{\frac{n}{2}-s}\left([0,2 \pi], H^{\frac{n}{2}+s}(\Sigma)\right)$, puis majorons avec la propositions 2.3 .10 a) :

$$
\begin{aligned}
\left|\varepsilon^{-s}\langle D\rangle^{-s+\frac{n}{2}} U_{\varepsilon}^{\prime}\right|_{L^{2}}^{2} & \leqslant \sum_{|k| \geqslant 1}\left|\varepsilon^{-s}\langle D\rangle^{-s+\frac{n}{2}} \sigma_{\frac{k}{\varepsilon}}\left(e^{i k \frac{\theta}{\varepsilon}} u_{k}\right)\right|_{L^{2}}^{2} \\
& \leqslant C_{\theta} \sum_{|k| \geqslant 1}\left|u_{k}\right|_{H^{\frac{n}{2}+s}}^{2} \\
& \leqslant C_{\theta}|u|_{C^{0}\left(H^{\frac{n}{2}+s}\right)}^{2}
\end{aligned}
$$


La proposition $2.3 .12 \mathrm{~b}$ ) donne ensuite :

$$
\begin{aligned}
\left|\varepsilon^{-s}\langle D\rangle^{-s}\langle\varepsilon D\rangle^{\frac{n}{2}} U_{\varepsilon}^{\prime \prime}\right|_{\mathrm{BMO}}^{2} & \leqslant \sum_{|k| \geqslant 1} \varepsilon^{-2 s}\left|\langle D\rangle^{-s}\langle\varepsilon D\rangle^{\frac{n}{2}}\left(1-\sigma_{\frac{k}{\varepsilon}}\right)\left(e^{i k \frac{\theta}{\varepsilon}} u_{k}\right)\right|_{\mathrm{BMO}}^{2} \\
\leqslant & \sum_{|k| \geqslant 1} \varepsilon^{-2 s}\left|\langle D\rangle^{-s}\left(1-\sigma_{\frac{k}{\varepsilon}}\right)\left(e^{i k \frac{\theta}{\varepsilon}} u_{k}\right)\right|_{\mathrm{BMO}}^{2} \\
& \quad+\sum_{|k| \geqslant 1} \varepsilon^{-2 s+n} \mid\langle D\rangle^{-s+\frac{n}{2}}\left\langle\left.\left(1-\sigma_{\frac{k}{\varepsilon}}\right)\left(e^{i k \frac{\theta}{\varepsilon}} u_{k}\right)\right|_{\mathrm{BMO}} ^{2}\right. \\
& \leqslant C_{\theta} \sum_{|k| \geqslant 1} k^{-2 s+n}\left|u_{k}\right|_{H^{\frac{n}{2}+s}}^{2} \\
& \leqslant C_{\theta}|u|_{C^{\frac{n}{2}-s}\left(H^{\frac{n}{2}+s}\right)}^{2}
\end{aligned}
$$

En prenant le sup en $\varepsilon$, nous obtenons

$$
\left\|\varepsilon^{-s} U_{\varepsilon}^{\prime}\right\|_{-s+\frac{n}{2}, 0,2} \leqslant C_{\theta}|u(x, \omega)|_{C^{0}\left(H^{\frac{n}{2}+s}\right)}
$$

et

$$
\left\|\varepsilon^{-s} U_{\varepsilon}^{\prime \prime}\right\|_{-s, \frac{n}{2}, \infty} \leqslant C_{\theta}|u(x, \omega)|_{C^{\frac{n}{2}-s}\left(H^{\frac{n}{2}+s}\right)}
$$

Par le a) ci-dessus,

$$
\left\|\varepsilon^{-s} u\left(x, \frac{\theta(x)}{\varepsilon}\right)\right\|_{-s, \frac{n}{2}, 2} \leqslant C_{\theta}|u(x, \omega)|_{C^{\frac{n}{2}-s}\left(H^{\frac{n}{2}-s}\right)}
$$

Il en résulte que

$$
||\left|\varepsilon^{-s} u\left(x, \frac{\theta(x)}{\varepsilon}\right)\right| \|_{\left(-s, \frac{n}{2}\right)} \leqslant C_{\theta}|u(x, \omega)|_{C^{\frac{n}{2}-s}\left(H^{\frac{n}{2}+s}\right)}
$$





\section{CHAPITRE 3}

\section{PROLONGEMENT ASYMPTOTIQUE DES FAMILLES DE SOLUTIONS APPROCHÉES PAR DES FAMILLES DE SOLUTIONS EXACTES}

Le but de cette section est de prouver le résultat suivant :

THÉORÈmE 3 (Prolongement asymptotique d'une famille de solutions approchées par une famille de solutions exactes)

Soit

$$
\left\{\begin{array}{l}
(A, \phi)_{\varepsilon} \in C^{0}\left(\left[0, t_{0}\right], \mathcal{Z}_{\varepsilon}^{\left(\frac{1}{2}, \frac{n}{2}\right)}(\Sigma)\right) \cap C^{1}\left(\left[0, t_{0}\right], \mathcal{Z}_{\varepsilon}^{\left(-\frac{1}{2}, \frac{n}{2}\right)}(\Sigma)\right) \\
(\psi)_{\varepsilon} \in C^{0}\left(\left[0, t_{0}\right], \mathcal{Z}_{\varepsilon}^{\left(0, \frac{n}{2}\right)}(\Sigma)\right)
\end{array}\right.
$$

une solution approchée du système $(Y M)$ en jauge $J_{\lambda}, \lambda \in[0,1]$, à l'ordre $O\left(\varepsilon^{M / 2}\right)$, $M \geqslant n$ :

$$
\left\{\begin{array}{l}
(\mathcal{O})_{\varepsilon}=(L A-F(A, \phi, \psi))_{\varepsilon} \in C^{0}\left(\left[0, t_{0}\right], \varepsilon^{M / 2} \mathcal{W}_{\varepsilon}^{-\frac{1}{2}, \frac{n}{2}, 2}(\Sigma)\right) \\
(\mathcal{P})_{\varepsilon}=(\square \phi-G(A, \phi, \psi))_{\varepsilon} \in C^{0}\left(\left[0, t_{0}\right], \varepsilon^{M / 2} \mathcal{W}_{\varepsilon}^{-\frac{1}{2}, \frac{n}{2}, 2}(\Sigma)\right) \\
(\mathcal{Q})_{\varepsilon}=(\mathcal{D} \psi-h(A, \phi, \psi))_{\varepsilon} \in C^{0}\left(\left[0, t_{0}\right], \varepsilon^{M / 2} \mathcal{W}_{\varepsilon}^{0, \frac{n}{2}, 2}(\Sigma)\right)
\end{array}\right.
$$

et

$$
(\mathcal{J})_{\varepsilon}=\left(J_{\lambda} A\right)_{\varepsilon}=\lambda \alpha^{-2} \nabla_{0} \mathcal{J}_{0}+(1-\lambda) \nabla^{i}\left(\mathcal{J}_{\Sigma}\right)_{i}
$$

avec

$$
\left(\mathcal{J}_{0}, \mathcal{J}_{\Sigma}\right) \in C^{0}\left([0, t], \varepsilon^{M / 2} \mathcal{W}_{\varepsilon}^{\frac{3}{2}, \frac{n}{2}, 2}(\Sigma)\right) \cap C^{1}\left([0, t], \varepsilon^{M / 2} \mathcal{W}_{\varepsilon}^{\frac{1}{2}, \frac{n}{2}, 2}(\Sigma)\right)
$$

Toutes données résiduelles

$$
(Z, z)_{\varepsilon_{\mid t=0}} \in \varepsilon^{M / 2} \mathcal{W}_{\varepsilon}^{\frac{1}{2}, \frac{n}{2}, 2},\left(\nabla_{0} Z, \nabla_{0} z\right)_{\varepsilon_{\mid t=0}} \in \varepsilon^{M / 2} \mathcal{W}_{\varepsilon}^{-\frac{1}{2}, \frac{n}{2}, 2} \text { et } \zeta_{\left.\varepsilon\right|_{t=0}} \in \varepsilon^{M / 2} \mathcal{W}_{\varepsilon}^{0, \frac{n}{2}, 2}
$$

vérifiant les contraintes de compatibilité (67) et de jauge (68), se prolongent sur un intervalle $\left[0, t_{1}\right], 0<t_{1} \leqslant t_{0}$ indépendant de $\varepsilon$, en une unique famille

$$
\left\{\begin{array}{l}
(Z, z)_{\varepsilon} \in C^{0}\left(\left[0, t_{1}\right], \varepsilon^{M / 2} \mathcal{W}_{\varepsilon}^{\frac{1}{2}, \frac{n}{2}, 2}(\Sigma)\right) \cap C^{1}\left(\left[0, t_{1}\right], \varepsilon^{M / 2} \mathcal{W}_{\varepsilon}^{-\frac{1}{2}, \frac{n}{2}, 2}(\Sigma)\right) \\
(\zeta)_{\varepsilon} \in C^{0}\left(\left[0, t_{1}\right], \varepsilon^{M / 2} \mathcal{W}_{\varepsilon}^{0, \frac{n}{2}, 2}(\Sigma)\right)
\end{array}\right.
$$

telle que

$$
(\mathcal{A}, \Phi, \Psi)_{\varepsilon}=(A, \phi, \psi)_{\varepsilon}+(Z, z, \zeta)_{\varepsilon}
$$


forme une famille de solutions exactes de (Y.M.) en jauge $J_{\lambda}$. De plus, si

$$
\begin{aligned}
& \left\|\varepsilon^{-M / 2}(Z, z)_{\varepsilon}(t=0)\right\|_{\frac{1}{2}, \frac{n}{2}, 2} \\
& \quad+\left\|\varepsilon^{-M / 2}\left(\partial_{t} Z, \partial_{t} z\right)_{\varepsilon}(t=0)\right\|_{-\frac{1}{2}, \frac{n}{2}, 2}+\left\|\varepsilon^{-M / 2}(\zeta)_{\varepsilon}(t=0)\right\|_{0, \frac{n}{2}, 2} \leqslant \delta_{M}
\end{aligned}
$$

pour un certain $\delta_{M}$ assez petit, alors $t_{1}=t_{0}$. En particulier, si on prend soin de choisir $M>M^{\prime} \geqslant n$ assez grand pour que $\varepsilon_{0}^{M-M^{\prime}} \leqslant \delta_{M}$, alors le prolongement $(Z, z, \zeta)_{\varepsilon}$ existe et est unique sur $\left[0, t_{0}\right]$ tout entier, et vérifie :

$$
\left\{\begin{array}{l}
(Z, z)_{\varepsilon} \in C^{0}\left(\left[0, t_{0}\right], \varepsilon^{M^{\prime} / 2} \mathcal{W}_{\varepsilon}^{\frac{1}{2}, \frac{n}{2}, 2}(\Sigma)\right) \cap C^{1}\left(\left[0, t_{0}\right], \varepsilon^{M^{\prime} / 2} \mathcal{W}_{\varepsilon}^{-\frac{1}{2}, \frac{n}{2}, 2}(\Sigma)\right) \\
(\zeta)_{\varepsilon} \in C^{0}\left(\left[0, t_{0}\right], \varepsilon^{M^{\prime} / 2} \mathcal{W}_{\varepsilon}^{0, \frac{n}{2}, 2}(\Sigma)\right)
\end{array}\right.
$$

Ce théorème justifie la méthode d'optique géométrique mise en place dans la section précédente. En effet, pour tout réel $s, \varepsilon^{\frac{M}{2}} \mathcal{W}_{\varepsilon}^{s, \frac{n}{2}, 2} \hookrightarrow \varepsilon^{\frac{M}{2}} \mathcal{H}_{\varepsilon}^{s}$ tandis que $\mathcal{Z}_{\varepsilon}^{\left(s, \frac{n}{2}\right)} \hookrightarrow \mathcal{H}_{\varepsilon}^{s}$. Nous sommes donc assurés que le reste $(Z, z, \zeta)_{\varepsilon}$ généré pour résoudre exactement le système $(Y M)$ est toujours d'ordre $\varepsilon^{M / 2}$ dans $\mathcal{H}_{\varepsilon}^{1 / 2} \times \mathcal{H}_{\varepsilon}^{1 / 2} \times \mathcal{H}_{\varepsilon}^{0}$, et reste arbitrairement petit devant la solution approchée $(A, \phi, \psi)_{\varepsilon}$. La solution exacte est fidèlement décrite par le développement que nous en avons fait.

\subsection{Estimations bilinéaires}

La difficulté des résultats de cette section tient au fait que la famille de solutions approchées construite par l'optique géométrique n'est pas uniformément lipschitzienne en $\varepsilon$. Ceci rend délicate l'estimation des produits de type $Z_{\varepsilon} \cdot \partial A_{\varepsilon}$ ou $z_{\varepsilon} \cdot \partial \phi_{\varepsilon}$. Les majorations mises au point dans $[\mathbf{1 1}, \mathbf{1 0}]$, par exemple, ne suffisent plus. Nous pourrions travailler directement dans $\mathcal{H}_{\varepsilon}^{\frac{1}{2}+\frac{n}{2}} \times \mathcal{H}_{\varepsilon}^{\frac{1}{2}+\frac{n}{2}} \times \mathcal{H}_{\varepsilon}^{\frac{n}{2}}$ et utiliser des estimations extrapolées de celles du lemme B.1, mais nous perdons alors le bénéfice de la petitesse du reste devant la solution approchée construite, et réduisons à peu de choses les efforts déployés pour décrire le comportement du système $(Y M)$ par le biais de l'optique géométrique. La marge de manœuvre dont nous disposons est réduite à une demie dérivée, qu'il va falloir exploiter systématiquement par un argument d'analyse adapté. Le résultat central de cette section est exposé dans le lemme suivant :

Proposition 3.1.1 (Estimations bilinéaires uniformes). — Étant donné un entier $M \geqslant n$, un réel $s>0$, il existe une constante $C$ telle que :

a) $\operatorname{si}(U)_{\varepsilon} \in \varepsilon^{M / 2} \mathcal{W}_{\varepsilon}^{s, \frac{n}{2}, 2}$ et $(V)_{\varepsilon} \in \varepsilon^{M / 2} \mathcal{W}_{\varepsilon}^{-s, \frac{n}{2}, 2}$, alors le produit $(U V)_{\varepsilon} \in$ $\varepsilon^{M / 2} \mathcal{W}_{\varepsilon}^{-s, \frac{n}{2}, 2}$ et

$$
\left\|\varepsilon^{-M / 2} U_{\varepsilon} V_{\varepsilon}\right\|_{-s, \frac{n}{2}, 2} \leqslant C\left\|\varepsilon^{-M / 2} U_{\varepsilon}\right\|_{s, \frac{n}{2}, 2}\left\|\varepsilon^{-M / 2} V_{\varepsilon}\right\|_{-s, \frac{n}{2}, 2}
$$

b) $\operatorname{si}(U)_{\varepsilon} \in \varepsilon^{M / 2} \mathcal{W}_{\varepsilon}^{s, \frac{n}{2}, 2}$ et $(V)_{\varepsilon} \in \mathcal{Z}_{\varepsilon}^{\left(-s, \frac{n}{2}\right)}$, alors le produit $(U V)_{\varepsilon} \in \varepsilon^{M / 2} \mathcal{W}_{\varepsilon}^{-s, \frac{n}{2}, 2}$ et

$$
\left\|\varepsilon^{-M / 2} U_{\varepsilon} V_{\varepsilon}\right\|_{-s, \frac{n}{2}, 2} \leqslant C\left\|\varepsilon^{-M / 2} U_{\varepsilon}\right\|_{s, \frac{n}{2}, 2}\left\|\mid V_{\varepsilon}\right\|_{\left(-s, \frac{n}{2}\right)}
$$


c) $\operatorname{si}(U)_{\varepsilon} \in \varepsilon^{M / 2} \mathcal{W}_{\varepsilon}^{-s, \frac{n}{2}, 2}$ et $(V)_{\varepsilon} \in \mathcal{Z}_{\varepsilon}^{\left(s, \frac{n}{2}\right)}$, alors le produit $(U V)_{\varepsilon} \in \varepsilon^{M / 2} \mathcal{W}_{\varepsilon}^{-s, \frac{n}{2}, 2}$ et

$$
\left\|\varepsilon^{-M / 2} U_{\varepsilon} V_{\varepsilon}\right\|_{-s, \frac{n}{2}, 2} \leqslant C\left\|\varepsilon^{-M / 2} U_{\varepsilon}\right\|_{-s, \frac{n}{2}, 2}\|\| V_{\varepsilon} \|_{\left(s, \frac{n}{2}\right)}
$$

3.1.1. Résultats intermédiaires. - Afin d'éclaircir la proposition 3.1.1, nous détaillons un résultat intermédiaire ${ }^{(1)}$ qui fait abstraction du paramètre $\varepsilon$ :

Proposition 3.1.2. - Pour tout réel $s>0$,

a) Si $f \in H^{s}$ et $\langle D\rangle^{-s} g \in L^{\infty}$, alors le produit $f g \in H^{-s}$ et il existe une constante $C>0$ telle que :

$$
|f g|_{H^{-s}} \leqslant C|f|_{H^{s}}\left|\langle D\rangle^{-s} g\right|_{L^{\infty}}
$$

Si $f \in H^{s}$ et $\langle D\rangle^{-s} g \in \mathrm{BMO} \cap L^{2}$, alors le produit $f g \in H^{-s}$ et il existe une constante $C>0$ telle que :

$$
|f g|_{H^{-s}} \leqslant C|f|_{H^{s}}\left(\left|\langle D\rangle^{-s} g\right|_{\mathrm{BMO}}+\left|\langle D\rangle^{-s} g\right|_{L^{2}}\right)
$$

b) Si $f \in H^{-s}$ et $\langle D\rangle^{s} g \in L^{\infty}$, alors le produit $f g \in H^{-s}\left(\mathbb{R}^{n}\right)$ et il existe une constante $C>0$ telle que :

$$
|f g|_{H^{-s}} \leqslant C|f|_{H^{-s}}\left|\langle D\rangle^{s} g\right|_{L^{\infty}}
$$

Si $f \in H^{-s}$ et $\langle D\rangle^{s} g \in \mathrm{BMO} \cap L^{2}$, alors le produit $f g \in H^{-s}\left(\mathbb{R}^{n}\right)$ et il existe une constante $C>0$ telle que :

$$
|f g|_{H^{-s}} \leqslant C|f|_{H^{-s}}\left(\left|\langle D\rangle^{s} g\right|_{\mathrm{BMO}}+\left|\langle D\rangle^{s} g\right|_{L^{2}}\right)
$$

Nous utilisons dans toute cette partie les opérateurs de décomposition diadique introduits section 2.3.2. Nous complétons les résultats du lemme 2.3.5 par les estimations suivantes :

Lemme 3.1.3. - a) Pour tout $s \in \mathbb{R}$, il existe $C>0$, tel que pour tout $k \geqslant 0$ et $u \in \mathrm{BMO}$,

$$
\left|\Delta_{k}\langle D\rangle^{s} u\right|_{L^{\infty}} \leqslant C 2^{k s}\left(|u|_{\mathrm{BMO}}\right)
$$

b) Il existe $C>0$, tel que pour tout $k \geqslant-1$ et $u \in \mathrm{BMO}$,

$$
\left|S_{k} u\right|_{L^{\infty}} \leqslant\left|\Delta_{-1} u\right|_{L^{\infty}}+C k|u|_{\mathrm{BMO}}
$$

Pour tout $s>0$, il existe $C>0$, tel que pour tout $k \geqslant-1$ et $u \in \mathrm{BMO}$,

$$
\left.\left|S_{k}\langle D\rangle^{s} u\right|_{L^{\infty}} \leqslant\left|\Delta_{-1}\langle D\rangle^{s} u\right|_{L^{\infty}}+C 2^{k s}|u|_{\mathrm{BMO}}\right)
$$

Pour tout $s<0$, il existe $C>0$, tel que pour tout $k \geqslant-1$ et $u \in \mathrm{BMO}$,

$$
\left|S_{k}\langle D\rangle^{s} u\right|_{L^{\infty}} \leqslant\left|\Delta_{-1}\langle D\rangle^{s} u\right|_{L^{\infty}}+C|u|_{\mathrm{BMO}}
$$

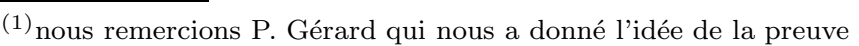


Démonstration. — C'est une conséquence de la caractérisation diadique de BMO. Se reporter directement à l'annexe $\mathrm{A}$.

Nous aurons aussi besoin d'un résultat un peu moins standard, que nous énonçons en élargissant légèrement le cadre des décompositions diadiques précédentes. C'est ici qu'apparaît de façon naturelle l'espace BMO.

Proposition 3.1.4 (Stein et Coifman-Meyer). - Soit $\widetilde{\phi}_{k}(\xi), k \geqslant 0$, une suite de fonctions vérifiant la condition de sommabilité $\sum_{k \geqslant 0}\left|\widetilde{\phi}_{k}\right|^{2} \leqslant \widetilde{C}$, et telle que la famille $\widetilde{\Phi}_{k}=\widetilde{\phi}_{k}\left(2^{k} \cdot\right)$ est supportée dans une couronne fixe $\{1 / R \leqslant|\xi| \leqslant R\}$ et reste uniformément bornée dans $C^{\infty}$. Soit $\widetilde{\psi}_{k}(\xi), k \geqslant 0$, une suite de fonctions telle que la famille $\widetilde{\Psi}_{k}=\widetilde{\psi}_{k}\left(2^{k} \cdot\right)$ est supportée dans une boule fixe $\{|\xi| \leqslant R\}$ et reste uniformément bornée dans $C^{\infty}$. Nous posons $\widetilde{S}_{k} u=\widetilde{\psi}_{k}(D) u$ et $\widetilde{\Delta}_{k} v=\widetilde{\phi}_{k}(D) v$. Si $u \in L^{2}$ et $v \in \mathrm{BMO}$, alors :

$$
\sum_{k} \int\left|\widetilde{\Delta}_{k}(D) v(y)\right|^{2}\left|\widetilde{S}_{k}(D) u(y)\right|^{2} d y \leqslant C|u|_{L^{2}}^{2}|v|_{\mathrm{BMO}}^{2}
$$

De plus, la constante $C$ ne dépend que d'un nombre fini de semi-normes des $\widetilde{\Phi}_{k}$ et $\widetilde{\Psi}_{k}$ dans $C_{0}^{\infty}$.

Démonstration de la proposition 3.1.4. — Cette proposition est énoncée dans [7], démonstration du théorème 33 , pour des décompositions diadiques dépendant d'un paramètre continu. La démonstration originale s'appuie sur [8], et peut-être lue dans [17]. Nous la reprenons dans l'Annexe A.

Démonstration de la proposition 3.1.2. - Le produit de deux fonctions $f$ et $g$ se décompose en :

$$
f g=T_{f} g+T_{g} f+R(f, g)
$$

où

$$
T_{f} g=\sum_{k \geqslant 2} S_{k-2} f \Delta_{k} g, \quad T_{g} f=\sum_{k \geqslant 2} S_{k-2} g \Delta_{k} f \quad \text { et } \quad R(f, g)=\sum_{\left|k-k^{\prime}\right| \leqslant 2} \Delta_{k^{\prime}} f \Delta_{k} g
$$

Démonstration $d u$ a). - Commençons par examiner le terme $T_{g} f$ (hautes fréquences en $f$, basses fréquences en $g$ ). Le spectre de chaque terme $S_{k-2} g \Delta_{k} f$ est supporté dans une couronne $2^{k-2} \leqslant|\xi| \leqslant 2^{k+2}$. En considérant une fonction test $h \in \mathcal{S},|h|_{L^{2}} \leqslant 1$, il vient

$$
\begin{aligned}
\left|\left\langle\sum_{k \geqslant 2} S_{k-2} g \Delta_{k} f, h\right\rangle\right| & \leqslant \sum_{\left|k-k^{\prime}\right| \leqslant 3}\left\langle\left|S_{k-2} g \Delta_{k} f\right|,\left|\Delta_{k^{\prime}} h\right|\right\rangle \\
& \leqslant C \sum_{\left|k-k^{\prime}\right| \leqslant 3}\left\langle\left|2^{k s} \Delta_{k} f 2^{-k s} S_{k-2} g\right|,\left|\Delta_{k^{\prime}} h\right|\right\rangle \\
& \leqslant C\left(\sum_{k}\left|2^{k s} \Delta_{k} f\right|_{L^{2}}^{2}\right)^{1 / 2}\left(\sum_{\left|k-k^{\prime}\right| \leqslant 5}\left|2^{-k s} S_{k} g \Delta_{k^{\prime}} h\right|_{L^{2}}^{2}\right)^{1 / 2}
\end{aligned}
$$


Par le lemme 3.1.3 b) lorsque $s>0$ :

$$
\begin{aligned}
\sum_{\left|k-k^{\prime}\right| \leqslant 5}\left|2^{-k s} S_{k} g \Delta_{k^{\prime}} h\right|_{L^{2}}^{2} & \leqslant C \sum_{\left|k-k^{\prime}\right| \leqslant 5}\left|2^{-k s} S_{k} g\right|_{L^{\infty}}^{2}\left|\Delta_{k^{\prime}} h\right|_{L^{2}}^{2} \\
& \leqslant\left(\left|\Delta_{-1}\langle D\rangle^{-s} g\right|_{L^{\infty}}+C\left|\langle D\rangle^{-s} g\right|_{\mathrm{BMO}}\right)^{2} \sum_{k}\left|\Delta_{k} h\right|_{L^{2}}^{2}
\end{aligned}
$$

Par le lemme 2.3.5 b), il vient que

$$
\sum_{k}\left|\Delta_{k} h\right|_{L^{2}}^{2} \leqslant C|h|_{L^{2}}^{2} \leqslant 1 \quad \text { et } \sum\left|2^{k s} \Delta_{k} f\right|_{L^{2}}^{2} \leqslant C|f|_{H^{s}}^{2}
$$

Selon l'estimation désirée, il reste à majorer

$$
\left|\Delta_{-1}\langle D\rangle^{-s} g\right|_{L^{\infty}}+C\left|\langle D\rangle^{-s} g\right|_{\mathrm{BMO}} \leqslant C\left|\langle D\rangle^{-s} g\right|_{L^{\infty}}
$$

ou

$$
\left|\Delta_{-1}\langle D\rangle^{-s} g\right|_{L^{\infty}} \leqslant C\left|\Delta_{-1}\langle D\rangle^{-s} g\right|_{H^{\frac{n}{2}+\epsilon}} \leqslant C\left|\langle D\rangle^{-s} g\right|_{L^{2}}
$$

nous en déduisons immédiatement

$$
\left|T_{g} f\right|_{L^{2}} \leqslant C|f|_{H^{s}}\left|\langle D\rangle^{-s} g\right|_{L^{\infty}}
$$

ou encore

$$
\left|T_{g} f\right|_{L^{2}} \leqslant C|f|_{H^{s}}\left(\left|\langle D\rangle^{-s} g\right|_{L^{2}}+\left|\langle D\rangle^{-s} g\right|_{\mathrm{BMO}}\right)
$$

Le terme de reste $R(f, g)$ (même ordre de fréquences pour $f$ et $g$ ) est nettement plus difficile à traiter. C'est ici qu'il est nécessaire d'introduire les estimations du lemme 3.1.4 et que l'on voit que $\langle D\rangle^{-s} g$ doit être bornée dans BMO. Considérons une fonction test $h \in \mathcal{S}$ vérifiant $|h|_{L^{2}} \leqslant 1$. Le spectre de chaque terme $\Delta_{k^{\prime}} f \Delta_{k} g$ est supporté dans une boule $|\xi| \leqslant 2^{k+4}$ lorsque $\left|k-k^{\prime}\right| \leqslant 2$, et il en résulte que :

$$
\begin{aligned}
\left|\left\langle\sum_{\left|k-k^{\prime}\right| \leqslant 2} \Delta_{k^{\prime}} f \Delta_{k} g, h\right\rangle\right| & \leqslant \sum_{\left|k-k^{\prime}\right| \leqslant 2}\left\langle\left|\Delta_{k^{\prime}} f \Delta_{k} g\right|,\left|S_{k+5} h\right|\right\rangle \\
& \leqslant C \sum_{\left|k-k^{\prime}\right| \leqslant 2}\left\langle\left|2^{k^{\prime} s} \Delta_{k^{\prime}} f 2^{-k s} \Delta_{k} g\right|,\left|S_{k+5} h\right|\right\rangle \\
& \left.\leqslant\left. C\left(\sum_{k} \mid 2^{k s} \Delta_{k} f\right)\right|_{L^{2}} ^{2}\right)^{1 / 2}\left(\sum_{k}\left|2^{-k s} \Delta_{k} g S_{k+5} h\right|_{L^{2}}^{2}\right)^{1 / 2}
\end{aligned}
$$

Pour tout $s \in \mathbb{R}$ et $k \geqslant 0$, posons $\widetilde{\phi}_{k}(\xi)=\left(2^{-2 k}+\left|2^{-k} \xi\right|^{2}\right)^{s / 2} \phi\left(2^{-k} \xi\right)$ et $\widetilde{\Delta}_{k} u=$ $\widetilde{\phi}_{k}(D) u$, de sorte que $2^{-k s} \Delta_{k} g=\widetilde{\Delta}_{k}\left(\langle D\rangle^{-s} g\right)$. Les fonctions $\widetilde{\Phi}_{k}(\xi)=\widetilde{\phi}_{k}\left(2^{k} \xi\right)$ sont uniformément bornées dans $C_{0}^{\infty}$ et supportées dans une couronne fixe. Nous définissons ainsi une décomposition diadique en couronnes légèrement modifiée, mais vérifiant toujours la bonne propriété de sommation : $\sum\left|\widetilde{\phi}_{k}(\xi)\right|^{2} \leqslant C$. Notons $S_{k+5} h=$ $\widetilde{S}_{k} h$. Nous sommes ainsi amenés à estimer

$$
\sum_{k}\left|\widetilde{\Delta}_{k}\left(\langle D\rangle^{-s} g\right) \widetilde{S}_{k} h\right|_{L^{2}}^{2}
$$


Or ce terme entre précisément dans le cadre du lemme 3.1.4 :

$$
\sum_{k}\left|\widetilde{\Delta}_{k}\left(\langle D\rangle^{-s} g\right) \widetilde{S}_{k} h\right|_{L^{2}}^{2} \leqslant C|h|_{L^{2}}^{2}\left|\langle D\rangle^{-s} g\right|_{\mathrm{BMO}}^{2}
$$

enfin,

$$
\sum_{k \geqslant 0}\left|2^{k s} \Delta_{k} f\right|_{L^{2}}^{2} \leqslant C|f|_{H^{s}}^{2}
$$

d'où l'estimation désirée :

$$
|R(f, g)|_{L^{2}} \leqslant C|f|_{H^{s}}\left|\langle D\rangle^{-s} g\right|_{\mathrm{BMO}}
$$

Pour le terme $T_{f} g$ (basses fréquences en $f$ et hautes fréquences en $g$ ), nous procédons de façon semblable avec une fonction test $h \in \mathcal{S},|h|_{H^{s}} \leqslant 1$, et en remarquant que le spectre de chaque terme $S_{k-2} f \Delta_{k} g$ est supporté dans une couronne $2^{k-2} \leqslant|\xi| \leqslant 2^{k+2}:$

$$
\begin{aligned}
\left|\left\langle\sum_{k \geqslant 2} S_{k-2} f \Delta_{k} g, h\right\rangle\right| & \leqslant C \sum_{\left|k-k^{\prime}\right| \leqslant 3}\left\langle\left|S_{k-2} f \Delta_{k} g\right|,\left|\Delta_{k}^{\prime} h\right|\right\rangle \\
& \leqslant C \sum_{\left|k-k^{\prime}\right| \leqslant 3}\left\langle\left|S_{k-2} f 2^{-k s} \Delta_{k} g\right|,\left|2^{k^{\prime} s} \Delta_{k}^{\prime} h\right|\right\rangle \\
& \leqslant C\left(\sum_{k \geqslant 2}\left|S_{k-2} f 2^{-k s} \Delta_{k} g\right|_{L^{2}}^{2}\right)^{1 / 2}\left(\sum_{k \geqslant 0}\left|2^{k s} \Delta_{k} h\right|_{L^{2}}^{2}\right)^{1 / 2}
\end{aligned}
$$

Comme précédemment (en notant cette fois $\widetilde{S}_{k}=S_{k-2}$, et sans changer la définition des $\left.\widetilde{\Delta}_{k}\right)$, nous appliquons le lemme 3.1.4 :

$$
\begin{aligned}
\sum_{k \geqslant 2}\left|S_{k-2} f 2^{-k s} \Delta_{k} g\right|_{L^{2}}^{2} & =\sum_{k}\left|\widetilde{S}_{k} f \widetilde{\Delta}_{k}\left(\langle D\rangle^{-s} g\right)\right|_{L^{2}}^{2} \\
& \leqslant C|f|_{L^{2}}^{2}\left|\langle D\rangle^{-s} g\right|_{\mathrm{BMO}}^{2}
\end{aligned}
$$

Enfin,

$$
\sum_{k \geqslant 0}\left|2^{k s} \Delta_{k} h\right|_{L^{2}}^{2} \leqslant C|h|_{H^{s}}^{2}
$$

et il vient

$$
\left|T_{f} g\right|_{H^{-s}} \leqslant C|f|_{L^{2}}\left|\langle D\rangle^{-s} g\right|_{\mathrm{BMO}}
$$

ce qui achève la preuve du a) de la proposition 3.1.2. La preuve du b) est symétrique.

Démonstration de la proposition 3.1.1. - Nous tenons compte, cette fois, de la dépendance en $\varepsilon$ des termes du produit. C'est ici que nous tirons bénéfice d'avoir résolu le système à l'ordre $\varepsilon^{M / 2}, M \geqslant n$. 
Démonstration $d u$ a). — Nous décomposons le produit $U_{\varepsilon} V_{\varepsilon}$ en :

$$
U_{\varepsilon} V_{\varepsilon}=T_{U_{\varepsilon}} V_{\varepsilon}+T_{V_{\varepsilon}} U_{\varepsilon}+R\left(U_{\varepsilon}, V_{\varepsilon}\right)
$$

Commençons par examiner le terme $T_{V_{\varepsilon}} U_{\varepsilon}$ (hautes fréquences en $U_{\varepsilon}$, basses fréquences en $\left.V_{\varepsilon}\right)$. Le spectre de chaque terme $S_{k-2} U_{\varepsilon} \Delta_{k} V_{\varepsilon}$ est supporté dans une couronne $2^{k-2} \leqslant|\xi| \leqslant 2^{k+2}$. En considérant une famille de fonctions test $(h)_{\varepsilon}$ de $\mathcal{S}$, vérifiant $\sup _{0<\varepsilon \leqslant \varepsilon_{0}}\left|\langle\varepsilon D\rangle^{-n / 2}\langle D\rangle^{s} h\right|_{L^{2}} \leqslant 1$, il vient pour tout $\left.\left.\varepsilon \in\right] 0, \varepsilon_{0}\right]$ :

$$
\begin{aligned}
& \left|\left\langle\sum_{k \geqslant 2} S_{k-2} V_{\varepsilon} \Delta_{k} U_{\varepsilon}, h_{\varepsilon}\right\rangle\right| \leqslant \sum_{\left|k-k^{\prime}\right| \leqslant 3}\left\langle\left|S_{k-2} V_{\varepsilon} \Delta_{k} U_{\varepsilon}\right|,\left|\Delta_{k^{\prime}} h_{\varepsilon}\right|\right\rangle \\
& \leqslant C \sum_{\left|k-k^{\prime}\right| \leqslant 3}\left\langle\left|2^{k s}\left\langle\varepsilon 2^{k}\right\rangle^{n / 2} \Delta_{k} U_{\varepsilon} 2^{-k s} S_{k-2} V_{\varepsilon}\right|,\left|\left\langle\varepsilon 2^{k^{\prime}}\right\rangle^{-n / 2} \Delta_{k^{\prime}} h_{\varepsilon}\right|\right\rangle \\
& \quad \leqslant C\left(\sum_{k}\left|2^{k s}\left\langle\varepsilon 2^{k}\right\rangle^{n / 2} \Delta_{k} U_{\varepsilon}\right|_{L^{2}}^{2}\right)^{1 / 2}\left(\sum_{\left|k-k^{\prime}\right| \leqslant 5}\left|2^{-k s} S_{k-2} V_{\varepsilon}\left\langle\varepsilon 2^{k^{\prime}}\right\rangle^{-n / 2} \Delta_{k^{\prime}} h_{\varepsilon}\right|_{L^{2}}^{2}\right)^{1 / 2}
\end{aligned}
$$

Par le lemme $3.1 .3 \mathrm{~b}$ ) (avec $s>0$ ) et 2.3 .5 b') :

$$
\begin{aligned}
\sum_{\left|k-k^{\prime}\right| \leqslant 5} & \left|2^{-k s} S_{k} V_{\varepsilon}\left\langle\varepsilon 2^{k^{\prime}}\right\rangle^{-n / 2} \Delta_{k^{\prime}} h_{\varepsilon}\right|_{L^{2}}^{2} \leqslant C \sum_{\left|k-k^{\prime}\right| \leqslant 5}\left|2^{-k s} S_{k-2} V_{\varepsilon}\right|_{L^{\infty}}^{2}\left|\Delta_{k^{\prime}}\langle\varepsilon D\rangle^{-n / 2} h_{\varepsilon}\right|_{L^{2}}^{2} \\
& \leqslant\left. C\left(\left|\Delta_{-1}\langle D\rangle^{-s} V_{\varepsilon}\right|_{L^{\infty}}+\left|\langle D\rangle^{-s} V_{\varepsilon}\right|_{\mathrm{BMO}}\right)^{2}\left|\sum_{k} k 2^{-2 k s}\right| \Delta_{k}\langle D\rangle^{s}\langle\varepsilon D\rangle^{-n / 2} h_{\varepsilon}\right|_{L^{2}} ^{2} \\
& \leqslant C\left(\left|\langle D\rangle^{-s} V_{\varepsilon}\right|_{L^{2}}+\left|\langle D\rangle^{-s} V_{\varepsilon}\right|_{\mathrm{BMO}}\right)^{2}\left|\langle D\rangle^{s}\langle\varepsilon D\rangle^{-n / 2} h_{\varepsilon}\right|_{L^{2}}^{2} \\
& \leqslant C\left(\left|\langle D\rangle^{-s} V_{\varepsilon}\right|_{L^{2}}+\left|\langle D\rangle^{-s} V_{\varepsilon}\right|_{\mathrm{BMO}}\right)^{2}
\end{aligned}
$$

Toujours par le lemme 2.3.5 b) et b'), nous savons que

$$
\sum\left|2^{k s}\left\langle\varepsilon 2^{k}\right\rangle^{n / 2} \Delta_{k} U_{\varepsilon}\right|_{L^{2}}^{2} \leqslant C\left|\langle\varepsilon D\rangle^{n / 2} U_{\varepsilon}\right|_{H^{s}}^{2}
$$

Il vient alors pour tout $\left.\varepsilon \in] 0, \varepsilon_{0}\right]$ :

$$
\left|\langle\varepsilon D\rangle^{n / 2}\left(T_{V_{\varepsilon}} U_{\varepsilon}\right)\right|_{H^{-s}} \leqslant C\left|\langle\varepsilon D\rangle^{n / 2} U_{\varepsilon}\right|_{H^{s}}\left(\left|\langle D\rangle^{-s} V_{\varepsilon}\right|_{L^{2}}+\left|\langle D\rangle^{-s} V_{\varepsilon}\right|_{\mathrm{BMO}}\right)
$$

Pour le terme de reste $R\left(U_{\varepsilon}, V_{\varepsilon}\right)$ (même ordre de fréquences pour $U_{\varepsilon}$ et $V_{\varepsilon}$ ), considérons une famille de fonctions test $(h)_{\varepsilon}$ de $\mathcal{S}$ vérifiant $\sup _{\left.\varepsilon \in] 0, \varepsilon_{0}\right]}\left|\langle\varepsilon D\rangle^{-n / 2} h\right|_{L^{2}} \leqslant 1$. Le spectre de chaque terme $\Delta_{k^{\prime}} U_{\varepsilon} \Delta_{k} V_{\varepsilon}$ est supporté dans une boule $|\xi| \leqslant 2^{k+4}$ lorsque $\left|k-k^{\prime}\right| \leqslant 2$, et il en résulte que :

$$
\begin{aligned}
& \left|\left\langle\sum_{\left|k-k^{\prime}\right| \leqslant 2} \Delta_{k} U_{\varepsilon} \Delta_{k^{\prime}} V_{\varepsilon}, h_{\varepsilon}\right\rangle\right| \leqslant \sum_{\left|k-k^{\prime}\right| \leqslant 2}\left\langle\left|\Delta_{k^{\prime}} U_{\varepsilon} \Delta_{k} V_{\varepsilon}\right|,\left|S_{k+5} h_{\varepsilon}\right|\right\rangle \\
& \leqslant C \sum_{\left|k-k^{\prime}\right| \leqslant 2}\left\langle\left|2^{k^{\prime} s}\left\langle\varepsilon 2^{k^{\prime}}\right\rangle^{\frac{n}{2}} \Delta_{k^{\prime}} U_{\varepsilon} 2^{-k s} \Delta_{k} V_{\varepsilon}\right|,\left|\left\langle\varepsilon 2^{k+5}\right\rangle^{-n / 2} S_{k+5} h_{\varepsilon}\right|\right\rangle \\
& \leqslant C\left(\sum_{k}\left|2^{k s}\left\langle\varepsilon 2^{k}\right\rangle^{\frac{n}{2}} \Delta_{k} U_{\varepsilon}\right|_{L^{2}}^{2}\right)^{1 / 2}\left(\sum_{k}\left|2^{-k s} \Delta_{k} V_{\varepsilon}\left\langle\varepsilon 2^{k+5}\right\rangle^{-n / 2} S_{k+5} h_{\varepsilon}\right|_{L^{2}}^{2}\right)^{1 / 2}
\end{aligned}
$$


Pour tout $s \in \mathbb{R}$ et $k \geqslant 0$, posons de nouveau $\widetilde{\phi}_{k}(\xi)=\left(2^{-2 k}+\left|2^{-k} \xi\right|^{2}\right)^{s / 2} \phi\left(2^{-k} \xi\right)$. Nous vérifions que $\sum_{k}\left|\widetilde{\phi}_{k}\right|^{2} \leqslant \widetilde{C}$. Les fonctions dilatées $\widetilde{\Phi}_{k}(\xi)=\widetilde{\phi}_{k}\left(2^{k} \xi\right)$ sont supportée dans une même couronne et restent bornées dans $C_{0}^{\infty}$, uniformément en $k$. Posons $\widetilde{\Delta}_{k} u=\widetilde{\phi}_{k}(D) u$, de sorte que $2^{-k s} \Delta_{k} V_{\varepsilon}=\widetilde{\Delta}_{k}\left(\langle D\rangle^{-s} V_{\varepsilon}\right)$. Nous introduisons une nouvelle famille de fonctions $\widetilde{\psi}_{k}(\xi)=\left\langle\varepsilon 2^{k+5}\right\rangle^{-n / 2}\langle\varepsilon \xi\rangle^{\frac{n}{2}} \psi\left(2^{-(k+5)} \xi\right)$, dépendant cette fois du paramètre $\varepsilon$. Les fonctions dilatées $\widetilde{\Psi}_{k}(\xi)=\widetilde{\psi}_{k}\left(2^{k} \xi\right)$ sont supportées dans une même boule et restent bornées dans $C_{0}^{\infty}$, uniformément en $k$ et en $\varepsilon$. Nous notons $\widetilde{S}_{k}=\widetilde{\psi}_{k}(D)$, de sorte que $\left\langle\varepsilon 2^{k+5}\right\rangle^{-n / 2} S_{k+5} h_{\varepsilon}=\widetilde{S}_{k}\left(\langle\varepsilon D\rangle^{-n / 2} h_{\varepsilon}\right)$. Nous sommes ainsi amenés à estimer

$$
\sum_{k}\left|\widetilde{\Delta}_{k}\left(\langle D\rangle^{-s} V_{\varepsilon}\right) \widetilde{S}_{k}\left(\langle\varepsilon D\rangle^{-n / 2} h_{\varepsilon}\right)\right|_{L^{2}}^{2}
$$

Or ce terme entre précisément dans le cadre du lemme 3.1.4, et il existe une constante $C$, ne dépendant que d'un nombre fini de semi-normes de $\widetilde{\Psi}_{k}$ et de $\widetilde{\Phi}_{k}$ dans $C_{0}^{\infty}$, donc indépendante de $\varepsilon$, telle que :

$$
\sum_{k}\left|\widetilde{\Delta}_{k}\left(\langle D\rangle^{-s} V_{\varepsilon}\right) \widetilde{S}_{k}\left(\langle\varepsilon D\rangle^{-n / 2} h_{\varepsilon}\right)\right|_{L^{2}}^{2} \leqslant C\left|\langle\varepsilon D\rangle^{-n / 2} h_{\varepsilon}\right|_{L^{2}}^{2}\left|\langle D\rangle^{-s} V_{\varepsilon}\right|_{\mathrm{BMO}}^{2}
$$

Par ailleurs, le lemme 2.3.5 b) et b') nous donne :

$$
\sum_{k \geqslant 0}\left|2^{k s}\left\langle\varepsilon 2^{k}\right\rangle^{\frac{n}{2}} \Delta_{k} U_{\varepsilon}\right|_{L^{2}}^{2} \leqslant C\left|\langle\varepsilon D\rangle^{\frac{n}{2}} U_{\varepsilon}\right|_{H^{s}}^{2}
$$

Nous obtenons alors l'estimation désirée :

$$
\left|\langle\varepsilon D\rangle^{\frac{n}{2}} R\left(U_{\varepsilon}, V_{\varepsilon}\right)\right|_{L^{2}} \leqslant C\left|\langle\varepsilon D\rangle^{\frac{n}{2}} U_{\varepsilon}\right|_{H^{s}}\left|\langle D\rangle^{-s} V_{\varepsilon}\right|_{\text {BMO }}
$$

Pour le terme $T_{U_{\varepsilon}} V_{\varepsilon}$ (basses fréquences en $U_{\varepsilon}$ et hautes fréquences en $V_{\varepsilon}$ ), nous considérons une famille de fonctions test $(h)_{\varepsilon}$ de $\mathcal{S}$, telle que

$$
\sup _{\left.\varepsilon \in] 0, \varepsilon_{0}\right]}\left|\langle D\rangle^{S}\langle\varepsilon D\rangle^{-n / 2} h\right|_{L^{2}} \leqslant 1 \text {. }
$$

Le spectre de chaque terme $S_{k-2} U_{\varepsilon} \Delta_{k} V_{\varepsilon}$ est supporté dans une couronne $2^{k-2} \leqslant$ $|\xi| \leqslant 2^{k+2}:$

$$
\begin{aligned}
& \left|\left\langle\sum_{k \geqslant 2} S_{k-2} U_{\varepsilon} \Delta_{k} V_{\varepsilon}, h_{\varepsilon}\right\rangle\right| \leqslant C \sum_{\left|k-k^{\prime}\right| \leqslant 3}\left\langle\left|S_{k-2} U_{\varepsilon} \Delta_{k} V_{\varepsilon}\right|,\left|\Delta_{k}^{\prime} h_{\varepsilon}\right|\right\rangle \\
& \quad \leqslant C \sum_{\left|k-k^{\prime}\right| \leqslant 3}\left\langle\left|S_{k-2} U_{\varepsilon} 2^{-k s}\left\langle\varepsilon 2^{k}\right\rangle^{\frac{n}{2}} \Delta_{k} V_{\varepsilon}\right|,\left|2^{k^{\prime} s}\left\langle\varepsilon 2^{k^{\prime}}\right\rangle^{-n / 2} \Delta_{k}^{\prime} h_{\varepsilon}\right|\right\rangle \\
& \quad \leqslant C\left(\sum_{k \geqslant 2}\left|S_{k-2} U_{\varepsilon} 2^{-k s}\left\langle\varepsilon 2^{k}\right\rangle^{\frac{n}{2}} \Delta_{k} V_{\varepsilon}\right|_{L^{2}}^{2}\right)^{1 / 2}\left(\sum_{k \geqslant 0}\left|2^{k s}\left\langle\varepsilon 2^{k}\right\rangle^{-n / 2} \Delta_{k} h_{\varepsilon}\right|_{L^{2}}^{2}\right)^{1 / 2}
\end{aligned}
$$

Par le lemme 2.3.5 d), b) et b') :

$$
\begin{aligned}
\sum_{k \geqslant 2}\left|S_{k-2} U_{\varepsilon} 2^{-k s}\left\langle\varepsilon 2^{k}\right\rangle^{\frac{n}{2}} \Delta_{k} V_{\varepsilon}\right|_{L^{2}}^{2} & \leqslant C\left|U_{\varepsilon}\right|_{L^{\infty}}^{2} \sum_{k}\left|\Delta_{k}\langle\varepsilon D\rangle^{\frac{n}{2}} 2^{-2 k s} V_{\varepsilon}\right|_{L^{2}}^{2} \\
& \leqslant C\left|U_{\varepsilon}\right|_{L^{\infty}}^{2}\left|\langle\varepsilon D\rangle^{\frac{n}{2}} V_{\varepsilon}\right|_{H^{-s}}^{2}
\end{aligned}
$$

MÉMOIRES DE LA SMF 90 
Par le lemme 2.3.5 b) et b') nous majorons de nouveau :

$$
\sum_{k}\left|2^{k s}\left\langle\varepsilon 2^{k}\right\rangle^{-n / 2} \Delta_{k} h_{\varepsilon}\right|_{L^{2}}^{2} \leqslant C\left|\langle\varepsilon D\rangle^{-n / 2} h_{\varepsilon}\right|_{H^{s}}^{2}
$$

et nous obtenons immédiatement l'estimation :

$$
\left|\langle\varepsilon D\rangle^{n / 2}\left(T_{U_{\varepsilon}} V_{\varepsilon}\right)\right|_{H^{-s}} \leqslant C\left|\langle\varepsilon D\rangle^{n / 2} V_{\varepsilon}\right|_{H^{-s}}\left|U_{\varepsilon}\right|_{L^{\infty}}
$$

En rassemblant les trois termes que nous venons d'estimer, nous disposons, pour tout $\left.\varepsilon \in] 0, \varepsilon_{0}\right]$, de l'estimation uniforme suivante :

$$
\begin{aligned}
& \quad\left|\langle\varepsilon D\rangle^{\frac{n}{2}}\left(U_{\varepsilon} V_{\varepsilon}\right)\right|_{H^{-s}} \\
& \quad \leqslant C\left(\left|\langle\varepsilon D\rangle^{\frac{n}{2}} U_{\varepsilon}\right|_{H^{s}}\left(\left|\langle D\rangle^{-s} V_{\varepsilon}\right|_{L^{2}}+\left|\langle D\rangle^{-s} V_{\varepsilon}\right|_{\mathrm{BMO}}\right)+\left|\langle\varepsilon D\rangle^{\frac{n}{2}} V_{\varepsilon}\right|_{H^{-s}}\left|U_{\varepsilon}\right|_{L^{\infty}}\right)
\end{aligned}
$$

Or $s>0$, donc on utilise l'injection de Sobolev ordinaire :

$$
\begin{aligned}
\left|U_{\varepsilon}\right|_{L^{\infty}} & \leqslant C\left|\langle D\rangle^{\frac{n}{2}+s} U_{\varepsilon}\right|_{L^{2}} \\
& \leqslant C \varepsilon^{-n / 2}\left|\langle\varepsilon D\rangle^{n / 2} U_{\varepsilon}\right|_{H^{s}}
\end{aligned}
$$

Puis on applique l'injection de Sobolev limite de $H^{n / 2}$ dans BMO :

$$
\begin{aligned}
\left|\langle D\rangle^{-s} V_{\varepsilon}\right|_{\mathrm{BMO}} & \leqslant C\left|\langle D\rangle^{n / 2}\langle D\rangle^{-s} V_{\varepsilon}\right|_{L^{2}} \\
& \leqslant C \varepsilon^{-n / 2}\left|\langle\varepsilon D\rangle^{n / 2}\langle D\rangle^{-s} V_{\varepsilon}\right|_{L^{2}}
\end{aligned}
$$

Nous obtenons ainsi, pour tout $\left.\varepsilon \in] 0, \varepsilon_{0}\right]$, l'estimation

$$
\begin{aligned}
& \left|\langle\varepsilon D\rangle^{\frac{n}{2}}\left(U_{\varepsilon} V_{\varepsilon}\right)\right|_{H^{-s}} \\
& \quad \leqslant C\left(\left|\langle\varepsilon D\rangle^{\frac{n}{2}} U_{\varepsilon}\right|_{H^{s}}\left|\varepsilon^{-n / 2}\langle\varepsilon D\rangle^{\frac{n}{2}} V_{\varepsilon}\right|_{H^{-s}}+\left|\langle\varepsilon D\rangle^{\frac{n}{2}} V_{\varepsilon}\right|_{H^{-s}}\left|\varepsilon^{-n / 2}\langle\varepsilon D\rangle^{\frac{n}{2}} U_{\varepsilon}\right|_{H^{s}}\right)
\end{aligned}
$$

En multipliant chaque membre par $\varepsilon^{-M / 2}, M \geqslant n$, puis en prenant le sup sur $\varepsilon \in$ ] $\left.0, \varepsilon_{0}\right]$, nous déduisons

$$
\left\|\varepsilon^{-M / 2} U_{\varepsilon} V_{\varepsilon}\right\|_{-s, \frac{n}{2}, 2} \leqslant C\left\|\varepsilon^{-M / 2} U_{\varepsilon}\right\|_{s, \frac{n}{2}, 2}\left\|\varepsilon^{-M / 2} V_{\varepsilon}\right\|_{-s, \frac{n}{2}, 2}
$$

Démonstration $d u b$ ). - Nous décomposons de nouveau le produit $U_{\varepsilon} V_{\varepsilon}$ en :

$$
U_{\varepsilon} V_{\varepsilon}=T_{U_{\varepsilon}} V_{\varepsilon}+T_{V_{\varepsilon}} U_{\varepsilon}+R\left(U_{\varepsilon}, V_{\varepsilon}\right)
$$

L'estimation des termes $T_{V_{\varepsilon}} U_{\varepsilon}$ et $R\left(U_{\varepsilon}, V_{\varepsilon}\right)$ se fait exactement comme dans le a), de sorte que :

$$
\left|\langle\varepsilon D\rangle^{n / 2}\left(T_{V_{\varepsilon}} U_{\varepsilon}\right)\right|_{H^{-s}} \leqslant C\left|\langle\varepsilon D\rangle^{n / 2} U_{\varepsilon}\right|_{H^{s}}\left(\left|\langle D\rangle^{-s} V_{\varepsilon}\right|_{L^{2}}+\left|\langle D\rangle^{-s} V_{\varepsilon}\right|_{\mathrm{BMO}}\right)
$$

et que :

$$
\left|\langle\varepsilon D\rangle^{\frac{n}{2}} R\left(U_{\varepsilon}, V_{\varepsilon}\right)\right|_{L^{2}} \leqslant C\left|\langle\varepsilon D\rangle^{\frac{n}{2}} U_{\varepsilon}\right|_{H^{s}}\left|\langle D\rangle^{-s} V_{\varepsilon}\right|_{\text {BMO }}
$$

En multipliant chaque inégalité par $\varepsilon^{-M / 2}, M \geqslant n$, puis en prenant le sup sur $\varepsilon \in$ ] $\left.0, \varepsilon_{0}\right]$, nous déduisons

$\left\|\varepsilon^{-M / 2} T_{V_{\varepsilon}} U_{\varepsilon}+\varepsilon^{-M / 2} R\left(U_{\varepsilon}, V_{\varepsilon}\right)\right\|_{-s, \frac{n}{2}, 2} \leqslant C\left\|\varepsilon^{-M / 2} U_{\varepsilon}\right\|_{s, \frac{n}{2}, 2}\left(\left\|V_{\varepsilon}\right\|_{-s, 0, \infty}+\left\|V_{\varepsilon}\right\|_{-s, \frac{n}{2}, 2}\right)$ 
et puisque le lemme 2.3.8 nous garantit que $\mathcal{Z}_{\varepsilon}^{\left(-s, \frac{n}{2}\right)} \hookrightarrow \mathcal{W}_{\varepsilon}^{-s, 0, \infty}$, il vient finalement

$$
\left\|\varepsilon^{-M / 2} T_{V_{\varepsilon}} U_{\varepsilon}+\varepsilon^{-M / 2} R\left(U_{\varepsilon}, V_{\varepsilon}\right)\right\|_{-s, \frac{n}{2}, 2} \leqslant C\left\|\varepsilon^{-M / 2} U_{\varepsilon}\right\|_{s, \frac{n}{2}, 2}\left\|V_{\varepsilon}\right\|_{\left(-s, \frac{n}{2}\right)}
$$

Seul le terme $T_{U_{\varepsilon}} V_{\varepsilon}$ (basses fréquences en $U_{\varepsilon}$ et hautes fréquences en $V_{\varepsilon}$ ) doit être estimé de façon différente. Nous considérons une famille de fonctions test $(h)_{\varepsilon}$ de $\mathcal{S}$, telle que $\sup _{\left.\varepsilon \in] 0, \varepsilon_{0}\right]}\left|\langle\varepsilon D\rangle^{-n / 2} h\right|_{H^{s}} \leqslant 1$. Le spectre de chaque terme $S_{k-2} U_{\varepsilon} \Delta_{k} V_{\varepsilon}$ est supporté dans une couronne $2^{k-2} \leqslant|\xi| \leqslant 2^{k+2}$ :

$$
\begin{aligned}
& \left|\left\langle\sum_{k \geqslant 2} S_{k-2} U_{\varepsilon} \Delta_{k} V_{\varepsilon}, h_{\varepsilon}\right\rangle\right| \leqslant C \sum_{\left|k-k^{\prime}\right| \leqslant 3}\left\langle\left|S_{k-2} U_{\varepsilon} \Delta_{k} V_{\varepsilon}\right|,\left|\Delta_{k}^{\prime} h_{\varepsilon}\right|\right\rangle \\
& \leqslant C \sum_{\left|k-k^{\prime}\right| \leqslant 3}\left\langle\left|S_{k-2} U_{\varepsilon} 2^{-k s}\left\langle\varepsilon 2^{k}\right\rangle^{\frac{n}{2}} \Delta_{k} V_{\varepsilon}\right|,\left|2^{k^{\prime} s}\left\langle\varepsilon 2^{k^{\prime}}\right\rangle^{-n / 2} \Delta_{k}^{\prime} h_{\varepsilon}\right|\right\rangle \\
& \leqslant C\left(\sum_{k \geqslant 2}\left|S_{k-2} U_{\varepsilon} 2^{-k s}\left\langle\varepsilon 2^{k}\right\rangle^{\frac{n}{2}} \Delta_{k} V_{\varepsilon}\right|_{L^{2}}^{2}\right)^{1 / 2}\left(\sum_{k \geqslant 0}\left|2^{k s}\left\langle\varepsilon 2^{k}\right\rangle^{-n / 2} \Delta_{k} h_{\varepsilon}\right|_{L^{2}}^{2}\right)^{1 / 2}
\end{aligned}
$$

Par le lemme 2.3.5 b) et b') nous majorons :

$$
\sum\left|2^{k s}\left\langle\varepsilon 2^{k}\right\rangle^{-n / 2} \Delta_{k} h_{\varepsilon}\right|_{L^{2}}^{2} \leqslant C\left|\langle D\rangle^{s}\langle\varepsilon D\rangle^{-n / 2} h_{\varepsilon}\right|_{L^{2}}^{2}
$$

Pour le terme restant, nous décomposons $V_{\varepsilon} \in \mathcal{Z}_{\varepsilon}^{\left(-s, \frac{n}{2}\right)}$ en

$$
V_{\varepsilon}=V_{\varepsilon}^{\prime}+V_{\varepsilon}^{\prime \prime}
$$

de sorte que

$$
\left\|V_{\varepsilon}^{\prime}\right\|_{-s+\frac{n}{2}, 0,2} \leqslant 2 \mid\left\|V_{\varepsilon}\right\|_{\left(-s, \frac{n}{2}\right)}
$$

et

$$
\left\|V_{\varepsilon}^{\prime \prime}\right\|_{-s, \frac{n}{2}, \infty} \leqslant 2\left\||| V_{\varepsilon}\right\|_{\left(-s, \frac{n}{2}\right)}
$$

Nous pouvons alors découper :

$$
\sum_{k \geqslant 2}\left|S_{k-2} U_{\varepsilon} 2^{-k s}\left\langle\varepsilon 2^{k}\right\rangle^{\frac{n}{2}} \Delta_{k} V_{\varepsilon}\right|_{L^{2}}^{2} \leqslant C\left(\Sigma_{1}^{\prime}+\Sigma_{2}^{\prime}+\Sigma^{\prime \prime}\right)
$$

avec

$$
\Sigma_{1}^{\prime}=\sum_{k \geqslant 2, \varepsilon 2^{k} \leqslant 1}\left|S_{k-2} U_{\varepsilon} 2^{-k s}\left\langle\varepsilon 2^{k}\right\rangle^{\frac{n}{2}} \Delta_{k} V_{\varepsilon}^{\prime}\right|_{L^{2}}^{2} \leqslant C \sum_{k \geqslant 2}\left|S_{k-2} U_{\varepsilon} 2^{-k s} \Delta_{k} V_{\varepsilon}^{\prime}\right|_{L^{2}}^{2}
$$

et

$$
\Sigma_{2}^{\prime}=\sum_{k \geqslant 2, \varepsilon 2^{k} \geqslant 1}\left|S_{k-2} U_{\varepsilon} 2^{-k s}\left\langle\varepsilon 2^{k}\right\rangle^{\frac{n}{2}} \Delta_{k} V_{\varepsilon}^{\prime}\right|_{L^{2}}^{2} \leqslant C \varepsilon^{n} \sum_{k \geqslant 2}\left|S_{k-2} U_{\varepsilon} 2^{k\left(-s+\frac{n}{2}\right)} \Delta_{k} V_{\varepsilon}^{\prime}\right|_{L^{2}}^{2}
$$

tandis que

$$
\Sigma^{\prime \prime}=\sum_{k \geqslant 2}\left|S_{k-2} U_{\varepsilon} 2^{-k s}\left\langle\varepsilon 2^{k}\right\rangle^{\frac{n}{2}} \Delta_{k} V_{\varepsilon}^{\prime \prime}\right|_{L^{2}}^{2}
$$

Pour $\Sigma^{\prime \prime}$, il faut de nouveau avoir recours au lemme 3.1.4. Pour tout $s \in \mathbb{R}$ et $k \geqslant 0$, définition la famille $\widetilde{\phi}_{k}(\xi)=\left\langle\varepsilon 2^{k}\right\rangle^{\frac{n}{2}}\langle\varepsilon \xi\rangle^{-n / 2} 2^{-k s}\langle\xi\rangle^{s} \phi\left(2^{-k} \xi\right)$, qui vérifie $\sum_{k \geqslant 0}\left|\widetilde{\phi}_{k}\right|^{2} \leqslant$ $\widetilde{C}$. La famille des dilatées $\widetilde{\Phi}_{k}(\xi)=\widetilde{\phi}_{k}\left(2^{k} \xi\right)$ est supportée dans une couronne fixe, et 
reste uniformément bornée dans $C_{0}^{\infty}$ par rapport à $k$ et à $\varepsilon$. Notons $\widetilde{\Delta}_{k} u=\widetilde{\phi}_{k}(D) u$, de sorte que

$$
\left\langle\varepsilon 2^{k}\right\rangle^{n / 2} 2^{-k s} \Delta_{k} V_{\varepsilon}^{\prime \prime}=\widetilde{\Delta}_{k}\left(\langle D\rangle^{-s}\langle\varepsilon D\rangle^{\frac{n}{2}} V_{\varepsilon}^{\prime \prime}\right) .
$$

Notons $\widetilde{S}_{k}=S_{k-2}$. Nous sommes ainsi amenés à estimer

$$
\sum_{k}\left|\widetilde{\Delta}_{k}\left(\langle\varepsilon D\rangle^{n / 2}\langle D\rangle^{-s} V_{\varepsilon}^{\prime \prime}\right) \widetilde{S}_{k} U_{\varepsilon}\right|_{L^{2}}^{2}
$$

Par le lemme 3.1.4, il existe une constante $C$ - indépendante de $\varepsilon$ - telle que :

$$
\sum_{k}\left|\widetilde{\Delta}_{k}\left(\langle\varepsilon D\rangle^{n / 2}\langle D\rangle^{-s} V_{\varepsilon}^{\prime \prime}\right) \widetilde{S}_{k} U_{\varepsilon}\right|_{L^{2}}^{2} \leqslant C\left|\langle\varepsilon D\rangle^{n / 2}\langle D\rangle^{-s} V_{\varepsilon}^{\prime \prime}\right|_{\mathrm{BMO}}^{2}\left|U_{\varepsilon}\right|_{L^{2}}^{2}
$$

Nous en déduisons l'estimation :

$$
\left|\langle\varepsilon D\rangle^{n / 2}\left(T_{U_{\varepsilon}} V_{\varepsilon}^{\prime \prime}\right)\right|_{H^{-s}} \leqslant C\left|\langle D\rangle^{-s}\langle\varepsilon D\rangle^{n / 2} V_{\varepsilon}^{\prime \prime}\right|_{\mathrm{BMO}}\left|U_{\varepsilon}\right|_{L^{2}}
$$

Pour $\Sigma_{1}^{\prime}$, nous procédons de même, en posant simplement $\widetilde{\phi}(\xi)=2^{-k s}\langle\xi\rangle^{s} \phi_{k}\left(2^{-k} \xi\right)$. Notons $\widetilde{\Delta}_{k} u=\widetilde{\phi}_{k}(D) u$, de sorte que $\left\langle\varepsilon 2^{k}\right\rangle^{n / 2} 2^{-k s} \Delta_{k} V_{\varepsilon}^{\prime}=\widetilde{\Delta}_{k}\left(\langle D\rangle^{-s} V_{\varepsilon}^{\prime}\right)$, et $\widetilde{S}_{k}=$ $S_{k-2}$. Nous devons estimer

$$
\sum_{k}\left|\widetilde{\Delta}_{k}\left(\langle D\rangle^{-s} V_{\varepsilon}^{\prime}\right) \widetilde{S}_{k} U_{\varepsilon}\right|_{L^{2}}^{2}
$$

Par le lemme 3.1.4, il existe une constante $C$ telle que :

$$
\sum_{k}\left|\widetilde{\Delta}_{k}\left(\langle D\rangle^{-s} V_{\varepsilon}^{\prime}\right) \widetilde{S}_{k} U_{\varepsilon}\right|_{L^{2}}^{2} \leqslant C\left|\langle D\rangle^{-s} V_{\varepsilon}^{\prime}\right|_{\mathrm{BMO}}^{2}\left|U_{\varepsilon}\right|_{L^{2}}^{2}
$$

par l'injection de Sobolev $H^{n / 2} \hookrightarrow$ BMO, nous en déduisons :

$$
\Sigma_{1}^{\prime} \leqslant C\left|V_{\varepsilon}^{\prime}\right|_{H^{-s+\frac{n}{2}}}^{2}\left|U_{\varepsilon}\right|_{L^{2}}^{2}
$$

Pour $\Sigma_{2}^{\prime}$, nous majorons directement, en utilisant l'injection de Sobolev ordinaire $H^{s+\frac{n}{2}} \hookrightarrow L^{\infty}$ lorsque $s>0$ :

$$
\begin{aligned}
\Sigma_{2}^{\prime} & \leqslant C \varepsilon^{n} \sum_{k \geqslant 2}\left|S_{k-2} U_{\varepsilon} 2^{k\left(-s+\frac{n}{2}\right)} \Delta_{k} V_{\varepsilon}^{\prime}\right|_{L^{2}}^{2} \\
& \leqslant C \varepsilon^{n} \sum_{k \geqslant 2}\left|S_{k-2} U_{\varepsilon}\right|_{L^{\infty}}^{2}\left|2^{k\left(-s+\frac{n}{2}\right)} \Delta_{k} V_{\varepsilon}^{\prime}\right|_{L^{2}}^{2} \\
& \leqslant C \varepsilon^{n}\left|U_{\varepsilon}\right|_{L^{\infty}}^{2}\left|V_{\varepsilon}^{\prime}\right|_{H^{-s+\frac{n}{2}}}^{2} \\
& \leqslant C \varepsilon^{n}\left|\langle D\rangle^{s+\frac{n}{2}} U_{\varepsilon}\right|_{L^{2}}^{2}\left|V_{\varepsilon}^{\prime}\right|_{H^{-s+\frac{n}{2}}}^{2} \\
& \leqslant C\left|\langle D\rangle^{s}\langle\varepsilon D\rangle^{\frac{n}{2}} U_{\varepsilon}\right|_{L^{2}}^{2}\left|V_{\varepsilon}^{\prime}\right|_{H^{-s+\frac{n}{2}}}^{2}
\end{aligned}
$$

Nous en déduisons l'estimation :

$$
\left|\langle\varepsilon D\rangle^{n / 2}\left(T_{U_{\varepsilon}} V_{\varepsilon}{ }^{\prime}\right)\right|_{H^{-s}} \leqslant C\left|V_{\varepsilon}{ }^{\prime}\right|_{H^{-s+\frac{n}{2}}}\left|\langle D\rangle^{s}\langle\varepsilon D\rangle^{\frac{n}{2}} U_{\varepsilon}\right|_{L^{2}}
$$

Pour tout $\left.\varepsilon \in] 0, \varepsilon_{0}\right]$, nous disposons de l'estimation uniforme suivante :

$$
\begin{aligned}
\left|\langle\varepsilon D\rangle^{\frac{n}{2}}\left(T_{U_{\varepsilon}} V_{\varepsilon}\right)\right|_{H^{-s}} & \leqslant C\left|\langle\varepsilon D\rangle^{\frac{n}{2}} U_{\varepsilon}\right|_{H^{s}}\left(\left|\langle D\rangle^{-s}\langle\varepsilon D\rangle^{n / 2} V_{\varepsilon}^{\prime \prime}\right|_{\mathrm{BMO}}+\left|V_{\varepsilon}^{\prime}\right|_{H^{-s+\frac{n}{2}}}\right) \\
& \leqslant\left. 2 C\left|\langle\varepsilon D\rangle^{\frac{n}{2}} U_{\varepsilon}\right|_{H^{s}}||\left|V_{\varepsilon}\right|\right|_{\left(-s, \frac{n}{2}\right)}
\end{aligned}
$$


En multipliant chaque membre par $\varepsilon^{-M / 2}, M \geqslant n$, puis en prenant le sup sur $\varepsilon \in$ ] $\left.0, \varepsilon_{0}\right]$, nous déduisons

$$
\left\|\varepsilon^{-M / 2} T_{U_{\varepsilon}} V_{\varepsilon}\right\|_{-s, \frac{n}{2}, 2} \leqslant C\left\|\varepsilon^{-M / 2} U_{\varepsilon}\right\|_{s, \frac{n}{2}, 2}\left\|\mid V_{\varepsilon}\right\|_{\left(-s, \frac{n}{2}\right)}
$$

Ceci achève la preuve du b) et constitue le résultat central de cette partie.

Nous procédons de façon symétrique pour démontrer le c). Nous reprenons la preuve pour être complets. Pour le terme $T_{V_{\varepsilon}} U_{\varepsilon}$, on considère une famille de fonctions test $(h)_{\varepsilon}$ de $\mathcal{S}$, vérifiant

$$
\sup _{\left.\varepsilon \in] 0, \varepsilon_{0}\right]}\left|\langle\varepsilon D\rangle^{-n / 2}\langle D\rangle^{s} h\right|_{L^{2}} \leqslant 1
$$

Il vient :

$$
\begin{aligned}
& \left|\left\langle\sum_{k \geqslant 2} S_{k-2} V_{\varepsilon} \Delta_{k} U_{\varepsilon}, h_{\varepsilon}\right\rangle\right| \leqslant \sum_{\left|k-k^{\prime}\right| \leqslant 3}\left\langle\left|S_{k-2} V_{\varepsilon} \Delta_{k} U_{\varepsilon}\right|,\left|\Delta_{k^{\prime}} h_{\varepsilon}\right|\right\rangle \\
& \quad \leqslant C\left(\sum_{\left|k-k^{\prime}\right| \leqslant 5}\left|S_{k-2} V_{\varepsilon} 2^{k s}\left\langle\varepsilon 2^{k^{\prime}}\right\rangle^{-n / 2} \Delta_{k^{\prime}} h_{\varepsilon}\right|_{L^{2}}^{2}\right)^{1 / 2}\left(\sum_{k}\left|2^{-k s}\left\langle\varepsilon 2^{k}\right\rangle^{\frac{n}{2}} \Delta_{k} U_{\varepsilon}\right|_{L^{2}}^{2}\right)^{1 / 2}
\end{aligned}
$$

Par le lemme 3.1 .3 b) et 2.3 .5 b') :

$$
\begin{aligned}
\sum_{\left|k-k^{\prime}\right| \leqslant 5} \mid S_{k-2} V_{\varepsilon} 2^{k s} & \left.\left\langle\varepsilon 2^{k^{\prime}}\right\rangle^{-n / 2} \Delta_{k^{\prime}} h_{\varepsilon}\right|_{L^{2}} ^{2} \leqslant C \sum_{\left|k-k^{\prime}\right| \leqslant 5}\left|S_{k-2} V_{\varepsilon}\right|_{L^{\infty}}^{2}\left|2^{k s}\left\langle\varepsilon 2^{k^{\prime}}\right\rangle^{-n / 2} \Delta_{k^{\prime}} h_{\varepsilon}\right|_{L^{2}}^{2} \\
& \leqslant C\left(\left|\Delta_{-1}\langle D\rangle^{s} V_{\varepsilon}\right|_{L^{\infty}}+\left|\langle D\rangle^{s} V_{\varepsilon}\right|_{\mathrm{BMO}}\right)^{2}\left|\langle\varepsilon D\rangle^{-n / 2}\langle D\rangle^{s} h\right|_{L^{2}}^{2} \\
& \leqslant C\left(\left|\langle D\rangle^{s} V_{\varepsilon}\right|_{L^{2}}+\left|\langle D\rangle^{s} V_{\varepsilon}\right|_{\mathrm{BMO}}\right)^{2}
\end{aligned}
$$

Toujours par le lemme 2.3.5 b) et b'), nous savons que

$$
\sum\left|2^{-k s}\left\langle\varepsilon 2^{k}\right\rangle^{n / 2} \Delta_{k} U_{\varepsilon}\right|_{L^{2}}^{2} \leqslant C\left|\langle\varepsilon D\rangle^{n / 2} U_{\varepsilon}\right|_{H^{-s}}^{2}
$$

Il vient alors pour tout $\left.\varepsilon \in] 0, \varepsilon_{0}\right]$ :

$$
\left|\langle\varepsilon D\rangle^{n / 2}\left(T_{V_{\varepsilon}} U_{\varepsilon}\right)\right|_{H^{-s}} \leqslant C\left|\langle\varepsilon D\rangle^{n / 2} U_{\varepsilon}\right|_{H^{-s}}\left(\left|\langle D\rangle^{s} V_{\varepsilon}\right|_{L^{2}}+\left|\langle D\rangle^{s} V_{\varepsilon}\right|_{\mathrm{BMO}}\right)
$$

En multipliant l'inégalité par $\varepsilon^{-M / 2}, M \geqslant n$, puis en prenant le sup sur $\left.\left.\varepsilon \in\right] 0, \varepsilon_{0}\right]$, nous déduisons

$$
\left\|\varepsilon^{-M / 2} T_{V_{\varepsilon}} U_{\varepsilon}\right\|_{-s, \frac{n}{2}, 2} \leqslant C\left\|\varepsilon^{-M / 2} U_{\varepsilon}\right\|_{-s, \frac{n}{2}, 2}\left(\left\|V_{\varepsilon}\right\|_{s, 0, \infty}+\left\|V_{\varepsilon}\right\|_{s, \frac{n}{2}, 2}\right)
$$

et puisque le lemme 2.3.8 nous garantit que $\mathcal{Z}_{\varepsilon}^{\left(s, \frac{n}{2}\right)} \hookrightarrow \mathcal{W}_{\varepsilon}^{s, 0, \infty}$, il vient finalement

$$
\left\|\varepsilon^{-M / 2} T_{V_{\varepsilon}} U_{\varepsilon}\right\|_{-s, \frac{n}{2}, 2} \leqslant C\left\|\varepsilon^{-M / 2} U_{\varepsilon}\right\|_{-s, \frac{n}{2}, 2}\left\|\mid V_{\varepsilon}\right\|_{\left(s, \frac{n}{2}\right)}
$$

Pour le terme de reste $R\left(U_{\varepsilon}, V_{\varepsilon}\right)$, considérons une famille de fonctions test $(h)_{\varepsilon}$ de $\mathcal{S}$ vérifiant

$$
\sup _{\left.\varepsilon \in] 0, \varepsilon_{0}\right]}\left|\langle\varepsilon D\rangle^{-n / 2} h\right|_{L^{2}} \leqslant 1
$$


Il vient :

$$
\begin{aligned}
& \left|\left\langle\sum_{\left|k-k^{\prime}\right| \leqslant 2} \Delta_{k} U_{\varepsilon} \Delta_{k^{\prime}} V_{\varepsilon}, h_{\varepsilon}\right\rangle\right| \\
& \quad \leqslant C\left(\sum_{k}\left|2^{-k s}\left\langle\varepsilon 2^{k}\right\rangle^{\frac{n}{2}} \Delta_{k} U_{\varepsilon}\right|_{L^{2}}^{2}\right)^{1 / 2}\left(\sum_{k}\left|2^{k s} \Delta_{k} V_{\varepsilon}\left\langle\varepsilon 2^{k+5}\right\rangle^{-n / 2} S_{k+5} h_{\varepsilon}\right|_{L^{2}}^{2}\right)^{1 / 2}
\end{aligned}
$$

Posons de nouveau $\widetilde{\phi}(\xi)=2^{2 k s}\langle D\rangle^{-s} \phi\left(2^{-k} \xi\right)$. Nous vérifions que $\sum_{k}|\widetilde{\phi}|^{2} \leqslant \widetilde{C}$. Les fonctions dilatées $\widetilde{\Phi}_{k}(\xi)=\widetilde{\phi}_{k}\left(2^{k} \xi\right)$ sont supportée dans une même couronne et restent uniformément bornées dans $C_{0}^{\infty}$. Posons $\widetilde{\Delta}_{k} u=\widetilde{\phi}_{k}(D) u$, de sorte que $2^{k s} \Delta_{k} V_{\varepsilon}=$ $\widetilde{\Delta}_{k}\left(\langle D\rangle^{s} V_{\varepsilon}\right)$. Nous prenons $\widetilde{\psi}_{k}(\xi)=\left\langle\varepsilon 2^{k+5}\right\rangle^{-n / 2}\langle\varepsilon \xi\rangle^{\frac{n}{2}} \psi\left(2^{-(k+5)} \xi\right)$, dépendant cette fois du paramètre $\varepsilon$. Les fonctions dilatées $\widetilde{\Psi}_{k}(\xi)=\widetilde{\psi}_{k}\left(2^{k} \xi\right)$ sont supportées dans une même boule et restent bornées dans $C_{0}^{\infty}$, uniformément en $k$ et en $\varepsilon$. Notons $\widetilde{S}_{k}=\widetilde{\psi}_{k}(D)$, de sorte que $\left\langle\varepsilon 2^{k+5}\right\rangle^{-n / 2} S_{k+5} h_{\varepsilon}=\widetilde{S}_{k}\left(\langle\varepsilon D\rangle^{-n / 2} h_{\varepsilon}\right)$. Nous sommes ainsi amenés à estimer

$$
\sum_{k}\left|\widetilde{\Delta}_{k}\left(\langle D\rangle^{s} V_{\varepsilon}\right) \widetilde{S}_{k}\left(\langle\varepsilon D\rangle^{-n / 2} h_{\varepsilon}\right)\right|_{L^{2}}^{2}
$$

Ce terme entre dans le cadre du lemme 3.1.4, et il existe une constante $C$, ne dépendant que d'un nombre fini de semi-normes de $\widetilde{\Psi}_{k}$ et de $\widetilde{\Phi}_{k}$ dans $C_{0}^{\infty}$, donc indépendante de $\varepsilon$, telle que :

$$
\sum_{k}\left|\widetilde{\Delta}_{k}\left(\langle D\rangle^{s} V_{\varepsilon}\right) \widetilde{S}_{k}\left(\langle\varepsilon D\rangle^{-n / 2} h_{\varepsilon}\right)\right|_{L^{2}}^{2} \leqslant C\left|\langle\varepsilon D\rangle^{-n / 2} h_{\varepsilon}\right|_{L^{2}}^{2}\left|\langle D\rangle^{s} V_{\varepsilon}\right|_{\mathrm{BMO}}^{2}
$$

Par ailleurs, le lemme 2.3.5 b) et b') nous donne :

$$
\sum_{k \geqslant 0}\left|2^{-k s}\left\langle\varepsilon 2^{k}\right\rangle^{\frac{n}{2}} \Delta_{k} U_{\varepsilon}\right|_{L^{2}}^{2} \leqslant C\left|\langle\varepsilon D\rangle^{\frac{n}{2}} U_{\varepsilon}\right|_{H^{-s}}^{2}
$$

Nous obtenons alors l'estimation désirée :

$$
\left|\langle\varepsilon D\rangle^{\frac{n}{2}} R\left(U_{\varepsilon}, V_{\varepsilon}\right)\right|_{L^{2}} \leqslant C\left|\langle\varepsilon D\rangle^{\frac{n}{2}} U_{\varepsilon}\right|_{H^{-s}}\left|\langle D\rangle^{s} V_{\varepsilon}\right|_{\mathrm{BMO}}
$$

En multipliant l'inégalité par $\varepsilon^{-M / 2}, M \geqslant n$, puis en prenant le sup sur $\left.\left.\varepsilon \in\right] 0, \varepsilon_{0}\right]$, nous déduisons

$$
\left\|\varepsilon^{-M / 2} R\left(U_{\varepsilon}, V_{\varepsilon}\right)\right\|_{-s, \frac{n}{2}, 2} \leqslant C\left\|\varepsilon^{-M / 2} U_{\varepsilon}\right\|_{-s, \frac{n}{2}, 2}\left\|V_{\varepsilon}\right\|_{s, 0, \infty}
$$

et puisque le lemme 2.3.8 nous garantit que $\mathcal{Z}_{\varepsilon}^{\left(s, \frac{n}{2}\right)} \hookrightarrow \mathcal{W}_{\varepsilon}^{s, 0, \infty}$, il vient finalement

$$
\left\|\varepsilon^{-M / 2} R\left(U_{\varepsilon}, V_{\varepsilon}\right)\right\|_{-s, \frac{n}{2}, 2} \leqslant C\left\|\varepsilon^{-M / 2} U_{\varepsilon}\right\|_{-s, \frac{n}{2}, 2}\left\|\mid V_{\varepsilon}\right\|_{\left(s, \frac{n}{2}\right)}
$$

Pour le terme $T_{U_{\varepsilon}} V_{\varepsilon}$, considérons une famille de fonctions test $(h)_{\varepsilon}$ de $\mathcal{S}$, telle que

$$
\sup _{\left.\varepsilon \in] 0, \varepsilon_{0}\right]}\left|\langle\varepsilon D\rangle^{-n / 2} h\right|_{H^{s}} \leqslant 1
$$


Il vient :

$$
\begin{aligned}
& \left|\left\langle\sum_{k \geqslant 2} S_{k-2} U_{\varepsilon} \Delta_{k} V_{\varepsilon}, h_{\varepsilon}\right\rangle\right| \leqslant C \sum_{\left|k-k^{\prime}\right| \leqslant 3}\left|\left\langle S_{k-2} U_{\varepsilon} \Delta_{k} V_{\varepsilon}, \Delta_{k}^{\prime} h_{\varepsilon}\right\rangle\right| \\
& \leqslant C \sum_{\left|k-k^{\prime}\right| \leqslant 3}\left|\left\langle 2^{-k s} S_{k-2} U_{\varepsilon}\left\langle\varepsilon 2^{k}\right\rangle^{\frac{n}{2}} \Delta_{k} V_{\varepsilon}, 2^{k^{\prime} s}\left\langle\varepsilon 2^{k^{\prime}}\right\rangle^{-n / 2} \Delta_{k}^{\prime} h_{\varepsilon}\right\rangle\right| \\
& \leqslant C\left(\sum_{k \geqslant 2}\left|2^{-k s} S_{k-2} U_{\varepsilon}\left\langle\varepsilon 2^{k}\right\rangle^{\frac{n}{2}} \Delta_{k} V_{\varepsilon}\right|_{L^{2}}^{2}\right)^{1 / 2}\left(\sum_{k \geqslant 0}\left|2^{k s}\left\langle\varepsilon 2^{k}\right\rangle^{-n / 2} \Delta_{k} h_{\varepsilon}\right|_{L^{2}}^{2}\right)^{1 / 2}
\end{aligned}
$$

Par le lemme 2.3.5 b) et b') nous majorons :

$$
\sum\left|2^{k s}\left\langle\varepsilon 2^{k}\right\rangle^{-n / 2} \Delta_{k} h_{\varepsilon}\right|_{L^{2}}^{2} \leqslant C\left|\langle D\rangle^{s}\langle\varepsilon D\rangle^{-n / 2} h_{\varepsilon}\right|_{L^{2}}^{2}
$$

Pour le terme restant, nous décomposons $V_{\varepsilon} \in \mathcal{Z}_{\varepsilon}^{\left(s, \frac{n}{2}\right)}$ en

$$
V_{\varepsilon}=V_{\varepsilon}^{\prime}+V_{\varepsilon}^{\prime \prime}
$$

de sorte que

$$
\left\|V_{\varepsilon}^{\prime}\right\|_{s+\frac{n}{2}, 0,2} \leqslant 2 \mid\left\|V_{\varepsilon}\right\|_{\left(s, \frac{n}{2}\right)} \quad \text { et } \quad\left\|V_{\varepsilon}^{\prime \prime}\right\|_{s, \frac{n}{2}, \infty} \leqslant\left. 2\left\||| V_{\varepsilon}\right\|\right|_{\left(s, \frac{n}{2}\right)}
$$

Nous pouvons alors découper :

$$
\sum_{k \geqslant 2}\left|2^{-(k-2) s} S_{k-2} U_{\varepsilon}\left\langle\varepsilon 2^{k}\right\rangle^{\frac{n}{2}} \Delta_{k} V_{\varepsilon}\right|_{L^{2}}^{2} \leqslant \Sigma_{1}^{\prime}+\Sigma_{2}^{\prime}+\Sigma^{\prime \prime}
$$

avec

$$
\Sigma_{1}^{\prime}=\sum_{k \geqslant 2, \varepsilon 2^{k} \leqslant 1}\left|2^{-(k-2) s} S_{k-2} U_{\varepsilon}\left\langle\varepsilon 2^{k}\right\rangle^{\frac{n}{2}} \Delta_{k} V_{\varepsilon}^{\prime}\right|_{L^{2}}^{2} \leqslant C \sum_{k \geqslant 2}\left|2^{-(k-2) s} S_{k-2} U_{\varepsilon} \Delta_{k} V_{\varepsilon}^{\prime}\right|_{L^{2}}^{2}
$$

et

$$
\begin{aligned}
\Sigma_{2}^{\prime}=\sum_{k \geqslant 2, \varepsilon 2^{k} \geqslant 1}\left|2^{-(k-2) s} S_{k-2} U_{\varepsilon}\left\langle\varepsilon 2^{k}\right\rangle^{\frac{n}{2}} \Delta_{k} V_{\varepsilon}^{\prime}\right|_{L^{2}}^{2} & \\
& \leqslant C \varepsilon^{n} \sum_{k \geqslant 2}\left|2^{-(k-2) s} S_{k-2} U_{\varepsilon} 2^{k \frac{n}{2}} \Delta_{k} V_{\varepsilon}^{\prime}\right|_{L^{2}}^{2}
\end{aligned}
$$

tandis que

$$
\Sigma^{\prime \prime}=\sum_{k \geqslant 2}\left|2^{-(k-2) s} S_{k-2} U_{\varepsilon}\left\langle\varepsilon 2^{k}\right\rangle^{\frac{n}{2}} \Delta_{k} V_{\varepsilon}^{\prime \prime}\right|_{L^{2}}^{2}
$$

Pour $\Sigma^{\prime \prime}$, il faut de nouveau avoir recours au lemme 3.1.4. Pour tout $s \in \mathbb{R}$ et $k \geqslant 0$, définissons la famille $\widetilde{\phi}_{k}(\xi)=\left\langle\varepsilon 2^{k}\right\rangle^{\frac{n}{2}}\langle\varepsilon \xi\rangle^{-n / 2} \phi\left(2^{-k} \xi\right)$, qui vérifie $\sum_{k \geqslant 0}\left|\widetilde{\phi}_{k}\right|^{2} \leqslant \widetilde{C}$. La famille des dilatées $\widetilde{\Phi}_{k}(\xi)=\widetilde{\phi}_{k}\left(2^{k} \xi\right)$ est supportée dans une couronne fixe, et reste uniformément bornée dans $C_{0}^{\infty}$, par rapport à $k$ et à $\varepsilon$. Notons $\widetilde{\Delta}_{k} u=\widetilde{\phi}_{k}(\underset{\sim}{D}) u$, de sorte que $\left\langle\varepsilon 2^{k}\right\rangle^{n / 2} \Delta_{k} V_{\varepsilon}^{\prime \prime}=\widetilde{\Delta}_{k}\left(\langle\varepsilon D\rangle^{\frac{n}{2}} V_{\varepsilon}^{\prime \prime}\right)$. Définissons également la famille $\widetilde{\psi}_{k}(\xi)=$ $2^{-(k-2) s}\langle\xi\rangle^{s} \psi\left(2^{-(k-2)} \xi\right)$. Les fonctions dilatées $\widetilde{\Psi}_{k}(\xi)=\widetilde{\psi}_{k}\left(2^{k} \xi\right)$ sont supportées dans 
une même boule et restent uniformément bornées dans $C_{0}^{\infty}$. Nous notons $\widetilde{S}_{k}=\widetilde{\psi}_{k}(D)$, de sorte que $2^{-(k-2) s} S_{k-2} U_{\varepsilon}=\widetilde{S}_{k}\left(\langle D\rangle^{-s} U_{\varepsilon}\right)$. Nous sommes ainsi amenés à estimer

$$
\sum_{k}\left|\widetilde{\Delta}_{k}\left(\langle\varepsilon D\rangle^{n / 2} V_{\varepsilon}^{\prime \prime}\right) \widetilde{S}_{k}\left(\langle D\rangle^{-s} U_{\varepsilon}\right)\right|_{L^{2}}^{2}
$$

Par le lemme 3.1.4, il existe une constante $C$ - indépendante de $\varepsilon$ - telle que

$$
\sum_{k}\left|\widetilde{\Delta}_{k}\left(\langle\varepsilon D\rangle^{n / 2} V_{\varepsilon}^{\prime \prime}\right) \widetilde{S}_{k}\left(\langle D\rangle^{-s} U_{\varepsilon}\right)\right|_{L^{2}}^{2} \leqslant C\left|\langle\varepsilon D\rangle^{n / 2} V_{\varepsilon}^{\prime \prime}\right|_{\text {BMO }}^{2}\left|U_{\varepsilon}\right|_{H^{-s}}^{2}
$$

Nous en déduisons l'estimation :

$$
\left|\langle\varepsilon D\rangle^{n / 2}\left(T_{U_{\varepsilon}} V_{\varepsilon}^{\prime \prime}\right)\right|_{H^{-s}} \leqslant C\left|\langle\varepsilon D\rangle^{n / 2} V_{\varepsilon}^{\prime \prime}\right|_{\mathrm{BMO}}\left|U_{\varepsilon}\right|_{H^{-s}}
$$

Pour $\Sigma_{1}^{\prime}$, nous procédons de même, en posant simplement $\widetilde{\Delta}_{k}=\Delta_{k}$, tandis que $\widetilde{S}_{k}$ est défini comme précédemment pour $\Sigma^{\prime \prime}$. Nous devons estimer

$$
\sum_{k}\left|\widetilde{\Delta}_{k} V_{\varepsilon}^{\prime} \widetilde{S}_{k}\left(\langle D\rangle^{-s} U_{\varepsilon}\right)\right|_{L^{2}}^{2}
$$

Par le lemme 3.1.4, il existe une constante $C$ telle que

$$
\sum_{k}\left|\widetilde{\Delta}_{k} V_{\varepsilon}^{\prime} \widetilde{S}_{k}\left(\langle D\rangle^{-s} U_{\varepsilon}\right)\right|_{L^{2}}^{2} \leqslant C\left|V_{\varepsilon}^{\prime}\right|_{\mathrm{BMO}}^{2}\left|U_{\varepsilon}\right|_{H^{-s}}^{2}
$$

par l'injection de Sobolev $H^{n / 2} \hookrightarrow$ BMO, nous en déduisons :

$$
\Sigma_{1}^{\prime} \leqslant C\left|V_{\varepsilon}^{\prime}\right|_{H^{\frac{n}{2}}}^{2}\left|U_{\varepsilon}\right|_{H^{-s}}^{2}
$$

Pour $\Sigma_{2}^{\prime}$, nous majorons directement, en utilisant le lemme 3.1.3, puis l'injection de Sobolev ordinaire $H^{\frac{n}{2}} \hookrightarrow$ BMO :

$$
\begin{aligned}
\Sigma_{2}^{\prime} & \leqslant C \varepsilon^{n} \sum_{k \geqslant 2}\left|2^{-k s} S_{k-2} U_{\varepsilon}\right|_{L^{\infty}}^{2}\left|2^{k \frac{n}{2}} \Delta_{k} V_{\varepsilon}^{\prime}\right|_{L^{2}}^{2} \\
& \leqslant C \varepsilon^{n} \sum_{k \geqslant 2}\left(\left|\Delta_{-1}\langle D\rangle^{-s} U_{\varepsilon}\right|_{L^{\infty}}+\left|\langle D\rangle^{-s} U_{\varepsilon}\right|_{\mathrm{BMO}}^{2}\right)\left|2^{k \frac{n}{2}} \Delta_{k} V_{\varepsilon}^{\prime}\right|_{L^{2}}^{2} \\
& \leqslant C \varepsilon^{n}\left|\langle D\rangle^{-s+\frac{n}{2}} U_{\varepsilon}\right|_{L^{2}}^{2}\left|V_{\varepsilon}^{\prime}\right|_{H^{\frac{n}{2}}}^{2} \\
& \leqslant C\left|\langle D\rangle^{-s}\langle\varepsilon D\rangle^{\frac{n}{2}} U_{\varepsilon}\right|_{L^{2}}^{2}\left|V_{\varepsilon}^{\prime}\right|_{H^{\frac{n}{2}}}^{2}
\end{aligned}
$$

Nous en déduisons l'estimation :

$$
\left|\langle\varepsilon D\rangle^{n / 2}\left(T_{U_{\varepsilon}} V_{\varepsilon}^{\prime}\right)\right|_{H^{-s}} \leqslant C\left|V_{\varepsilon}^{\prime}\right|_{H^{\frac{n}{2}}}\left|\langle\varepsilon D\rangle^{\frac{n}{2}} U_{\varepsilon}\right|_{H^{-s}}
$$

Pour tout $\left.\varepsilon \in] 0, \varepsilon_{0}\right]$, nous disposons de l'estimation uniforme suivante :

$$
\begin{aligned}
\left|\langle\varepsilon D\rangle^{\frac{n}{2}}\left(T_{U_{\varepsilon}} V_{\varepsilon}\right)\right|_{H^{-s}} & \leqslant C\left|\langle\varepsilon D\rangle^{\frac{n}{2}} U_{\varepsilon}\right|_{H^{-s}}\left(\left|\langle\varepsilon D\rangle^{n / 2} V_{\varepsilon}^{\prime \prime}\right|_{\mathrm{BMO}}+\left|V_{\varepsilon}^{\prime}\right|_{H^{\frac{n}{2}}}\right) \\
& \leqslant\left. 2 C\left|\langle\varepsilon D\rangle^{\frac{n}{2}} U_{\varepsilon}\right|_{H^{-s}}||\left|V_{\varepsilon}\right|\right|_{\left(s, \frac{n}{2}\right)}
\end{aligned}
$$

En multipliant chaque membre par $\varepsilon^{-M / 2}, M \geqslant n$, puis en prenant le sup sur $\varepsilon \epsilon$ ] $\left.0, \varepsilon_{0}\right]$, nous déduisons

$$
\left\|\varepsilon^{-M / 2} T_{U_{\varepsilon}} V_{\varepsilon}\right\|_{-s, \frac{n}{2}, 2} \leqslant C\left\|\varepsilon^{-M / 2} U_{\varepsilon}\right\|_{-s, \frac{n}{2}, 2}\left\|\left|V_{\varepsilon} \|\right|_{\left(s, \frac{n}{2}\right)}\right.
$$

La preuve de la proposition 3.1.1 est complète. 
3.1.2. Résultats complémentaires. - Nous aurons parfois besoin des estimations suivantes, qui prennent en compte des cas dissymétriques qui échappent à la proposition 3.1.1.

Proposition 3.1.5. - Étant donné un entier $M \geqslant n$, un réel $s>0$, il existe une constante $C$ telle que :

a) si $(U)_{\varepsilon} \in \varepsilon^{M / 2} \mathcal{W}_{\varepsilon}^{0, \frac{n}{2}, 2}$ et $(V)_{\varepsilon} \in \varepsilon^{M / 2} \mathcal{W}_{\varepsilon}^{0, \frac{n}{2}, 2}$, alors le produit $(U V)_{\varepsilon} \in$ $\varepsilon^{M / 2} \mathcal{W}_{\varepsilon}^{-s, \frac{n}{2}, 2}$ et

$$
\left\|\varepsilon^{-M / 2} U_{\varepsilon} V_{\varepsilon}\right\|_{-s, \frac{n}{2}, 2} \leqslant C\left\|\varepsilon^{-M / 2} U_{\varepsilon}\right\|_{0, \frac{n}{2}, 2}\left\|\varepsilon^{-M / 2} V_{\varepsilon}\right\|_{0, \frac{n}{2}, 2}
$$

b) $\operatorname{si}(U)_{\varepsilon} \in \varepsilon^{M / 2} \mathcal{W}_{\varepsilon}^{s, \frac{n}{2}, 2}$ et $(V)_{\varepsilon} \in \varepsilon^{M / 2} \mathcal{W}_{\varepsilon}^{0, \frac{n}{2}, 2}$, alors le produit $(U V)_{\varepsilon} \in$ $\varepsilon^{M / 2} \mathcal{W}_{\varepsilon}^{0, \frac{n}{2}, 2}$ et

$$
\left\|\varepsilon^{-M / 2} U_{\varepsilon} V_{\varepsilon}\right\|_{0, \frac{n}{2}, 2} \leqslant C\left\|\varepsilon^{-M / 2} U_{\varepsilon}\right\|_{s, \frac{n}{2}, 2}\left\|\varepsilon^{-M / 2} V_{\varepsilon}\right\|_{0, \frac{n}{2}, 2}
$$

c) $\operatorname{si}(U)_{\varepsilon} \in \varepsilon^{M / 2} \mathcal{W}_{\varepsilon}^{s, \frac{n}{2}, 2}$ et $(V)_{\varepsilon} \in \mathcal{Z}_{\varepsilon}^{\left(0, \frac{n}{2}\right)}$, alors le produit $(U V)_{\varepsilon} \in \varepsilon^{M / 2} \mathcal{W}_{\varepsilon}^{0, \frac{n}{2}, 2}$ et

$$
\left\|\varepsilon^{-M / 2} U_{\varepsilon} V_{\varepsilon}\right\|_{0, \frac{n}{2}, 2} \leqslant C\left\|\varepsilon^{-M / 2} U_{\varepsilon}\right\|_{s, \frac{n}{2}, 2}\left\|\mid V_{\varepsilon}\right\|_{\left(0, \frac{n}{2}\right)}
$$

d) $\operatorname{si}(U)_{\varepsilon} \in \varepsilon^{M / 2} \mathcal{W}_{\varepsilon}^{0, \frac{n}{2}, 2}$ et $(V)_{\varepsilon} \in \mathcal{Z}_{\varepsilon}^{\left(s, \frac{n}{2}\right)}$, alors le produit $(U V)_{\varepsilon} \in \varepsilon^{M / 2} \mathcal{W}_{\varepsilon}^{0, \frac{n}{2}, 2}$ et

$$
\left\|\varepsilon^{-M / 2} U_{\varepsilon} V_{\varepsilon}\right\|_{0, \frac{n}{2}, 2} \leqslant C\left\|\varepsilon^{-M / 2} U_{\varepsilon}\right\|_{0, \frac{n}{2}, 2}\left\|\mid V_{\varepsilon}\right\|_{\left(s, \frac{n}{2}\right)}
$$

e) $\operatorname{si}(U)_{\varepsilon} \in \varepsilon^{M / 2} \mathcal{W}_{\varepsilon}^{0, \frac{n}{2}, 2}$ et $(V)_{\varepsilon} \in \mathcal{Z}_{\varepsilon}^{\left(0, \frac{n}{2}\right)}$, alors le produit $(U V)_{\varepsilon} \in \varepsilon^{M / 2} \mathcal{W}_{\varepsilon}^{-s, \frac{n}{2}, 2}$ et

$$
\left\|\varepsilon^{-M / 2} U_{\varepsilon} V_{\varepsilon}\right\|_{-s, \frac{n}{2}, 2} \leqslant C\left\|\varepsilon^{-M / 2} U_{\varepsilon}\right\|_{0, \frac{n}{2}, 2}\left\|\mid V_{\varepsilon}\right\|_{\left(0, \frac{n}{2}\right)}
$$

Démonstration. - Elle ne présente pas de difficulté nouvelle, il faut reprendre presque mot pour mot celle de la proposition 3.1.1. Nous examinerons la preuve du a) et du c), les autres inégalités s'obtiennent avec les mêmes arguments pris dans un ordre différent.

Démonstration $d u$ a). — Pour le terme $T_{V_{\varepsilon}} U_{\varepsilon}$ en considérant une famille de fonctions test $(h)_{\varepsilon}$ de $\mathcal{S}$, vérifiant

$$
\sup _{\left.\varepsilon \in] 0, \varepsilon_{0}\right]}\left|\langle\varepsilon D\rangle^{-n / 2}\langle D\rangle^{s} h\right|_{L^{2}} \leqslant 1
$$

il vient pour tout $\left.\varepsilon \in] 0, \varepsilon_{0}\right]$ :

$$
\begin{aligned}
& \left|\left\langle\sum_{k \geqslant 2} S_{k-2} V_{\varepsilon} \Delta_{k} U_{\varepsilon}, h_{\varepsilon}\right\rangle\right| \\
& \quad \leqslant C\left(\sum_{k}\left|\left\langle\varepsilon 2^{k}\right\rangle^{n / 2} \Delta_{k} U_{\varepsilon}\right|_{L^{2}}^{2}\right)^{1 / 2}\left(\sum_{\left|k-k^{\prime}\right| \leqslant 5}\left|S_{k} V_{\varepsilon}\left\langle\varepsilon 2^{k^{\prime}}\right\rangle^{-n / 2} \Delta_{k^{\prime}} h_{\varepsilon}\right|_{L^{2}}^{2}\right)^{1 / 2}
\end{aligned}
$$


Par le lemme 3.1 .3 b) et 2.3 .5 b') :

$$
\begin{aligned}
& \sum_{\left|k-k^{\prime}\right| \leqslant 5}\left|S_{k} V_{\varepsilon}\left\langle\varepsilon 2^{k^{\prime}}\right\rangle^{-n / 2} \Delta_{k^{\prime}} h_{\varepsilon}\right|_{L^{2}}^{2} \leqslant C \sum_{\left|k-k^{\prime}\right| \leqslant 5}\left|S_{k} V_{\varepsilon}\right|_{L^{\infty}}^{2}\left|\Delta_{k^{\prime}}\langle\varepsilon D\rangle^{-n / 2} h_{\varepsilon}\right|_{L^{2}}^{2} \\
& \leqslant C\left(\left|\Delta_{-1} V_{\varepsilon}\right|_{L^{\infty}}+\left|V_{\varepsilon}\right|_{\mathrm{BMO}}\right)^{2} \sum_{k} k 2^{-2 k s}\left|\Delta_{k}\langle D\rangle^{s}\langle\varepsilon D\rangle^{-n / 2} h_{\varepsilon}\right|_{L^{2}}^{2} \\
& \leqslant C\left(\left|V_{\varepsilon}\right|_{L^{2}}+\left|V_{\varepsilon}\right|_{\mathrm{BMO}}\right)^{2}\left|\langle D\rangle^{s}\langle\varepsilon D\rangle^{-n / 2} h_{\varepsilon}\right|_{L^{2}}^{2} \\
& \leqslant C\left(\left|V_{\varepsilon}\right|_{L^{2}}+\left|V_{\varepsilon}\right|_{\mathrm{BMO}}\right)^{2}
\end{aligned}
$$

comme

$$
\sum\left|\left\langle\varepsilon 2^{k}\right\rangle^{n / 2} \Delta_{k} U_{\varepsilon}\right|_{L^{2}}^{2} \leqslant C\left|\langle\varepsilon D\rangle^{n / 2} U_{\varepsilon}\right|_{L^{2}}^{2}
$$

Il vient alors pour tout $\left.\varepsilon \in] 0, \varepsilon_{0}\right]$ :

$$
\left|\langle\varepsilon D\rangle^{n / 2}\left(T_{V_{\varepsilon}} U_{\varepsilon}\right)\right|_{H^{-s}} \leqslant C\left|\langle\varepsilon D\rangle^{n / 2} U_{\varepsilon}\right|_{L^{2}}\left(\left|V_{\varepsilon}\right|_{L^{2}}+\left|V_{\varepsilon}\right|_{\text {BMO }}\right)
$$

En permutant le rôle de $U$ et $V$, nous obtenons le terme symétrique :

$$
\left|\langle\varepsilon D\rangle^{n / 2}\left(T_{U_{\varepsilon}} V_{\varepsilon}\right)\right|_{H^{-s}} \leqslant C\left|\langle\varepsilon D\rangle^{n / 2} V_{\varepsilon}\right|_{L^{2}}\left(\left|U_{\varepsilon}\right|_{L^{2}}+\left|U_{\varepsilon}\right|_{\mathrm{BMO}}\right)
$$

Pour le terme de reste $R\left(U_{\varepsilon}, V_{\varepsilon}\right)$, considérons une famille de fonctions test $(h)_{\varepsilon}$ de $\mathcal{S}$ vérifiant

$$
\sup _{\left.\varepsilon \in] 0, \varepsilon_{0}\right]}\left|\langle\varepsilon D\rangle^{-n / 2} h\right|_{L^{2}} \leqslant 1
$$

Il vient :

$$
\begin{aligned}
& \left|\left\langle\sum_{\left|k-k^{\prime}\right| \leqslant 2} \Delta_{k} U_{\varepsilon} \Delta_{k^{\prime}} V_{\varepsilon}, h_{\varepsilon}\right\rangle\right| \\
& \quad \leqslant C\left(\sum_{k}\left|\left\langle\varepsilon 2^{k}\right\rangle^{\frac{n}{2}} \Delta_{k} U_{\varepsilon}\right|_{L^{2}}^{2}\right)^{1 / 2}\left(\sum_{k}\left|\Delta_{k} V_{\varepsilon}\left\langle\varepsilon 2^{k+5}\right\rangle^{-n / 2} S_{k+5} h_{\varepsilon}\right|_{L^{2}}^{2}\right)^{1 / 2}
\end{aligned}
$$

Posons simplement $\widetilde{\Delta}_{k} u=\widetilde{\phi}_{k}(D) u$, introduisons la famille

$$
\widetilde{\psi}_{k}(\xi)=\left\langle\varepsilon 2^{k+5}\right\rangle^{-n / 2}\langle\varepsilon \xi\rangle^{\frac{n}{2}} \psi\left(2^{-(k+5)} \xi\right) .
$$

Les fonctions dilatées $\widetilde{\Psi}_{k}(\xi)=\widetilde{\psi}_{k}\left(2^{k} \xi\right)$ sont supportées dans une même boule et restent bornées dans $C_{0}^{\infty}$, uniformément en $k$ et en $\varepsilon$. Nous notons $\widetilde{S}_{k}=\widetilde{\psi}_{k}(D)$, de sorte que $\left\langle\varepsilon 2^{k+5}\right\rangle^{-n / 2} S_{k+5} h_{\varepsilon}=\widetilde{S}_{k}\left(\langle\varepsilon D\rangle^{-n / 2} h_{\varepsilon}\right)$. Nous sommes amenés à estimer $\sum_{k}\left|\widetilde{\Delta}_{k} V_{\varepsilon} \widetilde{S}_{k}\left(\langle\varepsilon D\rangle^{-n / 2} h_{\varepsilon}\right)\right|_{L^{2}}^{2}$. Or ce terme entre dans le cadre du lemme 3.1.4, et il existe une constante $C$, indépendante de $\varepsilon$, telle que :

$$
\sum_{k}\left|\widetilde{\Delta}_{k} V_{\varepsilon} \widetilde{S}_{k}\left(\langle\varepsilon D\rangle^{-n / 2} h_{\varepsilon}\right)\right|_{L^{2}}^{2} \leqslant C\left|\langle\varepsilon D\rangle^{-n / 2} h_{\varepsilon}\right|_{L^{2}}^{2}\left|V_{\varepsilon}\right|_{\mathrm{BMO}}^{2}
$$

Par ailleurs :

$$
\sum_{k \geqslant 0}\left|\left\langle\varepsilon 2^{k}\right\rangle^{\frac{n}{2}} \Delta_{k} U_{\varepsilon}\right|_{L^{2}}^{2} \leqslant C\left|\langle\varepsilon D\rangle^{\frac{n}{2}} U_{\varepsilon}\right|_{L^{2}}^{2}
$$

Nous obtenons alors l'estimation désirée :

$$
\left|\langle\varepsilon D\rangle^{\frac{n}{2}} R\left(U_{\varepsilon}, V_{\varepsilon}\right)\right|_{L^{2}} \leqslant C\left|\langle\varepsilon D\rangle^{\frac{n}{2}} U_{\varepsilon}\right|_{L^{2}}\left|V_{\varepsilon}\right|_{\mathrm{BMO}}
$$


En rassemblant les trois inégalités obtenues, nous disposons, pour tout $\left.\varepsilon \in] 0, \varepsilon_{0}\right]$, de l'estimation uniforme suivante :

$$
\begin{aligned}
& \quad\left|\langle\varepsilon D\rangle^{\frac{n}{2}}\left(U_{\varepsilon} V_{\varepsilon}\right)\right|_{H^{-s}} \\
& \quad \leqslant C\left(\left|\langle\varepsilon D\rangle^{\frac{n}{2}} U_{\varepsilon}\right|_{L^{2}}\left|V_{\varepsilon}\right|_{\mathrm{BMO}}+\left|\langle\varepsilon D\rangle^{\frac{n}{2}} V_{\varepsilon}\right|_{L^{2}}\left|U_{\varepsilon}\right|_{\mathrm{BMO}}+\left|\langle\varepsilon D\rangle^{\frac{n}{2}} U_{\varepsilon}\right|_{L^{2}}\left|\langle\varepsilon D\rangle^{\frac{n}{2}} V_{\varepsilon}\right|_{L^{2}}\right)
\end{aligned}
$$

On applique l'injection de Sobolev limite de $H^{n / 2}$ dans BMO, et nous obtenons, pour tout $\left.\varepsilon \in] 0, \varepsilon_{0}\right]$, l'estimation

$$
\begin{aligned}
& \left|\langle\varepsilon D\rangle^{\frac{n}{2}}\left(U_{\varepsilon} V_{\varepsilon}\right)\right|_{H^{-s}} \\
& \quad \leqslant C\left(\left|\langle\varepsilon D\rangle^{\frac{n}{2}} U_{\varepsilon}\right|_{L^{2}}\left|\varepsilon^{-M / 2}\langle\varepsilon D\rangle^{\frac{n}{2}} V_{\varepsilon}\right|_{L^{2}}+\left|\langle\varepsilon D\rangle^{\frac{n}{2}} V_{\varepsilon}\right|_{L^{2}}\left|\varepsilon^{-M / 2}\langle\varepsilon D\rangle^{\frac{n}{2}} U_{\varepsilon}\right|_{L^{2}}\right)
\end{aligned}
$$

En multipliant chaque membre par $\varepsilon^{-M / 2}, M \geqslant n$, puis en prenant le sup sur $\varepsilon \in$ ] $\left.0, \varepsilon_{0}\right]$, nous déduisons

$$
\left\|\varepsilon^{-M / 2} U_{\varepsilon} V_{\varepsilon}\right\|_{-s, \frac{n}{2}, 2} \leqslant C\left\|\varepsilon^{-M / 2} U_{\varepsilon}\right\|_{0, \frac{n}{2}, 2} \cdot\left\|\varepsilon^{-M / 2} V_{\varepsilon}\right\|_{0, \frac{n}{2}, 2}
$$

Démonstration $d u c$ ). - Pour le terme $T_{V_{\varepsilon}} U_{\varepsilon}$ en considérant une famille de fonctions test $(h)_{\varepsilon}$ de $\mathcal{S}$, vérifiant

$$
\sup _{\left.\varepsilon \in] 0, \varepsilon_{0}\right]}\left|\langle\varepsilon D\rangle^{-n / 2} h\right|_{L^{2}} \leqslant 1
$$

il vient pour tout $\left.\varepsilon \in] 0, \varepsilon_{0}\right]$ :

$$
\begin{aligned}
& \left|\left\langle\sum_{k \geqslant 2} S_{k-2} V_{\varepsilon} \Delta_{k} U_{\varepsilon}, h_{\varepsilon}\right\rangle\right| \\
& \quad \leqslant C\left(\sum_{k}\left|2^{k s}\left\langle\varepsilon 2^{k}\right\rangle^{n / 2} \Delta_{k} U_{\varepsilon}\right|_{L^{2}}^{2}\right)^{1 / 2}\left(\sum_{\left|k-k^{\prime}\right| \leqslant 5}\left|S_{k} V_{\varepsilon} 2^{-k s}\left\langle\varepsilon 2^{k^{\prime}}\right\rangle^{-n / 2} \Delta_{k^{\prime}} h_{\varepsilon}\right|_{L^{2}}^{2}\right)^{1 / 2}
\end{aligned}
$$

Par le lemme $3.1 .3 \mathrm{~b}$ ) et $2.3 .5 \mathrm{~b}$ ') :

$$
\begin{aligned}
\sum_{\left|k-k^{\prime}\right| \leqslant 5} \mid S_{k} & \left.V_{\varepsilon} 2^{-k s}\left\langle\varepsilon 2^{k^{\prime}}\right\rangle^{-n / 2} \Delta_{k^{\prime}} h_{\varepsilon}\right|_{L^{2}} ^{2} \leqslant C \sum_{\left|k-k^{\prime}\right| \leqslant 5} 2^{-2 k s}\left|S_{k} V_{\varepsilon}\right|_{L^{\infty}}^{2}\left|\Delta_{k^{\prime}}\langle\varepsilon D\rangle^{-n / 2} h_{\varepsilon}\right|_{L^{2}}^{2} \\
& \leqslant C\left(\left|\Delta_{-1} V_{\varepsilon}\right|_{L^{\infty}}+\left|V_{\varepsilon}\right|_{\mathrm{BMO}}\right)^{2} \sum_{k} k 2^{-2 k s}\left|\Delta_{k}\langle\varepsilon D\rangle^{-n / 2} h_{\varepsilon}\right|_{L^{2}}^{2} \\
& \leqslant C\left(\left|V_{\varepsilon}\right|_{L^{2}}+\left|V_{\varepsilon}\right|_{\mathrm{BMO}}\right)^{2}\left|\langle\varepsilon D\rangle^{-n / 2} h_{\varepsilon}\right|_{L^{2}}^{2} \\
& \leqslant C\left(\left|V_{\varepsilon}\right|_{L^{2}}+\left|V_{\varepsilon}\right|_{\mathrm{BMO}}\right)^{2}
\end{aligned}
$$

comme

$$
\sum\left|2^{k s}\left\langle\varepsilon 2^{k}\right\rangle^{n / 2} \Delta_{k} U_{\varepsilon}\right|_{L^{2}}^{2} \leqslant C\left|\langle\varepsilon D\rangle^{n / 2} U_{\varepsilon}\right|_{H^{s}}^{2}
$$

Il vient alors pour tout $\left.\varepsilon \in] 0, \varepsilon_{0}\right]$ :

$$
\left|\langle\varepsilon D\rangle^{n / 2}\left(T_{V_{\varepsilon}} U_{\varepsilon}\right)\right|_{L^{2}} \leqslant C\left|\langle\varepsilon D\rangle^{n / 2} U_{\varepsilon}\right|_{H^{s}}\left(\left|V_{\varepsilon}\right|_{L^{2}}+\left|V_{\varepsilon}\right|_{\mathrm{BMO}}\right)^{2}
$$

Pour le terme de reste $R\left(U_{\varepsilon}, V_{\varepsilon}\right)$, nous procédons exactement comme au a) et obtenons

$$
\left|\langle\varepsilon D\rangle^{\frac{n}{2}} R\left(U_{\varepsilon}, V_{\varepsilon}\right)\right|_{L^{2}} \leqslant C\left|\langle\varepsilon D\rangle^{\frac{n}{2}} U_{\varepsilon}\right|_{L^{2}}\left|V_{\varepsilon}\right|_{\mathrm{BMO}}
$$


En multipliant chacune des deux inégalités obtenues par $\varepsilon^{-M / 2}, M \geqslant n$, puis en prenant le $\left.\sup \operatorname{sur} \varepsilon \in] 0, \varepsilon_{0}\right]$, nous déduisons

$$
\left\|\varepsilon^{-M / 2} T_{V_{\varepsilon}} U_{\varepsilon}+\varepsilon^{-M / 2} R\left(U_{\varepsilon}, V_{\varepsilon}\right)\right\|_{0, \frac{n}{2}, 2} \leqslant C\left\|\varepsilon^{-M / 2} U_{\varepsilon}\right\|_{s, \frac{n}{2}, 2}\left(\left\|V_{\varepsilon}\right\|_{0,0, \infty}+\left\|V_{\varepsilon}\right\|_{0,0,2}\right)
$$

et puisque le lemme 2.3 .8 nous garantit que $\mathcal{Z}_{\varepsilon}^{\left(-s, \frac{n}{2}\right)} \hookrightarrow \mathcal{W}_{\varepsilon}^{-s, 0, \infty}$, il vient finalement

$$
\left\|\varepsilon^{-M / 2} T_{V_{\varepsilon}} U_{\varepsilon}+\varepsilon^{-M / 2} R\left(U_{\varepsilon}, V_{\varepsilon}\right)\right\|_{0, \frac{n}{2}, 2} \leqslant C\left\|\varepsilon^{-M / 2} U_{\varepsilon}\right\|_{s, \frac{n}{2}, 2} \cdot\left\|\mid V_{\varepsilon}\right\|_{\left(0, \frac{n}{2}\right)}
$$

Pour le terme $T_{U_{\varepsilon}} V_{\varepsilon}$ nous considérons une famille de fonctions test $(h)_{\varepsilon}$ de $\mathcal{S}$, telle que

$$
\sup _{\left.\varepsilon \in] 0, \varepsilon_{0}\right]}\left|\langle\varepsilon D\rangle^{-n / 2} h\right|_{L^{2}} \leqslant 1
$$

Il vient :

$$
\begin{aligned}
& \left|\left\langle\sum_{k \geqslant 2} S_{k-2} U_{\varepsilon} \Delta_{k} V_{\varepsilon}, h_{\varepsilon}\right\rangle\right| \\
& \quad \leqslant C\left(\sum_{k \geqslant 2}\left|S_{k-2} U_{\varepsilon}\left\langle\varepsilon 2^{k}\right\rangle^{\frac{n}{2}} \Delta_{k} V_{\varepsilon}\right|_{L^{2}}^{2}\right)^{1 / 2}\left(\sum_{k \geqslant 0}\left|\left\langle\varepsilon 2^{k}\right\rangle^{-n / 2} \Delta_{k} h_{\varepsilon}\right|_{L^{2}}^{2}\right)^{1 / 2}
\end{aligned}
$$

nous majorons :

$$
\sum\left|\left\langle\varepsilon 2^{k}\right\rangle^{-n / 2} \Delta_{k} h_{\varepsilon}\right|_{L^{2}}^{2} \leqslant C\left|\langle\varepsilon D\rangle^{-n / 2} h_{\varepsilon}\right|_{L^{2}}^{2}
$$

Pour le terme restant, nous décomposons $V_{\varepsilon} \in \mathcal{Z}_{\varepsilon}^{\left(-0, \frac{n}{2}\right)}$ en

$$
V_{\varepsilon}=V_{\varepsilon}^{\prime}+V_{\varepsilon}^{\prime \prime}
$$

de sorte que

$$
\left\|V_{\varepsilon}^{\prime}\right\|_{\frac{n}{2}, 0,2} \leqslant 2\left\||| V_{\varepsilon} \mid\right\|_{\left(0, \frac{n}{2}\right)} \quad \text { et } \quad\left\|V_{\varepsilon}^{\prime \prime}\right\|_{0, \frac{n}{2}, \infty} \leqslant 2 \mid\left\|V_{\varepsilon}\right\|_{\left(0, \frac{n}{2}\right)}
$$

Nous pouvons alors découper :

$$
\sum_{k \geqslant 2}\left|S_{k-2} U_{\varepsilon}\left\langle\varepsilon 2^{k}\right\rangle^{\frac{n}{2}} \Delta_{k} V_{\varepsilon}\right|_{L^{2}}^{2} \leqslant \Sigma_{1}^{\prime}+\Sigma_{2}^{\prime}+\Sigma^{\prime \prime}
$$

avec

$$
\Sigma_{1}^{\prime}=\sum_{k \geqslant 2, \varepsilon 2^{k} \leqslant 1}\left|S_{k-2} U_{\varepsilon}\left\langle\varepsilon 2^{k}\right\rangle^{\frac{n}{2}} \Delta_{k} V_{\varepsilon}^{\prime}\right|_{L^{2}}^{2} \leqslant C \sum_{k \geqslant 2}\left|S_{k-2} U_{\varepsilon} \Delta_{k} V_{\varepsilon}^{\prime}\right|_{L^{2}}^{2}
$$

et

$$
\Sigma_{2}^{\prime}=\sum_{k \geqslant 2,2^{k} \geqslant 1}\left|S_{k-2} U_{\varepsilon}\left\langle\varepsilon 2^{k}\right\rangle^{\frac{n}{2}} \Delta_{k} V_{\varepsilon}^{\prime}\right|_{L^{2}}^{2} \leqslant C \varepsilon^{n} \sum_{k \geqslant 2}\left|S_{k-2} U_{\varepsilon} 2^{k \frac{n}{2}} \Delta_{k} V_{\varepsilon}^{\prime}\right|_{L^{2}}^{2}
$$

tandis que

$$
\Sigma^{\prime \prime}=\sum_{k \geqslant 2}\left|S_{k-2} U_{\varepsilon}\left\langle\varepsilon 2^{k}\right\rangle^{\frac{n}{2}} \Delta_{k} V_{\varepsilon}^{\prime \prime}\right|_{L^{2}}^{2}
$$

Pour $\Sigma^{\prime \prime}$ et $\Sigma_{1}^{\prime}$, il faut de nouveau avoir recours au lemme 3.1.4. Il existe des constantes $C$ - indépendantes de $\varepsilon$ - telles que :

$$
\Sigma^{\prime \prime} \leqslant C\left|\langle\varepsilon D\rangle^{n / 2} V_{\varepsilon}^{\prime \prime}\right|_{\mathrm{BMO}}^{2}\left|U_{\varepsilon}\right|_{L^{2}}^{2} \quad \text { et } \quad \Sigma_{1}^{\prime} \leqslant C\left|\langle D\rangle^{-s} V_{\varepsilon}^{\prime}\right|_{\mathrm{BMO}}^{2}\left|U_{\varepsilon}\right|_{L^{2}}^{2}
$$


par l'injection de Sobolev $H^{n / 2} \hookrightarrow$ BMO, nous en déduisons :

$$
\Sigma_{1}^{\prime} \leqslant C\left|V_{\varepsilon}^{\prime}\right|_{H^{-s+\frac{n}{2}}}^{2}\left|U_{\varepsilon}\right|_{L^{2}}^{2}
$$

Pour $\Sigma_{2}^{\prime}$, nous majorons directement, en utilisant l'injection de Sobolev ordinaire $H^{s+\frac{n}{2}} \hookrightarrow L^{\infty}$ lorsque $s>0$ :

$$
\begin{aligned}
\Sigma_{2}^{\prime} & \leqslant C \varepsilon^{n} \sum_{k \geqslant 2}\left|S_{k-2} U_{\varepsilon} 2^{k \frac{n}{2}} \Delta_{k} V_{\varepsilon}^{\prime}\right|_{L^{2}}^{2} \\
& \leqslant C \varepsilon^{n} \sum_{k \geqslant 2}\left|S_{k-2} U_{\varepsilon}\right|_{L^{\infty}}^{2}\left|2^{k \frac{n}{2}} \Delta_{k} V_{\varepsilon}^{\prime}\right|_{L^{2}}^{2} \\
& \leqslant C \varepsilon^{n}\left|U_{\varepsilon}\right|_{L^{\infty}}^{2}\left|V_{\varepsilon}^{\prime}\right|_{H^{\frac{n}{2}}}^{2} \\
& \leqslant C \varepsilon^{n}\left|\langle D\rangle^{s+\frac{n}{2}} U_{\varepsilon}\right|_{L^{2}}^{2}\left|V_{\varepsilon}^{\prime}\right|_{H^{\frac{n}{2}}}^{2} \\
& \leqslant C\left|\langle D\rangle^{s}\langle\varepsilon D\rangle^{\frac{n}{2}} U_{\varepsilon}\right|_{L^{2}}^{2}\left|V_{\varepsilon}^{\prime}\right|_{H^{\frac{n}{2}}}^{2}
\end{aligned}
$$

Pour tout $\left.\varepsilon \in] 0, \varepsilon_{0}\right]$, nous disposons de l'estimation uniforme suivante :

$$
\begin{aligned}
\left|\langle\varepsilon D\rangle^{\frac{n}{2}}\left(T_{U_{\varepsilon}} V_{\varepsilon}\right)\right|_{L^{2}} & \leqslant C\left|\langle\varepsilon D\rangle^{\frac{n}{2}} U_{\varepsilon}\right|_{H^{s}}\left(\left|\langle\varepsilon D\rangle^{n / 2} V_{\varepsilon}^{\prime \prime}\right|_{\mathrm{BMO}}+\left|V_{\varepsilon}^{\prime}\right|_{H^{\frac{n}{2}}}\right) \\
& \leqslant\left. 2 C\left|\langle\varepsilon D\rangle^{\frac{n}{2}} U_{\varepsilon}\right|_{H^{s}}||\left|V_{\varepsilon}\right|\right|_{\left(0, \frac{n}{2}\right)}
\end{aligned}
$$

En multipliant chaque membre par $\varepsilon^{-M / 2}, M \geqslant n$, puis en prenant le sup sur $\varepsilon \in$ ] $\left.0, \varepsilon_{0}\right]$, nous déduisons

$$
\left\|\varepsilon^{-M / 2} T_{U_{\varepsilon}} V_{\varepsilon}\right\|_{0, \frac{n}{2}, 2} \leqslant C\left\|\varepsilon^{-M / 2} U_{\varepsilon}\right\|_{s, \frac{n}{2}, 2} \mid\left\|V_{\varepsilon}\right\|_{\left(0, \frac{n}{2}\right)}
$$

Ceci achève la preuve du c). Nous procédons de façon similaire pour prouver les autres inégalités.

\subsection{Résultat d'existence pour un système modèle}

Nous complétons ce paragraphe par un résultat d'existence pour un système semilinéaire représentatif de ceux que nous devons résoudre pour prouver le théorème 3 . Ce résultat est tout à fait classique une fois établies les estimations bilinéaires de la section précédente :

Proposition 3.2.1. - Soit $M \geqslant n$. On considère des familles

$$
\begin{gathered}
(B)_{\varepsilon} \in C^{0}\left(\left[0, t_{0}\right], \mathcal{Z}_{\varepsilon}^{\left(\frac{1}{2}, \frac{n}{2}\right)}\right) \cap C^{1}\left(\left[0, t_{0}\right], \mathcal{Z}_{\varepsilon}^{\left(-\frac{1}{2}, \frac{n}{2}\right)}\right) \\
(\beta)_{\varepsilon} \in C^{0}\left(\left[0, t_{0}\right], \mathcal{Z}_{\varepsilon}^{\left(0, \frac{n}{2}\right)}\right)
\end{gathered}
$$

ainsi que

$$
\begin{gathered}
(P)_{\varepsilon} \in C^{0}\left(\left[0, t_{0}\right], \varepsilon^{M / 2} \mathcal{W}_{\varepsilon}^{-\frac{1}{2}, \frac{n}{2}, 2}\right) \\
(Q)_{\varepsilon} \in C^{0}\left(\left[0, t_{0}\right], \varepsilon^{M / 2} \mathcal{W}_{\varepsilon}^{0, \frac{n}{2}, 2}\right)
\end{gathered}
$$

Les fonctions $p(Z, \zeta, B, \beta)$ et $q(Z, \zeta, B, \beta)$ sont polynomiales de degré 3 , à coefficients $C^{\infty}$, et s'annulent au moins à l'ordre 1 en $(Z, \zeta)=(0,0)$. p est de degré au plus 1 en 
$\zeta$ et 2 en $\zeta, q$ est de degré au plus 1 en $\beta$ et $\zeta$. La notation « • désigne une forme bilinéaire arbitraire.

Si $(Z c)_{\varepsilon} \in \varepsilon^{M / 2} \mathcal{W}_{\varepsilon}^{\frac{1}{2}, \frac{n}{2}, 2},\left(Z c^{\prime}\right)_{\varepsilon} \in \varepsilon^{M / 2} \mathcal{W}_{\varepsilon}^{-\frac{1}{2}, \frac{n}{2}, 2}$ et $\left(\zeta_{c}\right)_{\varepsilon} \in \varepsilon^{M / 2} \mathcal{W}_{\varepsilon}^{0, \frac{n}{2}, 2}$, alors $i l$ existe un temps $0<t_{1} \leqslant t_{0}$ et une unique famille $(Z, \zeta)_{\varepsilon}$

$$
\begin{gathered}
(Z)_{\varepsilon} \in C^{0}\left(\left[0, t_{1}\right], \varepsilon^{M / 2} \mathcal{W}_{\varepsilon}^{\frac{1}{2}, \frac{n}{2}, 2}\right) \cap C^{1}\left(\left[0, t_{1}\right], \varepsilon^{M / 2} \mathcal{W}_{\varepsilon}^{-\frac{1}{2}, \frac{n}{2}, 2}\right) \\
(\zeta)_{\varepsilon} \in C^{0}\left(\left[0, t_{1}\right], \varepsilon^{M / 2} \mathcal{W}_{\varepsilon}^{0, \frac{n}{2}, 2}\right)
\end{gathered}
$$

solution pour tout $\varepsilon$ du problème de Cauchy suivant :

$$
\left\{\begin{aligned}
\square Z_{\varepsilon} & =Z \cdot \partial Z+Z \cdot \partial B+B \cdot \partial Z+p(Z, \zeta, B, \beta)+P_{\varepsilon} \\
\mathcal{D} \zeta & =q(Z, \zeta, B, \beta)+Q_{\varepsilon}
\end{aligned}\right.
$$

avec

$$
Z_{\varepsilon}(0, y)=Z c_{\varepsilon}(y), \partial_{t} Z_{\varepsilon}(0, y)=Z c_{\varepsilon}^{\prime}(y) \text { et } \zeta_{\varepsilon}(0, y)=\zeta_{c_{\varepsilon}}(y)
$$

De plus, si

$$
\begin{aligned}
& \left.\left|P_{\varepsilon}\right|_{L^{\infty}\left(\left[0, t^{\prime}\right], \varepsilon^{M / 2}\right.} \mathcal{W}_{\varepsilon}^{-\frac{1}{2}, \frac{n}{2}, 2}\right) \\
& \left.+\|\left. Q_{\varepsilon}\right|_{L^{\infty}\left(\left[0, t^{\prime}\right], \varepsilon^{M / 2}\right.} \mathcal{W}_{\varepsilon}^{0, \frac{n}{2}, 2}\right) \\
& +\left\|\varepsilon^{-M / 2} Z c_{\varepsilon}\right\|_{\frac{1}{2}, \frac{n}{2}, 2}+\left\|\varepsilon^{-M / 2} Z c_{\varepsilon}^{\prime}\right\|_{-\frac{1}{2}, \frac{n}{2}, 2}+\left\|\varepsilon^{-M / 2} \zeta c_{\varepsilon}\right\|_{0, \frac{n}{2}, 2} \leqslant \delta_{0}
\end{aligned}
$$

pour un certain $\delta_{0}$ assez petit, alors $t_{1}=t_{0}$.

En particulier, si on a pris soin de choisir $M>M^{\prime} \geqslant n$ assez grand pour que $\varepsilon_{0}^{M-M^{\prime}} \leqslant \delta_{0}$, alors il existe une unique solution à $(S)$, sur $\left[0, t_{0}\right]$ tout entier, et vérifiant :

$$
\begin{gathered}
(Z)_{\varepsilon} \in C^{0}\left(\left[0, t_{0}\right], \varepsilon^{M^{\prime} / 2} \mathcal{W}_{\varepsilon}^{\frac{1}{2}, \frac{n}{2}, 2}\right) \cap C^{1}\left(\left[0, t_{0}\right], \varepsilon^{M^{\prime} / 2} \mathcal{W}_{\varepsilon}^{-\frac{1}{2}, \frac{n}{2}, 2}\right) \\
(\zeta)_{\varepsilon} \in C^{0}\left(\left[0, t_{0}\right], \varepsilon^{M^{\prime} / 2} \mathcal{W}_{\varepsilon}^{0, \frac{n}{2}, 2}\right)
\end{gathered}
$$

Démonstration. - Nous résolvons par une méthode traditionnelle de point fixe. Le schéma itératif mis en œuvre est le suivant : dans un premier temps nous montrons qu'il existe une suite $(Z, \zeta)_{k}$ (nous omettons de noter la dépendance en $\varepsilon$ pour plus de lisibilité) solution des systèmes linéarisés

$(\text { S.Lin. })_{k}$

$$
\left\{\begin{aligned}
\square Z_{k+1} & =P_{k+1}^{l i n .}+P_{k}^{n . l .}+P_{\varepsilon} \\
\mathcal{D} \zeta_{k+1} & =Q_{k+1}^{l i n .}+Q_{k}^{n . l .}+Q_{\varepsilon}
\end{aligned}\right.
$$

avec les même données de Cauchy pour tout $k$ :

$$
Z_{k+1}(0, y)=Z c_{\varepsilon}(y), \partial_{t} Z_{k+1}(0, y)=Z c_{\varepsilon}^{\prime}(y) \text { et } \zeta_{k+1}(0, y)=\zeta_{c \varepsilon}(y)
$$


puis nous prouvons que cette suite converge uniformément en $\varepsilon$ vers la solution cherchée. Nous avons décomposé le second membre en ses termes linéaires et non linéaires :

$$
\begin{aligned}
P_{k+1}^{l i n .} & =Z_{k+1} . \partial B+B \cdot \partial Z_{k+1}+p^{1}\left(B_{\varepsilon}, \beta_{\varepsilon}\right) Z_{k+1}+p^{2}\left(B_{\varepsilon}, \beta_{\varepsilon}\right) \zeta_{k+1} \\
P_{k}^{n . l .} & =Z_{k} \partial Z_{k}+p^{3}\left(Z_{k}, \zeta_{k}, B_{\varepsilon}, \beta_{\varepsilon}\right) \\
Q_{k+1}^{l i n .} & =q^{1}\left(B_{\varepsilon}, \beta_{\varepsilon}\right) Z_{k+1}+q^{2}\left(B_{\varepsilon}, \beta_{\varepsilon}\right) \zeta_{k+1} \\
Q_{k}^{n . l .} & =q^{3}\left(Z_{k}, \zeta_{k}, B_{\varepsilon}, \beta_{\varepsilon}\right)
\end{aligned}
$$

où

$$
\begin{aligned}
p^{1}(B, \beta) Z & =\partial_{Z} p(0,0, B, \beta) Z \\
p^{2}(B, \beta) \zeta & =\partial_{\zeta} p(0,0, B, \beta) \zeta \\
p^{3}(Z, \zeta, B, \beta) & =p(Z, \zeta, B, \beta)-p^{1}(B, \beta) Z-p^{2}(B, \beta) \zeta \\
q^{1}(B, \beta) Z & =\partial_{Z} q(0,0, B, \beta) Z \\
q^{2}(B, \beta) \zeta & =\partial_{\zeta} q(0,0, B, \beta) \zeta \\
q^{3}(Z, \zeta, B, \beta) & =q(Z, \zeta, B, \beta)-q^{1}(B, \beta) Z-q^{2}(B, \beta) \zeta
\end{aligned}
$$

Nous prenons $(Z, \zeta)_{0}=0$. Tout les résultats nécessaires à la convergence du point fixe sont contenus dans la proposition 3.1.1. Nous utiliserons en fait le corollaire suivant :

Proposition 3.2.2. - Supposons que $M \geqslant n$ et

$$
\left|B_{\varepsilon}\right|_{L^{\infty}\left(\left[0, t_{0}\right], \mathcal{Z}_{\varepsilon}^{\left(\frac{1}{2}, \frac{n}{2}\right)}\right)}+\left|\partial_{t} B_{\varepsilon}\right|_{L^{\infty}\left(\left[0, t_{0}\right], \mathcal{Z}_{\varepsilon}^{\left(-\frac{1}{2}, \frac{n}{2}\right)}\right)}+\left|\beta_{\varepsilon}\right|_{L^{\infty}\left(\left[0, t_{0}\right], \mathcal{Z}_{\varepsilon}^{\left(0, \frac{n}{2}\right)}\right)} \leqslant \delta
$$

a) Il existe une constante positive $C(\delta)$, polynomiale en $\delta$ et vérifiant $C(0)=0$, telle que

$$
\begin{aligned}
& \left\|\varepsilon^{-M / 2} p_{k+1}^{\text {lin. }}\right\|_{-\frac{1}{2}, \frac{n}{2}, 2} \\
& \quad \leqslant C(\delta)\left(\left\|\varepsilon^{-M / 2} Z_{k+1}\right\|_{\frac{1}{2}, \frac{n}{2}, 2}+\left\|\varepsilon^{-M / 2} \partial_{t} Z_{k+1}\right\|_{-\frac{1}{2}, \frac{n}{2}, 2}+\left\|\varepsilon^{-M / 2} \zeta_{k+1}\right\|_{0, \frac{n}{2}, 2}\right) \\
& \left\|\varepsilon^{-M / 2} q_{k+1}^{\text {lin. }}\right\|_{0, \frac{n}{2}, 2} \\
& \quad \leqslant C(\delta)\left(\left\|\varepsilon^{-M / 2} Z_{k+1}\right\|_{\frac{1}{2}, \frac{n}{2}, 2}+\left\|\varepsilon^{-M / 2} \partial_{t} Z_{k+1}\right\|_{-\frac{1}{2}, \frac{n}{2}, 2}+\left\|\varepsilon^{-M / 2} \zeta_{k+1}\right\|_{0, \frac{n}{2}, 2}\right)
\end{aligned}
$$

b) $S i\left\|\varepsilon^{-M / 2} Z_{k}\right\|_{\frac{1}{2}, \frac{n}{2}, 2}+\left\|\varepsilon^{-M / 2} \partial_{t} Z_{k}\right\|_{-\frac{1}{2}, \frac{n}{2}, 2}+\left\|\varepsilon^{-M / 2} \zeta_{k}\right\|_{0, \frac{n}{2}, 2} \leqslant \delta^{\prime}$, alors il existe une constante positive $C\left(\delta, \delta^{\prime}\right)$, polynomiale en $\delta$ et $\delta^{\prime}$, et s'annulant à l'ordre 2 en $\delta^{\prime}=0$, telle que

$$
\left\|\varepsilon^{-M / 2} p_{k}^{n . l \cdot}\right\|_{-\frac{1}{2}, \frac{n}{2}, 2} \leqslant C\left(\delta, \delta^{\prime}\right), \quad\left\|\varepsilon^{-M / 2} q_{k}^{n . l \cdot}\right\|_{0, \frac{n}{2}, 2} \leqslant C\left(\delta, \delta^{\prime}\right)
$$

c) $S i$

$$
\left\|\varepsilon^{-M / 2} Z_{k}\right\|_{\frac{1}{2}, \frac{n}{2}, 2}+\left\|\varepsilon^{-M / 2} \partial_{t} Z_{k}\right\|_{-\frac{1}{2}, \frac{n}{2}, 2}+\left\|\varepsilon^{-M / 2} \zeta_{k}\right\|_{0, \frac{n}{2}, 2} \leqslant \delta^{\prime}
$$

et

$$
\left\|\varepsilon^{-M / 2} Z_{k-1}\right\|_{\frac{1}{2}, \frac{n}{2}, 2}+\left\|\varepsilon^{-M / 2} \partial_{t} Z_{k-1}\right\|_{-\frac{1}{2}, \frac{n}{2}, 2}+\left\|\varepsilon^{-M / 2} \zeta_{k-1}\right\|_{0, \frac{n}{2}, 2} \leqslant \delta^{\prime}
$$


alors il existe une constante positive $C\left(\delta, \delta^{\prime}\right)$, polynomiale en $\delta$ et $\delta^{\prime}$ et s'annulant à l'ordre 1 en $\delta^{\prime}=0$, telle que, en notant $E_{k}=Z_{k}-Z_{k-1}$ et $\xi_{k}=\zeta_{k}-\zeta_{k-1}$, on ait

$$
\begin{aligned}
& \left\|\varepsilon^{-M / 2}\left(p_{k}^{n . l .}-p_{k-1}^{n . l .}\right)\right\|_{-\frac{1}{2}, \frac{n}{2}, 2} \\
& \quad \leqslant C\left(\delta, \delta^{\prime}\right)\left(\left\|\varepsilon^{-M / 2} E_{k}\right\|_{\frac{1}{2}, \frac{n}{2}, 2}+\left\|\varepsilon^{-M / 2} \partial_{t} E_{k}\right\|_{-\frac{1}{2}, \frac{n}{2}, 2}+\left\|\varepsilon^{-M / 2} \xi_{k}\right\|_{0, \frac{n}{2}, 2}\right) \\
& \left\|\varepsilon^{-M / 2}\left(q_{k}^{n . l .}-q_{k-1}^{n . l}\right)\right\|_{0, \frac{n}{2}, 2} \\
& \quad \leqslant C\left(\delta, \delta^{\prime}\right)\left(\left\|\varepsilon^{-M / 2} E_{k}\right\|_{\frac{1}{2}, \frac{n}{2}, 2}+\left\|\varepsilon^{-M / 2} \partial_{t} E_{k}\right\|_{-\frac{1}{2}, \frac{n}{2}, 2}+\left\|\varepsilon^{-M / 2} \xi_{k}\right\|_{0, \frac{n}{2}, 2}\right) .
\end{aligned}
$$

Démonstration. - Il s'agit d'appliquer les propositions 3.1.1 et 3.1.5 aux systèmes linéarisés. La preuve est purement mécanique. Nous omettons les indices lorsqu'il n'y a pas risque d'ambiguïté.

a) Les termes à estimer sont de la forme $: Z \cdot \operatorname{grad} B$ et $Z \cdot \partial_{t} B$ qui se traitent par le b) de la proposition 3.1.1 de la façon suivante

$$
\left\|\varepsilon^{-M / 2} Z \cdot \operatorname{grad} B\right\|_{-\frac{1}{2}, \frac{n}{2}, 2} \leqslant C\left\|\varepsilon^{-M / 2} Z\right\|_{\frac{1}{2}, \frac{n}{2}, 2}\|\mid \operatorname{grad} B\|\left\|_{\left(-\frac{1}{2}, \frac{n}{2}\right)} \leqslant C(\delta)\right\| \varepsilon^{-M / 2} Z \|_{\frac{1}{2}, \frac{n}{2}, 2}
$$

et

$$
\left\|\varepsilon^{-M / 2} Z \cdot \partial_{t} B\right\|_{-\frac{1}{2}, \frac{n}{2}, 2} \leqslant C\left\|\varepsilon^{-M / 2} Z\right\|_{\frac{1}{2}, \frac{n}{2}, 2}\left\|\partial_{t} B\right\|_{\left(-\frac{1}{2}, \frac{n}{2}\right)} \leqslant C(\delta)\left\|\varepsilon^{-M / 2} Z\right\|_{\frac{1}{2}, \frac{n}{2}, 2}
$$

$B \cdot \partial_{t} Z$ et $B \cdot \operatorname{grad} Z$ qui se traitent par le c) de la proposition 3.1.1,

$$
\begin{aligned}
& \left\|\varepsilon^{-M / 2} B \cdot \operatorname{grad} Z\right\|_{-\frac{1}{2}, \frac{n}{2}, 2} \\
& \quad \leqslant C\left\|\varepsilon^{-M / 2} \operatorname{grad} Z\right\|_{-\frac{1}{2}, \frac{n}{2}, 2}\|B\|\left\|_{\left(\frac{1}{2}, \frac{n}{2}\right)} \leqslant C(\delta)\right\| \varepsilon^{-M / 2} Z \|_{\frac{1}{2}, \frac{n}{2}, 2} \\
& \left\|\varepsilon^{-M / 2} \partial_{t} Z \cdot B\right\|_{-\frac{1}{2}, \frac{n}{2}, 2} \\
& \quad \leqslant C\left\|\varepsilon^{-M / 2} \partial_{t} Z\right\|_{-\frac{1}{2}, \frac{n}{2}, 2}\|B\|_{\left(\frac{1}{2}, \frac{n}{2}\right)} \leqslant C(\delta)\left\|\varepsilon^{-M / 2} \partial_{t} Z\right\|_{-\frac{1}{2}, \frac{n}{2}, 2}
\end{aligned}
$$

Le terme polynomial $p^{1}(B, \beta) Z$ est de la forme $\sum_{\left|\alpha_{1}\right|+\left|\alpha_{2}\right| \leqslant 2} C_{\alpha} Z B^{\alpha_{1}} \beta^{\alpha_{2}}$. Nous utilisons en alternance les propositions 3.1.1 et 3.1.5, et obtenons successivement des majorations du type :

$\left\|\varepsilon^{-M / 2} Z B^{2}\right\|_{-\frac{1}{2}, \frac{n}{2}, 2} \leqslant C\left\|\varepsilon^{-M / 2} Z B\right\|_{-\frac{1}{2}, \frac{n}{2}, 2}|\|B\||_{\left(\frac{1}{2}, \frac{n}{2}\right)} \leqslant C\|\| B\left\|\left.\right|_{\left(\frac{1}{2}, \frac{n}{2}\right)} ^{2}\right\| \varepsilon^{-M / 2} Z \|_{\frac{1}{2}, \frac{n}{2}, 2}$ ou

$$
\begin{aligned}
& \left\|\varepsilon^{-M / 2} Z B \beta\right\|_{-\frac{1}{2}, \frac{n}{2}, 2} \\
& \quad \leqslant C\left\|\varepsilon^{-M / 2} Z \beta\right\|_{-\frac{1}{2}, \frac{n}{2}, 2}\|B\|\left\|_{\left(\frac{1}{2}, \frac{n}{2}\right)} \leqslant C\right\|\|B\|\left\|_{\left(\frac{1}{2}, \frac{n}{2}\right)} \mid\right\| \beta\|\|_{\left(0, \frac{n}{2}\right)}\left\|\varepsilon^{-M / 2} Z\right\|_{\frac{1}{2}, \frac{n}{2}, 2}
\end{aligned}
$$

ou encore

$$
\left\|\varepsilon^{-M / 2} Z \beta\right\|_{-\frac{1}{2}, \frac{n}{2}, 2} \leqslant C \mid\|\beta\|\left\|_{\left(0, \frac{n}{2}\right)}\right\| \varepsilon^{-M / 2} Z \|_{\frac{1}{2}, \frac{n}{2}, 2}
$$

et il en résulte

$$
\left\|\varepsilon^{-M / 2} p^{1}(B, \beta) Z\right\|_{-\frac{1}{2}, \frac{n}{2}, 2} \leqslant C(\delta)\left\|\varepsilon^{-M / 2} Z\right\|_{\frac{1}{2}, \frac{n}{2}, 2}
$$


Le terme polynomial $p^{2}(B, \beta) \zeta$ est de la forme $\sum_{\left|\alpha_{1}\right|+\left|\alpha_{2}\right| \leqslant 2,\left|\alpha_{2}\right| \leqslant 1} C_{\alpha} \zeta B^{\alpha_{1}} \beta^{\alpha_{2}}$. Nous utilisons en alternance les propositions 3.1.1 et 3.1.5, et obtenons successivement des majorations du type :

$$
\left\|\varepsilon^{-M / 2} \zeta B^{2}\right\|_{-\frac{1}{2}, \frac{n}{2}, 2} \leqslant C\left\|\varepsilon^{-M / 2} \zeta B\right\|_{-\frac{1}{2}, \frac{n}{2}, 2}\|B\|\left\|_{\left(\frac{1}{2}, \frac{n}{2}\right)} \leqslant C\right\|\|B\|\left\|_{\left(\frac{1}{2}, \frac{n}{2}\right)}^{2}\right\| \varepsilon^{-M / 2} \zeta \|_{0, \frac{n}{2}, 2}
$$

$\mathrm{ou}$

$$
\begin{aligned}
& \left\|\varepsilon^{-M / 2} \zeta B \beta\right\|_{-\frac{1}{2}, \frac{n}{2}, 2} \\
& \quad \leqslant C\left\|\varepsilon^{-M / 2} \zeta B\right\|_{0, \frac{n}{2}, 2}\|\beta\|\left\|_{\left(0, \frac{n}{2}\right)} \leqslant C \mid\right\| B\|\|_{\left(\frac{1}{2}, \frac{n}{2}\right)}\|\beta\|\left\|_{\left(0, \frac{n}{2}\right)}\right\| \varepsilon^{-M / 2} \zeta \|_{0, \frac{n}{2}, 2}
\end{aligned}
$$

et il en résulte

$$
\left\|\varepsilon^{-M / 2} p^{2}(B, \beta) \zeta\right\|_{-\frac{1}{2}, \frac{n}{2}, 2} \leqslant C(\delta)\left\|\varepsilon^{-M / 2} \zeta\right\|_{0, \frac{n}{2}, 2}
$$

Le terme polynomial $q^{1}(B, \beta) Z$ est de la forme $\sum_{\left|\alpha_{1}\right|+\left|\alpha_{2}\right| \leqslant 2,\left|\alpha_{2}\right| \leqslant 1} C_{\alpha} Z B^{\alpha_{1}} \beta^{\alpha_{2}}$. Nous utilisons en alternance les propositions 3.1.1 et 3.1.5, et obtenons successivement des majorations du type :

$$
\left\|\varepsilon^{-M / 2} Z B^{2}\right\|_{0, \frac{n}{2}, 2} \leqslant C\left\|\varepsilon^{-M / 2} Z B\right\|_{0, \frac{n}{2}, 2}\left|\|B\|\left\|_{\left(\frac{1}{2}, \frac{n}{2}\right)} \leqslant C \mid\right\| B\|\|_{\left(\frac{1}{2}, \frac{n}{2}\right)}^{2}\left\|\varepsilon^{-M / 2} Z\right\|_{\frac{1}{2}, \frac{n}{2}, 2}\right.
$$

$\mathrm{ou}$

$$
\begin{aligned}
& \left\|\varepsilon^{-M / 2} Z B \beta\right\|_{0, \frac{n}{2}, 2} \\
& \quad \leqslant C\left\|\varepsilon^{-M / 2} Z \beta\right\|_{0, \frac{n}{2}, 2}\|B\|\left\|_{\left(\frac{1}{2}, \frac{n}{2}\right)} \leqslant C\left|\|B\|\left\|_{\left(\frac{1}{2}, \frac{n}{2}\right)} \mid\right\| \beta\|\|_{\left(0, \frac{n}{2}\right)}\left\|\varepsilon^{-M / 2} Z\right\|_{\frac{1}{2}, \frac{n}{2}, 2}\right.\right.
\end{aligned}
$$

et il en résulte

$$
\left\|\varepsilon^{-M / 2} q^{1}(B, \beta) Z\right\|_{0, \frac{n}{2}, 2} \leqslant C(\delta)\left\|\varepsilon^{-M / 2} Z\right\|_{\frac{1}{2}, \frac{n}{2}, 2}
$$

Le terme polynomial $q^{2}(B) \zeta$ est de la forme $\sum_{\left|\alpha_{1}\right| \leqslant 2} C_{\alpha} Z B^{\alpha_{1}}$. Nous utilisons en alternance les propositions 3.1.1 et 3.1.5, et obtenons des majorations du type :

$$
\left\|\varepsilon^{-M / 2} \zeta B^{2}\right\|_{0, \frac{n}{2}, 2} \leqslant C\left\|\varepsilon^{-M / 2} \zeta B\right\|_{0, \frac{n}{2}, 2}\left|\left\|B \left|\left\|_{\left(\frac{1}{2}, \frac{n}{2}\right)} \leqslant C\left|\|B \mid\|_{\left(\frac{1}{2}, \frac{n}{2}\right)}^{2}\left\|\varepsilon^{-M / 2} \zeta\right\|_{0, \frac{n}{2}, 2}\right.\right.\right.\right.\right.
$$

donc en définitive

$$
\left\|\varepsilon^{-M / 2} q^{2}(B) \zeta\right\|_{0, \frac{n}{2}, 2} \leqslant C(\delta)\left\|\varepsilon^{-M / 2} \zeta\right\|_{0, \frac{n}{2}, 2}
$$

b) Les termes à estimer sont de la forme $Z \cdot \partial_{t} Z, Z \cdot \operatorname{grad} Z$ que l'on traite cette fois par le a) de la proposition 3.1.1. Il vient :

$$
\left\|\varepsilon^{-M / 2} Z \cdot \operatorname{grad} Z\right\|_{-\frac{1}{2}, \frac{n}{2}, 2} \leqslant C\left\|\varepsilon^{-M / 2} Z\right\|_{\frac{1}{2}, \frac{n}{2}, 2}^{2}
$$

et

$$
\left\|\varepsilon^{-M / 2} Z \cdot \partial_{t} Z\right\|_{-\frac{1}{2}, \frac{n}{2}, 2} \leqslant C\left\|\varepsilon^{-M / 2} Z\right\|_{\frac{1}{2}, \frac{n}{2}, 2} \cdot\left\|\varepsilon^{-M / 2} \partial_{t} Z\right\|_{-\frac{1}{2}, \frac{n}{2}, 2}
$$

Pour les termes polynomiaux, qui sont de la forme $\sum_{|\alpha| \leqslant 3} C_{\alpha} Z^{\alpha_{1}} \zeta^{\alpha_{2}} B^{\alpha_{3}} \beta^{\alpha_{4}}$, où le multi-indice $\alpha$ est de la forme $\left|\alpha_{1}\right|+\left|\alpha_{2}\right|=i>1$ et $\left|\alpha_{2}\right|+\left|\alpha_{4}\right| \leqslant 2$ ou $\left|\alpha_{2}\right|+\left|\alpha_{4}\right| \leqslant 2$ 
respectivement pour $p^{3}$ et $q^{3}$. On applique successivement les propositions 3.1.1 et 3.1.5 pour estimer les produits. Il vient

$$
\begin{aligned}
& \left\|\varepsilon^{-M / 2} p^{3}(Z, \zeta, B, \beta)\right\|_{-\frac{1}{2}, \frac{n}{2}, 2} \\
& \quad \leqslant \sum_{\alpha} C_{\alpha}\left\|\left|\|B\|_{\left(\frac{1}{2}, \frac{n}{2}\right)}^{\left|\alpha_{1}\right|}\|\beta\|\right|_{\left(0, \frac{n}{2}\right)}^{\left|\alpha_{2}\right|}\right\| \varepsilon^{-M / 2} Z\left\|_{\frac{1}{2}, \frac{n}{2}, 2}^{\left|\alpha_{3}\right|}\right\| \varepsilon^{-M / 2} \zeta \|_{0, \frac{n}{2}, 2}^{\left|\alpha_{4}\right|} \\
& \left\|\varepsilon^{-M / 2} q^{3}(Z, \zeta, B, \beta)\right\|_{0, \frac{n}{2}, 2} \\
& \quad \leqslant \sum_{\alpha} C_{\alpha}\left\||\|B\||_{\left(\frac{1}{2}, \frac{n}{2}\right)}^{\left|\alpha_{1}\right|}\right\| \beta\|\|_{\left(0, \frac{n}{2}\right)}^{\left|\alpha_{2}\right|}\left\|\varepsilon^{-M / 2} Z\right\|_{\frac{1}{2}, \frac{n}{2}, 2}^{\left|\alpha_{3}\right|}\left\|\varepsilon^{-M / 2} \zeta\right\|_{0, \frac{n}{2}, 2}^{\left|\alpha_{4}\right|}
\end{aligned}
$$

donc finalement

$$
\left\|\varepsilon^{-M / 2} p^{3}(Z, \zeta, B, \beta)\right\|_{-\frac{1}{2}, \frac{n}{2}, 2} \leqslant \sum_{i+j \leqslant 3} C_{i, j} \delta^{i} \delta^{\prime j}
$$

et

$$
\left\|\varepsilon^{-M / 2} q^{3}(Z, \zeta, B, \beta)\right\|_{0, \frac{n}{2}, 2} \leqslant \sum_{i+j \leqslant 3} C_{i, j} \delta^{i} \delta^{j}
$$

Nous en déduisons que

$$
\left\|\varepsilon^{-M / 2} p^{n . l \cdot}\right\|_{-\frac{1}{2}, \frac{n}{2}, 2} \leqslant C\left(\delta, \delta^{\prime}\right) \quad \text { et } \quad\left\|\varepsilon^{-M / 2} q^{n . l \cdot}\right\|_{0, \frac{n}{2}, 2} \leqslant C\left(\delta, \delta^{\prime}\right)
$$

c) Les termes à estimer sont de la forme $Z_{k} \cdot \partial_{t} Z_{k}-Z_{k-1} \partial_{t} Z_{k-1}=E_{k} \partial_{t} Z_{k}+$ $Z_{k-1} \partial_{t} E_{k}$ et $Z_{k} \cdot \operatorname{grad} Z_{k}-Z_{k-1} \cdot \operatorname{grad} Z_{k-1}=E_{k} \operatorname{grad} Z_{k}+Z_{k-1} \operatorname{grad} E_{k}$ que l'on traite par le a) de la proposition 3.1.1 :

$$
\left\|\varepsilon^{-M} E_{k} \partial_{t} Z_{k}\right\|_{-\frac{1}{2}, \frac{n}{2}, 2} \leqslant C\left\|\varepsilon^{-M} E_{k}\right\|_{\frac{1}{2}, \frac{n}{2}, 2}\left\|\varepsilon^{-M} \partial_{t} Z_{k}\right\|_{-\frac{1}{2}, \frac{n}{2}, 2} \leqslant C \delta^{\prime}\left\|\varepsilon^{-M} E_{k}\right\|_{\frac{1}{2}, \frac{n}{2}, 2}
$$

et

$$
\begin{aligned}
& \left\|\varepsilon^{-M} Z_{k-1} \partial_{t} E_{k}\right\|_{-\frac{1}{2}, \frac{n}{2}, 2} \\
& \quad \leqslant C\left\|\varepsilon^{-M} Z_{k-1}\right\|_{\frac{1}{2}, \frac{n}{2}, 2}\left\|\varepsilon^{-M} \partial_{t} E_{k}\right\|_{-\frac{1}{2}, \frac{n}{2}, 2} \leqslant C \delta^{\prime}\left\|\varepsilon^{-M} \partial_{t} E_{k}\right\|_{-\frac{1}{2}, \frac{n}{2}, 2}
\end{aligned}
$$

ainsi que

$$
\begin{aligned}
\left\|\varepsilon^{-M} E_{k} \operatorname{grad} Z_{k}\right\|_{-\frac{1}{2}, \frac{n}{2}, 2} & \\
& \leqslant C\left\|\varepsilon^{-M} E_{k}\right\|_{\frac{1}{2}, \frac{n}{2}, 2}\left\|\varepsilon^{-M} \operatorname{grad} Z_{k}\right\|_{-\frac{1}{2}, \frac{n}{2}, 2} \leqslant C \delta^{\prime}\left\|\varepsilon^{-M} E_{k}\right\|_{\frac{1}{2}, \frac{n}{2}, 2}
\end{aligned}
$$

et

$$
\begin{aligned}
& \left\|\varepsilon^{-M} Z_{k-1} \operatorname{grad} E_{k}\right\|_{-\frac{1}{2}, \frac{n}{2}, 2} \\
& \quad \leqslant C\left\|\varepsilon^{-M} Z_{k-1}\right\|_{\frac{1}{2}, \frac{n}{2}, 2}\left\|\varepsilon^{-M} \operatorname{grad} E_{k}\right\|_{-\frac{1}{2}, \frac{n}{2}, 2} \leqslant C \delta^{\prime}\left\|\varepsilon^{-M} E_{k}\right\|_{\frac{1}{2}, \frac{n}{2}, 2}
\end{aligned}
$$

Les termes polynomiaux

$$
p^{3}\left(Z_{k}, \zeta_{k}, B, \beta\right)-p^{3}\left(Z_{k-1}, \zeta_{k-1}, B, \beta\right) \quad \text { et } \quad q^{3}\left(Z_{k}, \zeta_{k}, B, \beta\right)-q^{3}\left(Z_{k-1}, \zeta_{k-1}, B, \beta\right)
$$

peuvent s'exprimer sous forme d'une somme de termes de type

$$
E_{k}^{\alpha^{\prime}}\left(Z_{k}^{\alpha_{1}} \zeta_{k}^{\alpha_{2}}+Z_{k-1}^{\alpha_{1}} \zeta_{k-1}^{\alpha_{2}}\right) B^{\alpha_{3}} \beta^{\alpha_{4}} \quad \text { et } \quad \xi_{k}^{\alpha^{\prime}}\left(Z_{k}^{\alpha_{1}} \zeta_{k}^{\alpha_{2}}+Z_{k-1}^{\alpha_{1}} \zeta_{k-1}^{\alpha_{2}}\right) B^{\alpha_{3}} \beta^{\alpha_{4}}
$$


où $\left|\alpha^{\prime}\right|=1,\left|\alpha_{1}\right|+\left|\alpha_{2}\right|+\left|\alpha_{3}\right|+\left|\alpha_{4}\right| \leqslant 2$, avec les restrictions correspondantes sur $\left|\alpha_{2}\right|+\left|\alpha_{4}\right|$. On estime ces termes comme précédemment, en appliquant à tour de rôle les propositions 3.1.1 et 3.1.5. On obtient sans difficulté particulière l'estimation désirée.

Nous pouvons désormais prouver le résultat d'existence et d'unicité du système linéarisé :

Proposition 3.2.3. - Si $M \geqslant n$,

$$
\begin{gathered}
\left(p_{k}^{n . l .}, \mathcal{P}\right)_{\varepsilon} \in L^{1}\left(\left[0, t_{1}\right], \varepsilon^{M / 2} \mathcal{W}_{\varepsilon}^{-\frac{1}{2}, \frac{n}{2}, 2}\right) \\
\left(q_{k}^{n . l .}, \mathcal{Q}\right)_{\varepsilon} \in L^{1}\left(\left[0, t_{1}\right], \varepsilon^{M / 2} \mathcal{W}_{\varepsilon}^{0, \frac{n}{2}, 2}\right)
\end{gathered}
$$

et si

$$
\left|B_{\varepsilon}\right|_{L^{\infty}\left(\left[0, t_{1}\right], \mathcal{Z}_{\varepsilon}^{\left(\frac{1}{2}, \frac{n}{2}\right)}\right)}+\left|\partial_{t} B_{\varepsilon}\right|_{L^{\infty}\left(\left[0, t_{1}\right], \mathcal{Z}_{\varepsilon}^{\left(\frac{1}{2}, \frac{n}{2}\right)}\right)}+\left|\beta_{\varepsilon}\right|_{L^{\infty}\left(\left[0, t_{1}\right], \mathcal{Z}_{\varepsilon}^{\left(\frac{1}{2}, \frac{n}{2}\right)}\right)} \leqslant \delta
$$

alors il existe une unique solution

$$
\begin{gathered}
(Z)_{k+1} \in C^{0}\left(\left[0, t_{1}\right], \varepsilon^{M / 2} \mathcal{W}_{\varepsilon}^{\frac{1}{2}, \frac{n}{2}, 2}\right) \cap C^{1}\left(\left[0, t_{1}\right], \varepsilon^{M / 2} \mathcal{W}_{\varepsilon}^{-\frac{1}{2}, \frac{n}{2}, 2}\right) \\
(\zeta)_{k+1} \in C^{0}\left(\left[0, t_{1}\right], \varepsilon^{M / 2} \mathcal{W}_{\varepsilon}^{0, \frac{n}{2}, 2}\right)
\end{gathered}
$$

du système linéarisé (S.Lin.) $)_{k}$ avec les données initiales

$$
\left(Z c, Z c^{\prime}, \zeta c\right)_{\varepsilon} \in \varepsilon^{M / 2} \mathcal{W}_{\varepsilon}^{\frac{1}{2}, \frac{n}{2}, 2} \times \varepsilon^{M / 2} \mathcal{W}_{\varepsilon}^{-\frac{1}{2}, \frac{n}{2}, 2} \times \varepsilon^{M / 2} \mathcal{W}_{\varepsilon}^{0, \frac{n}{2}, 2}
$$

et qui vérifie l'estimation d'énergie

$(*)$

$$
\begin{gathered}
\sup _{t \in\left[0, t_{1}\right]} e^{-\lambda(\delta) t}\left(\left\|\varepsilon^{-M / 2} Z_{k+1}\right\|_{\frac{1}{2}, \frac{n}{2}, 2}+\left\|\varepsilon^{-M / 2} \partial_{t} Z_{k+1}\right\|_{-\frac{1}{2}, \frac{n}{2}, 2}+\left\|\varepsilon^{-M / 2} \zeta_{k+1}\right\|_{0, \frac{n}{2}, 2}\right) \\
\leqslant\left\|\varepsilon^{-M / 2} Z c_{\varepsilon}\right\|_{\frac{1}{2}, \frac{n}{2}, 2}+\left\|\varepsilon^{-M / 2} Z c_{\varepsilon}^{\prime}\right\|_{-\frac{1}{2}, \frac{n}{2}, 2}+\left\|\varepsilon^{-M / 2} \zeta_{c_{\varepsilon}}\right\|_{0, \frac{n}{2}, 2} \\
+\int_{0}^{t_{1}} e^{-\lambda(\delta) t}\left(\left\|\varepsilon^{-M / 2} p_{k}^{n . l \cdot}\right\|_{-\frac{1}{2}, \frac{n}{2}, 2}+\left\|\varepsilon^{-M / 2} q_{k}^{n . l .}\right\|_{0, \frac{n}{2}, 2}\right. \\
+\left\|\varepsilon^{-M / 2} P_{\varepsilon}\right\|_{-\frac{1}{2}, \frac{n}{2}, 2} \\
\left.+\left\|\varepsilon^{-M / 2} Q_{\varepsilon}\right\|_{0, \frac{n}{2}, 2}\right) d t
\end{gathered}
$$

Démonstration. - Nous disposons pour le système de l'estimation a priori classique à $\varepsilon$ fixé :

$$
\begin{gathered}
\sup _{\varsigma \in[0, t]} e^{-C \varsigma}\left(\left|\langle D\rangle^{1 / 2}\langle\varepsilon D\rangle^{m} Z_{k+1}\right|_{L^{2}}+\left|\langle D\rangle^{-1 / 2}\langle\varepsilon D\rangle^{m} \partial_{t} Z_{k+1}\right|_{L^{2}}+\left|\langle\varepsilon D\rangle^{m} \zeta_{k+1}\right|_{L^{2}}\right) \\
\leqslant\left|\langle D\rangle^{1 / 2}\langle\varepsilon D\rangle^{m} Z c_{\varepsilon}\right|_{L^{2}}+\left|\langle D\rangle^{-1 / 2}\langle\varepsilon D\rangle^{m} Z c_{\varepsilon}^{\prime}\right|_{L^{2}}+\left|\langle\varepsilon D\rangle^{m} \zeta_{c_{\varepsilon}}\right|_{L^{2}} \\
+\int_{0}^{t} e^{-C \varsigma}\left(\left|\langle D\rangle^{-1 / 2}\langle\varepsilon D\rangle^{m}\left(p_{k+1}^{\text {lin. }}+p_{k}^{n . l .}+P_{\varepsilon}\right)\right|_{L^{2}}+\left|\langle\varepsilon D\rangle^{m}\left(q_{k+1}^{l i n .}+q_{k}^{n . l .}+Q_{\varepsilon}\right)\right|_{L^{2}}\right) d \varsigma
\end{gathered}
$$


Puis nous multiplions chaque membre par $\varepsilon^{-M / 2}$ et nous prenons le sup sur $\left.\left.\varepsilon \in\right] 0, \varepsilon_{0}\right]$, et nous obtenons une inégalité d'énergie uniforme en $\varepsilon$ :

$$
\begin{gathered}
\sup _{\varsigma \in[0, t]} e^{-C \varsigma}\left(\left\|\varepsilon^{-M / 2} Z_{k+1}\right\|_{\frac{1}{2}, \frac{n}{2}, 2}+\left\|\varepsilon^{-M / 2} \partial_{t} Z_{k+1}\right\|_{-\frac{1}{2}, \frac{n}{2}, 2}+\left\|\varepsilon^{-M / 2} \zeta_{k+1}\right\|_{0, \frac{n}{2}, 2}\right) \\
\leqslant\left\|\varepsilon^{-M / 2} Z c_{\varepsilon}\right\|_{\frac{1}{2}, \frac{n}{2}, 2}+\left\|\varepsilon^{-M / 2} Z c_{\varepsilon}^{\prime}\right\|_{-\frac{1}{2}, \frac{n}{2}, 2}+\left\|\varepsilon^{-M / 2} \zeta_{c_{\varepsilon}}\right\|_{0, \frac{n}{2}, 2} \\
\quad+\int_{0}^{t} e^{-C \varsigma}\left(\left\|\varepsilon^{-M / 2} p_{k+1}^{l i n}\right\|_{-\frac{1}{2}, \frac{n}{2}, 2}+\left\|\varepsilon^{-M / 2} q_{k+1}^{l i n .}\right\|_{0, \frac{n}{2}, 2}\right. \\
\left.+\left\|\varepsilon^{-M / 2} p_{k}^{n . l \cdot}\right\|_{-\frac{1}{2}, \frac{n}{2}, 2}+\left\|\varepsilon^{-M / 2} q_{k}^{n . l \cdot}\right\|_{0, \frac{n}{2}, 2}+\left\|\varepsilon^{-M / 2} P_{\varepsilon}\right\|_{-\frac{1}{2}, \frac{n}{2}, 2}+\left\|\varepsilon^{-M / 2} Q_{\varepsilon}\right\|_{0, \frac{n}{2}, 2}\right) d \varsigma
\end{gathered}
$$

Par ailleurs, la proposition 3.2.2 nous donne l'estimation suivante sur les termes linéaires :

$$
\begin{aligned}
& \left\|\varepsilon^{-M / 2} p_{k+1}^{l i n}\right\|_{-\frac{1}{2}, \frac{n}{2}, 2}^{\text {in }} \\
& \quad \leqslant C(\delta)\left(\left\|\varepsilon^{-M / 2} Z_{k+1}\right\|_{\frac{1}{2}, \frac{n}{2}, 2}+\left\|\varepsilon^{-M / 2} \partial_{t} Z_{k+1}\right\|_{-\frac{1}{2}, \frac{n}{2}, 2}+\left\|\varepsilon^{-M / 2} \zeta_{k+1}\right\|_{0, \frac{n}{2}, 2}\right) \\
& \left\|\varepsilon^{-M / 2} q_{k+1}^{l i n}\right\|_{0, \frac{n}{2}, 2} \\
& \quad \leqslant C(\delta)\left(\left\|\varepsilon^{-M / 2} Z_{k+1}\right\|_{\frac{1}{2}, \frac{n}{2}, 2}+\left\|\varepsilon^{-M / 2} \partial_{t} Z_{k+1}\right\|_{-\frac{1}{2}, \frac{n}{2}, 2}+\left\|\varepsilon^{-M / 2} \zeta_{k+1}\right\|_{0, \frac{n}{2}, 2}\right)
\end{aligned}
$$

En compensant avec un terme $e^{-\lambda(\delta) t}$, on réabsorbe ce terme dans le membre de gauche et on obtient l'estimation $(\star)$. Le reste de la preuve est classique.

Proposition 3.2.4. - Si $M \geqslant n$, et si

$$
\left|B_{\varepsilon}\right|_{L^{\infty}\left(\left[0, t_{0}\right], \mathcal{Z}_{\varepsilon}^{\left(\frac{1}{2}, \frac{n}{2}\right)}\right)}+\left|\partial_{t} B_{\varepsilon}\right|_{L^{\infty}\left(\left[0, t_{0}\right], \mathcal{Z}_{\varepsilon}^{\left(\frac{1}{2}, \frac{n}{2}\right)}\right)}+\left|\beta_{\varepsilon}\right|_{L^{\infty}\left(\left[0, t_{0}\right], \mathcal{Z}_{\varepsilon}^{\left(\frac{1}{2}, \frac{n}{2}\right)}\right)} \leqslant \delta
$$

et

$$
\begin{aligned}
& \left|P_{\varepsilon}\right|_{L^{\infty}\left(\left[0, t_{0}\right], \varepsilon^{M / 2}\right.} \mathcal{W}_{\varepsilon}^{\left.-\frac{1}{2, \frac{n}{2}, 2}\right)}+\left|Q_{\varepsilon}\right|_{L^{\infty}\left(\left[0, t_{0}\right], \varepsilon^{M / 2} \mathcal{W}_{\varepsilon}^{0, \frac{n}{2}, 2}\right)} \\
& +\left\|\varepsilon^{-M / 2} Z c_{\varepsilon}\right\|_{\frac{1}{2}, \frac{n}{2}, 2}+\left\|\varepsilon^{-M / 2} Z c_{\varepsilon}^{\prime}\right\|_{-\frac{1}{2}, \frac{n}{2}, 2}+\left\|\varepsilon^{-M / 2} \zeta c_{\varepsilon}\right\|_{0, \frac{n}{2}, 2} \leqslant \delta_{0}
\end{aligned}
$$

alors il existe un temps $t_{1}, 0<t_{1} \leqslant t_{0}$ et une constante $\delta^{\prime}$, tels que l'hypothèse de récurrence suivante est vérifiée pour tout $k \geqslant 0$ :

$\left(\mathcal{H}^{k}\right): \exists !(Z, z)_{k+1}$ vérifiant le système linéarisé $(\text { S.Lin. })_{k}$ avec pour données initiales $\left(Z c, Z c^{\prime}, \zeta c\right)_{\varepsilon}$, et tels que

$$
\begin{aligned}
\left.\left|Z_{k+1}\right|_{L^{\infty}\left(\left[0, t_{1}\right], \varepsilon^{M / 2} \mathcal{W}_{\varepsilon}^{\frac{1}{2}, \frac{n}{2}, 2}\right)}+\left|\partial_{t} Z_{k+1}\right|_{L^{\infty}\left(\left[0, t_{1}\right], \varepsilon^{M / 2}\right.} \mathcal{W}_{\varepsilon}^{-\frac{1}{2}, \frac{n}{2}, 2}\right) & \\
& +\left|\zeta_{k+1}\right|_{L^{\infty}\left(\left[0, t_{1}\right], \varepsilon^{M / 2} \mathcal{W}_{\varepsilon}^{0, \frac{n}{2}, 2}\right)} \leqslant \delta^{\prime}
\end{aligned}
$$

De plus, si $\delta_{0}$ est assez petit, alors on peut prendre $t_{1}=t_{0}$.

Démonstration. - Elle est presque entièrement contenue dans les propositions précédentes. Nous obtenons par la proposition 3.2.3 une unique solution $(Z, \zeta)_{1}$ de $(\text { S.Lin. })_{0}$ 
sur $\left[0, t_{0}\right]$, avec pour données initiales $\left(Z c, Z c^{\prime}, \zeta c\right)_{\varepsilon}$, et telle que

$$
\begin{gathered}
\left|Z_{1}\right|_{L^{\infty}\left(\left[0, t^{\prime}\right], \varepsilon^{M / 2} \mathcal{W}_{\varepsilon}^{\frac{1}{2}, \frac{n}{2}, 2}\right)}+\left|\partial_{t} Z_{1}\right|_{L^{\infty}\left(\left[0, t^{\prime}\right], \varepsilon^{M / 2} \mathcal{W}_{\varepsilon}^{-\frac{1}{2}, \frac{n}{2}, 2}\right)}+\left|\zeta_{1}\right|_{L^{\infty}\left(\left[0, t^{\prime}\right], \varepsilon^{M / 2} \mathcal{W}_{\varepsilon}^{0, \frac{n}{2}, 2}\right)} \\
\leqslant C_{0}\left(\left\|\varepsilon^{-M / 2} Z c_{\varepsilon}\right\|_{\frac{1}{2}, \frac{n}{2}, 2}+\left\|\varepsilon^{-M / 2} Z c_{\varepsilon}^{\prime}\right\|_{-\frac{1}{2}, \frac{n}{2}, 2}+\left\|\varepsilon^{-M / 2} \zeta_{c_{\varepsilon}}\right\|_{0, \frac{n}{2}, 2}\right. \\
\left.+\left|P_{\varepsilon}\right|_{L^{1}\left(\left[0, t^{\prime}\right], \varepsilon^{M / 2} \mathcal{W}_{\varepsilon}^{-\frac{1}{2}, \frac{n}{2}, 2}\right)}+\left|Q_{\varepsilon}\right|_{L^{1}\left(\left[0, t^{\prime}\right], \varepsilon^{M / 2} \mathcal{W}_{\varepsilon}^{0, \frac{n}{2}, 2}\right)}\right)
\end{gathered}
$$

avec $C_{0}=C_{0}\left(\delta, t_{0}\right)$. Du coup, $\left(\mathcal{H}^{0}\right)$ est trivialement vérifiée si nous choisissons

$$
\begin{aligned}
\delta^{\prime}=2 C_{0} \delta_{0} \geqslant C_{0}\left(\left\|\varepsilon^{-M / 2} Z c_{\varepsilon}\right\|_{\frac{1}{2}, \frac{n}{2}, 2}+\left\|\varepsilon^{-M / 2} Z c_{\varepsilon}^{\prime}\right\|_{-\frac{1}{2}, \frac{n}{2}, 2}+\left\|\varepsilon^{-M / 2} \zeta_{c_{\varepsilon}}\right\|_{0, \frac{n}{2}, 2}\right. \\
\left.+\left|P_{\varepsilon}\right|_{L^{1}\left(\left[0, t^{\prime}\right], \varepsilon^{M / 2} \mathcal{W}_{\varepsilon}^{-\frac{1}{2}, \frac{n}{2}, 2}\right)}+\left|Q_{\varepsilon}\right|_{L^{1}\left(\left[0, t^{\prime}\right], \varepsilon^{M / 2} \mathcal{W}_{\varepsilon}^{0, \frac{n}{2}, 2}\right)}\right)
\end{aligned}
$$

Supposons ensuite que les $\left(\mathcal{H}^{k}\right)$ sont vérifiées pour tout entier $0 \leqslant k<n$ et montrons $\left(\mathcal{H}^{n}\right)$ pour un choix adéquat de $t^{\prime}$. Nous appliquons la proposition 3.2.3, et obtenons $(Z, \zeta)_{n+1}$ solution de $(\text { S.Lin. })_{n}$ sur $\left[0, t_{1}\right]$, avec pour données initiales $\left(Z c, Z c^{\prime}, \zeta c\right)_{\varepsilon}$, et telle que

$$
\begin{gathered}
\left|Z_{k+1}\right|_{L^{\infty}\left(\left[0, t_{1}\right], \varepsilon^{M / 2} \mathcal{W}_{\varepsilon}^{\frac{1}{2,}, \frac{n}{2}, 2}\right)}+\left|\partial_{t} Z_{k+1}\right|_{L^{\infty}\left(\left[0, t_{1}\right], \varepsilon^{M / 2} \mathcal{W}_{\varepsilon}^{-\frac{1}{2}, \frac{n}{2}, 2}\right)} \\
+\left|\zeta_{k+1}\right|_{L^{\infty}\left(\left[0, t_{1}\right], \varepsilon^{M / 2} \mathcal{W}_{\varepsilon}^{0, \frac{n}{2}, 2}\right)} \\
\begin{array}{c}
C_{0}\left(\left\|\varepsilon^{-M / 2} Z c_{\varepsilon}\right\|_{\frac{1}{2}, \frac{n}{2}, 2}+\left\|\varepsilon^{-M / 2} Z c_{\varepsilon}^{\prime}\right\|_{-\frac{1}{2}, \frac{n}{2}, 2}+\left\|\varepsilon^{-M / 2} \zeta_{c \varepsilon}\right\|_{0, \frac{n}{2}, 2}\right. \\
\left.+\left|p_{k}^{n . l .}\right|_{L^{1}\left(\left[0, t_{1}\right], \varepsilon^{M / 2} \mathcal{W}_{\varepsilon}^{-\frac{1}{2}, \frac{n}{2}, 2}\right)}+\left|q_{k}^{n . l .}\right|_{L^{1}\left(\left[0, t_{1}\right], \varepsilon^{M / 2}\right.} \mathcal{W}_{\varepsilon}^{0, \frac{n}{2}, 2}\right) \\
\left.+\left|P_{\varepsilon}\right|_{L^{1}\left(\left[0, t_{1}\right], \varepsilon^{M / 2}\right.} \mathcal{W}_{\varepsilon}^{-\frac{1}{2}, \frac{n}{2}, 2}\right)
\end{array} \\
\left.+\left|Q_{\varepsilon}\right|_{L^{1}\left(\left[0, t_{1}\right], \varepsilon^{M / 2} \mathcal{W}_{\varepsilon}^{0, \frac{n}{2}, 2}\right)}\right)
\end{gathered}
$$

La proposition 3.2.2 nous donne

$$
\begin{gathered}
\left\|\varepsilon^{-M / 2} p_{k}^{n . l \cdot}\right\|_{-\frac{1}{2}, \frac{n}{2}, 2} \leqslant C\left(\delta, \delta^{\prime}\right) \\
\left\|\varepsilon^{-M / 2} q_{k}^{n . l \cdot}\right\|_{0, \frac{n}{2}, 2} \leqslant C\left(\delta, \delta^{\prime}\right)
\end{gathered}
$$

avec une constante positive $C\left(\delta, \delta^{\prime}\right)$ polynomiale en $\delta$ et en $\delta^{\prime}$, et s'annulant à l'ordre 2 en $\delta^{\prime}=0$. Si $t_{1}$ reste assez petit pour que $t_{1} C\left(\delta, \delta^{\prime}\right) C_{0} \leqslant \frac{\delta^{\prime}}{2}$ alors l'hypothèse $\left(\mathcal{H}^{n}\right)$ est vérifiée. $\mathrm{Si}$, de plus, $\delta^{\prime}=2 C_{0} \delta_{0}$ est assez petit pour que $t_{0} C\left(\delta, \delta^{\prime}\right) C_{0} \leqslant \operatorname{Cte}\left(\delta^{\prime}\right)^{2} \leqslant \frac{\delta^{\prime}}{2}$, alors il est toujours possible de prendre $t_{1}=t_{0}$.

Pour achever la preuve de la proposition 3.2.1, il reste à montrer la convergence de la suite $(Z, \zeta)_{k}$ vérifiant les hypothèses $\left(\mathcal{H}^{k}\right)$. Notons $E_{k}=Z_{k}-Z_{k-1}$ et $\xi_{k}=\zeta_{k}-\zeta_{k-1}$ pour tout entier $k \geqslant 1$.

Proposition 3.2.5. - Sous les hypothèses de la proposition 3.2.4, il existe un temps $t_{1}$ et une constante $c_{1}<1$ tels que sur l'intervalle $\left[0, t_{1}\right]$, pour tout entier $k \geqslant 1$,

$$
\begin{aligned}
\left\|\varepsilon^{-M / 2} E_{k+1}\right\|_{\frac{1}{2}, \frac{n}{2}, 2} & +\left\|\varepsilon^{-M / 2} \partial_{t} E_{k+1}\right\|_{-\frac{1}{2}, \frac{n}{2}, 2}+\left\|\varepsilon^{-M / 2} \xi_{k+1}\right\|_{0, \frac{n}{2}, 2} \\
\leqslant & c_{1}\left(\left\|\varepsilon^{-M / 2} \xi_{k}\right\|_{\frac{1}{2}, \frac{n}{2}, 2}+\left\|\varepsilon^{-M / 2} \partial_{t} \xi_{k}\right\|_{-\frac{1}{2}, \frac{n}{2}, 2}+\left\|\varepsilon^{-M / 2} \xi_{k}\right\|_{0, \frac{n}{2}, 2}\right)
\end{aligned}
$$

De plus, si $\delta_{0}$ est assez petit, on peut prendre $t_{1}=t_{0}$. 
Démonstration. - $(E, \xi)_{k+1}$ vérifie l'estimation a priori

$$
\begin{aligned}
& \left|E_{k+1}\right|_{L^{\infty}\left(\left[0, t_{1}\right], \varepsilon^{M / 2} \mathcal{W}_{\varepsilon}^{\frac{1}{2}, \frac{n}{2}, 2}\right)}+\left|\partial_{t} E_{k+1}\right|_{L^{\infty}\left(\left[0, t_{1}\right], \varepsilon^{M / 2} \mathcal{W}_{\varepsilon}^{-\frac{1}{2}, \frac{n}{2}, 2}\right)} \\
& +\left|\xi_{k+1}\right|_{L^{\infty}\left(\left[0, t_{1}\right], \varepsilon^{M / 2} \mathcal{W}_{\varepsilon}^{0, \frac{n}{2}, 2}\right)} \\
& \leqslant C_{0}\left(\left|p_{k}^{n . l .}-p_{k-1}^{n . l .}\right|_{L^{1}\left(\left[0, t_{1}\right], \varepsilon^{M / 2} \mathcal{W}_{\varepsilon}^{-\frac{1}{2}, \frac{n}{2}, 2}\right)}+\left|q_{k}^{n . l .}-q_{k-1}^{n . l .}\right|_{L^{1}\left(\left[0, t_{1}\right], \varepsilon^{M / 2} \mathcal{W}_{\varepsilon}^{0, \frac{n}{2}, 2}\right)}\right)
\end{aligned}
$$

Or la proposition 3.2.2 nous donne l'estimation

$$
\begin{aligned}
& \left\|\varepsilon^{-M / 2}\left(p_{k}^{n . l .}-p_{k-1}^{n . l .}\right)\right\|_{-\frac{1}{2}, \frac{n}{2}, 2} \\
& \quad \leqslant C\left(\delta, \delta^{\prime}\right)\left(\left\|\varepsilon^{-M / 2} E_{k}\right\|_{\frac{1}{2}, \frac{n}{2}, 2}+\left\|\varepsilon^{-M / 2} \partial_{t} E_{k}\right\|_{-\frac{1}{2}, \frac{n}{2}, 2}+\left\|\varepsilon^{-M / 2} \xi_{k}\right\|_{0, \frac{n}{2}, 2}\right)
\end{aligned}
$$

et

$$
\begin{aligned}
& \left\|\varepsilon^{-M / 2}\left(q_{k}^{n . l .}-q_{k-1}^{n . l .}\right)\right\|_{0, \frac{n}{2}, 2} \\
& \quad \leqslant C\left(\delta, \delta^{\prime}\right)\left(\left\|\varepsilon^{-M / 2} E_{k}\right\|_{\frac{1}{2}, \frac{n}{2}, 2}+\left\|\varepsilon^{-M / 2} \partial_{t} E_{k}\right\|_{-\frac{1}{2}, \frac{n}{2}, 2}+\left\|\varepsilon^{-M / 2} \xi_{k}\right\|_{0, \frac{n}{2}, 2}\right)
\end{aligned}
$$

Si nous choisissons $t_{1} C\left(\delta, \delta^{\prime}\right) C_{0} \leqslant c_{1}<1$, nous obtenons immédiatement l'inégalité annoncée. Si, de plus, on prend $\delta^{\prime}=2 C_{0} \delta_{0}$ assez petit pour que $t_{0} C\left(\delta, \delta^{\prime}\right) C_{0} \leqslant \mathrm{Cte} \delta^{\prime} \leqslant$ $c_{1}<1$, alors rien n'empêche de prendre $t_{1}=t_{0}$.

La série $\sum E_{k}$ converge donc normalement dans $\varepsilon^{M / 2} \mathcal{W}_{\varepsilon}^{\frac{1}{2}, \frac{n}{2}, 2}$ vers $(Z)_{\varepsilon}$ et $\sum \partial_{t} E_{k}$ dans $\varepsilon^{M / 2} \mathcal{W}_{\varepsilon}^{-\frac{1}{2}, \frac{n}{2}, 2}$ vers $\left(\partial_{t} Z\right)_{\varepsilon}$, tandis que $\sum \xi_{k}$ converge dans $\varepsilon^{M / 2} \mathcal{W}_{\varepsilon}^{0, \frac{n}{2}, 2}$ vers $(\zeta)_{\varepsilon}$. Par passage à la limite dans $\left(\mathcal{H}^{k}\right)$, nous obtenons en fait que

$$
\begin{gathered}
(Z)_{\varepsilon} \in C^{0}\left(\left[0, t^{\prime}\right], \varepsilon^{M / 2} \mathcal{W}_{\varepsilon}^{\frac{1}{2}, \frac{n}{2}, 2}\right) \cap C^{1}\left(\left[0, t^{\prime}\right], \varepsilon^{M / 2} \mathcal{W}_{\varepsilon}^{-\frac{1}{2}, \frac{n}{2}, 2}\right) \\
(\zeta)_{\varepsilon} \in C^{0}\left(\left[0, t^{\prime}\right], \varepsilon^{M / 2} \mathcal{W}_{\varepsilon}^{0, \frac{n}{2}, 2}\right)
\end{gathered}
$$

et

$$
\left\|\varepsilon^{-M / 2}(Z)_{\varepsilon}\right\|_{\frac{1}{2}, \frac{n}{2}, 2}+\left\|\varepsilon^{-M / 2} \partial_{t}(Z)_{\varepsilon}\right\|_{\frac{1}{2}, \frac{n}{2}, 2}+\left\|\varepsilon^{-M / 2}(\zeta)_{\varepsilon}\right\|_{0, \frac{n}{2}, 2} \leqslant \delta^{\prime}
$$

Puis par passage à la limite dans le schéma itératif, nous vérifions que $\left(Z_{\varepsilon}, \zeta_{\varepsilon}\right)$ est bien la solution cherchée au système semi-linéaire (S) pour tout $\left.\varepsilon \in] 0, \varepsilon_{0}\right]$. Nous en déduisons la proposition 3.2.1.

\subsection{Démonstration du théorème 3}

Nous posons

$$
\begin{aligned}
Z_{\varepsilon} & =\mathcal{A}_{\varepsilon}-A_{\varepsilon} \\
z_{\varepsilon} & =\Phi_{\varepsilon}-\phi_{\varepsilon} \\
\zeta_{\varepsilon} & =\Psi_{\varepsilon}-\psi_{\varepsilon}
\end{aligned}
$$

puis

$$
\begin{aligned}
& f^{r}\left(A_{\varepsilon}, \phi_{\varepsilon}, \psi_{\varepsilon}, Z-\varepsilon, z_{\varepsilon}, \zeta_{\varepsilon}\right)=f\left(A_{\varepsilon}+Z_{\varepsilon}, \phi_{\varepsilon}+z_{\varepsilon}, \psi_{\varepsilon}+\zeta_{\varepsilon}\right)-f\left(A_{\varepsilon}, \phi_{\varepsilon}, \psi_{\varepsilon}\right) \\
& g^{r}\left(A_{\varepsilon}, \phi_{\varepsilon}, \psi_{\varepsilon}, Z-\varepsilon, z_{\varepsilon}, \zeta_{\varepsilon}\right)=g\left(A_{\varepsilon}+Z_{\varepsilon}, \phi_{\varepsilon}+z_{\varepsilon}, \psi_{\varepsilon}+\zeta_{\varepsilon}\right)-g\left(A_{\varepsilon}, \phi_{\varepsilon}, \psi_{\varepsilon}\right)
\end{aligned}
$$


et

$$
h^{r}\left(A_{\varepsilon}, \phi_{\varepsilon}, \psi_{\varepsilon}, Z-\varepsilon, z_{\varepsilon}, \zeta_{\varepsilon}\right)=h\left(A_{\varepsilon}+Z_{\varepsilon}, \phi_{\varepsilon}+z_{\varepsilon}, \psi_{\varepsilon}+\zeta_{\varepsilon}\right)-h\left(A_{\varepsilon}, \phi_{\varepsilon}, \psi_{\varepsilon}\right)
$$

qui sont des polynômes à coefficients $C^{\infty}$, qui s'annulent au moins à l'ordre 1 en $(Z, z, \zeta)_{\varepsilon}=(0,0,0) . f_{r}$ et $g_{r}$ sont de degré au plus 1 en $\psi_{\varepsilon}$ et 2 en $\zeta_{\varepsilon} \cdot h_{r}$ est de degré au plus 1 en $\psi_{\varepsilon}$ et en $\zeta_{\varepsilon}$.

La famille $(\mathcal{A}, \Phi, \Psi)$ est solution de $(Y . M$.$) si et seulement si (Z, z, \zeta)_{\varepsilon}$ est solution du système :

$$
\left\{\begin{aligned}
L Z_{\varepsilon}= & F\left(A_{\varepsilon}+Z_{\varepsilon}, \phi_{\varepsilon}+z_{\varepsilon}, \psi_{\varepsilon}+\zeta_{\varepsilon}\right)-L A_{\varepsilon} \\
= & F\left(A_{\varepsilon}+Z_{\varepsilon}, \phi_{\varepsilon}+z_{\varepsilon}, \psi_{\varepsilon}+\zeta_{\varepsilon}\right)-F\left(A_{\varepsilon}, \phi_{\varepsilon}, \psi_{\varepsilon}\right)-\mathcal{O}_{\varepsilon} \\
= & F^{I}\left(Z_{\varepsilon}, \partial Z_{\varepsilon}\right)+F^{I I}\left(z_{\varepsilon}, \partial z_{\varepsilon}\right)+F^{I}\left(Z_{\varepsilon}, \partial A_{\varepsilon}\right)+F^{I}\left(A_{\varepsilon}, \partial Z_{\varepsilon}\right) \\
& +F^{I I}\left(z_{\varepsilon}, \partial \phi_{\varepsilon}\right)+F^{I I}\left(\phi_{\varepsilon}, \partial z_{\varepsilon}\right)+f^{r}\left(A_{\varepsilon}, \phi_{\varepsilon}, \psi_{\varepsilon}, Z_{\varepsilon}, z_{\varepsilon}, \zeta_{\varepsilon}\right)-\mathcal{O}_{\varepsilon} \\
\square z_{\varepsilon}= & G\left(A_{\varepsilon}+Z_{\varepsilon}, \phi_{\varepsilon}+z_{\varepsilon}, \psi_{\varepsilon}+\zeta_{\varepsilon}\right)-\square \phi_{\varepsilon} \\
= & G\left(A_{\varepsilon}+Z_{\varepsilon}, \phi_{\varepsilon}+z_{\varepsilon}, \psi_{\varepsilon}+\zeta_{\varepsilon}\right)-G\left(A_{\varepsilon}, \phi_{\varepsilon}, \psi_{\varepsilon}\right)-\mathcal{P}_{\varepsilon} \\
= & G^{I}\left(Z_{\varepsilon}, \partial z_{\varepsilon}\right)+G^{I I}\left(\partial Z_{\varepsilon}, z_{\varepsilon}\right)+G^{I}\left(Z_{\varepsilon}, \partial \phi_{\varepsilon}\right)+G^{I}\left(A_{\varepsilon}, \partial z_{\varepsilon}\right) \\
& +G^{I I}\left(\partial A_{\varepsilon}, z_{\varepsilon}\right)+G^{I I}\left(\partial Z_{\varepsilon}, \phi_{\varepsilon}\right)+g^{r}\left(A_{\varepsilon}, \phi_{\varepsilon}, \psi_{\varepsilon}, Z_{\varepsilon}, z_{\varepsilon}, \zeta_{\varepsilon}\right)-\mathcal{P}_{\varepsilon} \\
\mathcal{D} \zeta_{\varepsilon}= & h\left(A_{\varepsilon}+Z_{\varepsilon}, \phi_{\varepsilon}+z_{\varepsilon}, \psi_{\varepsilon}+\zeta_{\varepsilon}\right)-\mathcal{D} \psi_{\varepsilon} \\
= & h\left(A_{\varepsilon}+Z_{\varepsilon}, \phi_{\varepsilon}+z_{\varepsilon}, \psi_{\varepsilon}+\zeta_{\varepsilon}\right)-h\left(A_{\varepsilon}, \phi_{\varepsilon}, \psi_{\varepsilon}\right)-\mathcal{Q}_{\varepsilon} \\
= & h^{r}\left(A_{\varepsilon}, \phi_{\varepsilon}, \psi_{\varepsilon}, Z_{\varepsilon}, z_{\varepsilon}, \zeta_{\varepsilon}\right)-\mathcal{Q}_{\varepsilon}
\end{aligned}\right.
$$

Les données initiales

$$
\begin{gathered}
Z_{\varepsilon}(0, y)=Z c_{\varepsilon}(y) \in \varepsilon^{M / 2} \mathcal{W}_{\varepsilon}^{\frac{1}{2}, \frac{n}{2}, 2} \text { et } \nabla_{0}\left(Z_{\varepsilon}\right)_{\mu}(0, y)=\left(Z c_{\varepsilon}^{\prime}\right)_{\mu}(y) \in \varepsilon^{M / 2} \mathcal{W}_{\varepsilon}^{-\frac{1}{2}, \frac{n}{2}, 2} \\
z_{\varepsilon}(0, y)=z_{c \varepsilon}(y) \in \varepsilon^{M / 2} \mathcal{W}_{\varepsilon}^{\frac{1}{2}, \frac{n}{2}, 2} \text { et } \nabla_{0} z_{\varepsilon}(0, y)=z_{c_{\varepsilon}}^{\prime}(y) \in \varepsilon^{M / 2} \mathcal{W}_{\varepsilon}^{-\frac{1}{2}, \frac{n}{2}, 2} \\
\zeta_{\varepsilon}(0, y)=\zeta_{c \varepsilon}(y) \in \varepsilon^{M / 2} \mathcal{W}_{\varepsilon}^{0, \frac{n}{2}, 2}
\end{gathered}
$$

doivent vérifier la contrainte de compatibilité

$$
\begin{aligned}
& \quad \nabla^{j}\left(\nabla_{j}\left(Z c_{\varepsilon}\right)_{0}-\left(Z c_{\varepsilon}^{\prime}\right)_{j}\right)=\left(F^{I}\left(Z c_{\varepsilon}, \partial Z c_{\varepsilon}\right)+F^{I I}\left(z_{c \varepsilon}, \partial z_{c \varepsilon}\right)\right. \\
& +F^{I}\left(Z c_{\varepsilon}, \partial A_{\varepsilon \mid t=0}\right)+F^{I}\left(A_{\varepsilon \mid t=0}, \partial Z c_{\varepsilon}\right)+F^{I I}\left(z_{c \varepsilon}, \partial \phi_{\varepsilon \mid t=0}\right)+F^{I I}\left(\phi_{\varepsilon \mid t=0}, \partial z_{c \varepsilon}\right) \\
& \left.+f\left(Z c_{\varepsilon}, z_{c \varepsilon}, \zeta_{c_{\varepsilon}}\right)+f^{r}\left(A_{\varepsilon \mid t=0}, \phi_{\varepsilon \mid t=0}, \psi_{\varepsilon \mid t=0}, Z c_{\varepsilon}, z_{c \varepsilon}, \zeta_{c_{\varepsilon}}\right)-\mathcal{O}_{\varepsilon}\right)_{0}
\end{aligned}
$$

La résolution de ces contraintes sur les données de Cauchy se fait exactement comme dans le cas régulier, en substituant les propositions 3.1.1 et 3.1.5 aux estimations obtenues par le lemme B.1.

Le système (Y.M.) reste invariant par les changements de jauge de la forme $u_{\varepsilon}=$ $e^{\chi_{\varepsilon}}$, qui transforme

$$
\mathcal{A}_{\varepsilon} \stackrel{u_{\varepsilon}}{\rightsquigarrow} \mathcal{A}_{\varepsilon}^{\prime}=T \chi_{\varepsilon}+e^{-\chi_{\varepsilon}}\left(A_{\varepsilon}+Z_{\varepsilon}\right) e^{\chi_{\varepsilon}}
$$


Remarquons que $e^{\chi_{\varepsilon}}=1+\sum_{p \geqslant 1} \frac{\chi_{\varepsilon}^{p}}{p !}$ et que $\left\|\varepsilon^{-M / 2} \chi_{\varepsilon}^{p}\right\|_{\frac{1}{2}, \frac{n}{2}, 2} \leqslant C^{p}\left\|\varepsilon^{-M / 2} \chi_{\varepsilon}\right\|_{\frac{1}{2}, \frac{n}{2}, 2}^{p}$ pour tout entier $p \geqslant 1$ d'après les estimations bilinéaires de la section précédente, proposition 3.1.1. Il vient alors

$$
\left\|\varepsilon^{-M / 2} \sum_{p \geqslant 1} \frac{\chi_{\varepsilon}^{p}}{p !}\right\|_{\frac{1}{2}, \frac{n}{2}, 2} \leqslant \exp \left(C\left\|\varepsilon^{-M / 2} \chi_{\varepsilon}\right\|_{\frac{1}{2}, \frac{n}{2}, 2}\right)<\text { Cte }
$$

de même que

$$
\begin{aligned}
e^{-\chi_{\varepsilon}} A_{\varepsilon} e^{\chi_{\varepsilon}} & =A_{\varepsilon}+\left(\sum_{p \geqslant 1} \frac{(-1)^{p} \chi_{\varepsilon}^{p}}{p !}\right) A_{\varepsilon}+A_{\varepsilon}\left(\sum_{p \geqslant 1} \frac{\chi_{\varepsilon}^{p}}{p !}\right)+\left(\sum_{p \geqslant 1} \frac{(-1)^{p} \chi_{\varepsilon}^{p}}{p !}\right) A_{\varepsilon}\left(\sum_{p \geqslant 1} \frac{\chi_{\varepsilon}^{p}}{p !}\right) \\
& =A_{\varepsilon}+E\left(A_{\varepsilon}, \chi_{\varepsilon}\right)
\end{aligned}
$$

où

$$
\left\|\varepsilon^{-M / 2} E\left(A_{\varepsilon}, \chi_{\varepsilon}\right)\right\|_{\frac{1}{2}, \frac{n}{2}, 2} \leqslant \operatorname{Cte} \exp \left(2 C\left\|\varepsilon^{-M / 2} \chi_{\varepsilon}\right\|_{\frac{1}{2}, \frac{n}{2}, 2}\right)\left|\left\|A_{\varepsilon} \mid\right\|_{\left(\frac{1}{2}, \frac{n}{2}\right)}\right.
$$

compte tenu de la proposition 3.1.1. Enfin,

$$
\begin{aligned}
e^{-\chi_{\varepsilon}} Z_{\varepsilon} e^{\chi_{\varepsilon}} & =Z_{\varepsilon}+\left(\sum_{p \geqslant 1} \frac{(-1)^{p} \chi_{\varepsilon}^{p}}{p !}\right) Z_{\varepsilon}+Z_{\varepsilon}\left(\sum_{p \geqslant 1} \frac{\chi_{\varepsilon}^{p}}{p !}\right)+\left(\sum_{p \geqslant 1} \frac{(-1)^{p} \chi_{\varepsilon}^{p}}{p !}\right) Z_{\varepsilon}\left(\sum_{p \geqslant 1} \frac{\chi_{\varepsilon}^{p}}{p !}\right) \\
& =Z_{\varepsilon}+E\left(Z_{\varepsilon}, \chi_{\varepsilon}\right)
\end{aligned}
$$

avec

$$
\left\|\varepsilon^{-M / 2} E\left(Z_{\varepsilon}, \chi_{\varepsilon}\right)\right\|_{\frac{1}{2}, \frac{n}{2}, 2} \leqslant \operatorname{Cte} \exp \left(2 C\left\|\varepsilon^{-M / 2} \chi_{\varepsilon}\right\|_{\frac{1}{2}, \frac{n}{2}, 2}\right)\left\|\varepsilon^{-M / 2} Z_{\varepsilon}\right\|_{\frac{1}{2}, \frac{n}{2}, 2}
$$

Le même changement de jauge transforme

$$
\Phi_{\varepsilon} \stackrel{u_{\varepsilon}}{\rightsquigarrow} \Phi_{\varepsilon}^{\prime}=r\left(u_{\varepsilon}\right) \Phi_{\varepsilon} \quad \text { et } \quad \Psi_{\varepsilon} \stackrel{u_{\varepsilon}}{\rightsquigarrow} \Psi_{\varepsilon}^{\prime}=\rho\left(u_{\varepsilon}\right) \Psi_{\varepsilon}
$$

avec

$$
\begin{aligned}
& r\left(u_{\varepsilon}\right)=r\left(e^{\chi_{\varepsilon}}\right)=1+r^{\prime}(1) \sum_{p \geqslant 1} \frac{\chi_{\varepsilon}^{p}}{p !}+\cdots=1+r_{\varepsilon}, \quad \text { où } r_{\varepsilon} \in \varepsilon^{M / 2} \mathcal{W}_{\varepsilon}^{\frac{3}{2}, \frac{n}{2}, 2} \\
& \rho\left(u_{\varepsilon}\right)=\rho\left(e^{\chi_{\varepsilon}}\right)=1+\rho^{\prime}(1) \sum_{p \geqslant 1} \frac{\chi_{\varepsilon}^{p}}{p !}+\cdots=1+\rho_{\varepsilon}, \quad \text { où } \rho_{\varepsilon} \in \varepsilon^{M / 2} \mathcal{W}_{\varepsilon}^{\frac{3}{2}, \frac{n}{2}, 2} .
\end{aligned}
$$

Nous en déduisons que le système $(Y M \natural)$ reste invariant par changement de jauge $u_{\varepsilon}=e^{\chi_{\varepsilon}}$, avec $\chi_{\varepsilon} \in \varepsilon^{M / 2} \mathcal{W}_{\varepsilon}^{\frac{3}{2}, \frac{n}{2}, 2}$, qui transforme

$$
(A, \phi, \psi)_{\varepsilon} \stackrel{u_{\varepsilon}}{\rightsquigarrow}\left(A^{\prime}, \phi^{\prime}, \psi^{\prime}\right)_{\varepsilon}=(A, \phi, \psi)_{\varepsilon} \in \mathcal{Z}_{\varepsilon}^{\left(\frac{1}{2}, \frac{n}{2}\right)} \times \mathcal{Z}_{\varepsilon}^{\left(\frac{1}{2}, \frac{n}{2}\right)} \times \mathcal{Z}_{\varepsilon}^{\left(0, \frac{n}{2}\right)}
$$

et

$$
\begin{aligned}
Z_{\varepsilon} \stackrel{u_{\varepsilon}}{\rightsquigarrow} Z_{\varepsilon}^{\prime} & =T \chi_{\varepsilon}+Z_{\varepsilon}+E\left(A_{\varepsilon}, \chi_{\varepsilon}\right)+E\left(Z_{\varepsilon}, \chi_{\varepsilon}\right) \in \varepsilon^{M / 2} \mathcal{W}_{\varepsilon}^{\frac{1}{2}, \frac{n}{2}, 2} \\
& z_{\varepsilon} \stackrel{u_{\varepsilon}}{\rightsquigarrow} z_{\varepsilon}^{\prime}=z_{\varepsilon}+r_{\varepsilon}\left(\phi_{\varepsilon}+z_{\varepsilon}\right) \in \varepsilon^{M / 2} \mathcal{W}_{\varepsilon}^{\frac{1}{2}, \frac{n}{2}, 2} \\
& \zeta_{\varepsilon} \stackrel{u_{\varepsilon}}{\rightsquigarrow} \zeta_{\varepsilon}^{\prime}=\zeta_{\varepsilon}+\rho_{\varepsilon}\left(\psi_{\varepsilon}+\zeta_{\varepsilon}\right) \in \varepsilon^{M / 2} \mathcal{W}_{\varepsilon}^{0, \frac{n}{2}, 2}
\end{aligned}
$$

Nous dirons d'un tel changement de jauge qu'il est «admissible» pour ( $Y M \natural)$. Les conditions de jauges $J_{\lambda} \mathcal{A}_{\varepsilon}=0$ deviennent pour $Z_{\varepsilon}$ :

$$
J_{\lambda} Z_{\varepsilon}=-\mathcal{J}_{\varepsilon}=-J_{\lambda} A_{\varepsilon}=-\lambda \alpha^{-2} \nabla_{0} \mathcal{J}_{0}-(1-\lambda) \nabla^{i}\left(\mathcal{J}_{\Sigma}\right)_{i}
$$


avec

$$
\left(\mathcal{J}_{0}, \mathcal{J}_{\Sigma}\right) \in C^{0}\left(\left[0, t_{1}\right], \varepsilon^{M / 2} \mathcal{W}_{\varepsilon}^{\frac{3}{2}, \frac{n}{2}, 2}(\Sigma)\right) \cap C^{1}\left(\left[0, t_{1}\right], \varepsilon^{M / 2} \mathcal{W}_{\varepsilon}^{\frac{1}{2}, \frac{n}{2}, 2}(\Sigma)\right)
$$

Remarquons qu'il est toujours possible de se ramener au cas où $\mathcal{J}_{\varepsilon}=0$ en reposant le problème avec $A_{\varepsilon}^{\prime}=A_{\varepsilon}-\left(\mathcal{J}_{0}, \mathcal{J}_{\Sigma}\right)$ et $Z_{\varepsilon}^{\prime}(t=0)=Z_{\varepsilon}(t=0)+\left(\mathcal{J}_{0}, \mathcal{J}_{\Sigma}\right)(t=0)$. Les contraintes (67) sont invariantes par changement de jauge admissible à $t=0$, et il est donc possible de traduire les données de initiales sur $Z_{\varepsilon}$ dans une jauge $J_{\lambda}$ donnée et d'imposer la contrainte de jauge :

$$
\lambda \alpha^{-2}\left(Z c_{\varepsilon}^{\prime}\right)_{0}+(1-\lambda) \nabla^{j}\left(Z c_{\varepsilon}\right)_{j}=-J_{\lambda} A_{\left.\varepsilon\right|_{t=0}}=0
$$

avec

$$
\begin{aligned}
\sqrt{\frac{\lambda}{1-\lambda}}\left\|\varepsilon^{-M / 2}\left(Z c_{\varepsilon}\right)_{0}\right\|_{\frac{1}{2}, \frac{n}{2}, 2}+\sqrt{\frac{1-\lambda}{\lambda}}\left\|\varepsilon^{-M / 2} \nabla^{i}\left(Z c_{\varepsilon}^{\prime}\right)_{i}\right\|_{-\frac{3}{2}, \frac{n}{2}, 2} \\
\leqslant\left\|\varepsilon^{-M / 2}\left(Z c_{\varepsilon}\right)\right\|_{\frac{1}{2}, \frac{n}{2}, 2}+\left\|\varepsilon^{-M / 2}\left(Z c_{\varepsilon}^{\prime}\right)\right\|_{-\frac{1}{2}, \frac{n}{2}, 2}
\end{aligned}
$$

Pour résoudre $(Y M \natural)$, il suffit alors d'adapter les méthodes développées section 1.3.5 pour des données régulières, en remplaçant les estimations habituelles du lemme B.1, par des estimations uniformes en $\varepsilon$ de la proposition 3.1.1. Tout repose sur les résultats suivants :

Proposition 3.3.1 (Hyperbolicité de $Y M \natural)$ ). - Pour toute donnée

$$
(A)_{\varepsilon} \in C^{0}\left(\left[0, t_{0}\right], \mathcal{Z}_{\varepsilon}^{\left(\frac{1}{2}, \frac{n}{2}\right)}(\Sigma)\right) \cap C^{1}\left(\left[0, t_{0}\right], \mathcal{Z}_{\varepsilon}^{\left(-\frac{1}{2}, \frac{n}{2}\right)}(\Sigma)\right)
$$

la famille

$$
\left\{\begin{array}{l}
(Z, z)_{\varepsilon} \in C^{0}\left(\left[0, t_{1}\right], \varepsilon^{M / 2} \mathcal{W}_{\varepsilon}^{\frac{1}{2}, \frac{n}{2}, 2}(\Sigma)\right) \cap C^{1}\left(\left[0, t_{1}\right], \varepsilon^{M / 2} \mathcal{W}_{\varepsilon}^{-\frac{1}{2}, \frac{n}{2}, 2}(\Sigma)\right) \\
(\zeta)_{\varepsilon} \in C^{0}\left(\left[0, t_{1}\right], \varepsilon^{M / 2} \mathcal{W}_{\varepsilon}^{0, \frac{n}{2}, 2}(\Sigma)\right)
\end{array}\right.
$$

est solution du système $(Y M \natural)$ en jauge de Lorentz $R Z_{\varepsilon}=0$ si et seulement si elle vérifie le système hyperbolique :

$$
(Y M \downarrow, \text { hyp. })\left\{\begin{aligned}
(\square-\mathcal{R} i c .) Z_{\varepsilon}= & F^{I}\left(Z_{\varepsilon}, \partial Z_{\varepsilon}\right)+F^{I I}\left(z_{\varepsilon}, \partial z_{\varepsilon}\right)+F^{I}\left(Z_{\varepsilon}, \partial A_{\varepsilon}\right) \\
& +F^{I}\left(A_{\varepsilon}, \partial Z_{\varepsilon}\right)+F^{I I}\left(z_{\varepsilon}, \partial \phi_{\varepsilon}\right)+F^{I I}\left(\phi_{\varepsilon}, \partial z_{\varepsilon}\right) \\
& +f^{r}\left(A_{\varepsilon}, \phi_{\varepsilon}, \psi_{\varepsilon}, Z_{\varepsilon}, z_{\varepsilon}, \zeta_{\varepsilon}\right)-\mathcal{O}_{\varepsilon} \\
\square z_{\varepsilon}= & G^{I}\left(Z_{\varepsilon}, \partial z_{\varepsilon}\right)+G^{I I}\left(\partial Z_{\varepsilon}, z_{\varepsilon}\right)+G^{I}\left(Z_{\varepsilon}, \partial \phi_{\varepsilon}\right) \\
& +G^{I}\left(A_{\varepsilon}, \partial z_{\varepsilon}\right)+G^{I I}\left(\partial A_{\varepsilon}, z_{\varepsilon}\right)+G^{I I}\left(\partial Z_{\varepsilon}, \phi_{\varepsilon}\right) \\
& +g^{r}\left(A_{\varepsilon}, \phi_{\varepsilon}, \psi_{\varepsilon}, Z_{\varepsilon}, z_{\varepsilon}, \zeta_{\varepsilon}\right)-\mathcal{P}_{\varepsilon} \\
\mathcal{D} \zeta_{\varepsilon}= & h^{r}\left(A_{\varepsilon}, \phi_{\varepsilon}, \psi_{\varepsilon}, Z_{\varepsilon}, z_{\varepsilon}, \zeta_{\varepsilon}\right)-\mathcal{Q}_{\varepsilon}
\end{aligned}\right.
$$

et satisfait $\grave{a} t=0$ les contraintes

$$
\left\{\begin{array}{l}
(67) \\
\alpha^{-2}\left(Z c_{\varepsilon}^{\prime}\right)_{0}+\nabla^{j}\left(Z c_{\varepsilon}\right)_{j}=0
\end{array}\right.
$$


Démonstration. - A défaut de pouvoir résoudre directement $(Y M \natural)$, supposons que nous disposons d'une famille

$$
\left\{\begin{array}{l}
(Z, z)_{\varepsilon} \in C^{0}\left(\left[0, t_{1}\right], \varepsilon^{M / 2} \mathcal{W}_{\varepsilon}^{\frac{1}{2}, \frac{n}{2}, 2}(\Sigma)\right) \cap C^{1}\left(\left[0, t_{1}\right], \varepsilon^{M / 2} \mathcal{W}_{\varepsilon}^{-\frac{1}{2}, \frac{n}{2}, 2}(\Sigma)\right) \\
(\zeta)_{\varepsilon} \in C^{0}\left(\left[0, t_{1}\right], \varepsilon^{M / 2} \mathcal{W}_{\varepsilon}^{0, \frac{n}{2}, 2}(\Sigma)\right)
\end{array}\right.
$$

solution du système $(Y M \natural, h y p$.) et vérifiant à $t=0$ les contraintes (69). Nous en déduisons

$$
R Z_{\varepsilon \mid t=0}=0
$$

et

$\nabla_{0}\left(R Z_{\varepsilon \mid t=0}\right)=\left(\square\left(Z_{\varepsilon}\right)_{0 \mid t=0}-(\mathcal{R} i c .)_{0}{ }^{\nu}\left(Z_{\varepsilon}\right)_{\nu \mid t=0}\right)-\nabla^{i}\left(\nabla_{i}\left(Z_{\varepsilon}\right)_{\left.0\right|_{t=0}}-\nabla_{0}\left(Z_{\varepsilon}\right)_{i_{\mid t=0}}\right)=0$

Puisque $R L=R(\square-\mathcal{R} i c)-.R T R=0$, il vient

$$
\begin{aligned}
\square\left(R Z_{\varepsilon}\right) & =R T R Z_{\varepsilon} \\
& =R\left(\square-\mathcal{R} i c_{.}\right) Z_{\varepsilon} \\
& =R\left(F\left(A_{\varepsilon}+Z_{\varepsilon}, \phi_{\varepsilon}+z_{\varepsilon}, \psi_{\varepsilon}+\zeta_{\varepsilon}\right)-L A_{\varepsilon}\right) \\
& =R F\left(A_{\varepsilon}+Z_{\varepsilon}, \phi_{\varepsilon}+z_{\varepsilon}, \psi_{\varepsilon}+\zeta_{\varepsilon}\right)
\end{aligned}
$$

D'autre part, la relation de compatibilité du système (Y.M.) donne

$$
\begin{aligned}
R F\left(A_{\varepsilon}+Z_{\varepsilon}, \phi_{\varepsilon}+z_{\varepsilon},\right. & \left.\psi_{\varepsilon}+\zeta_{\varepsilon}\right) \\
=Q_{f} & \left.A_{\varepsilon}+Z_{\varepsilon}, L\left(A_{\varepsilon}+Z_{\varepsilon}\right)-F\left(A_{\varepsilon}+Z_{\varepsilon}, \phi_{\varepsilon}+z_{\varepsilon}, \psi_{\varepsilon}+\zeta_{\varepsilon}\right)\right) \\
& \quad+Q_{g}\left(\phi_{\varepsilon}+z_{\varepsilon}, \square\left(\phi_{\varepsilon}+z_{\varepsilon}\right)-G\left(A_{\varepsilon}+Z_{\varepsilon}, \phi_{\varepsilon}+z_{\varepsilon}, \psi_{\varepsilon}+\zeta_{\varepsilon}\right)\right) \\
& \quad+Q_{h}\left(\psi_{\varepsilon}+\zeta_{\varepsilon}, \mathcal{D}\left(\psi_{\varepsilon}+\zeta_{\varepsilon}\right)-h\left(A_{\varepsilon}+Z_{\varepsilon}, \phi_{\varepsilon}+z_{\varepsilon}, \psi_{\varepsilon}+\zeta_{\varepsilon}\right)\right. \\
=- & Q_{f}\left(A_{\varepsilon}+Z_{\varepsilon}, T\left(R Z_{\varepsilon}\right)\right)
\end{aligned}
$$

Il en résulte que $\left(R Z_{\varepsilon}\right)$ est solution du système

$$
\left\{\begin{aligned}
\square\left(R Z_{\varepsilon}\right) & =-Q_{f}\left(A_{\varepsilon}+Z_{\varepsilon}, T\left(R Z_{\varepsilon}\right)\right) \\
\left(R Z_{\varepsilon}\right)_{\mid t=0} & =0 \\
\nabla_{0}\left(R Z_{\varepsilon}\right)_{\mid t=0} & =0
\end{aligned}\right.
$$

Or $A \in C^{0}\left([0, t], \mathcal{Z}_{\varepsilon}^{\left(\frac{1}{2}, \frac{n}{2}\right)}\right) \cap C^{1}\left([0, t], \mathcal{Z}_{\varepsilon}^{\left(-\frac{1}{2}, \frac{n}{2}\right)}\right)$. En utilisant les estimations bilinéaires de la proposition 3.1.1, nous obtenons l'estimation a priori suivante :

$$
\begin{aligned}
\left|R Z_{\varepsilon}\right|_{L^{\infty}\left([0, t], \varepsilon^{M / 2} \mathcal{W}_{\varepsilon}^{\frac{1}{2}, \frac{n}{2}, 2}\right)} & +\left|\nabla_{0}\left(R Z_{\varepsilon}\right)\right|_{L^{\infty}\left([0, t], \varepsilon^{M / 2} \mathcal{W}_{\varepsilon}^{-\frac{1}{2}, \frac{n}{2}, 2}\right)} \\
\leqslant & \leqslant\left(\left|R Z_{\varepsilon \mid t=0}\right|_{\varepsilon^{M / 2} \mathcal{W}_{\varepsilon}^{\frac{1}{2}, \frac{n}{2}, 2}}+\mid \nabla_{0}\left(\left.\left.R Z_{\varepsilon}\right|_{\mid t=0}\right|_{\varepsilon^{M / 2} \mathcal{W}_{\varepsilon}^{-\frac{1}{2}, \frac{n}{2}, 2}}\right)\right.
\end{aligned}
$$

$\mathrm{Vu}$ la nullité des données initiales, il en résulte que $R Z_{\varepsilon} \equiv 0$ sur $[0, t]$. Le système (S.Hyp.) propage donc naturellement la jauge de Lorentz. Mais alors

$$
\square Z_{\varepsilon}-(\mathcal{R} i c .) Z_{\varepsilon}=L Z_{\varepsilon}+T R Z_{\varepsilon}=L Z_{\varepsilon}
$$


et

$$
(Y M \natural, h y p .)=(Y M \natural)
$$

Le système ( $Y M$ দ, hyp.) entre dans le cadre de la proposition 3.2.1 du paragraphe précédent, et nous pouvons affirmer que

Proposition 3.3.2 (corollaire de la proposition 3.2.1). - Toutes données initiales

$$
\begin{gathered}
Z_{\varepsilon}(0, y)=Z c_{\varepsilon}(y) \in \varepsilon^{M / 2} \mathcal{W}_{\varepsilon}^{\frac{1}{2}, \frac{n}{2}, 2} \text { et } \nabla_{0}\left(Z_{\varepsilon}\right)_{\mu}(0, y)=\left(Z c_{\varepsilon}^{\prime}\right)_{\mu}(y) \in \varepsilon^{M / 2} \mathcal{W}_{\varepsilon}^{-\frac{1}{2}, \frac{n}{2}, 2} \\
z_{\varepsilon}(0, y)=z_{c \varepsilon}(y) \in \varepsilon^{M / 2} \mathcal{W}_{\varepsilon}^{\frac{1}{2}, \frac{n}{2}, 2} \text { et } \nabla_{0} z_{\varepsilon}(0, y)=z_{c_{\varepsilon}}^{\prime}(y) \in \varepsilon^{M / 2} \mathcal{W}_{\varepsilon}^{-\frac{1}{2}, \frac{n}{2}, 2} \\
\zeta_{\varepsilon}(0, y)=\zeta_{c \varepsilon}(y) \in \varepsilon^{M / 2} \mathcal{W}_{\varepsilon}^{0, \frac{n}{2}, 2}
\end{gathered}
$$

se prolongent sur un intervalle $\left[0, t_{1}\right], 0<t_{1} \leqslant t_{0}$ indépendant de $\varepsilon$, en une unique solution

$$
\left\{\begin{array}{l}
(Z, z)_{\varepsilon} \in C^{0}\left(\left[0, t_{1}\right], \varepsilon^{M / 2} \mathcal{W}_{\varepsilon}^{\frac{1}{2}, \frac{n}{2}, 2}(\Sigma)\right) \cap C^{1}\left(\left[0, t_{1}\right], \varepsilon^{M / 2} \mathcal{W}_{\varepsilon}^{-\frac{1}{2}, \frac{n}{2}, 2}(\Sigma)\right) \\
(\zeta)_{\varepsilon} \in C^{0}\left(\left[0, t_{1}\right], \varepsilon^{M / 2} \mathcal{W}_{\varepsilon}^{0, \frac{n}{2}, 2}(\Sigma)\right)
\end{array}\right.
$$

du système hyperbolique (YMদ,hyp.). De plus, si

$$
\begin{aligned}
& \left\|\varepsilon^{-M / 2}(Z, z)_{\varepsilon}(t=0)\right\|_{\frac{1}{2}, \frac{n}{2}, 2}+\left\|\varepsilon^{-M / 2}\left(\partial_{t} Z, \partial_{t} z\right)_{\varepsilon}(t=0)\right\|_{-\frac{1}{2}, \frac{n}{2}, 2} \\
& +\left\|\varepsilon^{-M / 2}(\zeta)_{\varepsilon}(t=0)\right\|_{0, \frac{n}{2}, 2}+\sup _{t \in\left[0, t_{0}\right]}\left(\|\mid\|(A, \phi)_{\varepsilon}\|\|_{\left(\frac{1}{2}, \frac{n}{2}\right)}\right. \\
& \left.\quad+\left.\left\|\left(\partial_{t} A, \partial_{t} \phi\right)_{\varepsilon}\right\|\right|_{\left(-\frac{1}{2}, \frac{n}{2}\right)}+\left\|\mid \psi_{\varepsilon}\right\| \|_{\left(0, \frac{n}{2}\right)}\right) \leqslant \delta_{M}
\end{aligned}
$$

pour un certain $\delta_{M}$ assez petit, alors $t_{1}=t_{0}$.

Enfin, nous énonçons un résultat analogue à la proposition 1.3.6, section 1.3.5, pour le système $(Y M \natural)$ :

Proposition 3.3.3. - Toutes données initiales

$$
\begin{gathered}
Z_{\varepsilon}(0, y)=Z c_{\varepsilon}(y) \in \varepsilon^{M / 2} \mathcal{W}_{\varepsilon}^{\frac{1}{2}, \frac{n}{2}, 2} \text { et } \nabla_{0}\left(Z_{\varepsilon}\right)_{\mu}(0, y)=\left(Z c_{\varepsilon}^{\prime}\right)_{\mu}(y) \in \varepsilon^{M / 2} \mathcal{W}_{\varepsilon}^{-\frac{1}{2}, \frac{n}{2}, 2} \\
z_{\varepsilon}(0, y)=z_{c \varepsilon}(y) \in \varepsilon^{M / 2} \mathcal{W}_{\varepsilon}^{\frac{1}{2}, \frac{n}{2}, 2} \text { et } \nabla_{0} z_{\varepsilon}(0, y)=z_{c \varepsilon}^{\prime}(y) \in \varepsilon^{M / 2} \mathcal{W}_{\varepsilon}^{-\frac{1}{2}, \frac{n}{2}, 2} \\
\zeta_{\varepsilon}(0, y)=\zeta_{c_{\varepsilon}}(y) \in \varepsilon^{M / 2} \mathcal{W}_{\varepsilon}^{0, \frac{n}{2}, 2}
\end{gathered}
$$

vérifiant les contraintes de compatibilité (67) et de jauge (68) avec $\lambda \in[0,1]$, se prolongent sur un intervalle $\left[0, t_{1}\right], 0<t_{1} \leqslant t_{0}$ indépendant de $\varepsilon$ et de $\lambda$, en une unique famille

$$
\left\{\begin{array}{l}
(Z, z)_{\varepsilon} \in C^{0}\left(\left[0, t_{1}\right], \varepsilon^{M / 2} \mathcal{W}_{\varepsilon}^{\frac{1}{2}, \frac{n}{2}, 2}(\Sigma)\right) \cap C^{1}\left(\left[0, t_{1}\right], \varepsilon^{M / 2} \mathcal{W}_{\varepsilon}^{-\frac{1}{2}, \frac{n}{2}, 2}(\Sigma)\right) \\
(\zeta)_{\varepsilon} \in C^{0}\left(\left[0, t_{1}\right], \varepsilon^{M / 2} \mathcal{W}_{\varepsilon}^{0, \frac{n}{2}, 2}(\Sigma)\right)
\end{array}\right.
$$

solution du système $(Y M \natural$,$) et vérifiant la jauge$

$$
J_{\lambda} Z_{\varepsilon}=0
$$


De plus, si

$$
\begin{aligned}
& \left\|\varepsilon^{-M / 2}(Z, z)_{\varepsilon}(t=0)\right\|_{\frac{1}{2}, \frac{n}{2}, 2}+\left\|\varepsilon^{-M / 2}\left(\partial_{t} Z, \partial_{t} z\right)_{\varepsilon}(t=0)\right\|_{-\frac{1}{2}, \frac{n}{2}, 2} \\
& \quad+\left\|\varepsilon^{-M / 2}(\zeta)_{\varepsilon}(t=0)\right\|_{0, \frac{n}{2}, 2}+\sup _{t \in\left[0, t_{0}\right]}\left(\left\|||(A, \phi)_{\varepsilon}\right\| \|_{\left(\frac{1}{2}, \frac{n}{2}\right)}\right. \\
& \left.\quad+\|\|\left(\partial_{t} A, \partial_{t} \phi\right)_{\varepsilon}\left\|\left.\right|_{\left(-\frac{1}{2}, \frac{n}{2}\right)}+\right\|\left\|\psi_{\varepsilon}\right\| \|_{\left(0, \frac{n}{2}\right)}\right) \leqslant \delta_{M}
\end{aligned}
$$

pour un certain $\delta_{M}$ assez petit, alors $t_{1}=t_{0}$.

Démonstration. - En partant de données initiales $\left.\left(Z^{\natural}, z^{\natural}\right)_{\varepsilon}\right|_{t=0} \in \varepsilon^{M / 2} \mathcal{W}_{\varepsilon}^{\frac{1}{2}, \frac{n}{2}, 2}$, $\left.\left(\nabla_{0} Z^{\natural}, \nabla_{0} z^{\natural}\right)_{\varepsilon}\right|_{t=0} \in \varepsilon^{M / 2} \mathcal{W}_{\varepsilon}^{-\frac{1}{2}, \frac{n}{2}, 2}$ et $\left.\zeta_{\varepsilon}^{\natural}\right|_{t=0} \in \varepsilon^{M / 2} \mathcal{W}_{\varepsilon}^{0, \frac{n}{2}, 2}$ vérifiant les contraintes de compatibilité (67) et de jauge (68), nous souhaitons obtenir une solution $\left(Z^{\natural}, z^{\natural}, \zeta^{\natural}\right)_{\varepsilon}$ de $(Y M \natural)$ en jauge $J_{\lambda} Z_{\varepsilon}^{\natural}=0$. Nous posons à $t=0$ :

$$
\chi_{\varepsilon \mid t=0}=\nabla_{0} \chi_{\varepsilon \mid t=0}=0 \text { et } \alpha^{-2} \nabla_{0}^{2} \chi_{\varepsilon \mid t=0}=R Z_{\varepsilon \mid t=0}^{\natural}
$$

nous obtenons des données initiales :

$$
\begin{aligned}
\left(Z_{\varepsilon}^{b}\right)_{0 \mid t=0} & =\left(Z_{\varepsilon}^{\natural}\right)_{0_{\mid t=0}} \in \varepsilon^{M / 2} \mathcal{W}_{\varepsilon}^{\frac{1}{2}, \frac{n}{2}, 2} \\
\left(Z_{\varepsilon}^{b}\right)_{\Sigma_{\mid t=0}} & =A_{\Sigma \mid t=0}^{\natural} \in \varepsilon^{M / 2} \mathcal{W}_{\varepsilon}^{\frac{1}{2}, \frac{n}{2}, 2} \\
\nabla_{0}\left(Z_{\varepsilon}^{b}\right)_{0 \mid t=0} & =\nabla_{0}\left(Z_{\varepsilon}^{\natural}\right)_{0_{\mid t=0}}-\nabla_{0}^{2} \chi_{\varepsilon \mid t=0} \in \varepsilon^{M / 2} \mathcal{W}_{\varepsilon}^{-\frac{1}{2}, \frac{n}{2}, 2} \\
\nabla_{0}\left(Z_{\varepsilon}^{b}\right)_{\Sigma_{\mid t=0}} & =\nabla_{0}\left(Z_{\varepsilon}^{\natural}\right)_{\Sigma_{\mid t=0}} \in \varepsilon^{M / 2} \mathcal{W}_{\varepsilon}^{-\frac{1}{2}, \frac{n}{2}, 2}
\end{aligned}
$$

qui vérifient la jauge de Lorentz :

$$
R Z_{\varepsilon \mid t=0}^{b}=\alpha^{-2} \nabla_{0}\left(Z_{\varepsilon}^{\natural}\right)_{0 \mid t=0}+\nabla^{j}\left(Z_{\varepsilon}^{\natural}\right)_{\Sigma_{j \mid t=0}}-\alpha^{-2} \nabla_{0}^{2} \chi_{\varepsilon \mid t=0}=0
$$

ainsi que la formule de changement de jauge :

$$
Z_{\varepsilon \mid t=0}^{b} \stackrel{u_{\varepsilon}=e_{\mid t=0}^{\chi \chi_{\varepsilon}}}{\rightsquigarrow} Z_{\varepsilon \mid t=0}^{\natural}=T \chi_{\varepsilon \mid t=0}+Z_{\varepsilon \mid t=0}^{b}+E\left(A_{\varepsilon}+Z_{\varepsilon}^{b}, \chi_{\varepsilon}\right)_{\mid t=0}
$$

Puisque $(Y M \emptyset)$ est invariant par ce changement de jauge admissible à $t=0$, ces données restent compatibles et vérifient en définitive les contraintes (69). La proposition 3.3.1 s'applique, et la proposition 3.3.2 nous permet de prolonger ces nouvelles données par une unique famille

$$
\left\{\begin{array}{l}
\left(Z^{b}, z^{b}\right)_{\varepsilon} \in C^{0}\left(\left[0, t_{1}\right], \varepsilon^{M / 2} \mathcal{W}_{\varepsilon}^{\frac{1}{2}, \frac{n}{2}, 2}(\Sigma)\right) \cap C^{1}\left(\left[0, t_{1}\right], \varepsilon^{M / 2} \mathcal{W}_{\varepsilon}^{-\frac{1}{2}, \frac{n}{2}, 2}(\Sigma)\right) \\
\left(\zeta^{b}\right)_{\varepsilon} \in C^{0}\left(\left[0, t_{1}\right], \varepsilon^{M / 2} \mathcal{W}_{\varepsilon}^{0, \frac{n}{2}, 2}(\Sigma)\right)
\end{array}\right.
$$

solution du système $(Y M \natural)$ et vérifiant la jauge $R Z_{\varepsilon}^{b}=0$, ou de façon équivalente

$$
J_{\lambda} Z_{\varepsilon}^{b}=(2 \lambda-1) \nabla_{0}\left(Z_{\varepsilon}^{b}\right)_{0}
$$

Pour revenir à une famille de solutions $\left(Z^{\natural}, z^{\natural}, \zeta^{\natural}\right)_{\varepsilon}$ de $(Y M \natural$,$) en jauge J_{\lambda} Z_{\varepsilon}^{\natural}=0$, il faut résoudre l'équation suivante sur $\chi_{\varepsilon}$ :

$$
J_{\lambda} T \chi_{\varepsilon}=-J_{\lambda} Z_{\varepsilon}^{b}-J_{\lambda} E\left(A_{\varepsilon}+Z_{\varepsilon}^{b}, \chi_{\varepsilon}\right)
$$


Nous finissons la preuve comme pour la proposition 1.3.6, en prenant soin de remplacer les estimations $H^{s}, s \geqslant \frac{n}{2}+\frac{1}{2}$, par les estimations uniformes en $\varepsilon$ de la proposition 3.1.1.

\subsection{Conclusion, stabilité des solutions régulières de $(Y M)$ par perturba- tions oscillantes}

Le théorème 1 s'obtient naturellement en mettant bout à bout les théorèmes 2 et 3 , à condition de prendre des données de Cauchy

$$
(A, \phi, \psi)_{\varepsilon}(0, y) \in \mathcal{H}_{\varepsilon}^{1 / 2} \times \mathcal{H}_{\varepsilon}^{1 / 2} \times \mathcal{H}_{\varepsilon}^{0}(\Sigma) \text { et }\left(\partial_{t} A, \partial_{t} \phi\right)_{\varepsilon}(0, y) \in \mathcal{H}_{\varepsilon}^{-1 / 2} \times \mathcal{H}_{\varepsilon}^{-1 / 2}(\Sigma)
$$

admettant un développement oscillant à un ordre $\varepsilon^{M / 2}$ suffisant, $M \geqslant n$ :

$$
\left\{\begin{array}{l}
A_{\varepsilon}(0, y)=\sum_{i=0}^{M-1} \varepsilon^{i / 2}\left(\widetilde{A} c_{i}(y)+\varepsilon^{1 / 2} \stackrel{\star}{A} c_{i+1}\left(y, \frac{\theta(0, y)}{\varepsilon}\right)\right)+\varepsilon^{M / 2} Z_{c \varepsilon} \\
\phi_{\varepsilon}(0, y)=\sum_{i=0}^{M-1} \varepsilon^{i / 2}\left(\widetilde{\phi} c_{i}(y)+\varepsilon^{1 / 2} \stackrel{\star}{\phi} c_{i+1}\left(y, \frac{\theta(0, y)}{\varepsilon}\right)\right)+\varepsilon^{M / 2} z_{c \varepsilon} \\
\psi_{\varepsilon}(0, y)=\sum_{i=0}^{M-1} \varepsilon^{i / 2}\left(\widetilde{\psi} c_{i}(y)+\stackrel{\star}{\psi} c_{i+1}\left(y, \frac{\theta(0, y)}{\varepsilon}\right)\right)+\varepsilon^{M / 2} \zeta_{c_{\varepsilon}}
\end{array}\right.
$$

et

$$
\left\{\begin{array}{l}
\partial_{t} A_{\varepsilon}(0, y)= \\
\sum_{i=0}^{M-1} \varepsilon^{i / 2}\left(\varepsilon^{-1 / 2} \partial_{t} \theta \partial_{\omega} \stackrel{\star}{A} c_{i+1}\left(y, \frac{\theta(0, y)}{\varepsilon}\right)+\widetilde{A} c_{i}^{\prime}(y)+\varepsilon^{1 / 2} \partial_{t} \stackrel{\star}{A} c_{i+1}\left(y, \frac{\theta(0, y)}{\varepsilon}\right)\right)+\varepsilon^{M / 2} Z_{c \varepsilon}^{\prime} \\
\partial_{t} \phi_{\varepsilon}(0, y)= \\
\sum_{i=0}^{M-1} \varepsilon^{i / 2}\left(\varepsilon^{-1 / 2} \partial_{t} \theta \partial_{\omega} \stackrel{\star}{\phi} c_{i+1}\left(y, \frac{\theta(0, y)}{\varepsilon}\right)+\widetilde{\phi} c_{i}^{\prime}(y)+\varepsilon^{1 / 2} \partial_{t} \stackrel{\star}{\phi} c_{i+1}\left(y, \frac{\theta(0, y)}{\varepsilon}\right)\right)+\varepsilon^{M / 2} z_{c \varepsilon}^{\prime}
\end{array}\right.
$$

La compatibilité de ces données est acquise si nous respectons le choix suivant :

HYPothèSE 3.4.1. - Nous considérons pour le théorème 1 des données initiales qui sont la somme

- de données de Cauchy oscillantes à un ordre $\varepsilon^{M / 2}, M \geqslant n$, compatibles avec la phase $\theta$ retenue, choisies en conformité avec les hypothèses 2.3 .1 et vérifiant la clause de régularité (59) du théorème 2 , et

- d'un reste à l'ordre $\varepsilon^{M / 2}$ qui satisfait à la contrainte de compatibilité (67) et vérifiant la clause de régularité (66).

Nous achevons cette étude en montrant que le théorème 1 donne lieu à un résultat de stabilité du système $Y M$ par perturbations oscillantes, au voisinage de solutions régulières données. 
Proposition 3.4.1 (stabilité du système $(Y M)$ par perturbations oscillantes) a) Le terme dominant $\left(A_{0}, \phi_{0}, \widetilde{\psi}_{0}\right)$ des solutions oscillantes obtenues par le théorème 1 est lui-même solution de $(Y M)$ si et seulement si il l'est à $t=0$, i.e. vérifie la contrainte :

$$
\left(\triangle\left(A_{0}\right)_{0}-\operatorname{div} \nabla_{0}\left(A_{0}\right)^{\Sigma}\right)_{\mid(t=0)}=(F)_{0}\left(A_{0}, \partial A_{0}, \phi_{0}, \partial \phi_{0}, \widetilde{\psi}_{0}\right)_{\mid(t=0)}
$$

Cette condition équivaut à la nullité, au premier ordre, de la charge moyenne induite par les oscillations.

b) $S i\left(A_{0}, \phi_{0}, \widetilde{\psi}_{0}\right)$ est une solution régulière de $(Y M)$ sur $\left[0, t_{0}\right]$, alors il est possible de prendre $t_{1}=t_{0}$ dans le théorème 1 , c'est-à-dire que l'optique géométrique garde sa précision jusqu'à apparition de caustiques.

Pour démontrer cette dernière proposition, nous revenons directement sur l'expression des premières équations de profil, en coordonnées locales. Nous sommes maintenant assurés, par le biais du théorème 2, qu'elles décrivent fidèlement au premier ordre la solution exacte étudiée et que le reste ne risque pas de venir occulter la partie dominante des développements. Il n'est alors pas vain d'affiner nos calculs.

$\left(C_{0}\right)$

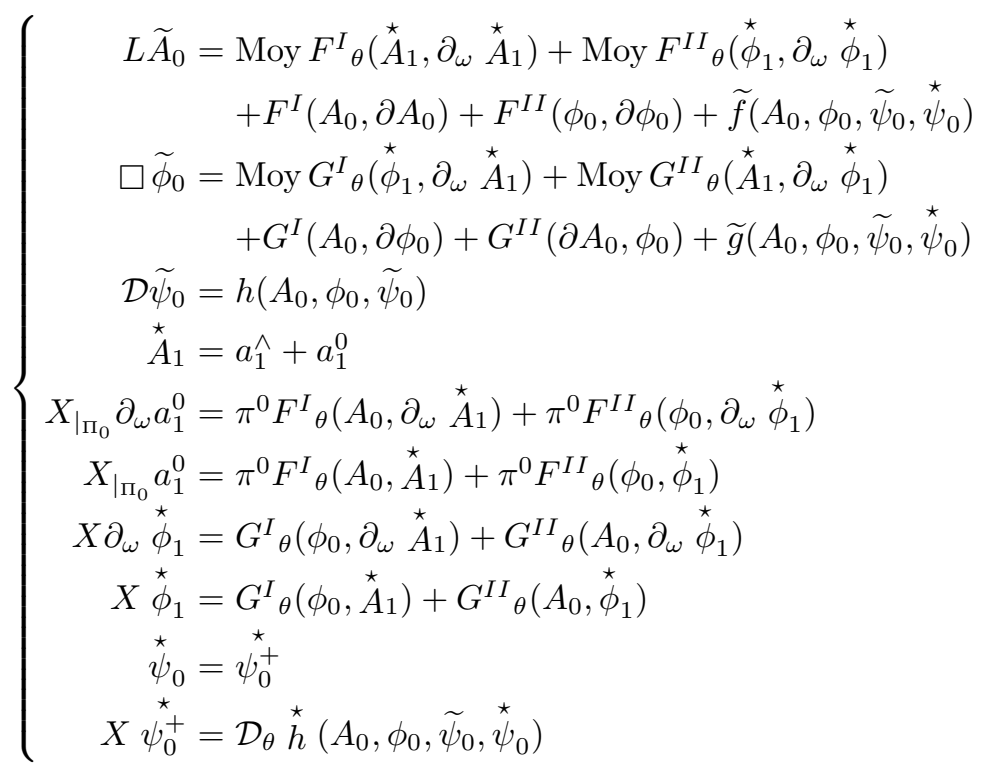

Comme $R_{\theta} \stackrel{\star}{A} 1=\nabla^{\nu}(\stackrel{\star}{A})_{\nu}=0$, il vient :

$$
\begin{aligned}
\operatorname{Moy} F_{\theta}^{I}\left(\stackrel{\star}{A}_{1}, \partial_{\omega} \stackrel{\star}{A_{1}}\right)_{\mu}= & -\operatorname{Moy}\left(2\left[\left(\stackrel{\star}{A_{1}}\right)^{\nu}, \nabla_{\nu} \theta \partial_{\omega}\left(\stackrel{\star}{A}_{1}\right)_{\mu}\right]+\left[\left(\stackrel{\star}{A}_{1}\right)^{\nu}, \nabla_{\mu} \theta \partial_{\omega}\left(\stackrel{\star}{A}_{1}\right)_{\nu}\right]\right. \\
& \left.+\left[\left(\stackrel{\star}{A_{1}}\right)_{\mu}, \nabla^{\nu} \theta \partial_{\omega}\left(\stackrel{\star}{A_{1}}\right)_{\nu}\right]\right) \\
= & \nabla_{\mu} \theta \operatorname{Moy}\left[\left(a_{1}^{0}\right)^{\nu}, \partial_{\omega}\left(a_{1}^{0}\right)_{\nu}\right]
\end{aligned}
$$


puis

$$
\begin{aligned}
\operatorname{Moy} F_{\theta}^{I I}\left(\stackrel{\star}{\phi}_{1}, \partial_{\omega} \stackrel{\star}{\phi}_{1}\right)_{\mu} & =-\operatorname{Moy}\left(\stackrel{\star}{\phi}_{1}{ }^{t} r^{\prime}(1)^{*} \nabla_{\mu} \theta \partial_{\omega} \stackrel{\star}{\phi}_{1}+\stackrel{\star}{\phi_{1}} r^{\prime}(1) \nabla_{\mu} \theta \partial_{\omega} \stackrel{\star}{\bar{\phi}_{1}}\right) \\
& =\nabla_{\mu} \theta \operatorname{Moy}\left(\bar{\star}_{1}{ }^{t} r^{\prime}(1)^{*} \partial_{\omega} \stackrel{\star}{\phi}_{1}+\stackrel{\star}{\phi}_{1} r^{\prime}(1) \partial_{\omega} \stackrel{\star}{\phi}_{1}\right)
\end{aligned}
$$

enfin

$$
\begin{aligned}
g_{\mu \nu} \gamma^{0} \gamma^{\nu} \pi^{+} & =g_{\mu \nu}{ }^{t} \gamma^{0} \gamma^{\nu} \frac{\alpha^{2}}{2 \nabla_{0} \theta} \mathcal{D}_{\theta} \gamma^{0} \\
& =\frac{\alpha^{2}}{2 \nabla_{0} \theta} \nabla_{\lambda} \theta g_{\mu \nu} \gamma^{0} \gamma^{\nu} \gamma^{\lambda} \gamma^{0} \\
& =\frac{1}{\nabla_{0} \theta} g_{\mu \nu} g^{\nu \lambda} \nabla_{\lambda} \theta \\
& =\nabla_{\mu} \theta\left(\nabla_{0} \theta\right)^{-1}
\end{aligned}
$$

du coup

$$
\begin{aligned}
\widetilde{f}\left(A_{0}, \phi_{0}, \widetilde{\psi}_{0}, \stackrel{\star}{\hat{\psi}_{0}}\right)_{\mu} & =\operatorname{Moy}\left(g_{\mu \nu}{ }^{t}\left(\left(_{0}^{*}\right)^{*} \gamma^{0} \gamma^{\nu} \stackrel{\star}{\psi_{0}^{+}}\right)+f\left(A_{0}, \phi_{0}, \widetilde{\psi}_{0}\right)_{\mu}\right. \\
& =\nabla_{\mu} \theta\left(\nabla_{0} \theta\right)^{-1} \operatorname{Moy}\left({ }^{t}\left(\psi_{0}^{+}\right)^{*} \rho^{\prime}(1) \psi_{0}^{+}\right)+f\left(A_{0}, \phi_{0}, \widetilde{\psi}_{0}\right)_{\mu}
\end{aligned}
$$

Toujours en utilisant $R_{\theta} \stackrel{\star}{A}{ }_{1}=\nabla^{\nu}\left(\stackrel{\star}{A_{1}}\right)_{\nu}=0$, il vient

$$
\operatorname{Moy} G_{\theta}^{I}\left({ }^{\star} \phi_{1}, \partial_{\omega} \stackrel{\star}{A} A_{1}\right)=-r^{\prime}(1) \cdot \nabla^{\nu} \theta \operatorname{Moy}\left(\partial_{\omega} \stackrel{\star}{A_{1}}\right)_{\nu} \stackrel{\star}{\phi}_{1}=0
$$

et

$$
\operatorname{Moy} G^{I I}\left({ }_{\theta}\left(\stackrel{\star}{A}_{1}, \partial_{\omega} \stackrel{\star}{\phi_{1}}\right)=-2 r^{\prime}(1) . \operatorname{Moy}\left(\stackrel{\star}{A}_{1}\right)^{\nu} \nabla_{\nu} \theta \partial_{\omega} \stackrel{\star}{\phi}_{1}=0\right.
$$

Calculons également

$$
\begin{aligned}
\left(\overline{\pi^{+}}\right) \gamma^{0} \pi^{+} & =\frac{\alpha^{4}}{4\left(\nabla_{0} \theta\right)^{2}} \nabla_{\nu} \theta \gamma^{0} \gamma^{0} \gamma^{\nu} \gamma^{0} \gamma^{0} \gamma^{\mu} \gamma^{0} \nabla_{\mu} \theta \\
& =\frac{1}{2\left(\nabla_{0} \theta\right)^{2}} g^{\mu \nu} \nabla_{\nu} \theta \nabla_{\mu} \theta \gamma^{0} \\
& =0
\end{aligned}
$$

d'où

Nous poursuivons avec

$$
{ }^{t}\left(\stackrel{\star}{\psi_{0}^{+}}\right)^{*} \gamma^{0 t} \mathbb{C}^{*} \stackrel{\star}{\psi_{0}^{+}}=0
$$

$$
\begin{aligned}
\pi^{0} F^{I}{ }_{\theta}\left(A_{0}, \partial_{\omega} \stackrel{\star}{A_{1}}\right)_{\mu}= & -2\left[\left(A_{0}\right)^{\nu}, \nabla_{\nu} \theta \partial_{\omega}\left(\stackrel{\star}{A_{1}}\right)_{\mu}\right]+\left[\left(A_{0}\right)^{\nu}, \nabla_{\mu} \theta \partial_{\omega}\left(\stackrel{\star}{A}_{1}\right)_{\nu}\right] \\
& \left.+\left[\left(A_{0}\right)_{\mu}, \nabla^{\nu} \theta \partial_{\omega}\left(\stackrel{\star}{A}_{1}\right)_{\nu}\right]\right) \\
= & -2\left[\nabla^{\nu} \theta\left(A_{0}\right)_{\nu}, \partial_{\omega} a_{1}^{0}\right]
\end{aligned}
$$

puis

$$
\begin{aligned}
\pi^{0} F^{I I}{ }_{\theta}\left(\phi_{0}, \partial_{\omega} \stackrel{\star}{\phi}_{1}\right) & =\pi^{0} T_{\theta}\left(\bar{\phi}_{0}{ }^{t} r^{\prime}(1)^{*} \partial_{\omega} \stackrel{\star}{\phi_{1}}+\phi_{0} r^{\prime}(1) \partial_{\omega} \stackrel{\star}{\phi}_{1}\right) \\
& =0
\end{aligned}
$$


de même

$$
\begin{aligned}
G^{I}{ }_{\theta}\left(\phi_{0}, \partial_{\omega} \stackrel{\star}{A_{1}}\right) & =-r^{\prime}(1) \cdot \nabla^{\nu} \theta\left(\partial_{\omega} \stackrel{\star}{A_{1}}\right)_{\nu} \stackrel{\star}{\phi_{0}}=0 \\
G^{I I}{ }_{\theta}\left(A_{0}, \partial_{\omega} \stackrel{\star}{\phi_{1}}\right) & =-2 r^{\prime}(1) \cdot \nabla^{\nu} \theta\left(A_{0}\right)_{\nu} \partial_{\omega} \stackrel{\star}{\phi_{1}}
\end{aligned}
$$

et

$$
\begin{aligned}
\mathcal{D}_{\theta} \stackrel{\star}{h}\left(A_{0}, \phi_{0}, \widetilde{\psi}_{0}, \stackrel{\star}{\psi_{0}}\right) & =-\rho^{\prime}(1) \cdot \nabla_{\nu} \theta \gamma^{\nu} \gamma^{\mu}\left(A_{0}\right)_{\mu} \stackrel{\star}{\star}_{0}^{+}+i\left(\mathbb{C} \phi_{0}+\bar{\phi}_{0}^{t} \mathbb{C}^{*}\right) \mathcal{D}_{\theta} \psi_{0}^{+} \\
& =-2 \rho^{\prime}(1) \cdot \nabla^{\nu} \theta\left(A_{0}\right)_{\nu} \psi_{0}^{+}
\end{aligned}
$$

Nommons

$$
\begin{aligned}
\mathcal{J}^{\%}= & T_{\theta} \operatorname{Moy}\left[\left(a_{1}^{0}\right)^{\nu}, \partial_{\omega}\left(a_{1}^{0}\right)_{\nu}\right] \\
& +T_{\theta} \operatorname{Moy}\left(\stackrel{\star}{\phi}_{1}{ }^{t} r^{\prime}(1)^{*} \partial_{\omega}{ }^{\star}{ }_{1}+\stackrel{\star}{\phi}_{1} r^{\prime}(1) \partial_{\omega} \stackrel{\star}{\phi_{1}}\right) \\
& +T_{\theta}\left(\nabla_{0} \theta\right)^{-1} \operatorname{Moy}\left({ }^{t}\left(\psi_{0}^{+}\right)^{*} \rho^{\prime}(1) \psi_{0}^{+}\right)
\end{aligned}
$$

le courant moyen induit par les premiers termes oscillants. Nous pouvons alors réécrire le premier système des équations de profils sous sa forme explicite :

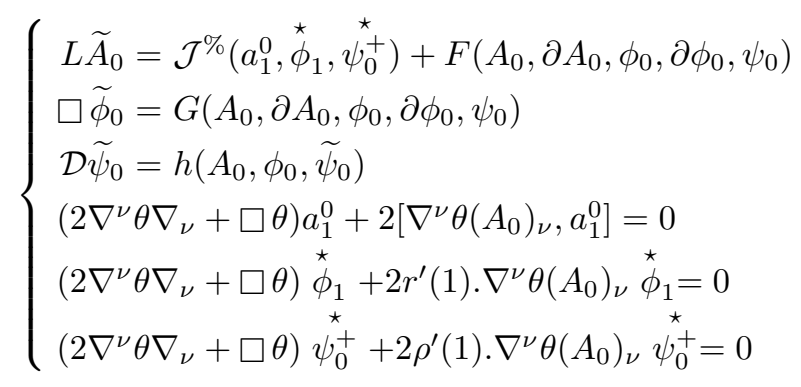

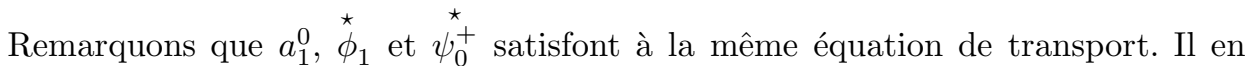
résulte que $\mathcal{J}^{\%}$ vérifie aussi une équation de transport linéaire très semblable le long des géodésiques nulles qui engendrent les surfaces de phase :

$$
\left(\nabla^{\nu} \theta \nabla_{\nu}+\square \theta\right) \mathcal{J}^{\%}+\left[\nabla^{\nu} \theta\left(A_{0}\right)_{\nu}, \mathcal{J}^{\%}\right]=0
$$

Si $\mathcal{J}_{(\mid t=0)}^{\%}=0$, la seul solution de l'équation précédente est bien entendu $\mathcal{J}^{\%}=0$ sur tout $\left[0, t_{0}\right]$. Le système $(C, 0)$ est donc découplé et les termes moyens $\left(A_{0}, \phi_{0}, \widetilde{\phi}_{0}\right)$ restent nécessairement solution du système $(Y M)$. Compte tenu de la contrainte (45) qui pèse sur les données de Cauchy de $(\mathrm{C}, 0)$, la condition $\mathcal{J}_{(\mid t=0)}^{\%}=0$ revient à imposer

$$
\left(\triangle\left(A_{0}\right)_{0}-\operatorname{div} \nabla_{0}\left(A_{0}\right)_{\Sigma}\right)_{\mid(t=0)}=(F)_{0}\left(A_{0}, \partial A_{0}, \phi_{0}, \partial \phi_{0}, \widetilde{\psi}_{0}\right)_{\mid(t=0)}
$$

qui est simplement la condition de compatibilité d'origine de $(Y M)$ pour les données non-oscillantes $\left(A_{0}, \phi_{0}, \widetilde{\psi}_{0}\right)$. 
Enfin, la condition $\mathcal{J}_{(\mid t=0)}^{\%}=0$ peut également s'interpréter comme la nullité au premier ordre de la charge moyenne engendrée par oscillations ${ }^{(2)}$. La solution oscillante que nous obtenons s'apparente alors à une perturbation linéaire d'une solution régulière donnée de système $(Y M)$. La «petitesse » de la perturbation devant la solution perturbée est donc à prendre au sens de «petitesse de la charge » moyenne induite initialement par la perturbation. L'amplitude maximale des oscillations n'est en définitive qu'un paramètre subordonné à cette condition de charge.

Nous en déduisons le résultat de stabilité a). Pour le b), si l'on suppose que $\left(A_{0}, \Phi_{0}, \psi_{0}\right)$ est solution de $(Y M)$ sur $\left[0, t_{0}\right]$, alors tous les $(C, k), 0 \leqslant k \leqslant M$ sont linéaires, et le temps d'existence de la solution approchée est $t_{0}$. Pour montrer que la solution approchée se prolonge bien en une solution exacte sur le même intervalle, il suffit de pousser le développement oscillant à l'ordre $M+2$ et de prendre $\varepsilon_{0}$ plus petit que le $\delta_{0}$ du théorème 3 .

\footnotetext{
${ }^{(2)} \mathcal{J}_{(\mid t=0)}^{\%}=0$ n'est pas une quantité définie positive, et il suffit par exemple de jouer sur la parité des coefficients de Fourrier de $\stackrel{\star}{\phi}_{1}(., \omega)$ pour garantir la nullité de l'expression. Quoi qu'il en soit, cette contrainte supplémentaire ne réduit pas notablement la généralité des données de Cauchy oscillantes admissibles.
} 


\section{APPENDICE A}

La preuve de la proposition 3.1.4 est détaillée dans [17, ch. II.2 et ch. IV.4.3], mais le cadre des décompositions diadiques utilisées diffère légèrement de celles introduites section 2.3.2. Nous la reprenons donc ici dans ses grandes lignes.

LEMme A.1. - Soit $\widetilde{\phi}_{k}$ une suite de fonctions vérifiant la condition de sommabilité $\sum_{k}\left|\widetilde{\phi}_{k}\right|^{2} \leqslant \widetilde{C}$, et telle que les fonctions $\widetilde{\Phi}_{k}(\xi)=\widetilde{\phi}_{k}\left(2^{k} \xi\right)$ sont supportées dans une couronne fixe $\left\{R^{-1} \leqslant|\xi| \leqslant R\right\}$, et sont uniformément bornées dans $C_{0}^{\infty}\left(\mathbb{R}^{n}-\{0\}\right)$. Notons $\delta_{2^{k}}(t)$ la masse de Dirac au point $t=2^{k}$ ainsi que $\widetilde{\Delta}_{k}=\widetilde{\phi}_{k}(D)$. Si $g \in \mathrm{BMO}$, alors la mesure

$$
d \mu=\sum_{k \geqslant 0}\left|\widetilde{\Delta}_{k} g(y)\right| d y \delta_{2^{k}}(t)
$$

est une mesure de Carleson, c'est-à-dire que pour tout $y_{0} \in \mathbb{R}^{n}$ et pour tout $\epsilon \geqslant 0$,

$$
\sum_{2^{-k} \geqslant \epsilon} \int_{\left|y-y_{0}\right| \leqslant \epsilon}\left|\widetilde{\Delta}_{k} g(y)\right|^{2} d y \leqslant C \epsilon^{n}|g|_{\text {BMO }}^{2}
$$

De plus, la constante $C$ ne dépend que d'un nombre fini de semi-normes des $\widetilde{\Phi}_{k}$ dans $C_{0}^{\infty}$, et de $\widetilde{C}$.

Démonstration du lemme A.1. - Nous notons Cte des constantes indépendantes des $\widetilde{\Phi}_{k}$. Pour toute boule $B=B\left(y_{0}, \epsilon\right), B^{*}$ désigne le double de $B, \chi_{B^{*}}$ la fonction indicatrice de $B^{*}, \chi^{c} B^{*}$ celle du complémentaire de $B^{*}$, et $g_{B^{*}}$ la moyenne de $g$ sur $B$. Il est alors toujours possible de décomposer $g=g_{1}+g_{2}+g_{B^{*}}$, avec $g_{1}=\left(g-g_{B^{*}}\right) \chi_{B^{*}}$ et $g_{2}=\left(g-g_{B^{*}}\right) \chi_{c^{c^{*}}}$. Puisque les $\widetilde{\phi}_{k}$ sont identiquement nulles dans un voisinage de 0 et que $g_{B^{*}}$ est une constante, il s'avère que $\widetilde{\Delta}_{k} g_{B^{*}}=0$ pour tout $k \geqslant 0$. Par conséquent $d \mu \leqslant d \mu_{1}+d \mu_{2}$, où $d \mu_{1}=\sum_{k}\left|\widetilde{\Delta}_{k} g_{1}(y)\right|^{2} d y \delta_{2^{k}}(t)$ et $d \mu_{2}=\sum_{k}\left|\widetilde{\Delta}_{k} g_{2}(y)\right|^{2} d y \delta_{2^{k}}(t)$. Nous cherchons alors à montrer que $d \mu_{1}$ et $d \mu_{2}$ sont des mesures de Carleson.

Nous avons supposé que $\sum_{k}\left|\widetilde{\phi}_{k}(\xi)\right|^{2} \leqslant \widetilde{C}$. En multipliant cette inégalité par ${\widehat{g_{1}}}^{2}(\xi)$ et en intégrant sur $\xi$, il en résulte que $\sum_{k}\left|\widetilde{\Delta}_{k} g_{1}\right|_{L^{2}}^{2} \leqslant \widetilde{C}\left|g_{1}\right|_{L^{2}}^{2}$. Nous pouvons donc 
estimer :

$$
\begin{aligned}
\sum_{2^{k} \leqslant \epsilon^{-1}} \int_{B}\left|\widetilde{\Delta}_{k} g_{1}(y)\right|^{2} d y & \leqslant \sum_{k \in \mathbb{N}}\left|\widetilde{\Delta}_{k} g_{1}\right|_{L^{2}}^{2} \\
& \leqslant \widetilde{C}\left|g_{1}\right|_{L^{2}}^{2} \\
& \leqslant \widetilde{C} \int_{B^{*}}\left|g(y)-g_{B^{*}}\right|^{2} d y \\
& \leqslant \widetilde{C}|B||g|_{\mathrm{BMO}}^{2}
\end{aligned}
$$

Nous concluons que $d \mu_{1}$ est bien une mesure de Carleson.

Notons $\widetilde{m}_{k}(y)$ et $\widetilde{M}_{k}(y)$ les transformées de Fourier inverses, respectivement, de $\widetilde{\phi}_{k}(\xi)$ et de $\widetilde{\Phi}_{k}(\xi)$. Puisque les $\widetilde{\Phi}_{k}$ sont uniformément bornées dans $C_{0}^{\infty}, \widetilde{M}_{k}$ l'est dans $\mathcal{S}$. Il existe une constante $C$ ne dépendant que d'un nombre fini de semi-normes des $\widetilde{\Phi}_{k}$ dans $C_{0}^{\infty}$, donc indépendante de $k$, telle que :

$$
\left|\widetilde{M}_{k}(y)\right| \leqslant \frac{C}{(1+|y|)^{n+1}}
$$

Comme $\widetilde{\phi}_{k}(\xi)=\widetilde{\Phi}_{k}\left(2^{-k} \xi\right)$, il vient que $\widetilde{m}_{k}(y)=2^{k n} \widetilde{M}_{k}\left(2^{k} y\right)$, et immédiatement, avec la même constante $C$,

$$
\left|\widetilde{m}_{k}(y)\right| \leqslant C \frac{2^{-k}}{\left(2^{-k}+|y|\right)^{n+1}}
$$

Si $y^{\prime} \notin B^{*}$, et si $y \in B$, alors $\left|y^{\prime}-y\right| \geqslant\left|y^{\prime}-y_{0}\right| / 2$ et $\left|y^{\prime}-y\right| \geqslant \epsilon \operatorname{donc}\left(2^{-k}+\left|y^{\prime}-y\right|\right) \geqslant$ $\left(\epsilon+\left|y^{\prime}-y_{0}\right|\right) / 3$. Nous en déduisons

$$
\begin{aligned}
\left|\widetilde{\Delta}_{k} g_{2}(y)\right|=\left|\widetilde{m}_{k} * g_{2}(y)\right| & \leqslant C \int_{y^{\prime} \in B^{*} B^{*}} \frac{2^{-k}\left|g\left(y^{\prime}\right)-g_{B^{*}}\right|}{\left(2^{-k}+\left|y^{\prime}-y\right|\right)^{n+1}} d y^{\prime} \\
& \leqslant \operatorname{Cte} C \int_{y^{\prime} \in B^{c} B^{*}} \frac{2^{-k}\left|g\left(y^{\prime}\right)-g_{B^{*}}\right|}{\left(\epsilon+\left|y^{\prime}-y_{0}\right|\right)^{n+1}} d y^{\prime} \\
& \leqslant \operatorname{Cte} C\left(\frac{2^{-k}}{\epsilon}\right) \int_{y^{\prime} \in \mathbb{R}^{n}} \frac{\epsilon\left|g\left(y^{\prime}\right)-g_{B^{*}}\right|}{\left(\epsilon+\left|y^{\prime}-y_{0}\right|\right)^{n+1}} d y^{\prime}
\end{aligned}
$$

Le fait que $g$ soit dans BMO implique ([17], ch. IV,1.1.4.)

$$
\int_{\mathbb{R}^{n}}\left|g\left(y^{\prime}\right)-g_{B(0,1)}\right|\left(1+\left|y^{\prime}\right|\right)^{-n-1} d y^{\prime} \leqslant \text { Cte }|g|_{\text {BMO }}
$$

Puisque l'espace BMO est invariant par translation et dilatation, nous en déduisons

$$
\int_{\mathbb{R}^{n}}\left|g\left(y^{\prime}\right)-g_{B\left(y_{0}, \epsilon\right)}\right| \frac{\epsilon}{\left(\epsilon+\left|y^{\prime}-y_{0}\right|\right)^{n+1}} d y^{\prime} \leqslant \text { Cte }|g|_{\text {BMO }}
$$

Il en résulte que pour tout $y \in B$ :

$$
\left|\widetilde{\Delta}_{k} g_{2}(y)\right| \leqslant \operatorname{Cte} C\left(\frac{2^{-k}}{\epsilon}\right)|g|_{\text {BMO }}
$$


En sommant, il vient :

$$
\sum_{2^{-k} \leqslant \epsilon} \int_{B}\left|\widetilde{\Delta}_{k} g_{2}(y)\right|^{2} d y \leqslant \operatorname{Cte} C|B||g|_{\mathrm{BMO}}^{2} \sum_{2^{-k} \leqslant \epsilon}\left(\frac{2^{-k}}{\epsilon}\right)^{2} \leqslant \operatorname{Cte} C|B||g|_{\mathrm{BMO}}^{2}
$$

Nous concluons que $d \mu_{2}$ est aussi une mesure de Carleson. Ceci achève la preuve du lemme A.1. Comme corollaire de cette preuve, nous obtenons le lemme 3.1.3.

Démonstration du lemme 3.1.3

a) Nous reprenons la preuve précédente, avec $\epsilon=2^{-k}, k \geqslant 0$, et $\widetilde{\Delta}_{k}$ satisfaisant aux hypothèses de la proposition A.1. La fonction $g_{B^{*}}$ est constante, donc $\widetilde{\Delta}_{k} g=$ $\widetilde{\Delta}_{k} g_{1}+\widetilde{\Delta}_{k} g_{2}$. L'inégalité (70) ci-dessus donne

$$
\left|\widetilde{\Delta}_{k} g_{2}\right| \leqslant \operatorname{Cte} C|g|_{\text {BMO }}
$$

tandis que $\left|\widetilde{\Delta}_{k} g_{1}\right|_{L_{\infty}} \leqslant C\left|\left(g-g_{B^{*}}\right) \chi_{B^{*}}\right|_{L^{\infty}}$. Or $\left|B^{*}\right|=2^{-(k-1) n} \leqslant 2^{n}$, et pour tout $y$ :

$$
\left|\left(g(y)-g_{B^{*}}\right) \chi_{B^{*}}(y)\right| \leqslant \int_{B^{*}}\left|g\left(y^{\prime}\right)-g_{B^{*}}\right| d y^{\prime} \leqslant C|g|_{\mathrm{BMO}}
$$

Il en résulte que pour tout $k \geqslant 0$,

$$
\left|\widetilde{\Delta}_{k} g\right|_{L^{\infty}} \leqslant C|g|_{\mathrm{BMO}}
$$

Si l'on pose $\widetilde{\phi}_{k}=2^{-k s}\langle\xi\rangle^{s} \phi 2^{-k \xi}, s \in \mathbb{R}$, et $\widetilde{\Delta}_{k}=\widetilde{\phi}_{k}(D)$, nous restons dans le cadre des hypothèses de la proposition A.1. Il vient $\widetilde{\Delta_{k}}\langle D\rangle^{s}=2^{k s} \widetilde{\Delta}_{k}$, et l'inégalité précédente devient

$$
\left|\widetilde{\Delta}_{k}\langle D\rangle^{s} g\right|_{L^{\infty}} \leqslant C 2^{k s}|g|_{\mathrm{BMO}}
$$

b) Nous majorons

$$
\left|S_{k}\langle D\rangle^{s} g\right|_{L^{\infty}} \leqslant\left|\Delta_{-1}\langle D\rangle^{s} g\right|_{L^{\infty}}+\sum_{j \geqslant 0}\left|\Delta_{j}\langle D\rangle^{s} g\right|_{L^{\infty}}
$$

En sommant les inégalités obtenues au a), il vient :

- si $s=0$ :

$$
\left|S_{k}\langle D\rangle^{s} g\right|_{L^{\infty}} \leqslant\left|\Delta_{-1} g\right|_{L^{\infty}}+C k|g|_{\mathrm{BMO}}
$$

- si $s<0$

$$
\left|S_{k}\langle D\rangle^{s} g\right|_{L^{\infty}} \leqslant\left|\Delta_{-1}\langle D\rangle^{s} g\right|_{L^{\infty}}+C|g|_{\mathrm{BMO}}
$$

- si $s>0$ :

$$
\left|S_{k}\langle D\rangle^{s} g\right|_{L^{\infty}} \leqslant\left|\Delta_{-1}\langle D\rangle^{s} g\right|_{L^{\infty}}+C 2^{k s}|g|_{\mathrm{BMO}}
$$

La preuve du lemme 3.1.3 est complète.

Nous notons $\mathcal{M} f(y)=\sup _{\epsilon>0} \epsilon^{-n} \int_{\left|y^{\prime}\right|<\epsilon}\left|f\left(y^{\prime}-y\right)\right| d y$ la Fonction Maximale de HardyLittlewood associée à $f$.

LEMme A.2. - Soit $\widetilde{\psi}_{k}$ une suite de fonctions telle que la famille $\widetilde{\Psi}_{k}(\xi)=\widetilde{\psi}_{k}\left(2^{k} \xi\right)$ est supportée dans une boule fixe et uniformément bornée dans $C_{0}^{\infty}\left(\mathbb{R}^{n}\right)$. Notons $\widetilde{S}_{k}=$ 
$\widetilde{\psi}_{k}(D)$. Si $\mathcal{M} f<\infty$, alors il existe une constante $C$, ne dépendant que d'un nombre fini de semi-normes des $\widetilde{\Psi}_{k}$ dans $C_{0}^{\infty}$, telle que :

$$
\sup _{\left|y^{\prime}-y\right|<2^{-k}}\left|\widetilde{S}_{k} f\left(y^{\prime}\right)\right| \leqslant C \mathcal{M} f(y)
$$

Démonstration du lemme A.2. - Nous notons Cte des constantes indépendantes des $\widetilde{\Psi}_{k}$. Nous commençons par remarquer que toute fonction $F(y)$ radiale, positive, décroissante et d'intégrale finie s'écrit comme la limite d'une somme $\sum_{i \leqslant N} c_{i} \chi_{i}$, où les $\chi_{i}$ sont les fonctions indicatrices de boules $B\left(0, R_{i}\right)$, centrées à l'origine, et les $c_{i}$ sont des constantes positives vérifiant $\lim _{N \rightarrow+\infty} \sum_{i \leqslant N} c_{i} \leqslant \int_{\mathbb{R}^{n}} F(y) d y$. Par ailleurs,

$$
\left(f * \chi_{i}\right)(y) \leqslant\left|B\left(0, R_{i}\right)\right| \mathcal{M} f(y)
$$

soit en somment sur $i \leqslant N$, puis en passant à la limite dans l'inégalité :

$$
(f * F)(y) \leqslant \mathcal{M} f(y) \cdot \int_{\mathbb{R}^{n}} F\left(y^{\prime}\right) d y^{\prime}
$$

Comme précédemment, nous notons $\widetilde{m}_{k}(y)$ et $\widetilde{M}_{k}(y)$ les transformées de Fourier inverses respectives de $\widetilde{\psi}(\xi)$ et $\widetilde{\Psi}_{k}(\xi)$. Les $\widetilde{\Psi}_{k}$ sont uniformément bornées dans $C_{0}^{\infty}$, donc les $\widetilde{M}_{k}$ le sont dans $\mathcal{S}$. Comme $\widetilde{m}_{k}(y)=2^{k n} \widetilde{M}_{k}\left(2^{k} y\right)$, il existe une constante $C$ ne dépendant que d'un nombre fini de semi-normes des $\widetilde{\Psi}_{k}$ dans $C_{0}^{\infty}$, telle que :

$$
\left|\widetilde{m}_{k}(y)\right| \leqslant C \frac{2^{-k}}{\left(2^{-k}+|y|\right)^{n+1}}=F_{k}(y)
$$

Les $F_{k}$ sont radiales, positives, décroissantes, et nous vérifions que :

$$
\int_{\mathbb{R}^{n}} F_{k}(y) d y \leqslant C\left(\int_{|y| \leqslant 2^{-k}} 2^{n k} d y+\int_{|y| \geqslant 2^{-k}} 2^{-k}|y|^{-n-1} d y\right) \leqslant \operatorname{Cte} C
$$

Le résultat ci-dessus s'applique à $F_{k} *|f|$, et

$$
\sup _{k \geqslant 0}\left(F_{k} *|f|\right)(y) \leqslant \operatorname{Cte} C \mathcal{M} f(y)
$$

Modifions légèrement les fonctions $F_{k}$ et posons

$$
\left\{\begin{array}{l}
\widetilde{F}_{k}(y)=F_{k}(0)=C 2^{n k} \text { lorsque }|y| \leqslant 2^{-k} \\
\widetilde{F}_{k}(y)=F_{k}\left(|y|-2^{-k}\right)=C 2^{-k}|y|^{-n-1} \text { si }|y| \geqslant 2^{-k}
\end{array}\right.
$$

Nous vérifions de nouveau que pour tout $k \geqslant 0$ :

$$
\int_{\mathbb{R}^{n}} \widetilde{F}_{k}(y) d y=C\left(\int_{|y| \leqslant 2^{-k}} 2^{k n} d y+\int_{\mathbb{R}^{n}} 2^{-k}|y|^{-n-1} d y\right) \leqslant \text { Cte } C
$$

Il en résulte que pour tout $\left|y-y_{0}\right| \leqslant 2^{-k}$ :

$$
\left|\widetilde{S}_{k} f(y)\right| \leqslant\left(|f| * F_{k}\right)(y) \leqslant\left(|f| * \widetilde{F}_{k}\right)\left(y_{0}\right) \mid \leqslant \operatorname{Cte} C \mathcal{M} f\left(y_{0}\right)
$$

ce qui correspond exactement à l'énoncé du lemme A.2. 
Démonstration de la proposition 3.1.4. - Il est bien connu ([17], ch.. II,2.2. th.2) que pour toute mesure de Carleson $d \mu(y, t)$ et toute fonction $F(y, t)$,

$$
\left|\int_{\mathbb{R}^{n} \times \mathbb{R}^{+}} F(x, t) d \mu(y, t)\right| \leqslant C \int_{\mathbb{R}^{n}} F^{*}(y, t) C(d \mu)(y) d y
$$

où

$$
F^{*}(y)=\sup _{\left|y^{\prime}-y\right| \leqslant t}\left|F\left(y^{\prime}, t\right)\right|
$$

et

$$
C(d \mu)(y)=\sup _{B\left(y_{0}, \epsilon\right) \ni y} \frac{1}{|B|} \int_{\left|y^{\prime}-y_{0}\right| \leqslant \epsilon-t}\left|d \mu\left(y^{\prime}, t\right)\right| d y^{\prime} d t
$$

En prenant pour mesure de Carleson $d \mu(y, t)=\sum_{k}\left|\widetilde{\Delta}_{k} g\right|^{2} d y \delta_{2^{k}}(t)$, et en appliquant le résultat à $F\left(y, 2^{k}\right)=\left|\widetilde{S}_{k} f(y)\right|^{2}$, nous obtenons

$$
\sum_{k} \int_{\mathbb{R}^{n}}\left|\widetilde{S}_{k} f\right|^{2}\left|\widetilde{\Delta}_{k} g\right|^{2} d y \leqslant C|g|_{\mathrm{BMO}}^{2} \int_{\mathbb{R}^{n}}\left|\sup _{\left|y^{\prime}-y\right| \leqslant 2^{-k}} \widetilde{S}_{k} f\left(y^{\prime}\right)\right|^{2} d y
$$

Grâce à la proposition A.2, il vient

$$
\int_{\mathbb{R}^{n}}\left|\sup _{\left|y^{\prime}-y\right| \leqslant 2^{-k}} \widetilde{S}_{k} f\left(y^{\prime}\right)\right|^{2} d y \leqslant C \int_{\mathbb{R}^{n}}|\mathcal{M} f(y)|^{2} d y \leqslant C|f|_{L^{2}}^{2}
$$

avec une constante $C$ qui ne dépend que d'un nombre fini de semi-normes des $\widetilde{\Phi}_{k}$ et des $\widetilde{\Psi}_{k}$ dans $C_{0}^{\infty}$, ainsi que de $\widetilde{C}$. La proposition 3.1.4 est prouvée. 



\section{APPENDICE B}

Nous rappelons ici un lemme de multiplication très classique, de type «GagliardoNirenberg », qui nous sert tout au long de l'article. Nous donnons la preuve à titre de comparaison avec les estimations bilinéaires des propositions 3.1.1 et 3.1.2.

Lemme B.1. - Soient $s^{\prime}, s^{\prime \prime}$ et $s$ trois réels, tels que $s<s^{\prime}+s^{\prime \prime}-\frac{n}{2}, s \leqslant \inf \left(s^{\prime}, s^{\prime \prime}\right)$ et $s^{\prime}+s^{\prime \prime} \geqslant 0$. Si $f \in H^{s^{\prime}}$ et $g \in H^{s^{\prime \prime}}$, alors le produit $f g \in H^{s}$ et il existe une constante $C>0$ telle que :

$$
|f g|_{H^{s}} \leqslant C|f|_{H^{s^{\prime}}}|g|_{H^{s^{\prime \prime}}}
$$

Démonstration. - Nous utilisons les opérateurs de décomposition diadique introduits section 2.3.2. Le produit de deux fonctions $f$ et $g$ se décompose en :

$$
f g=T_{f} g+T_{g} f+R(f, g)
$$

où

$$
T_{f} g=\sum_{k \geqslant 2} S_{k-2} f \Delta_{k} g, T_{g} f=\sum_{k \geqslant 2} S_{k-2} g \Delta_{k} f \text { et } R(f, g)=\sum_{\left|k-k^{\prime}\right| \leqslant 2} \Delta_{k^{\prime}} f \Delta_{k} g
$$

Commençons par examiner le terme $T_{g} f$ (hautes fréquences en $f$, basses fréquences en $g$ ). Le spectre de chaque terme $S_{k-2} g \Delta_{k} f$ est supporté dans une couronne $2^{k-2} \leqslant$ $|\xi| \leqslant 2^{k+2}$. Considérons une fonction test $h \in \mathcal{S}$, vérifiant $|h|_{H^{-s}} \leqslant 1$ :

$$
\begin{aligned}
& \left|\left\langle\sum_{k \geqslant 2} S_{k-2} g \Delta_{k} f, h\right\rangle\right| \leqslant C \sum_{\left|k-k^{\prime}\right| \leqslant 2}\left\langle\left|2^{k s^{\prime}} \Delta_{k} f 2^{k\left(s-s^{\prime}\right)} S_{k-2} g\right|,\left|2^{-k^{\prime} s} \Delta_{k^{\prime}} h\right|\right\rangle \\
& \leqslant C\left(\sum_{k}\left|2^{k s^{\prime}} \Delta_{k} f\right|_{L^{2}}^{2}\right)^{1 / 2}\left(\sum_{\left|k-k^{\prime}\right| \leqslant 5}\left|2^{k\left(s-s^{\prime}\right)} S_{k-2} g\right|_{L^{\infty}}^{2}\left|2^{k^{\prime} s} \Delta_{k^{\prime}} h\right|_{L^{2}}^{2}\right)^{1 / 2} \\
& \leqslant C|f|_{H^{s^{\prime}}}|h|_{H^{-s} \sup _{k}\left|2^{k\left(s-s^{\prime}\right)} S_{k} g\right|_{L^{\infty}}}
\end{aligned}
$$

Par injection de Sobolev ordinaire :

$$
\left|2^{k\left(s-s^{\prime}\right)} S_{k} g\right|_{L^{\infty}} \leqslant C\left|2^{k\left(s-s^{\prime}\right)} S_{k} g\right|_{H^{\frac{n}{2}+\epsilon}}
$$


pour tout $\epsilon>0$. D'autre part, $s-s^{\prime} \leqslant 0$ par hypothèse, et le lemme 2.3.5 c) nous donne

$$
\sup _{k}\left|2^{k\left(s-s^{\prime}\right)} S_{k} g\right|_{H^{\frac{n}{2}+\epsilon}} \leqslant C|g|_{H^{\frac{n}{2}+s-s^{\prime}+\epsilon}}
$$

Par hypothèses, $\frac{n}{2}+s-s^{\prime}<s^{\prime \prime}$, donc il existe $\epsilon>0$ tel que $\frac{n}{2}+s-s^{\prime}+\epsilon<s^{\prime \prime}$. Du coup,

Un calcul symétrique nous donne

$$
\left|T_{g} f\right|_{H^{s}} \leqslant C|f|_{H^{s^{\prime}}}|G|_{H^{s^{\prime \prime}}}
$$

$$
\left|T_{f} g\right|_{H^{s}} \leqslant C|f|_{H^{s^{\prime}}}|G|_{H^{s^{\prime \prime}}}
$$

Traitons enfin le terme de reste $R(f, g)$ (même ordre de fréquences pour $f$ et $g$ ). Le spectre de chaque terme $\Delta_{k^{\prime}} U_{\varepsilon} \Delta_{k} g$ est supporté dans une boule $|\xi| \leqslant 2^{k+4}$ lorsque $\left|k-k^{\prime}\right| \leqslant 2$. Considérons une fonction test $h \in \mathcal{S}$ vérifiant $|h|_{H^{s-\frac{n}{2}}} \leqslant 1$ :

$$
\begin{aligned}
& \left|\left\langle\sum_{\left|k-k^{\prime}\right| \leqslant 2} \Delta_{k} f \Delta_{k^{\prime}} g, h\right\rangle\right| \leqslant \sum_{\left|k-k^{\prime}\right| \leqslant 2}<\left|2^{k s^{\prime}} \Delta_{k^{\prime}} f 2^{k s^{\prime \prime}} \Delta_{k} g\right|,\left|2^{-k\left(s^{\prime}+s^{\prime \prime}\right)} S_{k+5} h\right|> \\
& \leqslant C\left(\sum_{k}\left|2^{k s^{\prime}} \Delta_{k} f\right|_{L^{2}}^{2}\right)^{1 / 2}\left(\sum_{k}\left|2^{k s^{\prime \prime}} \Delta_{k} g 2^{-(k+5)\left(s^{\prime}+s^{\prime \prime}\right)} S_{k+5} h\right|_{L^{2}}^{2}\right)^{1 / 2} \\
& \leqslant C|f|_{H^{s^{\prime}}}|g|_{H^{s^{\prime \prime}}} \sup _{k}\left|2^{-k\left(s^{\prime}+s^{\prime \prime}\right)} S_{k} h\right|_{L^{\infty}}
\end{aligned}
$$

Par injection de Sobolev ordinaire :

$$
\left|2^{-k\left(s^{\prime}+s^{\prime \prime}\right)} S_{k} h\right|_{L^{\infty}} \leqslant C\left|2^{-k\left(s^{\prime}+s^{\prime \prime}\right)} S_{k} h\right|_{H^{\frac{n}{2}+\epsilon}}
$$

pour tout $\epsilon>0$. D'autre part, $s^{\prime}+s^{\prime \prime} \geqslant 0$ par hypothèse, et le lemme 2.3.5 c) nous donne

$$
\sup _{k}\left|2^{-k\left(s^{\prime}+s^{\prime \prime}\right)} S_{k} g\right|_{H^{\frac{n}{2}+\epsilon}} \leqslant C|g|_{H^{\frac{n}{2}-s^{\prime}-s^{\prime \prime}+\epsilon}}
$$

Par hypothèses, $\frac{n}{2}-s^{\prime}-s^{\prime \prime}<-s$, donc il existe $\epsilon>0$ tel que $\frac{n}{2}-s^{\prime}-s^{\prime \prime}+\epsilon<-s$. Du coup,

Ceci achève la preuve du lemme.

$$
|R(f, g)|_{H^{s}} \leqslant C|f|_{H^{s^{\prime}}}|G|_{H^{s^{\prime \prime}}}
$$




\section{BIBLIOGRAPHIE}

[1] S. Alinhac \& P. GÉRARD - Opérateurs pseudodifférentiels et théorème de NashMoser, Savoirs Actuels, CNRS Éditions \& EDP Sciences, Paris, 1991.

[2] M. BEALS \& M. BÉZARD - «Low regularity local solutions for field equations 1-2 », Comm. Partial Differential Equations (1996), p. 79-124.

[3] Y. Choquet-Bruhat - «Ondes asymptotiques et approchées pour des systèmes d'équations aux dérivées partielles non linéaires », J. Math. Pures Appl. 48 (1969), p. 117-158.

[4] Y. Choquet-Bruhat \& D. Christodoulou - « Existence global solutions of the Yang-Mills, Higgs and spinor field in 3+1 dimensions », Ann. scient. Éc. Norm. Sup. $4^{\mathrm{e}}$ série 14 (1981), p. 581-606.

[5] Y. Choquet-Bruhat, D. Christodoulou \& M. Francaviglia - « On the wave equation in curved space-time», Ann. Inst. H. Poincaré. Phys. Théor. 31 (1979), p. 399-414.

[6] Y. Choquet-Bruhat \& A. Greco - « High frequency asymptotic solutions of Y.M. and associated fields », J. Math. Phys. 24 (1983), p. 337-379.

[7] R. Coifman \& Y. Meyer - Au delà des opérateurs pseudodifférentiels, Astérisque, vol. 57, Soc. Math. France, 1978.

[8] C. Fefferman \& E. Stein - « $H^{p}$ spaces of several variables », Acta Math. 129 (1972), p. 137-193.

[9] R. Geroch - « Spinor structure of space-times in general relativity 1-2, \& The domain of dependence », J. Math. Phys. 9,11 (1968,1970).

[10] O. Gù̀s - «Ondes multidimensionnelles $\varepsilon$-stratifiées et oscillations », Duke Math. J. 68 (1992), p. 401-446.

[11] _ _ «éveloppements asymptotiques de solutions exactes de systèmes hyperboliques quasilinéaires », Asymptotic Analysis 6 (1993), p. 635-678. 
[12] J.-L. Joly, G. MÉtivier \& J. RAUCH - « Generic rigourous asymptotic expentions for weakly nonlinear multidimentioneal oscillatory waves », Duke Math. J. 70 (1993), p. 373-404.

[13] J.-L. JoLY \& J. RAUCH - « Justification of multidimensional single phase semilinear geometric optics », Trans. Amer. Math. Soc. 330 (1992), p. 599-623.

[14] S. Klainerman \& M. Machedon - «On the M.K.G. equation with finite energy », Duke Math. J. 74 (1994), p. 19-44.

[15] _ « Finite energy solutions for Y.M. equations in $\mathrm{R}^{3+1}$ », Ann. of Math. (2) 142 (1995), p. 39-119.

[16] J.-P. NiCOLAS - «Dirac fields on asymptotically flat space-times », Preprint nº 99-22, Centre de Mathématiques, École polytechnique UMR 7640 CNRS, 1999.

[17] E. Stein - Harmonic analysis, Princeton University Press, 1993. 